
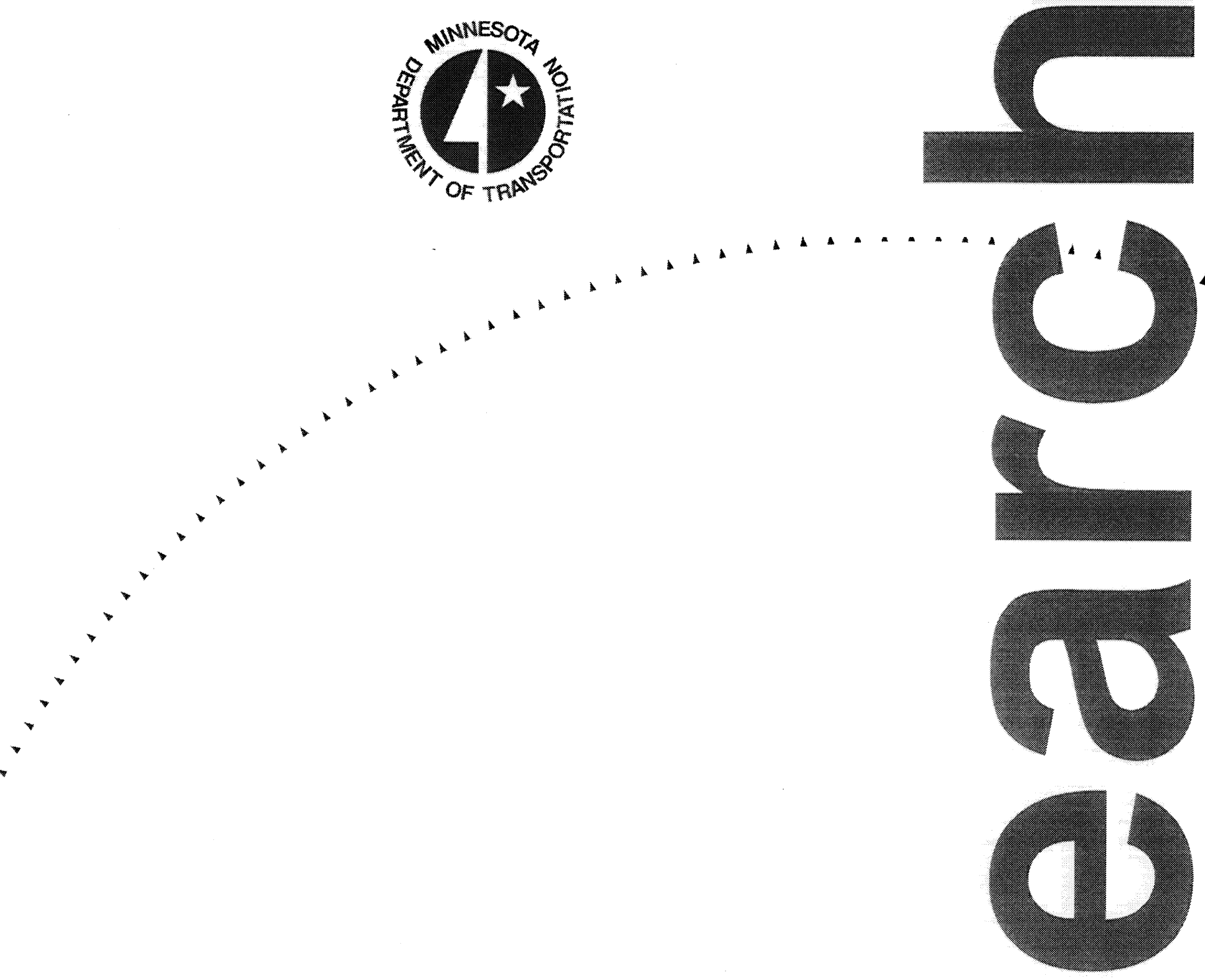

Superpave Level One Mix Design at the Local Govermment Level

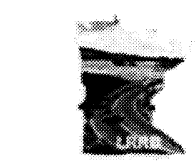

Minnesota Local

Road Research

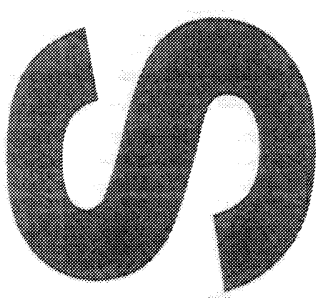

$$
\text { Board }
$$

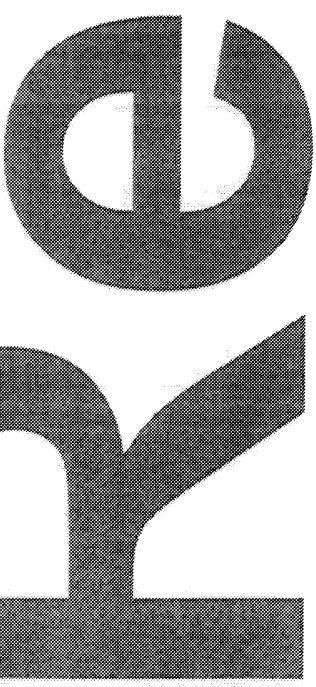




\begin{tabular}{|c|c|c|c|}
\hline $\begin{array}{l}\text { 1. Report No. } \\
\mathrm{MN} / \mathrm{RC}-2002-19\end{array}$ & 2. & \multicolumn{2}{|c|}{ 3. Recipients Accession No. } \\
\hline \multirow{2}{*}{\multicolumn{2}{|c|}{$\begin{array}{l}\text { 4. Title and Subtitle } \\
\text { SUPERPAVE LEVEL ONE MIX DESIGN AT THE LOCAL } \\
\text { GOVERNMENT LEVEL }\end{array}$}} & \multicolumn{2}{|l|}{$\begin{array}{l}\text { 5. Report Date } \\
\text { July } 2001\end{array}$} \\
\hline & & \multicolumn{2}{|l|}{6.} \\
\hline \multicolumn{2}{|c|}{$\begin{array}{l}\text { Timothy R. Clyne, Mark P. Hanson, Bruce Chadbourn, } \\
\text { Andrew Drescher and David E. Newcomb }\end{array}$} & \multicolumn{2}{|c|}{ 8. Performing Organization Report No. } \\
\hline \multirow{2}{*}{\multicolumn{2}{|c|}{$\begin{array}{l}\text { 9. Performing Organization Name and Address } \\
\text { Department of Civil Engineering } \\
\text { University of Minnesota } \\
\text { 500 Pillsbury Drive SE } \\
\text { Minneapolis, MN 55455-0116 }\end{array}$}} & \multicolumn{2}{|c|}{ 10. Project/Task/Work Unit No. } \\
\hline & & \multicolumn{2}{|c|}{$\begin{array}{l}\text { 11. Contract (C) or Grant (G) No. } \\
\text { C) } 74708 \text { wo) } 40\end{array}$} \\
\hline \multirow{3}{*}{\multicolumn{2}{|c|}{$\begin{array}{l}\text { 12. Sponsoring Organization Name and Address } \\
\text { Minnesota Department of Transportation } \\
\text { 395 John Ireland Boulevard Mail Stop } 330 \\
\text { St. Paul, Minnesota } 55155\end{array}$}} & \multicolumn{2}{|c|}{ 13. Type of Report and Period Covered } \\
\hline & & \\
\hline & & \multicolumn{2}{|c|}{ 14. Sponsoring Agency Code } \\
\hline \multicolumn{4}{|l|}{ 15. Supplementary Notes } \\
\hline \multirow{2}{*}{\multicolumn{4}{|c|}{$\begin{array}{l}\text { This report presents the results of an investigation into the use of the Superpave asphalt mix design methodology at } \\
\text { the local government level in Minnesota. } \\
\text { In the project, researchers combined low-cost natural sand with locally available aggregates from four sources: } \\
\text { limestone, quartzite, and partially crushed river gravel, and granite. They evaluated coarse and fine aggregate } \\
\text { gradations, along with the use of two asphalt grades. }\end{array}$}} \\
\hline & & & \\
\hline \multicolumn{4}{|c|}{$\begin{array}{l}\text { It was difficult to achieve the Superpave volumetric requirements of voids in mineral aggregate (VMA) and voids } \\
\text { filled with asphalt (VFA) at } 4 \text { percent air voids, regardless of the gradation. A target air void content of } 3 \text { percent } \\
\text { satisfied the VFA requirement, even though the VMA requirement could not be fulfilled. The fine aggregate } \\
\text { gradations produced densities indicating that the mixtures might be tender during construction, but not necessarily } \\
\text { be susceptible to rutting. The coarse-graded mixtures did not show the tenderness problem, but did show that they } \\
\text { might be susceptible to rutting. }\end{array}$} \\
\hline \multicolumn{4}{|c|}{$\begin{array}{l}\text { Resilient modulus testing showed little or no difference in the mixtures, regardless of aggregate source or gradation. } \\
\text { The difference in resilient modulus due to asphalt grade was apparent only at the intermediate temperatures, and not } \\
\text { at the highest or lowest test temperatures. Moisture sensitivity testing showed that all the mixtures studied had } \\
\text { adequate durability. Indirect tensile creep and APA rut testing indicated that resistance to low temperature cracking } \\
\text { and rutting may be improved by decreasing the lower PG binder grade and increasing the upper PG binder grade, } \\
\text { respectively. }\end{array}$} \\
\hline \multicolumn{2}{|l|}{ 17. Document Analysis/Descriptors } & \multirow{3}{*}{\multicolumn{2}{|c|}{$\begin{array}{l}\text { 18. Availability Statement } \\
\text { No restrictions. Document available from: } \\
\text { National Technical Information Services, } \\
\text { Springfield, Virginia } 22161\end{array}$}} \\
\hline Asphalt Mixture Design & Volumetric Asphalt Mixture Design & & \\
\hline Low-Volume Roads & Superpave & & \\
\hline 19. Security Class (this report) & 20. Security Class (this page) & 21. No. of Pages & 22. Price \\
\hline Unclassified & Unclassified & & \\
\hline
\end{tabular}




\title{
SUPERPAVE LEVEL ONE MIX DESIGN AT THE LOCAL GOVERNMENT LEVEL
}

\author{
Final Report \\ Prepared by: \\ Timothy R. Clyne \\ Mark P. Hanson \\ Bruce A. Chadbourn \\ Andrew Drescher \\ David E. Newcomb \\ University of Minnesota \\ Department of Civil Engineering \\ 500 Pillsbury Dr. SE \\ Minneapolis, MN 55455-0116 \\ July 2001 \\ Prepared for \\ Minnesota Department of Transportation
}

This report represents the results of research conducted by the authors and does not necessarily represent the views or policy of the Minnesota Department of Transportation. 


\section{ACKNOWLEDGEMENTS}

The authors gratefully acknowledge the financial and technical support provided by the Local Road Research Board and the Minnesota Department of Transportation (Mn/DOT). Alan Forsberg of Blue Earth County, and Roger Olson, John Isackson and Jim Schmidt of the $\mathrm{Mn} / \mathrm{DOT}$ Office of Materials and Road Research provided technical guidance to the project team. Koch Materials Company provided the asphalt binders and performed the rut testing, for which the project team is very thankful. Aggregates were provided by Commercial Asphalt, Meridian Aggregates, and Blue Earth County. Finally, the contribution of the undergraduate research assistants, Steve Kummer, Jennifer Matlock and Neal Dawes, was critical to the success of this project. 


\section{TABLE OF CONTENTS}

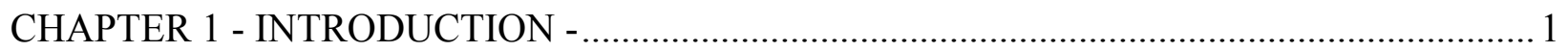

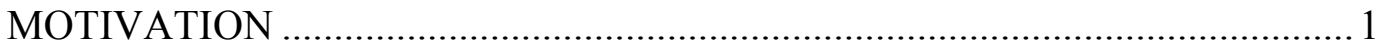

RELATIONSHIP TO PREVIOUS WORK ………............................................ 1

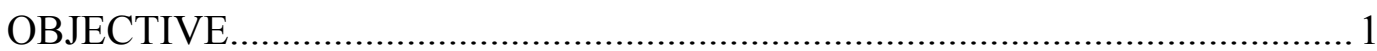

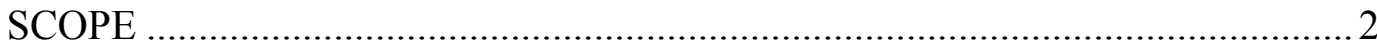

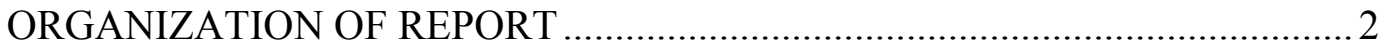

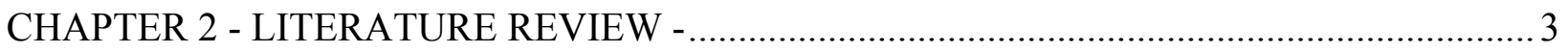

INTRODUCTION

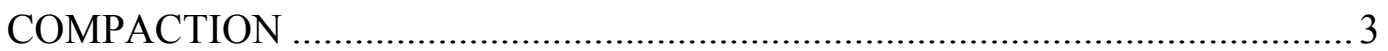

MATERIALS EVALUATION AND THEIR AFFECT ON COMPACTION .......6

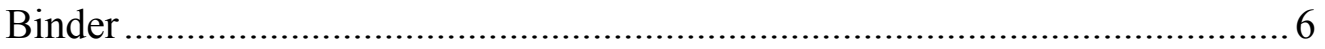

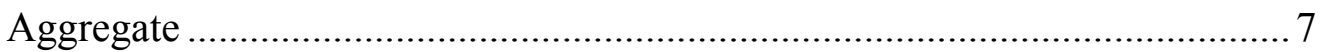

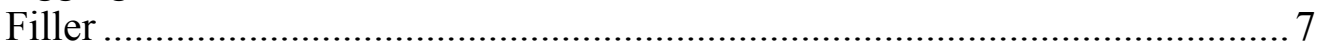

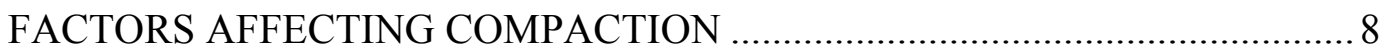

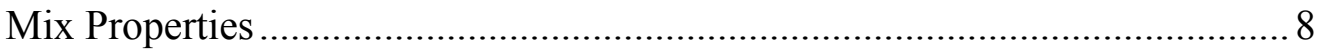

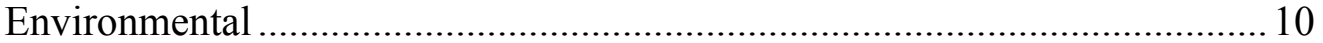

Layer (Lift) Thickness............................................................................ 10

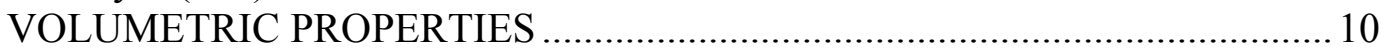

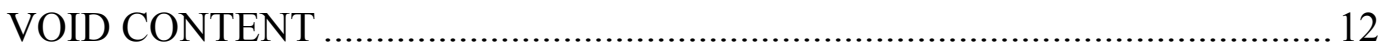

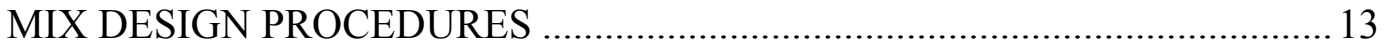

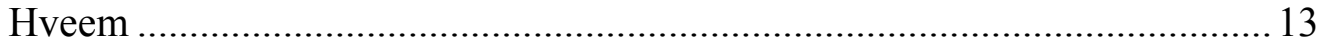

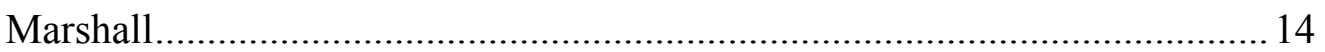

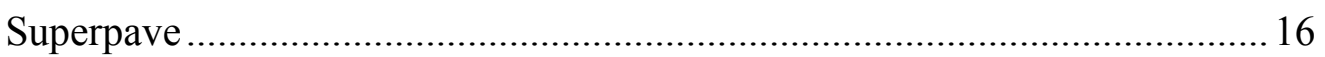

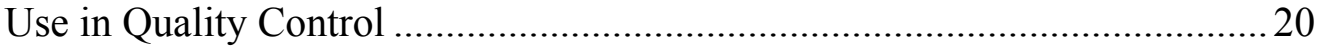

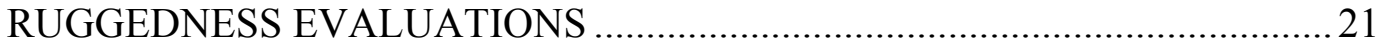

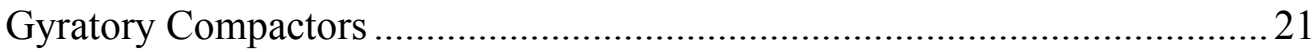

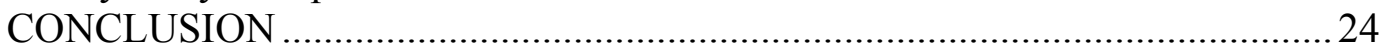

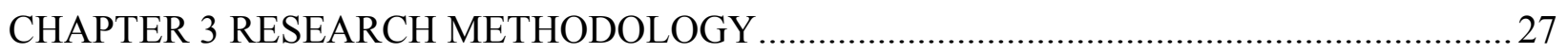

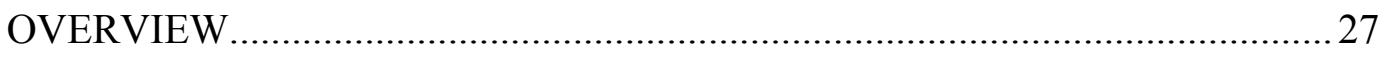

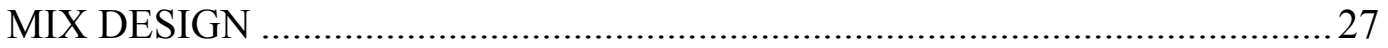

Summary of Laboratory Mixes .................................................................. 27

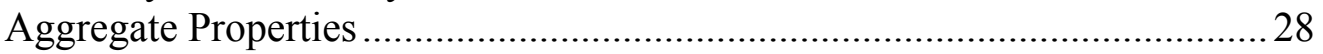

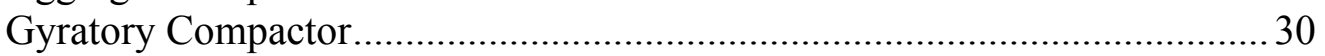

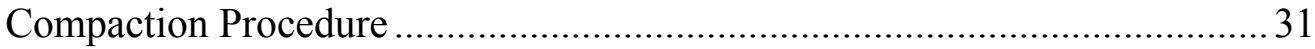

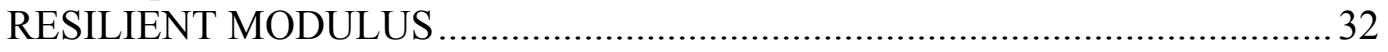

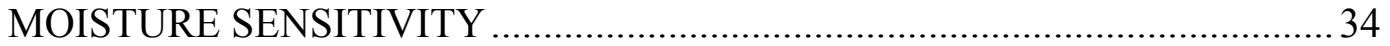

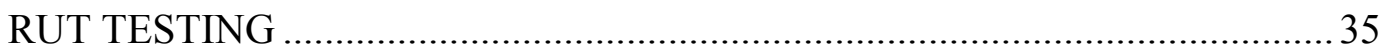

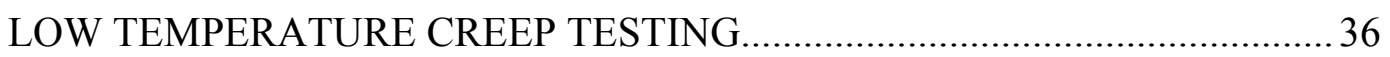

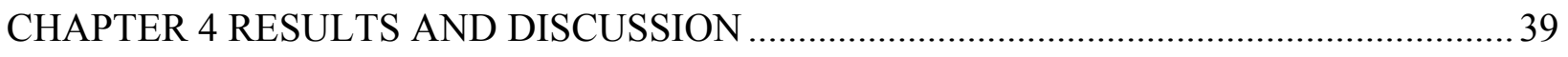

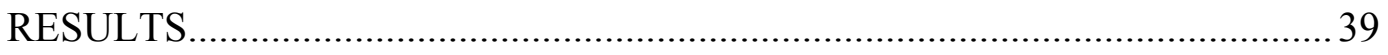

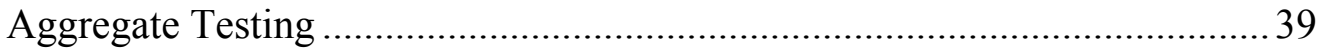




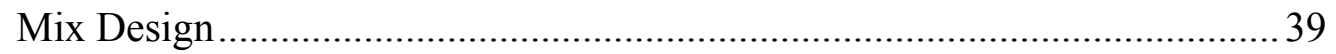

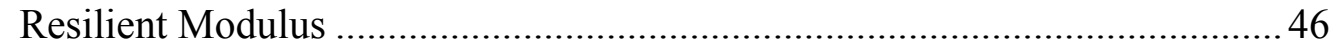

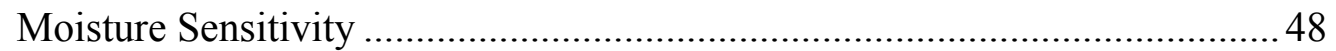

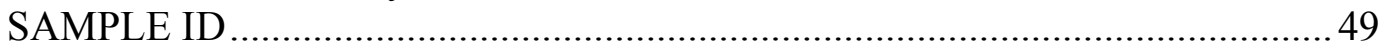

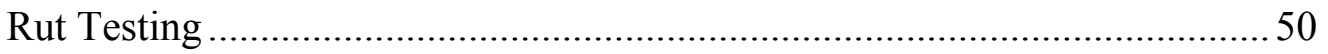

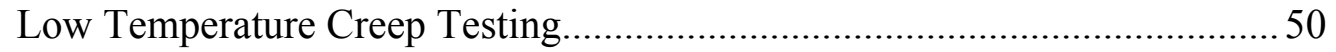

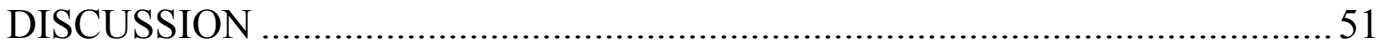

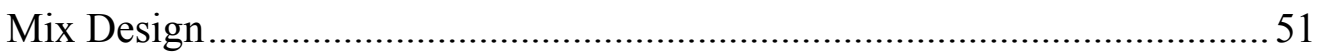

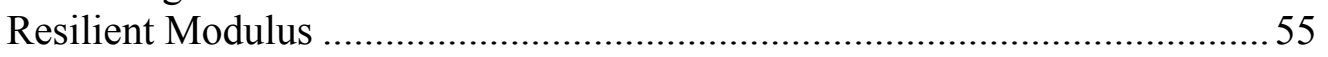

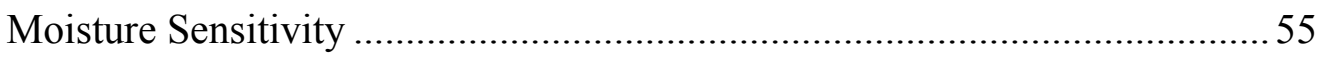

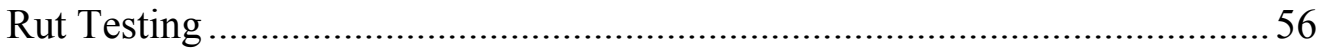

Low Temperature Creep Testing...........................................................56

CHAPTER 5 - CONCLUSIONS AND RECOMMENDATIONS - ....................................59

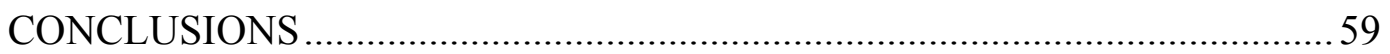

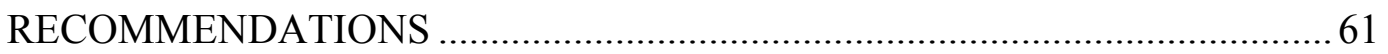

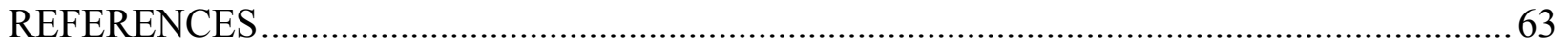

$\begin{array}{ll}\text { APPENDIX A } & \text { MIX DESIGN } \\ \text { APPENDIX B } & \text { RESILIENT MODULUS DATA } \\ \text { APPENDIX C } & \text { APA RUT TEST PROCEDURE } \\ \text { APPENDIX D } & \text { APA RUT DATA } \\ \text { APPENDIX E } & \text { IDT CREEP DATA } \\ \text { APPENDIX F } & \text { IDT STRENGTH DATA }\end{array}$




\section{LIST OF TABLES}

Table 2.1 Summary of Influence on Compaction (Asphalt Institute [15]) ............................... 11

Table 2.2 Effect of Compaction on Hot Mix Asphalt Pavements (Bell, et al [17]) ................... 12

Table 2.3 Superpave Design Gyratory Compactive Effort (Asphalt Institute [7]) ..................... 19

Table 2.4 Summary of Design and Compaction Methods (D'Angelo, et al [19]) ..................... 21

Table 2.5 Main Factors Evaluated in Ruggedness Experiment (McGennis, et al [20])...............22

Table 2.6 Variable Combinations used in Ruggedness Experiment (McGennis, et al [20]) ........ 22

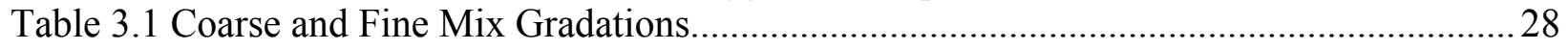

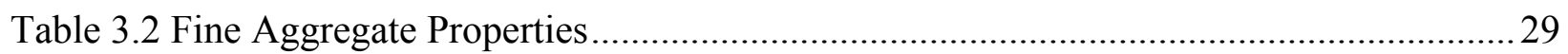

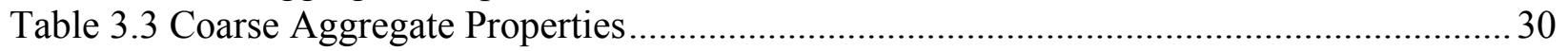

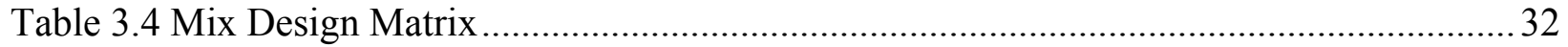

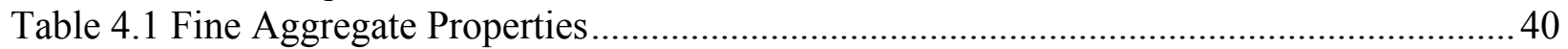

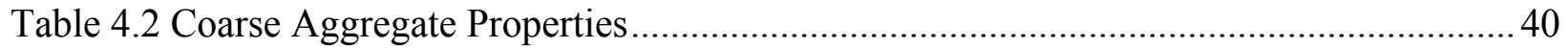

Table 4.3 Summarized Mixed Design Results @ 4\% Air Voids* .............................................. 45

Table 4.4 Summarized Mixed Design Results @ 3\% Air Voids* ............................................. 45

Table 4.5 Average Resilient Modulus Test Results ............................................................... 47

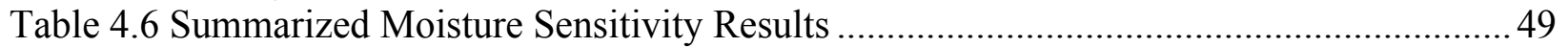

Table 4.7 Total Surface Area Calculation for Coarse Gradation ..............................................53

Table 4.8 Total Surface Area Calculation for Fine Gradation ..............................................53

Table 4.9 Asphalt Film Thickness Calculations........................................................................ 54

Table A.1 Mix Design Data for Granite Falls Coarse Gradation............................................A-1

Table A.2 Mix Design Data for Granite Falls Fine Gradation.................................................A-2

Table A.3 Mix Design Data for New Ulm Quartzite Coarse Gradation.......................................3

Table A.4 Mix Design Data for New Ulm Quartzite Fine Gradation ......................................A-4

Table A.5 Mix Design Data for Kasota Limestone Coarse Gradation........................................

Table A.6 Mix Design Data for Kasota Limestone Fine Gradation..........................................A-6

Table A.7 Mix Design Data for Cedar Grove Gravel Coarse Gradation ...................................A-7

Table A.8 Mix Design Data for Cedar Grove Gravel Fine Gradation ........................................ A-8

Table A.9 Mix Design Data for New Ulm Quartzite Fine Gradation (PG 58-40) ...................... A-9

Table A.10 Mix Design Data for Kasota Limestone Fine Gradation (PG 58-40) ...................A-10

Table B.1 Resilient Modulus Results for $0^{\circ} \mathrm{F}$ Tests ........................................................... B-1

Table B.2 Resilient Modulus Results for $34^{\circ} \mathrm{F}$ Tests ............................................................ B-3

Table B. 3 Resilient Modulus Results for $77^{\circ} \mathrm{F}$ Tests ........................................................ B-5

Table B.4 Resilient Modulus Results for $104^{\circ} \mathrm{F}$ Tests ...................................................... B-7

Table D.1 Summary of University of Minnesota APA Rut Depths (mm) ............................. D-1

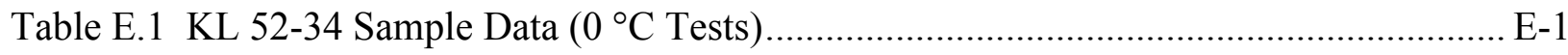

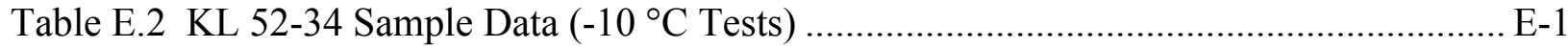

Table E.3 KL 52-34 Sample Data $\left(-20^{\circ} \mathrm{C}\right.$ Tests) ............................................................... E-1

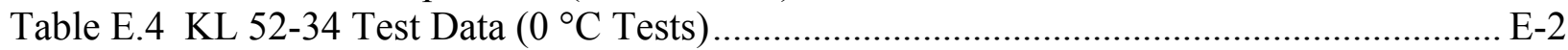

Table E.5 KL 52-34 Test Data $\left(-10^{\circ} \mathrm{C}\right.$ Tests) ................................................................... E-5

Table E.6 KL 52-34 Test Data $\left(-20^{\circ} \mathrm{C}\right.$ Tests) ................................................................. E-8 


\section{LIST OF TABLES (continued)}

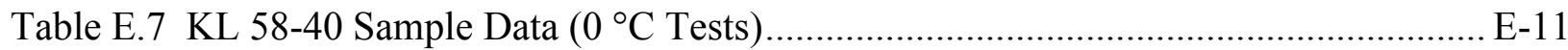

Table E.8 KL 58-40 Sample Data $\left(-10^{\circ} \mathrm{C}\right.$ Tests $)$.............................................................. E-11

Table E.9 KL 58-40 Sample Data $\left(-20^{\circ} \mathrm{C}\right.$ Tests $)$............................................................ E-11

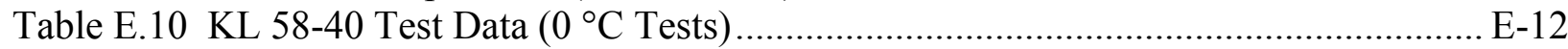

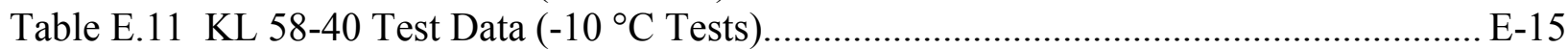

Table E.12 KL 58-40 Test Data $\left(-20^{\circ} \mathrm{C}\right.$ Tests $)$................................................................. E-18

Table E.13 NU 52-34 Sample Data $\left(0^{\circ} \mathrm{C}\right.$ Tests $)$............................................................ E-21

Table E.14 NU 52-34 Sample Data $\left(-10^{\circ} \mathrm{C}\right.$ Tests) …………........................................... E-21

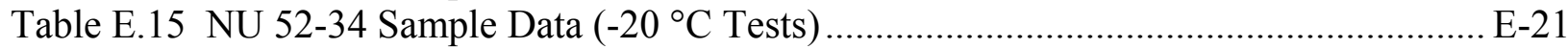

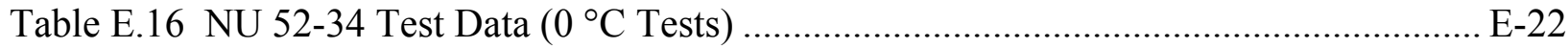

Table E.17 NU 52-34 Test Data $\left(-10^{\circ} \mathrm{C}\right.$ Tests) ................................................................... E-25

Table E.18 NU 52-34 Test Data $\left(-20^{\circ} \mathrm{C}\right.$ Tests $)$............................................................. E-28

Table E.19 NU 58-40 Sample Data $\left(0^{\circ} \mathrm{C}\right.$ Tests) ............................................................... E-31

Table E.20 NU 58-40 Sample Data $\left(-10^{\circ} \mathrm{C}\right.$ Tests) ………………………………............ E-31

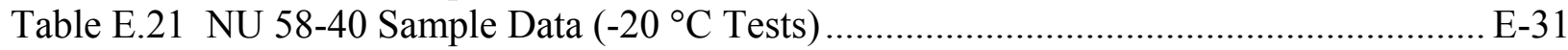

Table E.22 NU 58-40 Test Data $\left(0^{\circ} \mathrm{C}\right.$ Tests $)$.................................................................. E-32

Table E.23 NU 58-40 Test Data $\left(-10^{\circ} \mathrm{C}\right.$ Tests $)$.................................................................... E-35

Table E.24 NU 58-40 Test Data $\left(-20^{\circ} \mathrm{C}\right.$ Tests $)$.............................................................. E-38

Table F.1 IDT Specimen Geometry ………………….................................................. F-1

Table F.2 IDT Strength Data (KL 52-34 \#2) ………................................................. F-2

Table F.3 IDT Strength Data (KL 52-34 \#5) ………................................................... F-7

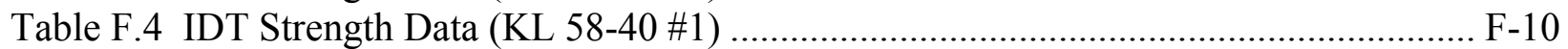

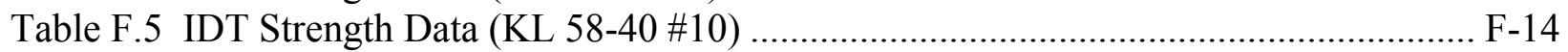

Table F.6 IDT Strength Data (KL 58-40 \#12) ............................................................... F-19

Table F.7 IDT Strength Data (KL 58-40 \#8) …………................................................. F-24

Table F.8 IDT Strength Data (NU 52-34 \#6) ................................................................. F-29

Table F.9 IDT Strength Data (NU 52-34 \#8) ........................................................... F-32

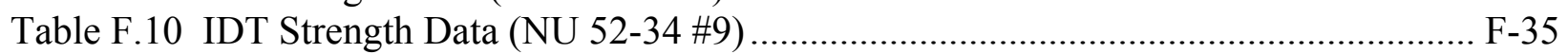

Table F.11 IDT Strength Data (NU 58-40 \#11) ................................................................ F-38

Table F.12 IDT Strength Data (NU 58-40 \#12) ............................................................. F-43

Table F.13 IDT Strength Data (NU 58-40 \#9) …………................................................ F-48 


\section{LIST OF FIGURES}

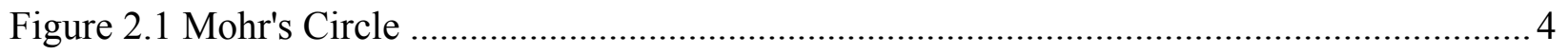

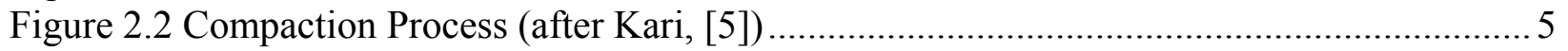

Figure 2.3 Understressed and Overstressed Conditions (after Kari,[5]) .................................. 6

Figure 2.4 Influence of Filler on Compactive Effort (after Santucci \& Schmidt [8])................... 8

Figure 2.5 Influence of Compaction Temperature on Percent Air Voids (after Parker [11]) ......... 9

Figure 3.1 Experimental Mix Gradations.............................................................................. 29

Figure 3.2 Shear Displacement During Gyratory Compaction ........................................... 31

Figure 4.1 Air Voids vs. Asphalt Content for Coarse Mixes ................................................... 40

Figure 4.2 Air Voids vs Asphalt Content for Fine Mixes ...................................................... 41

Figure 4.3 Air Voids vs Asphalt Content for PG 58-40 Mixes................................................. 41

Figure 4.4 VMA @ $\mathrm{N}_{\text {des }}$ vs. Asphalt Content for Coarse Mixes ................................................. 42

Figure 4.5 VMA @ $\mathrm{N}_{\text {des }}$ vs. Asphalt Content for Fine Mixes ..................................................... 42

Figure 4.6 VMA @ $\mathrm{N}_{\text {des }}$ vs. Asphalt Content for PG 58-40 Mixes ............................................. 43

Figure 4.7 VFA@ $\mathrm{N}_{\text {des }}$ Vs. Asphalt Content for Coarse Mixes ................................................... 43

Figure 4.8 VFA@ $\mathrm{N}_{\text {des }}$ vs. Asphalt Content for Fine Mixes....................................................... 44

Figure 4.9 VFA @ $\mathrm{N}_{\text {des }}$ vs. Asphalt Content for PG 58-40 Mixes............................................... 44

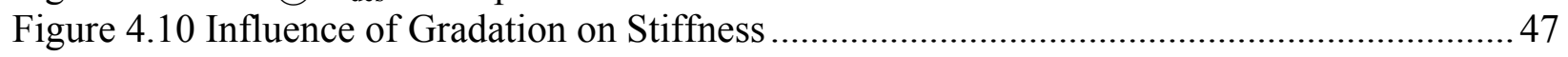

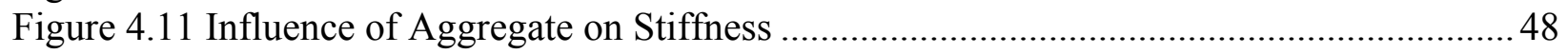

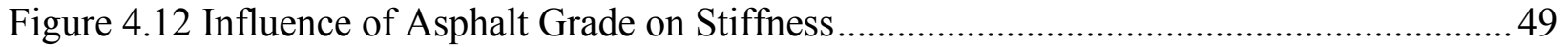




\section{EXECUTIVE SUMMARY}

This report presents the results of an investigation into the use of the Superpave asphalt mix design methodology at the local government level in Minnesota. It is commonly believed that the use of Superpave criteria will result in more costly asphalt mixtures, which may be prohibitive for cities and counties. A low-cost natural sand was combined with locally available aggregates from four sources: a limestone, a quartzite, a partially-crushed river gravel and a granite. The fine aggregate was combined with the coarse aggregates to produce coarse and fine aggregate gradations for evaluation. Additionally, the use of two asphalt grades, PG 52-34 and PG 58-40 were investigated.

The mix design effort showed that it was difficult to achieve the Superpave volumetric requirements of voids in mineral aggregate (VMA) and voids filled with asphalt at 4 percent air voids for a 12.5-mm maximum aggregate size, regardless of whether the gradation was coarse or fine. It was found that a target air void content of 3 percent allowed the VFA requirement to be satisfied, even though the VMA requirement could not be fulfilled. Compaction data showed that the fine aggregate gradations produced densities which would indicate that the mixtures might be tender during construction, but which would not necessarily be susceptible to rutting. The coarse-graded mixtures did not show the tenderness problem, but did show that they might be susceptible to rutting.

Resilient modulus testing showed little or no difference in the mixtures, regardless of aggregate source or gradation. The difference in resilient modulus due to asphalt grade was apparent only at the intermediate temperatures, and not at the highest or lowest test temperatures. Moisture sensitivity testing showed that all the mixtures studied had adequate durability. This was likely due to higher asphalt content.

Low temperature creep compliance testing indicated that mixtures made with PG 58-40 binder may be less susceptible to low temperature cracking than those made with PG 52-34 binder. Asphalt Pavement Analyzer (APA) rut testing indicated that PG 58-40 mixtures may also be less susceptible to rutting in the asphalt layer than PG 52-34 mixtures. While aggregate type had little effect on the measured rut depth in PG 58-40 mixtures, PG 52-34 limestone mixtures had almost twice the rut depth of PG 52-34 quartzite mixtures. 
For low-volume roads, it is recommended that if VMA requirements cannot be met using standard Superpave procedures, a lower design air void content of three percent be used with a fine aggregate gradation to ensure durability. Although this may result in tenderness problems, these may normally be overcome by adjustments in field compaction procedures.

In addition, decreasing the lower binder grade and increasing the upper PG binder grade may be effective in improving the low temperature cracking resistance and rut resistance of a pavement, respectively. 


\section{CHAPTER 1 \\ - INTRODUCTION -}

\section{MOTIVATION}

The Strategic Highway Research Program (SHRP) Superpave asphalt mixture design system, with its stringent material requirements was specifically developed to produce pavements to meet the expectations of the American public. The Superpave system includes a performance graded asphalt binder system, criteria for aggregate properties, a new mix design procedure using the Superpave gyratory compactor, and refined procedures and requirements for mixture analysis. To date, the Superpave system has primarily been used on medium to high volume roadways, where funding is more readily available. Issues concerning aggregate availability and local economy have limited its use on low-volume roads at the local government level. Therefore, the question is: Can the material and testing requirements of the Superpave system be economically applied at the local government level without compromising pavement performance? To answer the above question, the two key requirements of the Superpave system, material requirements and testing requirements, were evaluated.

\section{RELATIONSHIP TO PREVIOUS WORK}

The Superpave system has existed since 1987 when it was developed by the Strategic Highway Research Program (Asphalt Institute, 1996). It has been used with varying degrees of success by several State Departments of Transportation on high-volume roads such as urban interstates. Due to its high material and construction costs relative to other mix design systems, however, its use at the local government level has yet to be widely accepted. The results of this research presents a slightly modified Superpave system for use at the local level for low-volume roadways in the state of Minnesota.

\section{OBJECTIVE}

The intent of this research was to investigate the feasibility of using the Superpave Level One mix design system at the local government level for use on low-volume roadways in the state of Minnesota. The effects of different gradations, aggregate sources, and asphalt performance grades were evaluated. 


\section{SCOPE}

Two different aggregate gradations were evaluated: coarse and fine. The fine aggregate (passing the $9.5 \mathrm{~mm}$ (3/8 in.) sieve) in all mixes was composed solely of a sand from Lakeland, Minnesota - a very readily available, low-cost aggregate. Four different coarse aggregates (retained on the $12.5 \mathrm{~mm}(1 / 2 \mathrm{in}$.$) and 9.5 \mathrm{~mm}$ (3/8 in.) sieves) were evaluated: Granite Falls granite, New Ulm quartzite, Kasota limestone, and Cedar Grove gravel—all readily available at varying costs. Lastly, the effects of two different asphalt performance grades (PG 52-34 and PG 58-40) were evaluated. A Brovold gyratory compactor was used to prepare all samples.

\section{ORGANIZATION OF REPORT}

This report is arranged into five sections: Introduction, Literature Review, Research Methodology, Results and Discussion, and Conclusions and Recommendations. The Literature Review provides a background on the mechanics of asphalt compaction, the importance of volumetrics, and current mix design methods - with special emphasis on the Superpave method. Research Methodology discusses the aggregate properties, laboratory mixtures, gyratory compactor, compaction procedure, test methods and data analysis methods. Results and Discussion presents the results of the mix design, resilient modulus tests, moisture sensitivity tests, low-temperature behavior and rutting characteristics and discusses their significance. The report closes with some final conclusions and recommendations. Literature sources used as supporting references are cited in the bibliography and additional summarized test data are provided in the appendices. 


\section{CHAPTER 2 \\ - LITERATURE REVIEW -}

\section{INTRODUCTION}

The purpose of compaction is to densify an asphalt pavement. Resistance to shear deformation cannot be developed without close contact of the aggregate particles in the mix. The close contact of the particles allows the development of interparticle friction necessary to resist displacement of the mix under load. Likewise, the development of a high degree of impermeability results only when a well-designed and manufactured mix is thoroughly compacted. It has been conclusively shown that the durability of the pavement is directly related to permeability - the amount of air and water passing through the mix. Exposure to air may cause oxidation of the asphalt leading to premature hardening of the pavement and a susceptibility to cracking and stripping. According to Marker [1], unless the compactive effort has placed the particles close enough together, the tensile strength of a mix cannot be developed by the cohesiveness of the asphalt films coating each particle. Simply stated, a tough, durable, smooth pavement can only be accomplished with proper compaction.

\section{COMPACTION}

A pavement's resistance to shear is a function of the cohesive, internal friction and confining forces within the asphalt mix. These forces are best illustrated by Mohr's Circle (Figure 2.1), where Coulomb's equation is used to calculate shear strength:

$$
\tau=c+\sigma \tan \phi
$$

Where:

$\tau=$ shear stress

$c=$ cohesion

$\sigma=$ confining pressure

$\phi=$ angle of internal friction

The basic principles of asphalt compaction are similar to soil compaction. Sowers [2] lists three ways that soil compaction can occur: reorientation of particles; fracture of the bonds between them (followed by reorientation); and distortion of the particles and their adsorbed layers. Cohesive soil densification is primarily the result of distortion and particle reorientation. The 
fracturing and reorientation of particles enables densification of cohesionless soils such as crushed rock. The internal friction between particles, however, resists particle reorientation. Therefore, increasing aggregate angularity increases the material's resistance to densification.

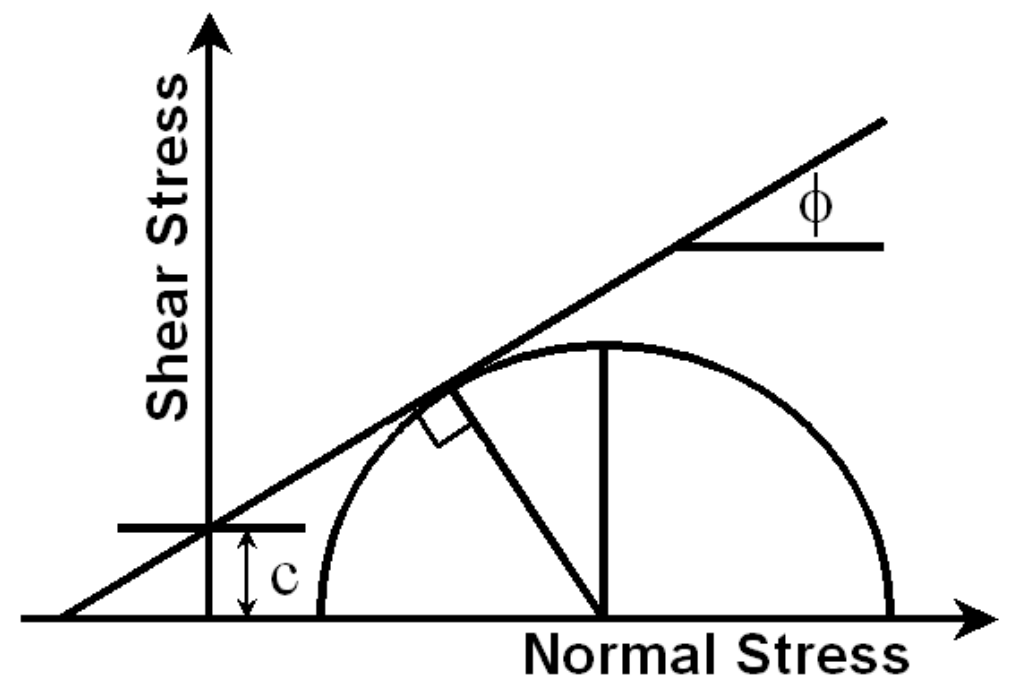

Figure 2.1 Mohr's Circle

The moisture content of soils, similar to asphalt content in hot mix asphalt, plays an important role in densification. In cohesive materials, the interparticle cohesive forces decrease as moisture content increases. Increasing moisture content in cohesionless materials cause the capillary tension between particle grains to decrease. Sowers [2] stated that the resulting decrease in interparticle contact pressures decreases the internal friction of the soil. Increases in moisture contents to optimum levels prior to compaction results in the most effective compactive effort.

Another important element of effective compaction is lateral confinement of the material. In the laboratory, confinement of the mixture is achieved via the mold. In the field, the flow properties of the material must enable adequate resistance to lateral flow. Geller [3] demonstrated that without lateral flow confinement, vertical compression cannot take place. The confining ability of pneumatic (rubber-tire) rollers makes them ideal for compacting tender mixtures.

Nijboer [4] explained the three primary forces resisting compaction within hot mix asphalt:

1. the angle of internal friction (frictional resistance)

2. the initial resistance (cohesive and interlocking resistances) 
3. the viscous resistance (viscosity of the mix times rate of flow)

The first resistance, angle of internal friction, is primarily a function of the aggregate properties. The second, initial resistance, is a function of the bitumen and filler properties acting as a thin film coating the aggregate, the interlocking action of the particle shapes comes into effect toward the completion of the compaction process. Viscous resistance is a function of both aggregate and binder properties.

Kari [5] explained how in the field, the rolling of a hot asphalt concrete pavement provides a means of applying vertical pressure and kneading action to a mix enabling densification to occur. The conditions existing under a moving roller are shown in Figure 2.2. The roller wheel or tires sink into the asphalt mix until the contact area is large enough to reduce the contact pressure of the wheel to approximately that of the mix's bearing capacity. The roller wheel's motion creates shear forces within the asphalt. The horizontal shear forces developed in the front and rear of the roller create zones of decompaction within the pavement. The vertical shear forces developed directly underneath the roller wheel create a zone of compaction.

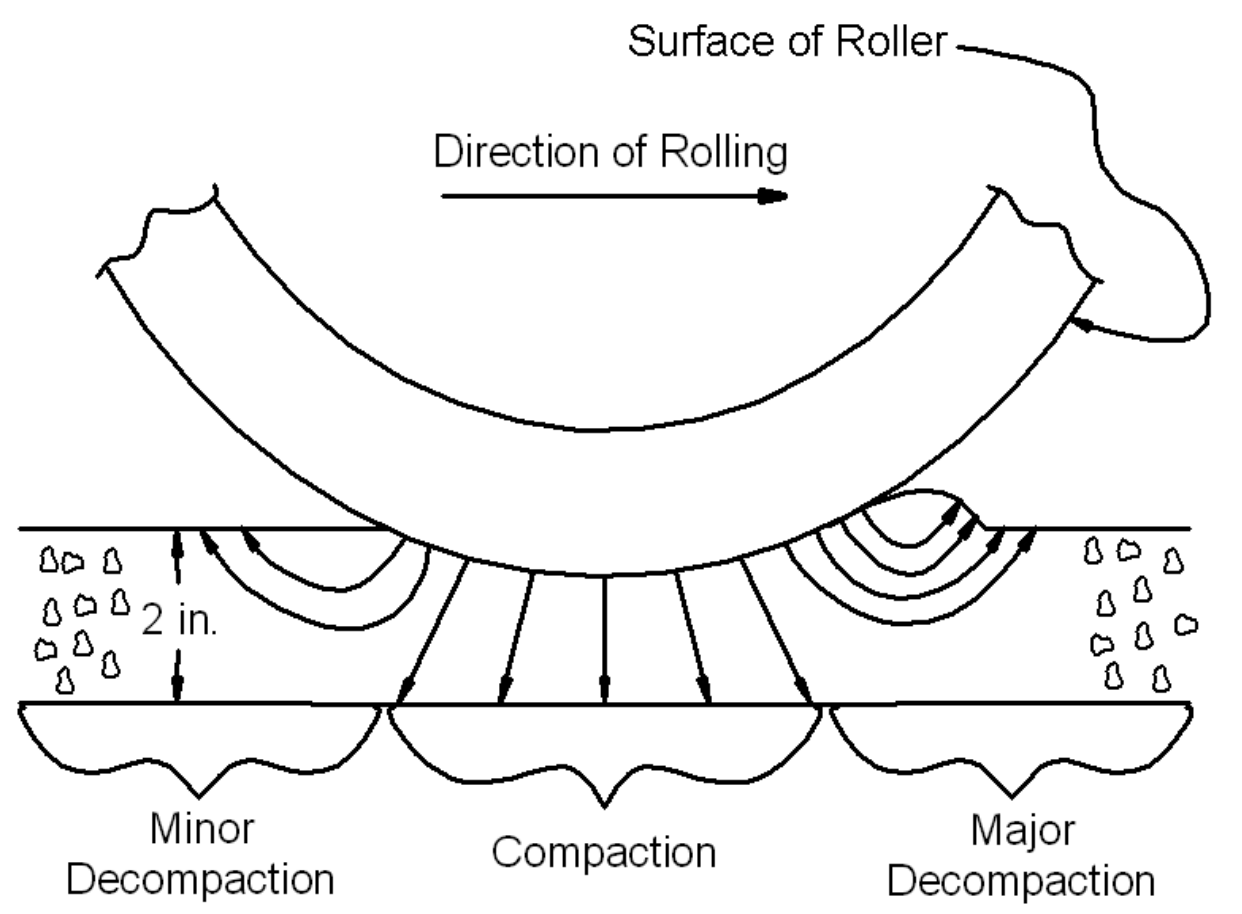

Figure 2.2 Compaction Process (after Kari, [5])

Kari [5] described two unfavorable compaction conditions: understressed and overstressed. A mix is understressed when the bearing capacity of the mixture is greater than the contact pressure 
applied by the roller - the roller simply rides on top of the mix without any compaction taking place (Figure 2.3). A mix is overstressed when it cannot support the weight of the roller-the roller sinks deep into the mix resulting in shoving and severe cracking but little to no densification. Thus, the bearing capacity of the mix and the roller weight and configuration must complement each other to achieve maximum density and toughness.
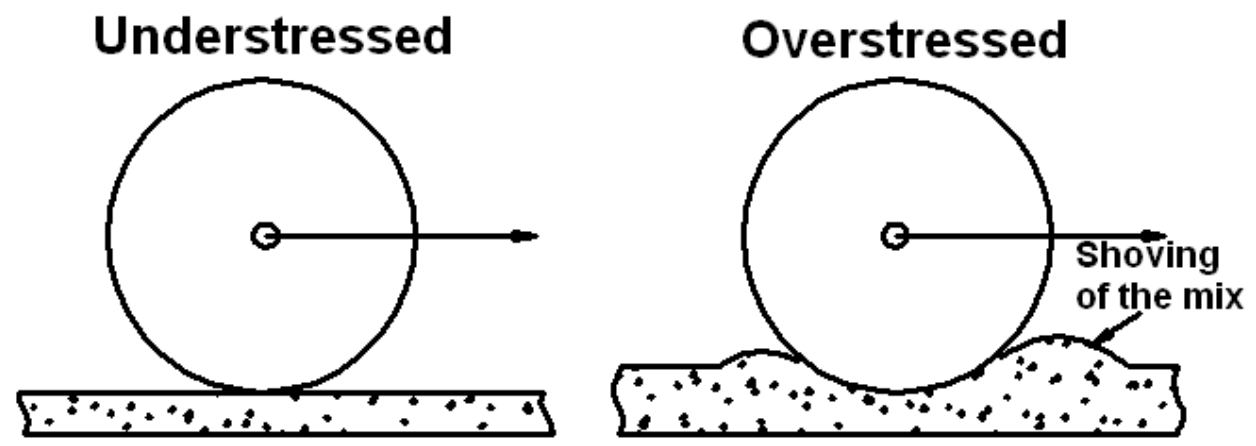

Figure 2.3 Understressed and Overstressed Conditions (after Kari,[5]) MATERIALS EVALUATION AND THEIR AFFECT ON COMPACTION

\section{Binder}

The asphalt binder is considered a viscoelastic material because it exhibits properties of both a viscous and an elastic material. As such, the asphalt binder affects compaction in a variety of ways. The viscosity of asphalt is extremely temperature dependent: at room temperature asphalt is virtually a solid, above $121^{\circ} \mathrm{C}$ it is a fluid. Increasing the temperature of a mixture decreases the binder's viscosity causing a reduction in the overall stiffness of the mix. If a mix is too hot, it will be tender and move laterally from underneath the roller. Conversely, as the mix cools, it stiffens, requiring a greater compactive effort to densify it.

The influence of the binder on an asphalt mixture's resistance to compaction was shown in a study by McLeod [6]. A high viscosity asphalt cement at a typical placement temperature of $135^{\circ} \mathrm{C}$ has a viscosity of approximately 5 poise. The viscosity of the same asphalt cement at $63{ }^{\circ} \mathrm{C}$, when rolling often ends, was 5000 poise - a 1000-fold increase. The respective Marshall stabilities of the mix at the two temperatures were $667 \mathrm{~N}$ and $6672 \mathrm{~N}$ - a 10-fold increase. In only a $72{ }^{\circ} \mathrm{C}$ temperature difference, a 1000 -fold increase in the binder's viscosity resulted in a 10 -fold increase in the mix's strength. 


\section{Aggregate}

Gradation, surface texture and angularity are the primary aggregate characteristics affecting the workability and resistance to compaction of a mix. Larger aggregate sizes and/or higher coarse aggregate percentages result in lower workability and higher compactive efforts. Likewise, a rough surface texture, as opposed to a smooth, glassy texture, results in a stiffer, less workable mix. Using highly angular coarse and fine aggregate results in a high degree of internal friction (and thus, high shear strength), increasing the resistance of the mix to permanent deformation. According to the Asphalt Institute [7], limiting the percentage of elongated particles minimizes the potential for aggregate crushing during mixing and construction.

If workability is too low, rounded sands are often added to increase the mix's workability. However, too much rounded sand results in tender mixes - mixes with high workability but low stability. Tender mixes are often easily overstressed by heavy rollers and over-rolling resulting in the lateral movement of the mix from under the roller.

\section{Filler}

Fines, or filler content, affect the compactibility of a mix because they combine with the asphalt cement to provide the binding, cohesive forces of the mix. Filler material increases the effective viscosity of the binder matrix, effectively creating a mastic.

Santucci and Schmidt [8] explained that there exists an optimum filler content for maximum compaction (Figure 2.4). A study by Bissada [9] showed higher filler contents resulted in higher stiffness values achieved at lower resistances to compaction. Additionally, filler will help offset the tenderness of mixes with too much sand. However, too much filler results in "gummy" mixes that are difficult to compact. 


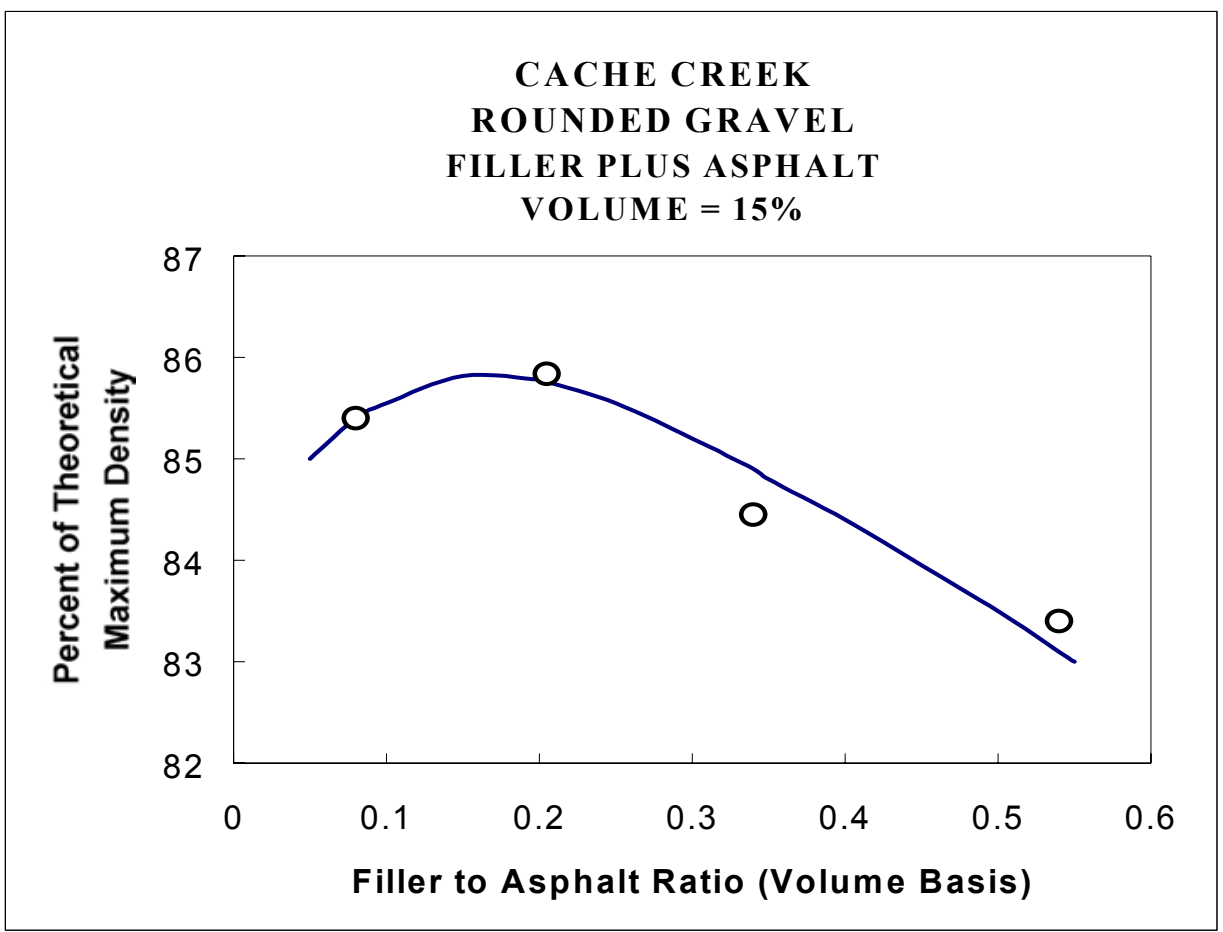

Figure 2.4 Influence of Filler on Compactive Effort (after Santucci \& Schmidt [8]) FACTORS AFFECTING COMPACTION

\section{Mix Properties}

At higher temperatures, the lower viscosity of the asphalt cement causes it to act as a liquid, allowing the aggregate particles to effectively interlock. At slightly lower temperatures, the binder acts as a lubricant permitting the aggregate to shift and densify during compaction. Further reduction in temperature results in a stiffening of the binder where its cohesion will prevent further densification.

As the asphalt content increases, so does the film thickness of the asphalt on the aggregate. At compaction temperatures, the thicker films increase the lubricating effect of the asphalt. Additionally, a study by Harvey and Tsai [10] showed pavement overlay life increased 10 to 20 percent with each $0.5 \%$ increase in asphalt content (when compacted to the same air void content) with respect to fatigue. If asphalt contents are excessive, however, the resulting tender mix will bleed. 
The temperature of the mix affects the compaction process in much the same way as asphalt content. As previously discussed, the workability of the mix increases as the temperature of the mix increases. The upper limit for mix temperature is approximately $150^{\circ} \mathrm{C}\left(300^{\circ} \mathrm{F}\right)$; temperatures above $150{ }^{\circ} \mathrm{C}$ may result in damage to the asphalt by accelerated hardening. The lower limit for effective compaction is approximately $85^{\circ} \mathrm{C}\left(185^{\circ} \mathrm{F}\right)$; below which great compactive effort is required for little to no gain in densification of the mix. Figure 2.5 shows the effect of compaction temperature on void content using a Marshall compactor at 50-blows per side. Parker's [11] work showed compaction at $150^{\circ} \mathrm{C}\left(300^{\circ} \mathrm{F}\right)$ yielded an air void content four times greater than compaction at $135^{\circ} \mathrm{C}\left(275^{\circ} \mathrm{F}\right)$.

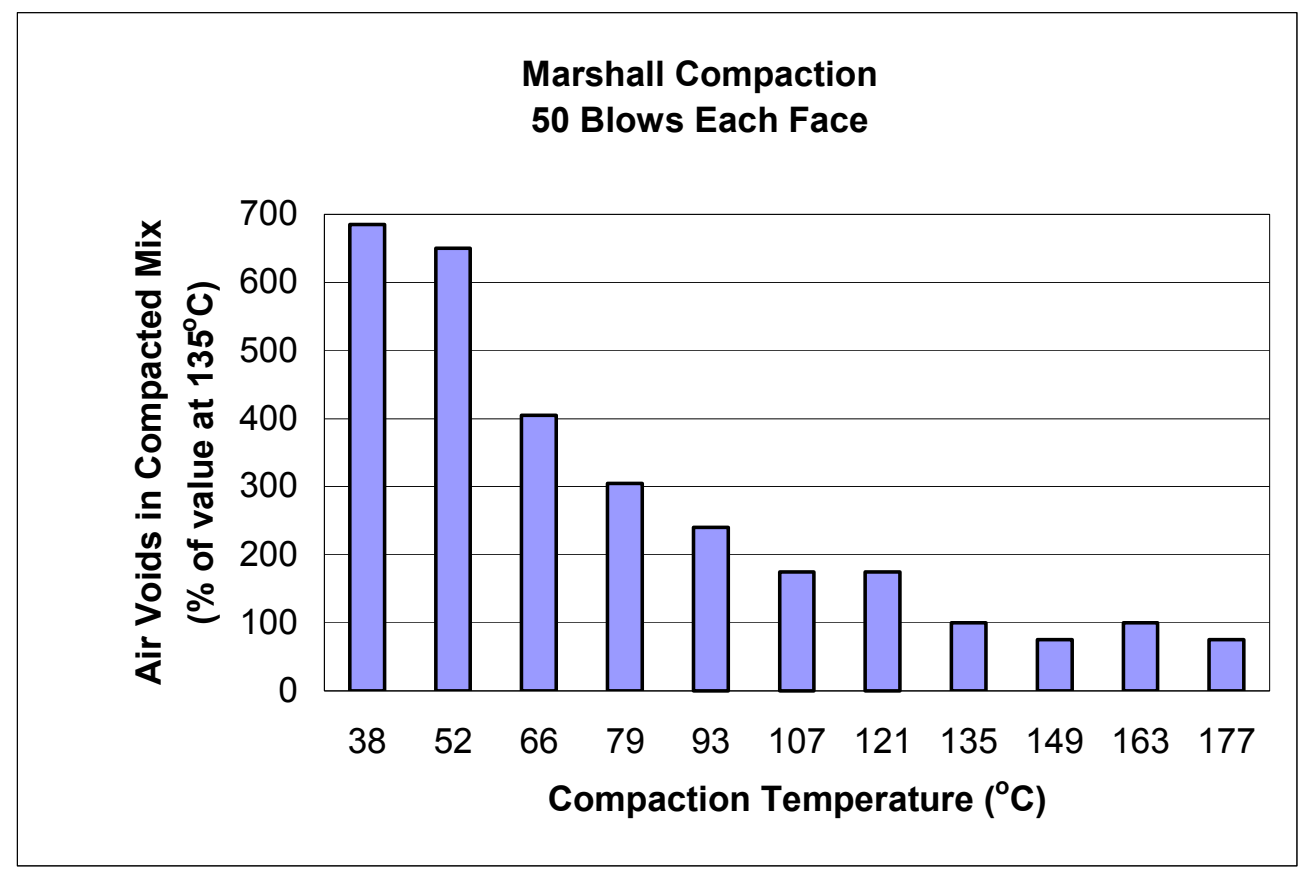

Figure 2.5 Influence of Compaction Temperature on Percent Air Voids (after Parker [11])

A study by Kennedy, et al [12] showed that low temperatures during compaction have an adverse effect on the engineering properties such as tensile strength, resilient modulus and Marshal stability of an asphalt concrete resulting in reduced pavement performance. Hadley, et al [13] found that of seven factors studied (aggregate type, aggregate gradation, asphalt cement, asphalt content, mixing temperature, compaction temperature, and curing temperature), compaction temperature dominated the results. Kennedy, et al concluded tensile strength, static and resilient moduli, Marshall stability, and Hveem stability of asphalt are all reduced when compaction occurs at lower temperatures. Brown [14] reported that temperatures in excess of $155^{\circ} \mathrm{C}$ 
$\left(310^{\circ} \mathrm{F}\right)$, however, can cause compaction problems (lateral movement) and increase oxidation of the binder which can result in hard and brittle pavements.

\section{Environmental}

According to the Asphalt Institute [15], the rate at which an asphalt mix cools directly affects the length of time during which density can and must be achieved. The ambient air temperature, humidity, wind velocity and the surface temperature under the mix all affect the rate of cooling of a freshly placed asphalt layer. Cool air temperatures, high humidity, strong winds, and cool surfaces either alone or together adversely shrink the effective compaction window.

\section{Layer (Lift) Thickness}

Thicker asphalt layers retain heat longer. Therefore, less compactive effort is required to achieve target density in thicker asphalt layers than in thinner layers. The heat-retaining ability of larger lifts make their use desirable when placing stiffer mixes or when paving in adverse environmental conditions. The Asphalt Institute [15] stated that retained heat of thicker lifts also permits lower paving temperatures, so either lower mixing temperatures or longer hauling distances are possible. A summary of the factors affecting compaction and their corrective actions can be found in Table 2.1 .

\section{VOLUMETRIC PROPERTIES}

Characterization of asphalt mixtures generally consists of several volumetric properties including voids in the total mix (VTM), voids in the mineral aggregate (VMA) and voids filled with asphalt (VFA). The most important property in construction is VTM, or air voids, a direct relation to density. A mix having $4 \%$ air voids has a density of $96 \%$ of maximum. Research and past performance have shown a final compacted void content of $4 \%$ is ideal for most densegraded mixtures. Generally, mixes having low compacted air voids (less than 3\%) will be unstable and exhibit premature rutting. Mixes having high air voids (greater than $8 \%$ ) will generally be permeable to water leading to an increased oxidation rate resulting in premature raveling and/or cracking. It is important to note, however, that these figures are nothing more than generalizations. It is quite possible to have an outstanding, long-lasting pavement that compacted to $98 \%$ density ( $2 \%$ air voids). 
Table 2.1 Summary of Influence on Compaction (Asphalt Institute [15])

\begin{tabular}{|c|c|c|}
\hline ITEM & EFFECT & CORRECTIONS* \\
\hline \multicolumn{3}{|c|}{ Aggregate } \\
\hline Smooth Surfaced & Low interparticle friction & \begin{tabular}{|l|} 
Use light rollers \\
Lower mix temperature
\end{tabular} \\
\hline Rough Surfaced & High interparticle friction & Use heavy rollers \\
\hline Unsound & Breaks under steel-wheeled rollers & $\begin{array}{l}\text { Use sound aggregate } \\
\text { Use pneumatic rollers }\end{array}$ \\
\hline Absorptive & Dries mix—difficult to compact & Increase asphalt in mix \\
\hline \multicolumn{3}{|c|}{ Asphalt } \\
\hline Viscosity-High & Particle movement restricted & $\begin{array}{l}\text { Use heavy rollers } \\
\text { Increase temperature }\end{array}$ \\
\hline Viscosity-Low & $\begin{array}{l}\text { Particles move easily during } \\
\text { compaction }\end{array}$ & \begin{tabular}{|l|} 
Use light rollers \\
Decrease temperature
\end{tabular} \\
\hline Quantity-High & Unstable and plastic under roller & Decrease asphalt in mix \\
\hline Quantity-Low & $\begin{array}{l}\text { Reduced lubrication-difficult } \\
\text { compaction }\end{array}$ & $\begin{array}{l}\text { Increase asphalt in mix } \\
\text { Use heavy rollers }\end{array}$ \\
\hline \multicolumn{3}{|c|}{ Mix } \\
\hline Excess Coarse Aggregate & Harsh mix—difficult to compact & Use heavy rollers \\
\hline Oversanded & Too workable_-difficult to compact & $\begin{array}{l}\text { Reduce sand in mix } \\
\text { Use light rollers } \\
\end{array}$ \\
\hline Too Much Filler & Stiffens mix-difficult to compact & $\begin{array}{l}\text { Reduce filler in mix } \\
\text { Use heavy rollers }\end{array}$ \\
\hline Too Little Filler & Low cohesion-mix may come apart & Increase filler in mix \\
\hline \multicolumn{3}{|c|}{ Mix Temperature } \\
\hline High & $\begin{array}{l}\text { Difficult to compact-mix lacks } \\
\text { cohesion }\end{array}$ & Decrease mixing temperature \\
\hline Low & Difficult to compact—mix too stiff & Increase mixing temperature \\
\hline \multicolumn{3}{|l|}{ Coarse Thickness } \\
\hline Thick Lifts & Hold heat-more time to compact & Roll normally \\
\hline Thin Lifts & Lose heat—less time to compact & $\begin{array}{l}\text { Roll before mix cools } \\
\text { Increase mix temperature }\end{array}$ \\
\hline \multicolumn{3}{|c|}{ Weather Conditions } \\
\hline Low Air Temperature & Cools mix rapidly & $\begin{array}{l}\text { Roll before mix cools } \\
\text { Increase mix temperature } \\
\text { Increase lift thickness }\end{array}$ \\
\hline Low Surface Temperature & Cools mix rapidly & $\begin{array}{l}\text { Roll before mix cools } \\
\text { Increase mix temperature } \\
\text { Increase lift thickness }\end{array}$ \\
\hline Wind & Cools mix-crusts surface & $\begin{array}{l}\text { Roll before mix cools } \\
\text { Increase mix temperature } \\
\text { Increase lift thickness }\end{array}$ \\
\hline
\end{tabular}


VMA, the void space in the aggregate, and probably the most important volumetric property in design, is primarily a function of aggregate gradation, particle shape and surface texture. Since VMA includes air voids (it is the sum of VTM and VFA), low VMA indicates low film coating on the aggregate because there is not enough void space for the asphalt to adequately coat the particles without overfilling the void space. Since overfilling the void space is the same as having a low VTM, premature and excessive rutting can result.

Although of great importance in volumetric proportioning, as the difference between VTM and VMA, VFA is typically not mentioned in volumetric discussions. However, for the purposes of generalities, typical VFA values as reported by Roberts, et al [16], range from 50-70\%. When VFA exceeds $80-85 \%$, the voids are considered overfilled (with asphalt) resulting in the low stability problems explained above.

\section{VOID CONTENT}

A study by Bell, et al [17], showed that percent compaction (or void content) was the most significant factor affecting mix performance. As shown in Table 2.2, an increase in void content is associated with a decrease in modulus, fatigue life, and resistance to permanent deformation.

Table 2.2 Effect of Compaction on Hot Mix Asphalt Pavements (Bell, et al [17])

\begin{tabular}{|c|c|c|c|c|c|c|}
\cline { 4 - 7 } \multicolumn{2}{c|}{} & \multicolumn{4}{c|}{$10^{7}$ ESAL Normal Design Life } \\
\hline $\begin{array}{c}\text { Compaction } \\
\text { Rating }\end{array}$ & $\begin{array}{c}\text { Voids } \\
\text { Content, } \\
\%\end{array}$ & $\begin{array}{c}\text { Resilient } \\
\text { Modulus, } \\
\text { MPa }\end{array}$ & $\begin{array}{c}\text { Horizontal } \\
\text { Strain at } \\
\text { ACC Bottom }\end{array}$ & $\begin{array}{c}\text { Est. Fatigue } \\
\text { Life, \# Loads to } \\
\text { Fail, } 10^{6}\end{array}$ & $\begin{array}{c}\text { Vertical Strain } \\
\text { at Subgrade } \\
\text { Surface }\end{array}$ & $\begin{array}{c}\text { Est. Perm } \\
\text { Deform Life, \# } \\
\text { Loads to Fail, } 10^{6}\end{array}$ \\
\hline Excellent & 4 & 3370 & 75 & 110 & 200 & 48 \\
\hline Good & 8 & 2060 & 100 & 12 & 245 & 21 \\
\hline Poor & 12 & 1430 & 120 & 2.6 & 280 & 12 \\
\hline
\end{tabular}

High stiffness (resilient modulus) values are essential to long-lasting, superior performing hot mix asphalt pavements. The stiffness of a pavement is directly related to the resulting horizontal and vertical strains in the pavement resulting from vehicle loads. Pavements with higher stiffness values exhibit lower strains under the same vehicle loads. Horizontal and vertical strains are important in predicting pavement performance because they directly correlate to fatigue and permanent. The accurate estimation of fundamental engineering properties by the 
consistent simulation of field compaction is key to a laboratory compaction method's (Hveem, Marshall, gyratory) value in the prediction of long-term pavement performance.

The Asphalt Institute found that changes in stiffness and void content affected fatigue life according to the following expression:

$$
N_{f}=18.4(C) \times\left(4.32 \times 10^{-3} \varepsilon_{t}^{-3.29} E^{-0.854}\right)
$$

Where:

$N_{f}=$ number of load applications to failure

$C=$ a factor dependent on the asphalt and void contents

$\varepsilon_{t}=$ tensile strain

$E=$ modulus of asphalt mixture

Bell, et al [17] used the above equation to calculate the fatigue values shown in Table 2.2. The table clearly shows the profound effect void content has on fatigue life. A 50\% reduction in void content from eight to four percent air voids results in nearly a 10-fold increase in fatigue life.

Permanent deformation of flexible pavement may be due to either densification or shear deformation. Densification, or further compaction by traffic, can be reduced by ensuring good compaction during construction. Shear deformation occurs when one or more pavement layers lack bearing capacity. Vertical pressure in unstable layers can be reduced by using stiffer mixes resulting from better compaction. Bell et al [17] found a two-fold increase in the estimated permanent deformation lives of a pavement when reducing the void content from eight to four percent (see Table 2.2).

\section{DESIGN PROCEDURES}

\section{Hveem}

The basic philosophy of the Hveem method of mix design is summarized by Roberts et al [16]:

1. It should provide sufficient asphalt cement for aggregate absorption and to produce an optimum film content of asphalt cement on the aggregate.

2. It should produce a compacted aggregate-asphalt cement mixture with sufficient stability to resist traffic.

3. It should contain enough asphalt cement for durability from weathering including the effects of oxidation and moisture susceptibility. 
In short, the Hveem method of mix design attempts to maximize durability by selecting the highest asphalt content while still exceeding the minimum stability requirements. The Hveem method has two primary advantages. First, the kneading action of laboratory densification (achieved by a rotating ram having about $1 / 4$ the contact area of the $101.6 \mathrm{~mm}$ (4") diameter mold) simulates the densification characteristics of hot mix asphalt compacted in the field. Second, Hveem stability is a direct measurement of the internal friction component of shear strength because it measures the ability of a test specimen to resist lateral displacement from application of a vertical load. However, the Hveem compactor is somewhat expensive, large, and not very portable. Furthermore, the important mixture volumetric properties described above are not routinely determined as part of the Hveem procedure.

The Hveem method uses the oil soak and Centrifuge Kerosene Equivalent (CKE) tests to aid in determining fine and coarse aggregate absorption for use in estimating the initial asphalt requirements of the mix. Once the initial asphalt content (IAC) is determined, test specimens are prepared containing the IAC, $0.5 \%$ and $1.0 \%$ above the IAC and $0.5 \%$ below the IAC. Compacted samples are put through stabilometer and cohesiometer tests to measure stiffness, a swell test to measure the mix's resistance to moisture, and a density-voids analysis. The optimum asphalt content is determined via a convoluted process involving a highly complex chart requiring several inputs and correction factors.

\section{Marshall}

A primary advantage of the Marshall method is the attention given to density and voids properties of asphalt mixtures. This analysis ensures the proper volumetric proportions of mixture materials for achieving a durable hot mix asphalt. Additionally, the required equipment is inexpensive and portable thereby lending itself to quality control operations. The Marshall hammer used in the Marshall method is repeatedly dropped onto a sample a prescribed number of times dependent on the estimated traffic level. However, without a kneading action imparting the horizontal shear forces created by rollers, the Marshall hammer does not simulate mixture densification as it occurs in the field. Furthermore, as explained by Brown, et al [18], the high variability of results and limited ability to simulate field conditions (temperature, load rate, tire pressures, etc.) of Marshall stability does not adequately estimate the shear strength of hot mix 
asphalt. The Asphalt Institute [7] stated that these two situations make it difficult to ensure rutting resistance of the designed mixture.

The two principle features of the Marshall method of mix design are a density-voids analysis and a stability-flow test of the compacted test specimens. The stability of the test specimens is the maximum load resistance in Newtons (lb.) that the standard test specimen will develop at $60{ }^{\circ} \mathrm{C}$ $\left(140^{\circ} \mathrm{F}\right)$. The flow value is the total movement or strain, in units of $0.25 \mathrm{~mm}(1 / 100 \mathrm{in}$.), occurring in the specimen between no load and maximum load during the stability. After determination of an optimum asphalt content, the density-voids analyses and the stability-flow tests are completed on five sets of three samples containing the optimum content, and $0.5 \%$ and $1.0 \%$ above and below optimum.

In the Marshall method, the mix is compacted using a $101.6 \mathrm{~mm}$ (4 in.) diameter by $75 \mathrm{~mm}(3$ in.) high mold and a $4.5 \mathrm{~kg}$ (10 lb.) compaction hammer constructed to obtain a $457 \mathrm{~mm}(18 \mathrm{in}$.) drop height. Depending on design traffic load, the weight is dropped from its $457 \mathrm{~mm}$ height 35 times (light traffic), 50 times (medium traffic) or 75 times (heavy traffic). The mold is inverted and the same number of blows are repeated.

Brown [14] found that the advent of the mechanical Marshall hammer actually decreased the effectiveness of Marshall compaction in simulating field compaction. Prior to the advent of mechanical compactors, the top of the hammer was held with one hand while the hammer was raised and dropped with the other. The inability to keep the hammer perfectly vertical resulted in a kneading action. Guides on mechanical hammers reduce the kneading action resulting in substantially different laboratory densities. The Marshall compactor is effective in achieving densification from grain fracturing and particle layer distortion but without a kneading action, densification through particle reorientation is minimal. On examining density-voids relationships of airfield pavements in Kuwait, Bissada [9] found that even the 75-blow Marshall compaction effort was inadequate as a realistic standard for predicting future densification under traffic. The characteristics of Marshall compacted specimens were not necessarily representative of their lifetime service performance. 


\section{Superpave}

The Strategic Highway Research Program (SHRP) spent five years developing a new mix design methodology, named Superior Performing Pavements, or Superpave. Roberts, et al [16] described several differences between Superpave and the Marshall and Hveem methods: it uses a new "Performance Grade" system for grading asphalt cement; it uses consensus properties for aggregate selection; and it contains new mix design and mixture analysis procedures. The Asphalt Institute [8] described Superpave as a performance-based system because the mixture tests and analyses have direct relationships to field performance.

Traditional grading of asphalts, such as penetration or viscosity graded asphalts, were based on physical properties at standard temperatures. However, such grading systems have two important shortcomings. First, their empirical nature limits their applicability beyond those conditions in which it was developed. A second limitation of previous grading systems is the lack of performance testing over the same temperature range the asphalt will likely see in the field. Superpave's performance graded asphalt system differs from previous grading systems in that the tests measure physical properties that can be directly related to field performance by engineering principles. Another unique feature of the Superpave binder specification system is that instead of performing a test at a constant temperature and varying the specified value, the specified value is constant and the temperature at which this value must be achieved is varied. The result is an identification system comprised of two numbers: the high temperature grade and the low temperature. For example, a PG 52-34 asphalt binder must posses adequate physical properties at the high temperature, $52^{\circ} \mathrm{C}\left(126^{\circ} \mathrm{F}\right)$, and at the low temperature, $-34^{\circ} \mathrm{C}\left(-29^{\circ} \mathrm{F}\right)$.

Another new feature of the Superpave mix design system is the concept of a restricted zone in the aggregate gradation. Brown, et al [14] described the purpose of the restricted zone as a way to help ensure that too much rounded, natural sand is not used in the mixture and to help ensure that the minimum VMA requirement is achieved. For blends with nominal sizes $25 \mathrm{~mm}(1 \mathrm{in}$.) and greater, the restricted zone boundaries are placed on the $4.75 \mathrm{~mm}$ (No. 4), $2.36 \mathrm{~mm}$ (No. 8), $1.18 \mathrm{~mm}$ (No. 16), $0.60 \mathrm{~mm}$ (No. 30), and $0.30 \mathrm{~mm}$ (No. 50) sieve sizes. For blends with nominal maximum aggregate sizes of $19 \mathrm{~mm}$ (3/4 in.) and less, the $4.75 \mathrm{~mm}$ (No. 4) sieve limits are omitted. It is important to note, however, that the restricted zone is just a guide. It is possible to use aggregate blends that pass through the restricted zone that still function 
satisfactorily. The Superpave system also uses upper and lower control points on the $0.075 \mathrm{~mm}$ (No. 200), $2.36 \mathrm{~mm}$ (No. 8), and the nominal sieve size of the blend. Additionally, a lower control limit is placed on the sieve size one size lower than the nominal size. Control and restricted zone limits for all nominal maximum aggregate sizes can be found in sources such as the Asphalt Institute's Superpave Series No. 2 (SP-2) manual [7].

Recognizing the importance of volumetric proportioning, Superpave incorporated aggregate criteria directly into its design procedures. Superpave has two forms of aggregate criteria: consensus properties (aggregate angularity, flat and elongated particles, and sand equivalent or clay content) and source properties (toughness, soundness and deleterious materials). Following is the rationale behind determining the aggregate properties and the test procedures used to determine the properties as given by the SP-2 manual. A complete listing of the minimum required values for the following consensus property tests can be found in the SP-2 manual.

Fine aggregate angularity (FAA) testing is done to ensure a high degree of internal friction and rutting resistance. FAA is defined by the percent of air voids in loosely compacted aggregate smaller than the $2.36 \mathrm{~mm}$ (No. 8) sieve. The procedure for FAA testing is outlined in Amercian Association of State Highway and Transportation Officials (AASHTO) TP 33, "Test Method for Uncompacted Void Content of Fine Aggregate (as Influenced by Particle Shape, Surface Texture, \& Grading)" (ASTM C1252). A sample of fine washed aggregate is poured into a small, calibrated cylinder through a standard funnel. By measuring the mass of fine aggregate in the filled cylinder of known volume, the void content can be calculated as the difference between the cylinder volume and fine aggregate volume collected in the cylinder. Superpave requires a minimum FAA value of 40 for use in mixes placed less than $100 \mathrm{~mm}$ (4 in.) from the surface.

Coarse aggregate angularity (fractured faces), ensures a high degree of aggregate internal friction and rutting resistance. It is defined as the percent by weight of aggregate larger than the 4.75 $\mathrm{mm}$ (No. 4) sieve with one or more fractured faces. The procedure for determining coarse aggregate angularity is given in ASTM D5821-95, "Determining the Percentage of Fractured Particles in Coarse Aggregate." The value is typically expressed as the percent with one or more fractured faces over the percent with two or more fractured faces. Superpave minimum CAA 
requirements range from 55/(unspecified) for low volume roads to $100 / 100$ for high volume roads.

Flat and elongated coarse aggregate particles are undesirable because they have a tendency to break during construction and under traffic. The fracturing of aggregate is a concern because it can reduce mixture stability and in extreme situations may actually make the gradation finer affecting the optimum asphalt content. The flat and elongated coarse aggregate property is expressed as the percentage by mass of coarse aggregate having a maximum to minimum dimension ratio greater than five to one. It is determined according to ASTM D4791, "Flat or Elongated Particles in Coarse Aggregate" on particles larger than $4.75 \mathrm{~mm}$ (0.187 in.). Superpave does not limit the percent of flat and elongated particles for low-volume mix designs, but limits their use to ten percent for all other design levels.

The sand equivalency test is a measure of the clay content in the fraction of the fine aggregate smaller than the $4.75 \mathrm{~mm}$ (No. 4) sieve. Sand equivalency is determined by the method in AASHTO T176, "Plastic Fines in Graded Aggregates and Soils by Use of the Sand Equivalent Test" (ASTM D2419). In the sand equivalency test, a sample of fine aggregate is mixed with a flocculating solution in a graduated cylinder and agitated to loosen the clayey fines and force them into suspension above the granular aggregate. After a settling period, the cylinder heights of the suspended clay and settled sand are measured. The sand equivalent value is the ratio of sand to clay height readings. Minimum Superpave requirements range from 40 percent for low volume roads to 50 percent for high volume.

Samples are compacted in the Superpave gyratory compactor (SGC) and the asphalt content is selected on the basis of volumetric design requirements (the goal being $4 \%$ air voids). In its SP-2 manual, the Asphalt Institute [7] identifies several goals of the SHRP efforts in designing the SGC:

- Realistically compact mix specimens to densities achieved under actual pavement climate and loading conditions.

- Accommodate large aggregate sizes.

- Measure compactibility so potential tender mix behavior could be identified.

- Portable enough for use in mixing facility quality control operations. 
The loading ram on an SGC produces a constant $600 \mathrm{kPa}(87 \mathrm{psi})$ vertical compaction pressure on a sample contained in a 150-mm (6 in.) diameter mold (which can accommodate mixes having up to $50-\mathrm{mm}$ ( 2 in.) maximum size aggregate). The base of the SGC rotates at a constant rate of 30 revolutions per minute with the mold positioned at a $1.25^{\circ}$ compaction angle. Density can be estimated at any time during the compaction process because the position of the ram is continually recorded as it compacts the specimen (the mass of the mix inside the mold and the mold's diameter are constant). The $1.25^{\circ}$ compaction angle coupled with the revolving base enables the SGC to impart a kneading action on the specimen much like it would undergo in the field. The kneading action enables further densification of the specimen through rearrangement of the aggregate particles.

Superpave mixes are designed at a specific level of compactive effort - the number of gyrations necessary, called $\mathrm{N}$-design $\left(\mathrm{N}_{\mathrm{des}}\right)$, to compact the mixture to $4 \%$ air voids. $\mathrm{N}_{\text {des }}$ is a function of climate and traffic levels. Climate is represented by the average design high air temperature and traffic level is represented by the design ESALs (equivalent $80 \mathrm{kN}(18,000 \mathrm{lb}$.) single axle loads). The range of values for $\mathrm{N}_{\text {des }}$ is show in Table 2.3. The two other values shown in Table 2.3, $\mathrm{N}$-initial $\left(\mathrm{N}_{\text {ini }}\right)$ and $\mathrm{N}$-maximum $\left(\mathrm{N}_{\max }\right.$, also play important roles in the Superpave design process. $\mathrm{N}_{\text {ini }}$ is considered a measure of mixture compactibility. At $\mathrm{N}_{\text {ini }}$ gyrations the density of the sample must be greater than $89 \%$ of the maximum density. Mixes that compact too quickly (less than $11 \%$ air voids at $\mathrm{N}_{\text {ini }}$ ) will probably be tender and unstable. $\mathrm{N}_{\max }$ is a possible measure of a mix's susceptibility to rutting. The density at Nmax must be less than $98 \%$. Mixes that compact to greater than $98 \%$ air voids at Nmax may exhibit premature or excessive rutting.

Table 2.3 Superpave Design Gyratory Compactive Effort (Asphalt Institute [7])

\begin{tabular}{|c|c|c|c|c|c|c|c|c|c|c|c|c|}
\hline \multirow{3}{*}{$\begin{array}{c}\text { Design } \\
\text { ESALs } \\
\text { (millions) }\end{array}$} & \multicolumn{12}{|c|}{ Average Design High Air Temperature } \\
\hline & \multicolumn{3}{|c|}{$<39^{\circ} \mathrm{C}$} & \multicolumn{3}{|c|}{$39-40^{\circ} \mathrm{C}$} & \multicolumn{3}{|c|}{$41-42^{\circ} \mathrm{C}$} & \multicolumn{3}{|c|}{$43-44^{\circ} \mathrm{C}$} \\
\hline & $\underline{N_{\text {ini }}}$ & $\underline{N_{\text {des }}}$ & $\underline{\mathrm{N}_{\text {max }}}$ & $\underline{N_{\text {ini }}}$ & $\underline{N_{\text {des }}}$ & $\underline{\underline{N_{\text {max }}}}$ & $\underline{\mathrm{N}_{\text {ini }}}$ & $\underline{N_{\text {des }}}$ & $\underline{\mathrm{N}_{\max }}$ & $\underline{\mathrm{N}_{\text {ini }}}$ & $\underline{N_{\text {des }}}$ & $\underline{N_{\text {max }}}$ \\
\hline$<0.3$ & 7 & 68 & 104 & 7 & 74 & 114 & 7 & 78 & 121 & 7 & 82 & 127 \\
\hline $0.3-1$ & 7 & 76 & 117 & 7 & 83 & 129 & 7 & 88 & 138 & 8 & 93 & 146 \\
\hline $1-3$ & 7 & 86 & 134 & 8 & 95 & 150 & 8 & 100 & 158 & 8 & 105 & 167 \\
\hline $3-10$ & 8 & 96 & 152 & 8 & 106 & 169 & 8 & 113 & 181 & 9 & 119 & 192 \\
\hline $10-30$ & 8 & 109 & 174 & 9 & 121 & 195 & 9 & 128 & 208 & 9 & 135 & 220 \\
\hline $30-100$ & 9 & 126 & 204 & 9 & 139 & 228 & 9 & 146 & 240 & 10 & 153 & 253 \\
\hline$>100$ & 9 & 143 & 235 & 10 & 158 & 262 & 10 & 165 & 275 & 10 & 172 & 288 \\
\hline
\end{tabular}




\section{Use in Quality Control}

A key part of any mixture design system is its ease and reliability when used in the construction process as part of production quality control. Roberts, et al [16] cited the Federal Highway Administration (FHWA) Demonstration Project No. 74's clear indication that significant differences exist between the volumetric properties of the laboratory designed and plant produced mixtures. Consequently, production quality control is performed by the contractor (typically in on-site laboratories) to ensure the plant is performing as anticipated, and by the owner (typically in mobile laboratories) to ensure production of a consistent, quality product. The first step in production quality control consists of periodic sampling of the material from either behind the paver (preferred) or from the bed of a hauling truck. The sample is then taken to the lab where it is compacted and its volumetric properties are determined. The values are then used to ensure mix production remains within set control limits, and to look for trends signifying the production is out of control (unacceptably large variations) and/or tending towards exceeding a control limit. Without production quality control, there is no means of verifying the product is indeed the consistent, high quality product desired.

When first instituted, the large size and expense of Hveem compactors made efficient production quality control difficult. Furthermore, the absence of routine volumetric property determination in the Hveem method exacerbated problems when volumetric analyses became the primary means of production quality control. The smaller, less costly Marshall hammer, however, was much more suited to on-site laboratories. This situation was acceptable when the majority of mixes were designed using the Marshall method. However, the increasing use of Superpave in the design of asphalt mixtures has brought with it an important question in the quality control process: Can a Marshall compactor (still found in most on-site laboratories) be used to perform production quality control of a Superpave job? D'Angelo, et al [19] conducted a study to determine whether a Marshall hammer could be used to adequately perform quality control on a Superpave mix and vice-versa. The study examined five different mixes from five different

plants by compacting each mix with both a mechanical Marshall hammer and a Superpave Gyratory Compactor. Table 2.4 summarizes the design and compaction methods used in the study. 
Table 2.4 Summary of Design and Compaction Methods (D’Angelo, et al [19])

\begin{tabular}{|c|c|c|c|c|}
\hline $\begin{array}{c}\text { Study } \\
\text { Number }\end{array}$ & $\begin{array}{l}\text { Design } \\
\text { Method }\end{array}$ & Compaction Effort & $\begin{array}{c}\text { Control } \\
\text { Compactor }\end{array}$ & $\begin{array}{c}\text { Compaction } \\
\text { Effort }\end{array}$ \\
\hline \# 539 & $\begin{array}{l}\text { Superpave } \\
\text { Level } 1\end{array}$ & $\begin{array}{l}\text { SGC 150x115mm } \\
\mathrm{N}_{\mathrm{d}}=100 \quad \mathrm{~N}_{\mathrm{m}}=158\end{array}$ & $\begin{array}{l}\text { 6-in. (152mm) } \\
\text { S. Marshall }\end{array}$ & 112 blows/side \\
\hline \# 540 & $\begin{array}{l}\text { 6-in. (152-mm) } \\
\text { S. Marshall }\end{array}$ & 112 blows/side & $\mathrm{SGC}$ & $\mathrm{N}_{\mathrm{d}}=100 \quad \mathrm{~N}_{\mathrm{m}}=158$ \\
\hline \#641 & $\begin{array}{c}\text { 4-in. (102-mm) } \\
\text { S. Marshall }\end{array}$ & 50 blows/side & SGC & $\mathrm{N}_{\mathrm{d}}=126 \quad \mathrm{~N}_{\mathrm{m}}=204$ \\
\hline \# 9401A & $\begin{array}{c}\text { 4-in. (102-mm) } \\
\text { S. Marshall }\end{array}$ & 75 blows/side & SGC & $\mathrm{N}_{\mathrm{d}}=109 \quad \mathrm{~N}_{\mathrm{m}}=174$ \\
\hline \# 9407A & $\begin{array}{l}\text { Superpave } \\
\text { Level } 1\end{array}$ & $\begin{array}{l}\text { SGC 150x115mm } \\
\mathrm{N}_{\mathrm{d}}=100 \quad \mathrm{~N}_{\mathrm{m}}=158\end{array}$ & $\begin{array}{l}\text { 4-in. }(102 \mathrm{~mm}) \\
\text { S. Marshall }\end{array}$ & 50 blows/side \\
\hline
\end{tabular}

The volumetric properties of the mixtures were evaluated to determine if the compaction devices were interchangeable or if the results were dependent on the compaction device used. D'Angelo, et al [19] concluded that when evaluating voids in the total mix (VTM) as the control criterion, the two compactors were interchangeable. Voids in the mineral aggregate (VMA), however, is actually a better criterion to evaluate quality control because it provides a better indication of the aggregate structure within the mix. When using VMA as the criterion, D'Angelo, et al [19] found that the two compactor were not interchangeable.

In two of the mixes, where the SGC indicated a continued increase in VMA with increasing asphalt content, the use of the Marshall hammer resulted in a decrease in VMA. The SGC indicates the additional binder has filled the void space between the particles forcing them apart. With the Marshall hammer, the additional binder lubricated the aggregate allowing the hammer to compact the mixture more densely. The results clearly indicate that when a mix is designed using Superpave, an SGC must be used for production quality control.

\section{RUGGEDNESS EVALUATIONS}

\section{Gyratory Compactors}

The Marshall and Hveem methods of mix design were developed over 50 years ago. Their strengths and shortcomings are well documented, but Superpave is still relatively new. Its reaction to variabilities in materials and conditions are not well documented. McGennis, et al [20], in cooperation with the FHWA expert task group, conducted a ruggedness test of the 
American Association of State Highway and Transportation Officials (AASHTO) Test Method TP4 to evaluate the extent to which variations in test parameters cause variations in test results. AASHTO TP4 is the provisional standard governing the preparation of test specimens with the Superpave Gyratory Compactor. The experiment was conducted using two SGCs that FHWA experiments determined were substantially equivalent: the Pine and Troxler SGCs (D'Angelo, 1995). Table 2.5 shows the seven primary factors and their levels of variation evaluated in the experiment. Table 2.6 lists the eight combinations of variables used in the experiment.

Table 2.5 Main Factors Evaluated in Ruggedness Experiment (McGennis, et al [20])

\begin{tabular}{|l|l|}
\hline Factor & Levels \\
\hline Angle of Gyration, degrees & Low Range (1.22 to 1.24) and High Range (1.26 to 1.28) \\
\hline Mold Loading Procedure & Transfer Bowl Method and Direct Loading Method \\
\hline Compaction Pressure, $\mathrm{kPa}$ & 582 and 618 \\
\hline Precompaction & None and 10 thrusts with Standard Rod \\
\hline Compaction Temperature, ${ }^{\circ} \mathrm{C}$ & $@ 0.250$ Pa-s viscosity and @ 0.310 Pa-s viscosity \\
\hline Specimen Height, $\mathrm{mm}$ & Low (around $110 \mathrm{~mm})$ and High (around $120 \mathrm{~mm}$ ) \\
\hline Aging Period @ $135^{\circ} \mathrm{C}, \mathrm{hrs}$ & 3.5 and 4 \\
\hline
\end{tabular}

Table 2.6 Variable Combinations used in Ruggedness Experiment (McGennis, et al [20])

\begin{tabular}{|l|c|c|c|c|c|c|c|c|}
\hline & \multicolumn{7}{|c|}{ Combination } \\
\hline Variable & $\mathbf{1}$ & $\mathbf{2}$ & $\mathbf{3}$ & $\mathbf{4}$ & $\mathbf{5}$ & $\mathbf{6}$ & $\mathbf{7}$ & $\mathbf{8}$ \\
\hline Angle of Gyration, degrees & 1.23 & 1.23 & 1.23 & 1.23 & 1.27 & 1.27 & 1.27 & 1.27 \\
\hline Mold Loading Procedure & $\mathrm{TB}$ & $\mathrm{TB}$ & $\mathrm{DL}$ & $\mathrm{DL}$ & $\mathrm{TB}$ & $\mathrm{TB}$ & $\mathrm{DL}$ & $\mathrm{DL}$ \\
\hline Compaction Pressure, $\mathrm{kPa}$ & 618 & 582 & 618 & 582 & 618 & 582 & 618 & 582 \\
\hline Precompaction & $\mathrm{Y}$ & $\mathrm{Y}$ & $\mathrm{N}$ & $\mathrm{N}$ & $\mathrm{N}$ & $\mathrm{N}$ & $\mathrm{Y}$ & $\mathrm{Y}$ \\
\hline Compaction Temperature, ${ }^{\circ} \mathrm{C}$ & $\mathrm{H}$ & $\mathrm{L}$ & $\mathrm{H}$ & $\mathrm{L}$ & $\mathrm{L}$ & $\mathrm{H}$ & $\mathrm{L}$ & $\mathrm{H}$ \\
\hline Specimen Height, mm & $\mathrm{L}$ & $\mathrm{H}$ & $\mathrm{H}$ & $\mathrm{L}$ & $\mathrm{L}$ & $\mathrm{H}$ & $\mathrm{H}$ & $\mathrm{L}$ \\
\hline Aging Period @ $135^{\circ} \mathrm{C}, \mathrm{hrs}$ & 4.0 & 3.5 & 3.5 & 4.0 & 3.5 & 4.0 & 4.0 & 3.5 \\
\hline
\end{tabular}

The nominal angle of gyration in AASHTO TP 4 is $1.25^{\circ}$ with an allowable tolerance of $0.02^{\circ}$. The tolerance was established by FHWA because several experiments showed density was profoundly affected by small changes in angle. One study on a project in Arizona determined 
that a $0.25^{\circ}$ change in angle of gyration resulted in a four percent change in void content. However, across the $0.04^{\circ}$ range of compaction angles evaluated, the trend toward an increase in density was not significant. Less than one percent of the variation was explained by compaction angle.

Although AASHTO TP4 vaguely informs the operator to "place the mixture in the mold in one lift," experience by the Colorado and Texas DOTs has shown that the method of mold loading has a significant influence on specimen density. The experiences of McGennis, et al [20] suggested two extremes of mold loading: loading the bowl with a specially designed "gyro loader" transfer bowl; and using a scoop to load the mold directly from the aging pan. Results of this experiment indicate the mold loading procedure does not have a clear, consistent influence on SGC test specimens.

AASHTO TP4 requires a nominal compactive pressure of $600 \mathrm{kPa}(87 \mathrm{psi})$. The allowable three percent tolerance results in test pressures of 582 (84.4 psi) and $618 \mathrm{kPa}(89.6 \mathrm{psi})$. Results indicate a significant difference in density between the two pressures. However, pressure tolerance is not significant since SGCs have transducers enabling operators to set the pressure at exactly $600 \mathrm{kPa}(87 \mathrm{psi})$.

Although TP4 does not require precompaction (the rodding of the mixture prior to compaction), most previous mix design methods such as the Marshall and Hveem methods have required it. Consequently, it is very likely SGC operators would precompact the mix out of habit. Experience has shown it can take as many as 20 gyrations for the compaction pressure to stabilize at $600 \mathrm{kPa}(87 \mathrm{psi})$. It was hypothesized precompaction would enable quicker pressure stabilization resulting in different measured bulk specific gravity. However, results showed the two extremes (zero and 10 rodding strokes) had no significant effect.

AASHTO TP4 specifies mixtures be compacted within a temperature range that results in a binder viscosity between $0.250 \mathrm{~Pa}-\mathrm{s}$ (2.5 poise) and $0.310 \mathrm{~Pa}-\mathrm{s}$ (3.1 poise). For the binder in the McGinnis, et al [20] evaluation, a PG 64-22, the resulting compaction temperatures were $141{ }^{\circ} \mathrm{C}$ $\left(286^{\circ} \mathrm{F}\right)$ and $146^{\circ} \mathrm{C}\left(295^{\circ} \mathrm{F}\right)$. Results indicated compaction temperatures at the extremes of $141^{\circ} \mathrm{C}\left(286^{\circ} \mathrm{F}\right)$ and $146^{\circ} \mathrm{C}\left(295^{\circ} \mathrm{F}\right)$ do not have a significant effect. 
McGennis, et al [20] discovered the 100-mm (3.94 in.) nominal specimen height requirement of AASHTO TP4 (Edition 1B, September, 1993), is actually incorrect. Most SHRP research had been completed on specimens with a $115-\mathrm{mm}(4.53$ in.) nominal height. Additionally, the required $\pm 1 \mathrm{~mm}(0.0394 \mathrm{in}$.) tolerance is counter-productive as it is extremely difficult to achieve on the initial compaction and may simply be too stringent. Consequently, McGennis, et al [20] chose to use a tolerance level of $\pm 5 \mathrm{~mm}$ ( 0.2 in.). Results showed a significant variability when the height difference of fine graded mixtures exceeded $\pm 12 \mathrm{~mm}(0.47 \mathrm{in}$.). Coarse mixtures did not exhibit a significant variability with respect to height. McGennis, et al concluded a $\pm 5 \mathrm{~mm}(0.2 \mathrm{in}$.) tolerance ensures reasonable variability.

Although TP4 requires four hours of short-term oven aging at $135^{\circ} \mathrm{C}\left(275^{\circ} \mathrm{F}\right)$, required compaction temperatures may sometimes be higher than $135^{\circ} \mathrm{C}\left(275^{\circ} \mathrm{F}\right)$. To achieve compaction temperatures above $135^{\circ} \mathrm{C}\left(275^{\circ} \mathrm{F}\right)$, two ovens are often used. The first oven, set at $135^{\circ} \mathrm{C}\left(275^{\circ} \mathrm{F}\right)$, is used for short-term aging of the mix. The second oven is used to heat the specimen up to the required compaction temperature. Two acceptable procedures exist for achieving the increased temperature: placing the mixture in the second oven for up to the 30 allowable minutes after the four hours of short-term aging, or removing the mixture from the first oven such that the increased temperature can be obtained within the required four hours of shortterm aging. Therefore, McGennis, et al [20] used 3.5 and 4.0 hours for the extremes of shortterm aging. Results indicated the extremes of the short-term aging protocols had an insignificant effect. However, it is important to note this conclusion was reached based upon only one binder. McGennis, et al advise binders exhibiting rapid aging characteristics may be more susceptible to variations in short term aging times. In summary, McGennis, et al [20] concluded the Superpave Gyratory Compactor is a rugged, dependable system that is not very susceptible to operating variations.

\section{CONCLUSION}

The Marshall and Hveem methods of mix design are proven, 50 year-old design procedures. However, they do have their shortcomings. Their primary material characterization tests (Marshall stability and flow and Hveem stability) are not reliable when conditions are outside those in which the tests were developed (i.e. the continuing increase in axle loads and tire 
pressures). A hot mix asphalt design procedure that characterizes the mixed based on performance-related fundamental engineering properties is required.

Superpave's gyratory compactor plays an important role in producing laboratory- compacted hot mix asphalt samples that are representative of field compaction. Shear and tensile tests are useless if performed on a laboratory specimen that is not representative of a field specimen. Researchers have concluded that as a gyratory compactor, the SGC does a better job of simulating field compaction because its shearing action simulates the densification through particle reorientation achieved by rollers in the field. 


\section{CHAPTER 3 \\ RESEARCH METHODOLOGY}

\section{OVERVIEW}

The previous section discussed the importance of compaction, the influence of mix properties on volumetrics, and presented the Superpave method of mix design. This section describes the equipment, materials, and procedures used in the production and analysis of mix specimens. Once determined from the mix design, the optimum asphalt contents for the various mixtures were used throughout the remainder of the project. Samples were created at the optimum asphalt content (for 3 percent compacted air voids) for resilient modulus testing at $-18,0,25$, and $40{ }^{\circ} \mathrm{C}$ $\left(0,34,77\right.$, and $104^{\circ} \mathrm{F}$, respectively) to determine each mixture's susceptibility to temperature variation. Moisture sensitivity was evaluated by comparing the indirect tensile strengths between unconditioned, control samples and vacuum-saturated, conditioned samples.

\section{DESIGN}

\section{Summary of Laboratory Mixes}

Generally speaking, it is economically desirable to use low-cost, locally available materials for roads with low traffic volumes. The fine aggregate (FA - passing the $4.75 \mathrm{~mm}$ (No.4) sieve) used for every gradation was a sand from Lakeland, Minnesota. It is readily available at low cost, but has a relatively rounded shape. The coarse aggregate $(\mathrm{CA}$ - retained on the $9.5 \mathrm{~mm}$ (3/8 in.) and larger sieves) used in the project consisted of aggregate from four different sources: Granite Falls (GF) granite, New Ulm (NU) quartzite, Kasota (KL) limestone, and Cedar Grove (CG) gravel. Two different gradations, shown in Figure 3.1, were used in the project: a fine gradation which ran above the Superpave restricted zone; and a coarse gradation which ran below the restricted zone. Additionally, two different asphalt cement grades were evaluated: a PG 52-34, the primary grade for the project, was used in all 8 mixes; a PG 58-40 cement was used in the fine gradations for the New Ulm quartzite and Kasota limestone mixes.

Eight different aggregate gradations (four above, two through and two below the restricted zone) using Granite Falls granite (CA) and Lakeland gravel (FA) were evaluated in the attempt to satisfy the Superpave VMA criteria. None of the eight gradations resulted in a sample 
compacted to $4 \%$ air voids having a VMA above the Superpave minimum requirement of $14 \%$ for low volume roads $(300,000<$ ESALs $<1,000,000)$. In fact, the only time the VMA criterion was satisfied was when the $2.36 \mathrm{~mm}$ (No. 8) and $1.18 \mathrm{~mm}$ (No. 16) Lakeland aggregate was replaced with a Wisconsin, Dresser basalt aggregate having a greater degree of angularity. Since the purpose of the project, however, is to use economical, locally available aggregate, the Dresser aggregate was not used in this project. Instead, the coarse and fine gradations were chosen on the basis of obtaining the best possible VMA/VFA results using a natural sand aggregate source.

The gradation and aggregate sources used in the project are listed in Table 3.1 for both coarse and fine mixes. The Superpave restricted zone, gradation band, and design gradations are illustrated in Figure 3.1.

Table 3.1 Coarse and Fine Mix Gradations

\begin{tabular}{|c|c|c|c|}
\hline $\begin{array}{c}\text { Sieve Size } \\
(\mathbf{m m})\end{array}$ & $\begin{array}{c}\text { Percent Passing } \\
\text { (Coarse Gradation) }\end{array}$ & $\begin{array}{c}\text { Percent Passing } \\
\text { (Fine Gradation) }\end{array}$ & $\begin{array}{c}\text { Aggregate } \\
\text { Source }\end{array}$ \\
\hline 19 & 100.0 & 100.0 & \\
\hline 12.5 & 93.2 & 96.1 & GF/NU/KL/CG \\
\hline 9.5 & 66.2 & 83.1 & GF/NU/KL/CG \\
\hline 4.75 & 47.3 & 70.1 & Lakeland Gravel \\
\hline 2.36 & 33.8 & 57.1 & Lakeland Gravel \\
\hline 1.18 & 23 & 44.2 & Lakeland Gravel \\
\hline 0.600 & 16.2 & 31.2 & Lakeland Gravel \\
\hline 0.300 & 12.2 & 18.2 & Lakeland Gravel \\
\hline 0.150 & 8.1 & 7.8 & Lakeland Gravel \\
\hline 0.075 & 4.1 & 3.9 & Lakeland Gravel \\
\hline PAN & 0.0 & 0.0 & Baghouse Fines \\
\hline
\end{tabular}

\section{Aggregate Properties}

The fine aggregate (FA) used for every gradation was a Lakeland gravel. It is readily available at low cost, but has a relatively low fine aggregate angularity (FAA) of 0.40 . The coarse aggregates (CA) are Granite Falls Granite, New Ulm Quartzite, Kasota Limestone, and Cedar Grove Gravel. Tables 3.2 and 3.3 list the aggregate properties for the fine and coarse mixes, respectively. 


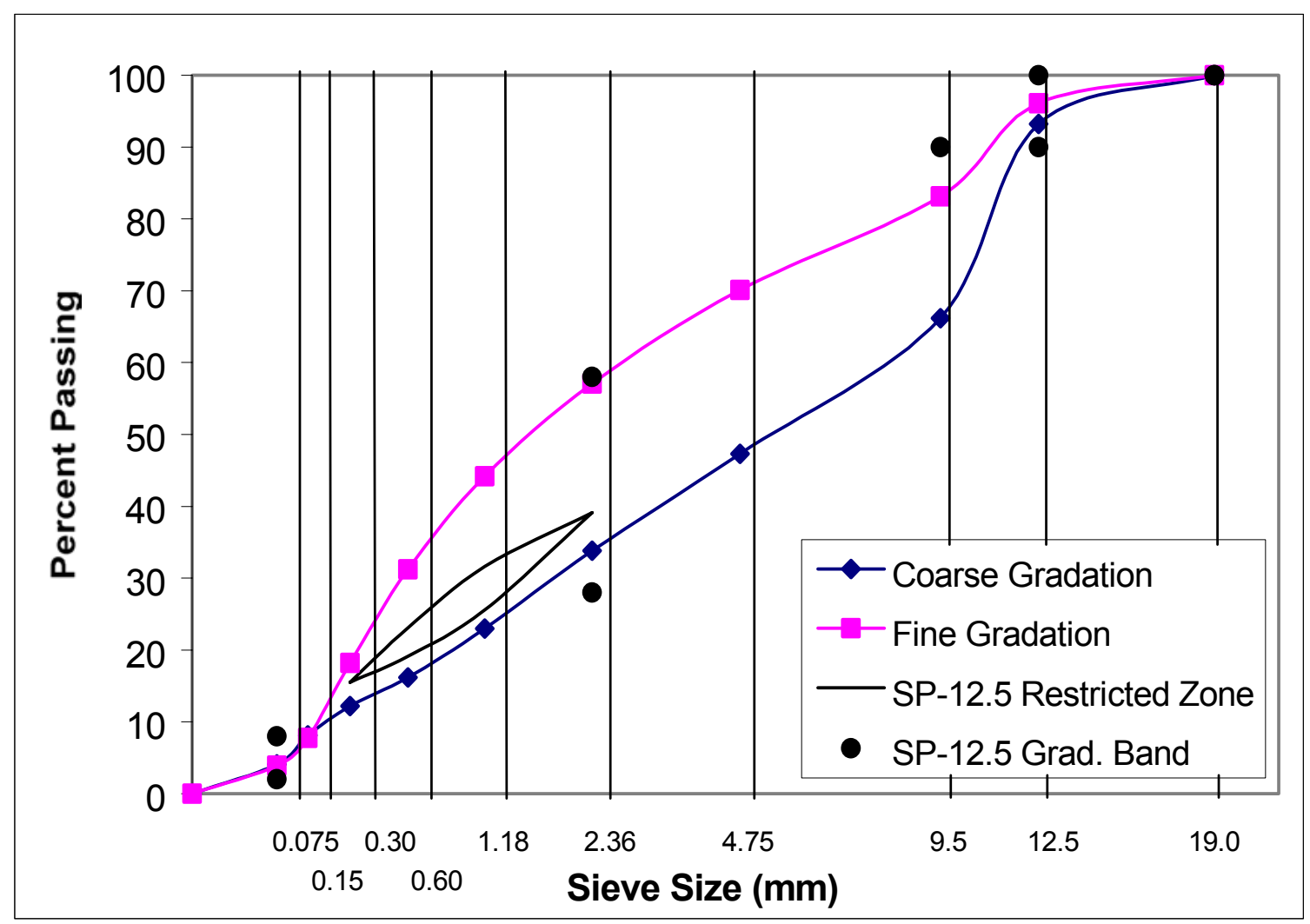

Figure 3.1 Experimental Mix Gradations

Table 3.2 Fine Aggregate Properties

\begin{tabular}{|c|c|}
\hline TEST & LAKELAND GRAVEL \\
\hline $\mathrm{G}_{\mathrm{sb}}$ & 2.602 \\
\hline $\mathrm{G}_{\mathrm{sa}}$ & 2.770 \\
\hline Wine Aggregate Angularity, $\%$ & 2.3 \\
\hline Sand Equivalent, $\%$ & 39.9 \\
\hline
\end{tabular}


Table 3.3 Coarse Aggregate Properties

\begin{tabular}{|c|c|c|c|c|}
\hline TEST & $\begin{array}{c}\text { Granite Falls } \\
\text { Granite }\end{array}$ & $\begin{array}{c}\text { New Ulm } \\
\text { Quartzite }\end{array}$ & $\begin{array}{c}\text { Kasota } \\
\text { Limestone }\end{array}$ & $\begin{array}{c}\text { Cedar Grove } \\
\text { Gravel }\end{array}$ \\
\hline $\mathrm{G}_{\mathrm{sb}}$ & 2.757 & 2.624 & 2.492 & 2.610 \\
\hline $\mathrm{G}_{\mathrm{sa}}$ & 2.797 & 2.661 & 2.770 & 2.731 \\
\hline Water Absorption, \% & 0.46 & 0.53 & 4.0 & 1.7 \\
\hline $\begin{array}{c}\text { Flat/Elongated Particles } \\
\%,(1: 3 \text { Ratio })\end{array}$ & 14.0 & 35.0 & 11.1 & 8.2 \\
\hline $\begin{array}{c}\text { Fractured Faces } \\
\%,(\geq 1 / \geq 2)\end{array}$ & $100 / 100$ & $100 / 100$ & $100 / 100$ & $64.7 / 38.6$ \\
\hline
\end{tabular}

All fine and coarse aggregate consensus property testing was done in accordance with Superpave specifications. The FAA of the Lakeland aggregate was determined using AASHTO TP33, "Test

Method for Uncompacted Void Content of Fine Aggregate (as Influenced by Particle Shape, Surface Texture, and Grading-Method A)." The Sand Equivalency of the Lakeland aggregate was determined using AASHTO T176, "Plastic Fines in Graded Aggregates and Soils by Use of the Sand Equivalent Test" (ASTM D2419).

The procedure used to determine the flat/elongated particle percentage of the Granite Falls, New Ulm, Kasota, and Cedar Grove aggregates was ASTM D4791, "Flat or Elongated Particles in Coarse Aggregate." The final aggregate property, coarse aggregate angularity, or fractured faces, was done in accordance with ASTM D5821-95, "Determining the Percentage of Fractured Particles in Coarse Aggregate."

\section{Gyratory Compactor}

The compactor used throughout the project was the Brovold gyratory compactor - a Superpave Gyratory Compactor manufactured by Test Quip. The Brovold compactor is considered an Intensive Compaction Tester (ICT) — operating on a "shear compaction" principle. Compaction occurs via two distinct elements: vertical pressure and shear displacement. These two elements combine to encourage the reorientation of aggregate particles-essential for the compaction of any particulate specimen. 
A piston pushing down on a plate resting on top the asphalt specimen inside the compaction mold supplies the vertical pressure. The Superpave standard of $600 \mathrm{kPa}$ ( $87 \mathrm{psi}$ ) was used throughout the project. The gyratory motion of the compactor creates the necessary shear force. Increasing the angle of the gyration increases the shear force created by the compactor. Superpave guidelines, however, set the gyratory angle at $1.25^{\circ} \pm 0.02^{\circ}$. Figure 3.2 illustrates the resulting shear displacement described above.

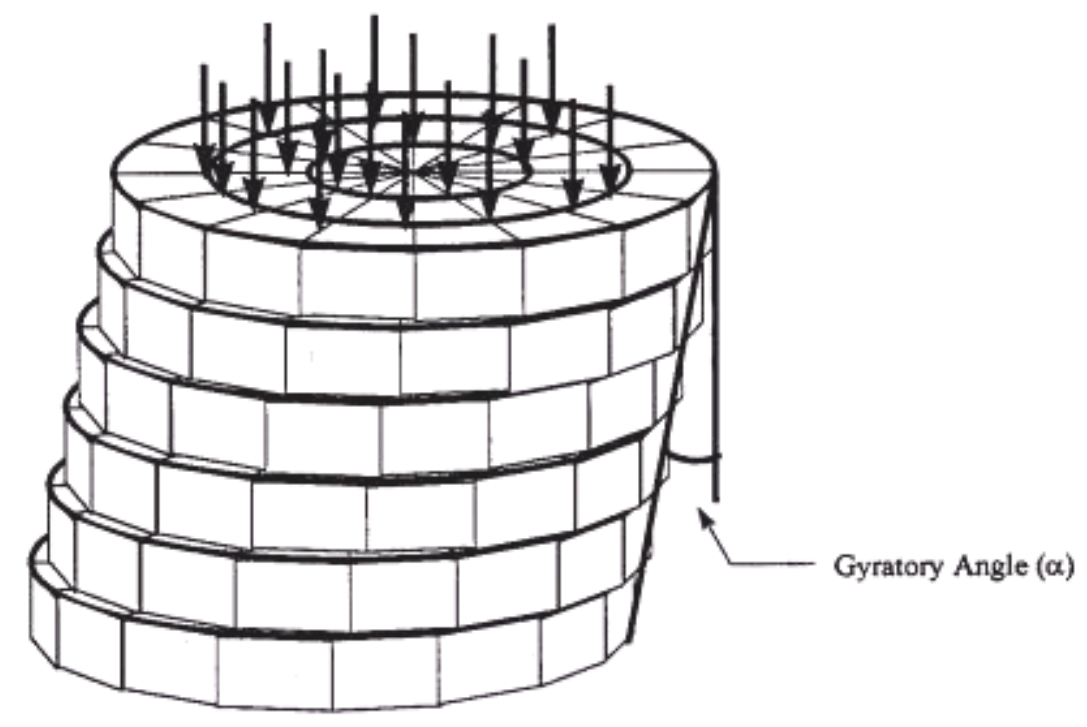

Figure 3.2 Shear Displacement During Gyratory Compaction

The height of the sample is continually recorded by a linear variable displacement transducer (LVDT). The density of the sample at each gyration is calculated using the current height and mass of the sample. The operator is able to control the compaction energy transmitted to the sample by inputting the desired number of gyrations. Superpave specifies the number of gyrations as a function of temperature and anticipated traffic.

\section{Compaction Procedure}

Prior to mixing, pre-batched, 12,000-gram (26.5 lb.) aggregate samples were placed in a forceddraft oven for a minimum of four hours to ensure adequate drying. Simultaneously, the asphalt binder was pre-heated to the appropriate mixing temperature, $138^{\circ}\left(280^{\circ}\right)$ for PG 52-34 and $145^{\circ}\left(293^{\circ}\right)$ for PG 58-40. The aggregate was first poured into a bucket mixer followed by the appropriate amount of asphalt cement. After adequate mixing in the bucket mixer, the mixture 
was placed in a large pan and mixed by hand to prevent segregation. Each batch was then split into two 4,800 g (10.6 lb.) samples for compaction, and two 1,000 g (2.2 lb.) samples for Theoretical Maximum Specific Gravity testing. Mix design samples were prepared according to the matrix shown in Table 3.4. The initial asphalt content $\left(\mathrm{P}_{\mathrm{b}}\right)$ was determined using the spreadsheet shown in Appendix A.

Table 3.4 Mix Design Matrix

\begin{tabular}{|c|c|c|c|c|c|}
\hline \multirow{3}{*}{$\begin{array}{l}\text { Aggregate } \\
\text { Type }\end{array}$} & \multirow{3}{*}{$\begin{array}{l}\text { Asphalt } \\
\text { Grade }\end{array}$} & \multicolumn{4}{|c|}{ Superpave Level I Mix Design } \\
\hline & & \multicolumn{4}{|c|}{ Asphalt Content } \\
\hline & & $\mathrm{P}_{\mathrm{b}}-0.5$ & $\mathrm{P}_{\mathrm{b}}$ & $\mathrm{P}_{\mathrm{b}}+0.5$ & $\mathrm{P}_{\mathrm{b}}-1.0$ \\
\hline GFC & PG 52-34 & $X$ & $X$ & $X$ & $X$ \\
\hline GFF & PG 52-34 & $\mathrm{X}$ & $\mathrm{X}$ & $\mathrm{X}$ & $\mathrm{X}$ \\
\hline $\mathrm{NUC}$ & PG 52-34 & $\mathrm{X}$ & $\mathrm{X}$ & $\mathrm{X}$ & $\mathrm{X}$ \\
\hline NUF & PG 52-34 & $\mathrm{X}$ & $\mathrm{X}$ & $\mathrm{X}$ & $\mathrm{X}$ \\
\hline $\mathrm{KLC}$ & PG 52-34 & $\mathrm{X}$ & $\mathrm{X}$ & $\mathrm{X}$ & $\mathrm{X}$ \\
\hline KLF & PG 52-34 & $\mathrm{X}$ & $\mathrm{X}$ & $\mathrm{X}$ & $\mathrm{X}$ \\
\hline $\mathrm{CGC}$ & PG 52-34 & $\mathrm{X}$ & $\mathrm{X}$ & $X$ & $\mathrm{X}$ \\
\hline CGF & PG 52-34 & $\mathrm{X}$ & $\mathrm{X}$ & $\mathrm{X}$ & $\mathrm{X}$ \\
\hline NUF & PG 58-40 & $X$ & $\mathrm{X}$ & $X$ & $X$ \\
\hline KLF & PG 58-40 & $X$ & $X$ & $X$ & $X$ \\
\hline
\end{tabular}

The samples were compacted using Test Quip's Brovold gyratory compactor. The PG 52-34 samples were compacted at $128^{\circ} \mathrm{C}\left(262.4^{\circ} \mathrm{F}\right)$. The PG 58-40 samples were compacted at $135^{\circ} \mathrm{C}\left(275^{\circ} \mathrm{F}\right)$. The required number of gyrations was based on low-volume, level two, traffic: 300,000 to 1,000,000 ESALs. Therefore, $\mathrm{Nini}=7, \mathrm{Ndes}=76$, and $\mathrm{Nmax}=117$.

\section{RESILIENT MODULUS}

Resilient modulus tests were conducted on all samples to determine the mixtures' susceptibility to temperature changes. The test was conducted in accordance with ASTM D 4123-82 (1987), "Standard Test Method for Indirect Tension Test for Resilient Modulus of Bituminous Mixtures." Samples were typically loaded to a stress level between 5 and 20 percent of indirect tensile strength (measured or estimated prior to conducting resilient modulus tests). Loads were applied in cycles consisting of 0.1 second load and 0.9 second no-load rest. After test completion, the resilient modulus was calculated via the following equation: 


$$
M_{R}=\frac{P}{H t} \times(0.27+\mu)
$$

Where:

$M_{R}=$ resilient modulus, $\mathrm{Pa}$

$P=$ applied load, Newtons

$H=$ horizontal deformation, $\mathrm{mm}$

$t=$ sample thickness, mm

$\mu=$ Poisson's ratio

Poisson's ration can be calculated as

$$
\begin{aligned}
& \mu=3.59 \frac{H}{V}-0.27 \quad \text { (for } 100 \mathrm{~mm} \text { samples) } \\
& \mu=4.09 \frac{H}{V}-0.27 \quad \text { (for } 150 \mathrm{~mm} \text { samples) }
\end{aligned}
$$

Where:

$\mu=$ Poisson's ratio

$H=$ horizontal deformation, $\mathrm{mm}$

$V=$ vertical deformation, $\mathrm{mm}$

The following Poisson's ratio values recommended by Brown, et al [18] were assumed: 0.25 for $5{ }^{\circ} \mathrm{C}\left(41^{\circ} \mathrm{F}\right) ; 0.35$ for $25^{\circ} \mathrm{C}\left(77^{\circ} \mathrm{F}\right)$, or 0.40 for $40{ }^{\circ} \mathrm{C}\left(104{ }^{\circ} \mathrm{F}\right)$.

There is much debate over the applicability of resilient modulus values in the prediction of longterm pavement performance. It was once commonly believed stiffer pavements (those with higher resilient modulus values) had greater resistance to permanent deformation. Roberts, et al [16] caution that, to date, there is no solid correlation between $M_{R}$ and rutting. However, $M_{R}$ at low temperatures is somewhat related to cracking as stiffer mixes (higher $M_{R}$ ) at low temperatures tend to crack earlier than more flexible mixtures (lower $\left.M_{R}\right)$.

For the Superpave project, three $3400 \mathrm{~g}$ (7.5 lb.) samples were made for each of the ten mixes. Each of the samples were tested at both zero and $90^{\circ}$ orientations. A minimum 2-hour waiting period was maintained between zero and $90^{\circ}$ testing to provide the samples time to recover from any distortion that might have resulted from the previous test. All 30 samples were tested at -18 , 0,25 , and $40{ }^{\circ} \mathrm{C}\left(0,34,77\right.$, and $104^{\circ} \mathrm{F}$, respectively). At each temperature, the samples were placed in temperature controlled environmental chambers for a minimum of 24 hours to ensure equilibrium had been reached at the respective temperature. Isopropyl alcohol was used to 
remove any ice accumulation from the extensometers. Due to the inherent high variability of resilient modulus testing, samples with suspected erroneous test results were immediately retested. Test results were continually examined to protect against inaccurate data resulting from damaged samples.

\section{MOISTURE SENSITIVITY}

The effects of moisture sensitivity can be as minor as the weakening of the bond between the asphalt cement and the aggregate or as drastic as the sudden peeling off of the asphalt so only bare aggregate remains. The more typical scenario is a gradual loss of strength over a period of years resulting in the development of rutting and shoving in the wheel paths. To help protect against moisture damage it is necessary to determine if a mixture is susceptible to water damage in the event of water penetration.

Moisture sensitivity tests were conducted in accordance with ASTM D4867. One set of six specimens for each mixture was compacted to $7 \pm 1$ percent air voids. Each set was then divided

into two subsets of approximate equal void content. One subset was maintained dry while the other subset was partially saturated with water and moisture conditioned. The samples were vacuum saturated to between 55 and 80 percent. After being partially saturated, the conditioned samples were placed in a $60^{\circ} \mathrm{C}\left(140^{\circ} \mathrm{F}\right)$ water bath for 24 hours. Both subsets were then subjected to the tensile splitting test and loaded with a diametral load until failure. The tensile strength of each subset was determined by equation 3.4.

$$
S_{t}=\frac{2000 P}{\pi t D}(\mathrm{kPa})
$$

Where:

$S_{t}=$ tensile strength, $\mathrm{kPa}$

$P=$ maximum load, $\mathrm{N}$

$t=$ specimen height before tensile test, $\mathrm{mm}$

$D=$ specimen diameter

The potential for moisture damage is indicated by the tensile strength ratio (TSR): the ratio of the tensile strength of the wet subset to that of the dry subset. The TSR for each mixture is calculated by equation 3.5 . 


$$
T S R=\frac{S_{t w}}{S_{t d}} \times 100
$$

Where:

TSR $=$ Tensile Strength Rati, $\%$

$\mathrm{Stw}=$ Moisture conditioned tensile strength, $\mathrm{kPa}$

$\mathrm{Std}=$ Dry tensile strength, $\mathrm{kPa}$

\section{PERFORMANCE TESTS}

Tests were conducted to determine susceptibility to rutting and low temperature cracking. Due to time and budget constraints, a subset of four mix types was selected for this testing. Table 3.5 shows the test matrix for performance testing. Only fine-graded mixtures were chosen because they are more representative of low volume pavements in Minnesota than coarse-graded mixtures. New Ulm quartzite and Kasota limestone were chosen to represent low and high asphalt absorption capacities, respectively. Both the unmodified (PG 52-34) and polymermodified (PG 58-40) were tested to determine if the expanded temperature range provided a significant reduction in rutting or cracking potential.

Table 3.5 Performance Testing Matrix

\begin{tabular}{|c|c|c|c|}
\hline \multirow{2}{*}{$\begin{array}{c}\text { Aggregate } \\
\text { Type }\end{array}$} & $\begin{array}{c}\text { Asphalt } \\
\text { Grade }\end{array}$ & APA Rut Tester & $\begin{array}{c}\text { PDT Low Temperature } \\
\text { Creep Compliance }\end{array}$ \\
\cline { 3 - 4 } & PG 52-34 & $\mathrm{X}$ & $\mathrm{X}$ \\
\hline NUF & PG 52-34 & $\mathrm{X}$ & $\mathrm{X}$ \\
\hline $\mathrm{KLF}$ & PG 58-40 & $\mathrm{X}$ & $\mathrm{X}$ \\
\hline $\mathrm{NUF}$ & PG 58-40 & $\mathrm{X}$ & $\mathrm{X}$ \\
\hline $\mathrm{KLF}$ & & & \\
\hline
\end{tabular}

\section{Rut Testing}

Laboratory rut testing was conducted on four of the asphalt mixes to determine their susceptibility to rutting. The tests were conducted in accordance with the "Standard Test Method for Determining Rutting Susceptibility Using the Asphalt Pavement Analyzer" (Pavement Technology, Inc. 1999). The Asphalt Pavement Analyzer (APA) is a Loaded Wheel Tester used for evaluating permanent deformation (rutting) in asphalt pavements. Rutting susceptibility of mixes is assessed by placing cylindrical samples under repetitive wheel loads 
and measuring the amount of permanent deformation under the wheel path. The APA features controllable wheel loads and contact pressure that are representative of actual field conditions, and an environmental chamber allows for samples to be tested under controlled temperature conditions.

The samples were preheated at $40{ }^{\circ} \mathrm{C}$ for 6 to 24 hours prior to testing. The testing chamber was also preheated to this temperature. The samples were subject to a total of 8,000 cycles by the APA. The rut depth was determined by subtracting the measurement for each cycle from the initial measurement. Six samples were tested at one time, and they were arranged in front and back and left, center, and right positions. The rut testing was performed by Koch Pavement Solutions in Wichita, Kansas.

\section{Low Temperature Creep Testing}

Indirect tensile tests were performed to evaluate the thermal cracking susceptibility of the asphalt mixtures. The Superpave Indirect Tensile Test (IDT) was used to determine the creep compliance and indirect tensile strength of asphalt mixtures at low pavement temperatures. The test results can be used in performance models to predict the low-temperature thermal cracking potential and intermediate-temperature fatigue cracking potential of asphalt pavements. The Superpave IDT consists of a vertical loading frame, specimen deformation measurement devices, an environmental chamber, and a data acquisition and control system. The IDT loads a cylindrical asphalt concrete specimen along its diametrical axis, and the resulting deformations are used to determine the viscoelastic material properties. The test procedures are described in the American Association of State Highway and Transportation Officials (AASHTO) provisional standard TP 9-96, "Standard Test Method for Determining the Creep Compliance and Strength of Hot Mix Asphalt (HMA) Using the Indirect Tensile Test Device."

Creep compliance was tested at three temperatures: $0{ }^{\circ} \mathrm{C},-10^{\circ} \mathrm{C}$, and $-20^{\circ} \mathrm{C}$. The indirect tensile strength was then tested at $-10^{\circ} \mathrm{C}$ for each sample.

For the creep compliance tests, the specimens were cooled to the desired temperature. A preconditioning cycle was applied to each specimen consisting of 100 cycles of haversine load with amplitude equivalent to $70 \mathrm{kPa}$ tension. One load cycle consisted of 0.1 seconds of 
haversine loading and 0.9 seconds of zero-load condition. The amplitude of the load was calculated by:

$$
P(N)=11 \times 10^{4} t D
$$

Where:

$\mathrm{t}=$ specimen thickness, $\mathrm{m}$

$\mathrm{D}=$ specimen diameter, $\mathrm{m}$

Then an axial load was applied with a ramp of $8 \mathrm{kN} / \mathrm{sec}$ until one of the horizontal extensometers reached a deformation of $0.002 \mathrm{~mm}$. The range of creep loads applied can be found in Table 1 . This compressive load was held constant for 100 seconds, and the time-dependent vertical and horizontal deformations of the specimens was measured using extensometers. A typical load history for a test specimen is shown in Figure 1. The data is shown at one-second intervals, so the preconditioning load is not detailed on this graph. Knowing the load and deformations as functions of time, the creep compliance of the asphalt concrete was calculated.

After the creep test was performed at the three temperatures, the indirect tensile strength test was performed on the samples. A vertical displacement was applied to the specimen at a rate of 12.5 $\mathrm{mm}$ per minute until the load decreased (10\% reduction from the peak value) from its maximum because of specimen failure. The data acquisition system measured the peak load that the specimen was subject to. 


\section{CHAPTER 4 \\ RESULTS AND DISCUSSION}

\section{RESULTS}

\section{Aggregate Testing}

Table 4.1 provides the results for the fine aggregate angularity and sand equivalency tests conducted on the fine aggregate and compares them with the Superpave criteria for low volume roads $(<1,000,000$ ESALs). Although easily meeting the Sand Equivalent minimum value, the Lakeland aggregate barely made the $40 \%$ minimum fine aggregate angularity value. As discussed in the Mix Design section, the low fine aggregate angularity of the Lakeland aggregate is suspected to be the primary cause of the inability to meet the VMA criterion. Table 4.2 provides the same comparison for the flat/elongated particles and fractured faces tests done on the coarse aggregate. A quick review of Table 4.2 shows that all four coarse aggregates are acceptable for use in Superpave low-volume mix designs. As is typical with any non-crushed aggregate such as gravels, the Cedar Grove aggregate had a very low fractured faces valuebarely meeting the $65 \%$ minimum. As will be discussed later in the Resilient Modulus and Moisture Sensitivity sections, however, the low fractured faces percentage of the Cedar Grove aggregate appeared to have no effect on its performance as compared to the other three aggregates evaluated.

\section{Mix Design}

Figures 4.1, 4.2, and 4.3 show the air voids versus asphalt content for the coarse, fine and PG 5840 mixes, respectively. Figures 4.4, 4.5, and 4.6 show the VMA versus asphalt content for the mixes, and VFA versus asphalt content is shown in Figures 4.7, 4.8, and 4.9. Table 4.3 summarizes the data for both three and four percent air voids. As shown in Table 4.3, the VMA criterion is not satisfied by any of the mixes at $4 \%$ compacted air voids. Additionally, at $4 \%$ voids, five of the ten mixes did not meet the VFA criteria $(65 \%<\mathrm{VFA}<78 \%)$, while the remaining five had only 66\% VFA. Low VMA and VFA may result in low aggregate film thickness which may lead to accelerated aggregate stripping and other related durability problems. 
Table 4.1 Fine Aggregate Properties

\begin{tabular}{|c|c|c|}
\hline TEST & LAKELAND SAND & $\begin{array}{c}\text { Superpave Criteria } \\
\left(<\mathbf{1 0}^{\mathbf{6}} \text { ESALS) }\right.\end{array}$ \\
\hline Fine Aggregate Angularity, $\%$ & 40 & $40(\mathrm{~min})$ \\
\hline Sand Equivalent, $\%$ & 48 & $40(\mathrm{~min})$ \\
\hline
\end{tabular}

Table 4.2 Coarse Aggregate Properties

\begin{tabular}{|c|c|c|c|c|c|}
\hline TEST & $\begin{array}{c}\text { Granite Falls } \\
\text { Granite }\end{array}$ & $\begin{array}{c}\text { New Ulm } \\
\text { Quartzite }\end{array}$ & $\begin{array}{c}\text { Kasota } \\
\text { Limestone }\end{array}$ & $\begin{array}{c}\text { Cedar Grove } \\
\text { Gravel }\end{array}$ & $\begin{array}{c}\text { SP Criteria } \\
\left(<10^{\mathbf{6}} \text { ESALS }\right)\end{array}$ \\
\hline $\begin{array}{c}\text { Flat/Elongated } \\
\text { Particles } \\
\%,(1: 3 \text { Ratio })\end{array}$ & 14 & 35 & 11 & 8 & None \\
\hline $\begin{array}{c}\text { Fractured Faces } \\
\%,(\geq 1 / \geq 2)\end{array}$ & $100 / 100$ & $100 / 100$ & $100 / 100$ & $65 / 39$ & $65 /-$ \\
\hline
\end{tabular}

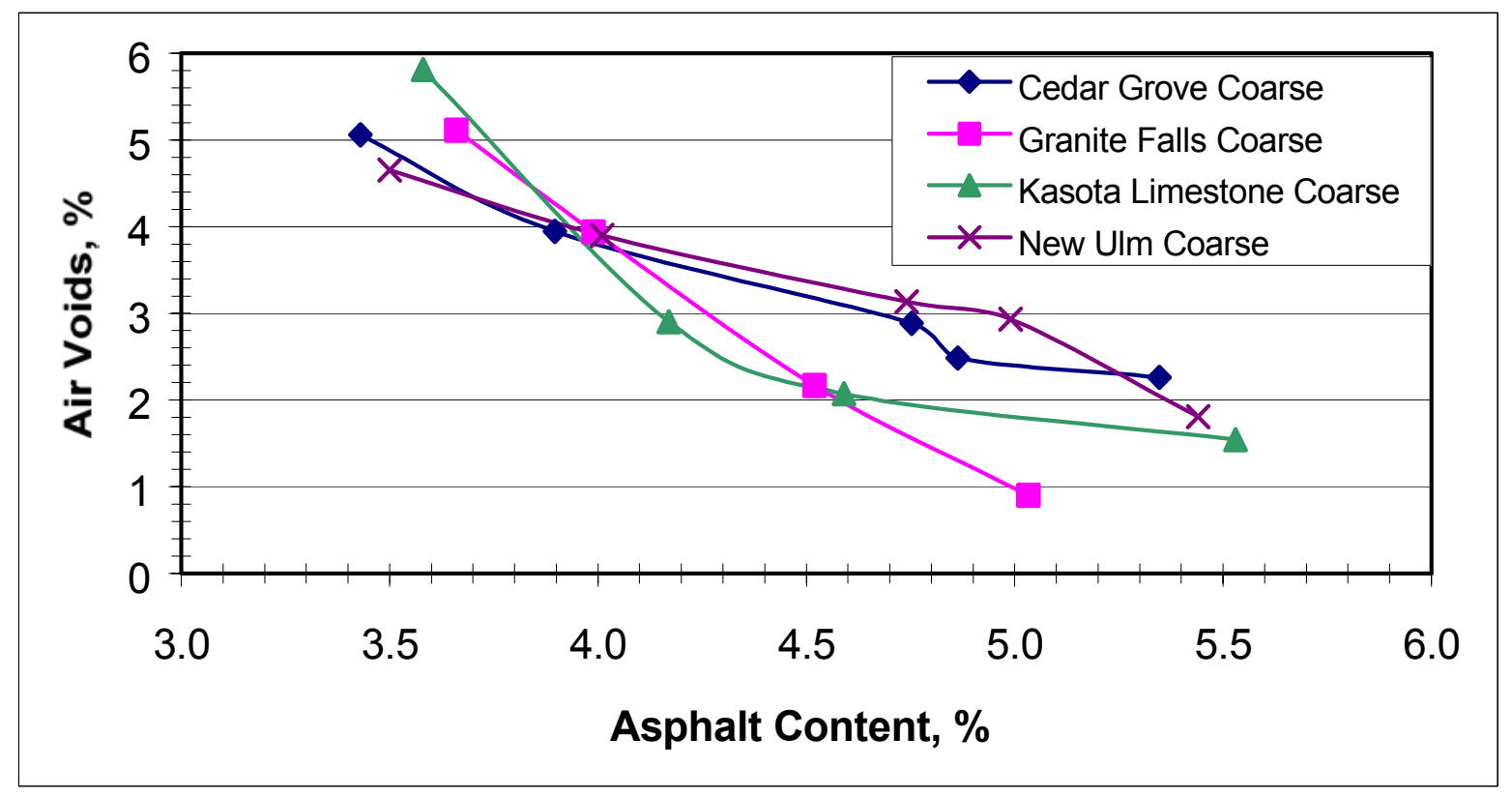

Figure 4.1 Air Voids vs. Asphalt Content for Coarse Mixes 


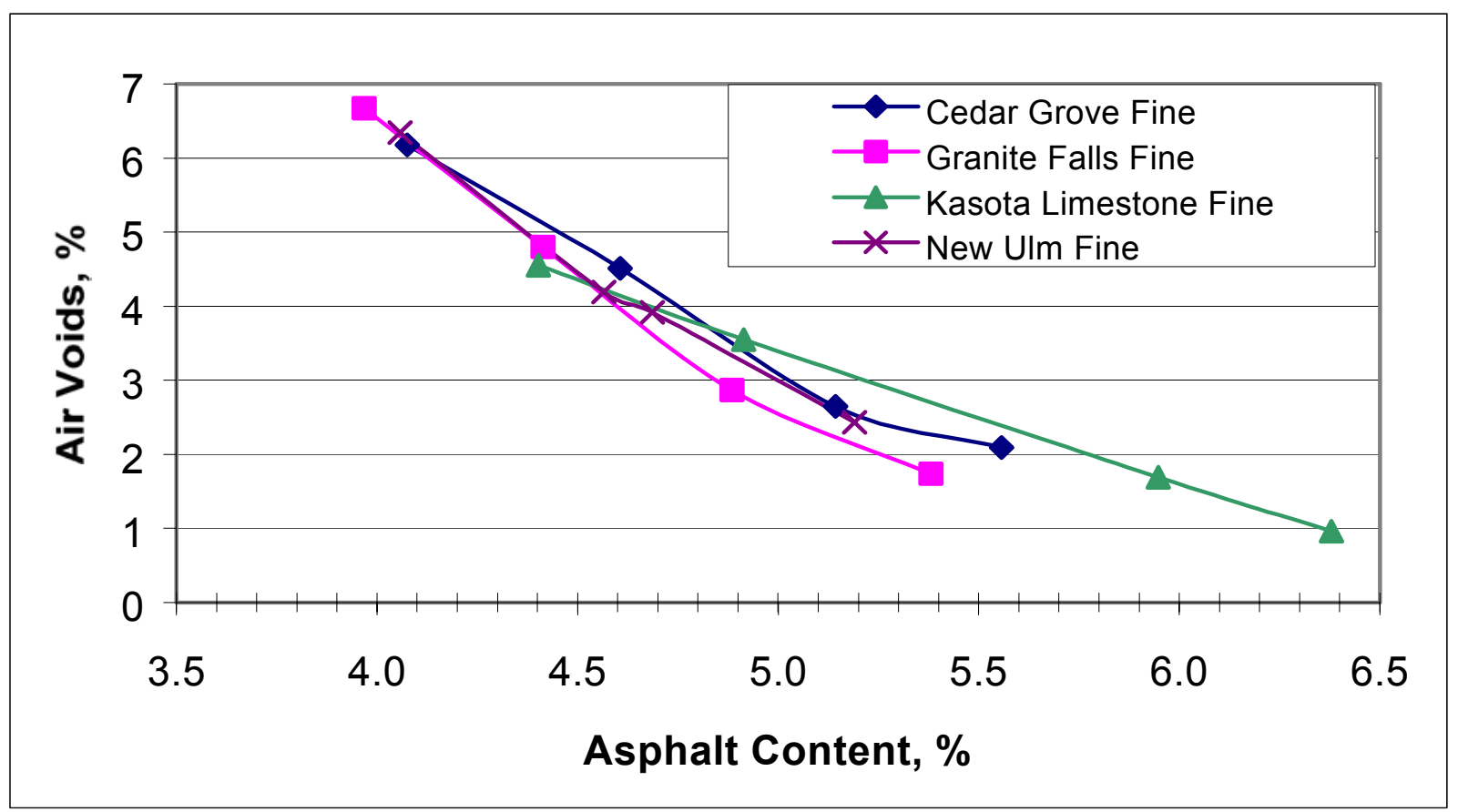

Figure 4.2 Air Voids vs Asphalt Content for Fine Mixes

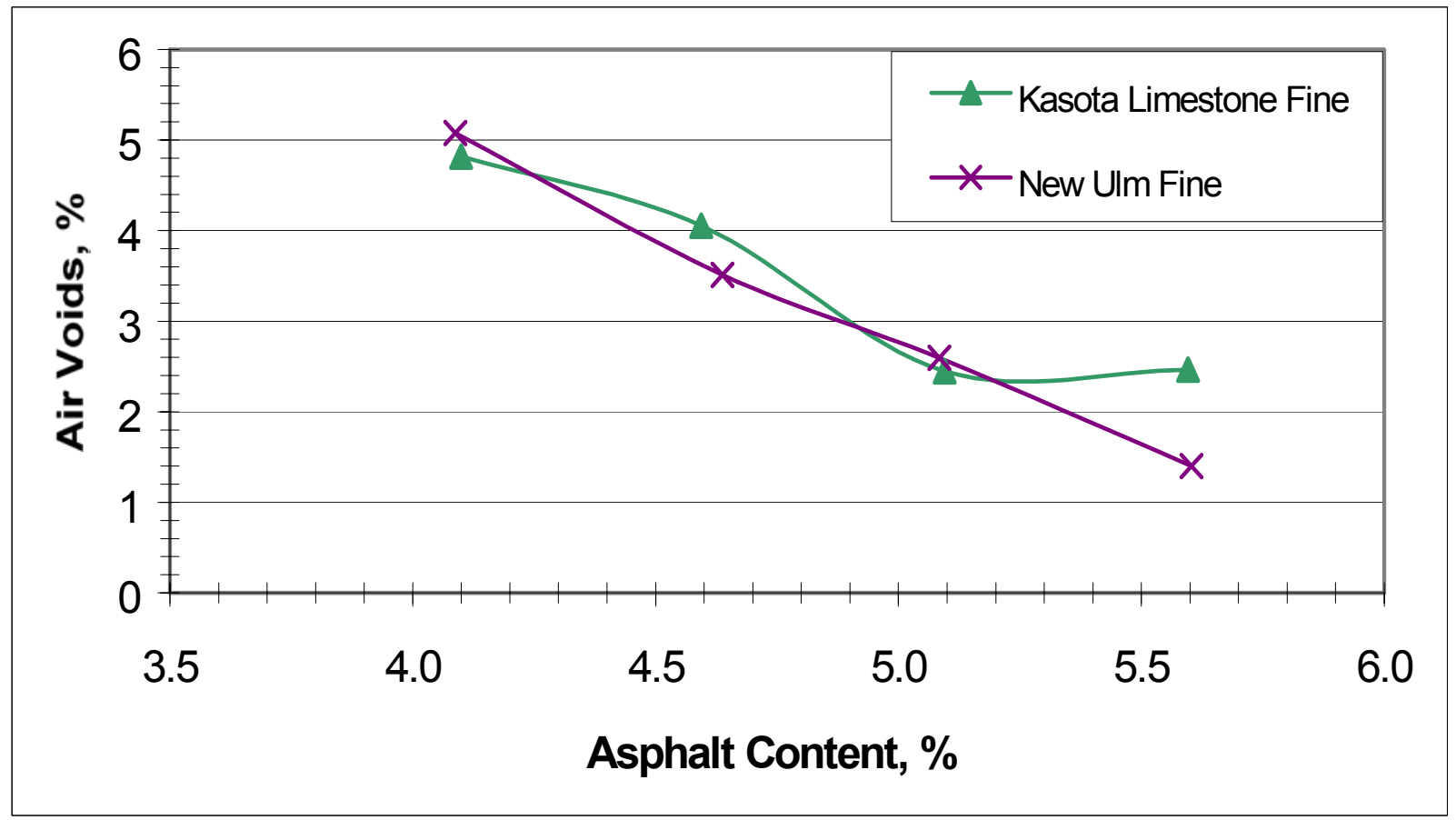

Figure 4.3 Air Voids vs Asphalt Content for PG 58-40 Mixes 


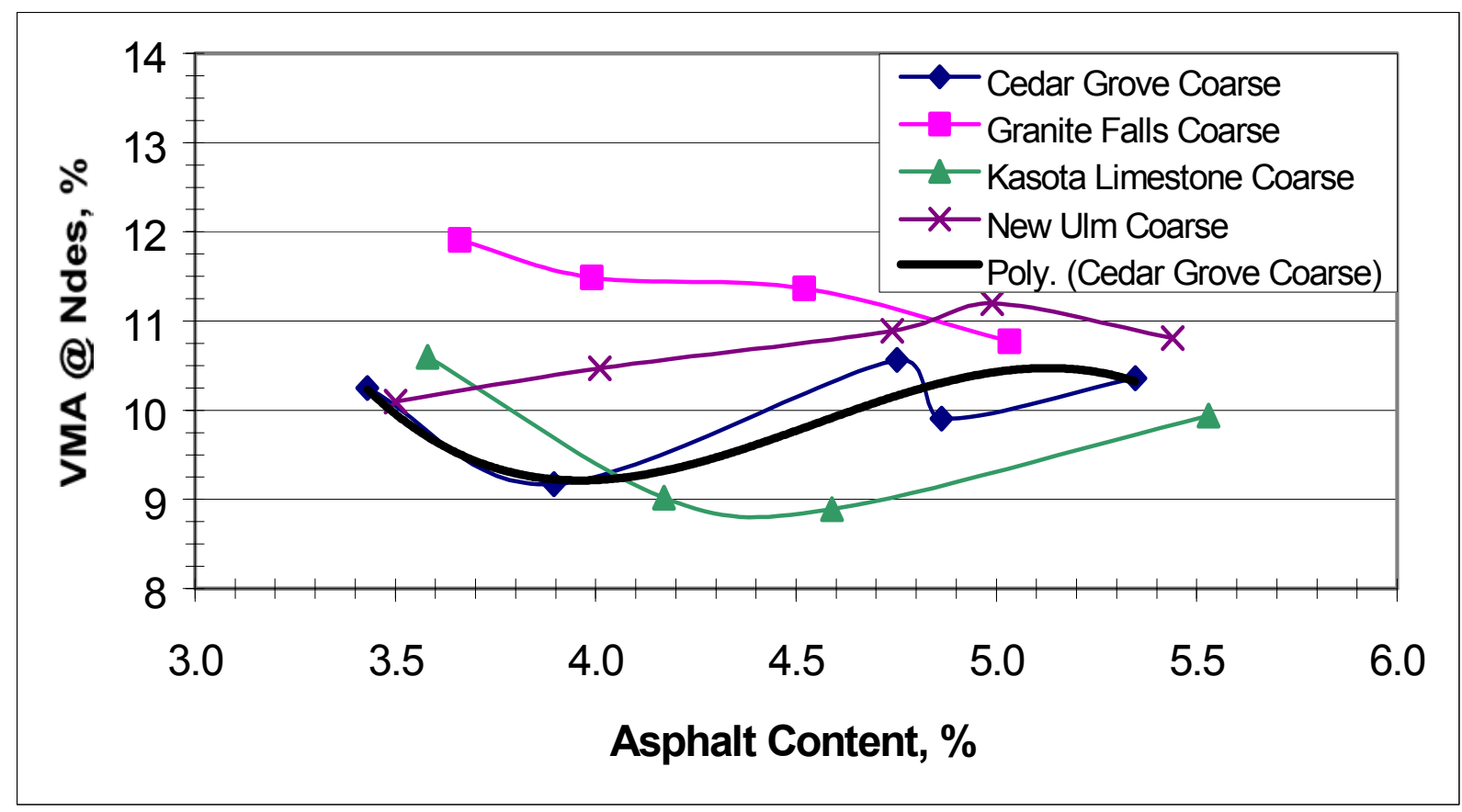

Figure 4.4 VMA@ $\mathbf{N}_{\text {des }}$ vs. Asphalt Content for Coarse Mixes

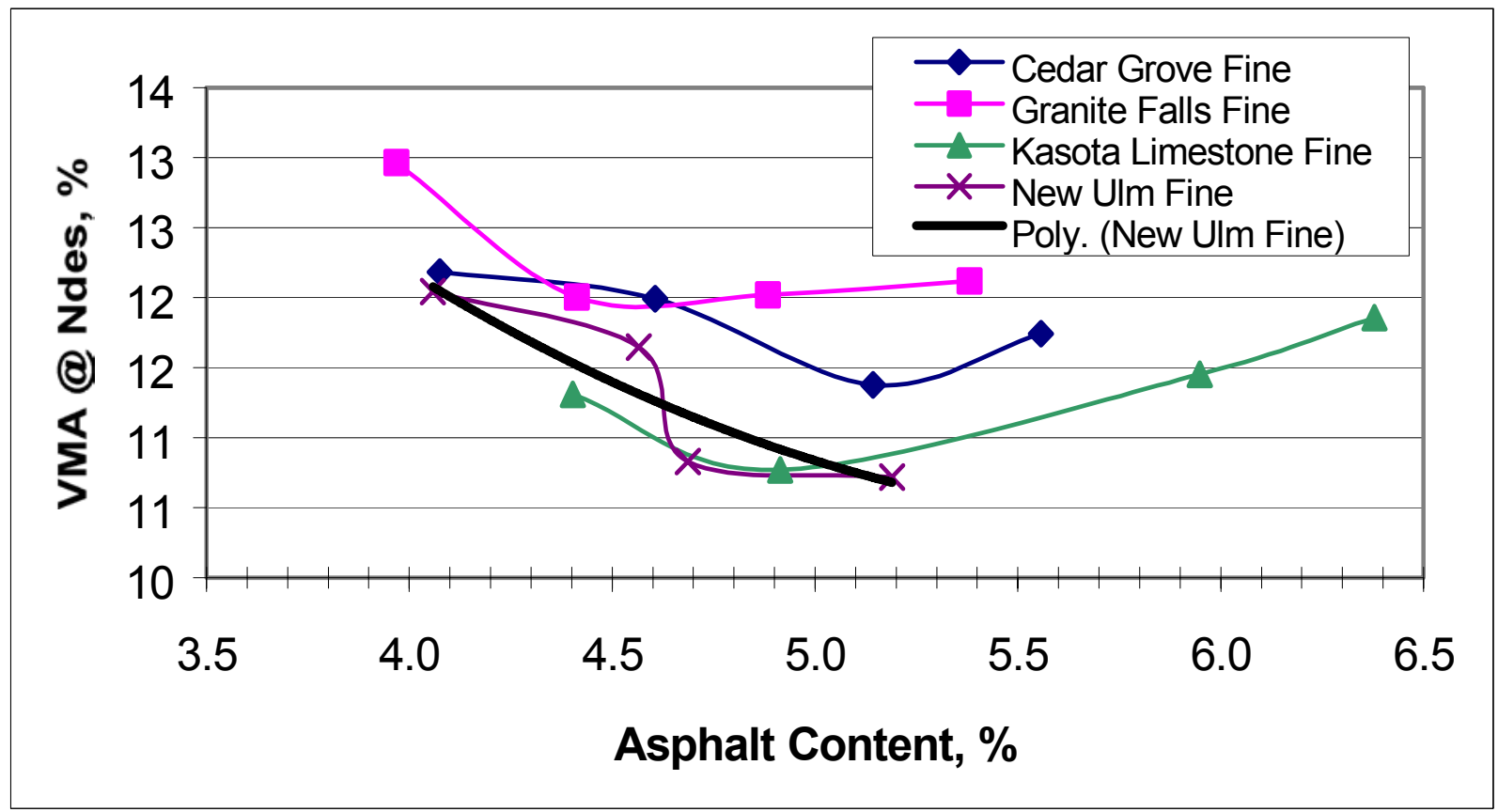

Figure 4.5 VMA@ $\mathbf{N}_{\text {des }}$ vs. Asphalt Content for Fine Mixes 


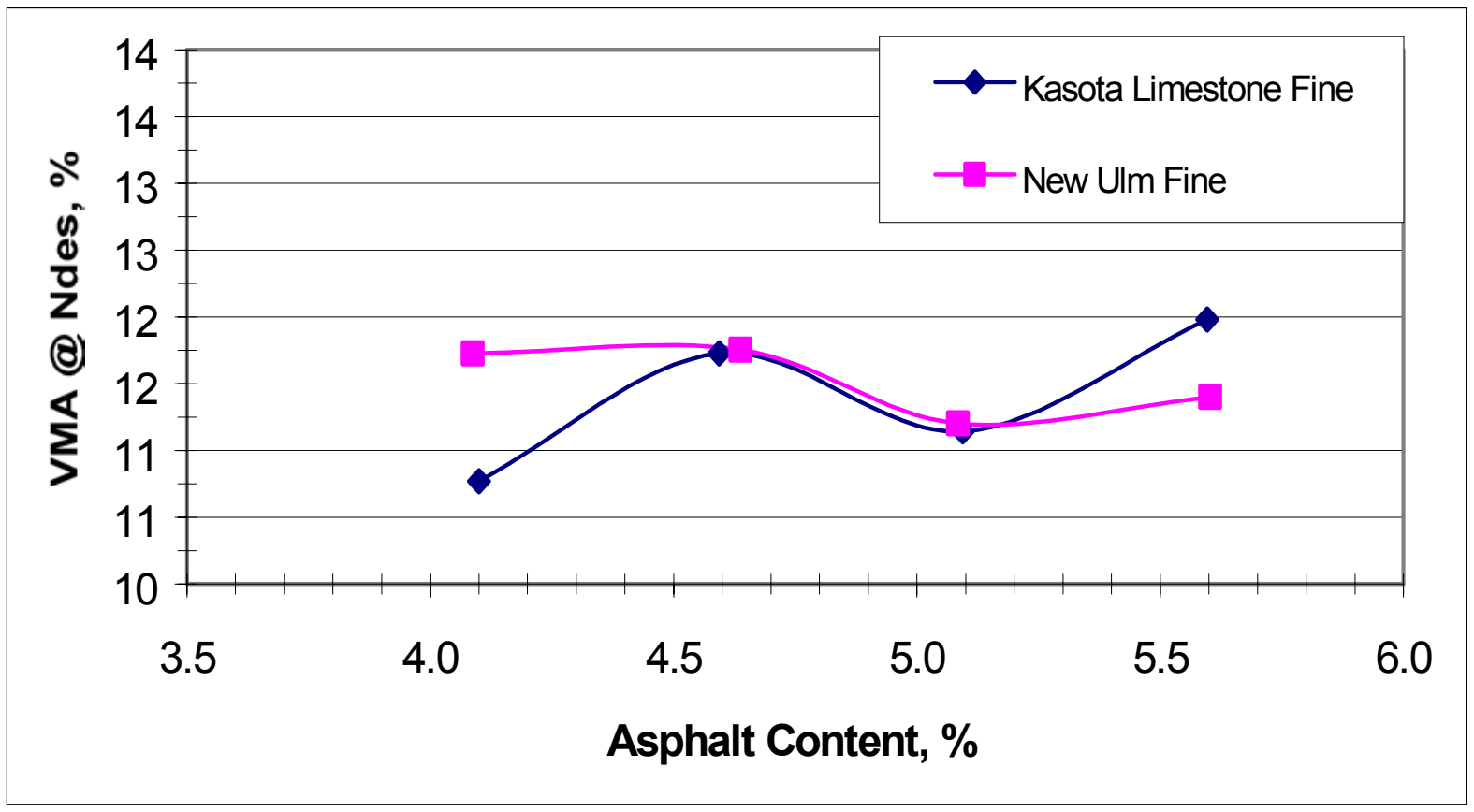

Figure 4.6 VMA@ $\mathbf{N}_{\text {des }}$ vs. Asphalt Content for PG 58-40 Mixes

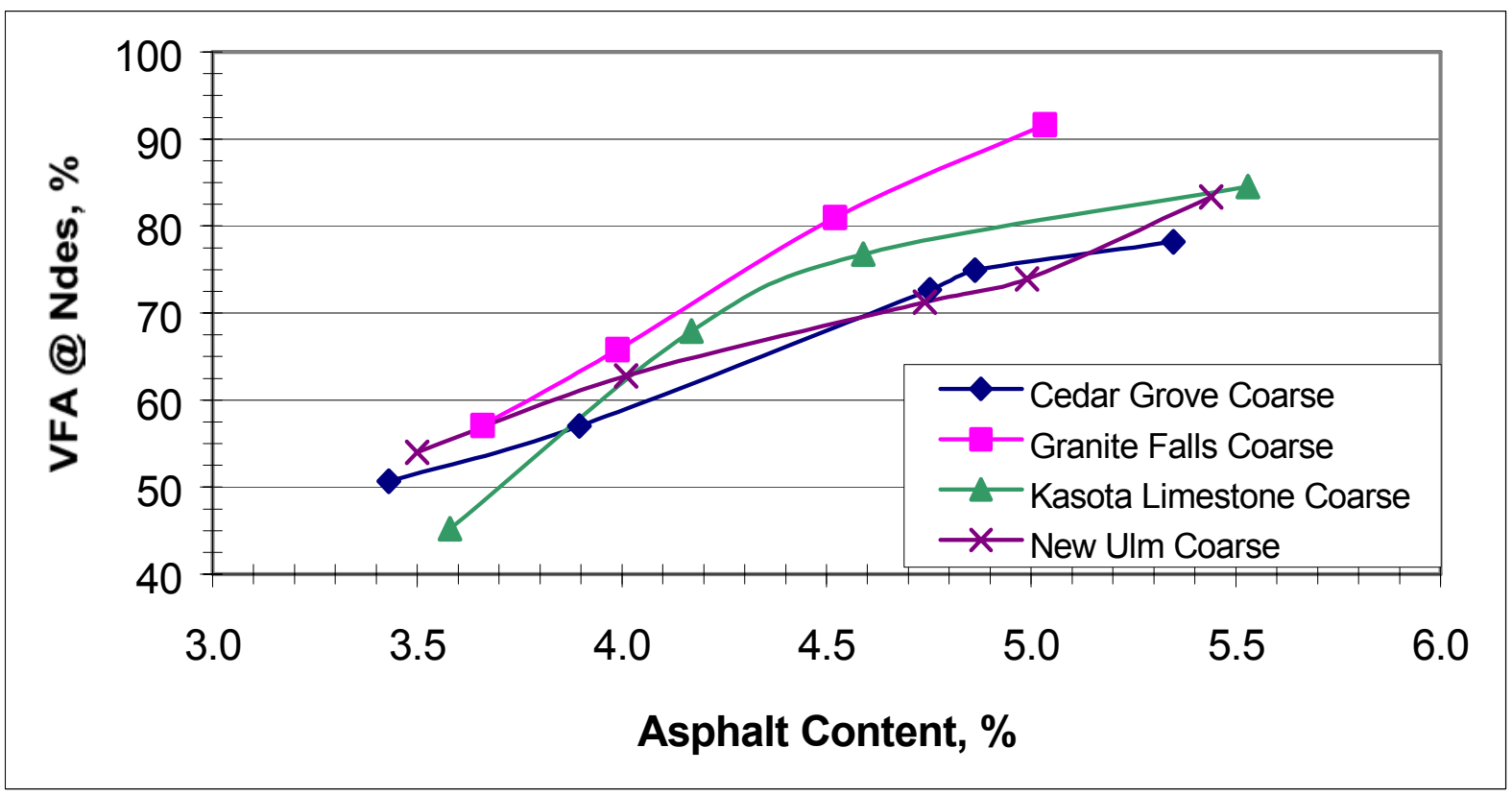

Figure 4.7 VFA@ $\mathbf{N}_{\text {des }}$ vs. Asphalt Content for Coarse Mixes 


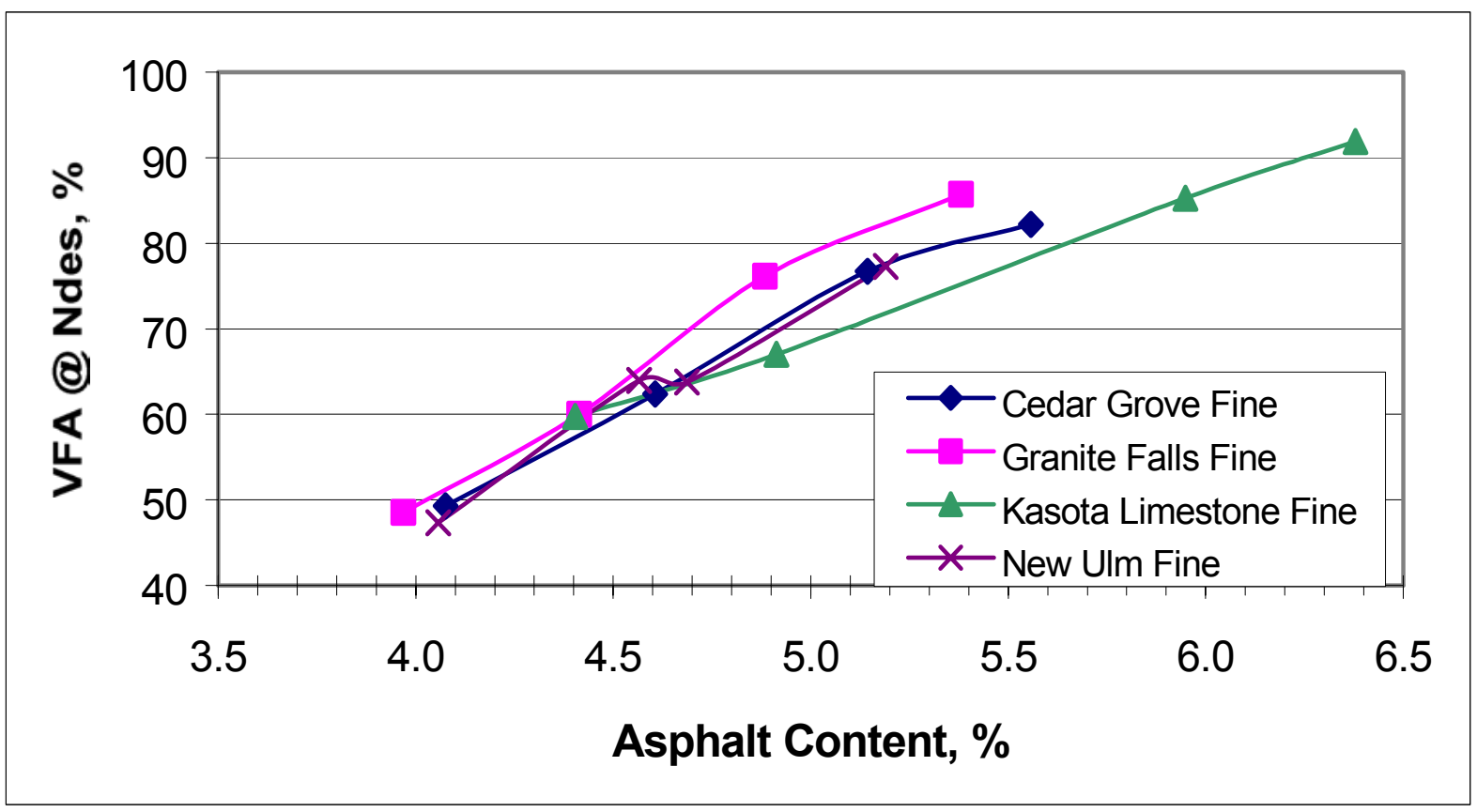

Figure 4.8 VFA@ $\mathbf{N}_{\text {des }}$ vs. Asphalt Content for Fine Mixes

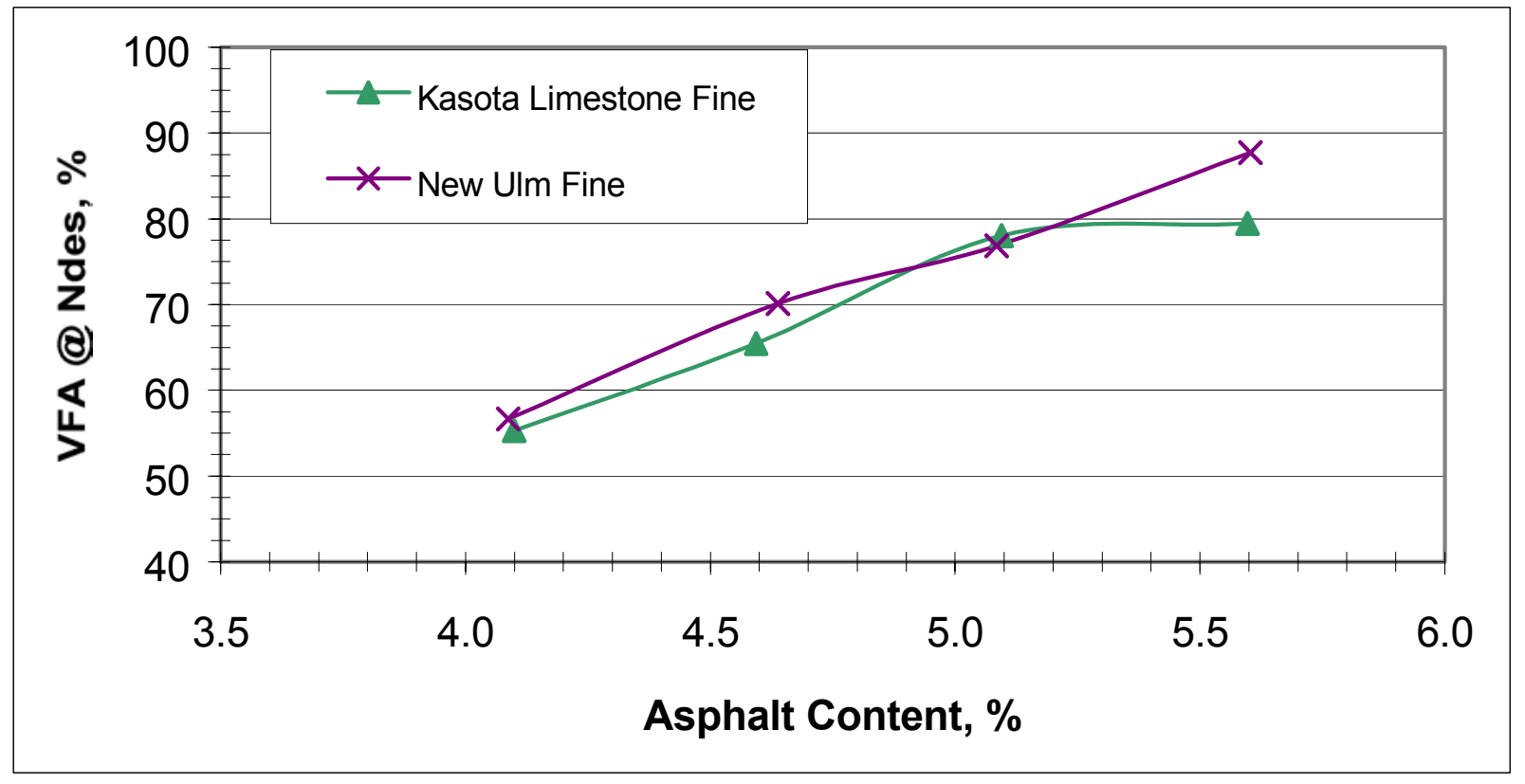

Figure 4.9 VFA@ $\mathbf{N}_{\text {des }}$ vs. Asphalt Content for PG 58-40 Mixes 
Table 4.3 Summarized Mixed Design Results @ 4\% Air Voids*

\begin{tabular}{|c|c|c|c|c|c|c|}
\hline Aggregate & $\begin{array}{c}\text { AC Content } \\
\text { @ 4\% Voids }\end{array}$ & $\begin{array}{c}\text { VMA, \% } \\
(\mathbf{1 4 \%})\end{array}$ & $\begin{array}{c}\text { VFA, \% } \\
\mathbf{( 6 5 - 7 8 \% )}\end{array}$ & $\begin{array}{c}\text { \% Gmm @ } \\
\text { Nini < 90.5\%? }\end{array}$ & $\begin{array}{c}\text { \% Gmm @ } \\
\text { Nmax < 98\%? }\end{array}$ & $\begin{array}{c}\text { Dust Prop b/w } \\
\mathbf{0 . 6} \text { and 1.2? }\end{array}$ \\
\hline GFC & 4.00 & $\underline{11.5}$ & 66 & 88.2 & 97.1 & 1.04 \\
\hline GFF & 4.60 & 11.9 & 66 & $\underline{90.8}$ & 96.7 & 0.87 \\
\hline NUC & 3.95 & $\underline{10.4}$ & $\underline{63}$ & 87.6 & 97.1 & 1.04 \\
\hline NUF & 4.65 & $\underline{10.8}$ & $\underline{64}$ & $\underline{90.8}$ & 96.7 & 0.87 \\
\hline KLC & 3.95 & $\underline{9.6}$ & $\underline{59}$ & 88.3 & 97.3 & 1.04 \\
\hline KLF & 4.65 & $\underline{10.8}$ & $\underline{64}$ & $\underline{90.9}$ & 96.8 & 0.87 \\
\hline CGC & 3.90 & $\underline{9.75}$ & $\underline{56}$ & 88.0 & 97.1 & 1.06 \\
\hline CGF & 4.75 & $\underline{11.8}$ & 66 & $\underline{90.7}$ & 96.7 & 0.84 \\
\hline NUF (58-40) & 4.45 & $\underline{11.6}$ & 66 & $\underline{90.9}$ & 96.6 & 0.89 \\
\hline KLF (58-40) & 4.60 & $\underline{11.5}$ & 66 & $\underline{90.8}$ & 96.7 & 0.87 \\
\hline
\end{tabular}

*Underlined values do not meet Superpave criteria.

Table 4.4 Summarized Mixed Design Results@3\% Air Voids*

\begin{tabular}{|c|c|c|c|c|c|c|}
\hline Aggregate & $\begin{array}{c}\text { AC Content } \\
\text { @ 4\% Voids }\end{array}$ & $\begin{array}{c}\text { VMA, \% } \\
(\mathbf{1 3} \%)\end{array}$ & $\begin{array}{c}\text { VFA, \% } \\
(\mathbf{6 5 - 7 8})\end{array}$ & $\begin{array}{c}\mathbf{\%} \text { Gmm @ } \\
\text { Nini }<\mathbf{9 0 . 5 \%} ?\end{array}$ & $\begin{array}{c}\text { \% Gmm @ } \\
\text { Nmax < 98\%? }\end{array}$ & $\begin{array}{c}\text { Dust Prop b/w } \\
\mathbf{0 . 6} \text { and 1.2? }\end{array}$ \\
\hline GFC & 4.30 & $\underline{11.5}$ & 74 & 90.1 & $\underline{98.2}$ & 0.97 \\
\hline GFF & 4.80 & $\underline{12.4}$ & 74 & $\underline{91.6}$ & 97.6 & 0.84 \\
\hline NUC & 4.95 & $\underline{11.3}$ & 73 & 88.4 & $\underline{98.1}$ & 0.82 \\
\hline NUF & 5.00 & $\underline{10.5}$ & 73 & $\underline{91.8}$ & 97.6 & 0.80 \\
\hline KLC & 4.20 & $\underline{9.0}$ & 70 & 89.0 & $\underline{98.1}$ & 0.97 \\
\hline KLF & 5.20 & $\underline{10.8}$ & 72 & $\underline{91.7}$ & 97.8 & 0.74 \\
\hline CGC & 4.70 & $\underline{10.5}$ & 72 & 88.7 & $\underline{98.2}$ & 0.86 \\
\hline CGF & 5.00 & $\underline{11.5}$ & 74 & $\underline{91.5}$ & 97.5 & 0.80 \\
\hline NUF (58-40) & 4.90 & $\underline{11.4}$ & 74 & $\underline{92.0}$ & 97.7 & 0.82 \\
\hline KLF (58-40) & 4.90 & $\underline{11.3}$ & 74 & $\underline{91.9}$ & 97.7 & 0.82 \\
\hline
\end{tabular}

*Underlined values do not meet Superpave criteria. 
An analysis of the mix design data at 3\% air voids shows no significant change in VMA but large increases in VFA. At 3\% voids, all 10 mixes satisfy the Superpave VFA criterion. The substantial increase in VFA at 3\% air voids should reduce the concern over aggregate film thickness. Therefore, it was determined the target asphalt content for the project would be based on $3 \%$ air voids.

As shown in Table 2.1, at 3\% air voids, none of the mixes satisfied both Superpave criteria for compacted densities at Nini and Nmax. None of the fine gradation mixes (including the two PG $58-40$ mixes) met the $<89 \%$ of maximum density criterion at Nini. Similarly, none of the coarse gradation mixes met the $>98 \%$ of maximum density criterion at Nmax. The implications associated with these observations will be addressed in the Discussions section. All 10 mixes met the Superpave dust proportion criterion of 0.6 to 1.2.

Although neither is significantly higher, the Granite Falls aggregate appeared to have the highest VMA of the four coarse aggregates and the PG 58-40 binder bettered the PG 52-34 binder. Another interesting observation shown in Table 4.4 concerns the optimum asphalt content of the coarse graded Kasota limestone mix. Although, the Kasota limestone has the highest water absorption of the four coarse aggregates (see Table 3.3), it had the lowest optimum asphalt content. Although no explanation is known, such a phenomenon is normally the result of either equipment or operator error.

\section{Resilient Modulus}

Three samples from each mix type were tested in accordance with ASTM D4123. The average values by temperature for each mix type are shown in Table 4.5. The average coefficient of variation $(\mathrm{CV})$ for each mix is also provided in Table 4.5. Complete test results are provided in Appendix B.

A comparison of coarse versus fine gradations is illustrated in Figure 4.10. Average values for the four coarse mixes and for the four fine mixes were used to make the comparison shown in Figure 4.10. As shown by Figure 4.10, the two gradations are virtually indistinguishable from one another. 
Table 4.5 Average Resilient Modulus Test Results

\begin{tabular}{|c|c|c|c|c|c|c|c|c|c|}
\hline \multirow[b]{2}{*}{ GRADATION } & \multirow{2}{*}{$\begin{array}{l}\text { Test Temperature } \\
\text { Freqency }=1.0 \mathrm{hz}\end{array}$} & \multicolumn{2}{|c|}{-18} & \multicolumn{2}{|c|}{0} & \multicolumn{2}{|c|}{25} & \multicolumn{2}{|c|}{40} \\
\hline & & $\begin{array}{c}\text { Res Mod } \\
(\mathrm{kPa})\end{array}$ & $\begin{array}{l}\text { Coef. of } \\
\text { Var. (\%) }\end{array}$ & $\begin{array}{l}\text { Res Mod } \\
\text { (kPa) }\end{array}$ & $\begin{array}{l}\text { Coef. of } \\
\text { Var. (\%) }\end{array}$ & $\begin{array}{c}\text { Res Mod } \\
(\mathrm{kPa})\end{array}$ & $\begin{array}{l}\text { Coef. of } \\
\text { Var. (\%) }\end{array}$ & $\begin{array}{l}\text { Res Mod } \\
\text { (kPa) }\end{array}$ & $\begin{array}{l}\text { Coef. of } \\
\text { Var. (\%) }\end{array}$ \\
\hline \multirow{4}{*}{ 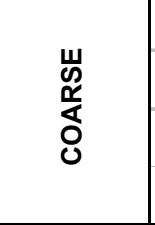 } & Granite Falls Granite & 12204 & 17.71 & 9273 & 3.06 & 1676 & 2.53 & 435 & 3.71 \\
\hline & New Ulm Quartzite & 14242 & 17.05 & 8160 & 5.15 & 1517 & 1.83 & 442 & 2.69 \\
\hline & Kasota Limestone & 11560 & 18.07 & 9579 & 3.72 & 2224 & 2.08 & 705 & 4.84 \\
\hline & Cedar Grove Gravel & 14228 & 23.32 & 9179 & 3.94 & 1835 & 1.74 & 526 & 3.50 \\
\hline \multirow{4}{*}{$\underset{\underline{L}}{\underline{\underline{L}}}$} & Granite Falls Granite & 12326 & 20.13 & 9446 & 3.60 & 1749 & 1.74 & 545 & 3.72 \\
\hline & New Ulm Quartzite & 13115 & 14.87 & 9429 & 4.66 & 1920 & 1.55 & 571 & 3.25 \\
\hline & Kasota Limestone & 12393 & 11.41 & 9356 & 4.54 & 1874 & 1.68 & 598 & 4.17 \\
\hline & Cedar Grove Gravel & 11841 & 16.42 & 9325 & 4.61 & 1777 & 1.95 & 508 & 4.79 \\
\hline \multirow{2}{*}{$\begin{array}{c}\text { FINE } \\
(58-40)\end{array}$} & New Ulm Quartzite & 11797 & 10.50 & 5950 & 2.78 & 1136 & 0.94 & 636 & 2.50 \\
\hline & Kasota Limestone & 12392 & 10.86 & 5812 & 3.68 & 1316 & 1.02 & 548 & 2.74 \\
\hline
\end{tabular}

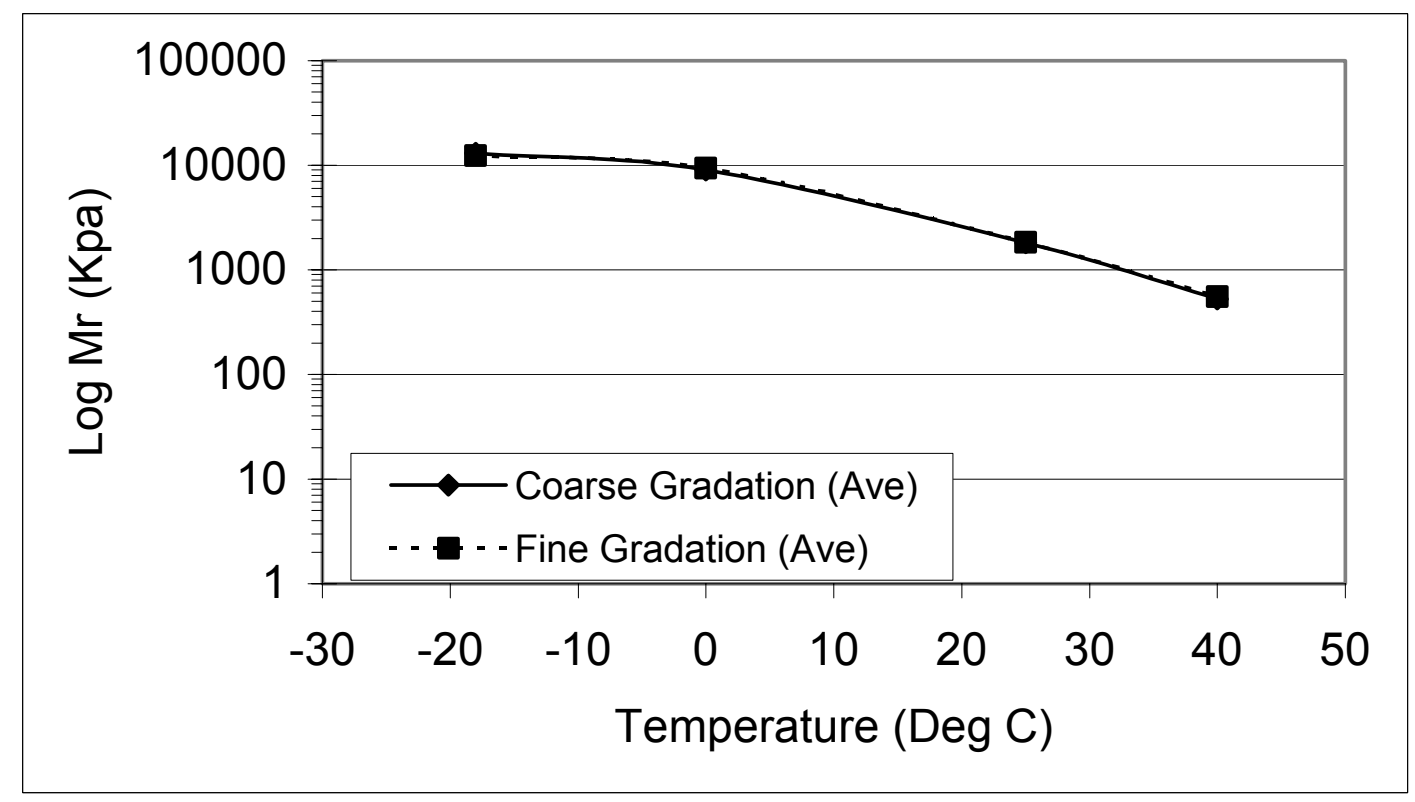

Figure 4.10 Influence of Gradation on Stiffness

The four aggregates (granite, quartzite, limestone, and gravel) are compared in Figure 4.11. Here, the coarse and fine gradations for each aggregate type were averaged together. The Kasota limestone has a slightly higher warm temperature resilient modulus, and the remaining three aggregate types are indistinguishable from one another. 


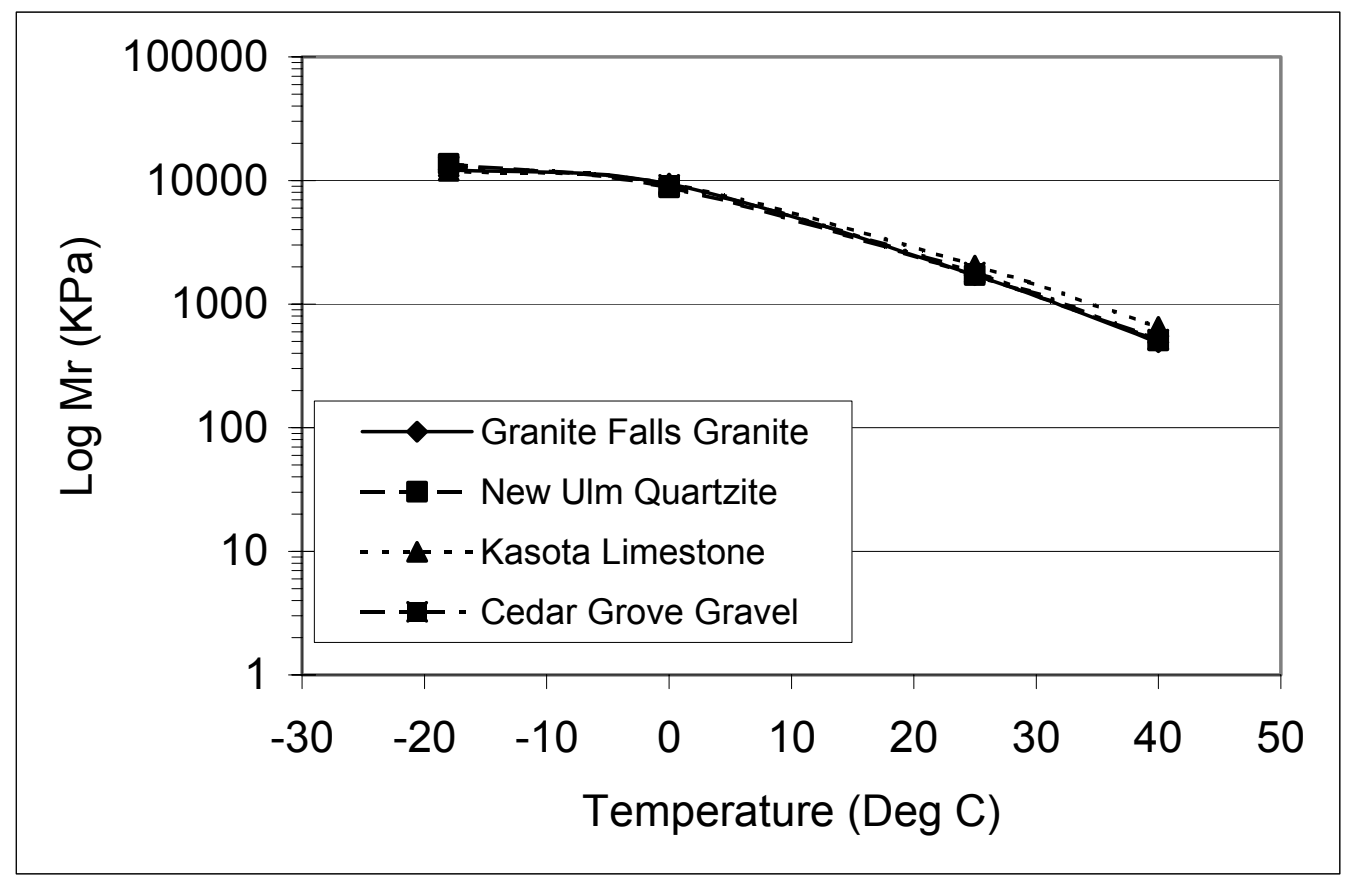

Figure 4.11 Influence of Aggregate on Stiffness

Finally, the two asphalt grades (PG 52-34 verses PG 58-40) are compared in Figure 4.12. This comparison was made by averaging the New Ulm and Kasota values together for each asphalt grade. The results of the performance grade comparison somewhat surprising. It was expected the two grades would have similar resilient moduli at the moderate temperatures and different moduli at the warmest and coldest temperatures. The purpose of the performance graded asphalt system is to ensure adequate pavement flexibility at cold temperatures to reduce cold temperature cracking and adequate stiffness at high tempures to reduce permanent deforemation. Therefore, had the samples been tested at temperature extremes closer to the PG 58-40 rating (i.e. $-40^{\circ} \mathrm{C}\left(-40^{\circ} \mathrm{F}\right)$ and $\left.58^{\circ} \mathrm{C}\left(136^{\circ} \mathrm{F}\right)\right)$, it is expected the PG $58-40$ would have had a lower resilient modulus at the cold extreme and a higher resilient modulus at the hot extreme than the PG 52-34.

\section{Moisture Sensitivity}

Table 4.6 provides a summary of the moisture sensitivity results. As expected, the effects of the vacuum saturation and the 24-hour warm water bath caused the samples to swell (increase in volume). Superpave criteria require a minimum tensile strength ratio of 80 percent. The TSRs for the 10 mixtures evaluated were all above 95 percent. Although, still debated, high TSR 
values such as these may indicate a lower susceptibility to moisture damage. Complete test results are provided in Appendix C.

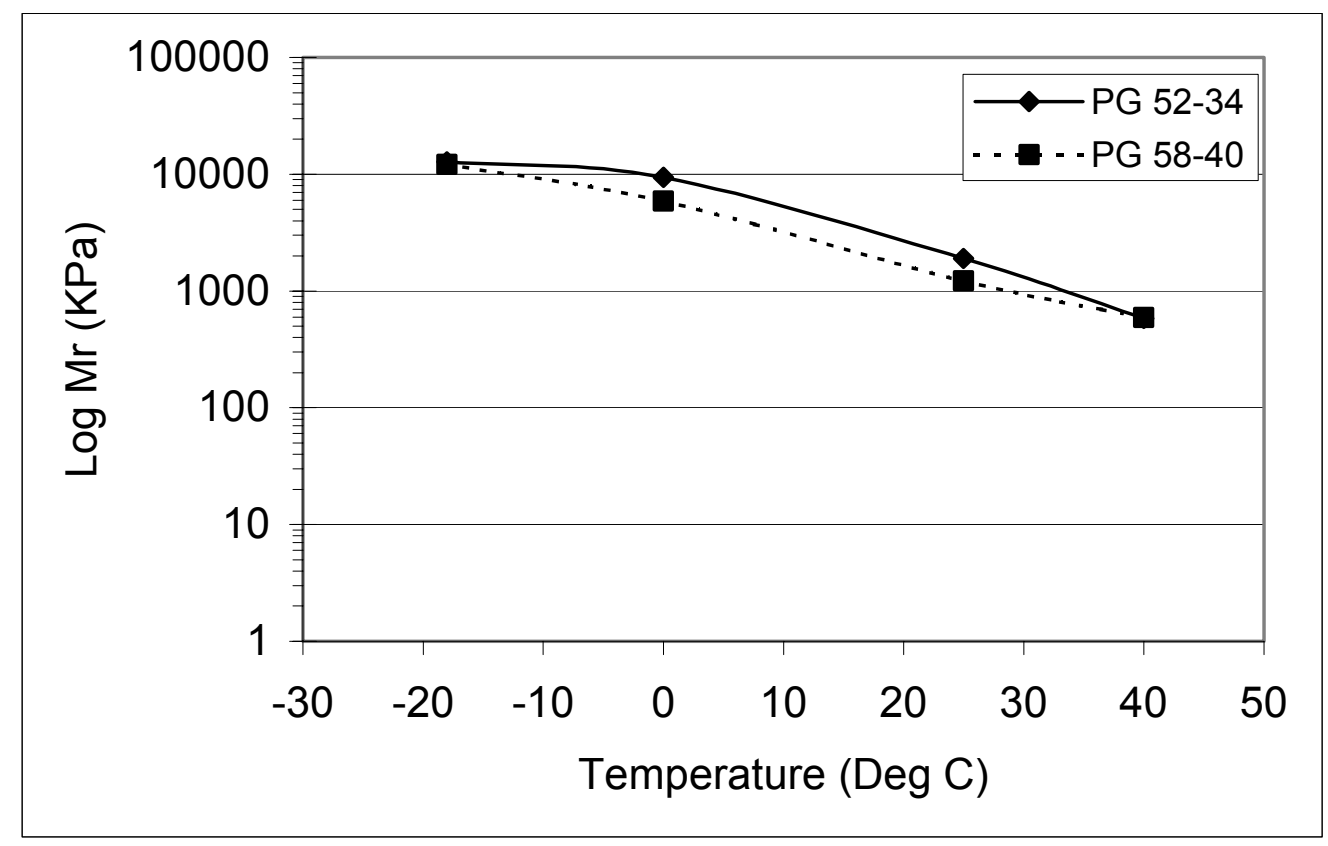

Figure 4.12 Influence of Asphalt Grade on Stiffness

Table 4.6 Summarized Moisture Sensitivity Results

\begin{tabular}{|c|c|c|c|c|c|c|c|c|c|c|c|}
\hline \multirow{2}{*}{$\begin{array}{l}\text { SAMPLE } \\
\text { ID }\end{array}$} & \multirow{2}{*}{ Measurement } & \multicolumn{4}{|c|}{ PG 52-34 Coarse Gradation } & \multicolumn{4}{|c|}{ PG 52-34 Fine Gradation } & \multicolumn{2}{|c|}{ PG 58-40 Fine } \\
\hline & & GFC & NUC & KLC & CGC & GFF & NUF & KLF & CGF & NUF & KLF \\
\hline \multirow{2}{*}{$\begin{array}{l}\text { Unconditioned } \\
\text { Samples }\end{array}$} & Load (lbf) & 4988.3 & 4970.8 & 4966.2 & 4945.7 & 4958.9 & 4957.0 & 4980.6 & 4962.7 & 4967.3 & 4957.7 \\
\hline & Dry Strength & 25779 & 25705 & 25094 & 25460 & 25082 & 25662 & 25434 & 25616 & 25275 & 24976 \\
\hline $\begin{array}{l}\text { Unconditioned } \\
\text { Samples }\end{array}$ & $\%$ Air Voids & 6.56 & 6.79 & 6.80 & 7.43 & 6.76 & 6.63 & 6.70 & 7.12 & 6.80 & 7.21 \\
\hline $\begin{array}{c}\text { Conditioned } \\
\text { Samples }\end{array}$ & $\%$ Air Voids & 6.66 & 7.01 & 6.73 & 7.60 & 6.77 & 6.46 & 6.67 & 7.51 & 6.83 & 7.37 \\
\hline \multirow{2}{*}{$\begin{array}{l}\text { After Vacuum } \\
\text { Saturation }\end{array}$} & $\%$ Saturation & 68.37 & 69.30 & 69.39 & 66.74 & 71.53 & 59.22 & 69.87 & 70.23 & 69.24 & 76.58 \\
\hline & $\%$ Swell & 3.41 & 4.32 & 3.78 & 3.84 & 3.03 & 3.01 & 3.32 & 4.59 & 3.53 & 3.93 \\
\hline \multirow{4}{*}{$\begin{array}{c}\text { After } 140^{\circ} \mathrm{F} \text { 24-hr } \\
\text { Water Bath }\end{array}$} & $\%$ Saturation & 86.58 & 104.43 & 79.99 & 82.09 & 99.84 & 79.23 & 86.70 & 82.56 & 89.13 & 108.33 \\
\hline & $\%$ Swell & 4.84 & 6.70 & 5.19 & 5.09 & 5.28 & 4.38 & 4.40 & 5.58 & 4.93 & 6.31 \\
\hline & Load (Ibf) & 4976.7 & 4981.3 & 4971.2 & 4971.9 & 4957.4 & 4996.2 & 4971.7 & 4973.2 & 4966.9 & 4966.9 \\
\hline & Wet Strength & 24814 & 25096 & 24325 & 25178 & 24338 & 25370 & 24699 & 25170 & 25030 & 24756 \\
\hline $\begin{array}{c}\text { Tensile Strength } \\
\text { Ratio }\end{array}$ & TSR & 96.73 & 97.62 & 96.93 & 98.88 & 96.97 & 99.46 & 97.11 & 98.26 & 99.03 & 99.34 \\
\hline
\end{tabular}




\section{Rut Testing}

The Asphalt Pavement Analyzer tested six samples from each mix type. The mixes were all tested at $40{ }^{\circ} \mathrm{C}$, even though there were two different grades of asphalt binder. The average rut depth measurements after 8000 cycles for each mix type are shown in Table 3. The average coefficient of variation (COV) for each mix is also provided in Table 3.

A comparison of the four mix types is illustrated in Figure 6. The six samples were averaged for each of the four mixes to make the rut depth comparisons in Figure 6. For the PG 58-40 binder, the New Ulm quartzite and Kasota limestone had virtually the same rut depth. They both had approximately $1 \mathrm{~mm}$ of rutting after 8000 cycles in the APA. The mixes using PG 52-34 asphalt binders showed significantly more rutting. This behavior is expected since the PG 52-34 is a lower grade binder. The Kasota Limestone PG 52-34 mix had the largest rut depths at over 6.5 $\mathrm{mm}$.

\section{Low Temperature Creep Testing}

The Superpave Indirect Tensile Test (IDT) assessed the creep compliance and indirect tensile strength on three samples of each of the four mixes. The creep compliance was tested at three temperatures: $0{ }^{\circ} \mathrm{C},-10{ }^{\circ} \mathrm{C}$, and $-20{ }^{\circ} \mathrm{C}$. The strength was then tested at $-10{ }^{\circ} \mathrm{C}$. For each of the four mixes, a plot of creep compliance vs. time was created. These plots are shown in Figures 25.

For all of the mixes, as the test temperature decreases, the creep compliance also decreases. This results in a stiffer mix at lower temperatures. In addition, Young's modulus increases as the temperature decreases. In the elastic range of the material (at time $t=0$ ), the modulus is the inverse of the creep compliance. Young's modulus was not actually calculated, but can be inferred qualitatively from Figures 2-5.

The indirect tensile strength of each mix is shown in Table 2. Also included is the Poisson's ratio calculated at half of the maximum load for each specimen. The indirect tensile strength was tested at $-10{ }^{\circ} \mathrm{C}$. 


\section{DISCUSSION}

\section{Mix Design}

One of the more important mix design issues to discuss is the difficulty in achieving the minimum voids in the mineral aggregate (VMA) criterion. As described in the mix design methodology, eight different gradations were tried in the attempt to meet the 14\% Superpave VMA criterion. In fact, this is not an isolated case. If there is a common theme in Superpave experiences, it is a difficulty in achieving the minimum VMA criterion. Kandhal, et al [21] attribute the problem to the increased compactive effort of the gyratory compactor and the increased use of coarser aggregate mixes.

McLeod [6] first presented the concept of volumetric proportioning to the Highway Research Board in 1956. He developed his volumetric criteria based on specimens compacted with a Marshall hammer (75 blows on each side). McLeod [6] concluded that to ensure adequate durability in a mixture compacted to 5 percent air voids, the mixture must contain a minimum VMA of 15 percent and a minimum asphalt content of 4.5 percent. Further work by McLeod [6] related minimum VMA criteria to nominal maximum particle size of the aggregate. Since VMA is the sum of air voids and voids filled with asphalt, the minimum VMA criteria can be extrapolated to 14 percent for four percent air voids, and 13 percent for three percent air voids. McLeod's original 15\% minimum VMA criterion was adopted by the Asphalt Institute in 1964 and revised to include the extrapolated values in 1993 for their MS-2 manual [22]. The revised minimum VMA requirements have also been included in the Superpave mix design. However, the VMA criteria were developed for denser aggregate gradations commonly used in the Marshall mix design. Therefore, it may be questionable to require coarser Superpave mixes to meet the same VMA criteria as denser Marshall mixes.

The rationale behind specifying a minimum VMA percentage is to ensure that the mix contains enough asphalt cement to adequately coat the aggregate particles. This asphalt coating of the aggregate is known as the asphalt film thickness. Adequate film thickness is essential for a longlasting, durable mix. Campen, et al [23] demonstrated that thicker films produced mixes that were flexible and durable. Thin films produced mixes that were brittle and exhibited excessive cracking, raveling, poor performance, and reduced longevity. Research by Campen, et al [23] 
showed an optimum film thickness of 6 to $8 \mu \mathrm{m}$. They also found that to achieve the desired 6 to $8 \mu \mathrm{m}$ thickness the asphalt binder requirement increased as surface areas increased, but at a much lower rate.

Recently, a new approach to ensuring adequate film thickness has surfaced. Rather than use VMA to indirectly ensure adequate film thickness, proponents of this new approach suggest estimating the film thickness directly. Unfortunately, however, there is an inherent problem in estimating aggregate film thickness - current methods of calculating film thickness assume an average thickness, but not every aggregate particle is going to have the same thickness. Goode and Lufsey [24] used a bitumen index to avoid the inference that all particles are coated with the same uniform thickness of asphalt cement. The bitumen index is defined as mass of asphalt cement per area of surface. Goode and Lufsey [24] concluded a minimum bitumen index of 6.0 $\mathrm{kg} / \mathrm{m}^{2}\left(0.00123 \mathrm{lb} / \mathrm{ft}^{2}\right.$, which correlates to $\left.6 \mu \mathrm{m}\right)$ was sufficient to ensure adequate film thickness.

Kandhal, et al [21] provided the following equations for the calculation of asphalt film thickness:

$$
\begin{gathered}
\text { Volume of Asphalt Binder, } V_{b}=V M A-V_{a} \\
\text { Mass of Asphalt Binder, } W_{b}=V_{b} \times \rho_{b} \times G_{b} \\
\text { Mass of Aggregate, } W_{a g g}=\frac{W_{b}}{P_{b}} \times\left(100-P_{b}\right) \\
\text { Mass of Asphalt per Kg of Aggregate, } W_{b / a g g}=\frac{W_{b}}{W_{a g g}} \\
\text { Asphalt Film Thickness, } A F T=\frac{W_{b / a g}}{S A_{a g g} \times \rho_{w} \times G_{b}}
\end{gathered}
$$

Where:

$V_{a}=$ Air voids

$\rho_{w}=$ Density of water $\left(1000 \mathrm{~kg} / \mathrm{m}^{3}\right)$

$G_{b}=$ Specific gravity of the Binder (1.02)

$P_{b}=$ Asphalt Content

$S A_{\text {agg }}=$ Total surface of the aggregate 
The total surface area of the aggregate is a function of the gradation. Aggregate surface area was calculated using the procedure outlined in the Asphalt Institute's MS-2 [22]. Results of the aggregate surface area computations are shown in Tables 4.7 and 4.8 for coarse and fine gradations, respectively.

Table 4.7 Total Surface Area Calculation for Coarse Gradation

\begin{tabular}{|c|c|c|c|}
\hline Sieve Size & Percent Passing & Surface Area Factor & Surface Area \\
\hline 19 & 100.0 & 0.41 & 0.410 \\
\hline 12.5 & 93.2 & & \\
\hline 9.5 & 66.2 & & 0.194 \\
\hline 4.75 & 47.3 & 0.41 & 0.277 \\
\hline 2.36 & 33.8 & 0.82 & 0.377 \\
\hline 1.18 & 23.0 & 1.64 & 0.465 \\
\hline 0.600 & 16.2 & 2.87 & 0.749 \\
\hline 0.300 & 12.2 & 6.14 & 0.995 \\
\hline 0.150 & 8.1 & 12.29 & 1.34 \\
\hline 0.075 & 4.1 & 32.77 & 4.81 \\
\hline & & Total: & \\
\hline
\end{tabular}

Table 4.8 Total Surface Area Calculation for Fine Gradation

\begin{tabular}{|c|c|c|c|}
\hline Sieve Size & Percent Passing & Surface Area Factor & Surface Area \\
\hline 19 & 100.0 & 0.41 & 0.410 \\
\hline 12.5 & 96.1 & & \\
\hline 9.5 & 83.1 & & 0.287 \\
\hline 4.75 & 70.1 & 0.41 & 0.468 \\
\hline 2.36 & 57.1 & 0.82 & 0.725 \\
\hline 1.18 & 44.2 & 1.64 & 0.895 \\
\hline 0.600 & 31.2 & 2.87 & 1.12 \\
\hline 0.300 & 18.2 & 6.14 & 0.959 \\
\hline 0.150 & 7.8 & 12.29 & 1.28 \\
\hline 0.075 & 3.9 & 32.77 & 6.14 \\
\hline & & Total: & \\
\hline
\end{tabular}

Equations 4.1 through 4.5 were used to calculate the asphalt film thicknesses for the mixtures in this project. The results are shown below in Table 4.9. It should be noted that all of the asphalt film thickness values exceed the minimum of $6 \mu \mathrm{m}$ recommended by Campen, et al [23]. In fact, most fell within the optimum range of $8-10 \mu \mathrm{m}$ recommended by Kandhal, et al [21] and by Kandhal and Chakraborty [25]. As expected, the coarse mixes have a higher AFT than the fines since they have fewer total voids and less surface area. 
Table 4.9 Asphalt Film Thickness Calculations

\begin{tabular}{|c|c|c|c|c|c|c|c|c|c|}
\hline Aggregate & $P_{b}(\%)$ & $\mathrm{V}_{\mathrm{a}}(\%)$ & VMA (\%) & $\begin{array}{l}\text { SA } A_{\text {agg }} \\
\left(\mathrm{m}^{2} / \mathrm{kg}\right)\end{array}$ & $V_{b}(\%)$ & $W_{b}(\mathbf{k g})$ & $W_{\text {agg }}(\mathbf{k g})$ & $\begin{array}{r}W_{\text {b/agg }} \\
(\mathrm{kg})\end{array}$ & AFT $(\mu \mathrm{m})$ \\
\hline GFC & 4.30 & 3.00 & 11.50 & 4.811 & 8.50 & 86.7 & 1929.6 & 0.0449 & 9.156 \\
\hline GFF & 4.80 & 3.00 & 12.40 & 6.140 & 9.40 & 95.88 & 1901.6 & 0.0504 & 8.051 \\
\hline NUC & 4.95 & 3.00 & 11.25 & 4.811 & 8.25 & 84.15 & 1615.9 & 0.0521 & 10.612 \\
\hline NUF & 5.00 & 3.00 & 10.50 & 6.140 & 7.50 & 76.5 & 1453.5 & 0.0526 & 8.404 \\
\hline KLC & 4.20 & 3.00 & 9.00 & 4.811 & 6.00 & 61.2 & 1395.9 & 0.0438 & 8.933 \\
\hline KLF & 5.20 & 3.00 & 10.80 & 6.140 & 7.80 & 79.56 & 1450.4 & 0.0549 & 8.758 \\
\hline CGC & 4.70 & 3.00 & 10.50 & 4.811 & 7.50 & 76.5 & 1551.2 & 0.0493 & 10.049 \\
\hline CGF & 5.00 & 3.00 & 11.50 & 6.140 & 8.50 & 86.7 & 1647.3 & 0.0526 & 8.404 \\
\hline NUF (58-40) & 4.90 & 3.00 & 11.40 & 6.140 & 8.40 & 85.68 & 1662.9 & 0.0515 & 8.227 \\
\hline KLF (58-40) & 4.90 & 3.00 & 11.30 & 6.140 & 8.30 & 84.66 & 1643.1 & 0.0515 & 8.227 \\
\hline
\end{tabular}

Kandhal et al. (1998) recommended lowering the Superpave minimum VMA criterion by 1.2 to 1.5 percent based on the results of their research, and specifying an $8 \mu \mathrm{m}$ minimum AFT. Unfortunately, while all the mixes in the Superpave project exceed the recommended $8 \mu \mathrm{m}$ minimum AFT, even lowering the VMA criteria by 1.5 percent would only allow the Granite Falls Coarse mixture to meet the $13 \%$ minimum.

Another interesting issue is the apparent susceptibility of the coarse gradations to rutting according to the compaction data. Brown, et al [18] showed that mixtures exceeding $98 \%$ of maximum density at $\mathrm{N}_{\max }$ may be more susceptible to rutting than those that remain below $98 \%$ of maximum density. Additionally, since all four coarse aggregates exceeded the $98 \%$ maximum density criterion, the problem is probably systemic to the gradation rather than any one specific aggregate. All six fine gradations have densities greater than the $89 \%$ maximum criterion at $\mathrm{N}_{\text {ini. }}$. Therefore, it is suspected the fine gradation might exhibit compactibility problems such as tenderness during construction and instability when subjected to traffic.

The concern over failing the $\mathrm{N}_{\text {ini }}$ and $\mathrm{N}_{\max }$ criteria is debatable. To date, there is no irrefutable research correlating the failure of the $\mathrm{N}_{\text {ini }}$ criterion with susceptibility to tenderness nor the failure of the $\mathrm{N}_{\max }$ criterion with increased rutting potential. In fact, mounting research by Brown, et al [18], Brown and Mallick [26], Habib, et al [27], and Huber, et al [28] is supporting the belief that all three Superpave gyration criteria $\left(\mathrm{N}_{\mathrm{ini}}, \mathrm{N}_{\mathrm{des}}, \mathrm{N}_{\max }\right)$ should be lowered (especially with low-volume designs). Superpave Team Leader, Paul Mack [29], addressed this concern by stating the problem is currently being evaluated in preparation of a possible revision to the Superpave N-design table. Mack [29] specifically addresses the failure of the $\mathrm{N}_{\mathrm{ini}}$ criterion 
by fine graded mixes stating such failure is common and should not eliminate their use, particularly on low-volume pavements. More concern over the validity of the density criteria arose when an evaluation of four different Superpave gyratory compactors (Texas, Pine, Troxler, and Rainhart) by McGennis, et al [30] showed a high degree of variability in $\mathrm{N}_{\text {ini }}$ results.

\section{Resilient Modulus}

Although the results of the resilient modulus testing were variable, some general conclusions can still be made. As expected, the resilient modulus values decrease considerably as temperature increases. The decreasing values are the result of the softening of the asphalt binder as temperatures increase. Except for the $0{ }^{\circ} \mathrm{C}$ temperature, the coefficient of variation values fell well below the 10-20 percent range recommended by Al-Sugair and Almudaiheem [31]. Additionally, the $\mathrm{CV}$ values follow the expected trend of increasing at the extreme temperatures. Stroup-Gardiner and Newcomb [32] attributed the increase in variability at the coldest and warmest temperatures as a function of sensor noise and the low stiffness of the binder, respectively.

The lack of a significant difference in the resilient modulus values between the coarse and fine gradations is surprising. It was expected the increased amount of crushed aggregate found in the coarse mixes (except for the Cedar Grove gravel) would have resulted in a stiffer mix. As stated earlier, the mix containing the Kasota limestone aggregate behaved differently than those made with other aggregates.

In Figure 4.12, the PG 58-40 grade asphalt has a slightly lesser slope of temperature susceptibility than the PG 52-34 asphalt. However, at the highest and lowest test temperatures the values were nearly identical. The effect of different asphalt grades on coarse gradations should be investigated.

\section{Moisture Sensitivity}

The range of the tensile strength ratio results was somewhat greater and less variable than expected. The TSR values shown in Table 4.6 would seem to indicate the differences in gradation, aggregate and asphalt grade had no significant effect on the moisture sensitivity of the mixtures. Such high TSR results may be explained by work done by McGennis et al. (1996) 
who concluded specimens compacted with a Superpave gyratory compactor resulted in significantly higher TSR values. Additionally, Brown, et al [18] noted the low reliability and lack of a satisfactory relationship between laboratory and field conditions as a chronic problem with moisture sensitivity tests.

\section{Rut Testing}

The asphalt mixes using PG 52-34 binder showed more rutting susceptibility than the mixes using PG 58-40. At elevated temperatures, the asphalt binder softens and leads to more permanent deformation. Except for the New Ulm Quartzite PG 52-34 mix, the coefficient of variation values were very high. We would like to see much more consistent test results with the APA.

It appears that after 8000 test cycles, the two PG 58-40 mixes have reached a plateau in their rut depths. Conversely, the two PG 52-34 mixes appear to continue rutting in a fairly linear trend with the number of cycles. The PG 58-40 mixes reached approximately $80 \%$ of their final rut depths after about 1000 cycles. They did not rut significantly more in the remaining 7000 cycles.

Another interesting observation to note is the variation due to the Asphalt Pavement Analyzer itself. For three out of the four mixes, the samples under the left wheel had the lowest rut depths while the samples under the right wheel had the highest rut depths. When the APA device was being developed, it went through a series of ruggedness studies at three different laboratories. Randy West (1999) pointed out similar differences between the left, center, and right rut depth measurements. He stated that although each wheel load is individually calibrated, the three wheel loads are not necessarily independent. Observations may indicate that the wheel positions do not apply uniform loads to the asphalt specimens.

\section{Low Temperature Creep Testing}

In holding the asphalt binder constant and varying the aggregate, there was virtually no difference in creep compliance at $-10^{\circ} \mathrm{C}$ and $-20^{\circ} \mathrm{C}$. For the PG 52-34 binder, the NU mix had

$40 \%$ lower creep compliance than the $\mathrm{KL} \operatorname{mix}$ at $0{ }^{\circ} \mathrm{C}$. For the PG 58-40 binder, the KL mix had $20 \%$ lower creep compliance than the NU mix at $0{ }^{\circ} \mathrm{C}$. 
In holding the aggregate constant and varying the asphalt binder, the PG 52-34 mix had lower creep compliance than the PG 58-40 mix. For the New Ulm quartzite aggregate, the PG 52-34 mix had a 50\% lower creep compliance than the PG 58-40 mix at all three low temperatures. For the Kasota limestone aggregate, the PG 52-34 mix had 30\%-40\% lower creep compliance than the PG 58-40 mix at $-10^{\circ} \mathrm{C}$ and $-20{ }^{\circ} \mathrm{C}$. At $0{ }^{\circ} \mathrm{C}$ there was virtually no difference in creep compliance between the two mixes.

From the above analysis, it was determined that the PG 52-34 mixes are stiffer at low temperatures than PG 58-40 mixes. This was an expected behavior, based on the binder grades. At $-10^{\circ} \mathrm{C}$ and $-20^{\circ} \mathrm{C}$, the aggregate was found to have little influence on the creep compliance. However, at $0{ }^{\circ} \mathrm{C}$ the aggregates did have an effect on the stiffness of the mix. In addition, the asphalt binder was found to have an effect on creep compliance at all three low temperatures. The PG 58-40 mixes have higher creep compliance than the PG 52-34 mixes.

From the IDT tests, Poisson's ratio was determined to be rather low. However, it was well within the range of possible values for $\mu$. For the PG 52-34 binder, the NU mix had a slightly higher strength than the KL mix. For the PG 58-40 binder, the two aggregates displayed the same strength. The softer binder (PG 58-40) was less affected by aggregate type than the stiffer binder (PG 52-34). 


\section{CHAPTER 5 \\ - CONCLUSIONS AND RECOMMENDATIONS -}

\section{CONCLUSIONS}

1. The locally available Minnesota aggregates Lakeland sand, Granite Falls granite, New Ulm quartzite, Kasota limestone, and Cedar Grove gravel meet Superpave aggregate consensus property criteria for low-volume roads (less than 1,000,000 ESALs). This corresponds to $\mathrm{Mn} / \mathrm{DOT}$ traffic levels 1 and 2.

2. Meeting Superpave's minimum VMA and VFA criteria was very difficult possibly due to the low angularity of the natural sand. Lowering the target air void content to $3 \%$ resulted in compliant VFA, but all mixes still failed the VMA requirement. Use of a more angular fine aggregate would help increase the VMA.

3. None of the six fine-graded mixtures met the $\mathrm{N}_{\text {ini }}$ criterion $(90.5$ for Level 2 traffic) indicating fine-graded mixtures produced with the natural sand may be susceptible to tenderness problems. There appeared to be no significant difference in tenderness susceptibility between the different coarse aggregates evaluated.

4. None of the coarse-graded mixtures met the $\mathrm{N}_{\max }$ criterion indicating coarse-graded mixtures produced with the Lakeland sand may experience premature permanent deformation. All of the fine-graded mixtures met the $\mathrm{N}_{\max }$ criterion. There appeared to be no significant difference in susceptibility to permanent deformation between the four coarse aggregates evaluated. Based on the gyratory compaction data, there was no significant difference in indications of susceptibility to either tenderness or permanent deformation between the two asphalt grades evaluated.

5. The results of the resilient modulus tests were highly variable. Although high variability is not uncommon to resilient modulus testing, the 1 to 23 percent average coefficients of variation range from this study was greater than the typical 6 to 20 percent range presented by Timm [33]. 
6. Except for the Kasota limestone which produced slightly greater resilient modulus values, the different coarse aggregates evaluated in this study had no significant effect on resilient modulus. To date, however, no direct correlation has been made between resilient modulus values and long-term susceptibility to permanent deformation.

7. There was no significant difference in resilient modulus values between the coarse and fine gradations evaluated in this study.

8. Mixes produced with the PG 52-34 asphalt binder had higher resilient modulus results at intermediate temperatures than those produced with the PG 58-40 binder. There was no significant difference in resilient modulus values between the two binders at the highest and lowest temperatures tested (40 and $-18^{\circ} \mathrm{C}$, respectively). The fact that this temperature range does not include the high and low temperature values for these PG binders may explain the failure of the resilient modulus test to differentiate between the two binders.

9. All mixes evaluated met the Superpave moisture sensitivity criterion. The higher asphalt contents associated with the relatively low 3\% design air void content was the probable cause for the high TSR values. High TSR values indicate the higher asphalt content associated with the lower target air void content may improve the durability of low-volume road mixtures.

10. There was no significant difference in moisture sensitivity results between the coarse and fine gradations, coarse aggregate types, or asphalt grades evaluated in this study (values ranged from 96.7 to $99.5 \%$ ). An examination of the dry and wet tensile strength values also revealed no significant differences - dry values ranged from 1110 to $1140 \mathrm{kPa}$ (161 to 166 psi) and wet values ranged from 1080 to $1130 \mathrm{kPa}$ (157 to $164 \mathrm{psi}$ ). As concluded by StroupGardiner and Newcomb [32], lack of a significant difference between material types indicates moisture sensitivity may be more dependent on volumetric parameters than on types of material.

11. The appropriate software for analyzing the low temperature creep compliance data was not available at the time this report was written. For all four mixes (see Table 3.5), as the test 
temperature decreased, the creep compliance also decreased and Young's Modulus based on the instantaneous loading portion of the creep compliance test increased.

12. Different aggregates had little effect on creep compliance. The PG 52-34 mixes had lower creep compliance than the PG 58-40 mixes which suggests that the PG 52-34 mixes may be more susceptible to low temperature cracking.

13. Based on the gyratory compaction data, there was no significant difference in indications of susceptibility to either tenderness or permanent deformation between the two asphalt grades evaluated (PG 52-34 and PG 58-40).

14. For a given coarse aggregate type, the PG 52-34 mixes showed more rutting than the PG 5840 mixes, indicating that increasing the upper grade of the binder may result in a more rutresistant pavement.

15. The type of aggregate had little effect on rutting in the PG 58-40 mixes. However, in the PG 52-34 mixes, the Kasota Limestone mixes had almost twice the rut depth of the New Ulm Quartzite mixes.

16. Based on the results of the limited testing performed in this study, decreasing the lower PG binder grade and increasing the upper PG binder grade can be effective in improving the low temperature cracking resistance and rut resistance of a pavement, respectively.

\section{RECOMMENDATIONS}

1. Consider using a target air void content of three percent when the quality of available aggregates makes it difficult to meet VMA requirements. The higher asphalt content associated with a lower air void target would increase the long-term durability of the pavement. The decrease in stability associated with pavements constructed with higher asphalt contents is offset by the lower volume of heavy-truck traffic experienced on lowvolume roads.

2. Since the low angularity of the natural sand resulted in difficulties meeting Superpave volumetric criteria, a more angular aggregate should be used to produce mixtures that can 
meet the volumetric criteria. A study should be conducted to investigate the benefit of using a more angular fine aggregate. This study should include an economic evaluation.

3. If volumetric criteria cannot be met, a possible solution would be to lower the target air void content and take the necessary precautions to overcome any tenderness problems that may result. Wolters [34] provides specific recommendations to overcome tenderness problems.

4. An economic analysis should be completed to determine whether the improvements in predicted long-term pavement performance due to the use higher quality materials.

5. A full low-temperature cracking analysis should be performed and master creep compliance curves generated when the software is available.

6. Performance testing (APA and creep compliance) should be conducted to evaluate the difference between coarse and fine aggregate gradations.

7. Further testing should be conducted to evaluate the benefits of decreasing the lower PG grade and increasing the upper PG grade. 


\section{REFERENCES}

1. Marker, V., "Introduction to the Compaction of Asphalt Concrete Symposium,"

Proceedings: Association of Asphalt Paving Technologists, Vol. 36, 1967, pp. 288-294.

2. Sowers, G.F., "Introductory Soil Mechanics and Foundations: Geotechnical

Engineering," 4th Ed., Macmillan Publishing Co., Inc., New York, 1979.

3. Geller, M.., "Compaction Equipment for Asphalt Mixtures," Placement and Compaction of Asphalt Mixtures, ASTM STP 829, F.T. Wagner, Ed., American Society for Testing and Materials, 1984, pp. 28-47.

4. Nijboer, L.W., Plasticity as a Factor in the Design of Dense Bituminous Road Carpets, Elsevier Publishing, New York, 1948.

5. Kari, W.J., "Mix Properties as They Affect Compaction," Proceedings: Association of Asphalt Paving Technologists, Vol. 36, 1967, pp. 295-309.

6. McLeod, N.W., "Influence of Viscosity of Asphalt Cements on Compaction of Paving Mixtures in the Field," Highway Research Record No. 158, Highway Research Board National Academy of Sciences, Washington D.C., 1960.

7. Asphalt Institute, "Superpave Series No. 2 (SP-2): Superpave Mix Design," The Asphalt Institute, Lexington, KY, 1996.

8. Santucci, L.E. and Schmidt, R.J., "Setting Rate of Asphalt Concrete," Highway Research Bulletin No. 333, Highway Research Board, Washington, D.C., 1962.

9. Bissada, A.F., "Resistance to Compaction of Asphalt Mixtures and Its Relationship to Stiffness," Placement and Compaction of Asphalt Mixtures, ASTM STP 829, F.T. Wagner, Ed., American Society for Testing and Materials, 1984, pp. 131-145.

10. Harvey, J.T., and Tsai, B-W., "Effects of Asphalt Content and Air Void Content on Mix Fatigue and Stiffness," Transportation Research Record 1543: Design and Construction of Asphalt Overlays and Hot-Mix Asphalt Construction Practices, Transportation Research Board, National Academy Press, Washington D.C., 1996, pp. 38-45.

11. Parker, C.F., "Steel-Tired Rollers," Highway Research Bulletin No. 246, Highway Research Board, Washington D.C., 1960.

12. Kennedy, T.W., Roberts, F.L., and McGennis, R.B., "Effects of Compaction Temperature and Effort on the Engineering Properties of Asphalt Concrete Mixtures," Placement and Compaction of Asphalt Mixtures, ASTM STP 829, F.T. Wagner, Ed., American Society for Testing and Materials, 1984, pp. 48-66.

13. Hadley, W.O., Hudson, W.R., Kennedy, T.W., "Correlation of Indirect Tensile Test Results with Stability and Cohesiometer Values for Asphalt-Treated Materials and 
Discussion,” Proceedings: Association of Asphalt Paving Technologists, Vol. 39, 1970, pp. 745-765.

14. Brown, E.R., "Experiences of Corps of Engineers in Compaction of Hot Asphalt Mixtures," Placement and Compaction of Asphalt Mixtures, ASTM STP 829, F.T. Wagner, Ed., American Society for Testing and Materials, 1984, pp. 67-79.

15. Asphalt Institute, "Manual Series No. 4 (MS-4), 1989 Ed.: The Asphalt Handbook," The Asphalt Institute, Lexington, KY, 1989.

16. Roberts, F.L., Kandhal, P.S., Brown, E.R., Lee, D., and Kennedy, T.W., "Hot Mix Asphalt Materials, Mixture Design, and Construction," 2nd Ed., NAPA Education Foundation, Lanhan Maryland, 1996.

17. Bell, C.A., Hicks, G.R., and Wilson, J.E., Effect of Percent Compaction on Asphalt Mixture Life," Placement and Compaction of Asphalt Mixtures, ASTM STP 829, F.T. Wagner, Ed., American Society for Testing and Materials, 1984, pp. 107-130.

18. Brown, E.R., Hanson, D.I., and Mallick, R.B., "Evaluation of Superpave Gyratory Compaction of Hot Mix Asphalt," Transportation Research Record 1543, Transportation Research Board, National Research Council, Washington, D.C., 1996, pp. 145-150.

19. D'Angelo, J.A., Paugh, C., Harman, T.P., and Bukowski, J., "Comnparison of the Superpave Gyratory Compactor to the Marshall for Field Quality Control," Proceedings: Association of Asphalt Paving Technologists, Vol. 64, 1995, pp. 611-635.

20. McGennis, R., Kennedy, T.W., Anderson V.L., and Perdomo, D., "Ruggedness Evaluation of AASHTO TP4 The Superpave Gyratory Compactor," Proceedings: Association of Asphalt Paving Technologists, Vol. 66, 1997, pp. 277-311.

21. Kandhal, P.S., Foo, K.Y., and Mallick, R.B., "Critical Review of Voids in Mineral Aggregate Requirements in Superpave," Transportation Research Record 1609, Transportation Research Board, National Research Council, Washington, D.C., 1998, pp. 21-27.

22. Asphalt Institute, "Manual Series No. 2 (MS-2), 6th Ed.: Mix Design Methods," The Asphalt Institute, Lexington, KY, 1989.

23. Campen, J.F., Smith, J.R., Erickson, L.G., and Mertz, L.R., "The Relationships Between Voids, Surface Area, Film Thickness and Stability in Bituminous Paving Mixtures," Proceedings: Association of Asphalt Paving Technologists, Vol. 28, 1959, pp. 149-178.

24. Goode, J.F. and Lufsey, L.A., "Voids, Permeability, Film Thickness vs. Asphalt Hardening," Proceedings: Association of Asphalt Paving Technologists, Vol. 34, 1965, pp. 430-463. 
25. Kandhal, P.S. and Chakraborty, S., "Effect of Asphalt Film Thickness on Short- and Long-Term Aging of Asphalt Paving Mixtures," Transportation Research Record 1535, Transportation Research Board, National Research Council, Washington, D.C., 1996, pp. 83-90.

26. Brown, E.R. and Mallick, R.B., "An Initial Evaluation for $\mathrm{N}_{\text {design }}$ Superpave Gyratory Compactor,", Proceedings: Association of Asphalt Paving Technologists, Vol. 67, 1998, pp. 101-124.

27. Habib, A., Hossain, M., Kaldate, R., and Fager, G.A., "Comparison of Superpave and Marshall Mixtures for Low-Volume Roads and Shoulders," Transportation Research Record 1609, Transportation Research Board, National Research Council, Washington, D.C., 1998, pp. 44-50.

28. Huber, G.A., Zhang, X., and Fontaine, R., "Superpave Models: Predicting Performance During Design and Construction," Transportation Research Record 1545, Transportation Research Board, National Research Council, Washington D.C., 1996, pp. 105-112.

29. Mack, P.J., "Newsbrief: 1998 AASHTO Superpave Lead State Guidance," Superpave Lead State Program, North Central Superpave Center, 10 Jun 1998.

30. McGennis, R.B., Anderson, R.M., Perdomo, D., and Turner, P., "Issues Pertaining to Use of Superpave Gyratory Compactor," Transportation Research Record 1543, Transportation Research Board, National Research Council, Washington D.C., 1996, pp. $139-144$.

31. Al-Sugair, F.H. and Almudaiheem, J.A., "Variations in Measured Resilient Modulus of Aspahlt Mixes," Journal of Materials in Civil Engineering, Vol. 4, No. 4, November 1992, pp. 343-352.

32. Stroup-Gardiner, M., and Newcomb, D., "Physio-Chemical Evaluation of AsphaltAggregate Interaction," University of Minnesota, Department of Civil Engineering, Minneapolis, MN, May 94.

33. Timm, D.H., "Incorporation of Reliability in Mechanistic-Empirical Flexible Pavement Thickness Design," University of Minnesota, Department of Civil Engineering, Minneapolis, MN, December 1997.

34. Wolters, R.O., "Tenderness of Hot Mix Asphalt," Minnesota Asphalt Pavement Association, Minneapolis, MN, September 1998. 


\section{APPENDIX A MIX DESIGN}


Table A.1 Mix Design Data for Granite Falls Coarse Gradation

\begin{tabular}{|c|c|c|c|c|c|c|c|c|c|}
\hline Project: & SUPE & PAVE & Technician: & & & & Date: & & \\
\hline CA Source: & GRA & ITE FALLS & FA Source: & LAKELA & & Blend Gsb: & 2.6560 & Blend Gse: & 2.7302 \\
\hline Gradation: & $\begin{array}{l}{[\mathrm{A}} \\
{[\mathrm{X}] \mathrm{B}}\end{array}$ & $\begin{array}{l}\text { ove (fine) } \\
\text { low (coarse) }\end{array}$ & the Restrictec & Zone & $\begin{array}{l}\text { Nom. Max. } \\
\text { Size (mm): }\end{array}$ & 12.5 & Batch & eight, Ws, (g): & 12000 \\
\hline Asphalt Sou & urse: & $\mathrm{KOCH}$ & & Grade: & PG 52 - 34 & Polymer: & None & $\mathrm{P}_{\mathrm{b}, \text { est }}=$ & 5.01 \\
\hline$T_{\text {mix }}: \quad 138$ & ${ }^{\circ} \mathrm{C}$ & $\mathrm{T}_{\text {comp: }}$ & $128{ }^{\circ} \mathrm{C}$ & Compact & Brovold & & Nini $\quad 7$ & $N_{\text {des }}$ & $N_{\max } \quad 117$ \\
\hline Target & & $\mathrm{Pb}_{\mathrm{b} \text {,est }}-0.5=$ & 4.5 & $\mathrm{P}_{\mathrm{b}, \text { est }}=$ & 5.0 & $\mathrm{P}_{\mathrm{b}, \text { est }}+0.5=$ & 5.5 & $\mathrm{~Pb}_{\mathrm{b}, \mathrm{est}}+1.0=$ & 6.0 \\
\hline Target W & $b(g)$ & & 6.8 & & 2.9 & 69 & 9.8 & 76 & 7.3 \\
\hline Actual W & $\mathrm{b}(\mathrm{g})$ & & 8.1 & & 36 & 70 & 3.5 & 76 & 9.5 \\
\hline Actual & & & 52 & & .03 & 5 & 54 & 6 . & 03 \\
\hline $\mathrm{Gmm}$ & & & 539 & & 517 & 2. & 509 & 2. & 187 \\
\hline Specimen & No. & 1 & 2 & 1 & 2 & 1 & 2 & 1 & 2 \\
\hline & 1 & 111.2 & 110.4 & 111.6 & 110.5 & 105.7 & 105.3 & 108.4 & 107.8 \\
\hline 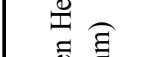 & 2 & 111.8 & 110.6 & $111 . .2$ & 110.4 & 105.8 & 105.5 & 108.8 & 108.2 \\
\hline$\Xi \Xi$ & 3 & 111.5 & 110.6 & 111.2 & 110.6 & 105.6 & 105.9 & 108.4 & 107.9 \\
\hline के & 4 & 111.3 & 110.6 & 111.5 & 110.8 & 105.6 & 105.8 & 108.8 & 107.9 \\
\hline Av & erage & 111.45 & 110.55 & 111.43 & 110.58 & 105.68 & 105.63 & 108.60 & 107.95 \\
\hline $\mathrm{W}_{\text {dry }}(\mathrm{g})$ & A & 4785.7 & 4762.1 & 4774.9 & 4713.7 & 4619.1 & 4593.9 & 4663.7 & 4686.6 \\
\hline Win water $(\mathrm{g})$ & $\mathrm{C}$ & 2825.8 & 2811.7 & 2823.9 & 2780.8 & 2735.2 & 2722.2 & 2752.5 & 2763.2 \\
\hline W SSD (g) & B & 4788.2 & 4764.8 & 4776.4 & 4715.6 & 4620.3 & 4597.0 & 4666.5 & 4687.5 \\
\hline $\mathrm{G}_{\mathrm{mb}}=\frac{}{(\mathrm{B}}$ & $\frac{A}{-C)}$ & 2.439 & 2.438 & 2.446 & 2.436 & 2.450 & 2.450 & 2.437 & 2.435 \\
\hline Corrected & $\mathrm{Nini}_{\mathrm{i}}$ & 2.219 & 2.210 & 2.240 & 2.245 & 2.242 & 2.226 & 2.200 & 2.232 \\
\hline $\mathrm{Gmb}$ & $\mathrm{N}_{\mathrm{des}}$ & 2.413 & 2.408 & 2.430 & 2.420 & 2.436 & 2.427 & 2.408 & 2.421 \\
\hline $\mathrm{V}_{\mathrm{a}} @ \mathrm{~N}_{\mathrm{c}}$ & & 4.98 & 5.18 & 3.44 & 3.84 & 2.9 & 3.27 & 3.18 & 2.63 \\
\hline Averag & & & 08 & & 64 & & 09 & 2. & 91 \\
\hline VMA@ & $\mathrm{N}_{\mathrm{des}}$ & 13.26 & 13.44 & 13.11 & 13.47 & 13.36 & 13.68 & 14.80 & 14.34 \\
\hline Averag & & & .35 & & 3.29 & & .52 & & .57 \\
\hline VFA@ & $\mathrm{N}_{\text {des }}$ & 62.43 & 61.45 & 73.77 & 71.50 & 78.30 & 76.10 & 78.51 & 81.66 \\
\hline Averag & & & .94 & & 2.63 & & .20 & & .09 \\
\hline \%Gmm@ & $\mathrm{N}_{\mathrm{ini}}$ & 87.40 & 87.04 & 88.99 & 89.19 & 89.36 & 88.72 & 88.46 & 89.75 \\
\hline Averag & & & .22 & & 9.09 & & .04 & & .10 \\
\hline \%Gmm@ & $\mathrm{N}_{\max }$ & 96.05 & 96.03 & 97.16 & 96.79 & 97.66 & 97.66 & 97.97 & 97.93 \\
\hline Averag & & & .04 & & 6.98 & & .66 & & .95 \\
\hline Dust Propo & ortion & & 49 & & 44 & & 40 & & 37 \\
\hline
\end{tabular}


Table A.2 Mix Design Data for Granite Falls Fine Gradation

\begin{tabular}{|c|c|c|c|c|c|c|c|c|c|}
\hline Project: & SUPE & PAVE & Technician: & & & & Date: & & \\
\hline CA Source: & GRA & ITE FALLS & FA Source: & LAKELA & & Blend Gsb: & 2.6287 & Blend Gse: & 2.7164 \\
\hline Gradation: & $\begin{array}{l}{[\mathrm{X}] \mathrm{A}} \\
{[\mathrm{B}}\end{array}$ & $\begin{array}{l}\text { ove (fine) } \\
\text { low (coarse) }\end{array}$ & the Restricte & Zone & $\begin{array}{l}\text { Nom. Max. } \\
\text { Size (mm): }\end{array}$ & 12.5 & Batch & eight, Ws, (g): & 12000 \\
\hline Asphalt Sou & Irse: & $\mathrm{KOCH}$ & & Grade: & PG 52 - 34 & Polymer: & None & $\mathrm{P}_{\mathrm{b}, \text { est }}=$ & 5.39 \\
\hline$T_{\text {mix }}: \quad 138$ & ${ }^{\circ} \mathrm{C}$ & $\mathrm{T}_{\text {comp: }}$ & $128{ }^{\circ} \mathrm{C}$ & Compact & Brovold & & Nini $\quad 7$ & $N_{\text {des }}$ & $N_{\max } \quad 117$ \\
\hline Target & & $\mathrm{P}_{\mathrm{b} \text {,est }}-0.5=$ & 4.9 & $\mathrm{P}_{\mathrm{b}, \text { est }}=$ & 5.4 & $\mathrm{P}_{\mathrm{b}, \text { est }}+0.5=$ & 5.9 & $\mathrm{~Pb}_{\mathrm{b}, \mathrm{est}}+1.0=$ & 6.4 \\
\hline Target W & $\mathrm{b}(\mathrm{g})$ & & 7.0 & & 3.6 & 75 & 1.0 & 81 & 9.1 \\
\hline Actual W & b (g) & & 6.2 & & 32.4 & 75 & 6.4 & 81 & 7.6 \\
\hline Actual & & & 88 & & .38 & 5 & 93 & 6 & 38 \\
\hline $\mathrm{Gmm}$ & & & 512 & & 511 & 2. & 482 & 2. & 173 \\
\hline Specimen & No. & 1 & 2 & 1 & 2 & 1 & 2 & 1 & 2 \\
\hline & 1 & 111.9 & 111.8 & 111.4 & 111.0 & 111.9 & 111.8 & 111.4 & 111.4 \\
\hline 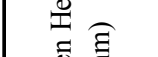 & 2 & 111.9 & 111.8 & 111.3 & 111.2 & 112.1 & 111.8 & 111.5 & 111.6 \\
\hline 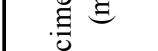 & 3 & 112.0 & 111.7 & 111.7 & 111.0 & 111.9 & 112.0 & 111.5 & 111.5 \\
\hline की & 4 & 111.9 & 111.9 & 111.7 & 111.1 & 112.1 & 112.2 & 111.5 & 111.4 \\
\hline $\mathrm{Av}$ & erage & 111.93 & 111.80 & 111.53 & 111.08 & 112.00 & 111.95 & 111.48 & 111.48 \\
\hline $\mathrm{W}_{\text {dry }}(\mathrm{g})$ & A & 4790.1 & 4796.4 & 4800.8 & 4779.3 & 4796.6 & 4790.2 & 4788.4 & 4786.6 \\
\hline Win water $(\mathrm{g})$ & $\mathrm{C}$ & 2790.8 & 2794.9 & 2805.5 & 2789.2 & 2779.9 & 2781.0 & 2779.7 & 2772.7 \\
\hline W SSD (g) & B & 4790.7 & 4797.9 & 4802.0 & 4781.5 & 4797.0 & 4790.5 & 4788.8 & 4786.8 \\
\hline $\mathrm{G}_{\mathrm{mb}}=\frac{}{(\mathrm{B}}$ & $\frac{A}{-C)}$ & 2.395 & 2.395 & 2.405 & 2.399 & 2.378 & 2.384 & 2.383 & 2.377 \\
\hline Corrected & $\mathrm{N}_{\text {ini }}$ & 2.256 & 2.255 & 2.259 & 2.251 & 2.223 & 2.230 & 2.248 & 2.242 \\
\hline $\mathrm{Gmb}$ & $\mathrm{N}_{\text {des }}$ & 2.380 & 2.378 & 2.388 & 2.382 & 2.359 & 2.367 & 2.377 & 2.371 \\
\hline $\mathrm{V}_{\mathrm{a}} @ \mathrm{~N}_{\mathrm{c}}$ & & 5.25 & 5.34 & 4.91 & 5.15 & 4.96 & 4.63 & 3.9 & 4.14 \\
\hline Averag & & & 30 & & .03 & & 80 & & 02 \\
\hline VMA@ & $\mathrm{N}_{\mathrm{des}}$ & 13.88 & 13.96 & 14.04 & 14.26 & 15.58 & 15.29 & 15.34 & 15.56 \\
\hline Averag & & & .92 & & 4.15 & & .44 & & .45 \\
\hline VFA@1 & $\mathrm{N}_{\text {des }}$ & 62.18 & 61.74 & 65.04 & 63.89 & 68.17 & 69.73 & 74.58 & 73.39 \\
\hline Averag & & & .96 & & 4.46 & & .95 & & .98 \\
\hline \%Gmm@ & $\mathrm{Nini}$ & 89.81 & 89.77 & 89.96 & 89.65 & 89.56 & 89.85 & 90.90 & 90.66 \\
\hline Averag & & & .79 & & 9.80 & & .71 & & .78 \\
\hline \%Gmm@ & $\mathrm{N}_{\max }$ & 95.35 & 95.33 & 95.76 & 95.54 & 95.81 & 96.04 & 96.38 & 96.10 \\
\hline Averag & & & .34 & & 5.65 & & .93 & & .24 \\
\hline Dust Propo & rrtion & & 44 & & .40 & & 36 & & 34 \\
\hline
\end{tabular}


Table A.3 Mix Design Data for New Ulm Quartzite Coarse Gradation

\begin{tabular}{|c|c|c|c|c|c|c|c|c|c|}
\hline Project: & SUPE & PAVE & Technician: & & & & Date: & & \\
\hline CA Source: & NEW & JLM & FA Source: & LAKEL & & Blend $\mathrm{G}_{\mathrm{sb}}$ : & 2.6099 & Blend Gse: & 2.6817 \\
\hline Gradation: & $\begin{array}{l}{[\mathrm{A}} \\
{[\mathrm{X}] \mathrm{B}}\end{array}$ & $\begin{array}{l}\text { ove (fine) } \\
\text { low (coarse }\end{array}$ & the Restricted & Zone & $\begin{array}{l}\text { Nom. Max. } \\
\text { Size (mm): }\end{array}$ & 12.5 & Batch & eight, Ws, (g): & 12000 \\
\hline Asphalt Sol & rse: & $\mathrm{KOCH}$ & & Grade: & PG 52-34 & Polymer: & none & $\mathrm{P}_{\mathrm{b}, \text { est }}=$ & 5.00 \\
\hline $\mathrm{T}_{\text {mix }}: \quad 138$ & $-{ }^{\circ} \mathrm{C}$ & $\mathrm{T}_{\text {comp: }}$ & $\underline{128}{ }^{\circ} \mathrm{C}$ & Compac & Brovold & & $\mathrm{N}_{\text {ini }}$ & $\mathrm{N}_{\text {des }} \quad 76$ & $N_{\max } \quad 117$ \\
\hline Target & & $\mathrm{P}_{\mathrm{b} \text {,est }}-0.5=$ & 4.5 & $\mathrm{P}_{\mathrm{b}, \mathrm{est}}=$ & 5.0 & $\mathrm{P}_{\mathrm{b}, \mathrm{est}}+0.5=$ & 5.5 & $\mathrm{P}_{\mathrm{b}, \mathrm{est}}+1.0=$ & 6.0 \\
\hline Target W & (g) & & 55.4 & & & 69 & 8.4 & 76 & 6.0 \\
\hline Actual W & $(g)$ & & 0.6 & & 0.0 & 73 & 0.8 & 86 & 4.3 \\
\hline Actual & & & 99 & & 44 & & 74 & 6. & 72 \\
\hline $\mathrm{G}$ & & & 513 & & 07 & 2. & 199 & 2.4 & 157 \\
\hline Specimen & No. & 1 & 2 & 1 & 2 & 1 & 2 & 1 & 2 \\
\hline & 1 & 112.0 & 111.6 & 109.8 & 109.9 & 109.65 & 110.05 & 108.53 & 108.40 \\
\hline$\stackrel{\widetilde{I}}{\exists}$ & 2 & 112.1 & 111.6 & 109.9 & 110.0 & 110.44 & 110.16 & 109.02 & 108.22 \\
\hline . & 3 & 112.2 & 111.6 & 110.0 & 110.1 & 110.44 & 109.96 & 108.08 & 108.01 \\
\hline की & 4 & 112.1 & 111.8 & 110.0 & 110.0 & 110.52 & 109.88 & 108.34 & 107.67 \\
\hline & erage & 112.1 & 111.7 & 109.9 & 110.0 & 110.26 & 110.01 & 108.49 & 108.08 \\
\hline $\mathrm{W}_{\text {dry }}(\mathrm{g})$ & A & 4792.5 & 4794.2 & 4769.3 & 4737.0 & 4756.4 & 4759.1 & 4666.2 & 4670.6 \\
\hline W in water $(\mathrm{g})$ & $\mathrm{C}$ & 2851.0 & 2854.3 & 2850.4 & 2830.0 & 2828.3 & 2831.4 & 2767.4 & 2764.1 \\
\hline WssD (g) & B & 4794.7 & 4794.0 & 4771.5 & 4739.2 & 4759.0 & 4761.6 & 4667.5 & 4673.1 \\
\hline $\mathrm{G}_{\mathrm{mb}}=\frac{}{(\mathrm{B}}$ & $\frac{A}{-C)}$ & 2.466 & 2.472 & 2.483 & 2.481 & 2.464 & 2.466 & 2.456 & 2.447 \\
\hline Corrected & $N_{\text {ini }}$ & 2.228 & 2.240 & 2.262 & 2.235 & 2.208 & 2.227 & 2.225 & 2.255 \\
\hline Gmb & $\mathrm{N}_{\text {des }}$ & 2.435 & 2.444 & 2.467 & 2.456 & 2.433 & 2.444 & 2.443 & 2.438 \\
\hline $\mathrm{Va} @ \mathrm{~N}$ & & 3.09 & 2.76 & 1.59 & 2.02 & 2.63 & 2.21 & 0.59 & 0.77 \\
\hline Avera & & & 93 & & 81 & & 42 & 0 . & 68 \\
\hline VMA@ & $J_{\text {des }}$ & 11.36 & 11.03 & 10.61 & 11.01 & 12.13 & 11.73 & 12.68 & 12.86 \\
\hline Avera & & & .20 & & .81 & & 93 & & .77 \\
\hline VFA@ & & 72.80 & 74.98 & 85.02 & 81.66 & 78.32 & 81.16 & 95.35 & 94.01 \\
\hline Avera & & & .89 & & .34 & & .74 & & 68 \\
\hline \%Gmm@ & $\mathrm{N}_{\text {ini }}$ & 88.66 & 89.14 & 90.23 & 89.15 & 88.36 & 89.12 & 90.56 & 91.78 \\
\hline Avera & & & .90 & & 69 & & .74 & & 17 \\
\hline \%Gmm@ & $J_{\max }$ & 98.12 & 98.35 & 99.03 & 98.97 & 98.58 & 98.66 & 99.95 & 99.58 \\
\hline Avera & & & .23 & & .00 & & 62 & & .76 \\
\hline Dust Prop & rtion & & 44 & & 41 & & 39 & 0. & 33 \\
\hline
\end{tabular}


Table A.4 Mix Design Data for New Ulm Quartzite Fine Gradation

\begin{tabular}{|c|c|c|c|c|c|c|c|c|c|}
\hline Project: & SUPE & PAVE & Technician: & & & & Date: & & \\
\hline CA Source: & NEW & JLM & FA Source: & LAKEL $A$ & & Blend Gsb: & 2.6059 & Blend Gse: & 2.6922 \\
\hline Gradation: & $\begin{array}{l}{[\mathrm{X}] \mathrm{A}} \\
{[\mathrm{B}}\end{array}$ & $\begin{array}{l}\text { ove (fine) } \\
\text { low (coarse }\end{array}$ & the Restrictec & Zone & $\begin{array}{l}\text { Nom. Max. } \\
\text { Size (mm): }\end{array}$ & 12.5 & Batch & ight, Ws, (g): & 12000 \\
\hline Asphalt Sou & rse: & $\mathrm{KOCH}$ & & Grade: & PG 52-34 & Polymer: & none & $\mathrm{P}_{\mathrm{b}, \text { est }}=$ & 5.19 \\
\hline$T_{\text {mix }}: \quad 138$ & $-{ }^{\circ} \mathrm{C}$ & $\mathrm{T}_{\text {comp: }}$ & $28{ }^{\circ} \mathrm{C}$ & Compac & Brovold & & $\mathrm{N}_{\text {ini }}$ & $\mathrm{N}_{\text {des }} \quad 76$ & $\mathrm{~N}_{\max } \quad 117$ \\
\hline Target & & $\mathrm{P}_{\mathrm{b} \text {,est }}-0.5=$ & 4.7 & $\mathrm{P}_{\mathrm{b}, \text { est }}=$ & 5.2 & $\mathrm{P}_{\mathrm{b}, \mathrm{est}}+0.5=$ & 5.7 & $\mathrm{P}_{\mathrm{b}, \mathrm{est}}+1.0=$ & 6.2 \\
\hline Target W & (g) & & 0.5 & & & 72 & 4.0 & 79 & 1.8 \\
\hline Actual W & $(g)$ & & 0.0 & & & 73 & 0.9 & 86 & 6.0 \\
\hline Actual & & & 69 & & 19 & & 74 & 6. & 73 \\
\hline $\mathrm{Gmm}$ & & & 37 & & 15 & 2. & 472 & 2.4 & 146 \\
\hline Specimen & No. & 1 & 2 & 1 & 2 & 1 & 2 & 1 & 2 \\
\hline & 1 & 111.42 & 111.58 & 110.69 & 110.41 & 110.27 & 110.92 & 109.32 & 110.29 \\
\hline$\stackrel{\mathscr{I}}{\approx}$ & 2 & 111.4 & 111.55 & 111.04 & 110.25 & 110.44 & 110.56 & 109.48 & 110.56 \\
\hline 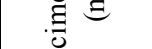 & 3 & 111.45 & 111.52 & 110.67 & 110.38 & 110.74 & 110.6 & 109.62 & 110.51 \\
\hline के & 4 & 111.38 & 111.46 & 110.73 & 110.44 & 110.42 & 110.48 & 109.93 & 110.29 \\
\hline $\mathrm{Av}$ & erage & 111.41 & 111.53 & 110.78 & 110.37 & 110.47 & 110.64 & 109.59 & 110.41 \\
\hline $\mathrm{W}_{\text {dry }}(\mathrm{g})$ & A & 4802.5 & 4805.2 & 4800.8 & 4796.1 & 4786.5 & 4794.4 & 4757.9 & 4772.1 \\
\hline Win water $(\mathrm{g})$ & $\mathrm{C}$ & 2847.8 & 2847.3 & 2852.2 & 2855.1 & 2838.1 & 2843.3 & 2810.8 & 2812.9 \\
\hline W SSD (g) & B & 4803.5 & 4805.6 & 4801.9 & 4796.9 & 4787.0 & 4794.9 & 4758.5 & 4772.5 \\
\hline $\mathrm{G}_{\mathrm{mb}}=\frac{}{(\mathrm{B}}$ & $\frac{A}{-C)}$ & 2.456 & 2.454 & 2.462 & 2.470 & 2.456 & 2.457 & 2.443 & 2.435 \\
\hline Corrected & $\mathrm{N}_{\text {ini }}$ & 2.307 & 2.303 & 2.326 & 2.327 & 2.326 & 2.321 & 2.362 & 2.369 \\
\hline $\mathrm{G}_{\mathrm{mb}}$ & $\mathrm{N}_{\text {des }}$ & 2.439 & 2.437 & 2.451 & 2.457 & 2.449 & 2.448 & 2.436 & 2.426 \\
\hline $\mathrm{Va} @ \mathrm{~N}$ & & 3.88 & 3.96 & 2.55 & 2.32 & 0.92 & 0.96 & 0.39 & 0.8 \\
\hline Averag & & & 92 & & 44 & & 94 & 0. & 60 \\
\hline VMA@ & $V_{\text {des }}$ & 10.79 & 10.86 & 10.83 & 10.61 & 11.42 & 11.45 & 12.81 & 13.17 \\
\hline Averag & & & .83 & & .72 & & .43 & 12. & 99 \\
\hline VFA@1 & & 64.04 & 63.55 & 76.45 & 78.13 & 91.94 & 91.62 & 96.96 & 93.93 \\
\hline Averag & & & .80 & & .29 & & .78 & 95 & .44 \\
\hline \%Gmm@ & $\mathrm{N}_{\text {ini }}$ & 90.93 & 90.78 & 92.49 & 92.52 & 94.09 & 93.89 & 96.57 & 96.85 \\
\hline Averag & & & .86 & & .50 & 93 & .99 & 96 & .71 \\
\hline \%Gmm@ & $V_{\max }$ & 96.79 & 96.72 & 97.91 & 98.21 & 99.35 & 99.38 & 99.87 & 99.56 \\
\hline Averag & & & 5.76 & & .06 & & .37 & 99 & .72 \\
\hline Dust Propc & rtion & & .46 & & 41 & & 37 & 0. & 32 \\
\hline
\end{tabular}


Table A.5 Mix Design Data for Kasota Limestone Coarse Gradation

\begin{tabular}{|c|c|c|c|c|c|c|c|c|c|}
\hline Project: & SUPE & PAVE & Technician: & & & & Date: & & \\
\hline CA Source: & KAS & TA & FA Source: & LAKEL & & Blend Gsb: & 2.561 & Blend Gse: & 2.686 \\
\hline Gradation: & $\begin{array}{l}{[\mathrm{A}} \\
{[\mathrm{X}] \mathrm{B}}\end{array}$ & $\begin{array}{l}\text { ove (fine) } \\
\text { low (coarse) }\end{array}$ & the Restricte & Zone & $\begin{array}{l}\text { Nom. Max. } \\
\text { Size (mm): }\end{array}$ & 12.5 & Batch & eight, Ws, (g): & 12000 \\
\hline Asphalt Sor & rse: & $\mathrm{KOCH}$ & & Grade: & PG 52-34 & Polymer: & none & $\mathrm{P}_{\mathrm{b} \text {,est }}=$ & 6.08 \\
\hline$T_{\text {mix }}: \quad 138$ & $-{ }^{\circ} \mathrm{C}$ & $\mathrm{T}_{\text {comp: }}$ & $28 \quad{ }^{\circ} \mathrm{C}$ & Compac & Brovold & & & & \\
\hline Target & & $\mathrm{P}_{\mathrm{b} \text {,est }}-0.5=$ & 5.6 & $\mathrm{P}_{\mathrm{b}, \mathrm{est}}=$ & 6.1 & $\mathrm{P}_{\mathrm{b}, \text { est }}+0.5=$ & 6.6 & $\mathrm{~Pb}_{\mathrm{b}, \mathrm{est}}+1.0=$ & 7.1 \\
\hline Target W & $(g)$ & & 9.2 & & 6.8 & 84 & 5.2 & 91 & \\
\hline Actual W & $\mathrm{b}(\mathrm{g})$ & & 7.9 & & 6.0 & 84 & 2.8 & 92 & \\
\hline Actual & & & 57 & & 07 & & 56 & 7. & \\
\hline Gmm & & & 485 & & 464 & 2. & 467 & 2. & \\
\hline Specimen & No. & 1 & 2 & 1 & 2 & 1 & 2 & 1 & 2 \\
\hline & 1 & 110.09 & 111.30 & 109.74 & 110.15 & 110.55 & 110.18 & 105.32 & 107.50 \\
\hline ฮี & 2 & 110.81 & 110.87 & 110.53 & 109.93 & 110.27 & 109.50 & 105.96 & 107.73 \\
\hline$\Xi \Xi$ & 3 & 110.78 & 110.94 & 110.52 & 109.80 & 110.46 & 109.73 & 105.64 & 107.57 \\
\hline की & 4 & 110.79 & 110.80 & 109.87 & 109.88 & 110.79 & 109.61 & 105.60 & 107.30 \\
\hline Av & erage & 110.62 & 110.98 & 110.17 & 109.94 & 110.52 & 109.76 & 105.63 & 107.53 \\
\hline $\mathrm{W}_{\text {dry }}(\mathrm{g})$ & A & 4769.2 & 4760.4 & 4706.7 & 4710.2 & 4692.5 & 4690.5 & 4495.8 & 4576.9 \\
\hline $\mathrm{W}_{\text {in water }}(\mathrm{g})$ & $\mathrm{C}$ & 2833.2 & 2825.4 & 2787.7 & 2788.6 & 2774.5 & 2772.0 & 2646.2 & 2690.1 \\
\hline WSSD (g) & B & 4769.9 & 4761.0 & 4707.9 & 4710.9 & 4694.0 & 4691.4 & 4497.1 & 4578.4 \\
\hline $\mathrm{G}_{\mathrm{mb}}=\frac{}{(\mathrm{B}}$ & $\frac{A}{-C)}$ & 2.463 & 2.459 & 2.451 & 2.450 & 2.445 & 2.444 & 2.429 & 2.424 \\
\hline Corrected & $\mathrm{N}_{\text {ini }}$ & 2.206 & 2.217 & 2.215 & 2.220 & 2.213 & 2.180 & 2.273 & 2.256 \\
\hline Gmb & $\mathrm{N}_{\text {des }}$ & 2.434 & 2.430 & 2.435 & 2.430 & 2.432 & 2.415 & 2.420 & 2.417 \\
\hline $\mathrm{V}_{\mathrm{a}} @ \mathrm{~N}$ & & 2.03 & 2.19 & 1.16 & 1.38 & 1.43 & 2.09 & 0.78 & 0.9 \\
\hline Averą & & & 11 & & .27 & & 76 & & \\
\hline VMA@ & $V_{\text {des }}$ & 10.25 & 10.40 & 10.70 & 10.88 & 11.27 & 11.89 & 12.30 & 12.40 \\
\hline Averą & & & .33 & & .79 & & .58 & 12 & \\
\hline VFA@ & & 80.20 & 78.94 & 89.15 & 87.31 & 87.31 & 82.42 & 93.66 & 92.74 \\
\hline Averą & & & .57 & & 3.23 & & 87 & & \\
\hline \%Gmm@ & $\mathrm{Nini}_{\mathrm{i}}$ & 88.77 & 89.22 & 89.89 & 90.10 & 89.70 & 88.37 & 93.19 & 92.50 \\
\hline Averą & & & .99 & & .00 & & .04 & 92 & \\
\hline \%Gmm@ & $\mathrm{N}_{\max }$ & 99.10 & 98.97 & 99.48 & 99.44 & 99.09 & 99.06 & 99.59 & 99.38 \\
\hline Averas & & & .03 & & .46 & & .08 & & \\
\hline Dust Prop & rtion & & 40 & & .37 & & 34 & & \\
\hline
\end{tabular}


Table A.6 Mix Design Data for Kasota Limestone Fine Gradation

\begin{tabular}{|c|c|c|c|c|c|c|c|c|c|}
\hline Project: & SUPE & PAVE & Technician: & & & & Date: & & \\
\hline CA Source: & KASC & & FA Source: & LAKELA & & Blend Gsb: & 2.581 & Blend Gse: & 2.695 \\
\hline Gradation: & $\begin{array}{l}{[\mathrm{X}] \mathrm{A}} \\
{[\mathrm{B}}\end{array}$ & $\begin{array}{l}\text { ove (fine) } \\
\text { low (coarse }\end{array}$ & the Restricted & Zone & $\begin{array}{l}\text { Nom. Max. } \\
\text { Size (mm): }\end{array}$ & 12.5 & Batch & ight, Ws, (g): & 12000 \\
\hline Asphalt Sol & rse: & $\mathrm{KOCH}$ & & Grade: & PG 52-34 & Polymer: & none & $\mathrm{P}_{\mathrm{b}, \text { est }}=$ & 5.38 \\
\hline$T_{\text {mix }}$ & $-{ }^{\circ} \mathrm{C}$ & $\mathrm{T}_{\text {comp: }}$ & $28{ }^{\circ} \mathrm{C}$ & Compact & Brovold & & $\mathrm{N}_{\text {ini }}$ & $\mathrm{N}_{\text {des }} \quad 76$ & $\mathrm{~N}_{\max } \quad 117$ \\
\hline Target & & $\mathrm{P}_{\mathrm{b} \text {,est }}-0.5=$ & 4.9 & $\mathrm{P}_{\mathrm{b}, \text { est }}=$ & 5.4 & $\mathrm{P}_{\mathrm{b}, \mathrm{est}}+0.5=$ & 5.9 & $\mathrm{P}_{\mathrm{b}, \mathrm{est}}+1.0=$ & 6.4 \\
\hline Target W & $(g)$ & & 5.6 & & & 74 & 9.7 & $81^{\prime}$ & 7.8 \\
\hline Actual W & $(g)$ & & & & & 75 & 8.9 & $81^{\prime}$ & 7.7 \\
\hline Actual & & & 08 & & 48 & 5 . & 95 & 6. & 38 \\
\hline Gmm & & & 502 & & 01 & 2. & 72 & 2.4 & 54 \\
\hline Specimen & No. & 1 & 2 & 1 & 2 & 1 & 2 & 1 & 2 \\
\hline & 1 & 113.48 & 112.79 & 103.34 & 104.38 & 112.02 & 112.06 & 110.09 & 112.83 \\
\hline$\stackrel{\mathscr{I}}{\approx}$ & 2 & 113.77 & 112.62 & 103.37 & 104.49 & 111.81 & 112.19 & 110.23 & 112.35 \\
\hline 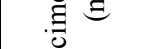 & 3 & 113.56 & 112.55 & 103.28 & 104.56 & 112.03 & 112.07 & 111.15 & 111.82 \\
\hline के & 4 & 113.42 & 112.49 & 103.12 & 104.52 & 111.81 & 111.9 & 110.44 & 112.17 \\
\hline & erage & 113.56 & 112.61 & 103.28 & 104.49 & 111.92 & 112.06 & 110.48 & 112.29 \\
\hline $\mathrm{W}_{\text {dry }}(\mathrm{g})$ & A & 4800.4 & 4744.2 & 4387.0 & 4442.7 & 4808.1 & 4807.4 & 4751.9 & 4829.5 \\
\hline Win water $(\mathrm{g})$ & $\mathrm{C}$ & 2820.2 & 2784.6 & 2582.2 & 2617.9 & 2838.1 & 2838.8 & 2802.9 & 2848.6 \\
\hline W SSD (g) & B & 4803.0 & 4748.4 & 4389.0 & 4444.1 & 4809.1 & 4807.9 & 4752.1 & 4829.9 \\
\hline $\mathrm{G}_{\mathrm{mb}}=\frac{}{(\mathrm{B}}$ & $\frac{A}{-C)}$ & 2.421 & 2.416 & 2.428 & 2.433 & 2.439 & 2.441 & 2.438 & 2.438 \\
\hline Corrected & $\mathrm{N}_{\text {ini }}$ & 2.254 & 2.250 & 2.265 & 2.267 & 2.298 & 2.288 & 2.323 & 2.320 \\
\hline Gmb & $\mathrm{N}_{\text {des }}$ & 2.402 & 2.395 & 2.412 & 2.411 & 2.430 & 2.430 & 2.431 & 2.429 \\
\hline $\mathrm{V}_{\mathrm{a}} @ \mathrm{~N}$ & & 4.00 & 4.29 & 3.57 & 3.59 & 1.69 & 1.69 & 0.92 & 1.00 \\
\hline Avera & & & 15 & & 58 & & 69 & 0. & 96 \\
\hline VMA@ & $V_{\text {des }}$ & 11.66 & 11.92 & 11.67 & 11.70 & 11.45 & 11.45 & 11.82 & 11.89 \\
\hline Avera & & & .79 & & 69 & & 45 & 11. & 86 \\
\hline VFA@ & & 65.70 & 64.01 & 69.40 & 69.33 & 85.24 & 85.24 & 92.22 & 91.59 \\
\hline Avera & & & 86 & & .36 & & 24 & 91 & 90 \\
\hline \%Gmm@ & $\mathrm{N}_{\text {ini }}$ & 90.09 & 89.93 & 90.56 & 90.64 & 92.96 & 92.56 & 94.66 & 94.54 \\
\hline Avera & & & .01 & & 60 & & .76 & 94 & 60 \\
\hline \%Gmm@ & $V_{\max }$ & 96.76 & 96.56 & 97.08 & 97.27 & 98.68 & 98.76 & 99.34 & 99.33 \\
\hline Avera & & & 66 & & .18 & & 72 & 99 & 34 \\
\hline Dust Prop & rtion & & 42 & & 39 & & 36 & 0. & 34 \\
\hline
\end{tabular}


Table A.7 Mix Design Data for Cedar Grove Gravel Coarse Gradation

\begin{tabular}{|c|c|c|c|c|c|c|c|c|c|}
\hline Project: & SUPE & PAVE & Technician: & & & & Date: & & \\
\hline CA Source: & CEDA & R GROVE & FA Source: & LAKEL $A$ & & Blend Gsb: & 2.6050 & Blend Gse: & 2.695 \\
\hline Gradation: & $\begin{array}{l}{[\mathrm{A}} \\
{[\mathrm{X}] \mathrm{B}}\end{array}$ & $\begin{array}{l}\text { ove (fine) } \\
\text { low (coarse) }\end{array}$ & the Restricted & Zone & $\begin{array}{l}\text { Nom. Max. } \\
\text { Size (mm): }\end{array}$ & 12.5 & Batch & ight, Ws, (g): & 12000 \\
\hline Asphalt Sou & rse: & $\mathrm{KOCH}$ & & Grade: & PG 52-34 & Polymer: & none & $\mathrm{P}_{\mathrm{b}, \text { est }}=$ & 5.36 \\
\hline$T_{\text {mix }}: \quad 138$ & $-{ }^{\circ} \mathrm{C}$ & $\mathrm{T}_{\text {comp: }}$ & $128{ }^{\circ} \mathrm{C}$ & Compac & Brovold & & $\mathrm{N}_{\text {ini }}$ & $\mathrm{N}_{\text {des }} \quad 76$ & $\mathrm{~N}_{\max } \quad 117$ \\
\hline Target & & $\mathrm{Pb}_{\mathrm{b}, \mathrm{est}}-0.5=$ & 4.9 & $\mathrm{P}_{\mathrm{b}, \text { est }}=$ & 5.4 & $\mathrm{P}_{\mathrm{b}, \mathrm{est}}+0.5=$ & 5.9 & $\mathrm{P}_{\mathrm{b}, \mathrm{est}}+1.0=$ & 6.4 \\
\hline Target W & (g) & & 3.0 & & & 74 & 7.0 & 81 & 5.0 \\
\hline Actual W & $(g)$ & & 3.4 & & & 74 & 9.5 & 82 & 0.7 \\
\hline Actual & & & 86 & & 35 & & 88 & 6. & 40 \\
\hline $\mathrm{Gmm}$ & & & 530 & & 24 & 2. & 475 & 2.4 & 74 \\
\hline Specimen & No. & 1 & 2 & 1 & 2 & 1 & 2 & 1 & 2 \\
\hline & 1 & 110.35 & 110.74 & 110.37 & 110.74 & 108.69 & 108.51 & 105.81 & 106.10 \\
\hline$\stackrel{\mathscr{I}}{\approx}$ & 2 & 110.40 & 110.71 & 110.47 & 110.94 & 108.44 & 108.48 & 105.69 & 105.84 \\
\hline . & 3 & 110.36 & 110.66 & 110.30 & 110.83 & 108.42 & 108.46 & 105.62 & 106.16 \\
\hline के & 4 & 110.36 & 110.75 & 109.93 & 110.79 & 108.40 & 108.61 & 105.67 & 105.83 \\
\hline $\mathrm{Av}$ & erage & 110.37 & 110.72 & 110.27 & 110.83 & 108.49 & 108.52 & 105.70 & 105.98 \\
\hline $\mathrm{W}_{\text {dry }}(\mathrm{g})$ & A & 4789.5 & 4793.1 & 4783.5 & 4786.7 & 4706.5 & 4706.3 & 4574.4 & 4600.5 \\
\hline Win water $(\mathrm{g})$ & $\mathrm{C}$ & 2869.7 & 2866.2 & 2863.4 & 2864.9 & 2804.6 & 2807.6 & 2724.1 & 2747.1 \\
\hline W SSD (g) & B & 4790.5 & 4794.4 & 4783.8 & 4787.5 & 4707.1 & 4706.9 & 4574.9 & 4600.9 \\
\hline $\mathrm{G}_{\mathrm{mb}}=\frac{}{(\mathrm{B}}$ & $\frac{A}{-C)}$ & 2.493 & 2.486 & 2.491 & 2.490 & 2.474 & 2.478 & 2.472 & 2.482 \\
\hline Corrected & $\mathrm{N}_{\text {ini }}$ & 2.275 & 2.242 & 2.256 & 2.273 & 2.300 & 2.270 & 2.304 & 2.296 \\
\hline $\mathrm{G}_{\mathrm{mb}}$ & $\mathrm{N}_{\text {des }}$ & 2.473 & 2.461 & 2.464 & 2.470 & 2.465 & 2.467 & 2.464 & 2.473 \\
\hline $\mathrm{Va} @ \mathrm{~N}$ & & 2.26 & 2.71 & 2.37 & 2.15 & 0.41 & 0.34 & 0.40 & 0.05 \\
\hline Averag & & & 49 & & 26 & & 38 & 0. & 23 \\
\hline VMA@ & $V_{\text {des }}$ & 9.68 & 10.12 & 10.47 & 10.25 & 10.94 & 10.86 & 11.47 & 11.14 \\
\hline Averag & & & 90 & & .36 & & 90 & 11. & 31 \\
\hline VFA@1 & & 76.66 & 73.23 & 77.36 & 79.03 & 96.25 & 96.87 & 96.51 & 99.55 \\
\hline Averag & & & 1.94 & & .20 & & .56 & 98 & 03 \\
\hline \%Gmm@ & $\mathrm{N}_{\text {ini }}$ & 89.92 & 88.62 & 89.38 & 90.06 & 92.93 & 91.72 & 93.13 & 92.81 \\
\hline Averag & & & .27 & & .72 & & .32 & 92 & 97 \\
\hline \%Gmm@ & $V_{\max }$ & 98.56 & 98.25 & 98.69 & 98.64 & 99.95 & 100.12 & 99.90 & 100.31 \\
\hline Averag & & & .40 & & 66 & & .04 & 100 & .11 \\
\hline Dust Prope & rtion & & 46 & & 42 & & 38 & 0. & 35 \\
\hline
\end{tabular}


Table A.8 Mix Design Data for Cedar Grove Gravel Fine Gradation

\begin{tabular}{|c|c|c|c|c|c|c|c|c|c|}
\hline Project: & SUPEI & PAVE & & & & & & $6 / 21 / 1$ & 999 \\
\hline CA Source: & Cedar & Grove Gravel & FA Source: & LAKELA & & Blend Gsb: & 2.6030 & Blend Gse: & 2.699 \\
\hline Gradation: & $\begin{array}{l}{[\mathrm{X}] \mathrm{A}} \\
{[\mathrm{B}}\end{array}$ & $\begin{array}{l}\text { ove (fine) } \\
\text { low (coarse) }\end{array}$ & the Restricted & Zone & $\begin{array}{l}\text { Nom. Max. } \\
\text { Size (mm): }\end{array}$ & 12.5 & Batch & eight, Ws, (g): & 12000 \\
\hline Asphalt Sou & Irse: & $\mathrm{KOCH}$ & & Grade: & PG 52-34 & Polymer: & none & $\mathrm{Pb}_{\mathrm{b} \text { est }}=$ & 4.60 \\
\hline $\mathrm{T}_{\text {mix }}: \quad 138$ & $-{ }^{\circ} \mathrm{C}$ & $\mathrm{T}_{\text {comp: }}$ & $\underline{128}{ }^{\circ} \mathrm{C}$ & Compact & Brovold & & Nini & $N_{\text {des }} \quad 76$ & $N_{\max } \quad 117$ \\
\hline Target & & $\mathrm{Pb}_{\text {,est }}-0.5=$ & 4.1 & $\mathrm{P}_{\mathrm{b}, \mathrm{est}}=$ & 4.6 & $\mathrm{P}_{\mathrm{b}, \mathrm{est}}+0.5=$ & 5.1 & $\mathrm{P}_{\mathrm{b}, \text { est }}+1.0=$ & 5.6 \\
\hline Target W & b (g) & & 3.0 & & 8.6 & 64 & 4.9 & 711 & 1.9 \\
\hline Actual W & $b(g)$ & & 9.8 & & 79.4 & 65 & 0.7 & 706 & \\
\hline Actual & & & 08 & & .61 & 5. & 14 & 5.5 & \\
\hline $\mathrm{Gmm}$ & & & 54 & & 515 & 2.4 & 499 & 2.4 & \\
\hline Specimen & No. & 1 & 2 & 1 & 2 & 1 & 2 & 1 & 2 \\
\hline & 1 & & & & & & & & \\
\hline$\stackrel{\stackrel{\Xi}{\exists}}{\Xi}$ & 2 & & & & & & & & \\
\hline हี हี & 3 & & & & & & & & \\
\hline के & 4 & & & & & & & & \\
\hline $\mathrm{Av}$ & erage & & & & & & & & \\
\hline $\mathrm{W}_{\text {dry }}(\mathrm{g})$ & A & 4802.4 & 4809.9 & 4804.6 & 4807.2 & 4789.1 & 4797.5 & 4811.0 & 4800.6 \\
\hline Win water $(\mathrm{g})$ & $\mathrm{C}$ & 2806.0 & 2815.8 & 2818.1 & 2823.2 & 2838.0 & 2838.9 & 2846.9 & 2839.7 \\
\hline W SSD (g) & B & 4807.6 & 4814.3 & 4807.0 & 4809.1 & 4790.0 & 4799.2 & 4811.8 & 4801.2 \\
\hline $\mathrm{G}_{\mathrm{mb}}=\frac{}{(\mathrm{B}}$ & $\frac{A}{-C)}$ & 2.399 & 2.407 & 2.416 & 2.421 & 2.453 & 2.447 & 2.448 & 2.447 \\
\hline Corrected & $\mathrm{N}_{\text {ini }}$ & 2.239 & 2.258 & 2.272 & 2.274 & 2.301 & 2.294 & 2.301 & 2.290 \\
\hline $\mathrm{Gmb}$ & $\mathrm{N}_{\text {des }}$ & 2.378 & 2.388 & 2.399 & 2.404 & 2.435 & 2.429 & 2.433 & 2.432 \\
\hline $\mathrm{V}_{\mathrm{a}} @ \mathrm{~N}_{\mathrm{c}}$ & & 6.38 & 5.98 & 4.61 & 4.42 & 2.53 & 2.76 & 2.07 & 2.11 \\
\hline Averag & & & 18 & & .52 & 2. & 65 & 2.0 & \\
\hline VMA@ & $\mathrm{N}_{\mathrm{des}}$ & 12.37 & 12.00 & 12.08 & 11.90 & 11.27 & 11.48 & 11.72 & 11.76 \\
\hline Averag & & & 18 & & 1.99 & 11. & 38 & 11. & \\
\hline VFA@1 & $\mathrm{N}_{\text {des }}$ & 48.41 & 50.16 & 61.84 & 62.85 & 77.54 & 75.97 & 82.34 & 82.06 \\
\hline Averag & & & .28 & & 2.35 & 76. & .75 & 82. & .20 \\
\hline \%Gmm@ & $\mathrm{N}_{\text {ini }}$ & 88.15 & 88.90 & 90.34 & 90.42 & 92.10 & 91.82 & 92.63 & 92.19 \\
\hline Averag & & & .52 & & 0.38 & 91. & 96 & 92. & \\
\hline \%Gmm@ & $N_{\max }$ & 94.46 & 94.75 & 96.05 & 96.25 & 98.20 & 97.95 & 98.57 & 98.53 \\
\hline Averag & & & 61 & & 6.15 & 98. & .07 & 98. & \\
\hline Dust Prope & rition & & 53 & & 46 & 0. & 42 & 0.3 & \\
\hline
\end{tabular}


Table A.9 Mix Design Data for New Ulm Quartzite Fine Gradation (PG 58-40)

\begin{tabular}{|c|c|c|c|c|c|c|c|c|c|}
\hline Project: & SUPEF & PAVE & Technician: & & & & Date: & $6 / 21 / 19$ & \\
\hline CA Source: & New L & Im Quartzite & FA Source: & LAKELA & & Blend Gsb: & 2.6060 & Blend Gse: & 2.6922 \\
\hline Gradation: & $\begin{array}{l}{[\mathrm{X}] \mathrm{A}} \\
{[\mathrm{B}}\end{array}$ & $\begin{array}{l}\text { ove (fine) } \\
\text { ow (coarse) }\end{array}$ & the Restrictec & Zone & $\begin{array}{l}\text { Nom. Max. } \\
\text { Size (mm): }\end{array}$ & 12.5 & Batch & eight, Ws, (g): & 12000 \\
\hline Asphalt Sor & rse: & $\mathrm{KOCH}$ & & Grade: & PG 58-40 & Polymer: & yes & $\mathrm{P}_{\mathrm{b}, \text { est }}=$ & 4.60 \\
\hline$T_{\text {mix }}: \quad 145$ & $-{ }^{\circ} \mathrm{C}$ & $\mathrm{T}_{\text {comp: }}$ & $-{ }^{\circ} \mathrm{C}$ & Compact & Brovold & & $\mathrm{N}_{\text {ini }} \quad 7$ & $\mathrm{~N}_{\text {des }} \quad 76$ & $\mathrm{~N}_{\max } \quad 117$ \\
\hline Target & & $\mathrm{P}_{\mathrm{b}, \text { est }}-0.5=$ & 4.1 & $\mathrm{P}_{\mathrm{b}, \text { est }}=$ & 4.6 & $\mathrm{P}_{\mathrm{b}, \mathrm{est}}+0.5=$ & 5.1 & $\mathrm{P}_{\mathrm{b}, \mathrm{est}}+1.0=$ & 5.6 \\
\hline Target W & $(g)$ & & 3.0 & & 8.6 & 64 & 4.9 & 71 & 1.9 \\
\hline Actual W & $b(g)$ & & 1.4 & & 3.6 & 64 & 2.8 & 71 & 2.3 \\
\hline Actual & & & 09 & & 64 & & 08 & 5. & 60 \\
\hline $\mathrm{G}_{\mathrm{mm}}$ & & & 527 & & 99 & 2.5 & 03 & 2.4 & 481 \\
\hline Specimen & No. & 1 & 2 & 1 & 2 & 1 & 2 & 1 & 2 \\
\hline & 1 & 113.67 & 113.42 & 112.97 & 112.93 & 111.87 & 111.78 & 111.16 & 111.35 \\
\hline$\stackrel{\mathscr{E}}{\Xi} \cong$ & 2 & 113.66 & 113.55 & 112.93 & 112.97 & 111.66 & 111.92 & 111.01 & 111.04 \\
\hline 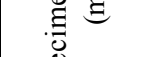 & 3 & 113.87 & 113.59 & 112.84 & 112.76 & 111.79 & 111.70 & 111.11 & 111.06 \\
\hline के & 4 & 113.94 & 113.75 & 113.22 & 112.83 & 111.78 & 111.75 & 111.16 & 111.17 \\
\hline & erage & 113.79 & 113.58 & 112.99 & 112.87 & 111.78 & 111.79 & 111.11 & 111.16 \\
\hline $\mathrm{W}_{\text {dry }}(\mathrm{g})$ & A & 4808.5 & 4799.0 & 4805.6 & 4800.2 & 4799.2 & 4820.5 & 4789.5 & 4793.7 \\
\hline W in water $(\mathrm{g})$ & $\mathrm{C}$ & 2825.3 & 2819.6 & 2830.7 & 2829.3 & 2843.1 & 2863.3 & 2839.1 & 2842.9 \\
\hline$W_{\text {SSD }}(\mathrm{g})$ & B & 4817.7 & 4807.3 & 4810.2 & 4805.7 & 4802.3 & 4823.7 & 4791.6 & 4796.8 \\
\hline $\mathrm{G}_{\mathrm{mb}}=\frac{}{(\mathrm{B}}$ & $\frac{A}{-C)}$ & 2.413 & 2.414 & 2.428 & 2.429 & 2.450 & 2.459 & 2.453 & 2.453 \\
\hline Corrected & $\mathrm{Nini}_{\mathrm{i}}$ & 2.272 & 2.269 & 2.284 & 2.286 & 2.308 & 2.321 & 2.329 & 2.331 \\
\hline Gmb & $\mathrm{N}_{\text {des }}$ & 2.398 & 2.399 & 2.411 & 2.412 & 2.432 & 2.444 & 2.446 & 2.446 \\
\hline $\mathrm{V} \mathrm{a} @ \mathrm{~N}$ & & 5.1 & 5.06 & 3.53 & 3.49 & 2.82 & 2.37 & 1.40 & 1.40 \\
\hline Avera & & & 08 & & 51 & & 60 & 1. & 40 \\
\hline VMA@ & $\mathrm{N}_{\mathrm{des}}$ & 11.74 & 11.71 & 11.77 & 11.74 & 11.42 & 10.98 & 11.40 & 11.40 \\
\hline Avera & & & .72 & & 76 & 11 & 20 & & .40 \\
\hline VFA@. & $\mathrm{J}_{\mathrm{des}}$ & 56.57 & 56.77 & 70.02 & 70.26 & 75.31 & 78.42 & 87.72 & 87.72 \\
\hline Avera & & & 67 & & 14 & 76 & 87 & 87 & .72 \\
\hline \%Gmm@ & $\mathrm{Nini}_{\mathrm{i}}$ & 89.91 & 89.79 & 91.40 & 91.48 & 92.21 & 92.73 & 93.87 & 93.95 \\
\hline Avera & & & .85 & & 44 & & 47 & 93 & .91 \\
\hline \%Gmm@ & $\mathrm{N}_{\max }$ & 95.51 & 95.54 & 97.15 & 97.19 & 97.87 & 98.24 & 98.87 & 98.89 \\
\hline Avera & & & .52 & & 17 & & .05 & 98 & .88 \\
\hline Dust Prop & rtion & & 52 & & 46 & & 42 & & 38 \\
\hline
\end{tabular}


Table A.10 Mix Design Data for Kasota Limestone Fine Gradation (PG58-40)

\begin{tabular}{|c|c|c|c|c|c|c|c|c|c|}
\hline Project: & SUPE & PAVE & Technician: & & & & Date: & $6 / 21 / 19$ & \\
\hline CA Source: & Kaso & Limestone & FA Source: & LAKEL $A$ & & Blend Gsb: & 2.5810 & Blend Gse: & 2.695 \\
\hline Gradation: & $\begin{array}{l}{[\mathrm{X}] \mathrm{A}} \\
{[\mathrm{B}}\end{array}$ & $\begin{array}{l}\text { ove (fine) } \\
\text { low (coarse) }\end{array}$ & the Restricted & Zone & $\begin{array}{l}\text { Nom. Max. } \\
\text { Size (mm): }\end{array}$ & 12.5 & Batch & ight, Ws, (g): & 12000 \\
\hline Asphalt Sol & rse: & $\mathrm{KOCH}$ & & Grade: & PG 58-40 & Polymer: & yes & $\mathrm{P}_{\mathrm{b}, \text { est }}=$ & 4.60 \\
\hline$T_{\text {mix }}: \quad 145$ & $-{ }^{\circ} \mathrm{C}$ & $\mathrm{T}_{\text {comp: }}$ & $135{ }^{\circ} \mathrm{C}$ & Compac & Brovold & & $\mathrm{N}_{\text {ini }}$ & $\mathrm{N}_{\text {des }} \quad 76$ & $\mathrm{~N}_{\max } \quad 117$ \\
\hline Target & & $\mathrm{Pb}_{\mathrm{b}, \mathrm{est}}-0.5=$ & 4.1 & $\mathrm{P}_{\mathrm{b}, \text { est }}=$ & 4.6 & $\mathrm{P}_{\mathrm{b}, \mathrm{est}}+0.5=$ & 5.1 & $\mathrm{P}_{\mathrm{b}, \mathrm{est}}+1.0=$ & 5.6 \\
\hline Target W & $(g)$ & & 3.0 & & & 64 & 4.9 & 71 & 1.9 \\
\hline Actual W & $(g)$ & & 3.0 & & & 64 & 4.2 & 71 & 1.4 \\
\hline Actual & & & 10 & & 59 & & 09 & 5. & 60 \\
\hline Gmm & & & 523 & & 89 & 2. & 77 & 2.4 & 67 \\
\hline Specimen & No. & 1 & 2 & 1 & 2 & 1 & 2 & 1 & 2 \\
\hline & 1 & 113.41 & 113.49 & 113.88 & 113.81 & 112.46 & 112.27 & 112.52 & 111.18 \\
\hline$\stackrel{\mathscr{I}}{\approx}$ & 2 & 113.54 & 113.49 & 113.80 & 113.68 & 112.55 & 112.11 & 112.56 & 110.75 \\
\hline . & 3 & 113.08 & 113.65 & 113.80 & 113.60 & 112.54 & 112.52 & 112.62 & 110.62 \\
\hline के & 4 & 113.18 & 113.54 & 113.82 & 113.76 & 112.39 & 112.51 & 112.7 & 111.43 \\
\hline & erage & 113.30 & 113.54 & 113.83 & 113.71 & 112.49 & 112.35 & 112.60 & 111.00 \\
\hline $\mathrm{W}_{\text {dry }}(\mathrm{g})$ & A & 4795.7 & 4807.5 & 4791.0 & 4791.0 & 4789.6 & 4787.4 & 4789.6 & 4689.9 \\
\hline W in water $(\mathrm{g})$ & $\mathrm{C}$ & 2817.4 & 2824.3 & 2801.4 & 2806.8 & 2823.0 & 2822.5 & 2819.7 & 2757.4 \\
\hline W SSD (g) & B & 4800.7 & 4813.4 & 4794.4 & 4796.4 & 4793.0 & 4790.7 & 4793.0 & 4695.1 \\
\hline $\mathrm{G}_{\mathrm{mb}}=\frac{}{(\mathrm{B}}$ & $\frac{A}{-C)}$ & 2.418 & 2.417 & 2.404 & 2.408 & 2.431 & 2.432 & 2.427 & 2.420 \\
\hline Corrected & $\mathrm{N}_{\text {ini }}$ & 2.276 & 2.278 & 2.258 & 2.264 & 2.292 & 2.294 & 2.274 & 2.272 \\
\hline $\mathrm{G}_{\mathrm{mb}}$ & $\mathrm{N}_{\text {des }}$ & 2.401 & 2.402 & 2.385 & 2.391 & 2.416 & 2.417 & 2.410 & 2.403 \\
\hline $\mathrm{Va} @ \mathrm{~N}$ & & 4.84 & 4.79 & 4.18 & 3.93 & 2.47 & 2.43 & 2.32 & 2.61 \\
\hline Avera & & & 82 & & 06 & & 45 & 2. & 47 \\
\hline VMA@ & $V_{\text {des }}$ & 10.79 & 10.75 & 11.84 & 11.62 & 11.16 & 11.13 & 11.85 & 12.11 \\
\hline Avera & & & .77 & & .73 & & 14 & 11. & 98 \\
\hline VFA@ & $J_{d e s}$ & 55.13 & 55.44 & 64.69 & 66.17 & 77.87 & 78.16 & 80.42 & 78.44 \\
\hline Avera & & & .29 & & .43 & & 01 & 79 & 43 \\
\hline \%Gmm@ & $N_{\text {ini }}$ & 90.21 & 90.29 & 90.72 & 90.96 & 92.53 & 92.61 & 92.18 & 92.10 \\
\hline Avera & & & .25 & & .84 & & .57 & 92 & 14 \\
\hline \%Gmm@ & $V_{\max }$ & 95.84 & 95.80 & 96.58 & 96.75 & 98.15 & 98.20 & 98.39 & 98.11 \\
\hline Avera & & & .82 & & 66 & & 18 & 98 & 25 \\
\hline Dust Prop & rtion & & 52 & & 47 & & 42 & 0. & 38 \\
\hline
\end{tabular}




\section{APPENDIX B RESILIENT MODULUS DATA}


Table B.1 Resilient Modulus Results for $0{ }^{\circ} \mathrm{F}$ Tests

\begin{tabular}{|c|c|c|c|c|c|c|c|c|c|c|}
\hline \multicolumn{2}{|c|}{ Temp $=0 \mathrm{~F}$} & & \multicolumn{2}{|c|}{ Res. Mod } & & Std. Dev. & \multirow[b]{2}{*}{1} & \multirow[b]{2}{*}{0.33} & \multicolumn{2}{|c|}{ CV (\%) } \\
\hline Freqency & ID (deg) & 0.33 & 0.5 & 1 & 0.33 & 0.5 & & & 0.5 & 1 \\
\hline & $1(0)$ & 2172.63 & 2283.94 & 2190.33 & 279.04 & 349.10 & 146.02 & 12.84 & 15.28 & 6.67 \\
\hline & $1(90)$ & 1391.62 & 1583.59 & 1618.22 & 127.71 & 246.06 & 224.29 & 9.18 & 15.54 & 13.86 \\
\hline GFC & $2(0)$ & 1214.23 & 1565.29 & 1577.36 & 265.28 & 456.89 & 515.05 & 21.85 & 29.19 & 32.65 \\
\hline$(4.5 \%)$ & $2(90)$ & 1492.35 & 1187.01 & 1412.87 & 55.65 & 209.98 & 262.60 & 3.73 & 17.69 & 18.59 \\
\hline & $3(0)$ & 2095.81 & 1936.28 & 2218.58 & 346.64 & 434.46 & 238.61 & 16.54 & 22.44 & 10.75 \\
\hline & $3(90)$ & 1393.97 & 1584.06 & 1602.93 & 251.37 & 222.49 & 380.62 & 18.03 & 14.05 & 23.74 \\
\hline & $1(0)$ & 1993.84 & 2309.81 & 2302.62 & 227.40 & 165.50 & 116.69 & 11.40 & 7.17 & 5.07 \\
\hline & $1(90)$ & 1368.26 & 1284.13 & 1191.02 & 166.10 & 128.12 & 49.01 & 12.14 & 9.98 & 4.11 \\
\hline GFF & $2(0)$ & 1473.61 & 1904.68 & 1459.01 & 326.77 & 419.95 & 374.44 & 22.17 & 22.05 & 25.66 \\
\hline ( $5.1 \%)$ & $2(90)$ & 1361.09 & 1667.67 & 1742.96 & 165.13 & 325.95 & 280.07 & 12.13 & 19.55 & 16.07 \\
\hline & $3(0)$ & 2119.95 & 1775.52 & 2123.50 & 529.17 & 332.36 & 1269.45 & 24.96 & 18.72 & 59.78 \\
\hline & $3(90)$ & 1661.60 & 1764.19 & 1907.43 & 234.31 & 214.35 & 192.63 & 14.10 & 12.15 & 10.10 \\
\hline & $1(0)$ & 1608.95 & 1563.11 & 1456.97 & 449.82 & 477.04 & 644.16 & 27.96 & 30.52 & 44.21 \\
\hline & $1(90)$ & 2106.77 & 2747.99 & 2456.10 & 105.65 & 246.34 & 335.72 & 5.01 & 8.96 & 13.67 \\
\hline NUC & $2(0)$ & 2116.92 & 2063.80 & 2099.05 & 431.04 & 374.70 & 193.24 & 20.36 & 18.16 & 9.21 \\
\hline$(4.7 \%)$ & $2(90)$ & 1643.43 & 1518.49 & 1518.89 & 303.73 & 283.12 & 245.65 & 18.48 & 18.64 & 16.17 \\
\hline & $3(0)$ & 1541.95 & 2173.93 & 2711.84 & 142.61 & 276.18 & 351.04 & 9.25 & 12.70 & 12.94 \\
\hline & $3(90)$ & 1850.71 & 2141.86 & 2151.38 & 220.13 & 245.63 & 130.79 & 11.89 & 11.47 & 6.08 \\
\hline & $1(0)$ & 1985.00 & 2068.07 & 1996.98 & 259.03 & 271.19 & 139.74 & 13.05 & 13.11 & 7.00 \\
\hline & $1(90)$ & 1714.72 & 1761.84 & 1969.99 & 287.21 & 308.75 & 593.95 & 16.75 & 17.52 & 30.15 \\
\hline NUF & $2(0)$ & 1374.27 & 1473.09 & 1563.87 & 223.00 & 68.85 & 80.99 & 16.23 & 4.67 & 5.18 \\
\hline$(5.1 \%)$ & $2(90)$ & 1663.11 & 1689.10 & 1683.02 & 314.84 & 231.39 & 204.83 & 18.93 & 13.70 & 12.17 \\
\hline & $3(0)$ & 1709.02 & 2115.77 & 1843.76 & 223.76 & 185.10 & 299.73 & 13.09 & 8.75 & 16.26 \\
\hline & $3(90)$ & 1893.65 & 1862.69 & 2355.57 & 402.18 & 234.12 & 435.04 & 21.24 & 12.57 & 18.47 \\
\hline & $1(0)$ & 1402.17 & 1403.41 & 1387.74 & 164.14 & 70.54 & 115.45 & 11.71 & 5.03 & 8.32 \\
\hline & $1(90)$ & 1686.70 & 1625.26 & 1867.27 & 171.31 & 168.12 & 185.71 & 10.16 & 10.34 & 9.95 \\
\hline KLC & $2(0)$ & 1370.36 & 1602.38 & 1504.30 & 288.72 & 341.35 & 360.36 & 21.07 & 21.30 & 23.96 \\
\hline$(4.4 \%)$ & $2(90)$ & 1414.69 & 1592.67 & 1684.07 & 237.50 & 231.46 & 274.47 & 16.79 & 14.53 & 16.30 \\
\hline & $3(0)$ & 1905.47 & 1951.13 & 1932.02 & 422.76 & 464.76 & 490.34 & 22.19 & 23.82 & 25.38 \\
\hline & $3(90)$ & 1648.07 & 1595.88 & 1684.02 & 289.64 & 372.33 & 412.53 & 17.57 & 23.33 & 24.50 \\
\hline & $1(0)$ & 1866.45 & 1986.83 & 1784.56 & 202.28 & 289.10 & 172.60 & 10.84 & 14.55 & 9.67 \\
\hline & $1(90)$ & 1499.52 & 1389.03 & 1465.26 & 186.05 & 134.70 & 100.87 & 12.41 & 9.70 & 6.88 \\
\hline KLF & $2(0)$ & 2207.94 & 1774.98 & 1809.16 & 1099.44 & 173.92 & 297.18 & 49.79 & 9.80 & 16.43 \\
\hline$(5.1 \%)$ & $2(90)$ & 2249.48 & 2229.46 & 2323.93 & 139.10 & 459.21 & 140.06 & 6.18 & 20.60 & 6.03 \\
\hline & $3(0)$ & 1764.55 & 2050.67 & 1923.62 & 564.42 & 393.72 & 424.68 & 31.99 & 19.20 & 22.08 \\
\hline & $3(90)$ & 1825.91 & 1879.79 & 1478.32 & 156.18 & 245.23 & 108.58 & 8.55 & 13.05 & 7.35 \\
\hline & $1(0)$ & 1299.18 & 1511.62 & 1539.62 & 483.44 & 359.96 & 350.13 & 37.21 & 23.81 & 22.74 \\
\hline & $1(90)$ & 2111.77 & 3613.15 & 2303.79 & 426.76 & 625.29 & 586.81 & 20.21 & 17.31 & 25.47 \\
\hline CGC & $2(0)$ & 1299.69 & 2308.48 & 2627.92 & 201.73 & 245.90 & 399.42 & 15.52 & 10.65 & 15.20 \\
\hline$(4.6 \%)$ & $2(90)$ & 1524.06 & 1693.85 & 1814.87 & 278.77 & 266.90 & 423.50 & 18.29 & 15.76 & 23.33 \\
\hline & $3(0)$ & 1318.51 & 1269.66 & 1543.28 & 170.94 & 307.04 & 168.84 & 12.96 & 24.18 & 10.94 \\
\hline & $3(90)$ & 2441.10 & 2232.04 & 2552.04 & 327.86 & 622.82 & 1078.33 & 13.43 & 27.90 & 42.25 \\
\hline & $1(0)$ & 1645.13 & 1634.44 & 1943.62 & 130.20 & 137.77 & 304.36 & 7.91 & 8.43 & 15.66 \\
\hline & $1(90)$ & 1633.10 & 1177.91 & 1413.31 & 234.75 & 207.59 & 338.02 & 14.37 & 17.62 & 23.92 \\
\hline CGF & $2(0)$ & 1569.45 & 1670.77 & 1670.86 & 170.42 & 204.32 & 257.28 & 10.86 & 12.23 & 15.40 \\
\hline$(5.0 \%)$ & $2(90)$ & 1506.24 & 2188.26 & 2463.16 & 178.43 & 207.99 & 248.63 & 11.85 & 9.50 & 10.09 \\
\hline & $3(0)$ & 1488.94 & 1370.55 & 1494.91 & 249.85 & 22.30 & 233.93 & 16.78 & 1.63 & 15.65 \\
\hline & $3(90)$ & 1437.06 & 1115.99 & 1318.76 & 262.98 & 147.68 & 234.53 & 18.30 & 13.23 & 17.78 \\
\hline
\end{tabular}


Table B.1 Resilient Modulus Results for $0{ }^{\circ} \mathrm{F}$ Tests, continued

\begin{tabular}{|c|c|c|c|c|c|c|c|c|c|c|}
\hline \multicolumn{2}{|c|}{ Temp = 0F } & \multicolumn{2}{c|}{ Res. Mod } & & \multicolumn{2}{|c|}{ Std. Dev. } & \multicolumn{2}{|c|}{ CV (\%) } \\
\hline Freqency & ID (deg) & $\mathbf{0 . 3 3}$ & $\mathbf{0 . 5}$ & $\mathbf{1}$ & $\mathbf{0 . 3 3}$ & $\mathbf{0 . 5}$ & $\mathbf{1}$ & $\mathbf{0 . 3 3}$ & $\mathbf{0 . 5}$ & $\mathbf{1}$ \\
\hline & $1(0)$ & 1859.03 & 1680.71 & 1923.09 & 696.01 & 228.76 & 200.71 & 37.44 & 13.61 & 10.44 \\
\hline & $1(90)$ & 1723.73 & 1561.61 & 1221.76 & 351.40 & 521.99 & 116.91 & 20.39 & 33.43 & 9.57 \\
\hline NUF & $2(0)$ & 1308.80 & 1177.77 & 1412.09 & 104.70 & 128.39 & 153.85 & 8.00 & 10.90 & 10.89 \\
\hline$(58-40)$ & $2(90)$ & 1577.23 & 1553.64 & 1669.22 & 97.46 & 150.10 & 186.69 & 6.18 & 9.66 & 11.18 \\
\hline$(4.9 \%)$ & $3(0)$ & 2100.99 & 2167.08 & 2196.59 & 252.89 & 97.41 & 282.55 & 12.04 & 4.49 & 12.86 \\
\hline & $3(90)$ & 1800.88 & 1792.76 & 1842.92 & 218.50 & 161.63 & 148.51 & 12.13 & 9.02 & 8.06 \\
\hline & $1(0)$ & 1846.81 & 1836.68 & 1926.72 & 180.38 & 159.19 & 229.46 & 9.77 & 8.67 & 11.91 \\
\hline & $1(90)$ & 1864.91 & 2114.13 & 2103.62 & 103.07 & 140.20 & 86.16 & 5.53 & 6.63 & 4.10 \\
\hline KLF & $2(0)$ & 1830.09 & 1699.25 & 1864.09 & 254.89 & 177.33 & 221.06 & 13.93 & 10.44 & 11.86 \\
\hline$(58-40)$ & $2(90)$ & 1941.27 & 1895.34 & 1820.74 & 251.63 & 176.33 & 64.18 & 12.96 & 9.30 & 3.52 \\
\hline$(4.9 \%)$ & $3(0)$ & 1427.18 & 1116.37 & 1173.38 & 358.89 & 235.26 & 257.48 & 25.15 & 21.07 & 21.94 \\
\hline & $3(90)$ & 1697.17 & 2005.81 & 1895.63 & 247.75 & 770.66 & 224.73 & 14.60 & 38.42 & 11.86 \\
\hline
\end{tabular}

Key

\begin{tabular}{|c|l|c|}
\hline Abbreviation & Mix Type & Binder \\
\hline GFC & Granite Falls granite, coarse gradation, & PG 52-34 \\
\hline GFF & Granite Falls granite, fine gradation, & PG 52-34 \\
\hline NUC & New Ulm quartzite, coarse gradation & PG 52-34 \\
\hline NUF & New Ulm quartzite, fine gradation & PG 52-34 \\
\hline KLC & Kasota limestone, coarse gradation & PG 52-34 \\
\hline KLF & Kasota limestone, fine gradation & PG 52-34 \\
\hline CGC & Cedar Grove gravel, coarse gradation & PG 52-34 \\
\hline CGF & Cedar Grove gravel, fine gradation & PG 52-34 \\
\hline NUF (58-40) & New Ulm quartzite, fine gradation & PG 58-40 \\
\hline KLF (58-40) & Kasota limestone, fine gradation & PG 58-40 \\
\hline
\end{tabular}


Table B.2 Resilient Modulus Results for $34^{\circ} \mathrm{F}$ Tests

\begin{tabular}{|c|c|c|c|c|c|c|c|c|c|c|}
\hline \multicolumn{2}{|c|}{ Temp = 34F } & \multirow[b]{2}{*}{0.33} & \multicolumn{2}{|c|}{ Res. Mod } & \multirow[b]{2}{*}{0.33} & \multirow{2}{*}{$\begin{array}{c}\text { Std. Dev. } \\
0.5\end{array}$} & \multirow[b]{2}{*}{1} & \multirow[b]{2}{*}{0.33} & \multicolumn{2}{|c|}{$\begin{array}{l}\text { Coeficient of } \\
\text { Variance }(\%)\end{array}$} \\
\hline Freqency & ID (deg) & & 0.5 & 1 & & & & & 0.5 & 1 \\
\hline & $1(0)$ & 1231.73 & 1261.85 & 1243.96 & 143.05 & 45.76 & 47.32 & 11.61 & 3.63 & 3.80 \\
\hline & $1(90)$ & 1189.42 & 1316.83 & 1308.97 & 116.78 & 91.91 & 30.40 & 9.82 & 6.98 & 2.32 \\
\hline GFC & $2(0)$ & 1141.11 & 979.11 & 1182.30 & 154.92 & 64.99 & 26.54 & 13.58 & 6.64 & 2.25 \\
\hline \multirow[t]{5}{*}{$(4.5 \%)$} & $2(90)$ & 1484.32 & 1480.81 & 1558.65 & 120.14 & 123.41 & 64.09 & 8.09 & 8.33 & 4.11 \\
\hline & $3(0)$ & 1222.55 & 1078.15 & 1117.57 & 77.68 & 94.31 & 32.81 & 6.35 & 8.75 & 2.94 \\
\hline & $3(90)$ & 1701.06 & 1600.36 & 1658.16 & 215.56 & 87.50 & 48.82 & 12.67 & 5.47 & 2.94 \\
\hline & $1(0)$ & 1303.46 & 1290.13 & 1313.70 & 196.80 & 95.45 & 79.21 & 15.10 & 7.40 & 6.03 \\
\hline & $1(90)$ & 1273.38 & 1279.33 & 1356.05 & 109.36 & 46.21 & 77.45 & 8.59 & 3.61 & 5.71 \\
\hline GFF & $2(0)$ & 1342.22 & 1324.13 & 1289.75 & 107.01 & 76.76 & 19.37 & 7.97 & 5.80 & 1.50 \\
\hline \multirow[t]{5}{*}{$(5.1 \%)$} & $2(90)$ & 1449.08 & 1522.79 & 1441.38 & 51.49 & 74.49 & 28.50 & 3.55 & 4.89 & 1.98 \\
\hline & $3(0)$ & 1570.86 & 1441.20 & 1460.77 & 321.31 & 100.96 & 49.34 & 20.45 & 7.01 & 3.38 \\
\hline & $3(90)$ & 1288.46 & 1268.12 & 1358.32 & 104.87 & 34.84 & 40.80 & 8.14 & 2.75 & 3.00 \\
\hline & $1(0)$ & 1048.69 & 1083.96 & 1095.61 & 50.65 & 46.79 & 22.89 & 4.83 & 4.32 & 2.09 \\
\hline & $1(90)$ & 1179.38 & 1151.28 & 1211.22 & 100.84 & 54.62 & 37.74 & 8.55 & 4.74 & 3.12 \\
\hline NUC & $2(0)$ & 1183.74 & 1111.77 & 1135.64 & 76.33 & 44.88 & 25.40 & 6.45 & 4.04 & 2.24 \\
\hline \multirow[t]{5}{*}{$(4.7 \%)$} & $2(90)$ & 1333.05 & 1425.85 & 1329.57 & 204.10 & 117.76 & 154.90 & 15.31 & 8.26 & 11.65 \\
\hline & $3(0)$ & 1193.35 & 1217.75 & 1208.64 & 87.71 & 55.12 & 53.88 & 7.35 & 4.53 & 4.46 \\
\hline & $3(90)$ & 1195.34 & 1119.76 & 1120.18 & 95.10 & 68.53 & 82.08 & 7.96 & 6.12 & 7.33 \\
\hline & $1(0)$ & 1286.75 & 1305.75 & 1290.82 & 91.41 & 152.07 & 93.28 & 7.10 & 11.65 & 7.23 \\
\hline & $1(90)$ & 1232.36 & 1297.36 & 1325.87 & 119.49 & 116.24 & 85.61 & 9.70 & 8.96 & 6.46 \\
\hline NUF & $2(0)$ & 1306.14 & 1343.82 & 1348.11 & 103.55 & 58.77 & 38.56 & 7.93 & 4.37 & 2.86 \\
\hline \multirow[t]{5}{*}{$(5.1 \%)$} & $2(90)$ & 1480.50 & 1435.83 & 1496.42 & 92.86 & 104.15 & 50.91 & 6.27 & 7.25 & 3.40 \\
\hline & $3(0)$ & 1419.75 & 1350.29 & 1386.60 & 196.15 & 85.71 & 63.88 & 13.82 & 6.35 & 4.61 \\
\hline & $3(90)$ & 1413.14 & 1348.87 & 1357.96 & 97.46 & 128.01 & 46.35 & 6.90 & 9.49 & 3.41 \\
\hline & $1(0)$ & 1407.99 & 1311.37 & 1377.39 & 101.90 & 138.25 & 43.88 & 7.24 & 10.54 & 3.19 \\
\hline & $1(90)$ & 1496.87 & 1528.41 & 1580.33 & 283.65 & 97.81 & 87.86 & 18.95 & 6.40 & 5.56 \\
\hline KLC & $2(0)$ & 1223.46 & 1290.74 & 1254.71 & 100.64 & 68.53 & 43.97 & 8.23 & 5.31 & 3.50 \\
\hline \multirow[t]{5}{*}{ (4.4\%) } & $2(90)$ & 1374.99 & 1297.12 & 1386.99 & 98.95 & 72.15 & 67.60 & 7.20 & 5.56 & 4.87 \\
\hline & $3(0)$ & 1324.96 & 1321.98 & 1393.36 & 110.24 & 121.39 & 22.23 & 8.32 & 9.18 & 1.60 \\
\hline & $3(90)$ & 1420.88 & 1437.80 & 1343.29 & 99.49 & 100.97 & 48.59 & 7.00 & 7.02 & 3.62 \\
\hline & $1(0)$ & 1414.75 & 1474.61 & 1516.36 & 95.84 & 56.80 & 50.17 & 6.77 & 3.85 & 3.31 \\
\hline & $1(90)$ & 1235.29 & 1304.08 & 1360.78 & 61.52 & 67.11 & 54.98 & 4.98 & 5.15 & 4.04 \\
\hline KLF & $2(0)$ & 1333.81 & 1326.25 & 1446.78 & 77.87 & 75.28 & 80.78 & 5.84 & 5.68 & 5.58 \\
\hline \multirow[t]{5}{*}{$(5.1 \%)$} & $2(90)$ & 1160.16 & 1208.38 & 1173.19 & 80.64 & 134.93 & 39.03 & 6.95 & 11.17 & 3.33 \\
\hline & $3(0)$ & 1368.82 & 1383.66 & 1423.98 & 77.12 & 72.23 & 81.37 & 5.63 & 5.22 & 5.71 \\
\hline & $3(90)$ & 1229.29 & 1227.13 & 1220.99 & 94.21 & 71.68 & 64.08 & 7.66 & 5.84 & 5.25 \\
\hline & $1(0)$ & 1322.69 & 1358.04 & 1385.72 & 133.60 & 37.05 & 28.06 & 10.10 & 2.73 & 2.03 \\
\hline & $1(90)$ & 1353.11 & 1370.87 & 1402.66 & 79.33 & 76.52 & 45.46 & 5.86 & 5.58 & 3.24 \\
\hline CGC & $2(0)$ & 1383.31 & 1357.07 & 1378.15 & 122.36 & 43.72 & 36.77 & 8.85 & 3.22 & 2.67 \\
\hline \multirow[t]{5}{*}{$(4.6 \%)$} & $2(90)$ & 1190.26 & 1198.69 & 1230.81 & 109.52 & 97.29 & 79.14 & 9.20 & 8.12 & 6.43 \\
\hline & $3(0)$ & 1257.28 & 1222.32 & 1270.63 & 82.05 & 58.97 & 47.62 & 6.53 & 4.82 & 3.75 \\
\hline & $3(90)$ & 1252.99 & 1284.41 & 1319.83 & 104.77 & 94.35 & 73.25 & 8.36 & 7.35 & 5.55 \\
\hline & $1(0)$ & 1268.80 & 1239.35 & 1245.56 & 190.05 & 82.21 & 62.77 & 14.98 & 6.63 & 5.04 \\
\hline & $1(90)$ & 1273.26 & 1175.71 & 1377.03 & 118.27 & 66.29 & 59.38 & 9.29 & 5.64 & 4.31 \\
\hline CGF & $2(0)$ & 1457.37 & 1439.52 & 1445.20 & 95.42 & 90.11 & 87.12 & 6.55 & 6.26 & 6.03 \\
\hline \multirow[t]{3}{*}{$(5.0 \%)$} & $2(90)$ & 1272.35 & 1269.28 & 1259.43 & 38.81 & 81.64 & 79.15 & 3.05 & 6.43 & 6.28 \\
\hline & $3(0)$ & 1260.31 & 1284.51 & 1356.33 & 26.27 & 88.78 & 34.98 & 2.08 & 6.91 & 2.58 \\
\hline & $3(90)$ & 1379.65 & 1402.81 & 1431.20 & 23.24 & 37.80 & 49.28 & 1.68 & 2.69 & 3.44 \\
\hline
\end{tabular}


Table B.2 Resilient Modulus Results for $34^{\circ} \mathrm{F}$ Tests, continued

\begin{tabular}{|c|c|c|c|c|c|c|c|c|c|c|}
\hline \multicolumn{2}{|c|}{ Temp = 34F } & & \multicolumn{2}{|c|}{ Res. Mod } & & \multirow{2}{*}{$\begin{array}{c}\text { Std. Dev. } \\
0.5\end{array}$} & \multirow[b]{2}{*}{1} & \multirow[b]{2}{*}{0.33} & \multicolumn{2}{|c|}{$\begin{array}{l}\text { Coeficient of } \\
\text { Variance (\%) }\end{array}$} \\
\hline Freqency & ID (deg) & 0.33 & 0.5 & 1 & 0.33 & & & & 0.5 & 1 \\
\hline & $1(0)$ & 791.27 & 824.33 & 856.41 & 54.52 & 47.47 & 40.21 & 6.89 & 5.76 & 4.70 \\
\hline & $1(90)$ & 849.43 & 813.27 & 807.09 & 73.31 & 48.14 & 17.29 & 8.63 & 5.92 & 2.14 \\
\hline NUF & $2(0)$ & 921.29 & 947.65 & 969.95 & 48.13 & 28.26 & 38.74 & 5.22 & 2.98 & 3.99 \\
\hline$(58-40)$ & $2(90)$ & 768.31 & 786.17 & 780.62 & 46.44 & 76.95 & 23.11 & 6.04 & 9.79 & 2.96 \\
\hline$(4.9 \%)$ & $3(0)$ & 894.92 & 920.48 & 966.41 & 25.84 & 39.96 & 22.47 & 2.89 & 4.34 & 2.33 \\
\hline & $3(90)$ & 829.44 & 824.79 & 797.74 & 32.90 & 40.17 & 4.47 & 3.97 & 4.87 & 0.56 \\
\hline & $1(0)$ & 832.51 & 838.55 & 828.51 & 26.36 & 33.46 & 31.44 & 3.17 & 3.99 & 3.80 \\
\hline & $1(90)$ & 829.29 & 852.16 & 842.86 & 36.36 & 33.78 & 14.72 & 4.38 & 3.96 & 1.75 \\
\hline KLF & $2(0)$ & 873.79 & 857.24 & 867.73 & 49.11 & 33.90 & 43.88 & 5.62 & 3.95 & 5.06 \\
\hline$(58-40)$ & $2(90)$ & 826.74 & 806.20 & 832.60 & 65.47 & 21.58 & 42.65 & 7.92 & 2.68 & 5.12 \\
\hline$(4.9 \%)$ & $3(0)$ & 763.24 & 750.92 & 793.58 & 34.30 & 18.60 & 30.54 & 4.49 & 2.48 & 3.85 \\
\hline & $3(90)$ & 875.57 & 863.51 & 892.52 & 84.09 & 78.24 & 22.44 & 9.60 & 9.06 & 2.51 \\
\hline
\end{tabular}

Key

\begin{tabular}{|c|l|c|}
\hline Abbreviation & Mix Type & Binder \\
\hline GFC & Granite Falls granite, coarse gradation, & PG 52-34 \\
\hline GFF & Granite Falls granite, fine gradation, & PG 52-34 \\
\hline NUC & New Ulm quartzite, coarse gradation & PG 52-34 \\
\hline NUF & New Ulm quartzite, fine gradation & PG 52-34 \\
\hline KLC & Kasota limestone, coarse gradation & PG 52-34 \\
\hline KLF & Kasota limestone, fine gradation & PG 52-34 \\
\hline CGC & Cedar Grove gravel, coarse gradation & PG 52-34 \\
\hline CGF & Cedar Grove gravel, fine gradation & PG 52-34 \\
\hline NUF (58-40) & New Ulm quartzite, fine gradation & PG 58-40 \\
\hline KLF (58-40) & Kasota limestone, fine gradation & PG 58-40 \\
\hline
\end{tabular}


Table B.3 Resilient Modulus Results for $77^{\circ} \mathrm{F}$ Tests

\begin{tabular}{|c|c|c|c|c|c|c|c|c|c|c|}
\hline \multicolumn{2}{|c|}{ Temp = 77F } & \multirow[b]{2}{*}{0.33} & \multicolumn{2}{|c|}{ Res. Mod } & \multirow[b]{2}{*}{0.33} & \multirow{2}{*}{$\begin{array}{c}\text { Std. Dev. } \\
0.5\end{array}$} & \multirow[b]{2}{*}{1} & \multirow[b]{2}{*}{0.33} & \multicolumn{2}{|c|}{$\begin{array}{l}\text { Coeficient of } \\
\text { Variance }(\%)\end{array}$} \\
\hline Freqency & ID (deg) & & 0.5 & 1 & & & & & 0.5 & 1 \\
\hline & $1(0)$ & 251.85 & 242.58 & 234.73 & 18.19 & 7.13 & 7.13 & 7.22 & 2.94 & 3.04 \\
\hline & $1(90)$ & 239.87 & 221.38 & 221.83 & 3.21 & 8.78 & 4.67 & 1.34 & 3.97 & 2.11 \\
\hline GFC & $2(0)$ & 238.41 & 242.51 & 239.24 & 7.54 & 3.55 & 7.70 & 3.16 & 1.46 & 3.22 \\
\hline \multirow[t]{5}{*}{$(4.5 \%)$} & $2(90)$ & 233.54 & 233.72 & 239.04 & 4.71 & 4.76 & 3.51 & 2.02 & 2.04 & 1.47 \\
\hline & $3(0)$ & 270.17 & 271.58 & 274.13 & 8.46 & 1.79 & 4.08 & 3.13 & 0.66 & 1.49 \\
\hline & $3(90)$ & 277.64 & 261.67 & 249.85 & 19.82 & 12.84 & 9.61 & 7.14 & 4.91 & 3.84 \\
\hline & $1(0)$ & 251.49 & 249.56 & 246.45 & 5.33 & 6.55 & 3.35 & 2.12 & 2.63 & 1.36 \\
\hline & $1(90)$ & 206.45 & 202.03 & 200.71 & 3.49 & 9.46 & 2.63 & 1.69 & 4.68 & 1.31 \\
\hline GFF & $2(0)$ & 309.70 & 298.91 & 304.82 & 6.14 & 5.67 & 7.83 & 1.98 & 1.90 & 2.57 \\
\hline \multirow[t]{5}{*}{$(5.1 \%)$} & $2(90)$ & 283.92 & 283.94 & 281.28 & 11.70 & 5.21 & 8.01 & 4.12 & 1.83 & 2.85 \\
\hline & $3(0)$ & 251.65 & 246.25 & 250.02 & 2.16 & 5.23 & 3.12 & 0.86 & 2.12 & 1.25 \\
\hline & $3(90)$ & 235.10 & 237.68 & 238.90 & 11.78 & 4.33 & 2.66 & 5.01 & 1.82 & 1.11 \\
\hline & $1(0)$ & 188.03 & 189.26 & 186.30 & 2.87 & 2.83 & 3.48 & 1.53 & 1.49 & 1.87 \\
\hline & $1(90)$ & 199.29 & 205.21 & 199.97 & 15.44 & 5.61 & 2.26 & 7.75 & 2.73 & 1.13 \\
\hline NUC & $2(0)$ & 220.47 & 223.47 & 222.45 & 3.69 & 4.13 & 7.91 & 1.67 & 1.85 & 3.56 \\
\hline \multirow[t]{5}{*}{$(4.7 \%)$} & $2(90)$ & 257.63 & 243.91 & 244.77 & 5.45 & 10.31 & 5.95 & 2.12 & 4.23 & 2.43 \\
\hline & $3(0)$ & 217.12 & 215.11 & 217.56 & 2.16 & 4.42 & 2.96 & 0.99 & 2.05 & 1.36 \\
\hline & $3(90)$ & 259.61 & 247.39 & 249.13 & 15.88 & 7.03 & 1.53 & 6.12 & 2.84 & 0.61 \\
\hline & $1(0)$ & 283.76 & 273.31 & 273.77 & 3.72 & 8.38 & 6.22 & 1.31 & 3.07 & 2.27 \\
\hline & $1(90)$ & 255.25 & 257.82 & 262.69 & 9.45 & 2.16 & 2.63 & 3.70 & 0.84 & 1.00 \\
\hline NUF & $2(0)$ & 280.32 & 280.24 & 284.68 & 1.71 & 2.81 & 4.57 & 0.61 & 1.00 & 1.61 \\
\hline \multirow[t]{5}{*}{$(5.0 \%)$} & $2(90)$ & 280.21 & 281.28 & 277.37 & 10.59 & 5.67 & 6.55 & 3.78 & 2.02 & 2.36 \\
\hline & $3(0)$ & 266.46 & 268.56 & 273.82 & 5.25 & 5.32 & 2.25 & 1.97 & 1.98 & 0.82 \\
\hline & $3(90)$ & 298.43 & 297.23 & 298.41 & 1.44 & 4.15 & 3.72 & 0.48 & 1.40 & 1.25 \\
\hline & $1(0)$ & 335.25 & 338.88 & 334.55 & 8.34 & 5.40 & 4.25 & 2.49 & 1.59 & 1.27 \\
\hline & $1(90)$ & 314.42 & 315.10 & 316.09 & 6.83 & 29.69 & 4.97 & 2.17 & 9.42 & 1.57 \\
\hline KLC & $2(0)$ & 339.71 & 344.83 & 349.74 & 6.98 & 7.17 & 7.74 & 2.05 & 2.08 & 2.21 \\
\hline \multirow[t]{5}{*}{ (4.4\%) } & $2(90)$ & 311.06 & 306.02 & 311.30 & 7.13 & 7.19 & 5.47 & 2.29 & 2.35 & 1.76 \\
\hline & $3(0)$ & 303.25 & 310.25 & 310.72 & 4.05 & 9.20 & 10.65 & 1.34 & 2.97 & 3.43 \\
\hline & $3(90)$ & 327.45 & 310.00 & 312.61 & 22.22 & 7.69 & 6.95 & 6.79 & 2.48 & 2.22 \\
\hline & $1(0)$ & 265.97 & 269.21 & 273.93 & 3.78 & 2.40 & 5.92 & 1.42 & 0.89 & 2.16 \\
\hline & $1(90)$ & 286.29 & 279.05 & 281.12 & 3.43 & 9.79 & 4.38 & 1.20 & 3.51 & 1.56 \\
\hline KLF & $2(0)$ & 260.75 & 267.00 & 267.74 & 5.03 & 3.13 & 2.42 & 1.93 & 1.17 & 0.90 \\
\hline \multirow[t]{5}{*}{$(5.1 \%)$} & $2(90)$ & 274.41 & 269.92 & 260.68 & 2.98 & 4.25 & 5.93 & 1.09 & 1.57 & 2.27 \\
\hline & $3(0)$ & 255.57 & 260.69 & 264.34 & 2.58 & 3.92 & 3.20 & 1.01 & 1.51 & 1.21 \\
\hline & $3(90)$ & 292.41 & 285.36 & 282.71 & 4.01 & 3.30 & 5.52 & 1.37 & 1.16 & 1.95 \\
\hline & $1(0)$ & 274.65 & 269.23 & 265.69 & 3.24 & 5.88 & 2.83 & 1.18 & 2.19 & 1.07 \\
\hline & $1(90)$ & 279.13 & 281.54 & 281.14 & 10.41 & 6.73 & 8.29 & 3.73 & 2.39 & 2.95 \\
\hline CGC & $2(0)$ & 296.50 & 286.83 & 274.82 & 3.82 & 2.55 & 7.39 & 1.29 & 0.89 & 2.69 \\
\hline \multirow[t]{5}{*}{$(4.6 \%)$} & $2(90)$ & 293.97 & 293.03 & 287.49 & 5.63 & 5.61 & 1.26 & 1.91 & 1.91 & 0.44 \\
\hline & $3(0)$ & 252.81 & 246.89 & 245.83 & 7.00 & 4.59 & 6.36 & 2.77 & 1.86 & 2.59 \\
\hline & $3(90)$ & 238.98 & 241.08 & 241.47 & 11.65 & 1.27 & 1.71 & 4.87 & 0.53 & 0.71 \\
\hline & $1(0)$ & 255.92 & 263.13 & 264.80 & 6.18 & 4.15 & 5.84 & 2.41 & 1.58 & 2.20 \\
\hline & $1(90)$ & 281.07 & 268.34 & 269.52 & 3.13 & 14.16 & 6.62 & 1.11 & 5.28 & 2.46 \\
\hline CGF & $2(0)$ & 267.74 & 266.02 & 263.86 & 9.94 & 6.36 & 6.49 & 3.71 & 2.39 & 2.46 \\
\hline \multirow[t]{3}{*}{$(5.0 \%)$} & $2(90)$ & 262.33 & 260.23 & 259.35 & 9.17 & 3.10 & 2.42 & 3.49 & 1.19 & 0.93 \\
\hline & $3(0)$ & 244.37 & 240.56 & 241.24 & 2.76 & 2.12 & 6.45 & 1.13 & 0.88 & 2.67 \\
\hline & $3(90)$ & 251.85 & 245.98 & 247.44 & 3.83 & 4.11 & 2.38 & 1.52 & 1.67 & 0.96 \\
\hline
\end{tabular}


Table B.3 Resilient Modulus Results for $77^{\circ} \mathrm{F}$ Tests, continued

\begin{tabular}{|c|c|c|c|c|c|c|c|c|c|c|}
\hline \multicolumn{2}{|c|}{ Temp = 77F } & & \multicolumn{2}{|c|}{ Res. Mod } & \multicolumn{3}{|c|}{ Std. Dev. } & & \multicolumn{2}{|c|}{$\begin{array}{l}\text { Coeficient of } \\
\text { Variance (\%) }\end{array}$} \\
\hline Freqency & ID (deg) & 0.33 & 0.5 & 1 & 0.33 & 0.5 & 1 & 0.33 & 0.5 & 1 \\
\hline & $1(0)$ & 158.15 & 159.38 & 164.03 & 2.94 & 2.32 & 0.35 & 1.86 & 1.45 & 0.21 \\
\hline & $1(90)$ & 166.26 & 164.04 & 165.88 & 1.27 & 2.94 & 1.04 & 0.76 & 1.79 & 0.62 \\
\hline NUF & $2(0)$ & 169.20 & 172.30 & 169.70 & 6.21 & 0.80 & 1.52 & 3.67 & 0.46 & 0.89 \\
\hline$(58-40)$ & $2(90)$ & 161.25 & 165.93 & 164.69 & 2.87 & 2.96 & 2.46 & 1.78 & 1.78 & 1.49 \\
\hline \multirow[t]{4}{*}{$(4.9 \%)$} & $3(0)$ & 151.70 & 155.86 & 156.40 & 1.02 & 0.74 & 0.78 & 0.68 & 0.48 & 0.50 \\
\hline & $3(90)$ & 163.38 & 164.07 & 167.23 & 9.08 & 3.65 & 3.18 & 5.56 & 2.22 & 1.90 \\
\hline & $1(0)$ & 173.99 & 175.18 & 180.01 & 4.11 & 4.10 & 2.24 & 2.36 & 2.34 & 1.24 \\
\hline & $1(90)$ & 176.72 & 176.03 & 177.59 & 3.16 & 2.63 & 2.45 & 1.79 & 1.49 & 1.38 \\
\hline KLF & $2(0)$ & 193.18 & 197.13 & 197.78 & 7.06 & 2.00 & 1.48 & 3.66 & 1.01 & 0.75 \\
\hline$(58-40)$ & $2(90)$ & 194.56 & 191.79 & 192.88 & 1.93 & 3.31 & 2.31 & 0.99 & 1.73 & 1.20 \\
\hline \multirow[t]{2}{*}{$(4.9 \%)$} & $3(0)$ & 200.62 & 204.18 & 204.12 & 6.23 & 1.97 & 1.88 & 3.11 & 0.97 & 0.92 \\
\hline & $3(90)$ & 191.50 & 187.49 & 192.46 & 3.25 & 4.67 & 1.21 & 1.70 & 2.49 & 0.63 \\
\hline
\end{tabular}

Key

\begin{tabular}{|c|l|c|}
\hline Abbreviation & Mix Type & Binder \\
\hline GFC & Granite Falls granite, coarse gradation, & PG 52-34 \\
\hline GFF & Granite Falls granite, fine gradation, & PG 52-34 \\
\hline NUC & New Ulm quartzite, coarse gradation & PG 52-34 \\
\hline NUF & New Ulm quartzite, fine gradation & PG 52-34 \\
\hline KLC & Kasota limestone, coarse gradation & PG 52-34 \\
\hline KLF & Kasota limestone, fine gradation & PG 52-34 \\
\hline CGC & Cedar Grove gravel, coarse gradation & PG 52-34 \\
\hline CGF & Cedar Grove gravel, fine gradation & PG 52-34 \\
\hline NUF (58-40) & New Ulm quartzite, fine gradation & PG 58-40 \\
\hline KLF (58-40) & Kasota limestone, fine gradation & PG 58-40 \\
\hline
\end{tabular}


Table B.4 Resilient Modulus Results for $104{ }^{\circ} \mathrm{F}$ Tests

\begin{tabular}{|c|c|c|c|c|c|c|c|c|c|c|}
\hline \multicolumn{2}{|c|}{ Temp $=104 \mathrm{~F}$} & \multirow[b]{2}{*}{0.33} & \multicolumn{2}{|c|}{ Res. Mod } & \multirow[b]{2}{*}{0.33} & \multirow{2}{*}{$\begin{array}{c}\text { Std. Dev. } \\
0.5\end{array}$} & \multirow[b]{2}{*}{1} & \multirow[b]{2}{*}{0.33} & \multicolumn{2}{|c|}{$\begin{array}{l}\text { Coeficient of } \\
\text { Variance }(\%)\end{array}$} \\
\hline Freqency & ID (deg) & & 0.5 & 1 & & & & & 0.5 & 1 \\
\hline & $1(0)$ & 63.86 & 61.74 & 60.36 & 6.83 & 3.89 & 1.93 & 10.70 & 6.30 & 3.20 \\
\hline & $1(90)$ & 62.96 & 56.42 & 56.59 & 6.72 & 3.77 & 2.04 & 10.68 & 6.68 & 3.60 \\
\hline GFC & $2(0)$ & 69.09 & 64.05 & 65.62 & 3.70 & 2.61 & 2.27 & 5.35 & 4.07 & 3.45 \\
\hline \multirow[t]{5}{*}{$(4.5 \%)$} & $2(90)$ & 67.14 & 62.59 & 65.17 & 8.96 & 6.19 & 1.60 & 13.34 & 9.89 & 2.46 \\
\hline & $3(0)$ & 71.51 & 66.77 & 64.68 & 5.93 & 4.57 & 4.86 & 8.29 & 6.84 & 7.51 \\
\hline & $3(90)$ & 67.80 & 69.41 & 65.82 & 6.29 & 3.37 & 1.34 & 9.28 & 4.85 & 2.03 \\
\hline & $1(0)$ & 75.56 & 73.70 & 73.76 & 3.11 & 4.47 & 2.56 & 4.12 & 6.07 & 3.47 \\
\hline & $1(90)$ & 96.09 & 89.32 & 85.87 & 11.36 & 8.20 & 1.62 & 11.82 & 9.18 & 1.89 \\
\hline GFF & $2(0)$ & 85.18 & 93.68 & 92.34 & 15.96 & 6.16 & 5.69 & 18.74 & 6.57 & 6.16 \\
\hline \multirow[t]{5}{*}{$(5.1 \%)$} & $2(90)$ & 95.53 & 84.90 & 86.52 & 3.41 & 5.24 & 2.82 & 3.57 & 6.17 & 3.26 \\
\hline & $3(0)$ & 69.78 & 65.65 & 70.01 & 8.76 & 4.38 & 2.38 & 12.55 & 6.67 & 3.40 \\
\hline & $3(90)$ & 65.67 & 66.28 & 65.66 & 4.12 & 1.92 & 2.71 & 6.28 & 2.90 & 4.13 \\
\hline & $1(0)$ & 60.77 & 62.52 & 59.66 & 6.57 & 1.06 & 1.08 & 10.81 & 1.70 & 1.81 \\
\hline & $1(90)$ & 46.44 & 46.71 & 46.26 & 1.69 & 1.22 & 1.01 & 3.64 & 2.62 & 2.19 \\
\hline NUC & $2(0)$ & 81.52 & 62.21 & 71.67 & 2.11 & 27.65 & 1.50 & 2.58 & 44.44 & 2.09 \\
\hline \multirow[t]{5}{*}{$(4.7 \%)$} & $2(90)$ & 76.29 & 67.69 & 67.42 & 1.72 & 2.33 & 2.18 & 2.26 & 3.44 & 3.23 \\
\hline & $3(0)$ & 76.87 & 72.81 & 70.83 & 5.70 & 2.74 & 2.31 & 7.42 & 3.76 & 3.26 \\
\hline & $3(90)$ & 75.46 & 68.25 & 69.09 & 8.60 & 4.33 & 2.46 & 11.40 & 6.34 & 3.56 \\
\hline & $1(0)$ & 90.28 & 83.73 & 85.69 & 2.93 & 6.42 & 2.46 & 3.24 & 7.67 & 2.87 \\
\hline & $1(90)$ & 80.95 & 75.03 & 77.47 & 11.37 & 8.41 & 2.88 & 14.05 & 11.20 & 3.71 \\
\hline NUF & $2(0)$ & 91.06 & 70.88 & 84.73 & 6.58 & 32.21 & 2.66 & 7.23 & 45.43 & 3.14 \\
\hline \multirow[t]{5}{*}{$(5.0 \%)$} & $2(90)$ & 85.72 & 83.75 & 77.10 & 10.99 & 2.51 & 3.52 & 12.82 & 2.99 & 4.56 \\
\hline & $3(0)$ & 94.72 & 85.13 & 85.97 & 5.21 & 5.23 & 2.67 & 5.50 & 6.14 & 3.11 \\
\hline & $3(90)$ & 92.62 & 84.54 & 86.05 & 11.08 & 3.97 & 1.80 & 11.97 & 4.69 & 2.09 \\
\hline & $1(0)$ & 112.32 & 105.64 & 105.66 & 13.94 & 4.44 & 4.64 & 12.41 & 4.20 & 4.39 \\
\hline & $1(90)$ & 89.56 & 92.57 & 95.18 & 6.15 & 2.71 & 4.78 & 6.87 & 2.93 & 5.02 \\
\hline $\mathrm{KLC}$ & $2(0)$ & 115.08 & 111.49 & 108.85 & 7.78 & 8.49 & 5.28 & 6.76 & 7.61 & 4.85 \\
\hline \multirow[t]{5}{*}{$(4.4 \%)$} & $2(90)$ & 119.82 & 102.29 & 100.72 & 8.97 & 5.89 & 4.20 & 7.49 & 5.76 & 4.17 \\
\hline & $3(0)$ & 112.73 & 106.52 & 102.64 & 9.28 & 3.47 & 4.85 & 8.24 & 3.26 & 4.72 \\
\hline & $3(90)$ & 112.37 & 98.18 & 100.83 & 7.65 & 5.49 & 5.92 & 6.81 & 5.59 & 5.87 \\
\hline & $1(0)$ & 103.27 & 99.38 & 98.52 & 14.56 & 2.71 & 5.26 & 14.10 & 2.72 & 5.34 \\
\hline & $1(90)$ & 97.87 & 87.48 & 87.97 & 3.63 & 4.12 & 5.06 & 3.71 & 4.71 & 5.75 \\
\hline KLF & $2(0)$ & 84.01 & 80.08 & 80.29 & 2.73 & 1.69 & 4.00 & 3.25 & 2.12 & 4.98 \\
\hline \multirow[t]{5}{*}{ (5.1\%) } & $2(90)$ & 86.29 & 75.17 & 77.06 & 8.24 & 7.20 & 3.54 & 9.55 & 9.58 & 4.59 \\
\hline & $3(0)$ & 88.28 & 90.40 & 88.82 & 5.98 & 4.29 & 2.50 & 6.77 & 4.74 & 2.81 \\
\hline & $3(90)$ & 93.78 & 90.04 & 87.36 & 11.18 & 4.46 & 1.34 & 11.92 & 4.95 & 1.54 \\
\hline & $1(0)$ & 89.48 & 85.11 & 84.09 & 7.67 & 4.22 & 1.52 & 8.58 & 4.96 & 1.81 \\
\hline & $1(90)$ & 88.65 & 80.71 & 79.87 & 5.71 & 3.68 & 3.07 & 6.44 & 4.56 & 3.84 \\
\hline CGC & $2(0)$ & 89.88 & 83.63 & 82.81 & 2.86 & 2.51 & 2.84 & 3.18 & 3.00 & 3.43 \\
\hline \multirow[t]{5}{*}{$(4.6 \%)$} & $2(90)$ & 93.86 & 87.11 & 79.64 & 1.88 & 1.46 & 2.98 & 2.00 & 1.68 & 3.75 \\
\hline & $3(0)$ & 75.22 & 70.99 & 68.91 & 2.21 & 2.24 & 1.68 & 2.94 & 3.16 & 2.43 \\
\hline & $3(90)$ & 72.32 & 69.62 & 62.23 & 9.31 & 2.98 & 3.59 & 12.88 & 4.27 & 5.76 \\
\hline & $1(0)$ & 81.27 & 81.80 & 77.77 & 6.84 & 6.86 & 5.20 & 8.42 & 8.39 & 6.69 \\
\hline & $1(90)$ & 86.36 & 80.05 & 77.01 & 9.76 & 7.30 & 2.12 & 11.31 & 9.12 & 2.75 \\
\hline CGF & $2(0)$ & 75.70 & 76.68 & 73.10 & 5.97 & 2.57 & 3.15 & 7.89 & 3.36 & 4.31 \\
\hline \multirow[t]{3}{*}{$(5.0 \%)$} & $2(90)$ & 75.01 & 78.02 & 72.60 & 12.04 & 2.72 & 5.57 & 16.05 & 3.49 & 7.67 \\
\hline & $3(0)$ & 80.64 & 74.12 & 73.25 & 2.70 & 2.65 & 3.54 & 3.35 & 3.57 & 4.84 \\
\hline & $3(90)$ & 77.05 & 72.19 & 68.52 & 10.65 & 3.66 & 1.69 & 13.83 & 5.07 & 2.47 \\
\hline
\end{tabular}


Table B.4 Resilient Modulus Results for $104{ }^{\circ} \mathrm{F}$ Tests, continued

\begin{tabular}{|c|c|c|c|c|c|c|c|c|c|c|}
\hline \multicolumn{2}{|c|}{ Temp $=104 \mathrm{~F}$} & & \multicolumn{2}{|c|}{ Res. Mod } & & \multicolumn{2}{|l|}{ Std. Dev. } & & \multicolumn{2}{|c|}{$\begin{array}{l}\text { Coeficient of } \\
\text { Variance (\%) }\end{array}$} \\
\hline Freqency & ID (deg) & 0.33 & 0.5 & 1 & 0.33 & 0.5 & 1 & 0.33 & 0.5 & 1 \\
\hline & $1(0)$ & 85.97 & 89.70 & 87.32 & 4.89 & 4.49 & 2.10 & 5.69 & 5.01 & 2.40 \\
\hline & $1(90)$ & 81.32 & 88.28 & 87.30 & 3.97 & 4.37 & 3.02 & 4.88 & 4.95 & 3.46 \\
\hline KLF & $2(0)$ & 88.55 & 93.66 & 90.22 & 3.09 & 3.06 & 3.20 & 3.49 & 3.27 & 3.55 \\
\hline$(58-40)$ & $2(90)$ & 95.74 & 91.24 & 94.47 & 2.14 & 2.53 & 1.84 & 2.23 & 2.77 & 1.95 \\
\hline$(4.9 \%)$ & $3(0)$ & 99.34 & 95.91 & 98.31 & 7.41 & 4.40 & 1.79 & 7.46 & 4.59 & 1.82 \\
\hline & $3(90)$ & 90.56 & 96.73 & 95.81 & 6.26 & 3.09 & 1.77 & 6.92 & 3.19 & 1.84 \\
\hline & $1(0)$ & 75.17 & 77.63 & 76.49 & 5.17 & 3.24 & 2.58 & 6.88 & 4.18 & 3.38 \\
\hline & $1(90)$ & 74.93 & 73.17 & 73.50 & 2.94 & 1.66 & 1.56 & 3.92 & 2.27 & 2.12 \\
\hline NUF & $2(0)$ & 78.49 & 74.84 & 77.66 & 4.85 & 1.89 & 2.35 & 6.18 & 2.53 & 3.03 \\
\hline$(58-40)$ & $2(90)$ & 76.33 & 79.40 & 81.06 & 4.12 & 2.78 & 2.20 & 5.39 & 3.50 & 2.71 \\
\hline$(4.9 \%)$ & $3(0)$ & 82.63 & 88.11 & 86.60 & 5.72 & 1.93 & 1.37 & 6.92 & 2.19 & 1.58 \\
\hline & $3(90)$ & 73.04 & 78.78 & 81.38 & 10.15 & 2.74 & 2.95 & 13.89 & 3.48 & 3.63 \\
\hline
\end{tabular}

Key

\begin{tabular}{|c|l|c|}
\hline Abbreviation & Mix Type & Binder \\
\hline GFC & Granite Falls granite, coarse gradation, & PG 52-34 \\
\hline GFF & Granite Falls granite, fine gradation, & PG 52-34 \\
\hline NUC & New Ulm quartzite, coarse gradation & PG 52-34 \\
\hline NUF & New Ulm quartzite, fine gradation & PG 52-34 \\
\hline KLC & Kasota limestone, coarse gradation & PG 52-34 \\
\hline KLF & Kasota limestone, fine gradation & PG 52-34 \\
\hline CGC & Cedar Grove gravel, coarse gradation & PG 52-34 \\
\hline CGF & Cedar Grove gravel, fine gradation & PG 52-34 \\
\hline NUF (58-40) & New Ulm quartzite, fine gradation & PG 58-40 \\
\hline KLF (58-40) & Kasota limestone, fine gradation & PG 58-40 \\
\hline
\end{tabular}




\section{APPENDIX C}

\section{APA RUT TEST PROCEDURE}


III-115M

Page 1 of 8

\section{GDT-115M \\ METHOD OF TEST FOR DETERMINING \\ RUTTING SUSCEPTIBILITY \\ USING THE ASPHALT PAVEMENT ANALYZER}

\section{A. SCOPE:}

The Asphalt Pavement Analyzer will be used to test the rutting susceptibility of asphalt concrete mixtures.

\section{B. APPARATUS:}

1. 16,000 gram scale, accurate to 0.1 gram.

2. Mixer.

3. Mixing utensils (bowls, spoon, spatula).

4. Astec Vibratory Compactor (Model AVC II).

5. $125 \mathrm{~mm}$ wide $\mathrm{x} 300 \mathrm{~mm}$ long beam mold with base plate and $150 \mathrm{~mm}$ diameter mold with base plate for AVC II.

6. $125 \mathrm{~mm} \times 300 \mathrm{~mm}$ beam specimen compaction head and $150 \mathrm{~mm}$ diameter cylinder compaction head for AVC II.

7. $125 \mathrm{~mm} \times 300 \mathrm{~mm} \times 2 \mathrm{~mm}$ beam sample support plates for beam specimens and 150 $\mathrm{mm}$ diameter $\mathrm{x} 2 \mathrm{~mm}$ sample support plates for cylindrical specimens.

8. $75 \mathrm{~mm}$ high calibration blocks for beam and pill compaction heads for the AVC II

9. Astec Asphalt Pavement Analyzer II.

10. Heating ovens for aggregate and asphalt cement. Capable of maintaining at least 200 ${ }^{\circ} \mathrm{C}$ and holding a set temperature $\pm 2{ }^{\circ} \mathrm{C}$.

11. Polyethylene restraint molds for sample testing.

12. Measuring template for rut measurement.

13. Digital Indicator (accurate to $0.1 \mathrm{~mm}$ ).

14. $150 \mathrm{~mm}$ diameter paper disks for cylindrical AVC II Molds. 


\section{Preparation of Sample:}

1. Asphalt mixes should be batched with asphalt and aggregate to meet actual laboratory density at optimum asphalt cement content according to Section $\mathrm{F}$ below. The target voids of the compacted mix should be $7.0 \% \pm 1.0 \%$, unless specified otherwise.

2. Preheat aggregate, liquid asphalt, compaction molds, and utensils to the specified temperatures. Temperatures for heating and mixing aggregate and asphalt cement should conform to the Superpave Performance Grade mixing and compaction binder requirement.

3. Dry mix, aggregates and hydrated lime or other admixtures, if required, then add the optimum percentage of asphalt cement and mix.

4. If additional heating is necessary, place entire mixture in one gallon cans, close lids and place in an oven set on approximately $176{ }^{\circ} \mathrm{C}$. Heat until mixture meets required compaction temperature. The mixture shall not be heated at the compaction temperature for more than one (1) hour.

\section{Sample Compaction:}

\section{Astec Vibratory Compactor (Model AVC II)}

a. The following steps should be completed before starting the specimen compaction operation:

1) To set the height of specimen to be compacted at $75 \mathrm{~mm}$, place the calibration block under the compaction head on the specimen supporting base. On the control unit, with the VIBRATION switch to OFF and the MODE switch to MANUAL, simultaneously press the GREEN PALM buttons to lower the compaction head onto the calibration block. For height calibration, the compaction head should be making full contact with the face of the block. Hand tighten the compaction head assembly bolts in this position. Raise the compaction head assembly for adequate clearance to tighten the bolts to the appropriate torque.

2) Adjust the compaction force and the vibration time, depending on the type of mix. The amount of compaction effort that can be delivered by this compaction machine depends on the static compaction force and the duration of vibrating action which can be adjusted. The static compaction force can be controlled by the compaction pressure (system pressure on the right) and the counter balance pressure (on the left). The relationship between these two pressure readings and the net compaction pressure are shown in Table 3-1, below: 
Table 3-1

\author{
SPECIMEN \\ TYPE \\ $150 \mathrm{~mm}$ dia. \\ Cylinder specimen
}

$125 \mathrm{~mm} \times 300 \mathrm{~mm}$

beam specimen

\section{SYSTEM \\ PRESSURE}

$700 \mathrm{kPa}$

$700 \mathrm{kPa}$

\section{COUNTER BAL. VIBRATING \\ PRESSURE TIME, sec.*}

$420 \mathrm{kPa}$

$30-60$

* Vibrating time can be adjusted from the CYCLE TIME counter on the control unit. The VIBRATING TIME will be $50 \%$ of the maximum total CYCLE TIME.

3) Heat the specimen mold assembly in an oven to a temperature of $150{ }^{\circ} \mathrm{C}$. The mold needs to be heated once at the beginning of the specimen compaction operation.

b. The following specimen compaction procedures can be used for compacting cylinder specimen and beam specimen:

1) Place the specimen mold on top of a counter adjacent to the compaction machine. Insert a preheated sample support plate into the mold. Apply a light coat of release agent on the inside surfaces of the mold and the sample support plate. If compacting a cylinder specimen, place a paper disk in the bottom of the mold.

2) Place the entire batch of mixture in the mold and spade the mixture vigorously with a heated spatula twenty (20) times evenly around the perimeter and twenty (20) times evenly in the interior of the beam mold, or, ten (10) times around the perimeter and ten (10) times evenly in the interior of the cylindrical mold. Then form the sample surface to a dome shape. Measure the temperature in the mixture. Temperature at the start of the compaction procedure should be within $3{ }^{\circ} \mathrm{C}$ of the specified compaction temperature for the mixture. If it is not, place the sample and mold in oven until the specified compaction temperature is reached.

3) Transfer the specimen mold to the supporting base of the machine and fit it in the recessed area. Apply a light coat of release agent on the compaction head.

4) On the control unit, set CYCLE TIME from one (1) minute to two (2) minutes (depending on the VIBRATING TIME), turn MODE switch to AUTO, VIBRATING switch to AUTO, and pull up (disable) the EMERGENCY STOP. 
5) Depress both GREEN PALM buttons simultaneously (you do not need to hold down the palm buttons). This will cause the compaction head assembly to move downward automatically, and when it drops to a specified position, the vibrating action will be activated automatically. Under the static compression force and the vibrating actions, the compaction head will move downward to compact the loose asphalt mixture confined in the specimen mold for thirty (30) seconds to sixty (60) seconds. After the selected VIBRATING TIME (50\% of the CYCLE TIME), turn the VIBRATING switch "OFF", allowing the specimens to remain under static compression force only, for the remaining $50 \%$ of the CYCLE TIME. At the end of the CYCLE TIME, the compaction head assembly will automatically retract. Additional vibrating compaction can be applied by increasing the vibrating time.

6) Lift the specimen mold from the compaction position, move forward and slide the edges of the specimen mold under the restraining brackets and position the specimen mold in the recessed area at the extruding support base.

7) On the control unit, turn the MODE switch to EJECT. Press and hold down both GREEN PALM buttons to raise the extrusion cylinder head to extrude the specimen out from the mold. Allow the specimen to cool for a few minutes, then remove the compacted specimen with the sample support plate together from the base plate of the mold and place them on a firm counter top. Press the OPEN button on the control unit to retract the extrusion cylinder head. Then remove the specimen mold from the supporting base plate.

\section{E. Rut Testing Procedure}

1. Method "A" - Laboratory Compacted Specimen

a. Asphalt Pavement Analyzer II

1) Rut Testing (Dry)

a) Preset chamber temperature as follows: For SMA and Superpave mixes use $50{ }^{\circ} \mathrm{C}$, for conventional mixes use $40{ }^{\circ} \mathrm{C}$. During testing these temperatures are to be maintained within $\pm 1{ }^{\circ} \mathrm{C}$.

b) Open the front doors, unlock the toggle clamps and pull out the sample tray.

c) Loosen and remove the bolts and the restraining brackets at the front ends of the three PVC molds that have been secured on the sample tray. Remove the molds from the sample tray and place them on a firm counter top for installing asphalt samples in the sample mold. 
d) After installing the samples into the molds, transfer the sample molds to the sample tray, one at a time, making sure to fit the recessed area of the sample mold to the rear end restraining bracket. Do the same for all three molds. Place and tighten the restraining brackets on the front end of the sample molds, fitting the bracket into the recessed area of the sample mold to restrain the sample molds from lateral movement.

e) Allow the machine to stroke through a maximum of 100 cycles to assure that the specimen are seated well in the molds or between the restraint brackets and that there is no build-up asphalt binder film thickness on the sample surface.

f) Measure and record the initial gauge readings for each sample by taking indicator readings at all five slots for beam samples, or by taking indicator readings using the two end slots on each end of the template for cylindrical samples. Record the readings and average on the data sheet.

g) Perform the rutting test. It is assumed that the chamber temperature has been brought to the preset test temperature and the test samples have been conditioned under the test temperature for a minimum of four (4) hours.

h) After closing the front door, allow the test samples to be stabilized at the test temperature for ten (10) minutes before starting the rutting test. The STROKE ON DELAY timer can be used to set the delay start time.

i) Set the PRESET counter to the number of repetitions for the test to be run. Reset the TOTALIZING counters too zero, turn the cylinder operation mode control switch to AUTO, and check the pressure gauge readings to be corresponding to the wheel load. Do this for all three (3) cylinders.

j) Press the green rectangular STROKE TEST START button to start the rut testing. This will start the automatic rut testing sequence. After completing the preset number of repetitions, the machine automatically stops the reciprocating motion and retracts the wheels. A complete test will take approximately 3.5 hours.

k) Measure the rut depth and record the results on the data sheet as in step 1.e., above.

2) Rut Testing (Submerged)

a) Precondition samples by vacuum saturation in accordance with GDT-66. Calculate the percent saturation. 
b) Place and secure specimen in the sample molds, same as the procedure used in 1.a). to 1.e)., above.

c) Perform the rutting test. It is important to remember that the water tray should be empty before raising or lowering to prevent spillage of water inside the cabinet.

d) After closing the front door, raise the water tray and turn on the water pump to flood the test samples. Use the STROKE ON DELAY timer and set the soaking time at 30 minutes to allow the test samples to be stabilized at the test temperature before starting the rut testing.

e) Set the PRESET counter to the number of repetitions for the test to be run. Reset the TOTALIZING counter to zero, turn the cylinder operation mode control switch to AUTO and check the pressure gauge reading which should correspond to the specified test wheel load.

f) Press the green rectangular STROKE TEST START button to start the rut testing. This will start the automatic rut testing sequence. After completing the preset number of repetitions, the machine will automatically stop the reciprocating motion and retract the wheels.

g) Drain the water in the water tray then lower the water tray.

h) Open the front doors, release the toggle clamps and pull out the sample tray.

i) Measure the rut depth and record the results on the data sheet as in step 1.e., above.

3) Method "B" - Roadway Specimen

a) $300 \mathrm{~mm}$ or $150 \mathrm{~mm}$ roadway cores are used in this method for evaluation.

b) $150 \mathrm{~mm}$ diameter cores must be sawed to a thickness of $75 \mathrm{~mm}$ high. $300 \mathrm{~mm}$ core must be sawed to give a sample specimen $75 \mathrm{~mm}$ thickness $\mathrm{x}$ $125 \mathrm{~mm}$ wide $\mathrm{x} 225 \mathrm{~mm}$ long.

\section{F. Calculations:}

1. Calculation of Specimen Mass:

a. Volume of Specimen $\left[\mathrm{cm}^{3}\right]$ :

1) Beam:

$\mathrm{V}=\mathrm{L} \times \mathrm{w} \times \mathrm{h}$ 
2) Cylindrical Specimen:

$$
\mathrm{V}=\frac{\pi \mathrm{D}^{2}}{4} \times 0.001 \mathrm{~cm}^{3} / \mathrm{mm}^{3} \times \mathrm{h}
$$

b. Target Mass of Specimen [g]:

1) Beam:

$\mathrm{W}_{\mathrm{mb}}=\rho_{\text {opt }} \times \mathrm{V}$

2) Cylindrical Specimen:

$\mathrm{W}_{\mathrm{mb}}=\mathrm{G}_{\mathrm{mm}}\left(1 \mathrm{~g} / \mathrm{cm}^{3}\right) \times \% \mathrm{G}_{\mathrm{mm}} \times \mathrm{V}$

Where,

$\mathrm{L}=$ Length of beam specimen, $\mathrm{cm}$

$\mathrm{W}=$ Width of beam specimen, $\mathrm{cm}$

$\mathrm{h}=$ Height of specimen, $\mathrm{cm}(7.5 \mathrm{~cm})$

$\mathrm{D}=$ Diameter of cylindrical mold, $\mathrm{mm}(15.0 \mathrm{~cm})$

$\mathrm{V}=$ Volume of specimen, $\mathrm{cm}^{3}$ $\left(30.0 \mathrm{~cm} \times 12.5 \mathrm{~cm} \times 7.5 \mathrm{~cm}=2812.5 \mathrm{~cm}^{3}\right.$ for laboratory beam specimen, $22.5 \mathrm{~cm} \times 12.5 \mathrm{~cm} \times 7.5 \mathrm{~cm}=2109.4 \mathrm{~cm}^{3}$ for field beam specimen, $\left[\pi(15.0 \mathrm{~cm})^{2}\right] / 4 \times 7.5 \mathrm{~cm}=1325.4 \mathrm{~cm}^{3}$ for cylindrical specimen)

$\rho_{\mathrm{opt}} \quad=$ Density $@$ optimum asphalt content, g/cm $\mathrm{cm}^{3}$

$\mathrm{G}_{\mathrm{mm}}=$ Theoretical maximum specific gravity @ optimum asphalt content

$\% \mathrm{G}_{\mathrm{mm}}=1.0-$ Target $\%$ air voids 100

$\left(1 \mathrm{~g} / \mathrm{cm}^{3}\right)=$ Density of water

$\mathrm{W}_{\mathrm{mb}} \quad=$ Target mass of specimen, $\mathrm{g}$.

2. Calculation of Rut Depth:

a. The rut depth at each location is determined by subtracting the final measurement from the initial measurement.

b. Determine the average rut depth for each specimen.

c. The rut depth for the mixture is the average of the specimen rut depths as determined above in 2.b.

\section{G. Report:}

The test report shall include at least the following information:

a. The project name, location, date of sampling and date of testing. 
III-115M

Page 8 of 8

b. The mixture type and description (or mix I.D. no.).

c. The specimen type and method of compaction.

d. The asphaltic cement binder performance grade, percentage in sample and source.

e. The aggregate source and location.

f. The average air void content of the test specimens.

g. The test temperature.

h. The maximum theoretical specific gravity, $\mathrm{G}_{\mathrm{mm}}$, of the mixture.

i. The average rut depth to the nearest $0.1 \mathrm{~mm}$.

j. Description of any special conditioning or preparation for the test specimens (or references to a test procedure number).

If the average rut depth of the specimens exceeds $5.0 \mathrm{~mm}$ for Superpave or SMA asphalt mixes or $8.0 \mathrm{~mm}$ for conventional asphalt mixes, it shall be reported as failing. 
APPENDIX D

APA RUT DATA 
Table D.1 Summary of University of Minnesota APA Rut Depths (mm)

May 3, 2000 Koch Pavement Solutions

\begin{tabular}{|c|c|c|c|c|c|c|c|c|c|c|c|c|}
\hline Cycles & $\begin{array}{l}\mathrm{KL} 52-34 \\
\text { cores } 4 \& 1\end{array}$ & $\begin{array}{c}\mathrm{KL} 52-34 \\
\text { cores } 9 \& 6\end{array}$ & $\begin{array}{l}\text { KL 52-34 } \\
\text { cores } 8 \& 3\end{array}$ & $\begin{array}{l}\text { NU } 58-40 \\
\text { cores } 6 \& 2\end{array}$ & $\begin{array}{l}\text { NU } 58-40 \\
\text { cores } 5 \& 1\end{array}$ & $\begin{array}{l}\text { NU } 58-40 \\
\text { cores } 4 \& 3\end{array}$ & $\begin{array}{l}\text { NU } 52-34 \\
\text { cores } 2 \& 1\end{array}$ & $\begin{array}{l}\text { NU } 52-34 \\
\text { cores } 5 \& 4\end{array}$ & $\begin{array}{l}\text { NU } 52-34 \\
\text { Cores } 6 \& 3\end{array}$ & $\begin{array}{c}\mathrm{KL} 58-40 \\
\text { cores } 13 \& 5\end{array}$ & $\begin{array}{c}\mathrm{KL} 58-40 \\
\text { cores } 4 \& 2\end{array}$ & $\begin{array}{c}\mathrm{KL} 58-40 \\
\text { cores } 11 \& 9\end{array}$ \\
\hline 0 & 0.00000 & 0.00000 & 0.00000 & 0.00000 & 0.00000 & 0.00000 & 0.00000 & 0.00000 & 0.00000 & 0.00000 & 0.00000 & 0.00000 \\
\hline 1 & 0.00012 & 0.00005 & 0.00008 & 0.00003 & 0.00000 & 0.00004 & 0.00009 & 0.00010 & -0.00002 & 0.00005 & 0.00004 & -0.00002 \\
\hline 2 & 0.00023 & 0.00011 & 0.00015 & 0.00007 & 0.00000 & 0.00008 & 0.00018 & 0.00020 & -0.00003 & 0.00009 & 0.00008 & -0.00004 \\
\hline 3 & -0.01635 & -0.01654 & -0.01977 & -0.00980 & -0.00990 & -0.00288 & -0.01443 & -0.01440 & -0.00865 & -0.00696 & -0.00948 & -0.00626 \\
\hline 4 & -0.02893 & -0.02919 & -0.04300 & -0.01867 & -0.01880 & -0.00564 & -0.02675 & -0.02670 & -0.01756 & -0.01302 & -0.01804 & -0.01058 \\
\hline 5 & -0.04561 & -0.04593 & -0.06332 & -0.02984 & -0.03000 & -0.01560 & -0.03906 & -0.03901 & -0.02448 & -0.01967 & -0.02480 & -0.01710 \\
\hline 6 & -0.05550 & -0.05588 & -0.08244 & -0.03850 & -0.03870 & -0.02166 & -0.04837 & -0.04831 & -0.03179 & -0.02442 & -0.03286 & -0.02572 \\
\hline 7 & -0.06528 & -0.06573 & -0.09907 & -0.04497 & -0.04520 & -0.03072 & -0.05858 & -0.05851 & -0.03851 & -0.02958 & -0.03882 & -0.03144 \\
\hline 8 & -0.07406 & -0.07457 & $\begin{array}{c}-0.11709 \\
\end{array}$ & -0.05184 & -0.05210 & $\begin{array}{r}-0.03908 \\
\end{array}$ & -0.06829 & -0.06821 & -0.04513 & -0.03513 & -0.04688 & -0.03826 \\
\hline 9 & -0.08564 & -0.08622 & -0.13312 & -0.06040 & -0.06070 & -0.04264 & -0.07680 & -0.07671 & -0.05164 & -0.04029 & -0.05424 & -0.04438 \\
\hline 10 & -0.09323 & -0.09387 & -0.15024 & -0.06767 & -0.06800 & -0.04770 & -0.08682 & -0.08671 & -0.05946 & -0.04434 & -0.06100 & -0.05090 \\
\hline 20 & -0.18585 & -0.18713 & -0.29928 & -0.11824 & -0.11891 & -0.10261 & -0.14733 & -0.14712 & -0.12041 & -0.09108 & -0.12099 & -0.09200 \\
\hline 30 & -0.26248 & -0.26440 & -0.43292 & -0.14762 & -0.14861 & -0.14521 & -0.19685 & -0.19653 & -0.16217 & -0.12312 & -0.15339 & -0.12580 \\
\hline 40 & -0.32541 & -0.32797 & -0.53386 & -0.17929 & -0.18061 & -0.18592 & -0.24897 & -0.24854 & -0.19683 & -0.14636 & -0.18779 & -0.14779 \\
\hline 50 & -0.36973 & -0.37293 & -0.64070 & -0.20026 & -0.20192 & -0.21582 & -0.29408 & -0.29355 & -0.24728 & -0.17750 & -0.23168 & -0.16749 \\
\hline 60 & -0.41936 & -0.42320 & $\begin{array}{l}-0.74444 \\
\end{array}$ & -0.21413 & -0.21612 & -0.24713 & -0.32930 & -0.32866 & -0.28064 & -0.19834 & -0.26458 & -0.17439 \\
\hline 70 & -0.46628 & -0.47077 & -0.83509 & -0.22570 & -0.22802 & -0.28283 & -0.36471 & -0.36397 & -0.31370 & -0.22538 & -0.29907 & -0.19299 \\
\hline 80 & -0.51991 & -0.52504 & -0.91993 & -0.25227 & -0.25492 & -0.30014 & -0.39993 & -0.39908 & -0.33026 & -0.25372 & -0.31987 & -0.20919 \\
\hline 90 & -0.56524 & -0.57100 & -0.99017 & -0.27285 & -0.27583 & -0.30464 & -0.42435 & -0.42339 & -0.32731 & -0.28026 & -0.33737 & -0.22169 \\
\hline 100 & -0.59646 & -0.60287 & -1.07161 & -0.30232 & -0.30563 & -0.32255 & -0.45696 & -0.45590 & -0.33947 & -0.30100 & -0.34986 & -0.23488 \\
\hline 200 & -0.91383 & -0.92664 & $\begin{array}{l}-1.71192 \\
\end{array}$ & -0.48294 & -0.48956 & $\begin{array}{l}-0.39359 \\
\end{array}$ & -0.60523 & -0.60310 & -0.54864 & -0.37400 & -0.46903 & -0.30447 \\
\hline 300 & -1.12989 & -1.14911 & -2.17092 & -0.57615 & -0.58609 & -0.47494 & -0.76909 & -0.76590 & -0.62791 & -0.49899 & -0.53059 & -0.35225 \\
\hline 400 & -1.29885 & -1.32448 & -2.51543 & -0.61937 & -0.63262 & -0.42248 & -0.90706 & -0.90281 & $\begin{array}{r}-0.71998 \\
\end{array}$ & -0.55809 & -0.58185 & -0.35134 \\
\hline 500 & -1.47161 & -1.50364 & -2.78724 & -0.62699 & -0.64355 & -0.44753 & -0.93472 & -0.92941 & -0.80454 & -0.58099 & -0.62151 & -0.49382 \\
\hline 600 & -1.56538 & -1.60381 & -3.02745 & -0.77541 & -0.79528 & -0.44757 & -1.01178 & -1.00541 & -0.87111 & -0.59369 & $\begin{array}{l}-0.67798 \\
\end{array}$ & -0.53860 \\
\hline 700 & -1.69694 & -1.74178 & -3.24325 & -0.77552 & -0.79871 & -0.57722 & -1.12935 & -1.12191 & $\begin{array}{r}-1.03308 \\
\end{array}$ & -0.60428 & -0.83564 & -0.57139 \\
\hline 800 & -1.85670 & -1.90795 & -3.46266 & -0.77944 & -0.80594 & -0.61746 & -1.12981 & -1.12131 & -1.08155 & -0.61248 & -0.83970 & -0.59417 \\
\hline 900 & -2.00546 & -2.06312 & -3.66167 & -0.79006 & -0.81987 & -0.64381 & -1.17357 & -1.16401 & -1.10932 & -0.69968 & -0.84796 & -0.59705 \\
\hline 1000 & -2.14953 & -2.21359 & -3.84218 & -0.83288 & -0.86600 & -0.66735 & -1.26874 & -1.25811 & -1.14719 & -0.73388 & $\begin{array}{l}-0.87023 \\
\end{array}$ & -0.62774 \\
\hline 1500 & -2.82064 & -2.91673 & -4.50451 & -0.90906 & -0.95875 & -0.70848 & -1.49276 & -1.47682 & -1.46113 & -0.77726 & -0.91734 & -0.76366 \\
\hline 2000 & -3.37605 & -3.50418 & -5.01285 & -0.93745 & -1.00370 & -0.73920 & -1.76538 & -1.74413 & -1.57188 & -0.78145 & -0.91135 & -0.81318 \\
\hline 2500 & -3.84586 & -4.00602 & -5.34929 & -1.01924 & -1.10205 & -0.75283 & -1.94399 & -1.91743 & -1.81412 & -0.77414 & -0.93756 & -0.81239 \\
\hline 3000 & -4.24768 & -4.43986 & -5.69313 & -1.03103 & -1.13040 & -0.72885 & -2.18281 & -2.15094 & -1.91826 & -0.77383 & -0.97608 & -0.85931 \\
\hline 3500 & -4.53449 & -4.75871 & -5.97486 & -1.05461 & -1.17055 & $\begin{array}{l}-0.87998 \\
\end{array}$ & -2.44673 & -2.40954 & -2.25971 & $\begin{array}{l}-0.76721 \\
\end{array}$ & -0.93619 & -0.90083 \\
\hline 4000 & -4.78240 & -5.03865 & -6.20980 & -1.03910 & -1.17160 & -0.87530 & -2.61275 & -2.57025 & -2.37015 & -0.78660 & -0.97170 & -0.92285 \\
\hline 4500 & -4.94141 & -5.22969 & -6.51634 & -1.04549 & -1.19455 & -0.87532 & -2.78807 & -2.74026 & -2.55289 & -0.74679 & -0.98261 & -0.93187 \\
\hline 5000 & -5.17513 & -5.49544 & -6.73788 & -1.04918 & -1.21480 & -0.90295 & -2.96199 & -2.90886 & -2.76324 & -0.77768 & -0.92673 & -0.95789 \\
\hline 5500 & -5.21414 & -5.56648 & -6.95531 & -1.04806 & -1.23025 & -0.89288 & -3.11891 & -3.06047 & -2.91248 & -0.74296 & -0.92104 & -0.99481 \\
\hline 6000 & -5.34255 & -5.72693 & -7.19685 & -1.02155 & -1.22030 & -0.87730 & -3.25093 & -3.18718 & -3.06423 & -0.73665 & -0.93935 & -1.14033 \\
\hline 6500 & -5.43776 & -5.85417 & -7.34099 & -1.00314 & -1.21845 & -0.87362 & -3.32174 & -3.25268 & -3.30007 & -0.70454 & -0.90956 & -1.15724 \\
\hline 7000 & -5.44038 & -5.88881 & $\begin{array}{l}-7.54933 \\
\end{array}$ & -1.13753 & -1.36940 & -0.84925 & -3.41016 & -3.33579 & -3.45301 & -0.69013 & -0.87958 & -1.17436 \\
\hline 7500 & -5.56129 & -6.04176 & -7.73456 & -1.14751 & -1.39595 & -0.84107 & -3.49998 & -3.42029 & -3.69886 & -0.70791 & -0.90709 & -1.22998 \\
\hline 8000 & -5.61250 & -6.12500 & -7.85500 & -1.15500 & -1.42000 & -0.87000 & -3.51500 & -3.43000 & -3.83500 & -0.68000 & -0.88250 & -1.24250 \\
\hline
\end{tabular}




\section{APPENDIX E}

\section{IDT CREEP DATA}


Table E.1 KL 52-34 Sample Data $\left(0{ }^{\circ} \mathrm{C}\right.$ Tests)

\begin{tabular}{|c|c|c|c|}
\hline & Thickness & Diameter & Creep Load \\
\hline Sample & $\mathrm{mm}$ & $\mathrm{mm}$ & $\mathrm{kN}$ \\
\hline 2 & 76.88 & 150.4 & -0.49 \\
\hline 5 & 76.91 & 150.3 & -2.27 \\
\hline 7 & 76.87 & 150.4 & -0.48 \\
\hline & & & -1.08 \\
\hline average & 76.89 & 150.4 & \\
\hline
\end{tabular}

\begin{tabular}{|c|c|}
\hline Gage length, $\mathrm{m}$ & 0.038 \\
\hline $\mathrm{C}_{\text {empl }}$ low & 0.595 \\
\hline $\mathrm{C}_{\text {empl }}$ high & 1.466 \\
\hline
\end{tabular}

Table E.2 KL 52-34 Sample Data $\left(-10{ }^{\circ} \mathrm{C}\right.$ Tests)

\begin{tabular}{|c|c|c|c|}
\hline & Thickness & Diameter & Creep Load \\
\hline Sample & $\mathrm{mm}$ & $\mathrm{mm}$ & $\mathrm{kN}$ \\
\hline 2 & 76.88 & 150.4 & -1.50 \\
\hline 5 & 76.91 & 150.3 & -4.92 \\
\hline 7 & & & \\
\hline & & & -3.21 \\
\hline average & 76.90 & 150.4 & \\
\hline
\end{tabular}

\begin{tabular}{|c|c|}
\hline Gage length, $\mathrm{m}$ & 0.038 \\
\hline $\mathrm{C}_{\text {empl }}$ low & 0.595 \\
\hline $\mathrm{C}_{\text {empl }}$ high & 1.466 \\
\hline
\end{tabular}

Table E.3 KL 52-34 Sample Data $\left(-20{ }^{\circ} \mathrm{C}\right.$ Tests $)$

\begin{tabular}{|c|c|c|c|}
\hline & Thickness & Diameter & Creep Load \\
\hline Sample & $\mathrm{mm}$ & $\mathrm{mm}$ & $\mathrm{kN}$ \\
\hline 2 & 76.88 & 150.4 & -4.51 \\
\hline 5 & 76.91 & 150.3 & -7.44 \\
\hline 7 & 76.87 & 150.4 & -8.21 \\
\hline & & & -6.72 \\
\hline average & 76.89 & 150.4 & \\
\hline
\end{tabular}

\begin{tabular}{|c|c|}
\hline Gage length, $\mathrm{m}$ & 0.038 \\
\hline $\mathrm{C}_{\text {empl }}$ low & 0.595 \\
\hline $\mathrm{C}_{\text {empl }}$ high & 1.466 \\
\hline
\end{tabular}


Table E.4 KL 52-34 Test Data $\left(0^{\circ} \mathrm{C}\right.$ Tests)

\begin{tabular}{|c|c|c|c|c|c|c|c|c|c|c|c|c|c|c|c|c|c|c|}
\hline & & & Vorm & zed & ertic & nd & ontal & form & atio & rrays & & & & & & & & \\
\hline & & $52-34$ & Sample & & & $52-34$ & sample & & & $52-34$ & sample & & Trim & ned & & & Creep & Pois. \\
\hline & vert & vert & horz & horz & vert & vert & horz & horz & vert & \begin{tabular}{|l|} 
vert \\
\end{tabular} & horz & horz & $\mathrm{Me}$ & & \begin{tabular}{|l|} 
Ratio \\
\end{tabular} & & Comp & \begin{tabular}{|l|} 
Ratio \\
\end{tabular} \\
\hline Time & front & back & front & back & front & back & front & back & front & back & front & back & vert & horz & $\mathrm{X} / \mathrm{Y}$ & $\mathrm{C}_{\text {empl }}$ & $\mathrm{D}(\mathrm{t})$ & \\
\hline $\mathrm{sec}$ & $\mu \mathrm{m}$ & $\mu \mathrm{m}$ & $\mu \mathrm{m}$ & $\mu \mathrm{m}$ & $\mu \mathrm{m}$ & $\mu \mathrm{m}$ & $\mu \mathrm{m}$ & $\mu \mathrm{m}$ & $\mu \mathrm{m}$ & $\mu \mathrm{m}$ & $\mu \mathrm{m}$ & $\mu \mathrm{m}$ & $\mu \mathrm{m}$ & $\mu \mathrm{m}$ & & & $1 / \mathrm{kPa}$ & \\
\hline 0 & 0.0 & 0.0 & 0.0 & 0.0 & 0.0 & 0.0 & 0.0 & 0.0 & 0.0 & 0.0 & 0.0 & 0.0 & 0.0 & 0.0 & \begin{tabular}{|l|}
0.00 \\
\end{tabular} & 0.00 & 0 & 0.000 \\
\hline 1 & 1.2 & -5.9 & 0.0 & 3.2 & \begin{tabular}{|l|l|}
-1.9 \\
\end{tabular} & \begin{tabular}{|l|}
-1.1 \\
\end{tabular} & 0.5 & 0.8 & $\begin{array}{l}-1.7 \\
\end{array}$ & $\begin{array}{l}-0.6 \\
\end{array}$ & 0.6 & 0.7 & $\begin{array}{l}-1.3 \\
\end{array}$ & $\begin{array}{ll}0.5 \\
\end{array}$ & \begin{tabular}{|l}
-0.42 \\
\end{tabular} & $\begin{array}{l}-1.86 \\
\end{array}$ & 283 & \begin{tabular}{|l|l|}
0.120 \\
\end{tabular} \\
\hline 2 & 1.0 & $\begin{array}{l}-8.4 \\
\end{array}$ & 0.1 & 4.1 & $\begin{array}{l}-2.3 \\
\end{array}$ & \begin{tabular}{|l|}
-1.3 \\
\end{tabular} & 0.6 & 1.0 & $\begin{array}{l}-2.1 \\
\end{array}$ & $\begin{array}{l}-0.9 \\
\end{array}$ & 0.8 & 0.9 & $\begin{array}{l}-1.7 \\
\end{array}$ & \begin{tabular}{|l|l}
0.7 \\
\end{tabular} & \begin{tabular}{|l|}
-0.43 \\
\end{tabular} & $\begin{array}{l}-1.81 \\
\end{array}$ & 364 & \begin{tabular}{|l|l|}
0.137 \\
\end{tabular} \\
\hline 3 & 1.3 & $\begin{array}{l}-9.3 \\
\end{array}$ & 0.2 & 4.9 & -2.6 & $\begin{array}{l}-1.5 \\
\end{array}$ & 0.7 & 1.2 & -2.6 & $\begin{array}{l}-1.1 \\
\end{array}$ & 0.9 & 0.9 & $\begin{array}{l}-1.9 \\
\end{array}$ & $\begin{array}{l}0.8 \\
\end{array}$ & \begin{tabular}{|l|}
-0.40 \\
\end{tabular} & -1.91 & 419 & \begin{tabular}{|l|}
0.106 \\
\end{tabular} \\
\hline 4 & 1.2 & -10.3 & 0.3 & 5.7 & $\begin{array}{l}-2.9 \\
\end{array}$ & $\begin{array}{l}-1.7 \\
\end{array}$ & 0.8 & 1.3 & -2.4 & $\begin{array}{l}-0.9 \\
\end{array}$ & 0.9 & 1.1 & -2.0 & $\begin{array}{l}0.9 \\
\end{array}$ & \begin{tabular}{|l}
-0.45 \\
\end{tabular} & -1.74 & 438 & \begin{tabular}{|l|}
0.162 \\
\end{tabular} \\
\hline 5 & 1.5 & -11.5 & 0.3 & 6.3 & -3.1 & $\begin{array}{l}-1.9 \\
\end{array}$ & 0.8 & 1.4 & -2.7 & \begin{tabular}{|l|}
-1.1 \\
\end{tabular} & 1.1 & 1.2 & -2.2 & 1.0 & -0.45 & -1.73 & 485 & \begin{tabular}{|l|l|}
0.163 \\
\end{tabular} \\
\hline 6 & 1.3 & -12.4 & 0.4 & 6.8 & $\begin{array}{l}-3.3 \\
\end{array}$ & $\begin{array}{l}-2.1 \\
\end{array}$ & 0.9 & 1.5 & $\begin{array}{l}-3.2 \\
\end{array}$ & $\begin{array}{l}-1.5 \\
\end{array}$ & 1.0 & 1.2 & -2.5 & 1.0 & \begin{tabular}{|l|}
-0.41 \\
\end{tabular} & -1.88 & 547 & 0.116 \\
\hline 7 & 1.5 & -13.4 & 0.5 & 7.3 & $\begin{array}{l}-3.5 \\
\end{array}$ & -2.2 & 0.9 & 1.6 & $\begin{array}{l}-3.2 \\
\end{array}$ & $\begin{array}{l}-1.2 \\
\end{array}$ & 1.1 & 1.3 & -2.5 & $\begin{array}{l}1.1 \\
\end{array}$ & -0.45 & -1.75 & |556 & 0.155 \\
\hline 8 & 1.5 & -14.3 & 0.6 & 7.8 & -3.6 & -2.3 & 1.0 & 1.7 & -3.2 & $\begin{array}{l}-1.2 \\
\end{array}$ & 1.2 & 1.4 & -2.6 & 1.2 & \begin{tabular}{|c|}
-0.47 \\
\end{tabular} & -1.70 & \begin{tabular}{|l|}
576 \\
\end{tabular} & \begin{tabular}{|l|}
0.177 \\
\end{tabular} \\
\hline$\overline{9}$ & 1.5 & -15.2 & 0.6 & 8.2 & -3.8 & -2.5 & 1.0 & 1.7 & -3.6 & \begin{tabular}{|l|}
-1.4 \\
\end{tabular} & 1.3 & 1.4 & -2.8 & 1.3 & \begin{tabular}{|l|}
-0.44 \\
\end{tabular} & -1.76 & \begin{tabular}{l|l}
620 \\
\end{tabular} & \begin{tabular}{|l|}
0.152 \\
\end{tabular} \\
\hline 10 & 1.6 & -15.9 & 0.7 & 8.6 & $\begin{array}{l}-4.0 \\
\end{array}$ & $\begin{array}{l}-2.6 \\
\end{array}$ & 1.0 & 1.8 & -3.5 & \begin{tabular}{|l|}
-1.4 \\
\end{tabular} & 1.3 & 1.5 & -2.9 & 1.3 & -0.47 & -1.69 & \begin{tabular}{l|}
633 \\
\end{tabular} & 0.178 \\
\hline 11 & 1.6 & -16.5 & 0.7 & 9.1 & -4.1 & -2.7 & 1.1 & 1.9 & -3.8 & \begin{tabular}{|l|}
-1.5 \\
\end{tabular} & 1.3 & 1.6 & $\begin{array}{l}-3.0 \\
\end{array}$ & $\begin{array}{l}1.4 \\
\end{array}$ & -0.46 & -1.71 & 670 & 0.171 \\
\hline 12 & 1.5 & -17.1 & 0.8 & 9.6 & $\begin{array}{l}-4.2 \\
\end{array}$ & -2.8 & 1.1 & 2.0 & $\begin{array}{l}-3.8 \\
\end{array}$ & $\begin{array}{l}-1.5 \\
\end{array}$ & 1.4 & 1.6 & -3.1 & 1.4 & \begin{tabular}{|l|}
-0.47 \\
\end{tabular} & -1.69 & 684 & \begin{tabular}{|l}
0.179 \\
\end{tabular} \\
\hline 13 & 1.8 & -17.8 & 0.9 & 9.9 & -4.3 & -2.8 & 1.2 & 2.1 & -3.9 & -1.7 & 1.5 & 1.7 & -3.2 & $\begin{array}{l}1.5 \\
\end{array}$ & -0.47 & $\begin{array}{l}-1.67 \\
\end{array}$ & 713 & 0.186 \\
\hline 14 & 1.6 & -18.5 & 0.9 & 10.3 & $\begin{array}{l}-4.4 \\
\end{array}$ & -2.9 & 1.2 & 2.1 & $\begin{array}{l}-4.4 \\
\end{array}$ & $\begin{array}{l}-1.7 \\
\end{array}$ & 1.5 & 1.7 & -3.4 & 1.6 & \begin{tabular}{|c|c|}
-0.47 \\
\end{tabular} & -1.69 & 748 & 0.180 \\
\hline 15 & 1.5 & -19.1 & 0.9 & \begin{tabular}{|l|l}
10.7 \\
\end{tabular} & $\begin{array}{l}-4.6 \\
\end{array}$ & $\begin{array}{l}-3.0 \\
\end{array}$ & 1.2 & 2.2 & $\begin{array}{l}-4.2 \\
\end{array}$ & $\begin{array}{l}-1.7 \\
\end{array}$ & 1.5 & 1.8 & -3.4 & $\begin{array}{l}1.6 \\
\end{array}$ & \begin{tabular}{|l|}
-0.48 \\
\end{tabular} & -1.66 & 752 & \begin{tabular}{|l|l|}
0.194 \\
\end{tabular} \\
\hline 16 & 1.8 & -19.7 & 1.0 & 11.0 & $\begin{array}{l}-4.7 \\
\end{array}$ & $\begin{array}{l}-3.1 \\
\end{array}$ & 1.3 & 2.3 & $\begin{array}{l}-4.4 \\
\end{array}$ & $\begin{array}{l}-2.0 \\
\end{array}$ & 1.6 & 1.9 & $\begin{array}{l}-3.5 \\
\end{array}$ & $\begin{array}{l}1.7 \\
\end{array}$ & \begin{tabular}{|l}
-0.48 \\
\end{tabular} & $\begin{array}{l}-1.66 \\
\end{array}$ & \begin{tabular}{|c|}
788 \\
\end{tabular} & \begin{tabular}{|l|}
0.193 \\
\end{tabular} \\
\hline 17 & 1.8 & -20.3 & 1.1 & \begin{tabular}{|l|l|}
11.4 \\
\end{tabular} & \begin{tabular}{l|l}
-4.8 \\
\end{tabular} & -3.1 & 1.3 & 2.3 & -4.6 & $\begin{array}{l}-1.7 \\
\end{array}$ & 1.6 & 1.9 & -3.5 & 1.7 & \begin{tabular}{|l|}
-0.49 \\
\end{tabular} & -1.62 & \begin{tabular}{|l|}
792 \\
\end{tabular} & 0.210 \\
\hline 18 & 1.6 & -21.0 & 1.1 & 11.8 & -4.9 & -3.2 & 1.3 & 2.4 & -4.7 & -2.1 & 1.7 & 1.9 & -3.7 & 1.8 & -0.47 & -1.68 & 830 & 0.185 \\
\hline 19 & 1.8 & -21.5 & 1.2 & \begin{tabular}{|l|l}
12.2 \\
\end{tabular} & -4.9 & -3.3 & 1.3 & 2.5 & -4.7 & $\begin{array}{l}-2.0 \\
\end{array}$ & 1.7 & 1.9 & $\begin{array}{l}-3.7 \\
\end{array}$ & $\begin{array}{l}1.8 \\
\end{array}$ & -0.49 & -1.63 & 836 & 0.208 \\
\hline 20 & $\begin{array}{l}1.8 \\
\end{array}$ & -21.9 & 1.3 & \begin{tabular}{|l|l|}
12.4 \\
\end{tabular} & -5.0 & $\begin{array}{l}-3.3 \\
\end{array}$ & 1.4 & 2.5 & -5.0 & $\begin{array}{l}-2.1 \\
\end{array}$ & 1.7 & 2.1 & -3.8 & $\begin{array}{l}1.9 \\
\end{array}$ & \begin{tabular}{|l|}
-0.50 \\
\end{tabular} & -1.61 & 864 & \begin{tabular}{|l|}
0.214 \\
\end{tabular} \\
\hline 21 & 1.9 & -22.2 & 1.3 & 12.8 & -5.1 & -3.4 & 1.4 & 2.6 & -5.3 & $\begin{array}{l}-2.4 \\
\end{array}$ & 1.8 & 2.0 & -4.1 & 1.9 & \begin{tabular}{|c|}
-0.47 \\
\end{tabular} & -1.67 & $\begin{array}{l}903 \\
\end{array}$ & \begin{tabular}{|l|}
0.186 \\
\end{tabular} \\
\hline 22 & 1.8 & -23.1 & 1.4 & 13.0 & -5.1 & \begin{tabular}{|l|}
-3.5 \\
\end{tabular} & 1.4 & 2.7 & -5.5 & $\begin{array}{l}-2.6 \\
\end{array}$ & 1.7 & 2.1 & -4.2 & 2.0 & \begin{tabular}{|l}
-0.48 \\
\end{tabular} & -1.67 & 926 & \begin{tabular}{|l|}
0.189 \\
\end{tabular} \\
\hline 23 & 1.8 & -23.5 & 1.4 & 13.4 & $\begin{array}{l}-5.2 \\
\end{array}$ & $\begin{array}{l}-3.5 \\
\end{array}$ & 1.4 & 2.7 & $\begin{array}{l}-5.8 \\
\end{array}$ & $\begin{array}{l}-2.6 \\
\end{array}$ & 1.8 & 2.1 & -4.3 & 2.0 & \begin{tabular}{|l|}
-0.47 \\
\end{tabular} & $\begin{array}{l}-1.69 \\
\end{array}$ & 947 & \begin{tabular}{|l|}
0.181 \\
\end{tabular} \\
\hline 24 & 1.8 & -24.0 & 1.5 & \begin{tabular}{|l|l}
13.8 \\
\end{tabular} & -5.3 & $\begin{array}{l}-3.6 \\
\end{array}$ & 1.5 & 2.8 & $\begin{array}{l}-5.8 \\
\end{array}$ & $\begin{array}{l}-2.6 \\
\end{array}$ & 1.8 & 2.2 & -4.3 & 2.1 & -0.48 & -1.65 & \begin{tabular}{|l|}
963 \\
\end{tabular} & \begin{tabular}{|l|l|}
0.195 \\
\end{tabular} \\
\hline 25 & 1.6 & -24.4 & 1.5 & \begin{tabular}{|l|l|}
14.1 \\
\end{tabular} & $\begin{array}{l}-5.4 \\
\end{array}$ & $\begin{array}{l}-3.7 \\
\end{array}$ & 1.5 & 2.8 & -5.9 & $\begin{array}{l}-2.9 \\
\end{array}$ & 1.8 & 2.4 & -4.5 & \begin{tabular}{|l|l}
2.1 \\
\end{tabular} & \begin{tabular}{|c|c|}
-0.48 \\
\end{tabular} & -1.65 & 997 & \begin{tabular}{|l|}
0.195 \\
\end{tabular} \\
\hline 26 & 2.1 & -25.0 & 1.6 & \begin{tabular}{|l|l|}
14.3 \\
\end{tabular} & $\begin{array}{l}-5.4 \\
\end{array}$ & -3.6 & 1.5 & 2.9 & -6.1 & \begin{tabular}{|l|}
-2.9 \\
\end{tabular} & 1.9 & 2.2 & -4.5 & 2.1 & \begin{tabular}{|l|}
-0.48 \\
\end{tabular} & -1.66 & 1000 & \begin{tabular}{|l|l|}
0.194 \\
\end{tabular} \\
\hline 27 & 1.8 & -25.3 & 1.6 & \begin{tabular}{|l|}
14.6 \\
\end{tabular} & -5.5 & -3.8 & 1.5 & 3.0 & -6.2 & $\begin{array}{l}-2.7 \\
\end{array}$ & 1.9 & 2.2 & -4.5 & 2.2 & -0.48 & -1.66 & 1015 & \begin{tabular}{|l}
0.193 \\
\end{tabular} \\
\hline 28 & 1.8 & -25.9 & 1.7 & \begin{tabular}{|l|l|}
14.9 \\
\end{tabular} & $\begin{array}{l}-5.5 \\
\end{array}$ & $\begin{array}{l}-3.8 \\
\end{array}$ & 1.6 & 3.0 & $\begin{array}{l}-6.1 \\
\end{array}$ & $\begin{array}{l}-2.9 \\
\end{array}$ & 1.9 & 2.3 & -4.6 & 2.2 & -0.49 & -1.64 & 1026 & 0.204 \\
\hline 29 & 1.9 & -26.3 & 1.7 & \begin{tabular}{|l|}
15.2 \\
\end{tabular} & $\begin{array}{l}-5.6 \\
\end{array}$ & $\begin{array}{l}-3.8 \\
\end{array}$ & 1.6 & 3.1 & $\begin{array}{l}-6.4 \\
\end{array}$ & $\begin{array}{l}-3.0 \\
\end{array}$ & 1.9 & 2.4 & -4.7 & 2.3 & \begin{tabular}{|l|}
-0.49 \\
\end{tabular} & -1.64 & 1053 & \begin{tabular}{|l|}
0.203 \\
\end{tabular} \\
\hline 30 & 2.2 & -26.9 & 1.8 & 15.6 & $\begin{array}{l}-5.7 \\
\end{array}$ & $\begin{array}{l}-3.9 \\
\end{array}$ & 1.6 & 3.1 & $\begin{array}{l}-6.4 \\
\end{array}$ & $\begin{array}{l}-3.2 \\
\end{array}$ & 1.9 & 2.4 & -4.8 & 2.3 & \begin{tabular}{|c|c|}
-0.48 \\
\end{tabular} & -1.65 & \begin{tabular}{|l|}
1072 \\
\end{tabular} & \begin{tabular}{|l|l|}
0.196 \\
\end{tabular} \\
\hline 31 & 1.9 & -27.2 & 1.8 & 15.8 & -5.8 & -4.0 & 1.6 & 3.2 & -6.7 & $\begin{array}{l}-3.5 \\
\end{array}$ & 2.0 & 2.2 & -5.0 & 2.3 & -0.47 & -1.70 & \begin{tabular}{|l|}
1103 \\
\end{tabular} & \begin{tabular}{|l|}
0.176 \\
\end{tabular} \\
\hline 32 & 2.1 & -27.8 & 1.9 & \begin{tabular}{|l|l}
16.0 \\
\end{tabular} & $\begin{array}{l}-5.8 \\
\end{array}$ & -4.0 & 1.6 & 3.3 & -6.5 & $\begin{array}{l}-3.2 \\
\end{array}$ & 2.1 & 2.5 & -4.9 & 2.4 & -0.50 & -1.61 & 1098 & 0.215 \\
\hline 33 & 2.2 & -28.1 & 1.9 & \begin{tabular}{|l|l|}
16.3 \\
\end{tabular} & -5.9 & -4.1 & 1.7 & 3.3 & -6.7 & $\begin{array}{l}-3.0 \\
\end{array}$ & 2.1 & 2.6 & -4.9 & 2.5 & \begin{tabular}{|l|}
-0.50 \\
\end{tabular} & -1.59 & 1112 & \begin{tabular}{|l|}
0.224 \\
\end{tabular} \\
\hline
\end{tabular}


Table E.4 KL 52-34 Test Data $\left(0{ }^{\circ} \mathrm{C}\right.$ Tests $)$, continued

\begin{tabular}{|c|c|c|c|c|c|c|c|c|c|c|c|c|c|c|c|c|c|c|}
\hline & & & Norm & & & & t & Deforr & $\overline{\text { atio }}$ & rrays & & & & & & & & \\
\hline & & $52-34$ & Sample & & & $52-34$ & sample & & & $52-34$ & ample & & Trim & ned & & & Creep & Pois. \\
\hline & $\begin{array}{l}\text { vert } \\
\end{array}$ & vert & horz & horz & vert & vert & horz & horz & vert & \begin{tabular}{l|l|} 
vert \\
\end{tabular} & horz & horz & $\overline{\mathrm{Me}}$ & & Ratio & & Comp & \begin{tabular}{|l|} 
Ratio \\
\end{tabular} \\
\hline Time & front & back & front & back & front & back & front & back & front & back & front & back & vert & horz & $\mathrm{X} / \mathrm{Y}$ & $\mathrm{C}_{\mathrm{empl}}$ & $\mathrm{D}(\mathrm{t})$ & \\
\hline $\mathrm{sec}$ & $\mu \mathrm{m}$ & $\mu \mathrm{m}$ & $\mu \mathrm{m}$ & $\mu \mathrm{m}$ & $\mu \mathrm{m}$ & $\mu \mathrm{m}$ & $\mu \mathrm{m}$ & $\mu \mathrm{m}$ & $\mu \mathrm{m}$ & $\mu \mathrm{m}$ & $\mu \mathrm{m}$ & $\mu \mathrm{m}$ & $\mu \mathrm{m}$ & $\mu \mathrm{m}$ & & & $1 / \mathrm{kPa}$ & \\
\hline 34 & 2.1 & -28.7 & 1.9 & 16.6 & -5.9 & -4.1 & 1.7 & 3.4 & -6.7 & \begin{tabular}{|l|}
-3.3 \\
\end{tabular} & 2.1 & 2.6 & -5.0 & 2.5 & -0.50 & -1.60 & 1130 & 0.222 \\
\hline 35 & 2.1 & -29.0 & 2.0 & 16.8 & -6.0 & -4.2 & 1.7 & 3.4 & -6.8 & $\begin{array}{l}-3.2 \\
\end{array}$ & 2.2 & 2.6 & -5.0 & 2.6 & -0.51 & -1.59 & 1138 & 0.228 \\
\hline 36 & 2.2 & -29.6 & 2.1 & \begin{tabular}{|l|l|}
17.1 \\
\end{tabular} & $\begin{array}{l}-6.0 \\
\end{array}$ & $\begin{array}{l}-4.2 \\
\end{array}$ & 1.7 & 3.5 & $\begin{array}{l}-6.8 \\
\end{array}$ & $\begin{array}{l}-3.2 \\
\end{array}$ & 2.2 & 2.8 & $\begin{array}{l}-5.1 \\
\end{array}$ & 2.6 & \begin{tabular}{|l}
-0.52 \\
\end{tabular} & -1.55 & 1150 & \begin{tabular}{|l|}
0.246 \\
\end{tabular} \\
\hline 37 & 1.9 & -30.3 & 2.1 & \begin{tabular}{|l|l|}
17.4 \\
\end{tabular} & $\begin{array}{l}-6.1 \\
\end{array}$ & -4.2 & 1.7 & 3.5 & -6.7 & \begin{tabular}{|l|}
-3.0 \\
\end{tabular} & 2.2 & 2.7 & $\begin{array}{l}-5.0 \\
\end{array}$ & 2.6 & \begin{tabular}{|l|}
-0.53 \\
\end{tabular} & -1.54 & 1142 & \begin{tabular}{|l|}
0.253 \\
\end{tabular} \\
\hline 38 & 1.9 & -30.5 & 2.2 & \begin{tabular}{|l|l|}
17.7 \\
\end{tabular} & -6.2 & -4.3 & 1.8 & 3.6 & -6.8 & $\begin{array}{l}-3.2 \\
\end{array}$ & 2.3 & 2.8 & $\begin{array}{l}-5.1 \\
\end{array}$ & 2.7 & \begin{tabular}{|l|}
-0.52 \\
\end{tabular} & -1.54 & \begin{tabular}{|l|}
1168 \\
\end{tabular} & \begin{tabular}{|l|}
0.251 \\
\end{tabular} \\
\hline 39 & $\begin{array}{l}2.1 \\
\end{array}$ & -30.8 & 2.2 & \begin{tabular}{|l|l|}
17.9 \\
\end{tabular} & $\begin{array}{l}-6.2 \\
\end{array}$ & $\begin{array}{l}-4.4 \\
\end{array}$ & 1.8 & 3.6 & -6.7 & $\begin{array}{l}-2.9 \\
\end{array}$ & 2.4 & 2.9 & $\begin{array}{l}-5.0 \\
\end{array}$ & 2.8 & \begin{tabular}{|l|}
-0.55 \\
\end{tabular} & -1.49 & \begin{tabular}{|l|}
1159 \\
\end{tabular} & \begin{tabular}{|l|}
0.285 \\
\end{tabular} \\
\hline 40 & 1.9 & -31.2 & 2.3 & 18.1 & $\begin{array}{l}-6.3 \\
\end{array}$ & -4.4 & 1.8 & 3.7 & \begin{tabular}{|c|}
-7.0 \\
\end{tabular} & -3.0 & 2.4 & 2.8 & -5.2 & 2.8 & -0.54 & -1.52 & \begin{tabular}{|l|}
1185 \\
\end{tabular} & 0.268 \\
\hline 41 & 1.9 & -31.5 & 2.4 & \begin{tabular}{|l|l|}
18.4 \\
\end{tabular} & $\begin{array}{l}-6.3 \\
\end{array}$ & -4.5 & 1.8 & 3.7 & $\begin{array}{l}-6.8 \\
\end{array}$ & \begin{tabular}{l|l|}
-2.9 \\
\end{tabular} & 2.4 & 3.0 & $\begin{array}{l}-5.1 \\
\end{array}$ & 2.9 & \begin{tabular}{|c|c|}
-0.56 \\
\end{tabular} & -1.47 & \begin{tabular}{|l|}
1182 \\
\end{tabular} & \begin{tabular}{|l|}
0.301 \\
\end{tabular} \\
\hline 42 & 1.9 & -31.9 & 2.4 & \begin{tabular}{|l|l|}
18.6 \\
\end{tabular} & -6.4 & -4.5 & 1.8 & 3.7 & -6.8 & \begin{tabular}{|l|}
-2.9 \\
\end{tabular} & 2.4 & 3.0 & -5.2 & 2.9 & \begin{tabular}{|l|}
-0.56 \\
\end{tabular} & -1.47 & \begin{tabular}{|l|}
1190 \\
\end{tabular} & \begin{tabular}{|l|}
0.296 \\
\end{tabular} \\
\hline 43 & 1.9 & -32.5 & 2.4 & \begin{tabular}{|l|}
18.9 \\
\end{tabular} & -6.4 & -4.6 & 1.8 & 3.8 & -6.8 & -3.0 & 2.4 & 3.1 & -5.2 & 2.9 & \begin{tabular}{|l|}
-0.56 \\
\end{tabular} & -1.46 & \begin{tabular}{|l|}
1206 \\
\end{tabular} & \begin{tabular}{|l|}
0.302 \\
\end{tabular} \\
\hline 44 & 1.9 & -32.8 & 2.5 & \begin{tabular}{|l|}
19.2 \\
\end{tabular} & -6.6 & -4.7 & 1.9 & 3.8 & -7.0 & -2.4 & 2.5 & 3.1 & $\begin{array}{l}-5.2 \\
\end{array}$ & 3.0 & \begin{tabular}{|l|}
-0.58 \\
\end{tabular} & -1.43 & \begin{tabular}{|l|}
1202 \\
\end{tabular} & \begin{tabular}{|l|}
0.331 \\
\end{tabular} \\
\hline 45 & 2.1 & -33.3 & 2.5 & \begin{tabular}{|l|}
19.4 \\
\end{tabular} & -6.6 & -4.7 & 1.9 & 3.9 & -6.8 & -2.7 & 2.5 & 3.2 & -5.2 & 3.0 & -0.58 & -1.42 & 1213 & 0.337 \\
\hline 46 & 1.9 & -33.7 & 2.5 & \begin{tabular}{|l|}
19.6 \\
\end{tabular} & -6.7 & -4.7 & 1.9 & 3.9 & -6.7 & $\begin{array}{l}-2.6 \\
\end{array}$ & 2.6 & 3.3 & $\begin{array}{l}-5.2 \\
\end{array}$ & 3.1 & \begin{tabular}{|l|}
-0.60 \\
\end{tabular} & -1.39 & \begin{tabular}{|l|}
1212 \\
\end{tabular} & \begin{tabular}{|l|}
0.359 \\
\end{tabular} \\
\hline 47 & 1.9 & -34.0 & 2.6 & \begin{tabular}{|l|}
19.9 \\
\end{tabular} & $\begin{array}{l}-6.7 \\
\end{array}$ & -4.8 & 1.9 & 4.0 & -6.7 & $\begin{array}{l}-2.6 \\
\end{array}$ & 2.6 & 3.2 & -5.2 & 3.1 & \begin{tabular}{|l}
-0.59 \\
\end{tabular} & -1.41 & \begin{tabular}{|l|}
1217 \\
\end{tabular} & \begin{tabular}{|l|l}
0.347 \\
\end{tabular} \\
\hline 48 & 2.1 & -34.4 & 2.6 & 20.1 & -6.7 & -4.9 & 1.9 & 4.0 & -6.8 & -2.6 & 2.6 & 3.3 & \begin{tabular}{|l|}
-5.3 \\
\end{tabular} & 3.2 & \begin{tabular}{|l|}
-0.60 \\
\end{tabular} & -1.39 & \begin{tabular}{|l|}
1236 \\
\end{tabular} & \begin{tabular}{|l|}
0.359 \\
\end{tabular} \\
\hline 49 & 2.1 & -34.7 & 2.7 & \begin{tabular}{|l}
20.3 \\
\end{tabular} & $\begin{array}{l}-6.9 \\
\end{array}$ & $\begin{array}{l}-5.0 \\
\end{array}$ & 1.9 & 4.1 & $\begin{array}{l}-6.8 \\
\end{array}$ & $\begin{array}{l}-2.4 \\
\end{array}$ & 2.7 & 3.5 & $\begin{array}{l}-5.3 \\
\end{array}$ & 3.2 & \begin{tabular}{|l|}
-0.62 \\
\end{tabular} & $\begin{array}{l}-1.37 \\
\end{array}$ & \begin{tabular}{|l|}
1244 \\
\end{tabular} & \begin{tabular}{|l|}
0.383 \\
\end{tabular} \\
\hline$\overline{50}$ & 2.1 & -35.0 & 2.7 & 20.6 & $\begin{array}{l}-6.9 \\
\end{array}$ & $\begin{array}{l}-5.0 \\
\end{array}$ & 2.0 & 4.1 & -6.5 & \begin{tabular}{|l|}
-2.3 \\
\end{tabular} & 2.8 & 3.5 & $\begin{array}{l}-5.2 \\
\end{array}$ & 3.3 & \begin{tabular}{|l|}
-0.63 \\
\end{tabular} & -1.34 & \begin{tabular}{|l|}
1234 \\
\end{tabular} & \begin{tabular}{|l|}
0.408 \\
\end{tabular} \\
\hline 51 & 2.1 & -35.6 & 2.8 & \begin{tabular}{|l|}
20.9 \\
\end{tabular} & -7.0 & -5.1 & 2.0 & 4.1 & -6.5 & -2.3 & 2.8 & 3.8 & $\begin{array}{l}-5.2 \\
\end{array}$ & 3.4 & \begin{tabular}{|l|}
-0.65 \\
\end{tabular} & -1.32 & \begin{tabular}{|l|}
1248 \\
\end{tabular} & \begin{tabular}{|l|}
0.431 \\
\end{tabular} \\
\hline 52 & 2.1 & -36.2 & 2.8 & 21.0 & -7.1 & -5.1 & 2.0 & 4.2 & -6.5 & \begin{tabular}{l|l}
-2.1 \\
\end{tabular} & 2.8 & 3.7 & -5.2 & 3.4 & -0.65 & -1.31 & \begin{tabular}{|l|}
1244 \\
\end{tabular} & 0.435 \\
\hline 53 & 1.9 & -36.0 & 2.9 & 21.2 & -7.1 & -5.2 & 2.0 & 4.2 & -6.4 & -1.8 & 2.9 & 3.8 & -5.1 & 3.4 & \begin{tabular}{|c|c|}
-0.67 \\
\end{tabular} & -1.28 & \begin{tabular}{|l|}
1237 \\
\end{tabular} & \begin{tabular}{|l}
0.478 \\
\end{tabular} \\
\hline 54 & 1.8 & -37.1 & 2.9 & $\begin{array}{l}21.5 \\
\end{array}$ & $\begin{array}{l}-7.1 \\
\end{array}$ & $\begin{array}{l}-5.2 \\
\end{array}$ & 2.0 & 4.3 & $\begin{array}{l}-6.7 \\
\end{array}$ & $\begin{array}{l}-2.1 \\
\end{array}$ & 2.8 & 3.8 & $\begin{array}{l}-5.3 \\
\end{array}$ & $\begin{array}{l}3.4 \\
\end{array}$ & \begin{tabular}{|l|}
-0.65 \\
\end{tabular} & -1.31 & \begin{tabular}{|l|}
1267 \\
\end{tabular} & \begin{tabular}{|l|}
0.441 \\
\end{tabular} \\
\hline 55 & 2.1 & -37.1 & 3.0 & \begin{tabular}{|l|l}
21.7 \\
\end{tabular} & \begin{tabular}{l|l}
-7.3 \\
\end{tabular} & -5.3 & 2.0 & 4.3 & -6.4 & $\begin{array}{l}-2.1 \\
\end{array}$ & 2.8 & 3.9 & \begin{tabular}{|l|}
-5.3 \\
\end{tabular} & 3.5 & \begin{tabular}{|l|}
-0.66 \\
\end{tabular} & -1.29 & \begin{tabular}{|l|}
1270 \\
\end{tabular} & \begin{tabular}{|l|}
0.463 \\
\end{tabular} \\
\hline 56 & $\begin{array}{l}1.8 \\
\end{array}$ & -37.7 & 3.0 & \begin{tabular}{|l|}
22.0 \\
\end{tabular} & $\begin{array}{l}-7.3 \\
\end{array}$ & $\begin{array}{l}-5.4 \\
\end{array}$ & 2.0 & 4.3 & -6.7 & -2.0 & 2.9 & 3.8 & \begin{tabular}{|l|}
-5.3 \\
\end{tabular} & 3.5 & \begin{tabular}{|l|}
-0.66 \\
\end{tabular} & -1.30 & \begin{tabular}{|l|}
1280 \\
\end{tabular} & \begin{tabular}{|l|l}
0.451 \\
\end{tabular} \\
\hline 57 & 1.9 & -38.0 & 3.0 & 22.2 & $\begin{array}{l}-7.4 \\
\end{array}$ & $\begin{array}{l}-5.4 \\
\end{array}$ & 2.1 & 4.4 & $\begin{array}{l}-6.7 \\
\end{array}$ & \begin{tabular}{l|l|}
-2.1 \\
\end{tabular} & 2.9 & 3.9 & $\begin{array}{l}-5.4 \\
\end{array}$ & 3.6 & \begin{tabular}{|l|l|}
-0.66 \\
\end{tabular} & -1.30 & \begin{tabular}{|l|}
1296 \\
\end{tabular} & 0.455 \\
\hline$\overline{58}$ & 1.8 & -38.5 & 3.1 & \begin{tabular}{|l|}
22.4 \\
\end{tabular} & $\begin{array}{l}-7.4 \\
\end{array}$ & -5.5 & 2.1 & 4.4 & $\begin{array}{l}-6.7 \\
\end{array}$ & -2.3 & 3.0 & 3.9 & -5.5 & 3.6 & -0.66 & -1.30 & \begin{tabular}{|l|}
1312 \\
\end{tabular} & 0.453 \\
\hline 59 & $\begin{array}{l}1.8 \\
\end{array}$ & -39.0 & 3.1 & \begin{tabular}{|l|l}
22.7 \\
\end{tabular} & $\begin{array}{l}-7.6 \\
\end{array}$ & $\begin{array}{l}-5.6 \\
\end{array}$ & 2.1 & 4.4 & -6.8 & \begin{tabular}{|l|}
-2.1 \\
\end{tabular} & 3.0 & 4.0 & $\begin{array}{l}-5.5 \\
\end{array}$ & 3.6 & \begin{tabular}{|c|}
-0.66 \\
\end{tabular} & -1.30 & \begin{tabular}{|l|}
1326 \\
\end{tabular} & 0.448 \\
\hline 60 & 1.6 & -39.3 & 3.2 & \begin{tabular}{|l|}
22.9 \\
\end{tabular} & $\begin{array}{l}-7.6 \\
\end{array}$ & -5.6 & 2.1 & 4.5 & -6.8 & \begin{tabular}{|l|}
-2.1 \\
\end{tabular} & 2.9 & 3.9 & -5.5 & 3.6 & \begin{tabular}{|l|}
-0.66 \\
\end{tabular} & -1.30 & \begin{tabular}{|l|}
1328 \\
\end{tabular} & \begin{tabular}{|l|}
0.450 \\
\end{tabular} \\
\hline 61 & 1.3 & -40.2 & 3.2 & \begin{tabular}{|l|}
23.0 \\
\end{tabular} & -7.7 & -5.7 & 2.1 & 4.5 & -7.0 & -2.3 & 3.0 & 4.0 & $\begin{array}{l}-5.7 \\
\end{array}$ & 3.7 & -0.65 & -1.31 & \begin{tabular}{|l|}
1352 \\
\end{tabular} & 0.436 \\
\hline 62 & $\begin{array}{l}1.9 \\
\end{array}$ & -39.9 & 3.3 & 23.4 & $\begin{array}{l}-7.7 \\
\end{array}$ & -5.7 & 2.1 & 4.6 & -7.0 & \begin{tabular}{|l|}
-2.1 \\
\end{tabular} & 3.0 & 4.1 & $\begin{array}{l}-5.6 \\
\end{array}$ & 3.7 & \begin{tabular}{|c|}
-0.66 \\
\end{tabular} & -1.29 & \begin{tabular}{|l|}
1355 \\
\end{tabular} & \begin{tabular}{|l|}
0.462 \\
\end{tabular} \\
\hline 63 & 1.6 & -40.2 & 3.3 & \begin{tabular}{|l|l|}
23.6 \\
\end{tabular} & $\begin{array}{l}-7.8 \\
\end{array}$ & -5.8 & 2.1 & 4.6 & -7.3 & -2.4 & 3.0 & 4.1 & -5.8 & 3.8 & -0.65 & -1.32 & \begin{tabular}{|l|}
1393 \\
\end{tabular} & 0.433 \\
\hline 64 & 1.6 & -40.5 & 3.4 & \begin{tabular}{|l|l}
23.8 \\
\end{tabular} & -7.8 & -5.8 & 2.2 & 4.6 & $\begin{array}{l}-7.1 \\
\end{array}$ & $\begin{array}{l}-2.4 \\
\end{array}$ & 3.1 & 4.0 & $\begin{array}{l}-5.8 \\
\end{array}$ & 3.8 & -0.65 & -1.31 & \begin{tabular}{|c|}
1385 \\
\end{tabular} & \begin{tabular}{|l|l|}
0.443 \\
\end{tabular} \\
\hline 65 & 1.6 & -41.2 & 3.4 & 24.0 & -8.0 & -5.9 & 2.2 & 4.7 & -7.1 & -2.4 & 3.1 & 4.2 & -5.9 & 3.8 & -0.65 & -1.30 & 1405 & \begin{tabular}{|l|}
0.445 \\
\end{tabular} \\
\hline 66 & 1.8 & -41. & $\overline{3.5}$ & 24.2 & -8.0 & -6.0 & $\overline{2.2}$ & 4.7 & -7.1 & -2.6 & 3.1 & $\overline{4.2}$ & -5.9 & 3.9 & -0.65 & -1.31 & 1422 & 0.444 \\
\hline
\end{tabular}


Table E.4 KL 52-34 Test Data $\left(0^{\circ} \mathrm{C}\right.$ Tests), continued

\begin{tabular}{|c|c|c|c|c|c|c|c|c|c|c|c|c|c|c|c|c|c|c|}
\hline & \multicolumn{12}{|c|}{ Normalized Vertical and Horizontal Deformation Arrays } & \multirow{2}{*}{\multicolumn{2}{|c|}{ Trimmed }} & & \multirow{3}{*}{\multicolumn{2}{|c|}{\begin{tabular}{|l|} 
Creep \\
Comp
\end{tabular}}} & \multirow{3}{*}{$\begin{array}{l}\text { Pois. } \\
\text { Ratio }\end{array}$} \\
\hline & \multicolumn{4}{|c|}{ KL 52-34 Sample \#2 } & \multicolumn{4}{|c|}{ KL 52-34 Sample \#5 } & \multicolumn{4}{|c|}{ KL 52-34 Sample \#7 } & & & \multirow[b]{2}{*}{ Ratio } & & & \\
\hline & vert & vert & horz & horz & vert & vert & horz & horz & vert & vert & horz & horz & & & & & & \\
\hline Time & front & back & front & back & front & back & front & back & front & back & front & back & vert & horz & $\overline{X / Y}$ & $\mathrm{C}_{\mathrm{emp}}$ & $\mathrm{D}(\mathrm{t})$ & \\
\hline $\mathrm{sec}$ & $\mu \mathrm{m}$ & $\mu \mathrm{m}$ & $\mu \mathrm{m}$ & $\mu \mathrm{m}$ & $\mu \mathrm{m}$ & $\mu \mathrm{m}$ & $\mu \mathrm{m}$ & $\mu \mathrm{m}$ & $\mu \mathrm{m}$ & $\mu \mathrm{m}$ & $\mu \mathrm{m}$ & $\mu \mathrm{m}$ & $\mu \mathrm{m}$ & $\mu \mathrm{m}$ & & & $1 / \mathrm{kPa}$ & \\
\hline 67 & 1.2 & -42.1 & 3.4 & 24.3 & -8.0 & -6.0 & 2.2 & 4.7 & -7.6 & -2.6 & 3.1 & 4.1 & -6.0 & 3.9 & -0.64 & -1.33 & \begin{tabular}{|c|}
1439 \\
\end{tabular} & 0.421 \\
\hline 68 & 1.8 & -42.1 & 3.5 & 24.7 & $\begin{array}{l}-8.1 \\
\end{array}$ & -6.1 & 2.2 & 4.8 & -7.4 & -2.6 & 3.1 & 4.3 & -6.0 & 4.0 & -0.65 & -1.30 & \begin{tabular}{|l|l}
1448 \\
\end{tabular} & 0.446 \\
\hline 69 & 1.6 & -42.4 & 3.6 & 24.8 & $\begin{array}{l}-8.1 \\
\end{array}$ & -6.1 & 2.2 & 4.8 & -7.4 & -2.6 & 3.2 & 4.3 & -6.0 & 4.0 & -0.66 & -1.30 & 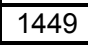 & 0.450 \\
\hline 70 & 1.8 & -43.0 & 3.6 & 25.1 & $\begin{array}{l}-8.1 \\
\end{array}$ & -6.2 & 2.2 & 4.8 & -7.9 & -2.9 & 3.2 & 4.2 & $\begin{array}{l}-6.3 \\
\end{array}$ & 4.0 & -0.63 & -1.34 & \begin{tabular}{|l}
1492 \\
\end{tabular} & 0.410 \\
\hline 71 & 1.0 & -43.7 & 3.6 & 25.2 & $\begin{array}{l}-8.2 \\
\end{array}$ & -6.3 & 2.2 & 4.9 & -7.7 & -2.7 & 3.2 & 4.4 & $\begin{array}{l}-6.2 \\
\end{array}$ & 4.0 & -0.64 & -1.32 & \begin{tabular}{|l|}
1490 \\
\end{tabular} & 0.426 \\
\hline 72 & 1.6 & -43.6 & 3.7 & 25.4 & $\begin{array}{l}-8.2 \\
\end{array}$ & -6.2 & 2.2 & 4.9 & -7.9 & $\begin{array}{l}-2.6 \\
\end{array}$ & 3.2 & 4.4 & $\begin{array}{l}-6.2 \\
\end{array}$ & $\begin{array}{l}4.1 \\
\end{array}$ & -0.65 & $\begin{array}{l}-1.31 \\
\end{array}$ & \begin{tabular}{|l|}
1494 \\
\end{tabular} & 0.444 \\
\hline 73 & 1.5 & -44.0 & 3.7 & 25.6 & \begin{tabular}{|l|}
-8.3 \\
\end{tabular} & -6.3 & 2.3 & . & -7.9 & -2.9 & 3.2 & 4.4 & $\begin{array}{l}-6.4 \\
\end{array}$ & 4.1 & -0.64 & -1.32 & \begin{tabular}{|l|}
1515 \\
\end{tabular} & 0.425 \\
\hline 74 & 1.6 & -44.3 & 3.7 & 25.9 & \begin{tabular}{|l|l|}
-8.4 \\
\end{tabular} & -6.4 & 2.3 & 5.0 & -8.0 & $\begin{array}{l}-2.9 \\
\end{array}$ & 3.3 & 4.4 & $\begin{array}{l}-6.4 \\
\end{array}$ & $\begin{array}{l}4.1 \\
\end{array}$ & -0.64 & $\begin{array}{l}-1.32 \\
\end{array}$ & 1529 & 0.424 \\
\hline 75 & 1.3 & -44.4 & 3.8 & 26.0 & $\begin{array}{l}-8.4 \\
\end{array}$ & -6.4 & 2.3 & 5.0 & -8.5 & $\begin{array}{l}-3.0 \\
\end{array}$ & 3.3 & 4.4 & $\begin{array}{l}-6.6 \\
\end{array}$ & 4.1 & -0.63 & -1.34 & \begin{tabular}{|c|}
1561 \\
\end{tabular} & 0.406 \\
\hline 76 & 1.5 & -44.9 & 3.8 & 26.2 & $\begin{array}{l}-8.5 \\
\end{array}$ & -6.4 & 2.3 & 5.1 & -8.2 & -3.2 & 3.4 & 4.6 & -6.6 & 4.2 & -0.64 & -1.32 & 1565 & 0.423 \\
\hline 77 & 0.9 & -45.9 & 3.8 & 26.3 & \begin{tabular}{|l|}
-8.5 \\
\end{tabular} & -6.5 & 2.3 & 5.1 & -8.3 & -3.0 & 3.4 & 4.5 & -6.6 & 4.2 & -0.64 & -1.33 & \begin{tabular}{|l}
1566 \\
\end{tabular} & 0.421 \\
\hline 78 & 1.5 & -45.6 & 3.9 & 26.7 & -8.5 & -6.5 & 2.3 & 5.2 & -8.8 & -3.6 & 3.3 & 4.4 & $\begin{array}{l}-6.9 \\
\end{array}$ & 4.2 & -0.61 & -1.37 & \begin{tabular}{|l|}
1619 \\
\end{tabular} & 0.374 \\
\hline 79 & 1.3 & -46.1 & 3.9 & 26.8 & $\begin{array}{l}-8.5 \\
\end{array}$ & -6.6 & 2.3 & 5.2 & -8.3 & $\begin{array}{l}-3.3 \\
\end{array}$ & $\begin{array}{l}3.4 \\
\end{array}$ & 4.6 & $\begin{array}{l}-6.7 \\
\end{array}$ & 4.3 & -0.64 & $\begin{array}{l}-1.33 \\
\end{array}$ & 1594 & 0.422 \\
\hline 80 & 1.3 & -46.3 & 4.0 & 27.0 & $\begin{array}{l}-8.6 \\
\end{array}$ & -6.6 & 2.4 & 5.2 & -8.5 & $\begin{array}{l}-3.2 \\
\end{array}$ & 3.4 & 4.6 & $\begin{array}{l}-6.7 \\
\end{array}$ & 4.3 & -0.64 & -1.32 & \begin{tabular}{|l|}
1600 \\
\end{tabular} & 0.427 \\
\hline 81 & 1. & -46.8 & 4.0 & 27.2 & $\begin{array}{l}-8.7 \\
\end{array}$ & -6.6 & 2.4 & 5. & -8.5 & \begin{tabular}{|l|}
-3.3 \\
\end{tabular} & 3.5 & 4.6 & $\begin{array}{l}-6.8 \\
\end{array}$ & 4.3 & -0.64 & -1.33 & \begin{tabular}{|l|}
1616 \\
\end{tabular} & 0.419 \\
\hline 82 & 1. & -47.1 & 4.0 & 27.4 & $\begin{array}{l}-8.7 \\
\end{array}$ & -6.7 & 2.4 & 5. & -9.0 & \begin{tabular}{|l|}
-3.5 \\
\end{tabular} & 3.5 & 4.7 & -7.0 & 4.4 & -0.63 & -1.34 & \begin{tabular}{|l|}
1650 \\
\end{tabular} & 0.405 \\
\hline 83 & 1.2 & -47.5 & 4.0 & 27.5 & $\begin{array}{l}-8.8 \\
\end{array}$ & -6.7 & 2.4 & 5. & -9.0 & $\begin{array}{l}-3.3 \\
\end{array}$ & 3.4 & 4. & -6.9 & 4. & -0.63 & -1.34 & 1644 & 0.403 \\
\hline 84 & 0. & -48.1 & 4.0 & 27.7 & $\begin{array}{l}-8.8 \\
\end{array}$ & -6.7 & 2.4 & 5.4 & -8.7 & $\begin{array}{l}-3.3 \\
\end{array}$ & 3.5 & 4.1 & -6.9 & 4.4 & -0.64 & -1.32 & \begin{tabular}{|l|l}
1638 \\
\end{tabular} & 0.424 \\
\hline 85 & 0. & -48.1 & 4.1 & 27.9 & $\begin{array}{l}-8.8 \\
\end{array}$ & -6.8 & 2.4 & 5.4 & \begin{tabular}{|l|}
-9.0 \\
\end{tabular} & $\begin{array}{l}-3.2 \\
\end{array}$ & 3.5 & 4. & -6.9 & 4.4 & -0.63 & -1.33 & \begin{tabular}{|l|}
1646 \\
\end{tabular} & 0.413 \\
\hline 86 & 0.9 & -48.7 & 4.2 & 28.0 & -8.8 & -6.8 & 2.4 & 5. & -9.0 & -3.5 & 3.5 & 4.7 & -7.0 & 4.4 & -0.63 & -1.33 & \begin{tabular}{|l|}
1667 \\
\end{tabular} & 0.414 \\
\hline 87 & 0.9 & -48.8 & 4.2 & 28.2 & $\begin{array}{l}-8.9 \\
\end{array}$ & -6.8 & 2.4 & 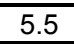 & -9.1 & $\begin{array}{l}-3.5 \\
\end{array}$ & 3.5 & 4. & -7. & $\overline{4}$ & -0.64 & -1.33 & \begin{tabular}{|l|}
1682 \\
\end{tabular} & 0.415 \\
\hline 88 & 0.9 & -49.1 & 4.3 & 28.4 & \begin{tabular}{|c|}
-9.0 \\
\end{tabular} & -6.8 & 2.5 & 5.5 & -9.1 & $\begin{array}{l}-3.6 \\
\end{array}$ & 3.6 & 4.6 & \begin{tabular}{l|l}
-7.1 \\
\end{tabular} & 4.5 & -0.63 & -1.34 & \begin{tabular}{|l|}
1696 \\
\end{tabular} & 0.407 \\
\hline 89 & 0.9 & -49.7 & 4.3 & 28.7 & -9.0 & -6.9 & 2.5 & 5. & -9.1 & -3.6 & 3.5 & 4.7 & -7.1 & 4.5 & -0.64 & -1.33 & 1701 & 0.416 \\
\hline 90 & 0.9 & -49.9 & 4.3 & 28.8 & -9.0 & -6.9 & 2.5 & 5. & -9.4 & -3.6 & 3.6 & 4. & $\begin{array}{l}-7.2 \\
\end{array}$ & 4.6 & -0.63 & -1.34 & \begin{tabular}{|l|}
1721 \\
\end{tabular} & 0.407 \\
\hline 91 & 0. & -50.2 & 4.4 & 29.0 & $\begin{array}{l}-9.0 \\
\end{array}$ & -7.0 & 2.5 & 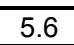 & -9.3 & -3.5 & 3.6 & 5.6 & -7.2 & 4.6 & -0.65 & -1.32 & \begin{tabular}{|l|}
1718 \\
\end{tabular} & 0.433 \\
\hline 92 & 0.6 & -50.8 & 4.4 & 29.2 & \begin{tabular}{|l|}
-9.0 \\
\end{tabular} & $\begin{array}{l}-7.0 \\
\end{array}$ & 2.5 & 5.7 & -9.3 & $\begin{array}{l}-3.5 \\
\end{array}$ & 3.6 & 4.9 & $\begin{array}{l}-7.2 \\
\end{array}$ & $\begin{array}{l}4.7 \\
\end{array}$ & -0.65 & $\begin{array}{l}-1.32 \\
\end{array}$ & \begin{tabular}{|l|}
1721 \\
\end{tabular} & 0.432 \\
\hline 93 & 0. & -50.9 & 4.4 & 29.3 & $\begin{array}{l}-9.1 \\
\end{array}$ & -7.0 & 2. & 5. & -9.3 & $\begin{array}{l}-3.6 \\
\end{array}$ & 3.7 & 4. & $\begin{array}{l}-7.3 \\
\end{array}$ & 1.7 & -0.64 & -1.32 & \begin{tabular}{|l}
1732 \\
\end{tabular} & 0.428 \\
\hline 94 & 0.7 & -51.3 & 4.5 & 29.5 & -9.1 & -7.0 & 2.5 & $\overline{5 . \varepsilon}$ & -9.3 & -3.6 & 3.7 & 4.9 & -7.2 & 4.7 & -0.65 & -1.31 & \begin{tabular}{|l|}
1732 \\
\end{tabular} & 0.438 \\
\hline 95 & 0.6 & -51.6 & 4.5 & 29.8 & $\begin{array}{l}-9.1 \\
\end{array}$ & -7.1 & 2.5 & 5.8 & -9.4 & -3.6 & 3.7 & 4. & $\begin{array}{l}-7.3 \\
\end{array}$ & 4.7 & -0.65 & -1.31 & \begin{tabular}{|l|l|}
1749 \\
\end{tabular} & 0.437 \\
\hline 96 & 0. & -51.9 & 4.5 & 29.9 & $\begin{array}{l}-9.1 \\
\end{array}$ & -7.1 & 2.6 & 3. & -9.4 & -3.6 & 3.8 & $\begin{array}{ll}4 . \\
\end{array}$ & $\begin{array}{l}-7.3 \\
\end{array}$ & & -0.65 & -1.31 & \begin{tabular}{|l|l}
1750 \\
\end{tabular} & 0.441 \\
\hline 97 & 0.4 & -52.4 & 4.5 & 30.1 & $\begin{array}{l}-9.2 \\
\end{array}$ & \begin{tabular}{|l|}
-7.1 \\
\end{tabular} & 2.6 & 5.0 & -9.6 & $\begin{array}{l}-3.6 \\
\end{array}$ & 3.7 & 5. & $\begin{array}{l}-7.4 \\
\end{array}$ & 4.8 & -0.66 & $\begin{array}{l}-1.30 \\
\end{array}$ & \begin{tabular}{|l|}
1766 \\
\end{tabular} & 0.448 \\
\hline 98 & 0.3 & -52.7 & 4.6 & 30.2 & $\begin{array}{l}-9.1 \\
\end{array}$ & -7.1 & 2.6 & 5. & -9.6 & $\begin{array}{l}-3.6 \\
\end{array}$ & 3.8 & 5. & $\begin{array}{l}-7.4 \\
\end{array}$ & 4.8 & -0.66 & -1.30 & \begin{tabular}{|l|l}
1765 \\
\end{tabular} & 0.455 \\
\hline 99 & 0.4 & -52.8 & 4.7 & 30.4 & -9.2 & -7.1 & 2.6 & 6. & -9.6 & -3 & 3.8 & 5. & -7.3 & 4.9 & -0.67 & $\begin{array}{l}-1.27 \\
\end{array}$ & \begin{tabular}{|l|l|}
1761 \\
\end{tabular} & 0.481 \\
\hline 100 & 0.3 & -53.1 & 4.7 & 30.5 & -9.2 & -7.1 & 2.6 & 6.0 & -9.6 & -3.5 & 3.9 & 5.2 & $\begin{array}{l}-7.4 \\
\end{array}$ & 4.9 & -0.67 & -1.28 & \begin{tabular}{|l|}
1774 \\
\end{tabular} & 0.47 \\
\hline
\end{tabular}


Table E.5 KL 52-34 Test Data $\left(-10{ }^{\circ} \mathrm{C}\right.$ Tests)

\begin{tabular}{|c|c|c|c|c|c|c|c|c|c|c|c|c|c|c|c|c|c|c|}
\hline & \multicolumn{12}{|c|}{ Normalized Vertical and Horizontal Deformation Arrays } & \multirow{2}{*}{\multicolumn{2}{|c|}{ Trimmed }} & \multirow[b]{3}{*}{ Ratio } & & \multirow{3}{*}{$\begin{array}{l}\text { Creep } \\
\text { Comp }\end{array}$} & \multirow{3}{*}{$\begin{array}{l}\text { Pois. } \\
\text { Ratio }\end{array}$} \\
\hline & \multicolumn{4}{|c|}{ KL 52-34 Sample \#2 } & \multicolumn{4}{|c|}{ KL 52-34 Sample \#5 } & \multicolumn{4}{|c|}{ KL 52-34 Sample \#7 } & & & & & & \\
\hline & vert & vert & horz & horz & vert & vert & horz & horz & vert & vert & horz & horz & & & & & & \\
\hline Time & front & back & front & back & front & back & front & back & front & back & front & back & vert & horz & $\mathrm{X} / \mathrm{Y}$ & $\mathrm{C}_{\mathrm{empl}}$ & $\overline{D(t)}$ & $\bar{v}$ \\
\hline $\mathrm{sec}$ & $\mu \mathrm{m}$ & $\mu \mathrm{m}$ & $\mu \mathrm{m}$ & $\mu \mathrm{m}$ & $\mu \mathrm{m}$ & $\mu \mathrm{m}$ & $\mu \mathrm{m}$ & $\mu \mathrm{m}$ & $\mu \mathrm{m}$ & $\mu \mathrm{m}$ & $\mu \mathrm{m}$ & $\mu \mathrm{m}$ & $\mu \mathrm{m}$ & $\mu \mathrm{m}$ & & & $1 / \mathrm{kPa}$ & \\
\hline 0 & 0.0 & 0.0 & 0.0 & 0.0 & 0.0 & 0.0 & 0.0 & 0.0 & $\mathrm{~N} / \mathrm{A}$ & $\mathrm{N} / \mathrm{A}$ & $\mathrm{N} / \mathrm{A}$ & $\mathrm{N} / \mathrm{A}$ & 0.0 & 0.0 & 0.00 & 0.00 & 0 & 0.000 \\
\hline 1 & 1.4 & -7.6 & -0.1 & 3.4 & -1.7 & -1.5 & 0.4 & 1.0 & $\mathrm{~N} / \mathrm{A}$ & $\mathrm{N} / \mathrm{A}$ & $\mathrm{N} / \mathrm{A}$ & $\mathrm{N} / \mathrm{A}$ & -1.6 & 0.4 & -0.28 & -2.64 & 110 & 0.000 \\
\hline 2 & 1.6 & -8.8 & 0.0 & 4.2 & \begin{tabular}{|l}
-1.9 \\
\end{tabular} & -1.7 & 0.5 & 1.1 & $\mathrm{~N} / \mathrm{A}$ & $\mathrm{N} / \mathrm{A}$ & $\mathrm{N} / \mathrm{A}$ & $\mathrm{N} / \mathrm{A}$ & -1.8 & 0.5 & -0.30 & -2.48 & 127 & 0.012 \\
\hline 3 & 1.6 & -9.9 & 0.0 & 4.7 & -2.1 & -1.9 & 0.5 & 1.2 & $\mathrm{~N} / \mathrm{A}$ & $\mathrm{N} / \mathrm{A}$ & $\mathrm{N} / \mathrm{A}$ & $\mathrm{N} / \mathrm{A}$ & -2.0 & 0.6 & -0.29 & -2.51 & 138 & 0.009 \\
\hline 4 & 1.6 & -10.6 & -0.1 & 5.1 & -2.1 & -2.0 & 0.6 & 1.3 & $\mathrm{~N} / \mathrm{A}$ & $\mathrm{N} / \mathrm{A}$ & $\mathrm{N} / \mathrm{A}$ & $\mathrm{N} / \mathrm{A}$ & -2.0 & 0.6 & -0.30 & -2.44 & 141 & 0.016 \\
\hline 5 & 1.7 & -11.4 & 0.0 & 5.6 & -2.1 & -2.0 & 0.6 & 1.3 & N/A & $\mathrm{N} / \mathrm{A}$ & $\mathrm{N} / \mathrm{A}$ & $\mathrm{N} / \mathrm{A}$ & -2.1 & 0.7 & -0.33 & -2.24 & 147 & 0.041 \\
\hline 6 & 1.9 & -11.9 & 0.0 & 5.9 & -2.2 & -2.1 & 0.6 & 1.4 & $\mathrm{~N} / \mathrm{A}$ & $\mathrm{N} / \mathrm{A}$ & $\mathrm{N} / \mathrm{A}$ & $\mathrm{N} / \mathrm{A}$ & -2.1 & 0.7 & -0.33 & -2.28 & 151 & 0.036 \\
\hline 7 & 1.9 & -12.5 & 0.0 & 6.2 & -2.3 & -2.2 & 0.6 & 1.4 & $\mathrm{~N} / \mathrm{A}$ & $\mathrm{N} / \mathrm{A}$ & $\mathrm{N} / \mathrm{A}$ & $\mathrm{N} / \mathrm{A}$ & -2.2 & 0.7 & -0.33 & -2.25 & 158 & 0.040 \\
\hline 8 & 2.0 & -13.1 & 0.1 & 6.5 & -2.5 & -2.4 & 0.7 & 1.5 & $\mathrm{~N} / \mathrm{A}$ & $\mathrm{N} / \mathrm{A}$ & $\mathrm{N} / \mathrm{A}$ & $\mathrm{N} / \mathrm{A}$ & -2.4 & 0.8 & -0.31 & -2.38 & 170 & 0.023 \\
\hline 9 & 2.2 & -13.5 & $\overline{0.1}$ & 6.8 & -2.5 & -2.4 & 0.7 & 1.5 & $\mathrm{~N} / \mathrm{A}$ & $\mathrm{N} / \mathrm{A}$ & $\mathrm{N} / \mathrm{A}$ & $\mathrm{N} / \mathrm{A}$ & -2.5 & 0.8 & -0.33 & -2.28 & 173 & 0.037 \\
\hline 10 & 2.2 & -14.0 & 0.1 & 6.9 & -2.4 & -2.4 & 0.7 & 1.5 & $\mathrm{~N} / \mathrm{A}$ & $\mathrm{N} / \mathrm{A}$ & $\mathrm{N} / \mathrm{A}$ & N/A & -2.4 & 0.8 & -0.35 & -2.16 & 168 & 0.054 \\
\hline 11 & 2.0 & -14.4 & 0.0 & 7.2 & -2.4 & -2.3 & 0.7 & 1.6 & N/A & $\mathrm{N} / \mathrm{A}$ & $\mathrm{N} / \mathrm{A}$ & $\mathrm{N} / \mathrm{A}$ & -2.3 & 0.8 & -0.35 & -2.17 & 167 & 0.053 \\
\hline 12 & 2.2 & -14.7 & 0.1 & 7.4 & -2.5 & -2.4 & 0.7 & 1.6 & $\mathrm{~N} / \mathrm{A}$ & $\mathrm{N} / \mathrm{A}$ & $\mathrm{N} / \mathrm{A}$ & $\mathrm{N} / \mathrm{A}$ & -2.4 & 0.9 & -0.36 & -2.08 & 173 & 0.068 \\
\hline 13 & 2.0 & -15.2 & 0.2 & 7.6 & -2.5 & -2.4 & 0.7 & 1.7 & $\mathrm{~N} / \mathrm{A}$ & $\mathrm{N} / \mathrm{A}$ & $\mathrm{N} / \mathrm{A}$ & $\mathrm{N} / \mathrm{A}$ & -2.5 & 0.9 & -0.37 & -2.06 & 178 & 0.072 \\
\hline 14 & 2.0 & -15.4 & 0.1 & 7.8 & -2.5 & -2.3 & 0.7 & 1.7 & $\mathrm{~N} / \mathrm{A}$ & $\mathrm{N} / \mathrm{A}$ & $\mathrm{N} / \mathrm{A}$ & N/A & -2.4 & 0.9 & -0.37 & -2.05 & 175 & 0.075 \\
\hline 15 & 2.0 & -15.8 & 0.2 & 8.0 & -2.5 & -2.5 & 0.8 & 1.7 & $\mathrm{~N} / \mathrm{A}$ & $\mathrm{N} / \mathrm{A}$ & $\mathrm{N} / \mathrm{A}$ & $\mathrm{N} / \mathrm{A}$ & -2.5 & 0.9 & -0.38 & -2.02 & 180 & 0.080 \\
\hline 16 & 2.2 & -16.0 & 0.3 & 8.2 & -2.5 & -2.5 & 0.8 & 1.8 & $\mathrm{~N} / \mathrm{A}$ & $\mathrm{N} / \mathrm{A}$ & $\mathrm{N} / \mathrm{A}$ & $\mathrm{N} / \mathrm{A}$ & -2.5 & 1.0 & -0.40 & -1.91 & 182 & 0.108 \\
\hline 17 & 2.4 & -16.4 & 0.3 & 8.5 & -2.6 & -2.5 & 0.8 & 1.8 & $\mathrm{~N} / \mathrm{A}$ & $\mathrm{N} / \mathrm{A}$ & $\mathrm{N} / \mathrm{A}$ & $\mathrm{N} / \mathrm{A}$ & -2.5 & 1.0 & -0.40 & -1.90 & 184 & 0.109 \\
\hline 18 & 2.4 & -16.4 & 0.3 & 8.7 & -2.6 & -2.5 & 0.8 & 1.8 & $\mathrm{~N} / \mathrm{A}$ & $\mathrm{N} / \mathrm{A}$ & $\mathrm{N} / \mathrm{A}$ & $\mathrm{N} / \mathrm{A}$ & -2.6 & 1.0 & -0.40 & -1.92 & 188 & 0.104 \\
\hline 19 & 2.7 & -16.5 & 0.3 & 8.9 & -2.5 & -2.5 & 0.8 & 1.9 & $\mathrm{~N} / \mathrm{A}$ & $\mathrm{N} / \mathrm{A}$ & $\mathrm{N} / \mathrm{A}$ & $\mathrm{N} / \mathrm{A}$ & -2.5 & 1.1 & -0.44 & -1.79 & 186 & 0.142 \\
\hline 20 & 2.6 & -16.8 & 0.3 & 9.2 & -2.6 & -2.6 & 0.8 & 1.9 & $\mathrm{~N} / \mathrm{A}$ & $\mathrm{N} / \mathrm{A}$ & $\mathrm{N} / \mathrm{A}$ & $\mathrm{N} / \mathrm{A}$ & -2.6 & 1.1 & -0.43 & -1.82 & 191 & 0.134 \\
\hline 21 & 2.7 & -17.0 & 0.4 & 9.3 & -2.7 & -2.6 & 0.8 & 1.9 & $\mathrm{~N} / \mathrm{A}$ & $\mathrm{N} / \mathrm{A}$ & $\mathrm{N} / \mathrm{A}$ & $\mathrm{N} / \mathrm{A}$ & -2.6 & 1.1 & -0.43 & -1.81 & 196 & 0.137 \\
\hline 22 & 2.7 & -17.3 & 0.4 & 9.4 & -2.6 & -2.6 & 0.8 & 1.9 & $\mathrm{~N} / \mathrm{A}$ & $\mathrm{N} / \mathrm{A}$ & $\mathrm{N} / \mathrm{A}$ & $\mathrm{N} / \mathrm{A}$ & -2.6 & 1.2 & -0.44 & -1.77 & 195 & 0.149 \\
\hline 23 & 2.9 & -17.1 & 0.4 & 9.7 & -2.6 & -2.6 & 0.9 & 1.9 & $\mathrm{~N} / \mathrm{A}$ & $\mathrm{N} / \mathrm{A}$ & $\mathrm{N} / \mathrm{A}$ & N/A & -2.6 & 1.2 & -0.45 & -1.76 & 195 & 0.154 \\
\hline 24 & 3.3 & -17.6 & 0.4 & 9.8 & -2.6 & -2.6 & 0.9 & 2.0 & N/A & $\mathrm{N} / \mathrm{A}$ & $\mathrm{N} / \mathrm{A}$ & $\mathrm{N} / \mathrm{A}$ & -2.6 & 1.2 & -0.46 & -1.72 & 196 & 0.167 \\
\hline 25 & 3.2 & -17.8 & 0.4 & 10.0 & -2.7 & -2.6 & 0.9 & 2.0 & $\mathrm{~N} / \mathrm{A}$ & $\mathrm{N} / \mathrm{A}$ & $\mathrm{N} / \mathrm{A}$ & N/A & -2.6 & 1.2 & -0.45 & -1.73 & 198 & 0.163 \\
\hline 26 & 3.5 & -17.8 & 0.5 & 10.1 & -2.7 & -2.8 & 0.9 & 2.0 & $\mathrm{~N} / \mathrm{A}$ & $\mathrm{N} / \mathrm{A}$ & $\mathrm{N} / \mathrm{A}$ & $\mathrm{N} / \mathrm{A}$ & -2.7 & 1.2 & -0.45 & -1.74 & 204 & 0.162 \\
\hline 27 & 3.3 & -18.1 & 0.5 & 10.3 & -2.6 & -2.7 & 0.9 & 2.0 & N/A & $\mathrm{N} / \mathrm{A}$ & $\mathrm{N} / \mathrm{A}$ & N/A & -2.7 & 1.3 & -0.48 & -1.66 & 201 & 0.191 \\
\hline 28 & 3.5 & -18.1 & 0.5 & 10.4 & -2.7 & -2.7 & 0.9 & 2.1 & $\mathrm{~N} / \mathrm{A}$ & $\mathrm{N} / \mathrm{A}$ & $\mathrm{N} / \mathrm{A}$ & $\mathrm{N} / \mathrm{A}$ & -2.7 & 1.3 & -0.49 & -1.63 & 202 & 0.204 \\
\hline 29 & 3.6 & -18.6 & 0.6 & 10.6 & -2.7 & -2.7 & 0.9 & 2.1 & $\mathrm{~N} / \mathrm{A}$ & $\mathrm{N} / \mathrm{A}$ & $\mathrm{N} / \mathrm{A}$ & $\mathrm{N} / \mathrm{A}$ & -2.7 & 1.3 & -0.49 & -1.62 & 204 & 0.210 \\
\hline 30 & 3.5 & -18.7 & 0.6 & 10.8 & -2.7 & -2.7 & 0.9 & 2.1 & $\mathrm{~N} / \mathrm{A}$ & $\mathrm{N} / \mathrm{A}$ & $\mathrm{N} / \mathrm{A}$ & $\mathrm{N} / \mathrm{A}$ & -2.7 & 1.3 & -0.50 & -1.61 & 205 & 0.218 \\
\hline 31 & 3.6 & -18.8 & 0.6 & 11.0 & -2.7 & -2.7 & 0.9 & 2.1 & $\mathrm{~N} / \mathrm{A}$ & $\mathrm{N} / \mathrm{A}$ & $\mathrm{N} / \mathrm{A}$ & $\mathrm{N} / \mathrm{A}$ & -2.7 & 1.4 & -0.51 & -1.59 & 205 & 0.228 \\
\hline 32 & 3.9 & -18.8 & 0.6 & 11.0 & -2.7 & -2.7 & 0.9 & 2.1 & N/A & $\mathrm{N} / \mathrm{A}$ & $\mathrm{N} / \mathrm{A}$ & $\mathrm{N} / \mathrm{A}$ & -2.7 & 1.4 & -0.50 & -1.59 & 207 & 0.225 \\
\hline 33 & 3.7 & -19.3 & 0.6 & 11.2 & -2.7 & -2.8 & 1.0 & 2.2 & $\mathrm{~N} / \mathrm{A}$ & $\mathrm{N} / \mathrm{A}$ & $\mathrm{N} / \mathrm{A}$ & N/A & -2.7 & 1.4 & -0.52 & -1.57 & 208 & 0.239 \\
\hline
\end{tabular}


Table E.5 KL 52-34 Test Data $\left(-10{ }^{\circ} \mathrm{C}\right.$ Tests), continued

\begin{tabular}{|c|c|c|c|c|c|c|c|c|c|c|c|c|c|c|c|c|c|c|}
\hline & & & Norm & & & & & etor & atio & rra) & & & & & & & & \\
\hline & & $52-34$ & Sample & & & $52-34$ & sample & & & $52-34$ & ample & & Trim & ned & & & Creep & Pois. \\
\hline & $\begin{array}{l}\text { vert } \\
\end{array}$ & vert & horz & horz & vert & vert & horz & horz & vert & \begin{tabular}{l|l|} 
vert \\
\end{tabular} & horz & horz & & & Ratio & & Comp & \begin{tabular}{|l|} 
Ratio \\
\end{tabular} \\
\hline Time & front & back & front & back & front & back & front & back & front & back & front & back & vert & horz & $\mathrm{X} / \mathrm{Y}$ & $\mathrm{C}_{\mathrm{empl}}$ & $\mathrm{D}(\mathrm{t})$ & \\
\hline $\mathrm{sec}$ & $\mu \mathrm{m}$ & $\mu \mathrm{m}$ & $\mu \mathrm{m}$ & $\mu \mathrm{m}$ & $\mu \mathrm{m}$ & $\mu \mathrm{m}$ & $\mu \mathrm{m}$ & $\mu \mathrm{m}$ & $\mu \mathrm{m}$ & $\mu \mathrm{m}$ & $\mu \mathrm{m}$ & $\mu \mathrm{m}$ & $\mu \mathrm{m}$ & $\mu \mathrm{m}$ & & & $1 / \mathrm{kPa}$ & \\
\hline 34 & 3.6 & -19.6 & 0.7 & 11.4 & -2.7 & -2.8 & 1.0 & 2.2 & $\mathrm{~N} / \mathrm{A}$ & $\mathrm{N} / \mathrm{A}$ & $\mathrm{N} / \mathrm{A}$ & $\mathrm{N} / \mathrm{A}$ & -2.7 & 1.4 & -0.52 & -1.55 & 210 & 0.247 \\
\hline 35 & 3.6 & -19.6 & 0.7 & \begin{tabular}{|l|l}
11.5 \\
\end{tabular} & $\begin{array}{l}-2.7 \\
\end{array}$ & $\begin{array}{l}-2.8 \\
\end{array}$ & 1.0 & 2.2 & $\mathrm{~N} / \mathrm{A}$ & $\mathrm{N} / \mathrm{A}$ & $\mathrm{N} / \mathrm{A}$ & N/A & -2.7 & 1.5 & -0.54 & -1.51 & 210 & 0.270 \\
\hline 36 & $\begin{array}{l}3.7 \\
\end{array}$ & -19.7 & 0.7 & \begin{tabular}{|l|l|}
11.6 \\
\end{tabular} & $\begin{array}{l}-2.7 \\
\end{array}$ & $\begin{array}{l}-2.8 \\
\end{array}$ & 1.0 & 2.2 & $\mathrm{~N} / \mathrm{A}$ & N/A & $\mathrm{N} / \mathrm{A}$ & N/A & $\begin{array}{l}-2.7 \\
\end{array}$ & $\begin{array}{l}1.5 \\
\end{array}$ & \begin{tabular}{|l|}
-0.54 \\
\end{tabular} & -1.51 & 212 & \begin{tabular}{|l|}
0.274 \\
\end{tabular} \\
\hline 37 & 3.6 & -20.0 & 0.8 & \begin{tabular}{|l|l|}
11.8 \\
\end{tabular} & -2.8 & -2.8 & 1.0 & 2.3 & $\mathrm{~N} / \mathrm{A}$ & $\mathrm{N} / \mathrm{A}$ & $\mathrm{N} / \mathrm{A}$ & $\mathrm{N} / \mathrm{A}$ & $\begin{array}{l}-2.8 \\
\end{array}$ & 1.5 & \begin{tabular}{|l|}
-0.54 \\
\end{tabular} & -1.50 & 215 & \begin{tabular}{|l|}
0.279 \\
\end{tabular} \\
\hline 38 & 3.6 & -20.4 & 0.7 & \begin{tabular}{|l|}
11.9 \\
\end{tabular} & $\begin{array}{l}-2.7 \\
\end{array}$ & -2.8 & 1.0 & 2.3 & $\mathrm{~N} / \mathrm{A}$ & $\mathrm{N} / \mathrm{A}$ & $\mathrm{N} / \mathrm{A}$ & $\mathrm{N} / \mathrm{A}$ & $\begin{array}{l}-2.7 \\
\end{array}$ & $\begin{array}{l}1.5 \\
\end{array}$ & \begin{tabular}{|l|}
-0.56 \\
\end{tabular} & -1.48 & 211 & \begin{tabular}{|l|}
0.293 \\
\end{tabular} \\
\hline 39 & $\begin{array}{l}3.6 \\
\end{array}$ & -20.6 & 0.8 & \begin{tabular}{|l|l}
12.0 \\
\end{tabular} & $\begin{array}{l}-2.7 \\
\end{array}$ & $\begin{array}{l}-2.8 \\
\end{array}$ & 1.0 & 2.3 & $\mathrm{~N} / \mathrm{A}$ & $\mathrm{N} / \mathrm{A}$ & $\mathrm{N} / \mathrm{A}$ & $\mathrm{N} / \mathrm{A}$ & -2.8 & 1.5 & \begin{tabular}{|l|}
-0.55 \\
\end{tabular} & -1.48 & 214 & \begin{tabular}{|l|}
0.291 \\
\end{tabular} \\
\hline 40 & 3.6 & -20.9 & 0.8 & 12.1 & $\begin{array}{l}-2.7 \\
\end{array}$ & -2.8 & 1.0 & 2.3 & $\mathrm{~N} / \mathrm{A}$ & $\mathrm{N} / 1$ & N/A & $\mathrm{N} / \mathrm{A}$ & $\begin{array}{l}-2.8 \\
\end{array}$ & 1.5 & \begin{tabular}{|l|}
-0.56 \\
\end{tabular} & -1.47 & 215 & \begin{tabular}{|l|}
0.297 \\
\end{tabular} \\
\hline 41 & 3.6 & -21.1 & 0.8 & 12.1 & -2.7 & $\begin{array}{l}-2.8 \\
\end{array}$ & 1.0 & 2.4 & $\mathrm{~N} / \mathrm{A}$ & N/A & $\mathrm{N} / \mathrm{A}$ & $\mathrm{N} / \mathrm{A}$ & $\begin{array}{l}-2.8 \\
\end{array}$ & $\begin{array}{ll}1.6 \\
\end{array}$ & \begin{tabular}{|c|c|}
-0.58 \\
\end{tabular} & -1.43 & 216 & \begin{tabular}{|l|}
0.324 \\
\end{tabular} \\
\hline 42 & 3.6 & -21.1 & 0.8 & \begin{tabular}{|l|l}
12.3 \\
\end{tabular} & -2.7 & -2.8 & 1.0 & 2.4 & $\mathrm{~N} / \mathrm{A}$ & $\mathrm{N} / \mathrm{A}$ & $\mathrm{N} / \mathrm{A}$ & $\mathrm{N} / \mathrm{A}$ & -2.7 & $\begin{array}{l}1.6 \\
\end{array}$ & \begin{tabular}{|l|}
-0.59 \\
\end{tabular} & -1.42 & 214 & \begin{tabular}{|l|}
0.339 \\
\end{tabular} \\
\hline 43 & 3.5 & -21.4 & 0.8 & \begin{tabular}{|l|}
12.4 \\
\end{tabular} & -2.8 & -2.8 & 1.0 & 2.4 & $\mathrm{~N} / \mathrm{A}$ & $\mathrm{N} / \mathrm{A}$ & $\mathrm{N} / \mathrm{A}$ & $\mathrm{N} / \mathrm{A}$ & -2.8 & 1.6 & \begin{tabular}{|l|}
-0.56 \\
\end{tabular} & -1.46 & 219 & \begin{tabular}{|l|}
0.307 \\
\end{tabular} \\
\hline 44 & 3.5 & -21.9 & 0.8 & 12.5 & -2.8 & -2.8 & 1.0 & 2.4 & $\mathrm{~N} / \mathrm{A}$ & $\mathrm{N} / t$ & $\mathrm{~N} / \mathrm{A}$ & $\mathrm{N} / \mathrm{A}$ & $\begin{array}{l}-2.8 \\
\end{array}$ & 1.6 & \begin{tabular}{|l|}
-0.57 \\
\end{tabular} & -1.45 & 219 & 0.310 \\
\hline 45 & 3.3 & -22.2 & 0.8 & \begin{tabular}{|l|}
12.6 \\
\end{tabular} & -2.8 & -2.9 & 1.1 & 2.4 & $\mathrm{~N} / \mathrm{A}$ & $\mathrm{N} / \mathrm{A}$ & $\mathrm{N} / \mathrm{A}$ & N/A & -2.9 & $\begin{array}{l}1.6 \\
\end{array}$ & -0.57 & -1.45 & 224 & 0.309 \\
\hline 46 & 3.5 & -22.3 & 0.8 & 12.7 & $\begin{array}{l}-2.8 \\
\end{array}$ & $\begin{array}{l}-2.9 \\
\end{array}$ & 1.1 & 2.4 & $\mathrm{~N} / \mathrm{A}$ & N/A & $\mathrm{N} / \mathrm{A}$ & $\mathrm{N} / \mathrm{A}$ & $\begin{array}{l}-2.8 \\
\end{array}$ & 1.6 & -0.58 & -1.43 & 222 & 0.327 \\
\hline 47 & 3.3 & -22.6 & 0.8 & \begin{tabular}{|l|}
12.8 \\
\end{tabular} & $\begin{array}{l}-2.7 \\
\end{array}$ & -2.9 & 1.1 & 2.5 & $\mathrm{~N} / \mathrm{A}$ & $\mathrm{N} / \mathrm{A}$ & $\mathrm{N} / \mathrm{A}$ & $\mathrm{N} / \mathrm{A}$ & $\begin{array}{l}-2.8 \\
\end{array}$ & $\begin{array}{l}1.6 \\
\end{array}$ & \begin{tabular}{|l}
-0.59 \\
\end{tabular} & -1.41 & 221 & \begin{tabular}{|l|l}
0.340 \\
\end{tabular} \\
\hline 48 & 3.2 & -22.6 & 0.8 & 12.8 & -2.8 & -2.9 & 1.1 & 2.5 & $\mathrm{~N} / \mathrm{A}$ & $\mathrm{N} / \mathrm{A}$ & $\mathrm{N} / \mathrm{A}$ & N/A & -2.9 & 1.6 & -0.57 & -1.45 & 226 & 0.315 \\
\hline 49 & 3.2 & -23.0 & 0.9 & \begin{tabular}{|l|}
12.9 \\
\end{tabular} & $\begin{array}{l}-2.8 \\
\end{array}$ & $\begin{array}{l}-2.9 \\
\end{array}$ & 1.1 & 2.5 & $\mathrm{~N} / \mathrm{A}$ & N/A & $\mathrm{N} / \mathrm{A}$ & N/A & $\begin{array}{l}-2.9 \\
\end{array}$ & $\begin{array}{l}1.7 \\
\end{array}$ & \begin{tabular}{|l|}
-0.58 \\
\end{tabular} & $\begin{array}{l}-1.43 \\
\end{array}$ & 227 & \begin{tabular}{|l|}
0.330 \\
\end{tabular} \\
\hline$\overline{50}$ & 3.2 & -23.2 & 0.9 & \begin{tabular}{|l|l}
13.1 \\
\end{tabular} & -2.8 & $\begin{array}{l}-3.0 \\
\end{array}$ & 1.1 & 2.5 & $N / A$ & $\mathrm{~N} / \mathrm{A}$ & $\mathrm{N} / \mathrm{A}$ & $\mathrm{N} / \mathrm{A}$ & -2.9 & $\begin{array}{l}1.7 \\
\end{array}$ & \begin{tabular}{|c|c|}
-0.58 \\
\end{tabular} & -1.43 & \begin{tabular}{|l|}
229 \\
\end{tabular} & \begin{tabular}{|l|}
0.331 \\
\end{tabular} \\
\hline 51 & 2.9 & -23.5 & 0.9 & \begin{tabular}{|l|}
13.2 \\
\end{tabular} & -2.8 & -3.0 & 1.1 & 2.5 & $\mathrm{~N} / \mathrm{A}$ & $\mathrm{N} / \mathrm{A}$ & $\mathrm{N} / \mathrm{A}$ & $\mathrm{N} / \mathrm{A}$ & -2.9 & 1.7 & \begin{tabular}{|l|}
-0.59 \\
\end{tabular} & -1.40 & 230 & \begin{tabular}{|l|}
0.349 \\
\end{tabular} \\
\hline 52 & $\begin{array}{l}2.7 \\
\end{array}$ & -23.5 & 0.9 & \begin{tabular}{|l|l}
13.3 \\
\end{tabular} & \begin{tabular}{l|}
-2.8 \\
\end{tabular} & $\begin{array}{l}-3.0 \\
\end{array}$ & 1.1 & 2.5 & $\mathrm{~N} / \mathrm{A}$ & $\mathrm{N} / \mathrm{A}$ & $\mathrm{N} / \mathrm{A}$ & N/A & -2.9 & $\begin{array}{l}1.7 \\
\end{array}$ & \begin{tabular}{|l|}
-0.59 \\
\end{tabular} & -1.40 & 230 & \begin{tabular}{|l|}
0.352 \\
\end{tabular} \\
\hline 53 & 2.7 & -23.7 & 1.0 & 13.3 & -2.8 & -2.9 & 1.1 & 2.6 & $\mathrm{~N} / \mathrm{A}$ & $\mathrm{N} / \mathrm{A}$ & $\mathrm{N} / \mathrm{A}$ & $\mathrm{N} / \mathrm{A}$ & -2.8 & 1.8 & \begin{tabular}{|c|}
-0.62 \\
\end{tabular} & -1.36 & 225 & \begin{tabular}{|l|}
0.391 \\
\end{tabular} \\
\hline 54 & 2.9 & -23.7 & 1.0 & \begin{tabular}{|l|l|}
13.4 \\
\end{tabular} & $\begin{array}{l}-2.9 \\
\end{array}$ & $\begin{array}{l}-3.1 \\
\end{array}$ & 1.1 & 2.6 & $\mathrm{~N} / \mathrm{A}$ & N/A & $\mathrm{N} / \mathrm{A}$ & N/A & $\begin{array}{l}-3.0 \\
\end{array}$ & $\begin{array}{l}1.8 \\
\end{array}$ & \begin{tabular}{|c|}
-0.60 \\
\end{tabular} & -1.40 & 236 & \begin{tabular}{|l|}
0.356 \\
\end{tabular} \\
\hline 55 & 2.7 & -24.0 & 1.0 & 13.5 & -2.9 & -3.0 & 1.1 & 2.6 & $\mathrm{~N} / \mathrm{A}$ & $\mathrm{N} / \mathrm{A}$ & $\mathrm{N} / \mathrm{A}$ & $\mathrm{N} / \mathrm{A}$ & -3.0 & $\begin{array}{l}1.8 \\
\end{array}$ & \begin{tabular}{|l|}
-0.60 \\
\end{tabular} & -1.39 & \begin{tabular}{|l|}
234 \\
\end{tabular} & \begin{tabular}{|l|}
0.361 \\
\end{tabular} \\
\hline 56 & 3.0 & -24.0 & 1.0 & \begin{tabular}{|l|l|}
13.7 \\
\end{tabular} & -2.8 & $\begin{array}{l}-3.1 \\
\end{array}$ & 1.1 & 2.6 & $\mathrm{~N} / \mathrm{A}$ & $\mathrm{N} / \mathrm{A}$ & $\mathrm{N} / \mathrm{A}$ & $\mathrm{N} / \mathrm{A}$ & $\begin{array}{l}-3.0 \\
\end{array}$ & $\begin{array}{l}1.8 \\
\end{array}$ & \begin{tabular}{|l|}
-0.61 \\
\end{tabular} & -1.38 & \begin{tabular}{|l|}
235 \\
\end{tabular} & \begin{tabular}{|l|}
0.371 \\
\end{tabular} \\
\hline 57 & 2.7 & -24.6 & 1.1 & \begin{tabular}{|l|l|}
13.7 \\
\end{tabular} & -2.8 & -3.1 & 1.1 & 2.6 & $N / A$ & $\mathrm{~N} / \mathrm{A}$ & $\mathrm{N} / \mathrm{A}$ & $\mathrm{N} / \mathrm{A}$ & $\begin{array}{l}-3.0 \\
\end{array}$ & $\begin{array}{l}1.8 \\
\end{array}$ & \begin{tabular}{|l}
-0.62 \\
\end{tabular} & -1.36 & \begin{tabular}{l|}
236 \\
\end{tabular} & \begin{tabular}{|l|}
0.391 \\
\end{tabular} \\
\hline$\overline{58}$ & 2.7 & -24.9 & 1.0 & \begin{tabular}{|l|l}
13.8 \\
\end{tabular} & $\begin{array}{l}-2.9 \\
\end{array}$ & $\begin{array}{l}-3.1 \\
\end{array}$ & 1.1 & 2.6 & $\mathrm{~N} / \mathrm{A}$ & $\mathrm{N} / /$ & $\mathrm{N} / \mathrm{A}$ & N/A & $\begin{array}{l}-3.0 \\
\end{array}$ & $\begin{array}{l}1.8 \\
\end{array}$ & -0.61 & -1.37 & 237 & 0.379 \\
\hline 59 & 2.7 & -25.0 & 1.1 & \begin{tabular}{|l|l}
13.8 \\
\end{tabular} & $\begin{array}{l}-3.1 \\
\end{array}$ & -3.2 & 1.1 & 2.6 & $N / A$ & $\mathrm{~N} / \mathrm{A}$ & $\mathrm{N} / \mathrm{A}$ & $\mathrm{N} / \mathrm{A}$ & $\begin{array}{l}-3.1 \\
\end{array}$ & $\begin{array}{l}1.8 \\
\end{array}$ & \begin{tabular}{|l|}
-0.59 \\
\end{tabular} & -1.41 & 245 & \begin{tabular}{|l|}
0.346 \\
\end{tabular} \\
\hline 60 & 2.6 & -25.2 & 1.1 & \begin{tabular}{|l|l}
14.0 \\
\end{tabular} & -2.9 & -3.1 & 1.1 & 2.6 & $\mathrm{~N} / \mathrm{A}$ & $\mathrm{N} / \mathrm{A}$ & $\mathrm{N} / \mathrm{A}$ & $\mathrm{N} / \mathrm{A}$ & -3.0 & 1.9 & \begin{tabular}{|l|}
-0.61 \\
\end{tabular} & -1.37 & 241 & \begin{tabular}{|l|}
0.380 \\
\end{tabular} \\
\hline 61 & 2.9 & -25.2 & 1.1 & \begin{tabular}{|l|}
14.1 \\
\end{tabular} & -3.0 & -3.2 & 1.1 & 2.6 & $\mathrm{~N} / \mathrm{A}$ & $\mathrm{N} / \mathrm{t}$ & $\mathrm{N} / \mathrm{A}$ & $\mathrm{N} / \mathrm{A}$ & -3.1 & 1.9 & -0.61 & -1.37 & 244 & 0.378 \\
\hline 62 & 2.6 & -25.5 & 1.1 & \begin{tabular}{|l|l|}
14.3 \\
\end{tabular} & -3.0 & -3.1 & 1.1 & 2.6 & $\mathrm{~N} / \mathrm{A}$ & $\mathrm{N} / \mathrm{A}$ & $\mathrm{N} / \mathrm{A}$ & N/A & -3.0 & 1.9 & \begin{tabular}{|l|}
-0.62 \\
\end{tabular} & -1.35 & 243 & \begin{tabular}{|l|}
0.393 \\
\end{tabular} \\
\hline 63 & 2.7 & -25.8 & 1.2 & \begin{tabular}{|l|l|}
14.4 \\
\end{tabular} & -3.0 & $\begin{array}{l}-3.1 \\
\end{array}$ & 1.1 & 2.7 & $\mathrm{~N} / \mathrm{A}$ & $\mathrm{N} / \mathrm{A}$ & N/A & N/A & $\begin{array}{l}-3.0 \\
\end{array}$ & 1.9 & -0.63 & -1.35 & 244 & 0.400 \\
\hline 64 & 2.6 & -25.6 & 1.2 & 14.5 & -3.0 & -3.1 & 1.2 & 2.7 & $\mathrm{~N} / \mathrm{A}$ & $\mathrm{N} / \mathrm{A}$ & $\mathrm{N} / \mathrm{A}$ & $\mathrm{N} / \mathrm{A}$ & -3.0 & 1.9 & -0.63 & -1.33 & 242 & 0.413 \\
\hline 65 & 2.6 & -26.0 & 1.2 & \begin{tabular}{|l|}
14.5 \\
\end{tabular} & -3.0 & -3.2 & 1.2 & 2.7 & $\mathrm{~N} / \mathrm{A}$ & $\mathrm{N} / \mathrm{A}$ & $\mathrm{N} / \mathrm{A}$ & $\mathrm{N} / \mathrm{A}$ & -3.1 & 1.9 & \begin{tabular}{|l|}
-0.63 \\
\end{tabular} & -1.34 & 246 & \begin{tabular}{|l|}
0.408 \\
\end{tabular} \\
\hline 66 & 2.4 & -26.2 & 1.2 & 14.6 & -3.0 & -3.2 & 1.2 & 2.7 & $\mathrm{~N} / \mathrm{A}$ & $\mathrm{N} / \mathrm{A}$ & $\mathrm{N} / \mathrm{A}$ & $\mathrm{N} / \mathrm{A}$ & -3.1 & 1.9 & \begin{tabular}{|l|}
-0.63 \\
\end{tabular} & -1.34 & 247 & 0.404 \\
\hline
\end{tabular}


Table E.5 KL 52-34 Test Data $\left(-10{ }^{\circ} \mathrm{C}\right.$ Tests $)$, continued

\begin{tabular}{|c|c|c|c|c|c|c|c|c|c|c|c|c|c|c|c|c|c|c|}
\hline & \multicolumn{12}{|c|}{ Normalized Vertical and Horizontal Deformation Arrays } & \multirow{2}{*}{\multicolumn{2}{|c|}{ Trimmed }} & \multirow[b]{3}{*}{ Ratio } & & \multirow{3}{*}{\begin{tabular}{|l|} 
Creep \\
Comp \\
\end{tabular}} & \multirow{3}{*}{$\begin{array}{l}\text { Pois. } \\
\text { Ratio }\end{array}$} \\
\hline & \multicolumn{4}{|c|}{ KL 52-34 Sample \#2 } & \multicolumn{4}{|c|}{ KL 52-34 Sample \#5 } & \multicolumn{4}{|c|}{ KL 52-34 Sample \#7 } & & & & & & \\
\hline & vert & vert & horz & horz & vert & vert & horz & horz & vert & vert & horz & horz & & & & & & \\
\hline Time & front & back & front & back & front & back & front & back & front & back & front & back & vert & horz & $\mathrm{X} / \mathrm{Y}$ & $\mathrm{C}_{\mathrm{empl}}$ & $\mathrm{D}(\mathrm{t})$ & $\bar{v}$ \\
\hline $\mathrm{sec}$ & $\mu \mathrm{m}$ & $\mu \mathrm{m}$ & $\overline{\mu m}$ & $\mu \mathrm{m}$ & $\mu \mathrm{m}$ & $\mu \mathrm{m}$ & $\mu \mathrm{m}$ & $\mu \mathrm{m}$ & $\mu \mathrm{m}$ & $\mu \mathrm{m}$ & $\mu \mathrm{m}$ & $\mu \mathrm{m}$ & $\mu \mathrm{m}$ & $\mu \mathrm{m}$ & & & $1 / \mathrm{kPa}$ & \\
\hline 67 & 2.6 & -26.3 & $\overline{1.2}$ & 14.8 & -3.0 & -3.2 & $\overline{1.2}$ & 2.7 & $\mathrm{~N} / \mathrm{A}$ & $\mathrm{N} / \mathrm{A}$ & $\mathrm{N} / \mathrm{A}$ & $\mathrm{N} / \mathrm{A}$ & -3.1 & 2.0 & -0.64 & -1.33 & 246 & 0.420 \\
\hline 68 & 2.9 & -26.3 & 1.2 & 14.8 & -3.0 & -3.3 & 1.2 & 2.7 & $\mathrm{~N} / \mathrm{A}$ & $\mathrm{N} / \mathrm{A}$ & N/A & $\mathrm{N} / \mathrm{A}$ & -3.2 & 2.0 & -0.63 & $\begin{array}{l}-1.34 \\
\end{array}$ & 252 & 0.402 \\
\hline 69 & 2.6 & -26.6 & 1.2 & 14.9 & -3.0 & -3.2 & 1.2 & 2.7 & $\mathrm{~N} / \mathrm{A}$ & $\mathrm{N} / \mathrm{A}$ & $\mathrm{N} / \mathrm{A}$ & $\mathrm{N} / \mathrm{A}$ & -3.1 & 2.0 & \begin{tabular}{|l|}
-0.63 \\
\end{tabular} & -1.34 & 249 & 0.410 \\
\hline 70 & 2.6 & -26.8 & 1.2 & 14.9 & -3.0 & -3.3 & 1.2 & 2.7 & $\mathrm{~N} / \mathrm{A}$ & $\mathrm{N} / \mathrm{A}$ & $\mathrm{N} / \mathrm{A}$ & $\mathrm{N} / \mathrm{A}$ & -3.2 & 2.0 & \begin{tabular}{|l|}
-0.63 \\
\end{tabular} & -1.34 & 252 & 0.403 \\
\hline 71 & 2.7 & -26.8 & 1.3 & 15.0 & -3.1 & -3.3 & 1.2 & 2.8 & $\mathrm{~N} / \mathrm{A}$ & $\mathrm{N} / \mathrm{A}$ & $\mathrm{N} / \mathrm{A}$ & $\mathrm{N} / \mathrm{A}$ & -3.2 & 2.0 & \begin{tabular}{|l|}
-0.63 \\
\end{tabular} & -1.34 & 255 & 0.411 \\
\hline 72 & 2.9 & -26.9 & 1.3 & 15.1 & -3.0 & -3.3 & 1.2 & 2.8 & $\mathrm{~N} / \mathrm{A}$ & $\mathrm{N} / \mathrm{A}$ & N/A & $\mathrm{N} / \mathrm{A}$ & -3.2 & 2.0 & -0.64 & -1.32 & 254 & 0.430 \\
\hline 73 & 2.7 & -27.0 & 1.3 & 15.2 & -3.1 & $\begin{array}{l}-3.3 \\
\end{array}$ & 1.2 & 2.8 & $\mathrm{~N} / \mathrm{A}$ & $\mathrm{N} / \mathrm{A}$ & $\mathrm{N} / \mathrm{A}$ & $\mathrm{N} / \mathrm{A}$ & -3.2 & 2.0 & -0.64 & -1.32 & 256 & 0.426 \\
\hline 74 & 2.6 & -27.0 & 1.3 & 15.4 & -3.1 & -3.3 & 1.2 & 2.8 & $\mathrm{~N} / \mathrm{A}$ & $\mathrm{N} / \mathrm{A}$ & $\mathrm{N} / \mathrm{A}$ & $\mathrm{N} / \mathrm{A}$ & -3.2 & 2.0 & -0.64 & $\begin{array}{l}-1.33 \\
\end{array}$ & 257 & 0.422 \\
\hline 75 & 2.6 & -27.5 & 1.3 & 15.4 & -3.1 & -3.3 & 1.2 & 2.8 & $\mathrm{~N} / \mathrm{A}$ & $\mathrm{N} / \mathrm{A}$ & $\mathrm{N} / \mathrm{A}$ & $\mathrm{N} / \mathrm{A}$ & -3.2 & 2.1 & -0.65 & -1.32 & 258 & 0.432 \\
\hline 76 & 2.4 & -27.5 & 1.3 & 15.5 & -3.2 & -3.3 & 1.2 & 2.8 & $\mathrm{~N} / \mathrm{A}$ & $\mathrm{N} / \mathrm{A}$ & $\mathrm{N} / \mathrm{A}$ & $\mathrm{N} / \mathrm{A}$ & -3.2 & 2.1 & -0.64 & -1.32 & 261 & 0.427 \\
\hline 77 & 2.6 & -27.6 & 1.4 & 15.5 & -3.2 & -3.5 & 1.2 & 2.8 & $\mathrm{~N} / \mathrm{A}$ & $\mathrm{N} / \mathrm{A}$ & $\mathrm{N} / \mathrm{A}$ & $\mathrm{N} / \mathrm{A}$ & -3.3 & 2.1 & -0.63 & -1.33 & 265 & 0.413 \\
\hline 78 & 2.7 & -28.1 & 1.3 & 15.6 & -3.2 & -3.4 & 1.2 & 2.8 & $\mathrm{~N} / \mathrm{A}$ & $\mathrm{N} / \mathrm{A}$ & $\mathrm{N} / \mathrm{A}$ & $\mathrm{N} / \mathrm{A}$ & -3.3 & 2.1 & -0.64 & -1.33 & 262 & 0.422 \\
\hline 79 & 2.7 & -27.9 & 1.3 & 15.8 & -3.2 & -3.4 & 1.2 & 2.9 & $\mathrm{~N} / \mathrm{A}$ & $\mathrm{N} / \mathrm{A}$ & $\mathrm{N} / \mathrm{A}$ & $\mathrm{N} / \mathrm{A}$ & -3.3 & 2.1 & -0.64 & -1.32 & 263 & 0.430 \\
\hline 80 & 2.6 & -28.2 & 1.4 & 15.8 & -3.2 & -3.4 & 1.2 & 2.8 & $\mathrm{~N} / \mathrm{A}$ & $\mathrm{N} / \mathrm{A}$ & $\mathrm{N} / \mathrm{A}$ & $\mathrm{N} / \mathrm{A}$ & -3.3 & 2.1 & -0.64 & -1.32 & 266 & 0.423 \\
\hline 81 & 2.6 & -28.3 & 1.4 & 15.9 & -3.2 & -3.4 & 1.3 & 2.9 & $\mathrm{~N} / \mathrm{A}$ & $\mathrm{N} / \mathrm{A}$ & $\mathrm{N} / \mathrm{A}$ & $\mathrm{N} / \mathrm{A}$ & -3.3 & 2.1 & -0.65 & $\begin{array}{l}-1.30 \\
\end{array}$ & 264 & 0.447 \\
\hline 82 & 2.7 & -28.5 & 1.5 & 15.9 & -3.2 & -3.5 & 1.3 & 2.9 & $\mathrm{~N} / \mathrm{A}$ & $\mathrm{N} / \mathrm{A}$ & $\mathrm{N} / \mathrm{A}$ & $\mathrm{N} / \mathrm{A}$ & -3.3 & 2.2 & -0.65 & -1.31 & 270 & 0.437 \\
\hline 83 & 2.4 & -28.3 & 1.5 & 16.1 & -3.2 & -3.4 & 1.3 & 2.9 & $\mathrm{~N} / \mathrm{A}$ & $\mathrm{N} / \mathrm{A}$ & $\mathrm{N} / \mathrm{A}$ & $\mathrm{N} / \mathrm{A}$ & -3.3 & 2.2 & -0.66 & -1.29 & 268 & 0.460 \\
\hline 84 & 2.7 & -28.8 & 1.5 & 16.3 & -3.1 & -3.3 & 1.3 & 2.9 & $\mathrm{~N} / \mathrm{A}$ & $\mathrm{N} / \mathrm{A}$ & $\mathrm{N} / \mathrm{A}$ & $\mathrm{N} / \mathrm{A}$ & -3.2 & 2.2 & -0.70 & -1.24 & 261 & 0.523 \\
\hline 85 & 2.7 & -28.9 & 1.5 & 16.4 & -3.2 & -3.4 & 1.3 & 2.9 & $\mathrm{~N} / \mathrm{A}$ & $\mathrm{N} / \mathrm{A}$ & $\mathrm{N} / \mathrm{A}$ & $\mathrm{N} / \mathrm{A}$ & -3.3 & 2.2 & -0.68 & -1.26 & 267 & 0.492 \\
\hline 86 & 2.9 & -28.8 & 1.5 & 16.4 & -3.2 & -3.5 & 1.3 & 2.9 & $\mathrm{~N} / \mathrm{A}$ & $\mathrm{N} / \mathrm{A}$ & $\mathrm{N} / \mathrm{A}$ & $\mathrm{N} / \mathrm{A}$ & -3.3 & 2.2 & -0.66 & -1.29 & 272 & 0.462 \\
\hline 87 & 2.7 & -29.3 & 1.5 & 16.5 & -3.2 & -3.5 & 1.3 & 3.0 & $\mathrm{~N} / \mathrm{A}$ & $\mathrm{N} / \mathrm{A}$ & $\mathrm{N} / \mathrm{A}$ & $\mathrm{N} / \mathrm{A}$ & -3.3 & 2.2 & -0.66 & -1.29 & 272 & 0.461 \\
\hline 88 & 3.0 & -29.2 & 1.5 & 16.7 & -3.2 & -3.5 & 1.3 & 3.0 & $\mathrm{~N} / \mathrm{A}$ & $\mathrm{N} / \mathrm{A}$ & $\mathrm{N} / \mathrm{A}$ & $\mathrm{N} / \mathrm{A}$ & -3.3 & 2.3 & -0.67 & -1.28 & 273 & 0.476 \\
\hline 89 & 3.0 & -29.1 & 1.6 & 16.7 & -3.2 & -3.5 & 1.3 & 3.0 & $\mathrm{~N} / \mathrm{A}$ & $\mathrm{N} / \mathrm{A}$ & $\mathrm{N} / \mathrm{A}$ & $\mathrm{N} / \mathrm{A}$ & -3.4 & 2.3 & -0.68 & -1.27 & 275 & 0.486 \\
\hline 90 & 2.9 & -29.3 & 1.6 & 16.9 & -3.2 & -3.5 & 1.3 & 3.0 & $\mathrm{~N} / \mathrm{A}$ & $\mathrm{N} / \mathrm{A}$ & $\mathrm{N} / \mathrm{A}$ & $\mathrm{N} / \mathrm{A}$ & -3.4 & 2.3 & -0.68 & -1.27 & 276 & 0.491 \\
\hline 91 & 3.0 & -29.2 & 1.7 & 16.9 & -3.2 & -3.5 & 1.3 & 3.0 & $\mathrm{~N} / \mathrm{A}$ & $\mathrm{N} / \mathrm{A}$ & $\mathrm{N} / \mathrm{A}$ & $\mathrm{N} / \mathrm{A}$ & -3.4 & 2.3 & -0.69 & -1.25 & 277 & 0.508 \\
\hline 92 & 3.0 & -29.2 & 1.7 & 17.1 & -3.2 & -3.5 & 1.3 & 3.0 & $\mathrm{~N} / \mathrm{A}$ & $\mathrm{N} / \mathrm{A}$ & $\mathrm{N} / \mathrm{A}$ & $\mathrm{N} / \mathrm{A}$ & -3.4 & 2.4 & -0.70 & -1.25 & 279 & 0.518 \\
\hline 93 & 3.0 & -29.6 & 1.7 & 17.1 & -3.3 & -3.5 & 1.3 & 3.0 & $\mathrm{~N} / \mathrm{A}$ & $\mathrm{N} / \mathrm{A}$ & $\mathrm{N} / \mathrm{A}$ & $\mathrm{N} / \mathrm{A}$ & -3.4 & 2.4 & -0.69 & -1.25 & 280 & 0.507 \\
\hline 94 & 3.0 & -29.6 & 1.7 & 17.2 & -3.3 & -3.6 & 1.3 & 3.0 & $\mathrm{~N} / \mathrm{A}$ & $\mathrm{N} / \mathrm{A}$ & $\mathrm{N} / \mathrm{A}$ & $\mathrm{N} / \mathrm{A}$ & -3.5 & 2.4 & -0.69 & -1.26 & 283 & 0.502 \\
\hline 95 & 3.0 & -29.6 & 1.7 & 17.3 & -3.3 & -3.5 & 1.3 & 3.1 & $\mathrm{~N} / \mathrm{A}$ & $\mathrm{N} / \mathrm{A}$ & $\mathrm{N} / \mathrm{A}$ & $\mathrm{N} / \mathrm{A}$ & -3.4 & 2.4 & -0.70 & -1.24 & 281 & 0.525 \\
\hline 96 & 3.0 & -29.9 & 1.7 & 17.3 & -3.3 & -3.5 & 1.3 & 3.0 & $\mathrm{~N} / \mathrm{A}$ & $\mathrm{N} / \mathrm{A}$ & $\mathrm{N} / \mathrm{A}$ & $\mathrm{N} / \mathrm{A}$ & -3.4 & 2.4 & -0.70 & -1.24 & 281 & 0.522 \\
\hline 97 & 3.2 & -29.9 & 1.8 & 17.5 & -3.3 & -3.6 & 1.3 & 3.1 & N/A & $\mathrm{N} / \mathrm{A}$ & $\mathrm{N} / \mathrm{A}$ & $\mathrm{N} / \mathrm{A}$ & -3.5 & 2.4 & -0.70 & -1.24 & 285 & 0.526 \\
\hline 98 & 3.0 & -29.9 & 1.8 & 17.5 & -3.3 & -3.6 & 1.3 & 3.1 & $\mathrm{~N} / \mathrm{A}$ & $\mathrm{N} / \mathrm{A}$ & $\mathrm{N} / \mathrm{A}$ & $\mathrm{N} / \mathrm{A}$ & -3.5 & 2.4 & -0.70 & -1.24 & 286 & 0.518 \\
\hline 99 & 3.5 & -29.9 & 1.8 & 17.6 & -3.3 & -3.6 & 1.3 & 3.1 & $\mathrm{~N} / \mathrm{A}$ & $\mathrm{N} / \mathrm{A}$ & $\mathrm{N} / \mathrm{A}$ & $\mathrm{N} / \mathrm{A}$ & -3.5 & 2.5 & -0.71 & -1.23 & 286 & 0.546 \\
\hline 100 & 3.2 & -30.2 & 1.9 & 17.6 & -3.3 & -3.6 & 1.4 & 3.1 & $\mathrm{~N} / \mathrm{A}$ & $\mathrm{N} / \mathrm{A}$ & $\mathrm{N} / \mathrm{A}$ & $\mathrm{N} / \mathrm{A}$ & -3.4 & 2.5 & -0.72 & -1.21 & 285 & 0.562 \\
\hline
\end{tabular}


Table E.6 KL 52-34 Test Data $\left(-20{ }^{\circ} \mathrm{C}\right.$ Tests)

\begin{tabular}{|c|c|c|c|c|c|c|c|c|c|c|c|c|c|c|c|c|c|c|}
\hline & \multicolumn{12}{|c|}{ Normalized Vertical and Horizontal Deformation Arrays } & \multirow{2}{*}{\multicolumn{2}{|c|}{ Trimmed }} & \multirow[b]{3}{*}{ Ratio } & & \multirow{3}{*}{\begin{tabular}{|l|} 
Creep \\
Comp
\end{tabular}} & \multirow{3}{*}{$\begin{array}{l}\text { Pois. } \\
\text { Ratio }\end{array}$} \\
\hline & \multicolumn{4}{|c|}{ KL 52-34 Sample \#2 } & \multicolumn{4}{|c|}{ KL 52-34 Sample \#5 } & \multicolumn{4}{|c|}{ KL 52-34 Sample \#7 } & & & & & & \\
\hline & vert & vert & horz & horz & vert & vert & horz & horz & vert & vert & horz & horz & & & & & & \\
\hline Time & front & back & front & back & front & back & front & back & front & back & front & back & vert & horz & $\mathrm{X} / \mathrm{Y}$ & $\mathrm{C}$ empl $_{1}$ & $\mathrm{D}(\mathrm{t})$ & $v$ \\
\hline sec & $\mu \mathrm{m}$ & $\mu \mathrm{m}$ & $\mu \mathrm{m}$ & $\mu \mathrm{m}$ & $\mu \mathrm{m}$ & $\mu \mathrm{m}$ & $\mu \mathrm{m}$ & $\mu \mathrm{m}$ & $\mu \mathrm{m}$ & $\mu \mathrm{m}$ & $\mu \mathrm{m}$ & $\mu \mathrm{m}$ & $\mu \mathrm{m}$ & $\mu \mathrm{m}$ & & & $1 / \mathrm{kPa}$ & \\
\hline$\overline{0}$ & 0.0 & 0.0 & 0.0 & 0.0 & 0.0 & 0.0 & 0.0 & 0.0 & 0.0 & 0.0 & 0.0 & 0.0 & 0.0 & 0.0 & 0.00 & 0.00 & $\overline{0}$ & 0.000 \\
\hline 1 & 1.7 & -8.3 & 0.0 & 3.4 & -2.4 & -2.1 & 0.6 & 1.3 & -2.1 & -2.5 & 0.5 & 1.1 & -2.3 & 0.9 & -0.40 & -1.94 & 79 & 0.100 \\
\hline 2 & 1.9 & $\begin{array}{l}-9.0 \\
\end{array}$ & 0.0 & 3.9 & $\begin{array}{l}-2.7 \\
\end{array}$ & $\begin{array}{l}-2.4 \\
\end{array}$ & 0.7 & 1.4 & $\begin{array}{l}-2.5 \\
\end{array}$ & $\begin{array}{l}-2.8 \\
\end{array}$ & 0.6 & 1.3 & $\begin{array}{l}-2.6 \\
\end{array}$ & 1.0 & \begin{tabular}{|c|}
-0.39 \\
\end{tabular} & \begin{tabular}{|l|}
-1.96 \\
\end{tabular} & 89 & \begin{tabular}{|l|l|}
0.094 \\
\end{tabular} \\
\hline 3 & 1.9 & -9.7 & -0.1 & 4.3 & -2.9 & -2.6 & 0.7 & 1.5 & -2.6 & -2.9 & 0.7 & 1.4 & -2.7 & 1.1 & \begin{tabular}{|c|c|} 
\\
\end{tabular} & -1.95 & 95 & \begin{tabular}{|l|}
0.097 \\
\end{tabular} \\
\hline 4 & 2.0 & -10.1 & 0.0 & 4.5 & $\begin{array}{l}-3.1 \\
\end{array}$ & $\begin{array}{l}-3.0 \\
\end{array}$ & 0.7 & 1.5 & -2.8 & $\begin{array}{l}-3.0 \\
\end{array}$ & 0.7 & 1.5 & $\begin{array}{l}-3.0 \\
\end{array}$ & $\begin{array}{l}1.1 \\
\end{array}$ & -0.37 & -2.05 & 102 & 0.074 \\
\hline 5 & 2.1 & -10.6 & 0.0 & 4.7 & -3.1 & -2.8 & 0.8 & 1.6 & -2.8 & -3.0 & 0.7 & 1.5 & -2.9 & $\begin{array}{l}1.1 \\
\end{array}$ & -0.39 & -1.95 & 101 & \begin{tabular}{|l|}
0.096 \\
\end{tabular} \\
\hline 6 & 2.1 & -10.9 & $\overline{0.0}$ & 4.9 & -3.2 & $\begin{array}{l}-3.0 \\
\end{array}$ & 0.8 & 1.6 & -2.9 & $\begin{array}{l}-3.1 \\
\end{array}$ & 0.8 & 1.6 & $\begin{array}{l}-3.0 \\
\end{array}$ & 1.2 & -0.38 & -1.98 & 105 & 0.089 \\
\hline 7 & 2.3 & -11.2 & 0.0 & 5.1 & \begin{tabular}{|l|}
-3.3 \\
\end{tabular} & $\begin{array}{l}-3.1 \\
\end{array}$ & 0.8 & 1.6 & $\begin{array}{l}-2.9 \\
\end{array}$ & $\begin{array}{l}-3.2 \\
\end{array}$ & 0.8 & 1.6 & $\begin{array}{l}-3.1 \\
\end{array}$ & 1.2 & \begin{tabular}{|c|}
-0.38 \\
\end{tabular} & \begin{tabular}{|c|}
-2.00 \\
\end{tabular} & 108 & 0.085 \\
\hline 8 & 2.2 & -11.5 & -0.1 & 5.2 & -3.3 & -3.0 & 0.8 & 1.7 & -2.9 & -3.1 & 0.8 & 1.6 & -3.1 & 1.2 & -0.40 & -1.92 & 107 & 0.105 \\
\hline 9 & 2.3 & -11.7 & 0.0 & 5.3 & -3.5 & -3.1 & 0.8 & 1.7 & -3.0 & -3.2 & 0.8 & 1.6 & -3.2 & 1.2 & -0.38 & -1.99 & 111 & \begin{tabular}{|l|}
0.088 \\
\end{tabular} \\
\hline 10 & 2.3 & -11.8 & -0.1 & 5.5 & $\begin{array}{l}-3.5 \\
\end{array}$ & $\begin{array}{l}-3.2 \\
\end{array}$ & 0.8 & 1.7 & $\begin{array}{l}-3.0 \\
\end{array}$ & $\begin{array}{l}-3.2 \\
\end{array}$ & 0.8 & 1.7 & -3.2 & 1.3 & \begin{tabular}{|c|}
-0.39 \\
\end{tabular} & \begin{tabular}{c|}
-1.97 \\
\end{tabular} & 112 & 0.092 \\
\hline 11 & 2.5 & -11.9 & 0.0 & 5.6 & $\begin{array}{l}-3.6 \\
\end{array}$ & $\begin{array}{l}-3.2 \\
\end{array}$ & 0.8 & 1.7 & $\begin{array}{l}-3.1 \\
\end{array}$ & $\begin{array}{l}-3.4 \\
\end{array}$ & 0.8 & 1.7 & $\begin{array}{l}-3.3 \\
\end{array}$ & $\begin{array}{l}1.3 \\
\end{array}$ & \begin{tabular}{|l|}
-0.38 \\
\end{tabular} & \begin{tabular}{l|l}
-2.01 \\
\end{tabular} & 114 & \begin{tabular}{|l|l|}
0.084 \\
\end{tabular} \\
\hline 12 & $\begin{array}{l}2.4 \\
\end{array}$ & -12.3 & 0.0 & 5.7 & $\begin{array}{l}-3.6 \\
\end{array}$ & -3.4 & 0.8 & 1.7 & -3.0 & -3.2 & 0.9 & 1.7 & $\begin{array}{l}-3.3 \\
\end{array}$ & 1.3 & \begin{tabular}{|c|}
-0.38 \\
\end{tabular} & \begin{tabular}{|c|}
-1.98 \\
\end{tabular} & 114 & \begin{tabular}{|l|}
0.089 \\
\end{tabular} \\
\hline 13 & 2.6 & -12.5 & 0.0 & 5.8 & \begin{tabular}{|l|}
-3.6 \\
\end{tabular} & $\begin{array}{l}-3.4 \\
\end{array}$ & 0.8 & 1.7 & -3.1 & -3.4 & 0.9 & 1.7 & -3.4 & 1.3 & -0.38 & -2.00 & 117 & \begin{tabular}{|l|}
0.086 \\
\end{tabular} \\
\hline 14 & 2.6 & -12.6 & 0.0 & 6.0 & -3.7 & -3.5 & 0.8 & 1.7 & -3.1 & -3.4 & 0.9 & 1.7 & -3.4 & 1.3 & -0.38 & -2.02 & 118 & 0.080 \\
\hline 15 & 2.5 & -12.7 & 0.0 & 6.0 & $\begin{array}{l}-3.7 \\
\end{array}$ & $\begin{array}{l}-3.6 \\
\end{array}$ & 0.9 & 1.7 & -3.1 & -3.4 & 0.9 & 1.8 & -3.4 & 1.3 & -0.38 & -1.98 & 119 & \begin{tabular}{|l|}
0.089 \\
\end{tabular} \\
\hline 16 & 2.4 & -13.0 & 0.0 & 6.1 & $\begin{array}{l}-3.6 \\
\end{array}$ & $\begin{array}{l}-3.2 \\
\end{array}$ & 0.9 & 1.8 & $\begin{array}{l}-3.1 \\
\end{array}$ & $\begin{array}{l}-3.4 \\
\end{array}$ & 0.9 & 1.8 & $\begin{array}{l}-3.3 \\
\end{array}$ & 1.3 & \begin{tabular}{|c|c|}
-40 \\
\end{tabular} & \begin{tabular}{|l|}
-1.91 \\
\end{tabular} & 117 & \begin{tabular}{|l|}
0.106 \\
\end{tabular} \\
\hline 17 & 2.7 & -13.0 & 0.0 & 6.2 & $\begin{array}{l}-3.8 \\
\end{array}$ & $\begin{array}{l}-3.6 \\
\end{array}$ & 0.9 & 1.7 & $\begin{array}{l}-3.1 \\
\end{array}$ & $\begin{array}{l}-3.5 \\
\end{array}$ & 0.9 & 1.8 & $\begin{array}{l}-3.5 \\
\end{array}$ & 1.3 & \begin{tabular}{|c|}
-0.38 \\
\end{tabular} & -2.00 & 121 & 0.086 \\
\hline 18 & 2.7 & -13.2 & 0.0 & 6.3 & -3.8 & -3.6 & 0.9 & 1.8 & -3.2 & -3.5 & 0.9 & 1.8 & -3.5 & 1.4 & -0.39 & -1.97 & 121 & \begin{tabular}{|l|}
0.092 \\
\end{tabular} \\
\hline 19 & 2.5 & -13.3 & 0.0 & 6.4 & -3.7 & -3.6 & 0.9 & 1.8 & -3.2 & -3.5 & 0.9 & 1.8 & -3.5 & 1.4 & -0.40 & -1.94 & 121 & \begin{tabular}{|l|l}
0.101 \\
\end{tabular} \\
\hline 20 & 2.7 & -13.5 & 0.0 & 6.5 & -3.8 & \begin{tabular}{|l|}
-3.6 \\
\end{tabular} & 0.9 & 1.8 & -3.2 & -3.5 & 0.9 & 1. & \begin{tabular}{|l|}
-3.5 \\
\end{tabular} & 1.4 & -0.39 & -1.94 & 122 & \begin{tabular}{|l|}
0.098 \\
\end{tabular} \\
\hline 21 & 2.6 & -13.5 & 0.0 & 6.5 & $\begin{array}{l}-3.8 \\
\end{array}$ & $\begin{array}{l}-3.6 \\
\end{array}$ & 0.9 & 1.9 & -3.2 & -3.4 & 0.9 & 1.9 & -3.5 & 1.4 & -0.41 & -1.90 & 122 & 0.110 \\
\hline 22 & 2.7 & $\begin{array}{l}-13.7 \\
\end{array}$ & 0.0 & 6.6 & \begin{tabular}{|l|}
-3.8 \\
\end{tabular} & $\begin{array}{l}-3.6 \\
\end{array}$ & 0.9 & 1.9 & -3.2 & $\begin{array}{l}-3.5 \\
\end{array}$ & 0.9 & 1.9 & -3.5 & 1.4 & -0.40 & -1.91 & 123 & \begin{tabular}{|l|}
0.107 \\
\end{tabular} \\
\hline 23 & 2.7 & -13.7 & 0.0 & 6.7 & -3.8 & -3.6 & 0.9 & 1.9 & -3.2 & -3.5 & 1.0 & 1.9 & $\begin{array}{l}-3.5 \\
\end{array}$ & 1.4 & -0.41 & -1.87 & 122 & \begin{tabular}{|l|l}
0.117 \\
\end{tabular} \\
\hline 24 & 2.7 & -14.0 & 0.0 & 6.7 & $\begin{array}{l}-3.8 \\
\end{array}$ & $\begin{array}{l}-3.6 \\
\end{array}$ & 0.9 & 1.9 & $\begin{array}{l}-3.3 \\
\end{array}$ & $\begin{array}{l}-3.5 \\
\end{array}$ & 1.0 & 10 & $\begin{array}{l}-3.6 \\
\end{array}$ & $\begin{array}{l}1.4 \\
\end{array}$ & \begin{tabular}{l|l|}
-0.41 \\
\end{tabular} & \begin{tabular}{|c|}
-1.89 \\
\end{tabular} & 124 & 0.112 \\
\hline 25 & 2.7 & -14.1 & 0.0 & 6.8 & $\begin{array}{l}-3.8 \\
\end{array}$ & $\begin{array}{l}-3.6 \\
\end{array}$ & 1.0 & 2.0 & $\begin{array}{l}-3.3 \\
\end{array}$ & $\begin{array}{l}-3.6 \\
\end{array}$ & 1.0 & 2.0 & $\begin{array}{l}-3.6 \\
\end{array}$ & $\begin{array}{l}1.5 \\
\end{array}$ & -0.41 & \begin{tabular}{|c|}
-1.89 \\
\end{tabular} & 125 & \begin{tabular}{|l|l|}
0.113 \\
\end{tabular} \\
\hline 26 & 2.7 & -14.1 & 0.0 & 6.8 & -3.9 & $\begin{array}{l}-3.8 \\
\end{array}$ & 0.9 & 1.9 & $\begin{array}{l}-3.3 \\
\end{array}$ & -3.6 & 1.0 & 2.0 & -3.7 & 1.5 & -0.40 & \begin{tabular}{|c|}
-1.93 \\
\end{tabular} & 127 & \begin{tabular}{|l|l|}
0.102 \\
\end{tabular} \\
\hline 27 & 2.8 & -14.3 & 0.0 & 7.0 & -3.8 & -3.6 & 1.0 & 2.0 & -3.3 & -3.6 & 1.0 & 2.0 & $\begin{array}{l}-3.6 \\
\end{array}$ & 1.5 & -0.42 & -1.86 & 124 & \begin{tabular}{|l|}
0.121 \\
\end{tabular} \\
\hline 28 & $\begin{array}{l}2.7 \\
\end{array}$ & -14.4 & 0.0 & 7.0 & $\begin{array}{l}-3.7 \\
\end{array}$ & $\begin{array}{l}-3.6 \\
\end{array}$ & 1.0 & 2.0 & $\begin{array}{l}-3.3 \\
\end{array}$ & $\begin{array}{l}-3.6 \\
\end{array}$ & 1.0 & 2.0 & $\begin{array}{l}-3.5 \\
\end{array}$ & $\begin{array}{l}1.5 \\
\end{array}$ & $\begin{array}{l}-0.42 \\
\end{array}$ & \begin{tabular}{|c|}
-1.83 \\
\end{tabular} & 125 & 0.128 \\
\hline 29 & 2.7 & -14.5 & 0.0 & 7.0 & $\begin{array}{l}-3.7 \\
\end{array}$ & $\begin{array}{l}-3.6 \\
\end{array}$ & 1.0 & 2.1 & $\begin{array}{l}-3.4 \\
\end{array}$ & $\begin{array}{l}-3.6 \\
\end{array}$ & 1.0 & 2.0 & $\begin{array}{l}-3.6 \\
\end{array}$ & 1.5 & \begin{tabular}{|c|c|}
-0.43 \\
\end{tabular} & -1.82 & 125 & \begin{tabular}{|l|}
0.133 \\
\end{tabular} \\
\hline 30 & 2.8 & -14.6 & 0.0 & 7.1 & $\begin{array}{l}-3.6 \\
\end{array}$ & $\begin{array}{l}-3.5 \\
\end{array}$ & 1.0 & 2.1 & $\begin{array}{l}-3.3 \\
\end{array}$ & $\begin{array}{l}-3.6 \\
\end{array}$ & 1.0 & 2.0 & -3.5 & $\begin{array}{l}1.5 \\
\end{array}$ & \begin{tabular}{|c|c|}
-0.43 \\
\end{tabular} & -1.80 & 124 & \begin{tabular}{|l|}
0.140 \\
\end{tabular} \\
\hline 31 & 2.7 & -14.8 & 0.0 & 7.2 & $\begin{array}{l}-3.7 \\
\end{array}$ & \begin{tabular}{|l|}
-3.6 \\
\end{tabular} & 1.0 & 2.1 & -3.4 & -3.6 & 1.0 & 2.0 & -3.5 & $\begin{array}{l}1.5 \\
\end{array}$ & -0.43 & -1.81 & 125 & \begin{tabular}{|l|}
0.137 \\
\end{tabular} \\
\hline 32 & 2.8 & -15.0 & $\overline{0.0}$ & 7.2 & -3.7 & -3.5 & 1.0 & 2.1 & -3.4 & -3.6 & 1.0 & 2.0 & -3.5 & 1.5 & -0.44 & -1.79 & 125 & \\
\hline 33 & 2.7 & -14.9 & 0.1 & $\begin{array}{ll}7.3 \\
\end{array}$ & -3.7 & -3.5 & 1.0 & 2.1 & -3.4 & -3.6 & 1.1 & 2.0 & -3.6 & 1.6 & -0.44 & -1.79 & 126 & 0.143 \\
\hline
\end{tabular}


Table E.6 KL 52-34 Test Data $\left(-20{ }^{\circ} \mathrm{C}\right.$ Tests), continued

\begin{tabular}{|c|c|c|c|c|c|c|c|c|c|c|c|c|c|c|c|c|c|c|}
\hline & \multicolumn{12}{|c|}{ Normalized Vertical and Horizontal Deformation Arrays } & \multirow{2}{*}{\multicolumn{2}{|c|}{ Trimmed }} & & & \multirow{3}{*}{\begin{tabular}{|l|l|} 
Creep \\
Comp \\
\end{tabular}} & \multirow{3}{*}{\begin{tabular}{|l} 
Pois. \\
Ratio \\
\end{tabular}} \\
\hline & \multicolumn{4}{|c|}{ KL 52-34 Sample \#2 } & \multicolumn{4}{|c|}{ KL 52-34 Sample \#5 } & \multicolumn{4}{|c|}{ KL 52-34 Sample \#7 } & & & & & & \\
\hline & vert & vert & horz & horz & vert & vert & horz & horz & vert & vert & horz & horz & & & Ratio & & & \\
\hline Time & front & back & front & back & front & back & front & back & front & back & front & back & vert & horz & $\mathrm{X} / \mathrm{Y}$ & $\mathrm{C}_{\mathrm{empl}}$ & $\mathrm{D}(\mathrm{t})$ & \\
\hline $\mathrm{sec}$ & $\mu \mathrm{m}$ & $\mu \mathrm{m}$ & $\mu \mathrm{m}$ & $\mu \mathrm{m}$ & $\mu \mathrm{m}$ & $\mu \mathrm{m}$ & $\mu \mathrm{m}$ & $\mu \mathrm{m}$ & $\mu \mathrm{m}$ & $\mu \mathrm{m}$ & $\mu \mathrm{m}$ & $\mu \mathrm{m}$ & $\mu \mathrm{m}$ & $\mu \mathrm{m}$ & & & $1 / \mathrm{kPa}$ & \\
\hline 34 & 2.7 & -14.9 & 0.1 & 7.3 & -3.6 & -3.4 & 1.0 & 2.2 & -3.4 & -3.7 & 1.1 & 2.0 & -3.5 & 1.6 & -0.45 & -1.76 & 125 & 0.154 \\
\hline 35 & 2.7 & -15.0 & 0.1 & 7.4 & -3.6 & -3.3 & 1.0 & 2.2 & $\begin{array}{l}-3.4 \\
\end{array}$ & -3.6 & 1.1 & 2.1 & -3.5 & 1.6 & -0.45 & -1.73 & 124 & \begin{tabular}{|l|l|}
0.164 \\
\end{tabular} \\
\hline 36 & 3.0 & -15.0 & 0.1 & 7.5 & -3.6 & -3.5 & 1.0 & 2.2 & $\begin{array}{l}-3.4 \\
\end{array}$ & -3.6 & 1.1 & 2.1 & -3.5 & 1.6 & -0.45 & -1.74 & 125 & 0.162 \\
\hline 37 & 2.8 & -15.2 & 0.1 & 7.5 & -3.6 & $\begin{array}{l}-3.4 \\
\end{array}$ & 1.1 & 2.2 & $\begin{array}{l}-3.4 \\
\end{array}$ & -3.6 & 1.1 & 2.1 & \begin{tabular}{|l|}
-3.5 \\
\end{tabular} & 1.6 & \begin{tabular}{|l|}
-0.46 \\
\end{tabular} & $\begin{array}{l}-1.70 \\
\end{array}$ & 125 & \begin{tabular}{|l|l|}
0.174 \\
\end{tabular} \\
\hline 38 & 2.9 & -15.1 & 0.1 & 7.6 & $\begin{array}{l}-3.6 \\
\end{array}$ & -3.4 & 1.1 & 2.3 & $\begin{array}{l}-3.4 \\
\end{array}$ & $\begin{array}{l}-3.7 \\
\end{array}$ & 1.1 & 2.1 & -3.5 & 1.6 & \begin{tabular}{|l|l|}
-0.46 \\
\end{tabular} & \begin{tabular}{|l}
-1.71 \\
\end{tabular} & 126 & \begin{tabular}{|l|}
0.171 \\
\end{tabular} \\
\hline 39 & 2.9 & -15.2 & 0.1 & 7.6 & $\begin{array}{l}-3.5 \\
\end{array}$ & -3.5 & 1.1 & 2.3 & $\begin{array}{l}-3.4 \\
\end{array}$ & $\begin{array}{l}-3.7 \\
\end{array}$ & 1.1 & 2.1 & $\begin{array}{l}-3.5 \\
\end{array}$ & 1.6 & \begin{tabular}{|c|c|} 
\\
\end{tabular} & -1.69 & 126 & \begin{tabular}{|l|}
0.178 \\
\end{tabular} \\
\hline 40 & 2.9 & -15.4 & 0.1 & 7.6 & $\begin{array}{l}-3.6 \\
\end{array}$ & $\begin{array}{c}-3.4 \\
\end{array}$ & 1.1 & 2.2 & -3.5 & -3.7 & 1.1 & 2.1 & -3.5 & 1.6 & -0.46 & -1.71 & 126 & 0.172 \\
\hline 41 & 3.0 & -15.3 & 0.1 & 7.7 & $\begin{array}{l}-3.6 \\
\end{array}$ & -3.5 & 1.1 & 2.2 & \begin{tabular}{|l|}
-3.4 \\
\end{tabular} & $\begin{array}{l}-3.6 \\
\end{array}$ & 1.1 & 2.1 & $\begin{array}{l}-3.5 \\
\end{array}$ & 1.6 & \begin{tabular}{|c|}
-0.46 \\
\end{tabular} & \begin{tabular}{|l}
-1.72 \\
\end{tabular} & 126 & \begin{tabular}{|l|}
0.168 \\
\end{tabular} \\
\hline 42 & 3.0 & -15.4 & 0.1 & 7.8 & $\begin{array}{l}-3.6 \\
\end{array}$ & -3.5 & 1.1 & 2.2 & -3.4 & -3.6 & 1.1 & 2.1 & $\begin{array}{l}-3.5 \\
\end{array}$ & 1.6 & \begin{tabular}{|c|}
-0.46 \\
\end{tabular} & \begin{tabular}{|l}
-1.71 \\
\end{tabular} & 126 & \begin{tabular}{|l|}
0.171 \\
\end{tabular} \\
\hline 43 & 2.9 & -15.5 & 0.1 & 7.8 & -3.6 & -3.5 & 1.1 & 2.2 & -3.5 & -3.8 & 1.1 & 2.1 & -3.6 & 1.6 & \begin{tabular}{|l|}
-0.46 \\
\end{tabular} & -1.72 & 128 & \begin{tabular}{|l|}
0.166 \\
\end{tabular} \\
\hline 44 & 2.7 & -15.7 & 0.0 & 7.8 & -3.8 & -3.5 & 1.1 & 2.2 & $\begin{array}{l}-3.5 \\
\end{array}$ & -3.8 & 1.1 & 2.1 & -3.6 & 1.6 & \begin{tabular}{|l|}
-0.45 \\
\end{tabular} & $\begin{array}{l}-1.75 \\
\end{array}$ & 129 & \begin{tabular}{|l|}
0.157 \\
\end{tabular} \\
\hline 45 & 3.1 & -15.6 & 0.1 & 7.9 & -3.7 & -3.6 & 1.1 & 2.3 & -3.5 & -3.7 & 1.1 & 2.2 & -3.6 & 1.7 & \begin{tabular}{|l}
-0.46 \\
\end{tabular} & -1.72 & 129 & 0.168 \\
\hline 46 & 3.2 & -15.6 & 0.1 & 7.9 & -3.8 & -3.6 & 1.1 & 2.2 & $\begin{array}{l}-3.4 \\
\end{array}$ & $\begin{array}{l}-3.7 \\
\end{array}$ & 1.1 & 2.2 & $\begin{array}{l}-3.6 \\
\end{array}$ & 1.6 & -0.45 & \begin{tabular}{|l|}
-1.73 \\
\end{tabular} & 129 & 0.163 \\
\hline 47 & 3.1 & -15.7 & 0.1 & 8.0 & -3.8 & -3.6 & 1.1 & 2.3 & -3.5 & $\begin{array}{l}-3.8 \\
\end{array}$ & 1.1 & 2.2 & $\begin{array}{l}-3.7 \\
\end{array}$ & 1.7 & \begin{tabular}{|l}
-0.45 \\
\end{tabular} & \begin{tabular}{|l|}
-1.74 \\
\end{tabular} & 131 & \begin{tabular}{|l|l}
0.160 \\
\end{tabular} \\
\hline 48 & 3.0 & -15.8 & 0.1 & 8.1 & -3.8 & -3.6 & 1.1 & 2.3 & -3.4 & -3.7 & 1.1 & 2.2 & -3.7 & 1.7 & -0.46 & \begin{tabular}{|l|l} 
\\
\end{tabular} & 130 & \begin{tabular}{|l}
0.170 \\
\end{tabular} \\
\hline 49 & 2.9 & -16.0 & 0.1 & 8.1 & $\begin{array}{l}-3.8 \\
\end{array}$ & $\begin{array}{l}-3.7 \\
\end{array}$ & 1.1 & 2.3 & $\begin{array}{l}-3.5 \\
\end{array}$ & $\begin{array}{l}-3.7 \\
\end{array}$ & 1.1 & 2.2 & $\begin{array}{l}-3.7 \\
\end{array}$ & 1.7 & \begin{tabular}{|l|}
-0.46 \\
\end{tabular} & \begin{tabular}{|l|l|}
-1.72 \\
\end{tabular} & 131 & \begin{tabular}{|l|}
0.168 \\
\end{tabular} \\
\hline 50 & 3.1 & \begin{tabular}{|l|}
-16.0 \\
\end{tabular} & 0.1 & 8.2 & $\begin{array}{l}-3.9 \\
\end{array}$ & $\begin{array}{l}-3.7 \\
\end{array}$ & 1.1 & 2.3 & \begin{tabular}{|l|}
-3.4 \\
\end{tabular} & $\begin{array}{l}-3.8 \\
\end{array}$ & 1.1 & 2.2 & $\begin{array}{l}-3.7 \\
\end{array}$ & 1.7 & \begin{tabular}{|l}
-0.45 \\
\end{tabular} & \begin{tabular}{|l|l}
-1.73 \\
\end{tabular} & 131 & \begin{tabular}{|l|}
0.163 \\
\end{tabular} \\
\hline 51 & 3.0 & -16.0 & 0.1 & 8.3 & -3.9 & -3.8 & 1.1 & 2.3 & -3.4 & -3.7 & 1.1 & 2.2 & $\begin{array}{l}-3.7 \\
\end{array}$ & 1.7 & \begin{tabular}{|l|}
-0.45 \\
\end{tabular} & $\begin{array}{l}-1.74 \\
\end{array}$ & 132 & \begin{tabular}{|l|}
0.161 \\
\end{tabular} \\
\hline 52 & 3.0 & -16.0 & 0.1 & 8.3 & -4.1 & -3.9 & 1.1 & 2.3 & -3.4 & -3.6 & 1.2 & 2.2 & -3.7 & 1.7 & -0.45 & -1.73 & 133 & 0.162 \\
\hline 53 & 2.8 & -16.4 & 0.1 & 8.3 & -4.1 & -3.9 & 1.1 & 2.3 & -3.4 & -3.6 & 1.2 & 2.2 & -3.7 & 1.7 & -0.45 & -1.74 & 133 & \begin{tabular}{|l|l}
0.161 \\
\end{tabular} \\
\hline 54 & 3.1 & -16.3 & 0.2 & 8.3 & -4.1 & -3.9 & 1.1 & 2.3 & -3.4 & -3.7 & 1.2 & 2.3 & -3.8 & 1.7 & -0.45 & -1.74 & 134 & 0.160 \\
\hline 55 & 3.1 & -16.4 & 0.1 & 8.4 & -4.2 & -4.0 & 1.1 & 2.3 & $\begin{array}{l}-3.4 \\
\end{array}$ & -3.6 & 1.2 & 2.3 & -3.8 & 1.7 & \begin{tabular}{|l|}
-0.44 \\
\end{tabular} & \begin{tabular}{|l}
-1.76 \\
\end{tabular} & 135 & \begin{tabular}{|l|}
0.151 \\
\end{tabular} \\
\hline 56 & 3.1 & \begin{tabular}{|l|l} 
\\
\end{tabular} & 0.1 & 8.4 & -4.2 & -4.0 & 1.1 & 2.3 & -3.5 & $\begin{array}{l}-3.8 \\
\end{array}$ & 1.2 & 2.3 & -3.9 & 1.7 & \begin{tabular}{|l|l|}
-0.44 \\
\end{tabular} & \begin{tabular}{|l}
-1.79 \\
\end{tabular} & 138 & \begin{tabular}{|l|}
0.142 \\
\end{tabular} \\
\hline 57 & 3.0 & -16.5 & 0.2 & 8.4 & $\begin{array}{l}-4.2 \\
\end{array}$ & -4.1 & 1.1 & 2.3 & \begin{tabular}{|l|}
-3.4 \\
\end{tabular} & $\begin{array}{l}-3.6 \\
\end{array}$ & 1.2 & 2.3 & $\begin{array}{l}-3.8 \\
\end{array}$ & 1.7 & \begin{tabular}{|l|}
-0.45 \\
\end{tabular} & \begin{tabular}{|l|}
-1.73 \\
\end{tabular} & 135 & 0.162 \\
\hline 58 & 3.0 & -16.6 & 0.1 & 8.4 & $\begin{array}{l}-4.3 \\
\end{array}$ & -4.1 & 1.1 & 2.3 & -3.4 & $\begin{array}{l}-3.7 \\
\end{array}$ & 1.2 & 2.3 & -3.9 & 1.7 & -0.45 & -1.75 & 137 & \begin{tabular}{|l|}
0.156 \\
\end{tabular} \\
\hline 59 & 3.0 & \begin{tabular}{|l|l|}
-16.7 \\
\end{tabular} & 0.2 & 8.5 & -4.2 & -4.1 & 1.1 & 2.3 & \begin{tabular}{|l|}
-3.4 \\
\end{tabular} & -3.7 & 1.2 & 2.3 & $\begin{array}{l}-3.8 \\
\end{array}$ & 1.7 & \begin{tabular}{|l}
-0.45 \\
\end{tabular} & \begin{tabular}{|l}
-1.76 \\
\end{tabular} & 136 & \begin{tabular}{|l|}
0.154 \\
\end{tabular} \\
\hline 60 & 2.8 & -16.9 & 0.1 & 8.5 & -4.4 & -4.2 & 1.1 & 2.2 & -3.4 & -3.7 & 1.2 & 2.3 & $\begin{array}{l}-3.9 \\
\end{array}$ & 1.7 & \begin{tabular}{|l|}
-0.44 \\
\end{tabular} & \begin{tabular}{|l}
-1.78 \\
\end{tabular} & 138 & \begin{tabular}{|l|l}
0.147 \\
\end{tabular} \\
\hline 61 & 2.9 & -17.0 & 0.1 & 8.5 & -4.4 & -4.1 & 1.1 & 2.3 & -3.4 & -3.6 & 1.2 & 2.3 & -3.9 & 1.7 & -0.45 & -1.76 & 138 & 0.153 \\
\hline 62 & 2.8 & $\begin{array}{l}-17.1 \\
\end{array}$ & 0.1 & 8.5 & -4.4 & -4.2 & 1.1 & 2.3 & -3.4 & -3.7 & 1.2 & 2.3 & $\begin{array}{l}-3.9 \\
\end{array}$ & 1.7 & \begin{tabular}{|l|}
-0.45 \\
\end{tabular} & \begin{tabular}{|l}
-1.76 \\
\end{tabular} & 138 & \begin{tabular}{|l|}
0.154 \\
\end{tabular} \\
\hline 63 & 2.8 & -17.3 & 0.1 & 8.6 & -4.4 & -4.2 & 1.1 & 2.3 & $\begin{array}{l}-3.4 \\
\end{array}$ & -3.6 & 1.2 & 2.3 & -3.9 & 1.7 & -0.45 & -1.74 & 138 & \begin{tabular}{|l|l|}
0.159 \\
\end{tabular} \\
\hline 64 & 2.7 & -17.5 & 0.1 & 8.6 & -4.3 & -4.1 & 1.1 & 2.3 & \begin{tabular}{|c|}
-3.4 \\
\end{tabular} & -3.6 & 1.2 & 2.4 & \begin{tabular}{|c|} 
\\
\end{tabular} & 1.8 & \begin{tabular}{|c|c|}
-0.46 \\
\end{tabular} & \begin{tabular}{|l}
-1.71 \\
\end{tabular} & 138 & \begin{tabular}{|l|l|}
0.172 \\
\end{tabular} \\
\hline 65 & 2.6 & -17.5 & 0.1 & 8.6 & -4.2 & -3.9 & 1.1 & 2.4 & -3.4 & -3.6 & 1.2 & 2.4 & -3.8 & 1.8 & \begin{tabular}{|l}
-0.47 \\
\end{tabular} & -1.69 & 136 & \begin{tabular}{|l}
0.180 \\
\end{tabular} \\
\hline 66 & 2.6 & -17.7 & 0.1 & $\overline{8.6}$ & -4.4 & -4.2 & 1.1 & 2.4 & \begin{tabular}{|c|}
-3.4 \\
\end{tabular} & $\begin{array}{l}-3.6 \\
\end{array}$ & 1.2 & 2.4 & -3.9 & 1.8 & \begin{tabular}{|c|}
-0.46 \\
\end{tabular} & -1.71 & 138 & 0.170 \\
\hline
\end{tabular}


Table E.6 KL 52-34 Test Data $\left(-20{ }^{\circ} \mathrm{C}\right.$ Tests $)$, continued

\begin{tabular}{|c|c|c|c|c|c|c|c|c|c|c|c|c|c|c|c|c|c|c|}
\hline & \multicolumn{12}{|c|}{ Normalized Vertical and Horizontal Deformation Arrays } & \multirow{2}{*}{\multicolumn{2}{|c|}{ Trimmed }} & & & & \\
\hline & \multicolumn{4}{|c|}{ KL 52-34 Sample \#2 } & \multicolumn{4}{|c|}{ KL 52-34 Sample \#5 } & \multicolumn{4}{|c|}{ KL 52-34 Sample \#7 } & & & & & Creep & Pois. \\
\hline & vert & vert & horz & horz & vert & vert & horz & horz & vert & vert & horz & horz & & & Ratio & & Comp & Ratio \\
\hline Time & front & back & front & back & front & back & front & back & front & back & front & back & vert & horz & $\mathrm{X} / \mathrm{Y}$ & $\mathrm{C}_{\mathrm{empl}}$ & $D(t)$ & $\bar{v}$ \\
\hline $\mathrm{sec}$ & $\mu \mathrm{m}$ & $\mu \mathrm{m}$ & $\mu \mathrm{m}$ & $\mu \mathrm{m}$ & $\mu \mathrm{m}$ & $\mu \mathrm{m}$ & $\mu \mathrm{m}$ & $\mu \mathrm{m}$ & $\mu \mathrm{m}$ & $\mu \mathrm{m}$ & $\mu \mathrm{m}$ & $\mu \mathrm{m}$ & $\mu \mathrm{m}$ & $\mu \mathrm{m}$ & & & $1 / \mathrm{kPa}$ & \\
\hline 67 & 2.6 & -17.7 & 0.1 & 8.7 & -4.3 & -4.1 & 1.1 & $\overline{2.4}$ & -3.4 & -3.6 & 1.2 & 2.4 & -3.9 & 1.8 & -0.47 & -1.69 & 138 & 0.180 \\
\hline 68 & 2.5 & -17.8 & 0.1 & 8.7 & -4.4 & -4.2 & 1.2 & 2.4 & -3.4 & -3.8 & 1.2 & 2.4 & -3.9 & 1.8 & -0.46 & -1.72 & 140 & 0.167 \\
\hline 69 & 2.6 & -17.9 & 0.1 & 8.7 & -4.4 & -4.2 & 1.1 & 2.4 & -3.4 & -3.6 & 1.3 & 2.4 & -3.9 & 1.8 & -0.46 & -1.71 & 139 & 0.171 \\
\hline 70 & 2.6 & -17.9 & 0.1 & 8.8 & -4.4 & -4.2 & 1.1 & 2.4 & -3.4 & -3.6 & 1.2 & 2.5 & -3.9 & 1.8 & -0.46 & -1.70 & 139 & 0.175 \\
\hline 71 & 2.6 & -17.9 & 0.1 & 8.8 & -4.4 & -4.1 & 1.1 & 2.4 & -3.4 & -3.7 & 1.3 & 2.5 & -3.9 & 1.8 & -0.47 & -1.69 & 139 & 0.181 \\
\hline 72 & 2.3 & -18.1 & 0.1 & 8.8 & -4.2 & -4.1 & 1.2 & 2.4 & -3.5 & -3.6 & 1.2 & 2.4 & -3.9 & 1.8 & -0.47 & $\begin{array}{l}-1.68 \\
-1\end{array}$ & 139 & 0.184 \\
\hline 73 & 2.4 & -18.1 & 0.1 & 8.8 & -4.2 & -4.1 & 1.2 & 2.4 & -3.4 & -3.7 & 1.3 & 2.5 & -3.9 & 1.8 & -0.48 & -1.66 & 138 & 0.191 \\
\hline 74 & 2.5 & -18.2 & 0.1 & 8.9 & -4.2 & -4.2 & 1.2 & 2.4 & -3.4 & -3.7 & 1.3 & 2.5 & -3.9 & 1.8 & \begin{tabular}{|l|}
-0.47 \\
\end{tabular} & -1.68 & 139 & 0.184 \\
\hline 75 & 2.5 & -18.4 & 0.1 & 8.9 & -4.1 & -3.9 & 1.2 & 2.5 & -3.5 & -3.7 & 1.3 & 2.4 & -3.8 & 1.8 & -0.49 & -1.63 & 137 & 0.204 \\
\hline 76 & 2.5 & -18.2 & 0.1 & 9.0 & -4.1 & -3.9 & 1.2 & 2.5 & -3.4 & -3.6 & 1.3 & 2.4 & -3.7 & 1.9 & -0.50 & -1.61 & 136 & 0.213 \\
\hline 77 & 2.6 & -18.2 & 0.1 & 9.0 & -4.1 & -3.9 & 1.2 & 2.5 & -3.4 & -3.7 & 1.3 & 2.5 & -3.8 & 1.9 & -0.50 & -1.61 & 137 & 0.214 \\
\hline 78 & 2.5 & -18.4 & 0.1 & 9.1 & -3.9 & -3.8 & 1.2 & 2.6 & -3.4 & -3.7 & 1.3 & 2.5 & -3.7 & 1.9 & -0.51 & -1.57 & 135 & 0.234 \\
\hline 79 & 2.5 & -18.5 & 0.1 & 9.0 & -3.9 & -3.8 & 1.2 & 2.6 & -3.5 & -3.7 & 1.3 & 2.5 & -3.7 & 1.9 & -0.51 & -1.58 & 136 & 0.229 \\
\hline 80 & 2.6 & -18.5 & 0.1 & 9.0 & -3.9 & -3.8 & 1.2 & 2.6 & -3.4 & -3.8 & 1.3 & 2.5 & -3.7 & 1.9 & -0.51 & -1.58 & 136 & 0.228 \\
\hline 81 & 2.5 & -18.6 & 0.1 & 9.1 & -3.9 & -3.8 & 1.2 & 2.6 & -3.5 & $\begin{array}{l}-3.8 \\
\end{array}$ & 1.3 & 2.5 & -3.8 & 1.9 & -0.50 & -1.59 & 136 & 0.224 \\
\hline 82 & 2.5 & -18.6 & 0.1 & 9.1 & -3.9 & -3.8 & 1.2 & 2.6 & -3.5 & -3.7 & 1.3 & 2.5 & -3.7 & 1.9 & -0.51 & -1.57 & 135 & 0.237 \\
\hline 83 & 2.4 & -18.7 & 0.1 & 9.2 & -4.1 & -3.8 & 1.2 & 2.6 & -3.5 & -3.7 & 1.3 & 2.5 & -3.8 & 1.9 & -0.50 & -1.60 & 137 & 0.220 \\
\hline 84 & 2.5 & -18.8 & 0.1 & 9.2 & -3.9 & -3.9 & 1.2 & 2.6 & -3.5 & -3.8 & 1.3 & 2.5 & -3.8 & 1.9 & -0.51 & -1.58 & 137 & 0.229 \\
\hline 85 & 2.5 & -18.8 & 0.2 & 9.3 & -3.9 & \begin{tabular}{|l|}
-3.8 \\
\end{tabular} & 1.2 & 2.6 & -3.5 & $\begin{array}{l}-3.8 \\
\end{array}$ & 1.3 & 2.5 & -3.8 & 1.9 & -0.51 & -1.59 & 138 & 0.226 \\
\hline 86 & 2.6 & -18.6 & 0.2 & 9.3 & -4.1 & -3.9 & 1.2 & 2.6 & -3.5 & -3.8 & 1.3 & 2.5 & -3.8 & 1.9 & -0.50 & -1.60 & 138 & 0.220 \\
\hline 87 & 2.6 & -19.0 & 0.2 & 9.3 & -4.1 & -3.9 & 1.2 & 2.6 & -3.6 & -3.8 & 1.3 & 2.5 & -3.9 & 1.9 & -0.50 & -1.61 & 140 & 0.216 \\
\hline 88 & 2.7 & -18.8 & 0.2 & 9.4 & -4.1 & -3.9 & 1.2 & 2.6 & -3.6 & -3.9 & 1.3 & 2.5 & -3.9 & 1.9 & -0.49 & -1.62 & 141 & 0.212 \\
\hline 89 & 2.7 & -18.7 & 0.2 & 9.4 & -4.1 & -3.9 & 1.2 & 2.6 & -3.5 & -3.8 & 1.3 & 2.5 & -3.8 & 1.9 & -0.50 & -1.61 & 139 & 0.216 \\
\hline 90 & 2.7 & -18.8 & 0.2 & 9.5 & -4.1 & -4.0 & 1.2 & 2.6 & -3.6 & -3.8 & 1.3 & 2.5 & -3.9 & 1.9 & -0.49 & -1.62 & 141 & 0.210 \\
\hline 91 & 2.9 & -18.7 & 0.2 & 9.5 & -4.1 & -4.1 & 1.3 & 2.6 & -3.6 & -3.8 & 1.3 & 2.5 & -3.9 & 1.9 & -0.49 & -1.62 & 142 & 0.211 \\
\hline 92 & 3.0 & -18.5 & 0.2 & 9.6 & -4.3 & -4.3 & 1.2 & 2.6 & -3.7 & -3.9 & 1.3 & 2.6 & -4.0 & 1.9 & -0.47 & -1.67 & 145 & 0.187 \\
\hline 93 & 3.0 & -18.7 & 0.2 & 9.7 & -4.2 & -4.1 & 1.3 & 2.6 & -3.6 & -3.9 & 1.3 & 2.6 & -4.0 & 1.9 & -0.49 & -1.63 & 143 & 0.205 \\
\hline 94 & 3.0 & -18.8 & 0.2 & 9.7 & -4.4 & -4.1 & 1.3 & 2.6 & -3.6 & -4.0 & 1.3 & 2.6 & -4.0 & 1.9 & -0.48 & -1.65 & 145 & 0.196 \\
\hline 95 & 3.1 & -19.0 & 0.2 & 9.7 & -4.4 & -4.2 & 1.2 & 2.6 & -3.7 & -4.0 & 1.3 & 2.5 & -4.1 & 1.9 & -0.47 & -1.67 & 146 & 0.186 \\
\hline 96 & 3.2 & -18.9 & 0.2 & 9.7 & -4.4 & -4.2 & 1.3 & 2.6 & -3.8 & -4.0 & 1.3 & 2.5 & -4.1 & 1.9 & -0.47 & -1.69 & 147 & 0.181 \\
\hline 97 & 3.3 & -18.8 & 0.2 & 9.8 & -4.5 & -4.4 & 1.2 & 2.6 & -3.7 & -4.0 & 1.3 & 2.5 & -4.2 & 1.9 & -0.46 & -1.72 & 149 & 0.169 \\
\hline 98 & 3.1 & -19.0 & 0.2 & 9.8 & -4.6 & -4.5 & 1.2 & 2.5 & -3.8 & -4.0 & 1.3 & 2.6 & -4.2 & 1.9 & -0.45 & -1.74 & 150 & 0.161 \\
\hline 99 & 3.3 & -18.9 & 0.2 & 9.8 & -4.6 & -4.5 & 1.2 & 2.5 & -3.8 & -4.1 & 1.3 & 2.5 & -4.3 & 1.9 & -0.45 & -1.74 & 151 & 0.158 \\
\hline 100 & 3.1 & -19.0 & 0.2 & 9.8 & -4.5 & -4.4 & 1.2 & 2.5 & -3.8 & -4.1 & 1.3 & 2.5 & -4.2 & 1.9 & -0.46 & -1.72 & 149 & 0.167 \\
\hline
\end{tabular}


Table E.7 KL 58-40 Sample Data $\left(0{ }^{\circ} \mathrm{C}\right.$ Tests)

\begin{tabular}{|c|c|c|c|}
\hline & Thickness & Diameter & Creep Load \\
\hline Sample & $\mathrm{mm}$ & $\mathrm{mm}$ & $\mathrm{kN}$ \\
\hline 1 & 76.82 & 150.1 & -3.20 \\
\hline 10 & 76.74 & 150.2 & -2.11 \\
\hline 12 & 76.85 & 150.0 & -2.80 \\
\hline 8 & 76.80 & 150.0 & -3.48 \\
\hline average & 76.80 & 150.1 & -2.90 \\
\hline
\end{tabular}

\begin{tabular}{|c|c|}
\hline Gage length, $\mathrm{m}$ & 0.038 \\
\hline $\mathrm{C}_{\text {empl }}$ low & 0.595 \\
\hline $\mathrm{C}_{\text {empl }}$ high & 1.466 \\
\hline
\end{tabular}

Table E.8 KL 58-40 Sample Data $\left(-10{ }^{\circ} \mathrm{C}\right.$ Tests)

\begin{tabular}{|c|c|c|c|}
\hline & Thickness & Diameter & Creep Load \\
\hline Sample & $\mathrm{mm}$ & $\mathrm{mm}$ & $\mathrm{kN}$ \\
\hline 1 & 76.82 & 150.1 & -3.66 \\
\hline 10 & 76.74 & 150.2 & -3.59 \\
\hline 12 & 76.85 & 150.0 & -5.14 \\
\hline 8 & 76.80 & 150.0 & -3.24 \\
\hline average & 76.80 & 150.1 & -3.91 \\
\hline
\end{tabular}

\begin{tabular}{|c|c|}
\hline Gage length, $\mathrm{m}$ & 0.038 \\
\hline $\mathrm{C}_{\mathrm{empl}}$ low & 0.595 \\
\hline $\mathrm{C}_{\mathrm{empl}}$ high & 1.466 \\
\hline
\end{tabular}

Table E.9 KL 58-40 Sample Data $\left(-20{ }^{\circ} \mathrm{C}\right.$ Tests $)$

\begin{tabular}{|c|c|c|c|}
\hline & Thickness & Diameter & Creep Load \\
\hline Sample & $\mathrm{mm}$ & $\mathrm{mm}$ & $\mathrm{kN}$ \\
\hline 1 & 76.82 & 150.1 & -10.82 \\
\hline 10 & 76.74 & 150.2 & -5.73 \\
\hline 12 & 76.85 & 150.0 & -9.10 \\
\hline 8 & 76.80 & 150.0 & -5.31 \\
\hline average & 76.80 & 150.1 & -7.74 \\
\hline
\end{tabular}

\begin{tabular}{|c|c|}
\hline Gage length, $\mathrm{m}$ & 0.038 \\
\hline $\mathrm{C}_{\text {empl }}$ low & 0.595 \\
\hline $\mathrm{C}_{\text {empl }}$ high & 1.466 \\
\hline
\end{tabular}


Table E.10 KL 58-40 Test Data $\left(0^{\circ} \mathrm{C}\right.$ Tests)

\begin{tabular}{|c|c|c|c|c|c|c|c|c|c|c|c|c|c|c|c|c|c|c|c|c|c|c|}
\hline & & & & & & & & 10 & rizo & & & rrays & & & & & & & & & & \\
\hline & & $58 / 40$ & Sample & & & $58 / 40 \mathrm{~s}$ & ample & & $\mathrm{KL}$ & $58 / 40 \mathrm{~S}$ & ample & & & $58 / 40$ & Sample & & Trim & ned & & & Creep & Pois. \\
\hline & \begin{tabular}{l|l} 
vert \\
\end{tabular} & vert & horz & horz & vert & vert & horz & horz & $\begin{array}{l}\text { vert } \\
\end{array}$ & \begin{tabular}{l|l} 
vert \\
\end{tabular} & horz & horz & \begin{tabular}{|l|} 
vert \\
\end{tabular} & \begin{tabular}{|l|} 
vert \\
\end{tabular} & horz & horz & $\mathrm{Me}$ & & Ratio & & Comp & \begin{tabular}{|l|l} 
Ratio \\
\end{tabular} \\
\hline Time & front & back & front & back & front & back & front & back & \begin{tabular}{|l|} 
front \\
\end{tabular} & back & front & back & \begin{tabular}{|l|} 
front \\
\end{tabular} & \begin{tabular}{|l|} 
back \\
\end{tabular} & front & back & vert & horz & $\overline{X / Y}$ & $C_{\text {empl }}$ & $\mathrm{D}(\mathrm{t})$ & \\
\hline $\mathrm{sec}$ & $\mu \mathrm{m}$ & $\mu \mathrm{m}$ & $\mu \mathrm{m}$ & $\mu \mathrm{m}$ & $\mu \mathrm{m}$ & $\mu \mathrm{m}$ & $\mu \mathrm{m}$ & $\mu \mathrm{m}$ & \begin{tabular}{|l|}
$\mu \mathrm{m}$ \\
\end{tabular} & $\mu \mathrm{m}$ & $\mu \mathrm{m}$ & $\mu \mathrm{m}$ & $\mu \mathrm{m}$ & $\mu \mathrm{m}$ & $\mu \mathrm{m}$ & $\mu \mathrm{m}$ & $\mu \mathrm{m}$ & $\mu \mathrm{m}$ & & & $1 / \mathrm{kPa}$ & \\
\hline 0 & 0.0 & 0.0 & 0.0 & 0.0 & 0.0 & 0.0 & 0.0 & 0.0 & 0.0 & 0.0 & 0.0 & 0.0 & 0.0 & 0.0 & 0.0 & 0.0 & 0.0 & 0.0 & 0.00 & $\begin{array}{ll}0.00 \\
\end{array}$ & 0 & 0.000 \\
\hline 1 & -3.8 & $\begin{array}{l}-3.0 \\
\end{array}$ & 1.0 & 1.8 & -0.8 & $\begin{array}{l}-6.0 \\
\end{array}$ & 0.5 & 3.5 & \begin{tabular}{|l}
-10.5 \\
\end{tabular} & -1.9 & 2.6 & 1.4 & -3.4 & -3.6 & 0.9 & 2.5 & -3.6 & 2.1 & \begin{tabular}{|c|}
-0.57 \\
\end{tabular} & \begin{tabular}{|l}
-1.46 \\
\end{tabular} & 313 & 0.308 \\
\hline 2 & -5.0 & $\begin{array}{l}-4.0 \\
\end{array}$ & 1.3 & 2.2 & \begin{tabular}{|l|}
-1.0 \\
\end{tabular} & $\begin{array}{l}-7.2 \\
\end{array}$ & 0.7 & 4.2 & \begin{tabular}{|l}
-13.3 \\
\end{tabular} & -3.0 & 3.3 & 1.9 & -4.4 & -4.8 & 1.1 & 3.2 & -4.7 & 2.5 & \begin{tabular}{|l|}
-0.54 \\
\end{tabular} & \begin{tabular}{|l}
-1.52 \\
\end{tabular} & 402 & 0.268 \\
\hline 3 & -5.4 & -4.1 & 1.5 & 2.6 & $\begin{array}{l}-1.4 \\
\end{array}$ & $\begin{array}{l}-8.0 \\
\end{array}$ & 0.8 & 4.6 & \begin{tabular}{|l}
-15.1 \\
\end{tabular} & -3.9 & 3.9 & 2.3 & -4.9 & -5.3 & 1.2 & 3.7 & $\begin{array}{l}-5.3 \\
\end{array}$ & 2.9 & \begin{tabular}{|l|}
-0.55 \\
\end{tabular} & \begin{tabular}{|l}
-1.48 \\
\end{tabular} & 451 & 0.288 \\
\hline 4 & -5.9 & $\begin{array}{l}-4.6 \\
\end{array}$ & 1.6 & 2.8 & $\begin{array}{l}-1.7 \\
\end{array}$ & \begin{tabular}{|l|}
-8.9 \\
\end{tabular} & 0.9 & 5.0 & \begin{tabular}{|l}
-16.6 \\
\end{tabular} & -4.7 & 4.3 & 2.7 & -5.2 & -5.8 & 1.3 & 4.1 & -5.9 & 3.2 & \begin{tabular}{|l|}
-0.54 \\
\end{tabular} & \begin{tabular}{|l|}
-1.50 \\
\end{tabular} & 500 & \begin{tabular}{|l|}
0.277 \\
\end{tabular} \\
\hline 5 & $\begin{array}{l}-6.3 \\
\end{array}$ & -4.9 & 1.8 & 3.0 & $\begin{array}{l}-1.6 \\
\end{array}$ & $\begin{array}{l}-9.1 \\
\end{array}$ & 1.0 & 5.2 & \begin{tabular}{|l}
-17.7 \\
\end{tabular} & -5.1 & 4.6 & 3.1 & -5.6 & -6.4 & 1.4 & 4.5 & -6.3 & 3.4 & \begin{tabular}{|l|}
-0.55 \\
\end{tabular} & \begin{tabular}{|l|}
-1.50 \\
\end{tabular} & 535 & \begin{tabular}{|l|l|}
0.281 \\
\end{tabular} \\
\hline 6 & -6.7 & -5.2 & 1.9 & 3.2 & -1.8 & $\begin{array}{l}-9.6 \\
\end{array}$ & 1.0 & 5.6 & \begin{tabular}{|l}
-18.7 \\
\end{tabular} & -5.8 & 4.9 & 3.3 & -6.0 & -6.8 & 1.5 & 4.7 & -6.7 & 3.6 & \begin{tabular}{|l|}
-0.54 \\
\end{tabular} & \begin{tabular}{|l|}
-1.50 \\
\end{tabular} & 570 & 0.277 \\
\hline 7 & $\begin{array}{l}-7.1 \\
\end{array}$ & $\begin{array}{l}-5.7 \\
\end{array}$ & 2.0 & 3.3 & \begin{tabular}{|c|}
-1.9 \\
\end{tabular} & -10.1 & 1.1 & 5.8 & \begin{tabular}{|l}
-19.6 \\
\end{tabular} & -6.2 & 5.2 & 3.7 & $\begin{array}{l}-6.3 \\
\end{array}$ & -7.1 & 1.5 & 5.0 & -7.1 & 3.8 & \begin{tabular}{|l|}
-0.54 \\
\end{tabular} & $\begin{array}{l}-1.52 \\
\end{array}$ & 604 & 0.266 \\
\hline 8 & $\begin{array}{l}-7.3 \\
\end{array}$ & -5.7 & 2.1 & 3.5 & $\begin{array}{l}-2.1 \\
\end{array}$ & -10.4 & 1.2 & 6.1 & \begin{tabular}{|l}
-20.5 \\
\end{tabular} & -6.8 & 5.4 & 3.9 & -6.7 & -7.5 & 1.6 & 5.3 & -7.4 & 4.0 & \begin{tabular}{|l|}
-0.54 \\
\end{tabular} & \begin{tabular}{|l}
-1.51 \\
\end{tabular} & 633 & 0.273 \\
\hline$\overline{9}$ & -7.5 & -5.8 & 2.2 & 3.6 & -2.2 & -10.6 & 1.2 & 6.3 & \begin{tabular}{|l|l|}
-21.3 \\
\end{tabular} & -7.3 & 5.7 & 4.2 & -7.0 & -7.8 & 1.7 & 5.5 & -7.7 & 4.2 & \begin{tabular}{|l|}
-0.54 \\
\end{tabular} & \begin{tabular}{|l|}
-1.51 \\
\end{tabular} & 655 & \begin{tabular}{|l|}
0.273 \\
\end{tabular} \\
\hline 10 & $\begin{array}{l}-7.8 \\
\end{array}$ & $\begin{array}{l}-6.0 \\
\end{array}$ & 2.3 & 3.8 & -2.3 & -10.8 & 1.3 & 6.5 & \begin{tabular}{|l}
-21.9 \\
\end{tabular} & $\begin{array}{l}-7.7 \\
\end{array}$ & 5.9 & 4.4 & $\begin{array}{l}-7.1 \\
\end{array}$ & -8.1 & 1.8 & 5.8 & \begin{tabular}{|l|}
-7.9 \\
\end{tabular} & 4.3 & \begin{tabular}{|l|}
-0.55 \\
\end{tabular} & \begin{tabular}{|l}
-1.50 \\
\end{tabular} & 678 & \begin{tabular}{|l|}
0.280 \\
\end{tabular} \\
\hline 11 & $\begin{array}{l}-8.2 \\
\end{array}$ & $\begin{array}{l}-6.3 \\
\end{array}$ & 2.3 & 3.9 & $\begin{array}{l}-2.5 \\
\end{array}$ & -11.2 & 1.4 & 6.7 & \begin{tabular}{|l}
-22.6 \\
\end{tabular} & -8.2 & 6.1 & 4.7 & $\begin{array}{l}-7.4 \\
\end{array}$ & -8.3 & 1.8 & 5.9 & \begin{tabular}{|l}
-8.3 \\
\end{tabular} & 4.5 & \begin{tabular}{|l|}
-0.54 \\
\end{tabular} & \begin{tabular}{|l|}
-1.51 \\
\end{tabular} & 704 & 0.273 \\
\hline 12 & $\begin{array}{l}-8.5 \\
\end{array}$ & $\begin{array}{l}-6.4 \\
\end{array}$ & 2.4 & 4.1 & -2.5 & -11.4 & 1.4 & 6.9 & \begin{tabular}{|l}
-23.3 \\
\end{tabular} & -8.5 & 6.3 & 4.9 & $\begin{array}{l}-7.6 \\
\end{array}$ & -8.5 & 1.8 & 6.1 & -8.5 & 4.6 & \begin{tabular}{|l|}
-0.54 \\
\end{tabular} & \begin{tabular}{|l|l|} 
\\
\end{tabular} & 724 & 0.276 \\
\hline 13 & -8.6 & $\begin{array}{l}-6.6 \\
\end{array}$ & 2.5 & 4.2 & -2.5 & -11.5 & 1.5 & 7.0 & \begin{tabular}{|l}
-23.8 \\
\end{tabular} & -8.8 & 6.5 & 5.2 & -7.8 & -8.8 & 1.9 & 6.3 & -8.7 & 4.7 & \begin{tabular}{|l|}
-0.55 \\
\end{tabular} & \begin{tabular}{|l}
-1.50 \\
\end{tabular} & 741 & \begin{tabular}{|l|}
0.279 \\
\end{tabular} \\
\hline 14 & -8.9 & -6.8 & 2.6 & 4.3 & -2.5 & -11.8 & 1.5 & 7.2 & -24.4 & -9.2 & 6.6 & 5.3 & -8.0 & -9.1 & 2.0 & 6.5 & -9.0 & 4.9 & \begin{tabular}{|l|}
-0.54 \\
\end{tabular} & -1.50 & 764 & \begin{tabular}{|l|l}
0.275 \\
\end{tabular} \\
\hline 15 & $\begin{array}{l}-9.1 \\
\end{array}$ & $\begin{array}{l}-6.9 \\
\end{array}$ & 2.6 & 4.4 & $\begin{array}{l}-2.6 \\
\end{array}$ & -12.1 & 1.6 & 7.3 & \begin{tabular}{|l|}
-24.9 \\
\end{tabular} & -9.7 & 6.8 & 5.5 & -8.2 & -9.3 & 2.0 & 6.6 & -9.2 & 5.0 & \begin{tabular}{|l|}
-0.54 \\
\end{tabular} & \begin{tabular}{|l|}
-1.51 \\
\end{tabular} & 784 & \begin{tabular}{|l|l|}
0.272 \\
\end{tabular} \\
\hline 16 & $\begin{array}{l}-9.3 \\
\end{array}$ & \begin{tabular}{l|l}
-7.1 \\
\end{tabular} & 2.7 & 4.5 & $\begin{array}{l}-2.7 \\
\end{array}$ & -12.1 & 1.6 & 7.5 & \begin{tabular}{|l}
-25.5 \\
\end{tabular} & \begin{tabular}{|c|}
-10.0 \\
\end{tabular} & 6.9 & 5.7 & -8.3 & -9.4 & 2.1 & 6.8 & $\begin{array}{l}-9.4 \\
\end{array}$ & 5.1 & \begin{tabular}{|l|}
-0.54 \\
\end{tabular} & \begin{tabular}{|l|}
-1.50 \\
\end{tabular} & 801 & 0.276 \\
\hline 17 & $\begin{array}{l}-9.4 \\
\end{array}$ & $\begin{array}{l}-7.1 \\
\end{array}$ & 2.8 & 4.6 & -2.8 & -12.4 & 1.7 & 7.7 & -26.0 & \begin{tabular}{|c|}
-10.3 \\
\end{tabular} & 7.1 & 6.0 & -8.5 & -9.7 & 2.1 & 7.0 & -9.6 & 5.2 & \begin{tabular}{|l|}
-0.54 \\
\end{tabular} & \begin{tabular}{|l|}
-1.50 \\
\end{tabular} & 818 & \begin{tabular}{|l|}
0.277 \\
\end{tabular} \\
\hline 18 & -9.7 & $\begin{array}{l}-7.4 \\
\end{array}$ & 2.8 & 4.7 & $\begin{array}{l}-2.9 \\
\end{array}$ & -12.5 & 1.7 & 7.8 & \begin{tabular}{|l}
-26.5 \\
\end{tabular} & \begin{tabular}{|c|}
-10.8 \\
\end{tabular} & 7.2 & 6.1 & -8.7 & \begin{tabular}{|l|}
-10.0 \\
\end{tabular} & 2.1 & 7.1 & -9.8 & 5.3 & \begin{tabular}{|l|}
-0.54 \\
\end{tabular} & \begin{tabular}{|l|}
-1.51 \\
\end{tabular} & 838 & 0.269 \\
\hline 19 & -9.8 & $\begin{array}{l}-7.5 \\
\end{array}$ & 2.9 & 4.8 & -2.9 & $\begin{array}{l}-12.7 \\
\end{array}$ & 1.8 & $\overline{7.9}$ & $\begin{array}{l}-27.0 \\
\end{array}$ & -11.0 & 7.4 & 6.3 & -9.0 & \begin{tabular}{|l}
-10.3 \\
\end{tabular} & 2.2 & 7.3 & -10.1 & 5.4 & \begin{tabular}{|l|}
-0.54 \\
\end{tabular} & \begin{tabular}{|l}
-1.52 \\
\end{tabular} & 857 & 0.267 \\
\hline 20 & \begin{tabular}{|c|}
-10.0 \\
\end{tabular} & $\begin{array}{l}-7.8 \\
\end{array}$ & 2.9 & 4.9 & $\begin{array}{l}-2.9 \\
\end{array}$ & -12.9 & 1.8 & 8.0 & \begin{tabular}{|l}
-27.4 \\
\end{tabular} & -11.4 & 7.5 & 6.5 & -9.0 & \begin{tabular}{|l|}
-10.4 \\
\end{tabular} & 2.2 & 7.4 & \begin{tabular}{|l|}
-10.3 \\
\end{tabular} & 5.5 & \begin{tabular}{|l|}
-0.54 \\
\end{tabular} & \begin{tabular}{|l}
-1.52 \\
\end{tabular} & 874 & 0.266 \\
\hline 21 & -10.3 & $\begin{array}{l}-7.9 \\
\end{array}$ & 3.0 & 5.0 & -3.0 & -13.0 & 1.8 & 8.2 & \begin{tabular}{|l}
-27.8 \\
\end{tabular} & -11.6 & 7.6 & 6.7 & -9.2 & \begin{tabular}{|l}
-10.6 \\
\end{tabular} & 2.3 & 7.5 & -10.4 & 5.6 & \begin{tabular}{|l|}
-0.54 \\
\end{tabular} & \begin{tabular}{|l}
-1.52 \\
\end{tabular} & 888 & \begin{tabular}{|l|}
0.267 \\
\end{tabular} \\
\hline 22 & -10.3 & $\begin{array}{l}-8.0 \\
\end{array}$ & 3.0 & 5.1 & $\begin{array}{l}-3.2 \\
\end{array}$ & -13.2 & 1.9 & 8.3 & \begin{tabular}{|l}
-28.3 \\
\end{tabular} & -11.9 & 7.7 & 6.8 & -9.4 & \begin{tabular}{|l|}
-10.7 \\
\end{tabular} & 2.3 & 7.7 & -10.6 & 5.7 & \begin{tabular}{|l|}
-0.54 \\
\end{tabular} & \begin{tabular}{|l}
-1.51 \\
\end{tabular} & 902 & 0.269 \\
\hline 23 & \begin{tabular}{|c|}
-10.5 \\
\end{tabular} & $\begin{array}{l}-8.2 \\
\end{array}$ & 3.1 & 5.2 & $\begin{array}{l}-3.3 \\
\end{array}$ & -13.5 & 1.9 & 8.4 & \begin{tabular}{|l}
-28.7 \\
\end{tabular} & -12.3 & 7.9 & 7.0 & -9.7 & \begin{tabular}{|l}
-10.9 \\
\end{tabular} & 2.4 & 7.8 & \begin{tabular}{|l|}
-10.8 \\
\end{tabular} & 5.8 & \begin{tabular}{|l|}
-0.53 \\
\end{tabular} & \begin{tabular}{|l}
-1.52 \\
\end{tabular} & 922 & 0.264 \\
\hline 24 & -10.7 & \begin{tabular}{|l|}
-8.3 \\
\end{tabular} & 3.2 & 5.2 & \begin{tabular}{|l|}
-3.4 \\
\end{tabular} & -13.6 & 1.9 & 8.5 & \begin{tabular}{|l}
-29.1 \\
\end{tabular} & -12.5 & 8.0 & 7.1 & -9.7 & \begin{tabular}{|l}
-11.0 \\
\end{tabular} & 2.4 & 7.9 & -11.0 & 5.9 & \begin{tabular}{|l|}
-0.54 \\
\end{tabular} & \begin{tabular}{|l}
-1.52 \\
\end{tabular} & 934 & 0.266 \\
\hline 25 & \begin{tabular}{|c|}
-10.8 \\
\end{tabular} & \begin{tabular}{|l|}
-8.4 \\
\end{tabular} & 3.2 & 5.3 & $\begin{array}{l}-3.6 \\
\end{array}$ & -13.9 & 2.0 & 8.6 & \begin{tabular}{|l}
-29.4 \\
\end{tabular} & -12.8 & 8.1 & 7.3 & \begin{tabular}{|l|}
-9.9 \\
\end{tabular} & \begin{tabular}{|l|l} 
\\
\end{tabular} & 2.4 & 8.1 & -11.2 & 6.0 & \begin{tabular}{|l|}
-0.53 \\
\end{tabular} & \begin{tabular}{|l}
-1.52 \\
\end{tabular} & 951 & 0.262 \\
\hline 26 & -11.0 & $\begin{array}{l}-8.5 \\
\end{array}$ & 3.3 & 5.4 & -3.6 & -13.9 & 2.0 & 8.7 & \begin{tabular}{|l}
-29.8 \\
\end{tabular} & -13.0 & 8.2 & 7.5 & \begin{tabular}{|l|l}
-10.1 \\
\end{tabular} & \begin{tabular}{|l|l}
-11.4 \\
\end{tabular} & 2.5 & 8.2 & -11.3 & 6.1 & \begin{tabular}{|l|}
-0.54 \\
\end{tabular} & \begin{tabular}{|l}
-1.52 \\
\end{tabular} & 961 & 0.267 \\
\hline 27 & -11.1 & $\begin{array}{l}-8.6 \\
\end{array}$ & 3.3 & 5.5 & -3.6 & -14.0 & 2.1 & 8.8 & -30.2 & -13.3 & 8.3 & 7.6 & -10.2 & \begin{tabular}{|l|l}
-11.6 \\
\end{tabular} & 2.5 & 8.3 & -11.5 & 6.1 & \begin{tabular}{|l|}
-0.53 \\
\end{tabular} & -1.52 & 978 & \begin{tabular}{|l|l}
0.263 \\
\end{tabular} \\
\hline 28 & -11.3 & $\begin{array}{l}-8.8 \\
\end{array}$ & 3.3 & 5.6 & -3.9 & -14.4 & 2.1 & 8.8 & $\begin{array}{l}-30.5 \\
\end{array}$ & \begin{tabular}{|l|}
-13.5 \\
\end{tabular} & 8.5 & 7.8 & \begin{tabular}{|l|l}
-10.3 \\
\end{tabular} & \begin{tabular}{|l|l}
-11.7 \\
\end{tabular} & 2.5 & 8.4 & -11.7 & 6.2 & \begin{tabular}{|l|}
-0.53 \\
\end{tabular} & \begin{tabular}{|l}
-1.53 \\
\end{tabular} & 991 & 0.261 \\
\hline 29 & -11.6 & $\begin{array}{l}-8.9 \\
\end{array}$ & $\overline{3.4}$ & 5.7 & -4.1 & $\begin{array}{l}-14.7 \\
\end{array}$ & 2.1 & 8.9 & \begin{tabular}{|l}
-30.9 \\
\end{tabular} & -13.9 & 8.6 & 8.0 & -10.4 & \begin{tabular}{|l}
-11.8 \\
\end{tabular} & 2.6 & 8.6 & -11.9 & 6.3 & \begin{tabular}{|l|}
-0.53 \\
\end{tabular} & \begin{tabular}{|l|}
-1.53 \\
\end{tabular} & 1010 & 0.257 \\
\hline 30 & -11.6 & \begin{tabular}{l|l|}
-8.9 \\
\end{tabular} & 3.4 & 5.7 & -4.1 & -14.8 & 2.2 & 9.0 & \begin{tabular}{|l}
-31.3 \\
\end{tabular} & -14.2 & 8.7 & 8.1 & \begin{tabular}{|l|}
-10.6 \\
\end{tabular} & \begin{tabular}{|l|l}
-12.0 \\
\end{tabular} & 2.6 & 8.7 & -12.0 & 6.3 & \begin{tabular}{|l|}
-0.53 \\
\end{tabular} & \begin{tabular}{|l|}
-1.54 \\
\end{tabular} & \begin{tabular}{|l|l|}
1020 \\
\end{tabular} & 0.255 \\
\hline 31 & -11.8 & $\begin{array}{l}-9.0 \\
\end{array}$ & 3.5 & 5.8 & -4.2 & -15.2 & 2.2 & 9.1 & \begin{tabular}{|l}
-31.5 \\
\end{tabular} & -14.4 & 8.7 & 8.2 & -10.7 & \begin{tabular}{|l|}
-12.1 \\
\end{tabular} & 2.6 & 8.8 & -12.2 & 6.4 & \begin{tabular}{|l|}
-0.53 \\
\end{tabular} & -1.54 & \begin{tabular}{|l|l|}
1033 \\
\end{tabular} & \begin{tabular}{|l|}
0.253 \\
\end{tabular} \\
\hline 32 & -12.0 & $\begin{array}{l}-9.2 \\
\end{array}$ & 3.5 & 5.9 & -4.4 & -15.4 & 2.2 & 9.1 & -31.9 & -14.7 & 8.8 & 8.3 & -10.8 & \begin{tabular}{|l|}
-12.3 \\
\end{tabular} & 2.7 & 8.9 & -12.4 & 6.5 & \begin{tabular}{|l|}
-0.52 \\
\end{tabular} & -1.55 & \begin{tabular}{|l|}
1051 \\
\end{tabular} & \begin{tabular}{|l|}
0.248 \\
\end{tabular} \\
\hline 33 & -12.0 & -9.3 & 3.6 & 5.9 & -4.6 & -15.5 & 2.2 & 9.2 & \begin{tabular}{|l}
-32.1 \\
\end{tabular} & -14.8 & 9.0 & 8.6 & -11.0 & -12.5 & 2.7 & 9.0 & -12.5 & 6.6 & \begin{tabular}{|l|}
-0.52 \\
\end{tabular} & \begin{tabular}{|l|}
-1.55 \\
\end{tabular} & 1061 & 0.250 \\
\hline
\end{tabular}


Table E.10 KL 58-40 Test Data $\left(0^{\circ} \mathrm{C}\right.$ Tests), continued

\begin{tabular}{|c|c|c|c|c|c|c|c|c|c|c|c|c|c|c|c|c|c|c|c|c|c|c|}
\hline & & & & & & & & & & & & & & & & & & & & & & \\
\hline & & $58 / 40$ & $\mathrm{mple}$ & & $\overline{\mathrm{KL}}$ & $58 / 40 \mathrm{~s}$ & ample & & $\overline{\mathrm{KL}}$ & $58 / 40 \mathrm{~s}$ & Sample & $\# 12$ & & $58 / 40$ & Sample & & Trim & $\mathrm{med}$ & & & Creep & Pois. \\
\hline & vert & vert & horz & horz & vert & vert & horz & horz & vert & vert & horz & \begin{tabular}{|l} 
horz \\
\end{tabular} & vert & vert & horz & \begin{tabular}{|l|} 
horz \\
\end{tabular} & $\overline{\mathrm{Me}}$ & & Ratio & & Comp & \begin{tabular}{|l|} 
Ratio \\
\end{tabular} \\
\hline Time & front & back & front & back & front & back & front & back & front & back & front & back & front & back & front & back & vert & hol & $\mathrm{X} / \mathrm{Y}$ & $\overline{C_{t}}$ & $\mathrm{D}(\mathrm{t})$ & $v$ \\
\hline $\mathrm{sec}$ & $\mu \mathrm{m}$ & $\mu \mathrm{m}$ & $\mu \mathrm{m}$ & $\mu \mathrm{m}$ & $\mu \mathrm{m}$ & $\mu \mathrm{m}$ & $\mu \mathrm{m}$ & $\mu \mathrm{m}$ & $\mu \mathrm{m}$ & $\mu \mathrm{m}$ & $\mu \mathrm{m}$ & \begin{tabular}{|c|}
$\mu \mathrm{m}$ \\
\end{tabular} & $\mu \mathrm{m}$ & \begin{tabular}{|l|}
$\mu \mathrm{m}$ \\
\end{tabular} & $\mu \mathrm{m}$ & $\mu \mathrm{m}$ & $\mu \mathrm{m}$ & $\mu \mathrm{m}$ & & & $1 / \mathrm{kPa}$ & \\
\hline 34 & -12.5 & -9.9 & 3.6 & 5.9 & -4.8 & -15.8 & 2.3 & 9.3 & -32.4 & \begin{tabular}{|l|}
-15.1 \\
\end{tabular} & 9.1 & 8.6 & -11.1 & \begin{tabular}{|l}
-12.6 \\
\end{tabular} & 2.7 & 9.1 & -12.8 & 6.6 & -0.52 & -1.57 & 1082 & 0.239 \\
\hline 35 & \begin{tabular}{|l|}
-12.4 \\
\end{tabular} & -9.6 & 3.6 & - & -4.9 & \begin{tabular}{|l|}
-15.9 \\
\end{tabular} & 2.3 & $\begin{array}{l}9.4 \\
\end{array}$ & \begin{tabular}{|l}
-32.7 \\
\end{tabular} & \begin{tabular}{|l|}
-15.3 \\
\end{tabular} & 9.1 & 8.8 & -11.2 & \begin{tabular}{|l|}
-12.7 \\
\end{tabular} & 2.8 & 9.2 & \begin{tabular}{|l|}
-12.8 \\
\end{tabular} & 6.7 & -0.52 & -1.55 & 1087 & 0.246 \\
\hline 36 & -12.5 & $\begin{array}{l}-9.7 \\
\end{array}$ & 3.7 & 6.1 & -5.2 & -15.9 & 2.3 & 9.5 & \begin{tabular}{|l}
-33.0 \\
\end{tabular} & \begin{tabular}{|l}
-15.6 \\
\end{tabular} & 9.2 & 8.9 & -11.3 & \begin{tabular}{|l|}
-12.8 \\
\end{tabular} & 2. & 9.3 & -13.0 & 6.8 & -0.52 & -1.55 & 1098 & \begin{tabular}{|l|}
0.247 \\
\end{tabular} \\
\hline 37 & \begin{tabular}{|l}
-12.8 \\
\end{tabular} & -9.9 & 3.7 & 6.2 & -5.2 & \begin{tabular}{|l|}
-16.3 \\
\end{tabular} & 2.3 & $\begin{array}{l}9.5 \\
\end{array}$ & \begin{tabular}{|l}
-33.4 \\
\end{tabular} & \begin{tabular}{|l|}
-15.8 \\
\end{tabular} & 9.3 & 9.0 & -11.5 & \begin{tabular}{|l}
-12.9 \\
\end{tabular} & 2.8 & 9.4 & \begin{tabular}{|l|}
-13.2 \\
\end{tabular} & 6.8 & -0.52 & -1.56 & 1114 & 0.242 \\
\hline 38 & -12.9 & -9.9 & 3.7 & 6.2 & -5.3 & -16.4 & 2.4 & 9.6 & \begin{tabular}{|l}
-33.6 \\
\end{tabular} & $\mid-16.0$ & 9.4 & 9.2 & -11.7 & \begin{tabular}{|l|}
-13.3 \\
\end{tabular} & 2.8 & 9.4 & -13.4 & 6.9 & -0.51 & -1.57 & 1128 & \begin{tabular}{|l}
0.238 \\
\end{tabular} \\
\hline 39 & -13.1 & -10.2 & 3.8 & 6.3 & -5.4 & \begin{tabular}{|l|l}
-16.5 \\
\end{tabular} & 2.4 & 9.7 & $\mid-33.9$ & $\mid-16.2$ & 9.5 & 9.3 & -11.7 & \begin{tabular}{|l|}
-13.3 \\
\end{tabular} & 2.9 & 9.6 & \begin{tabular}{l|}
-13.5 \\
\end{tabular} & 7.0 & -0.52 & -1.56 & 1139 & 0.240 \\
\hline 40 & -13.2 & -10.3 & 3.8 & 6.3 & -5.4 & -16.5 & 2.4 & $\begin{array}{l}9.8 \\
\end{array}$ & \begin{tabular}{|l}
-34.3 \\
\end{tabular} & \begin{tabular}{|l|}
-16.4 \\
\end{tabular} & 9.6 & 9.5 & -11.8 & \begin{tabular}{|l}
-13.4 \\
\end{tabular} & 2.9 & 9.7 & $\mid-13.6$ & 7.0 & -0.52 & -1.56 & 1149 & 0.240 \\
\hline 41 & -13.3 & -10.3 & 3.8 & 6. & -5.5 & -16.8 & 2.4 & 9. & -34.5 & $\mid-16.6$ & 9.7 & 9.6 & -11.9 & \begin{tabular}{|l}
-13.5 \\
\end{tabular} & 2.9 & 9.7 & -13.7 & 7.1 & -0.51 & -1.57 & 1160 & \\
\hline 42 & \begin{tabular}{|l}
-13.4 \\
\end{tabular} & -10.5 & 3.9 & 6.5 & -5.6 & $\mid-17.0$ & 2.5 & 9.9 & \begin{tabular}{|l}
-34.8 \\
\end{tabular} & \begin{tabular}{|l|}
-16.9 \\
\end{tabular} & 9.8 & 9.7 & -12.0 & \begin{tabular}{|l}
-13.6 \\
\end{tabular} & 3.0 & 9.8 & \begin{tabular}{|c|}
-13.9 \\
\end{tabular} & 7. & -0.51 & -1.57 & 1173 & 0.236 \\
\hline 43 & \begin{tabular}{|l|}
-13.6 \\
\end{tabular} & $\begin{array}{l}-10.6 \\
\end{array}$ & 3.9 & 6.5 & -5.7 & -17.1 & 2.5 & 10.0 & \begin{tabular}{|l|}
-35.0 \\
\end{tabular} & \begin{tabular}{|l|}
-16.9 \\
\end{tabular} & 9.8 & 9.8 & -12.1 & \begin{tabular}{|l|}
-13.7 \\
\end{tabular} & 3.0 & 10.0 & $\mid-14.0$ & 7.2 & -0.51 & -1.57 & 1182 & 0.238 \\
\hline 44 & \begin{tabular}{|l}
-13.7 \\
\end{tabular} & -10.6 & 4.0 & 6.6 & -5.7 & \begin{tabular}{|l|l}
-17.2 \\
\end{tabular} & 2.5 & 10.1 & \begin{tabular}{|l}
-35.3 \\
\end{tabular} & \begin{tabular}{|l|l}
-17.2 \\
\end{tabular} & 9.9 & 10.0 & -12.3 & \begin{tabular}{|l|}
-13.8 \\
\end{tabular} & 3.0 & \begin{tabular}{|l|}
10.0 \\
\end{tabular} & \begin{tabular}{l|l}
-14.1 \\
\end{tabular} & 7. & -0.51 & -1.57 & 1193 & 0.236 \\
\hline 45 & -13.9 & \begin{tabular}{|l|}
-10.8 \\
\end{tabular} & 4.0 & 8.6 & -5.8 & -17.3 & 2.6 & 10.0 & \begin{tabular}{|l}
-35.5 \\
\end{tabular} & \begin{tabular}{|l}
-17.3 \\
\end{tabular} & 10.0 & 10.1 & -12.5 & \begin{tabular}{|l|}
-14.0 \\
\end{tabular} & 3.0 & \begin{tabular}{|l|}
10.1 \\
\end{tabular} & \begin{tabular}{|l|l} 
\\
\end{tabular} & 7. & -0.51 & \begin{tabular}{|l|l}
-1.58 \\
\end{tabular} & 1205 & \begin{tabular}{|l|}
0.233 \\
\end{tabular} \\
\hline 46 & \begin{tabular}{|l}
-13.9 \\
\end{tabular} & \begin{tabular}{|l|}
-10.9 \\
\end{tabular} & 4.0 & 6.7 & -5.9 & -17.4 & 2.6 & 10.1 & \begin{tabular}{|l}
-35.7 \\
\end{tabular} & \begin{tabular}{|l|l} 
\\
\end{tabular} & 10.1 & 10.2 & -12.5 & \begin{tabular}{|l}
-14.0 \\
\end{tabular} & 3. & \begin{tabular}{|l}
10.2 \\
\end{tabular} & -14.4 & 7. & -0.51 & -1.58 & 1216 & 0.233 \\
\hline 47 & \begin{tabular}{|l|l|}
-14. \\
\end{tabular} & $\mid-11.1$ & 4.1 & 6.7 & -5.9 & \begin{tabular}{|l|}
-17 \\
\end{tabular} & 2.6 & 10.2 & \begin{tabular}{|l|}
-35.9 \\
\end{tabular} & \begin{tabular}{|l|l} 
\\
\end{tabular} & 10.2 & 10.3 & -12.6 & \begin{tabular}{|l|l|}
-14.1 \\
\end{tabular} & 3. & \begin{tabular}{|l}
10.3 \\
\end{tabular} & -14.5 & 7. & -0.51 & -1.58 & 1224 & 0.233 \\
\hline 48 & -14.2 & -11.1 & 4.1 & 6.8 & -6.0 & -17.6 & 2.6 & 10.3 & \begin{tabular}{|l}
-36.2 \\
\end{tabular} & \begin{tabular}{|l|l|}
-18.0 \\
\end{tabular} & 10.2 & \begin{tabular}{|c|}
10.5 \\
\end{tabular} & -12.7 & \begin{tabular}{|l|l|}
-14. \\
\end{tabular} & 3.2 & \begin{tabular}{|l|l}
10.4 \\
\end{tabular} & \begin{tabular}{|l|}
-14.7 \\
\end{tabular} & 7.5 & -0.51 & -1.58 & 1237 & 0.233 \\
\hline 49 & -14.3 & \begin{tabular}{|l}
-11.3 \\
\end{tabular} & 4.1 & 6.9 & -6. & -17.6 & 2.7 & 10.4 & \begin{tabular}{|l}
-36.6 \\
\end{tabular} & \begin{tabular}{|l|l}
-18.4 \\
\end{tabular} & 10.3 & \begin{tabular}{|l|l}
10.5 \\
\end{tabular} & -12.8 & \begin{tabular}{|l|}
-14.4 \\
\end{tabular} & 3.1 & \begin{tabular}{|l}
10.5 \\
\end{tabular} & \begin{tabular}{|l}
-14.8 \\
\end{tabular} & 7. & -0.51 & \begin{tabular}{|l|l}
-1.58 \\
\end{tabular} & 1247 & \begin{tabular}{|l|}
0.233 \\
\end{tabular} \\
\hline 50 & $\begin{array}{l}-14.4 \\
\end{array}$ & \begin{tabular}{|l|}
-11.4 \\
\end{tabular} & 4.2 & 6. & -5 & \begin{tabular}{|l|l}
-17 \\
\end{tabular} & 2.7 & 10. & \begin{tabular}{|l}
-36 \\
\end{tabular} & \begin{tabular}{|l|l|}
-18.4 \\
\end{tabular} & 10.4 & 10.7 & -12.8 & \begin{tabular}{|l|l|}
-14. \\
\end{tabular} & 3. & \begin{tabular}{|l|l}
10.6 \\
\end{tabular} & \begin{tabular}{|l|l|}
-14.8 \\
\end{tabular} & 7 & -0.51 & \begin{tabular}{|l|}
-1.57 \\
\end{tabular} & 1252 & \begin{tabular}{|l|}
0.237 \\
\end{tabular} \\
\hline 51 & \begin{tabular}{|l}
-14.6 \\
\end{tabular} & $\mid-11.4$ & 4.2 & 7. & -6 & \begin{tabular}{|l|}
-17.7 \\
\end{tabular} & 2.7 & 10.5 & \begin{tabular}{|l}
-36.8 \\
\end{tabular} & \begin{tabular}{|l|l}
-18.5 \\
\end{tabular} & 10.4 & 10.8 & -13.1 & \begin{tabular}{|l|}
-14.7 \\
\end{tabular} & 3.2 & 10.6 & \begin{tabular}{|c|}
-15.0 \\
\end{tabular} & 7. & -0.51 & -1.58 & 1266 & \begin{tabular}{|l}
0.231 \\
\end{tabular} \\
\hline 52 & \begin{tabular}{|l|l|}
-14.7 \\
\end{tabular} & -11.6 & 4.2 & 7.0 & -6 & -17.6 & $\begin{array}{l}2.8 \\
\end{array}$ & 10.6 & \begin{tabular}{|l}
-37.1 \\
\end{tabular} & \begin{tabular}{|l|l} 
\\
\end{tabular} & 10.5 & \begin{tabular}{|l|}
10.9 \\
\end{tabular} & -13.2 & \begin{tabular}{|l|l|} 
\\
\end{tabular} & 3.2 & \begin{tabular}{|l}
10.7 \\
\end{tabular} & \begin{tabular}{|l|}
-15.1 \\
\end{tabular} & 7. & -0.51 & -1.58 & 1273 & 0.233 \\
\hline 53 & \begin{tabular}{|l}
-14.8 \\
\end{tabular} & -11.6 & 4.3 & 7.1 & -5 & \begin{tabular}{|l|}
-17.7 \\
\end{tabular} & 2.8 & 10.8 & \begin{tabular}{|l}
-37.3 \\
\end{tabular} & \begin{tabular}{|l|}
-19.0 \\
\end{tabular} & 10.6 & \begin{tabular}{|l|}
11.0 \\
\end{tabular} & -13.2 & \begin{tabular}{|l|}
-14.8 \\
\end{tabular} & 3.3 & \begin{tabular}{|l|}
10.8 \\
\end{tabular} & -15.2 & 7.8 & -0.51 & \begin{tabular}{|l|}
-1.57 \\
\end{tabular} & 1281 & 0.236 \\
\hline 54 & -14.8 & -11.6 & 4.3 & 7.1 & -5.9 & -17.8 & 2.8 & 10.8 & \begin{tabular}{|l}
-37.5 \\
\end{tabular} & -19.2 & 10.7 & \begin{tabular}{|l|}
11.1 \\
\end{tabular} & -13.5 & \begin{tabular}{|l|}
-15.1 \\
\end{tabular} & 3.3 & \begin{tabular}{|l}
10.8 \\
\end{tabular} & -15.3 & 7.8 & -0.51 & $\mid-1.58$ & 1293 & \begin{tabular}{|l|}
0.233 \\
\end{tabular} \\
\hline 55 & -14.9 & -11.7 & 4.3 & 7.2 & -5.8 & -17.8 & 2.9 & 10.9 & \begin{tabular}{|l}
-37.8 \\
\end{tabular} & \begin{tabular}{|l}
-19.3 \\
\end{tabular} & 10.7 & \begin{tabular}{|c|}
11.2 \\
\end{tabular} & -13.5 & \begin{tabular}{|l}
-15.0 \\
\end{tabular} & 3. & \begin{tabular}{|l}
11.0 \\
\end{tabular} & -15.4 & 1. & -0.51 & -1.57 & 1297 & \begin{tabular}{|l|}
0.237 \\
\end{tabular} \\
\hline 56 & -15.1 & $\mid-11.8$ & 4.3 & 7.2 & -5 & -17.8 & 2.9 & 10.9 & -38.0 & \begin{tabular}{|l}
-19.5 \\
\end{tabular} & 10.8 & \begin{tabular}{|l|}
11.4 \\
\end{tabular} & -13.6 & \begin{tabular}{|l|}
-15.2 \\
\end{tabular} & 3.3 & \begin{tabular}{|l|}
11.0 \\
\end{tabular} & -15.5 & 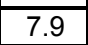 & -0.51 & -1.58 & 1308 & \begin{tabular}{|l|}
0.233 \\
\end{tabular} \\
\hline 57 & $\begin{array}{l}-15.2 \\
\end{array}$ & -12.0 & 4.4 & 7.3 & -5.9 & -17.9 & 3.0 & 11.0 & \begin{tabular}{|l}
-38.3 \\
\end{tabular} & \begin{tabular}{|l|l|}
-19.6 \\
\end{tabular} & 10.9 & \begin{tabular}{|c|}
11.4 \\
\end{tabular} & -13.6 & \begin{tabular}{|l|l|}
-15. \\
\end{tabular} & 3.4 & \begin{tabular}{|l|l}
11.1 \\
\end{tabular} & -15.6 & 8.0 & -0.51 & -1.57 & 1318 & 0.234 \\
\hline 58 & \begin{tabular}{|l}
-15.3 \\
\end{tabular} & -12.0 & 4.4 & 7.4 & -6.0 & $\mid-17.9$ & 3.0 & 11.1 & \begin{tabular}{|l}
-38.4 \\
\end{tabular} & \begin{tabular}{|l|}
-19.9 \\
\end{tabular} & 10.9 & \begin{tabular}{|l|}
11.6 \\
\end{tabular} & -13.7 & \begin{tabular}{|l}
-15.5 \\
\end{tabular} & 3.4 & \begin{tabular}{|l|}
11.2 \\
\end{tabular} & -15.7 & 8. & -0.51 & -1.57 & 1324 & 0.235 \\
\hline 59 & -15.3 & -12.0 & 4.4 & 7.4 & -6 & -18.1 & 3.0 & 11.2 & -38.6 & $\mid-20.1$ & 11.0 & \begin{tabular}{|l}
11.6 \\
\end{tabular} & -13.8 & -15.5 & 3.4 & \begin{tabular}{|l|}
11.3 \\
\end{tabular} & -15.8 & 8. & -0.51 & \begin{tabular}{|l|} 
\\
\end{tabular} & 1333 & \begin{tabular}{|l|l}
0.236 \\
\end{tabular} \\
\hline 60 & \begin{tabular}{|l}
-15.3 \\
\end{tabular} & $\mid-12.1$ & 4.5 & 7.4 & -6.0 & $\mid-18.1$ & 3.0 & 11.2 & \begin{tabular}{|l}
-38.7 \\
\end{tabular} & \begin{tabular}{|l|}
-20.1 \\
\end{tabular} & 11.1 & \begin{tabular}{|l|l}
11.8 \\
\end{tabular} & -13.9 & \begin{tabular}{|l}
-15.6 \\
\end{tabular} & 3.4 & \begin{tabular}{|l|}
11.3 \\
\end{tabular} & -15.9 & 8.2 & -0.51 & -1.57 & 1338 & \begin{tabular}{|l|l}
0.237 \\
\end{tabular} \\
\hline 61 & -15.5 & -12.2 & 4.5 & 7.5 & -6. & -18.3 & 3.0 & 11.2 & -38.9 & -20.3 & 11.1 & \begin{tabular}{|l|l|}
11.9 \\
\end{tabular} & -14.0 & \begin{tabular}{|l}
-15.7 \\
\end{tabular} & 3.4 & 11.4 & -16.0 & 8.2 & -0.51 & -1.57 & 1350 & 0.235 \\
\hline 62 & \begin{tabular}{|l}
-15.6 \\
\end{tabular} & -12.3 & 4.5 & 7.5 & -6 & \begin{tabular}{|l|}
-18.4 \\
\end{tabular} & 3.0 & 11.3 & \begin{tabular}{|l}
-39.2 \\
\end{tabular} & \begin{tabular}{|l}
-20.5 \\
\end{tabular} & 11.2 & \begin{tabular}{|l|}
12.0 \\
\end{tabular} & -14.1 & \begin{tabular}{|l}
-15.8 \\
\end{tabular} & 3.4 & \begin{tabular}{|l|}
11.4 \\
\end{tabular} & \begin{tabular}{|c|}
-16.1 \\
\end{tabular} & 8. & -0.51 & -1.57 & 1358 & 0.235 \\
\hline 63 & -15.7 & -12.3 & 4.6 & 7.6 & -6.5 & -18.5 & 3.1 & 11.4 & \begin{tabular}{|l}
-39.4 \\
\end{tabular} & $\mid-20.5$ & 11.3 & \begin{tabular}{|l|}
12.1 \\
\end{tabular} & -14.2 & \begin{tabular}{|l}
-16.0 \\
\end{tabular} & 3.5 & \begin{tabular}{|l|l}
11.5 \\
\end{tabular} & -16.2 & 8. & -0.51 & -1.57 & 1367 & \begin{tabular}{|l|}
0.235 \\
\end{tabular} \\
\hline 64 & -15.8 & -12.4 & 4.6 & 7.7 & -6.3 & -18.5 & 3.1 & 11.5 & -39.6 & $\mid-20.8$ & 11.3 & \begin{tabular}{|l|}
12.2 \\
\end{tabular} & -14.3 & $\mid-16.0$ & 3.5 & 11.6 & -16.3 & 8. & -0.51 & -1.57 & 1374 & \begin{tabular}{|l|l}
0.236 \\
\end{tabular} \\
\hline 65 & -15.8 & -12.3 & 4.6 & 7.7 & -6.3 & -18.8 & 3.1 & 11.6 & \begin{tabular}{|l}
-39.5 \\
\end{tabular} & $\mid-20.7$ & 11.4 & \begin{tabular}{|l|}
12.3 \\
\end{tabular} & -14.4 & $\mid-16.2$ & 3.5 & \begin{tabular}{|l|l}
11.7 \\
\end{tabular} & -16.4 & 8. & -0.51 & -1.57 & 1381 & \begin{tabular}{|l|}
0.238 \\
\end{tabular} \\
\hline 66 & 15.8 & -12.5 & 4.7 & 7.8 & -6.5 & $\mid-18.8$ & 3.2 & 11.6 & -39.9 & $\mid-21.1$ & 11.4 & \begin{tabular}{|l|}
12.3 \\
\end{tabular} & -14.4 & \begin{tabular}{|l}
-16.2 \\
\end{tabular} & 3.6 & 11.7 & -16.5 & 8.5 & -0.51 & -1.57 & 1389 & 0.23 \\
\hline
\end{tabular}


Table E.10 KL 58-40 Test Data $\left(0^{\circ} \mathrm{C}\right.$ Tests), continued

\begin{tabular}{|c|c|c|c|c|c|c|c|c|c|c|c|c|c|c|c|c|c|c|c|c|c|c|}
\hline & & & & & Norm & alized & Vertica & and $H$ & rizonta & IDeforr & nation & irrays & & & & & & & & & & \\
\hline & & $58 / 40$ & Sampl & & & $58 / 40 \mathrm{~s}$ & Sample & $\# 10$ & & $58 / 40 \mathrm{~s}$ & Sample & & & $58 / 40$ & Sample & & Trim & med & & & Creep & ois. \\
\hline & vert & vert & horz & horz & vert & vert & horz & horz & vert & vert & horz & horz & vert & \begin{tabular}{|l|} 
vert \\
\end{tabular} & horz & horz & $\bar{M}$ & & Ratio & & Comp & \begin{tabular}{|l|l|} 
Ratio \\
\end{tabular} \\
\hline Time & \begin{tabular}{|l|} 
front \\
\end{tabular} & back & front & back & \begin{tabular}{|l|} 
front \\
\end{tabular} & back & front & back & front & back & front & back & front & \begin{tabular}{|l|} 
back \\
\end{tabular} & front & back & vert & \begin{tabular}{|l|} 
horz \\
\end{tabular} & $\mathrm{X} / \mathrm{Y}$ & 0 & $\mathrm{D}(\mathrm{t})$ & $v$ \\
\hline $\mathrm{sec}$ & \begin{tabular}{|l|}
$\mu \mathrm{m}$ \\
\end{tabular} & $\mu \mathrm{m}$ & $\mu \mathrm{m}$ & $\mu \mathrm{m}$ & \begin{tabular}{|l|}
$\mu \mathrm{m}$ \\
\end{tabular} & $\mu \mathrm{m}$ & $\mu \mathrm{m}$ & $\mu \mathrm{m}$ & $\mu \mathrm{m}$ & $\mu \mathrm{m}$ & $\mu \mathrm{m}$ & $\mu \mathrm{m}$ & $\mu \mathrm{m}$ & $\mu \mathrm{m}$ & $\mu \mathrm{m}$ & $\mu \mathrm{m}$ & $\mu \mathrm{m}$ & $\mu \mathrm{m}$ & & & $1 / \mathrm{kPa}$ & \\
\hline 67 & -16.1 & -12.8 & 4.7 & $\begin{array}{l}7.8 \\
\end{array}$ & -6.6 & -18.8 & 3.2 & 11.7 & -40.0 & -21.4 & 11.5 & 12.4 & -14.6 & -16.3 & 3.6 & 11.8 & -16.7 & 8.5 & -0.51 & -1.58 & 1404 & 0.232 \\
\hline 68 & \begin{tabular}{|l|}
-16.0 \\
\end{tabular} & \begin{tabular}{|l|}
-12.5 \\
\end{tabular} & 4.7 & 7.9 & $\begin{array}{l}-6.6 \\
\end{array}$ & -18.9 & 3.2 & $\begin{array}{l}11.7 \\
\end{array}$ & \begin{tabular}{|l|}
-40.3 \\
\end{tabular} & -21.4 & 111.6 & 12.5 & -14.7 & \begin{tabular}{|l}
-16.4 \\
\end{tabular} & 3.6 & 11.9 & -16.7 & 8.6 & -0.51 & \begin{tabular}{|l|l|}
-1.57 \\
\end{tabular} & 1406 & 0.238 \\
\hline 69 & -16.1 & -12.6 & 4.8 & 8.0 & -6.7 & -19.0 & 3.2 & 11.7 & -40.5 & -21.7 & 11.6 & 12.6 & -14.8 & \begin{tabular}{|l|l}
-16.5 \\
\end{tabular} & 3.6 & 11.9 & -16.8 & 8.6 & -0.51 & -1.57 & 1415 & 0.236 \\
\hline 70 & \begin{tabular}{|l|}
-16.1 \\
\end{tabular} & -12.5 & 4.8 & 8.0 & $\begin{array}{l}-6.8 \\
\end{array}$ & -19.2 & 3.2 & $\begin{array}{l}11.8 \\
\end{array}$ & -40.7 & \begin{tabular}{|l|l} 
\\
\end{tabular} & 111.7 & $\begin{array}{l}12.7 \\
\end{array}$ & \begin{tabular}{|l}
-14.9 \\
\end{tabular} & \begin{tabular}{|l|}
-16.6 \\
\end{tabular} & 3.6 & 12.0 & -16.9 & 8.6 & -0.51 & -1.57 & 1422 & 0.236 \\
\hline 71 & \begin{tabular}{|l}
-16.2 \\
\end{tabular} & -12.7 & 4.8 & 8.1 & -6.7 & \begin{tabular}{|l|}
-19.2 \\
\end{tabular} & 3.2 & 11.9 & -40.8 & \begin{tabular}{|l|}
-22.0 \\
\end{tabular} & 11.8 & 12.8 & -15.0 & \begin{tabular}{|l}
-16.6 \\
\end{tabular} & 3.7 & 12.1 & -16.9 & 8.7 & -0.51 & -1.57 & 1430 & 0.238 \\
\hline 72 & -16.2 & -12.7 & 4.8 & 8.1 & -6.9 & -19.5 & 3.3 & 11.9 & -41.0 & $\mid-22.0$ & 11.8 & 12.9 & -15.0 & \begin{tabular}{|l|}
-16.8 \\
\end{tabular} & 3.7 & 12.1 & -17.0 & 8.7 & -0.51 & -1.57 & 1437 & \begin{tabular}{|l}
0.237 \\
\end{tabular} \\
\hline 73 & -16.2 & \begin{tabular}{|l}
-12.8 \\
\end{tabular} & 4.9 & 8.2 & -7.0 & \begin{tabular}{|l|}
-19.7 \\
\end{tabular} & 3.2 & 12.0 & -41.2 & \begin{tabular}{|l}
-22.2 \\
\end{tabular} & 11.8 & 13.0 & -15.1 & \begin{tabular}{|l}
-16.8 \\
\end{tabular} & 3.7 & 12.2 & -17.2 & 8.8 & -0.51 & -1.57 & 1447 & 0.235 \\
\hline 74 & -16.2 & \begin{tabular}{|l|l}
-12.8 \\
\end{tabular} & 4.9 & 8.2 & -7.2 & -19.9 & 3.3 & 12.0 & -41.4 & \begin{tabular}{|l|}
-22.4 \\
\end{tabular} & 11.9 & 13.1 & -15.2 & \begin{tabular}{|l|}
-16.9 \\
\end{tabular} & 3.7 & 12.3 & -17.2 & 8.8 & -0.51 & \begin{tabular}{|l|l}
-1.57 \\
\end{tabular} & 1454 & \begin{tabular}{|l}
0.236 \\
\end{tabular} \\
\hline 75 & \begin{tabular}{|l}
-16.3 \\
\end{tabular} & -13.0 & 4.9 & 8.3 & $\begin{array}{l}-7.2 \\
\end{array}$ & -20.0 & 3.3 & 12.1 & -41.5 & \begin{tabular}{|l}
-22.6 \\
\end{tabular} & 12.0 & 13.2 & \begin{tabular}{|l}
-15.3 \\
\end{tabular} & \begin{tabular}{|l|l|}
-17.0 \\
\end{tabular} & 3.8 & 12.3 & -17.3 & 8.9 & -0.51 & -1.57 & 1463 & 0.235 \\
\hline 76 & \begin{tabular}{|l}
-16.4 \\
\end{tabular} & -13.0 & 4.9 & 8.3 & \begin{tabular}{|l|}
-7.3 \\
\end{tabular} & \begin{tabular}{|l|}
-20.0 \\
\end{tabular} & 3.3 & 12.0 & -41.8 & \begin{tabular}{|l|}
-22.7 \\
\end{tabular} & 12.0 & 13.2 & \begin{tabular}{|l}
-15.5 \\
\end{tabular} & \begin{tabular}{|l|}
-17.1 \\
\end{tabular} & 3.8 & 12.4 & -17.5 & 8.9 & -0.51 & -1.58 & 1471 & 0.232 \\
\hline 77 & -16.4 & -13.0 & 5.0 & 8.4 & -7.5 & -20.0 & 3.3 & 12.1 & -42.0 & $\mid-22.8$ & 12.1 & 13.3 & -15.5 & \begin{tabular}{|l|}
-17.1 \\
\end{tabular} & 3.8 & 12.4 & -17.5 & 9.0 & -0.51 & -1.57 & 1475 & 0.235 \\
\hline 78 & \begin{tabular}{|l}
-16.5 \\
\end{tabular} & -13.1 & 5.0 & 8.4 & \begin{tabular}{|l|}
-7.6 \\
\end{tabular} & \begin{tabular}{|l|}
-20.3 \\
\end{tabular} & 3.3 & 12.2 & -42.1 & \begin{tabular}{|l|}
-22.9 \\
\end{tabular} & 12.1 & 13.4 & -15.6 & \begin{tabular}{|l|l}
-17.3 \\
\end{tabular} & 3.8 & 12.5 & -17.6 & 9.0 & -0.51 & -1.57 & 1485 & 0.234 \\
\hline 79 & -16.5 & \begin{tabular}{|l|}
-13.1 \\
\end{tabular} & 5.0 & 8.5 & -7.8 & -20.4 & 3.4 & 12.1 & -42.3 & -23.2 & 12.2 & 13.5 & -15.6 & \begin{tabular}{|l|}
-17.3 \\
\end{tabular} & 3.8 & 12.6 & -17.7 & 9.0 & -0.51 & \begin{tabular}{|l|l}
-1.58 \\
\end{tabular} & 1491 & \begin{tabular}{|l}
0.233 \\
\end{tabular} \\
\hline 80 & \begin{tabular}{|l}
-16.7 \\
\end{tabular} & -13.2 & 5.0 & 8.5 & -7.7 & \begin{tabular}{|l|}
-20.4 \\
\end{tabular} & 3.4 & 12.3 & -42.5 & \begin{tabular}{|l|}
-23.3 \\
\end{tabular} & 12.2 & 13.6 & \begin{tabular}{|l}
-15.7 \\
\end{tabular} & \begin{tabular}{|l|l|}
-17.4 \\
\end{tabular} & 3.8 & 12.7 & -17.8 & 9.1 & -0.51 & -1.57 & 1499 & 0.234 \\
\hline 81 & -16.7 & \begin{tabular}{|l}
-13.3 \\
\end{tabular} & 5.1 & 8.6 & -7.8 & -20.6 & 3.4 & 12.3 & -42.6 & -23.5 & 12.3 & $\begin{array}{l}13.7 \\
\end{array}$ & \begin{tabular}{|l}
-15.9 \\
\end{tabular} & \begin{tabular}{|l|}
-17.5 \\
\end{tabular} & 3.9 & 12.7 & -17.9 & 9.1 & -0.51 & \begin{tabular}{|l|l}
-1.58 \\
\end{tabular} & 1509 & \begin{tabular}{|l}
0.233 \\
\end{tabular} \\
\hline 82 & -16.9 & \begin{tabular}{|l}
-13.3 \\
\end{tabular} & 5.1 & 8.6 & -7.9 & -20.8 & 3.4 & 12.3 & -42.8 & -23.5 & 12.3 & 13.8 & -16.0 & \begin{tabular}{|l|l}
-17.6 \\
\end{tabular} & 3.9 & 12.8 & -18.0 & 9.2 & -0.51 & \begin{tabular}{|l|}
-1.58 \\
\end{tabular} & 1517 & \begin{tabular}{|l}
0.230 \\
\end{tabular} \\
\hline 83 & \begin{tabular}{|l|}
-16.8 \\
\end{tabular} & \begin{tabular}{|l|l}
-13.3 \\
\end{tabular} & 5.1 & 8.7 & \begin{tabular}{|l|}
-7.9 \\
\end{tabular} & \begin{tabular}{|l|}
-20.8 \\
\end{tabular} & 3.4 & $\begin{array}{l}12.4 \\
\end{array}$ & \begin{tabular}{|l|}
-42.9 \\
\end{tabular} & \begin{tabular}{|l|}
-23.7 \\
\end{tabular} & 12.4 & 13.9 & \begin{tabular}{|l|}
-16.1 \\
\end{tabular} & \begin{tabular}{|l|}
-17.6 \\
\end{tabular} & 3.9 & 12.8 & -18.0 & 9.2 & -0.51 & -1.57 & 1521 & 0.234 \\
\hline 84 & \begin{tabular}{|l}
-17.0 \\
\end{tabular} & \begin{tabular}{|l|}
-13.4 \\
\end{tabular} & 5.1 & 8.7 & -8.1 & -21.3 & 3.4 & 12.4 & -43.2 & \begin{tabular}{|l}
-23.9 \\
\end{tabular} & 12.5 & 13.9 & $\begin{array}{l}-16.1 \\
\end{array}$ & \begin{tabular}{|l|l}
-17.7 \\
\end{tabular} & 3.9 & 12.9 & -18.2 & 9.3 & -0.51 & -1.58 & 1534 & 0.229 \\
\hline 85 & \begin{tabular}{|l}
-16.9 \\
\end{tabular} & -13.3 & 5.2 & 8.8 & -8.0 & $\mid-21.1$ & 3.5 & 12.5 & -43.3 & $\mid-24.0$ & 12.5 & $\begin{array}{l}14.0 \\
\end{array}$ & $\begin{array}{l}-16.1 \\
\end{array}$ & \begin{tabular}{|l|}
-17.8 \\
\end{tabular} & 4.0 & 12.9 & -18.2 & 9.3 & -0.51 & \begin{tabular}{|l|}
-1.57 \\
\end{tabular} & 1533 & 0.234 \\
\hline 86 & \begin{tabular}{|l|}
-17.0 \\
\end{tabular} & \begin{tabular}{|l|}
-13.4 \\
\end{tabular} & 5.2 & 8.8 & -8.1 & -21.1 & 3.5 & 12.5 & \begin{tabular}{|l|}
-43.4 \\
\end{tabular} & \begin{tabular}{|l|l} 
\\
\end{tabular} & 12.6 & $\begin{array}{l}14.1 \\
\end{array}$ & \begin{tabular}{|l|l|} 
\\
\end{tabular} & \begin{tabular}{|l|l|}
-17.9 \\
\end{tabular} & 4.0 & 13.0 & -18.3 & 9.3 & -0.51 & \begin{tabular}{|l|}
-1.58 \\
\end{tabular} & 1541 & 0.233 \\
\hline 87 & \begin{tabular}{|l|l}
-17.1 \\
\end{tabular} & -13.4 & 5.2 & 8.8 & -8.4 & -21.3 & 3.5 & 12.5 & -43.6 & -24.2 & 12.6 & 14.2 & \begin{tabular}{|l|l}
-16.4 \\
\end{tabular} & \begin{tabular}{|l}
-18.0 \\
\end{tabular} & 4.0 & 13.0 & -18.4 & 9.4 & -0.51 & -1.58 & 1550 & 0.230 \\
\hline 88 & \begin{tabular}{|l|l|}
-17. \\
\end{tabular} & -13.5 & 5.3 & 8.9 & $\begin{array}{l}-8.2 \\
\end{array}$ & -21.3 & 3.6 & 12.6 & \begin{tabular}{|l|}
-43.7 \\
\end{tabular} & \begin{tabular}{|l} 
\\
\end{tabular} & 12.7 & $\begin{array}{l}14.3 \\
\end{array}$ & \begin{tabular}{|l|}
-16.4 \\
\end{tabular} & \begin{tabular}{|l|}
-18.0 \\
\end{tabular} & 4.0 & 13.1 & -18.4 & 9.4 & -0.51 & -1.58 & 1554 & 0.233 \\
\hline 89 & -17.2 & -13.6 & 5.3 & 8.9 & -8.3 & -21.3 & 3.6 & 12.7 & -43.9 & \begin{tabular}{|l}
-24.5 \\
\end{tabular} & 12.7 & 14.4 & -16.5 & \begin{tabular}{|l|}
-18.1 \\
\end{tabular} & 4.0 & 13.2 & -18.5 & 9.5 & -0.51 & \begin{tabular}{|l|}
-1.58 \\
\end{tabular} & 1562 & 0.232 \\
\hline 90 & -17.3 & $\mid-13.7$ & 5.3 & 9.0 & -8.3 & -21.4 & 3.6 & 12.7 & -44.2 & -24.7 & 12.7 & 14.4 & -16.5 & \begin{tabular}{|l|}
-18.3 \\
\end{tabular} & 4.0 & 13.2 & -18.6 & 9.5 & -0.51 & \begin{tabular}{|l|}
-1.58 \\
\end{tabular} & 1571 & 0.231 \\
\hline 91 & \begin{tabular}{|l|l} 
\\
\end{tabular} & \begin{tabular}{|l|}
-13.7 \\
\end{tabular} & 5.3 & 9.0 & -8.3 & -21.5 & 3.6 & 12.8 & -44.2 & \begin{tabular}{|l}
-24.9 \\
\end{tabular} & 12.8 & 14.5 & -16.6 & \begin{tabular}{|l}
-18.3 \\
\end{tabular} & 4.1 & 13.3 & -18.7 & 9.5 & -0.51 & \begin{tabular}{|l|}
-1.58 \\
\end{tabular} & 1577 & 0.232 \\
\hline 92 & -17.4 & $\mid-13.7$ & 5.4 & 9.1 & -8.5 & -21.6 & 3.6 & 12.8 & -44.4 & $\mid-25.0$ & 12.8 & $\begin{array}{l}14.6 \\
\end{array}$ & \begin{tabular}{|l}
-16.7 \\
\end{tabular} & \begin{tabular}{|l|}
-18.4 \\
\end{tabular} & 4.1 & 13.3 & -18.8 & 9.6 & -0.51 & -1.58 & 1583 & 0.231 \\
\hline 93 & $\begin{array}{l}-17.5 \\
\end{array}$ & \begin{tabular}{|l}
-13.9 \\
\end{tabular} & 5.4 & 9.1 & -8.6 & -21.8 & 3.6 & 12.8 & -44.5 & \begin{tabular}{|l}
-25.1 \\
\end{tabular} & 12.9 & $\begin{array}{l}14.7 \\
\end{array}$ & -16.7 & \begin{tabular}{|l|l|}
-18.5 \\
\end{tabular} & 4.1 & 13.4 & -18.9 & 9.6 & -0.51 & \begin{tabular}{|l|}
-1.58 \\
\end{tabular} & 1592 & 0.230 \\
\hline 94 & \begin{tabular}{|l}
-17.6 \\
\end{tabular} & $\mid-13.9$ & 5.4 & 9.1 & $\begin{array}{l}-8.7 \\
\end{array}$ & $\mid-21.8$ & 3.7 & 12.8 & -44.7 & \begin{tabular}{|l}
-25.3 \\
\end{tabular} & 13.0 & $\begin{array}{l}14.8 \\
\end{array}$ & -16.8 & \begin{tabular}{|l}
-18.6 \\
\end{tabular} & 4.1 & 13.4 & -19.0 & 9.6 & -0.51 & \begin{tabular}{|l|l} 
\\
\end{tabular} & 1599 & 0.228 \\
\hline 95 & -17.6 & -13.9 & 5.4 & $\overline{9.2}$ & -8.6 & -21.9 & 3.7 & 12.9 & -44.9 & $\mid-25.3$ & 13.0 & 14.8 & -16.9 & \begin{tabular}{|l}
-18.7 \\
\end{tabular} & - & 13.5 & -19.1 & 9.7 & -0.51 & \begin{tabular}{|l|}
-58 \\
\end{tabular} & 1604 & 0.230 \\
\hline 96 & \begin{tabular}{|l|}
-17.9 \\
\end{tabular} & \begin{tabular}{|l|l}
-14.1 \\
\end{tabular} & 5.4 & 9.2 & \begin{tabular}{|l}
-8.5 \\
\end{tabular} & \begin{tabular}{|l|l|}
-21. \\
\end{tabular} & 3.7 & 13.0 & \begin{tabular}{|l|}
-44.9 \\
\end{tabular} & \begin{tabular}{|l}
-25.5 \\
\end{tabular} & 13.1 & 14.9 & \begin{tabular}{|l|}
-17.0 \\
\end{tabular} & \begin{tabular}{|l|l|}
-18.7 \\
\end{tabular} & 4.1 & 13.5 & -19.2 & 9.7 & -0.51 & \begin{tabular}{|l|}
-1.58 \\
\end{tabular} & 1613 & 0.229 \\
\hline 97 & -17.8 & -14.1 & 5.5 & $\overline{9.2}$ & -8.7 & $\mid-22.0$ & 3.7 & 13.0 & -45.1 & -25.5 & 13.1 & 15.0 & -17.1 & \begin{tabular}{|l|}
-18.8 \\
\end{tabular} & 4.1 & 13.6 & -19.2 & 9.8 & -0.51 & -1.58 & 1617 & 0.230 \\
\hline 98 & -17.9 & -14.1 & 5.5 & 9.4 & -8.7 & \begin{tabular}{|l|}
-22.1 \\
\end{tabular} & 3.7 & 13.0 & -45.4 & \begin{tabular}{|l}
-25.8 \\
\end{tabular} & 13.1 & 15.0 & \begin{tabular}{|l|l} 
\\
\end{tabular} & \begin{tabular}{|l}
-18.9 \\
\end{tabular} & 4.2 & 13.6 & -19.3 & 9.8 & -0.51 & -1.58 & 1626 & 0.229 \\
\hline 99 & -17.9 & $\mid-14.2$ & 5.5 & 9.4 & -8.7 & -22.1 & 3.8 & 13.1 & -45.5 & -25.8 & 13.2 & 15.2 & -17.3 & \begin{tabular}{|l|}
-19.1 \\
\end{tabular} & 4.2 & 13.7 & -19.4 & 9.9 & -0.51 & $\mid-1.58$ & 1632 & 0.230 \\
\hline 100 & -17.9 & -14.2 & 5.5 & 9.4 & -8.7 & $\mid-21.9$ & 3.8 & 13.2 & -45.8 & $\mid-26.0$ & 13.2 & 15.2 & -17.3 & \begin{tabular}{|l}
-19.0 \\
\end{tabular} & 4.2 & 13.8 & -19.4 & 9.9 & -0.5 & -1.58 & 1634 & 0.23 \\
\hline
\end{tabular}


Table E.11 KL 58-40 Test Data $\left(-10{ }^{\circ} \mathrm{C}\right.$ Tests)

\begin{tabular}{|c|c|c|c|c|c|c|c|c|c|c|c|c|c|c|c|c|c|c|c|c|c|c|}
\hline & & & & & Norr & alized & ertica & and $\mathrm{Hc}$ & rizont & Deforn & lation & arays & & & & & & & & & & \\
\hline & & $58 / 40$ & $\overline{\mathrm{ample}}$ & & & $58 / 40$ & ample & $\# 10$ & & $58 / 40 \mathrm{~s}$ & ample & $\# 12$ & & $58 / 40$ & ample & & Trir & ned & & & Creep & Pois. \\
\hline & vert & vert & horz & horz & vert & vert & horz & horz & vert & vert & horz & horz & vert & vert & horz & horz & & & Ratio & & Comp & \begin{tabular}{|l|l|} 
Ratio \\
\end{tabular} \\
\hline Time & front & back & front & back & front & back & front & back & front & back & front & back & front & back & front & back & vert & horz & $\mathrm{X} / \mathrm{Y}$ & $\mathrm{C}_{\text {empl }}$ & $\mathrm{D}(\mathrm{t})$ & $v$ \\
\hline $\mathrm{sec}$ & $\mu \mathrm{m}$ & $\mu \mathrm{m}$ & $\mu \mathrm{m}$ & $\mu \mathrm{m}$ & $\mu \mathrm{m}$ & $\mu \mathrm{m}$ & $\mu \mathrm{m}$ & $\mu \mathrm{m}$ & $\mu \mathrm{m}$ & $\mu \mathrm{m}$ & $\mu \mathrm{m}$ & $\mu \mathrm{m}$ & $\mu \mathrm{m}$ & $\mu \mathrm{m}$ & $\mu \mathrm{m}$ & $\mu \mathrm{m}$ & $\mu \mathrm{m}$ & $\mu \mathrm{m}$ & & & $1 / \mathrm{kPa}$ & \\
\hline 0 & 0.0 & 0.0 & 0.0 & 0.0 & 0.0 & 0.0 & 0.0 & 0.0 & 0.0 & 0.0 & 0.0 & 0.0 & 0.0 & 0.0 & 0.0 & 0.0 & 0.0 & 0.0 & 0.00 & 0.00 & 0 & 0.000 \\
\hline 1 & -2.7 & -2.3 & 0.6 & 1.3 & -0.1 & -4.6 & 0.3 & 2.7 & -3.6 & -1.8 & 0.9 & 1.0 & -2.8 & -3.3 & 0.7 & 2.3 & -2.8 & 1.2 & -0.44 & -1.77 & 167 & 0.148 \\
\hline 2 & -3.0 & -2.6 & 0.8 & 1.5 & -0.5 & -5.5 & 0.4 & 3.0 & -4.2 & -2.2 & 1.1 & 1.2 & -3.4 & -4.0 & 0.8 & 2.8 & -3.2 & 1.4 & -0.43 & -1.80 & 196 & 0.139 \\
\hline 3 & $\begin{array}{l}-3.3 \\
\end{array}$ & -2.9 & 0.8 & 1.6 & -0.7 & -5.8 & 0.5 & 3.3 & -4.5 & -2.5 & 1.2 & 1.4 & -3.9 & -4.9 & 0.9 & 3.1 & -3.7 & 1.5 & -0.42 & -1.85 & 221 & 0.123 \\
\hline 4 & -3.4 & -2.9 & 0.9 & 1.7 & -0.7 & -6.1 & 0.5 & 3.5 & -4.8 & -2.7 & 1.3 & 1.5 & -4.0 & -4.9 & 1.0 & 3.4 & -3.8 & 1.6 & -0.43 & -1.80 & 229 & 0.140 \\
\hline 5 & -3.6 & -3.2 & 0.9 & 1.8 & $\begin{array}{l}-0.7 \\
\end{array}$ & -6.3 & 0.6 & 3.7 & -5.0 & \begin{tabular}{|c|}
-2.9 \\
\end{tabular} & 1.3 & 1.6 & -4.1 & $\begin{array}{c}-5.1 \\
\end{array}$ & 1.1 & 3.6 & -4.0 & 1.7 & -0.43 & -1.79 & 241 & 0.141 \\
\hline 6 & -3.7 & -3.2 & 1.0 & 1.9 & -1.0 & -6.6 & 0.6 & 3.8 & -5.2 & -3.1 & 1.4 & 1.6 & -4.5 & -5.3 & 1.2 & 3.8 & -4.1 & 1.8 & -0.43 & -1.79 & 250 & 0.141 \\
\hline 7 & -3.9 & -3.3 & 1.0 & 1.9 & -1.0 & -6.7 & 0.7 & 3.9 & -5.4 & -3.2 & 1.4 & 1.7 & -4.7 & -5.6 & 1.1 & 4.0 & -4.3 & 1.9 & -0.43 & -1.82 & 262 & 0.131 \\
\hline 8 & -3.9 & -3.3 & 1.0 & 2.0 & -1.2 & -7.0 & 0.7 & 4.0 & -5.5 & -3.3 & 1.5 & 1.7 & -4.8 & -5.7 & 1.2 & 4.1 & -4.4 & 1.9 & -0.43 & -1.80 & 267 & 0.139 \\
\hline 9 & -4.0 & -3.4 & 1.1 & 2.1 & -1.0 & -7.0 & 0.7 & 4.2 & -5.6 & -3.5 & 1.5 & 1.8 & -5.0 & -6.1 & 1.2 & 4.2 & -4.6 & 2.0 & -0.43 & -1.80 & 277 & 0.140 \\
\hline 10 & -4.2 & -3.4 & 1.1 & 2.2 & -1.1 & -7.1 & 0.8 & 4.3 & -5.7 & -3.5 & 1.6 & 1.9 & -5.1 & -6.2 & 1.3 & 4.3 & -4.7 & 2.1 & -0.44 & -1.78 & 283 & 0.146 \\
\hline 11 & -4.2 & -3.5 & 1.1 & 2.2 & -1.2 & -7.2 & 0.8 & 4.3 & -5.9 & -3.7 & 1.6 & 1.9 & -5.3 & -6.3 & 1.3 & 4.5 & -4.8 & 2.1 & -0.44 & -1.79 & 291 & 0.142 \\
\hline 12 & -4.3 & -3.7 & 1.2 & 2.2 & -1.1 & -7.4 & 0.8 & 4.4 & -5.9 & -3.7 & 1.7 & 2.0 & -5.3 & -6.6 & 1.4 & 4.6 & -4.9 & 2.1 & -0.44 & -1.79 & 297 & 0.144 \\
\hline 13 & -4.4 & -3.7 & 1.2 & 2.3 & -1.2 & -7.5 & 0.8 & 4.5 & -6.0 & -3.8 & 1.7 & 2.0 & -5.4 & -6.5 & 1.4 & 4.7 & -5.0 & 2.2 & -0.44 & -1.78 & 301 & 0.146 \\
\hline 14 & -4.5 & -3.7 & 1.2 & 2.3 & -1.2 & -7.5 & 0.9 & 4.6 & -6.1 & -3.9 & 1.7 & 2.1 & -5.5 & -6.7 & 1.4 & 4.8 & -5.1 & 2.2 & -0.44 & -1.78 & 307 & 0.147 \\
\hline 15 & -4.6 & -3.8 & 1.2 & 2.3 & -1.2 & -7.7 & 0.9 & 4.7 & -6.2 & -4.0 & 1.8 & 2.2 & -5.6 & -6.8 & 1.5 & 5.0 & -5.2 & 2.3 & -0.44 & -1.78 & 313 & 0.147 \\
\hline 16 & -4.6 & -3.8 & 1.3 & 2.4 & -1.2 & -7.7 & 0.9 & 4.7 & -6.3 & -4.0 & 1.8 & 2.2 & -5.7 & -7.0 & 1.5 & 5.0 & -5.2 & 2.3 & -0.44 & -1.77 & 317 & 0.148 \\
\hline 17 & -4.7 & -3.9 & 1.3 & 2.4 & -1.2 & -7.6 & 1.0 & 4.8 & -6.4 & -4.1 & 1.8 & 2.3 & -5.7 & -7.0 & 1.5 & 5.2 & -5.3 & 2.4 & -0.44 & -1.77 & 323 & 0.150 \\
\hline 18 & -4.8 & -3.9 & 1.3 & 2.5 & -1.4 & -7.9 & 1.0 & 4.9 & -6.6 & -4.2 & 1.8 & 2.3 & -6.1 & -7.6 & 1.5 & 5.2 & -5.5 & 2.4 & -0.43 & -1.81 & 334 & 0.134 \\
\hline 19 & -4.8 & -3.9 & 1.3 & 2.5 & -1.4 & -7.9 & 1.0 & 5.0 & -6.5 & -4.2 & 1.9 & 2.3 & -5.8 & -7.4 & 1.6 & 5.3 & -5.4 & 2.4 & -0.45 & -1.75 & 331 & 0.155 \\
\hline 20 & -4.9 & -4.1 & 1.3 & 2.5 & -1.5 & -8.0 & 1.0 & 5.0 & -6.6 & -4.3 & 1.9 & 2.4 & -6.0 & -7.4 & 1.6 & 5.4 & -5.5 & 2.4 & -0.44 & -1.77 & 337 & 0.149 \\
\hline 21 & -4.9 & -4.0 & 1.4 & 2.6 & -1.5 & -8.1 & 1.0 & 5.1 & -6.7 & -4.3 & 1.9 & 2.4 & -6.2 & -7.6 & 1.6 & 5.4 & -5.6 & 2.5 & -0.44 & -1.77 & 340 & 0.150 \\
\hline 22 & -4.9 & -4.1 & 1.4 & 2.6 & -1.5 & -8.3 & 1.1 & 5.1 & -6.7 & -4.4 & 1.9 & 2.4 & -6.2 & -7.7 & 1.6 & 5.5 & -5.7 & 2.5 & -0.44 & -1.76 & 345 & 0.152 \\
\hline 23 & -5.0 & -4.1 & 1.4 & 2.6 & -1.5 & -8.3 & 1.1 & 5.2 & -6.8 & -4.4 & 2.0 & 2.5 & -6.2 & -7.8 & 1.6 & 5.6 & -5.7 & 2.5 & -0.44 & -1.76 & 348 & 0.152 \\
\hline 24 & -5.1 & -4.1 & 1.4 & 2.6 & -1.5 & -8.4 & 1.1 & 5.2 & -6.9 & -4.5 & 2.0 & 2.5 & -6.4 & -7.9 & 1.7 & 5.7 & -5.8 & 2.6 & -0.44 & -1.77 & 354 & 0.148 \\
\hline 25 & -5.1 & -4.4 & 1.4 & 2.7 & -1.5 & -8.4 & 1.1 & 5.2 & -7.0 & -4.6 & 2.0 & 2.5 & -6.3 & -7.9 & 1.7 & 5.7 & -5.9 & 2.6 & -0.44 & -1.78 & 357 & 0.147 \\
\hline 26 & -5.2 & -4.2 & 1.4 & 2.7 & -1.7 & -8.4 & 1.1 & 5.3 & -7.0 & -4.7 & 2.0 & 2.6 & -6.3 & -8.1 & 1.7 & 5.8 & -5.9 & 2.6 & -0.45 & -1.76 & 360 & 0.153 \\
\hline 27 & -5.2 & -4.3 & 1.5 & 2.8 & -1.7 & -8.7 & 1.1 & 5.4 & -7.1 & -4.7 & 2.1 & 2.6 & -6.5 & -8.1 & 1.8 & 5.9 & -6.0 & 2.7 & -0.45 & -1.75 & 363 & 0.155 \\
\hline 28 & -5.3 & -4.3 & 1.5 & 2.8 & -1.6 & -8.7 & 1.2 & 5.4 & -7.3 & -4.7 & 2.1 & 2.6 & -6.6 & -8.3 & 1.7 & 5.9 & -6.1 & 2.7 & -0.44 & -1.77 & 369 & 0.148 \\
\hline 29 & -5.3 & -4.2 & 1.5 & 2.8 & -1.6 & -8.8 & 1.2 & 5.5 & -7.3 & -4.9 & 2.1 & 2.6 & -6.6 & -8.3 & 1.8 & 6.0 & -6.1 & 2.7 & -0.45 & -1.75 & 371 & 0.155 \\
\hline 30 & -5.4 & -4.4 & 1.5 & 2.8 & -1.8 & -8.9 & 1.2 & 5.5 & -7.3 & -4.8 & 2.1 & 2.7 & -6.8 & -8.4 & 1.8 & 6.1 & -6.2 & 2.7 & -0.44 & -1.76 & 375 & 0.151 \\
\hline 31 & -5.4 & -4.4 & 1.5 & 2.9 & -1.8 & -8.9 & 1.2 & 5.6 & -7.3 & -4.9 & 2.1 & 2.7 & -6.8 & -8.4 & 1.8 & 6.2 & -6.2 & 2.8 & -0.45 & -1.75 & 377 & 0.156 \\
\hline 32 & -5.3 & -4.5 & 1.5 & 2.9 & -1.8 & -9.0 & 1.2 & 5.6 & -7.4 & -4.9 & 2.1 & 2.7 & -6.9 & -8.6 & 1.8 & 6.2 & -6.3 & 2.8 & $\begin{array}{l}-0.44 \\
\end{array}$ & -1.76 & 381 & 0.152 \\
\hline 33 & -5.4 & -4.5 & 1.6 & 2.9 & -1.8 & -9.1 & 1.2 & 5.6 & -7.5 & -5.0 & 2.2 & 2.7 & -7.0 & -8.7 & 1.9 & 6.3 & -6.3 & 2.8 & -0.44 & -1.76 & 386 & 0.151 \\
\hline
\end{tabular}


Table E.11 KL 58-40 Test Data $\left(-10{ }^{\circ} \mathrm{C}\right.$ Tests), continued

\begin{tabular}{|c|c|c|c|c|c|c|c|c|c|c|c|c|c|c|c|c|c|c|c|c|c|c|}
\hline & & & & & & alized & ertical & & ontal & Deforr & ation & rrays & & & & & & & & & & \\
\hline & & $58 / 40$ & ampl & & $\overline{\mathrm{KL}}$ & $58 / 40 \mathrm{~s}$ & ample & & $\overline{\mathrm{KL}}$ & $88 / 40 \mathrm{~s}$ & ample & & & $58 / 40$ & Sample & & & $\mathrm{med}$ & & & Creep & Pois. \\
\hline & vert & vert & horz & horz & vert & vert & horz & horz & vert & vert & horz & horz & vert & vert & horz & \begin{tabular}{|l|} 
horz \\
\end{tabular} & & & Ratio & & Comp & \begin{tabular}{|l|} 
Ratio \\
\end{tabular} \\
\hline Time & front & back & front & back & front & back & front & back & front & back & front & back & front & back & \begin{tabular}{|l|l} 
front \\
\end{tabular} & back & vert & horz & $\mathrm{X} / \mathrm{Y}$ & $\mathrm{C}_{\mathrm{e}}$ & $\mathrm{D}(\mathrm{t})$ & $v$ \\
\hline $\mathrm{sec}$ & $\mu \mathrm{m}$ & $\mu \mathrm{m}$ & $\mu \mathrm{m}$ & $\mu \mathrm{m}$ & $\mu \mathrm{m}$ & $\mu \mathrm{m}$ & $\mu \mathrm{m}$ & $\mu \mathrm{m}$ & $\mu \mathrm{m}$ & $\mu \mathrm{m}$ & $\mu \mathrm{m}$ & $\mu \mathrm{m}$ & $\mu \mathrm{m}$ & \begin{tabular}{|l|}
$\mu \mathrm{m}$ \\
\end{tabular} & $\mu \mathrm{m}$ & $\mu \mathrm{m}$ & $\mu \mathrm{m}$ & $\mu \mathrm{m}$ & & & $1 / \mathrm{kPa}$ & \\
\hline 34 & -5.4 & -4.5 & 1.6 & 2.9 & -1.8 & -9.1 & 1.2 & 5.7 & -7.5 & -5.1 & 2.2 & 2.8 & -7.0 & -8.7 & 1.9 & 6.4 & -6.4 & 2.8 & -0.45 & -1.75 & 387 & 0.155 \\
\hline 35 & $\begin{array}{l}-5.4 \\
\end{array}$ & -4.5 & 1.6 & 3.0 & \begin{tabular}{|l|}
-1.9 \\
\end{tabular} & $\begin{array}{l}-9.2 \\
\end{array}$ & 1.3 & 5.8 & \begin{tabular}{|l|}
-7.6 \\
\end{tabular} & $\begin{array}{c}-5.2 \\
\end{array}$ & 2.2 & 2.8 & -7.1 & \begin{tabular}{|l|}
-8.8 \\
\end{tabular} & 1.9 & 6.4 & -6.4 & 2.9 & -0.45 & -1.76 & 392 & 0.153 \\
\hline 36 & -5.5 & -4.4 & 1.6 & 3.0 & -1.9 & -9.2 & 1.3 & 5.8 & \begin{tabular}{|l|}
-7.7 \\
\end{tabular} & -5.2 & 2.2 & 2.8 & -7.2 & -8.9 & 1.9 & 6.5 & -6.5 & 2.9 & -0.45 & \begin{tabular}{|l|}
-1.75 \\
\end{tabular} & 394 & \begin{tabular}{|l|}
0.156 \\
\end{tabular} \\
\hline 37 & $\begin{array}{l}-5.5 \\
\end{array}$ & -4.6 & 1.6 & 3.0 & \begin{tabular}{|l|}
-2.0 \\
\end{tabular} & \begin{tabular}{l|l|}
-9.4 \\
\end{tabular} & 1.3 & 5.8 & \begin{tabular}{|l|}
-7.7 \\
\end{tabular} & $\begin{array}{l}-5.3 \\
\end{array}$ & 2.2 & 2.9 & -7.2 & -9.0 & 1.9 & 6.5 & -6.5 & 2.9 & -0.45 & -1.76 & 398 & \begin{tabular}{|l|l|}
0.154 \\
\end{tabular} \\
\hline 38 & -5.6 & -4.6 & 1.6 & 3.0 & -2.1 & -9.6 & 1.3 & 5.8 & \begin{tabular}{|l}
-7.8 \\
\end{tabular} & -5.4 & 2.3 & 2.9 & \begin{tabular}{|l|}
-7.4 \\
\end{tabular} & -9.1 & 1.9 & 6.6 & -6.6 & 2.9 & -0.44 & -1.77 & 403 & \begin{tabular}{|l|l}
0.149 \\
\end{tabular} \\
\hline 39 & $\begin{array}{l}-5.6 \\
\end{array}$ & -4.7 & 1.6 & 3.0 & -2.0 & -9.4 & 1.3 & 5.9 & $\begin{array}{l}-7.9 \\
\end{array}$ & -5.5 & 2.3 & 2.9 & -7.4 & -9.1 & 2.0 & 6.6 & -6.7 & 3.0 & -0.44 & $\mid-1.77$ & 406 & 0.149 \\
\hline 40 & -5.7 & -4.7 & 1.7 & 3.1 & -2.0 & -9.4 & 1.3 & 6.0 & \begin{tabular}{|l|}
-7.9 \\
\end{tabular} & $\begin{array}{c}-5.4 \\
\end{array}$ & 2.3 & 2.9 & \begin{tabular}{|l|}
-7.4 \\
\end{tabular} & -9.1 & 2.0 & 6.7 & -6.7 & 3.0 & -0.45 & \begin{tabular}{|l|}
-1.75 \\
\end{tabular} & 406 & \begin{tabular}{|l|l}
0.156 \\
\end{tabular} \\
\hline 41 & -5.7 & -4.7 & 1.7 & 3.1 & -2.0 & -9.5 & 1.3 & 6. & \begin{tabular}{|l}
-8.0 \\
\end{tabular} & -5.6 & 2.3 & 2.9 & -7.6 & \begin{tabular}{|l|}
-9.3 \\
\end{tabular} & 2.0 & 6.7 & -6.8 & 3.0 & -0.44 & -1.78 & 414 & \begin{tabular}{|l|l}
0.146 \\
\end{tabular} \\
\hline 42 & -5.7 & -4.7 & 1.7 & 3.1 & -2.2 & -9.6 & 1.3 & 6.0 & -8.0 & \begin{tabular}{c|}
-5.6 \\
\end{tabular} & 2.3 & 3.0 & $\begin{array}{ll}-7.8 \\
\end{array}$ & -9.5 & 2.0 & 6.8 & -6.9 & 3.0 & -0.44 & -1.78 & 416 & \begin{tabular}{|l|l|}
0.147 \\
\end{tabular} \\
\hline 43 & $\begin{array}{l}-5.7 \\
\end{array}$ & $\begin{array}{l}-4.7 \\
\end{array}$ & 1.7 & 3.2 & -2.2 & -9.6 & 1.4 & 6.0 & \begin{tabular}{|l|l|}
-8.1 \\
\end{tabular} & -5.5 & 2.3 & 3.0 & -7.8 & $\begin{array}{l}-9.5 \\
\end{array}$ & 2.0 & 6.8 & -6.9 & 3.0 & -0.44 & \begin{tabular}{|l|l|}
-1.77 \\
\end{tabular} & 418 & $\begin{array}{l}0.150 \\
\end{array}$ \\
\hline 44 & $\begin{array}{l}-5.8 \\
\end{array}$ & -4.7 & 1.7 & 3.2 & -2.4 & -9.9 & 1.4 & 6.0 & \begin{tabular}{|l|}
-8.1 \\
\end{tabular} & $\begin{array}{l}-5.6 \\
\end{array}$ & 2.4 & 3.0 & -8.0 & -9.6 & 2.0 & 6.8 & -7.0 & 3.1 & -0.44 & \begin{tabular}{|l|}
-1.78 \\
\end{tabular} & 423 & \begin{tabular}{|l|}
0.145 \\
\end{tabular} \\
\hline 45 & -5.7 & -4.8 & 1.7 & 3.2 & -2.3 & -9.8 & 1.4 & 6.1 & \begin{tabular}{|l}
-8.2 \\
\end{tabular} & -5.7 & 2.4 & 3.1 & -8.1 & -9.8 & 2.0 & 6.9 & -7.0 & 3.1 & -0.44 & \begin{tabular}{|l|l}
-1.78 \\
\end{tabular} & 427 & \begin{tabular}{|l|}
0.145 \\
\end{tabular} \\
\hline 46 & $\begin{array}{l}-5.8 \\
\end{array}$ & -4.7 & 1.8 & 3.2 & -2.3 & -9.8 & 1.4 & 6. & \begin{tabular}{|l}
-8.2 \\
\end{tabular} & -5.7 & 2.4 & 3.1 & -8.0 & -9.6 & 2.1 & 7.0 & $\begin{array}{l}-7.0 \\
\end{array}$ & 3. & -0.44 & -1.77 & 426 & \begin{tabular}{|l|l}
0.151 \\
\end{tabular} \\
\hline 47 & $\begin{array}{l}-5.8 \\
\end{array}$ & $\begin{array}{l}-4.9 \\
\end{array}$ & 1.8 & 3.2 & $\begin{array}{l}-2.4 \\
\end{array}$ & -9.9 & 1.4 & 6.1 & \begin{tabular}{|l|l|}
-8.2 \\
\end{tabular} & -5.8 & 2.4 & 3.1 & -8.3 & $\begin{array}{l}-9.9 \\
\end{array}$ & 2.1 & 7.0 & $\begin{array}{l}-7.1 \\
\end{array}$ & 3. & -0.44 & -1.79 & 433 & 0.143 \\
\hline 48 & $\begin{array}{l}-5.8 \\
\end{array}$ & -4.8 & 1.8 & 3.2 & -2.4 & -10.0 & 1.4 & 6.2 & \begin{tabular}{|c|}
-8.2 \\
\end{tabular} & $\begin{array}{l}-5.7 \\
\end{array}$ & 2.4 & 3.1 & \begin{tabular}{|l|}
-8.3 \\
\end{tabular} & \begin{tabular}{|l}
-10.0 \\
\end{tabular} & 2.1 & 7.0 & \begin{tabular}{ll|}
-7.1 \\
\end{tabular} & 3.1 & -0.44 & \begin{tabular}{|l|}
-1.78 \\
\end{tabular} & 433 & \begin{tabular}{|l|l|}
0.147 \\
\end{tabular} \\
\hline 49 & -5.8 & -4.8 & 1.8 & 3.3 & -2.5 & -10.0 & 1.4 & 6.2 & \begin{tabular}{|l}
-8.3 \\
\end{tabular} & -5.8 & 2.4 & 3.2 & -8.4 & \begin{tabular}{|l|}
-10.3 \\
\end{tabular} & 2.1 & 7.0 & -7.2 & 3.2 & -0.44 & \begin{tabular}{|l|l|}
-1.79 \\
\end{tabular} & 439 & \begin{tabular}{|l|}
0.144 \\
\end{tabular} \\
\hline 50 & $\begin{array}{l}-5.9 \\
\end{array}$ & -4.9 & 1.8 & 3.3 & -2.6 & -10.0 & 1.4 & 6.2 & \begin{tabular}{|c|c|}
-8.4 \\
\end{tabular} & -5.9 & 2.5 & 3.2 & -8.3 & \begin{tabular}{|l}
-10.3 \\
\end{tabular} & 2.1 & 7.1 & -7.3 & 3. & -0.43 & \begin{tabular}{|l|l}
-1.79 \\
\end{tabular} & 441 & \begin{tabular}{|l|}
0.141 \\
\end{tabular} \\
\hline 51 & $\begin{array}{l}-5.9 \\
\end{array}$ & -5.0 & 1.8 & 3.3 & -2.6 & $\mid-10.2$ & 1.4 & 6.3 & $\begin{array}{l}-8.6 \\
\end{array}$ & -6.1 & 2.5 & 3.2 & -8.5 & \begin{tabular}{|l|l}
-10.4 \\
\end{tabular} & 2.1 & 7.1 & -7.4 & 3. & -0.43 & -1.82 & 448 & \begin{tabular}{|l|}
0.133 \\
\end{tabular} \\
\hline 52 & $\begin{array}{l}-5.9 \\
\end{array}$ & $\begin{array}{l}-4.9 \\
\end{array}$ & 1.8 & 3.3 & $\begin{array}{l}-2.6 \\
\end{array}$ & -10.3 & 1.4 & 6.3 & \begin{tabular}{|l}
-8.6 \\
\end{tabular} & $\begin{array}{l}-6.0 \\
\end{array}$ & 2.5 & 3.2 & $\begin{array}{l}-8.6 \\
\end{array}$ & \begin{tabular}{|l|l|l}
-10. \\
\end{tabular} & 2.2 & 7.2 & -7.4 & 3.2 & -0.43 & -1.80 & 448 & 0.139 \\
\hline 53 & $\begin{array}{l}-5.9 \\
\end{array}$ & -5.0 & 1.8 & 3.3 & -2.9 & -10.5 & 1.4 & 6.3 & \begin{tabular}{|c|}
-8.5 \\
\end{tabular} & $\begin{array}{l}-6.1 \\
\end{array}$ & 2.5 & 3.2 & -8.6 & \begin{tabular}{|l|l}
-10.4 \\
\end{tabular} & 2.1 & 7.2 & -7.4 & 3.2 & -0.43 & \begin{tabular}{|l|}
-1.81 \\
\end{tabular} & 450 & \begin{tabular}{|l|l}
0.137 \\
\end{tabular} \\
\hline 54 & -6.0 & -4.9 & 1.8 & 3.3 & -2.6 & -10.4 & 1.5 & 6.3 & \begin{tabular}{|l}
-8.7 \\
\end{tabular} & -6.2 & 2.5 & 3.2 & -8.7 & \begin{tabular}{|l|l}
-10.5 \\
\end{tabular} & 2.1 & 7.2 & -7.5 & 3.2 & -0.43 & \begin{tabular}{|l|}
-1.81 \\
\end{tabular} & 454 & \begin{tabular}{|l|}
0.137 \\
\end{tabular} \\
\hline 55 & -6.0 & -4.9 & 1.8 & 3.4 & \begin{tabular}{|c|}
-2.7 \\
\end{tabular} & -10.5 & 1.5 & 6.4 & \begin{tabular}{|l}
-8.7 \\
\end{tabular} & -6.2 & 2.5 & 3.3 & -8.8 & \begin{tabular}{|l}
-10.7 \\
\end{tabular} & 2.2 & 7.3 & -7.6 & 3. & -0.43 & \begin{tabular}{|l|}
-1.81 \\
\end{tabular} & 457 & \begin{tabular}{|l}
0.136 \\
\end{tabular} \\
\hline 56 & $\begin{array}{l}-6.1 \\
\end{array}$ & -5.0 & 1.9 & 3.4 & -2.7 & -10.5 & $1 . t$ & 6.4 & \begin{tabular}{|l}
-8.8 \\
\end{tabular} & -6.3 & 2.5 & 3.3 & -8.8 & \begin{tabular}{|l|}
-10.8 \\
\end{tabular} & 2.2 & 7.3 & -7.6 & 3 & -0.43 & -1.82 & 461 & \begin{tabular}{|l|}
0.134 \\
\end{tabular} \\
\hline 57 & $\begin{array}{l}-6.1 \\
\end{array}$ & -5.0 & 1.9 & 3.4 & -2.8 & -10.6 & 1.5 & 6.4 & \begin{tabular}{|l|l}
-8.7 \\
\end{tabular} & $\begin{array}{l}-6.3 \\
\end{array}$ & 2.5 & 3.3 & -8.8 & \begin{tabular}{|l}
-10.6 \\
\end{tabular} & 2.2 & 7.4 & $\begin{array}{l}-7.6 \\
\end{array}$ & 3.3 & -0.43 & \begin{tabular}{|l|l}
-1.80 \\
\end{tabular} & 460 & \begin{tabular}{|l|l}
0.139 \\
\end{tabular} \\
\hline 58 & \begin{tabular}{l|l}
-6.1 \\
\end{tabular} & -5.0 & 1.9 & 3.4 & -2.8 & \begin{tabular}{|l|}
-10.6 \\
\end{tabular} & 1.5 & 6.5 & \begin{tabular}{|l|}
-8.9 \\
\end{tabular} & $\begin{array}{l}-6.3 \\
\end{array}$ & 2.5 & 3.3 & -8.9 & \begin{tabular}{|l|}
-10.8 \\
\end{tabular} & 2.2 & 7.4 & -7.7 & 3.3 & -0.43 & -1.80 & 463 & \begin{tabular}{|l|}
0.138 \\
\end{tabular} \\
\hline 59 & -6.1 & -5.0 & 1.9 & 3.5 & -2.9 & -10.7 & 1.5 & 6.5 & -8.9 & -6.5 & 2.6 & 3.3 & -8.9 & -10.8 & 2.2 & 7.5 & -7.7 & 3.3 & -0.43 & -1.81 & 465 & \begin{tabular}{|l|l}
0.137 \\
\end{tabular} \\
\hline 60 & $\begin{array}{l}-6.2 \\
\end{array}$ & -5.1 & 1.9 & 3.5 & \begin{tabular}{|l|}
-2.9 \\
\end{tabular} & $\mid-10.7$ & 1.5 & 6.5 & \begin{tabular}{|l|}
-9.0 \\
\end{tabular} & $\begin{array}{l}-6.6 \\
\end{array}$ & 2.6 & 3.3 & -9.0 & \begin{tabular}{|l|}
-10.8 \\
\end{tabular} & 2.2 & 7.5 & -7.8 & 3.3 & -0.43 & -1.82 & 470 & \begin{tabular}{|l|}
0.134 \\
\end{tabular} \\
\hline 61 & $\begin{array}{l}-6.2 \\
\end{array}$ & -5.2 & 1.9 & 3.5 & -2.9 & \begin{tabular}{|l|}
-10.7 \\
\end{tabular} & 1.5 & 6. & -8.9 & -6.5 & 2.6 & 3.4 & -9.0 & \begin{tabular}{|l|l|}
-10. \\
\end{tabular} & 2.3 & 7.6 & $\begin{array}{l}-7.8 \\
\end{array}$ & 3.4 & -0.43 & -1.80 & 470 & 0.139 \\
\hline 62 & $\begin{array}{l}-6.2 \\
\end{array}$ & $\begin{array}{l}-5.1 \\
\end{array}$ & 1.9 & 3.5 & -3.1 & \begin{tabular}{|l|}
-10.8 \\
\end{tabular} & 1.6 & 6.5 & \begin{tabular}{|l|l}
-9.0 \\
\end{tabular} & $\begin{array}{l}-6.6 \\
\end{array}$ & 2.6 & 3.4 & -9.1 & \begin{tabular}{|l|l}
-10.8 \\
\end{tabular} & 2.3 & 7.7 & -7.8 & 3.4 & -0.43 & -1.80 & 472 & \begin{tabular}{|l|}
0.140 \\
\end{tabular} \\
\hline 63 & -6.2 & -5.0 & 2.0 & 3.5 & -3.1 & -10.9 & 1.5 & 6.5 & \begin{tabular}{|l}
-9.0 \\
\end{tabular} & -6.6 & 2.6 & 3.4 & -9.1 & \begin{tabular}{|l}
-11.0 \\
\end{tabular} & 2.3 & 7.7 & -7.8 & 3.4 & -0.43 & $\mid-1.80$ & 474 & \begin{tabular}{|l|l}
0.139 \\
\end{tabular} \\
\hline 64 & -6.2 & -5.2 & 2.0 & 3.5 & -3.4 & -11.2 & 1.5 & 6.5 & -9. & -6.7 & 2.6 & 3.4 & -9.1 & $\mid-11.0$ & 2.3 & 7.7 & -7.9 & 3.4 & -0.43 & -1.81 & 477 & \begin{tabular}{|l|l}
0.137 \\
\end{tabular} \\
\hline 65 & -6.2 & -5.2 & 2.0 & 3.6 & \begin{tabular}{|l|}
-3.1 \\
\end{tabular} & -11.0 & 1.6 & 6.6 & \begin{tabular}{|l}
-9.2 \\
\end{tabular} & -6.7 & 2.6 & 3.4 & -9.2 & $\mid-11.2$ & 2.3 & 7.8 & -8.0 & 3.4 & -0.43 & \begin{tabular}{|l|}
-1.81 \\
\end{tabular} & 480 & \begin{tabular}{|l|}
0.135 \\
\end{tabular} \\
\hline 66 & -6.3 & -5.3 & 2.0 & 3.6 & -3.1 & -11.1 & 1.6 & 6.6 & \begin{tabular}{|l|}
-9.1 \\
\end{tabular} & -6.7 & 2.7 & 3.4 & -9.1 & -11.1 & 2.3 & 7.8 & -8.0 & 3.4 & -0.43 & -1.80 & 481 & \begin{tabular}{|l|}
0.138 \\
\end{tabular} \\
\hline
\end{tabular}


Table E.11 KL 58-40 Test Data $\left(-10{ }^{\circ} \mathrm{C}\right.$ Tests $)$, continued

\begin{tabular}{|c|c|c|c|c|c|c|c|c|c|c|c|c|c|c|c|c|c|c|c|c|c|c|}
\hline & \multicolumn{16}{|c|}{ Normalized Vertical and Horizontal Deformation Arrays } & \multirow{2}{*}{\multicolumn{2}{|c|}{ Trimmed }} & & & & \\
\hline & & $58 / 40$ & Sample & & & $58 / 40 \mathrm{~s}$ & ample & $\$ 10$ & & $58 / 40$ & ample & $\$ 12$ & & $58 / 40$ & ample & & & & & & Creep & Pois. \\
\hline & vert & vert & horz & horz & vert & vert & horz & horz & vert & vert & horz & horz & vert & vert & horz & horz & & & Ratio & & Comp & Ratio \\
\hline Time & front & back & front & back & front & back & front & back & front & back & front & back & front & back & front & back & vert & horz & $\mathrm{X} / \mathrm{Y}$ & $\mathrm{C}_{\mathrm{empl}}$ & $\mathrm{D}(\mathrm{t})$ & $\bar{v}$ \\
\hline $\sec$ & $\mu \mathrm{m}$ & $\mu \mathrm{m}$ & $\mu \mathrm{m}$ & $\mu \mathrm{m}$ & $\mu \mathrm{m}$ & $\mu \mathrm{m}$ & $\mu \mathrm{m}$ & $\mu \mathrm{m}$ & $\mu \mathrm{m}$ & $\mu \mathrm{m}$ & $\mu \mathrm{m}$ & $\mu \mathrm{m}$ & $\mu \mathrm{m}$ & $\mu \mathrm{m}$ & $\mu \mathrm{m}$ & $\mu \mathrm{m}$ & $\mu \mathrm{m}$ & $\mu \mathrm{m}$ & & & $1 / \mathrm{kPa}$ & \\
\hline 67 & -6.4 & -5.3 & 2.0 & 3.6 & -3.0 & -10.8 & 1.6 & 6.7 & -9.3 & -6.8 & 2.7 & 3.5 & -9.2 & -11.1 & 2.3 & 7.9 & -8.0 & 3.5 & -0.43 & -1.81 & 485 & 0.137 \\
\hline 68 & -6.4 & -5.4 & 2.0 & 3.6 & -3.2 & -11.2 & 1.6 & 6.7 & -9.3 & -6.9 & 2.7 & 3.4 & -9.2 & -11.2 & 2.4 & 7.9 & -8.1 & 3.5 & -0.43 & -1.81 & 487 & 0.136 \\
\hline 69 & -6.3 & -5.2 & 2.0 & 3.6 & -3.1 & -11.2 & 1.6 & 6.7 & -9.3 & -6.9 & 2.7 & 3.5 & -9.4 & -11.2 & 2.4 & 8.0 & -8.1 & 3.5 & -0.43 & -1.80 & 487 & 0.138 \\
\hline 70 & -6.4 & -5.3 & 2.0 & 3.7 & -3.2 & -11.3 & 1.6 & 6.7 & -9.3 & -6.9 & 2.7 & 3.5 & -9.1 & -11.2 & 2.4 & 8.0 & -8.0 & 3.5 & -0.44 & -1.79 & 487 & 0.142 \\
\hline 71 & -6.4 & -5.3 & 2.0 & 3.7 & -3.3 & -11.4 & 1.6 & 6.7 & -9.4 & -6.9 & 2.7 & 3.5 & -9.3 & -11.2 & 2.4 & 8.1 & -8.1 & 3.5 & -0.43 & -1.80 & 490 & 0.140 \\
\hline 72 & -6.4 & -5.4 & 2.0 & 3.7 & -3.3 & -11.4 & 1.6 & 6.8 & -9.4 & -6.9 & 2.7 & 3.5 & -9.2 & -11.2 & 2.4 & 8.1 & -8.1 & 3.5 & -0.44 & -1.79 & 491 & 0.142 \\
\hline 73 & -6.5 & -5.4 & 2.0 & 3.7 & -3.1 & -11.3 & 1.7 & 6.9 & -9.6 & -7.0 & 2.7 & 3.6 & -9.3 & -11.2 & 2.5 & 8.2 & -8.2 & 3.6 & -0.44 & -1.79 & 494 & 0.142 \\
\hline 74 & -6.4 & -5.4 & 2.1 & 3.7 & -3.2 & -11.5 & 1.7 & 6.8 & -9.6 & -7.0 & 2.7 & 3.6 & -9.3 & -11.2 & 2.5 & 8.2 & -8.2 & 3.6 & -0.44 & -1.79 & 495 & 0.144 \\
\hline 75 & -6.4 & -5.4 & 2.1 & 3.7 & -3.3 & -11.6 & 1.7 & 6.8 & -9.6 & -7.0 & 2.8 & 3.6 & -9.4 & -11.3 & 2.5 & 8.2 & -8.2 & 3.6 & -0.44 & $\begin{array}{l}-1.79 \\
\end{array}$ & 497 & 0.143 \\
\hline 76 & -6.5 & -5.4 & 2.1 & 3.8 & -3.4 & -11.6 & 1.7 & 6.9 & -9.6 & -7.2 & 2.8 & 3.6 & -9.3 & -11.5 & 2.5 & 8.3 & -8.3 & 3.6 & -0.44 & -1.78 & 500 & 0.145 \\
\hline 77 & -6.6 & -5.4 & 2.1 & 3.8 & -3.4 & -11.6 & 1.7 & 6.9 & -9.7 & -7.1 & 2.8 & 3.7 & -9.3 & -11.3 & 2.5 & 8.3 & -8.2 & 3.6 & -0.44 & -1.78 & 499 & 0.145 \\
\hline 78 & -6.5 & -5.4 & 2.1 & 3.8 & -3.4 & -11.6 & 1.7 & 7.0 & -9.7 & -7.2 & 2.8 & 3.7 & -9.3 & -11.3 & 2.5 & 8.4 & -8.2 & 3.7 & -0.44 & -1.76 & 500 & 0.152 \\
\hline 79 & -6.6 & -5.4 & 2.1 & 3.8 & -3.5 & -11.7 & 1.7 & 6.9 & -9.7 & -7.2 & 2.8 & 3.7 & -9.2 & -11.5 & 2.5 & 8.4 & -8.3 & 3.6 & -0.44 & -1.78 & 502 & 0.146 \\
\hline 80 & -6.6 & -5.4 & 2.1 & 3.8 & -3.5 & -11.8 & 1.7 & 7.0 & -9.7 & -7.2 & 2.8 & 3.7 & -9.1 & -11.2 & 2.6 & 8.5 & -8.2 & 3.7 & -0.45 & -1.76 & 500 & 0.154 \\
\hline 81 & -6.7 & -5.4 & 2.1 & 3.8 & -3.5 & -11.7 & 1.7 & 7.0 & -9.9 & -7.4 & 2.8 & 3.7 & -9.3 & -11.6 & 2.6 & 8.5 & -8.4 & 3.7 & -0.44 & -1.78 & 508 & 0.145 \\
\hline 82 & -6.6 & -5.5 & 2.1 & 3.8 & -3.4 & -11.8 & 1.8 & 7.0 & -9.8 & -7.3 & 2.8 & 3.8 & -9.3 & -11.3 & 2.6 & 8.5 & -8.3 & 3.7 & -0.45 & -1.76 & 504 & 0.154 \\
\hline 83 & -6.6 & -5.5 & 2.1 & 3.9 & -3.6 & -11.8 & 1.7 & 7.1 & -9.9 & -7.3 & 2.8 & 3.8 & -9.2 & -11.3 & 2.6 & 8.6 & -8.3 & 3.7 & -0.45 & -1.75 & 505 & 0.156 \\
\hline 84 & -6.6 & -5.5 & 2.1 & 3.9 & -3.6 & -11.9 & 1.7 & 7.1 & -9.8 & -7.4 & 2.9 & 3.8 & -9.1 & -11.3 & 2.6 & 8.6 & -8.3 & 3.7 & -0.45 & -1.74 & 506 & 0.159 \\
\hline 85 & -6.7 & -5.5 & 2.1 & 3.9 & -3.6 & -11.9 & 1.8 & 7.1 & -9.9 & -7.4 & 2.9 & 3.8 & -9.2 & -11.3 & 2.7 & 8.7 & -8.3 & 3.7 & -0.45 & -1.75 & 508 & 0.158 \\
\hline 86 & -6.7 & -5.6 & 2.2 & 3.9 & -3.6 & -11.9 & 1.8 & 7.1 & -9.9 & -7.4 & 2.9 & 3.8 & -9.3 & -11.3 & 2.7 & 8.8 & -8.3 & 3.8 & -0.45 & -1.74 & 509 & 0.160 \\
\hline 87 & -6.8 & -5.6 & 2.2 & 3.9 & -3.6 & -12.1 & 1.8 & 7.1 & -10.0 & -7.5 & 2.9 & 3.8 & -9.3 & -11.6 & 2.7 & 8.8 & -8.4 & 3.8 & -0.45 & -1.75 & 514 & 0.155 \\
\hline 88 & -6.7 & -5.7 & 2.2 & 3.9 & $\begin{array}{l}-3.7 \\
\end{array}$ & -12.0 & 1.8 & 7.2 & -10.0 & $\begin{array}{l}-7.4 \\
\end{array}$ & 2.9 & 3.9 & -9.2 & -11.3 & 2.7 & 8.9 & -8.4 & 3.8 & -0.45 & -1.74 & 512 & 0.161 \\
\hline 89 & -6.8 & -5.7 & 2.2 & 4.0 & -3.7 & -12.0 & 1.8 & 7.2 & -10.0 & -7.5 & 2.9 & 3.9 & -9.3 & -11.5 & 2.7 & 8.9 & -8.5 & 3.8 & -0.45 & -1.74 & 516 & 0.159 \\
\hline 90 & -6.8 & -5.6 & 2.2 & 4.0 & -3.7 & -12.1 & 1.8 & 7.2 & -10.0 & -7.5 & 2.9 & 3.9 & -9.4 & -11.6 & 2.7 & 8.9 & -8.5 & 3.8 & -0.45 & -1.74 & 517 & 0.161 \\
\hline 91 & -6.7 & -5.6 & 2.2 & 4.0 & -3.7 & -12.1 & 1.8 & 7.2 & -10.1 & -7.5 & 2.9 & 3.9 & -9.3 & -11.6 & 2.7 & 8.9 & -8.5 & 3.8 & -0.45 & -1.73 & 517 & 0.163 \\
\hline 92 & -6.9 & -5.6 & 2.2 & 4.0 & -3.7 & -12.2 & 1.8 & 7.2 & \begin{tabular}{|l|}
-10.2 \\
\end{tabular} & -7.7 & 2.9 & 3.9 & -9.3 & -11.7 & 2.7 & 9.0 & -8.5 & 3.8 & -0.45 & -1.75 & 521 & 0.157 \\
\hline 93 & -6.8 & -5.6 & 2.2 & 4.1 & -3.7 & -12.1 & 1.8 & 7.3 & \begin{tabular}{|l|}
-10.2 \\
\end{tabular} & -7.6 & 3.0 & 4.0 & -9.4 & -11.7 & 2.7 & 9.0 & -8.5 & 3.9 & -0.45 & -1.73 & 520 & 0.163 \\
\hline 94 & -6.8 & -5.6 & 2.2 & 4.1 & -3.8 & -12.2 & 1.8 & 7.3 & \begin{tabular}{|l|}
-10.2 \\
\end{tabular} & -7.6 & 3.0 & 4.0 & -9.5 & -11.7 & 2.8 & 9.1 & -8.5 & 3.9 & -0.45 & -1.73 & 522 & 0.162 \\
\hline 95 & -6.9 & -5.7 & 2.2 & 4.1 & -3.7 & -12.3 & 1.8 & 7.3 & \begin{tabular}{|l|}
-10.1 \\
\end{tabular} & -7.6 & 3.0 & 4.0 & -9.5 & -11.7 & 2.8 & 9.1 & $\begin{array}{l}-8.6 \\
\end{array}$ & 3.9 & -0.45 & -1.73 & 524 & 0.164 \\
\hline 96 & -6.8 & -5.7 & 2.2 & 4.1 & -3.8 & $\begin{array}{l}-12.3 \\
\end{array}$ & 1.9 & 7.3 & -10.2 & -7.6 & 3.0 & 4.0 & -9.4 & -11.8 & 2.8 & 9.1 & -8.6 & 3.9 & -0.46 & $\begin{array}{l}-1.73 \\
\end{array}$ & 524 & 0.164 \\
\hline 97 & -6.9 & -5.7 & 2.3 & 4.1 & -3.8 & -12.2 & 1.9 & 7.4 & -10.1 & -7.6 & 3.0 & 4.0 & -9.5 & -11.9 & 2.8 & 9.2 & -8.6 & 3.9 & -0.46 & -1.73 & 526 & 0.164 \\
\hline 98 & -6.8 & -5.7 & 2.3 & 4.1 & -3.6 & -12.3 & 1.9 & 7.4 & \begin{tabular}{|l|l}
-10.2 \\
\end{tabular} & -7.6 & 3.0 & 4.0 & -9.5 & -11.9 & 2.8 & 9.2 & -8.6 & 3.9 & -0.46 & -1.72 & 527 & 0.166 \\
\hline 99 & -6.9 & -5.8 & 2.3 & 4.1 & -3.6 & -12.2 & 1.9 & 7.4 & -10.2 & -7.7 & 3.0 & 4.1 & -9.5 & -11.9 & 2.8 & 9.2 & -8.7 & 4.0 & -0.46 & -1.72 & 529 & 0.166 \\
\hline 100 & -6.9 & -5.8 & 2.3 & 4.1 & -3.7 & -12.1 & 1.9 & 7.5 & -10.2 & -7.7 & 3.0 & 4.1 & -9.6 & -11.9 & 2.8 & 9.2 & -8.7 & 4.0 & -0.46 & -1.72 & 531 & 0.166 \\
\hline
\end{tabular}


Table E.12 KL 58-40 Test Data $\left(-20{ }^{\circ} \mathrm{C}\right.$ Tests)

\begin{tabular}{|c|c|c|c|c|c|c|c|c|c|c|c|c|c|c|c|c|c|c|c|c|c|c|}
\hline & \multicolumn{16}{|c|}{ Normalized Vertical and Horizontal Deformation Arrays } & \multirow{2}{*}{\multicolumn{2}{|c|}{ Trimmed }} & \multirow[b]{3}{*}{ Ratio } & & \multirow{3}{*}{\begin{tabular}{|l|} 
Creep \\
Comp \\
\end{tabular}} & \multirow{3}{*}{\begin{tabular}{|l|} 
Pois. \\
Ratio \\
\end{tabular}} \\
\hline & \multicolumn{4}{|c|}{ KL 58/40 Sample \#1 } & \multicolumn{4}{|c|}{ KL 58/40 Sample \#10 } & \multicolumn{4}{|c|}{ KL 58/40 Sample \#12 } & \multicolumn{4}{|c|}{ KL 58/40 Sample \#8 } & & & & & & \\
\hline & vert & vert & horz & horz & vert & vert & horz & horz & vert & vert & horz & horz & vert & vert & horz & horz & & & & & & \\
\hline Time & front & back & front & back & front & back & front & back & front & back & front & back & front & back & front & back & vert & horz & $\mathrm{X} / \mathrm{Y}$ & $\mathrm{C}_{\mathrm{empl}}$ & $\mathrm{D}(\mathrm{t})$ & $\bar{v}$ \\
\hline $\mathrm{sec}$ & $\mu \mathrm{m}$ & $\mu \mathrm{m}$ & $\mu \mathrm{m}$ & $\mu \mathrm{m}$ & $\mu \mathrm{m}$ & $\mu \mathrm{m}$ & $\mu \mathrm{m}$ & $\mu \mathrm{m}$ & $\mu \mathrm{m}$ & $\mu \mathrm{m}$ & $\mu \mathrm{m}$ & $\mu \mathrm{m}$ & $\mu \mathrm{m}$ & $\mu \mathrm{m}$ & $\mu \mathrm{m}$ & $\mu \mathrm{m}$ & $\mu \mathrm{m}$ & $\mu \mathrm{m}$ & & & $1 / \mathrm{kPa}$ & \\
\hline 0 & 0.0 & 0.0 & 0.0 & 0.0 & 0.0 & 0.0 & 0.0 & 0.0 & 0.0 & 0.0 & 0.0 & 0.0 & 0.0 & 0.0 & 0.0 & 0.0 & 0.0 & 0.0 & 0.00 & 0.00 & 0 & 0.000 \\
\hline 1 & -2.4 & -2.2 & 0.6 & 1.1 & -1.2 & -4.9 & 0.5 & 2.5 & -3.8 & -1.4 & 1.0 & 1.0 & -2.8 & -3.7 & 0.7 & 2.2 & -2.7 & 1.1 & -0.42 & -1.85 & 83 & 0.123 \\
\hline 2 & -3.7 & -3.1 & 1.0 & 1.8 & -1.5 & -5.3 & 0.6 & 2.7 & -5.0 & -2.3 & 1.2 & 1.4 & -3.0 & -4.1 & 0.8 & 2.5 & -3.5 & 1.5 & -0.42 & -1.86 & 108 & 0.122 \\
\hline 3 & -3.9 & -3.3 & 1.0 & 2.0 & -1.5 & -5.5 & 0.7 & 2.9 & -5.3 & -2.5 & 1.3 & 1.5 & -3.3 & -4.3 & 0.9 & 2.6 & -3.8 & 1.6 & -0.43 & -1.81 & 115 & 0.135 \\
\hline 4 & -4.1 & -3.5 & 1.1 & 2.1 & -1.6 & -5.7 & 0.7 & 3.0 & -5.5 & -2.8 & 1.4 & 1.6 & -3.5 & -4.6 & 0.9 & 2.8 & -4.0 & 1.7 & -0.42 & -1.84 & 121 & 0.126 \\
\hline 5 & -4.3 & -3.6 & 1.1 & 2.2 & -1.9 & -6.2 & 0.7 & 3.0 & -5.7 & -3.0 & 1.4 & 1.7 & -3.5 & -4.7 & 0.9 & 2.9 & -4.1 & 1.7 & -0.42 & -1.85 & 125 & 0.125 \\
\hline 6 & -4.4 & -3.7 & 1.2 & 2.3 & -1.9 & -6.0 & 0.8 & 3.2 & -5.8 & -3.0 & 1.4 & 1.7 & -3.7 & -4.9 & 0.9 & 3.0 & -4.2 & 1.8 & -0.42 & -1.84 & 129 & 0.127 \\
\hline 7 & -4.5 & -3.9 & 1.2 & 2.3 & \begin{tabular}{|c|}
-1.8 \\
\end{tabular} & -6.2 & 0.8 & 3.3 & -5.8 & -3.1 & 1.5 & 1.8 & -3.7 & -4.9 & 1.0 & 3.1 & -4.3 & 1.8 & -0.43 & -1.82 & 132 & 0.132 \\
\hline 8 & -4.7 & -4.0 & 1.2 & 2.3 & -1.8 & -6.2 & 0.8 & 3.3 & -5.9 & -3.2 & 1.5 & 1.8 & -3.7 & -5.1 & 1.0 & 3.1 & -4.4 & 1.9 & -0.42 & -1.85 & 135 & 0.124 \\
\hline 9 & -4.8 & -4.1 & 1.2 & 2.4 & -2.2 & $\begin{array}{l}-6.4 \\
\end{array}$ & 0.8 & 3.4 & -6.1 & -3.3 & 1.6 & 1.9 & -3.9 & -4.9 & 1.0 & 3.2 & -4.5 & 1.9 & -0.42 & -1.84 & 137 & 0.127 \\
\hline 10 & -4.8 & -4.2 & 1.3 & 2.4 & -2.0 & -6.4 & 0.9 & 3.5 & -6.2 & -3.4 & 1.6 & 1.9 & -4.0 & -5.2 & 1.0 & 3.3 & -4.6 & 1.9 & -0.42 & -1.85 & 140 & 0.123 \\
\hline 11 & -4.9 & -4.3 & 1.3 & 2.4 & -2.0 & -6.5 & 0.9 & 3.5 & -6.2 & -3.4 & 1.6 & 2.0 & -3.9 & $\begin{array}{c}-5.4 \\
\end{array}$ & 1.0 & 3.3 & -4.7 & 2.0 & -0.42 & -1.85 & 143 & 0.125 \\
\hline 12 & -5.1 & -4.4 & 1.3 & 2.4 & -2.1 & -6.5 & 0.9 & 3.6 & -6.3 & -3.5 & 1.6 & 2.0 & -3.9 & -5.1 & 1.1 & 3.4 & -4.7 & 2.0 & $\begin{array}{l}-0.43 \\
\end{array}$ & -1.82 & 144 & 0.133 \\
\hline 13 & -5.2 & -4.5 & 1.3 & 2.5 & -2.0 & -6.6 & 0.9 & 3.6 & -6.3 & -3.6 & 1.7 & 2.1 & -4.0 & -5.4 & 1.1 & 3.4 & -4.8 & 2.0 & -0.42 & -1.84 & 147 & 0.127 \\
\hline 14 & -5.2 & -4.5 & 1.3 & 2.5 & -2.2 & -6.8 & 0.9 & 3.7 & -6.5 & -3.7 & 1.7 & 2.1 & -4.1 & -5.5 & 1.1 & 3.5 & -4.9 & 2.1 & -0.42 & -1.85 & 149 & 0.123 \\
\hline 15 & -5.4 & $\begin{array}{l}-4.6 \\
\end{array}$ & 1.3 & 2.5 & \begin{tabular}{|c|}
-2.3 \\
\end{tabular} & -6.7 & 0.9 & 3.7 & -6.5 & -3.7 & 1.7 & 2.1 & -4.1 & -5.5 & 1.1 & 3.5 & -5.0 & 2.1 & -0.42 & -1.83 & 151 & 0.128 \\
\hline 16 & -5.4 & -4.7 & 1.4 & 2.5 & -2.3 & -6.8 & 0.9 & 3.8 & -6.5 & -3.7 & 1.7 & 2.1 & -4.1 & -5.6 & 1.1 & 3.6 & -5.0 & 2.1 & -0.42 & -1.83 & 152 & 0.129 \\
\hline 17 & -5.5 & -4.8 & 1.4 & 2.6 & -2.3 & -6.9 & 0.9 & 3.9 & -6.6 & -3.7 & 1.8 & 2.2 & -4.2 & -5.7 & 1.1 & 3.6 & -5.1 & 2.1 & -0.42 & -1.84 & 154 & 0.127 \\
\hline 18 & -5.6 & -4.8 & 1.4 & 2.6 & -2.3 & -6.9 & 1.0 & 3.9 & -6.6 & -3.8 & 1.8 & 2.2 & -4.2 & -5.8 & 1.2 & 3.7 & -5.1 & 2.2 & -0.42 & -1.84 & 156 & 0.125 \\
\hline 19 & -5.6 & -4.9 & 1.4 & 2.6 & -2.3 & -7.0 & 1.0 & 3.9 & -6.7 & -3.8 & 1.8 & 2.2 & -4.3 & -5.7 & 1.2 & 3.7 & -5.2 & 2.2 & -0.42 & -1.83 & 157 & 0.128 \\
\hline 20 & -5.7 & -4.9 & 1.4 & 2.7 & -2.4 & -7.0 & 1.0 & 3.9 & -6.7 & -3.8 & 1.8 & 2.2 & -4.4 & -5.8 & 1.2 & 3.7 & -5.2 & 2.2 & -0.42 & -1.84 & 158 & 0.125 \\
\hline 21 & \begin{tabular}{|l|}
-5.7 \\
\end{tabular} & -5.0 & 1.4 & 2.7 & -2.4 & -7.0 & 1.0 & 4.0 & -6.8 & -3.8 & 1.8 & 2.2 & -4.4 & -5.9 & 1.2 & 3.8 & -5.3 & 2.2 & -0.42 & -1.84 & 160 & 0.126 \\
\hline 22 & -5.7 & -5.0 & 1.4 & 2.7 & -2.4 & -7.1 & 1.0 & 4.0 & -6.9 & -3.9 & 1.8 & 2.2 & -4.3 & -5.9 & 1.2 & 3.8 & -5.3 & 2.2 & -0.42 & -1.85 & 161 & 0.125 \\
\hline 23 & \begin{tabular}{|l|}
-5.9 \\
\end{tabular} & -5.0 & 1.4 & 2.7 & -2.4 & -7.1 & 1.0 & 4.0 & -7.0 & -4.1 & 1.8 & 2.3 & -4.4 & -5.9 & 1.2 & 3.8 & -5.4 & 2.2 & -0.42 & -1.85 & 163 & 0.122 \\
\hline 24 & \begin{tabular}{|l|}
-5.8 \\
\end{tabular} & -5.0 & 1.4 & 2.7 & -2.3 & -7.2 & 1.0 & 4.1 & -7.1 & -4.3 & 1.8 & 2.2 & -4.4 & -5.9 & 1.2 & 3.9 & -5.4 & 2.3 & -0.42 & -1.86 & 164 & 0.121 \\
\hline 25 & \begin{tabular}{|l|}
-5.9 \\
\end{tabular} & -5.1 & 1.5 & 2.7 & -2.4 & -7.3 & 1.0 & 4.1 & -7.0 & -4.0 & 1.9 & 2.3 & -4.4 & -6.0 & 1.2 & 3.9 & -5.4 & 2.3 & -0.42 & -1.83 & 164 & 0.129 \\
\hline 26 & -6.0 & -5.1 & 1.5 & 2.8 & -2.5 & -7.3 & 1.0 & 4.1 & -7.0 & -4.1 & 1.9 & 2.3 & -4.5 & -6.2 & 1.2 & 3.9 & -5.5 & 2.3 & -0.42 & -1.85 & 166 & 0.125 \\
\hline 27 & \begin{tabular}{|l|}
-5.9 \\
\end{tabular} & -5.1 & 1.5 & 2.8 & -2.4 & -7.3 & 1.1 & 4.2 & -7.0 & -4.1 & 1.9 & 2.3 & -4.6 & -6.1 & 1.2 & 4.0 & -5.5 & 2.3 & -0.42 & -1.83 & 166 & 0.130 \\
\hline 28 & \begin{tabular}{|l|}
-5.9 \\
\end{tabular} & -5.0 & 1.5 & 2.8 & -2.5 & -7.3 & 1.1 & 4.2 & -7.0 & -4.2 & 1.9 & 2.4 & -4.4 & -6.2 & 1.3 & 4.1 & -5.4 & 2.4 & -0.43 & -1.80 & 166 & 0.139 \\
\hline 29 & \begin{tabular}{|l|}
-5.9 \\
\end{tabular} & -5.0 & 1.5 & 2.9 & -2.4 & -7.3 & 1.1 & 4.2 & -7.1 & -4.2 & 1.9 & 2.4 & -4.5 & -6.0 & 1.3 & 4.0 & -5.4 & 2.4 & -0.43 & -1.80 & 166 & 0.140 \\
\hline 30 & -5.9 & -5.0 & 1.5 & 2.9 & -2.5 & -7.4 & 1.1 & 4.2 & -7.3 & -4.3 & 1.9 & 2.4 & -4.5 & -6.2 & 1.3 & 4.1 & -5.5 & 2.4 & -0.43 & -1.81 & 168 & 0.134 \\
\hline 31 & \begin{tabular}{|l|}
-5.9 \\
\end{tabular} & -4.9 & 1.6 & 2.9 & -2.5 & -7.3 & 1.1 & 4.2 & -7.4 & -4.5 & 1.9 & 2.4 & -4.6 & -6.2 & 1.4 & 4.1 & -5.6 & 2.4 & -0.43 & -1.82 & 170 & 0.134 \\
\hline 32 & \begin{tabular}{|l|}
-5.9 \\
\end{tabular} & -5.1 & 1.6 & 3.0 & -2.5 & -7.5 & 1.1 & 4.2 & -7.3 & -4.3 & 2.0 & 2.4 & -4.6 & -6.3 & 1.3 & 4.1 & -5.6 & 2.4 & -0.43 & -1.81 & 170 & 0.137 \\
\hline 33 & -5.9 & -5.0 & 1.6 & 3.0 & -2.5 & -7.5 & 1.1 & 4.2 & -7.3 & -4.4 & 2.0 & 2.4 & -4.6 & -6.3 & 1.3 & 4.2 & -5.6 & 2.4 & -0.43 & -1.80 & 170 & 0.139 \\
\hline
\end{tabular}


Table E.12 KL 58-40 Test Data $\left(-20^{\circ} \mathrm{C}\right.$ Tests), continued

\begin{tabular}{|c|c|c|c|c|c|c|c|c|c|c|c|c|c|c|c|c|c|c|c|c|c|c|}
\hline & & & & & & & & ( & 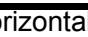 & & & rays & & & & & & & & & & \\
\hline & & $58 / 40$ & Sample & & $\overline{\mathrm{KL}}$ & $58 / 40$ & ample & & $\overline{\mathrm{KL}}$ & $58 / 40 \leqq$ & ample & & & $58 / 40$ & Sample & & Trim & med & & & Creep & \begin{tabular}{|l} 
Pois. \\
\end{tabular} \\
\hline & vert & vert & horz & \begin{tabular}{|l} 
horz \\
\end{tabular} & \begin{tabular}{|l|} 
vert \\
\end{tabular} & vert & horz & \begin{tabular}{|l|} 
horz \\
\end{tabular} & \begin{tabular}{|l|} 
vert \\
\end{tabular} & vert & horz & \begin{tabular}{|l} 
horz \\
\end{tabular} & \begin{tabular}{|l|} 
vert \\
\end{tabular} & \begin{tabular}{|l|} 
vert \\
\end{tabular} & horz & \begin{tabular}{|l|} 
horz \\
\end{tabular} & $\overline{\mathrm{Me}}$ & ean & Ratio & & Comp & \begin{tabular}{|l|l|} 
Ratio \\
\end{tabular} \\
\hline Time & front & back & front & back & front & back & front & back & \begin{tabular}{|l|} 
front \\
\end{tabular} & back & front & \begin{tabular}{|l|} 
back \\
\end{tabular} & \begin{tabular}{|l|} 
front \\
\end{tabular} & back & front & \begin{tabular}{|l|} 
back \\
\end{tabular} & vert & horz & $\mathrm{X} / \mathrm{Y}$ & $\mathrm{C}_{\text {empl }}$ & $\mathrm{D}(\mathrm{t})$ & \\
\hline $\mathrm{sec}$ & $\mu \mathrm{m}$ & $\mu \mathrm{m}$ & $\mu \mathrm{m}$ & $\mu \mathrm{m}$ & $\mu \mathrm{m}$ & $\mu \mathrm{m}$ & $\mu \mathrm{m}$ & $\mu \mathrm{m}$ & $\mu \mathrm{m}$ & $\mu \mathrm{m}$ & $\mu \mathrm{m}$ & $\mu \mathrm{m}$ & $\mu \mathrm{m}$ & $\mu \mathrm{m}$ & $\mu \mathrm{m}$ & $\mu \mathrm{m}$ & $\mu \mathrm{m}$ & \begin{tabular}{|l|}
$\mu \mathrm{m}$ \\
\end{tabular} & & & $1 / \mathrm{kPa}$ & \\
\hline 34 & -5.9 & -5.1 & 1.6 & 3.0 & -2.5 & -7.6 & 1.1 & 4.3 & -7.4 & -4.4 & 2.0 & 2.5 & -4.6 & -6.4 & 1.3 & 4.1 & -5.6 & 2.4 & -0.43 & -1.80 & 172 & 0.140 \\
\hline 35 & -5.9 & -5.0 & 1.6 & 3.0 & -2.5 & -7.6 & 1.1 & 4.4 & -7.4 & -4.4 & 2.0 & 2.5 & -4.9 & -6.5 & 1.3 & 4.2 & -5.7 & 2.5 & -0.43 & -1.79 & 174 & (14 \\
\hline 36 & -6.0 & -5.0 & 1.6 & 3.1 & -2.8 & -7.7 & 1.1 & 4.4 & -7.5 & -4.4 & 2.0 & 2.5 & -4.7 & -6.4 & 1.4 & 4.2 & -5.7 & 2.5 & -0.44 & -1.78 & 173 & \begin{tabular}{|l|l}
0.147 \\
\end{tabular} \\
\hline 37 & -6.0 & -5.1 & 1.6 & 3.1 & -2.6 & -7.7 & 1.1 & 4.4 & -7.5 & -4.5 & 2.0 & 2.5 & -4.9 & -6.5 & 1.3 & 4.2 & \begin{tabular}{|l|}
-5.7 \\
\end{tabular} & 2.5 & -0.43 & -1.80 & 175 & \begin{tabular}{|l|l}
0.140 \\
\end{tabular} \\
\hline 38 & -6.0 & -5.1 & 1.6 & 3.1 & -2.6 & -7.7 & 1.1 & 4.4 & \begin{tabular}{|l}
-7.5 \\
\end{tabular} & -4.5 & 2.0 & 2.5 & -4.9 & -6.4 & 1.3 & 4.2 & \begin{tabular}{|l}
-5.7 \\
\end{tabular} & 2.5 & -0.43 & -1.79 & 175 & 0.141 \\
\hline 39 & -6.0 & -5.0 & 1.6 & 3.1 & -2.6 & -7.8 & 1.1 & 4.4 & -7.5 & -4.6 & 2.0 & 2.6 & -4.9 & -6.6 & 1.3 & 4.3 & \begin{tabular}{|l|}
-5.7 \\
\end{tabular} & 2.5 & -0.44 & -1.78 & 176 & \begin{tabular}{|l|l}
0.146 \\
\end{tabular} \\
\hline 40 & -6.0 & -5.1 & 1.7 & 3.1 & -2.8 & -7.8 & 1.1 & 4.4 & -7.6 & -4.6 & 2.0 & 2.5 & -4.9 & -6.5 & 1.4 & 4.3 & -5.8 & 2.5 & -0.43 & -1.80 & 177 & 0.140 \\
\hline 41 & -6.1 & -5.1 & 1.7 & 3.1 & -2.8 & -7.9 & 1.2 & 4.4 & -7.6 & -4.6 & 2.1 & 2.6 & -5.0 & -6.6 & 1.4 & 4.4 & \begin{tabular}{|l}
-5.8 \\
\end{tabular} & 2.5 & -0.43 & -1.80 & 178 & 0.140 \\
\hline 42 & -6.1 & -5.2 & 1.7 & 3.2 & -2.9 & -7.9 & 1.2 & 4.4 & -7.7 & -4.7 & 2.1 & 2.6 & -4.9 & -6.6 & 1.4 & 4.3 & -5.9 & 2.6 & -0.44 & -1.79 & 179 & \begin{tabular}{|l|l|}
0.143 \\
\end{tabular} \\
\hline 43 & -6.1 & -5.2 & 1.7 & 3.2 & -2.9 & -7.9 & 1.2 & 4.4 & $\begin{array}{l}-7.8 \\
\end{array}$ & -4.8 & 2.1 & 2.6 & -5.0 & -6.6 & 1.4 & 4.4 & \begin{tabular}{|l|}
-5.9 \\
\end{tabular} & 2.6 & -0.43 & -1.81 & 181 & \begin{tabular}{|l|}
0.137 \\
\end{tabular} \\
\hline 44 & -6.2 & -5.2 & 1.7 & 3.2 & -2.8 & -8.0 & 1.2 & 4.5 & -7.8 & -5.0 & 2.1 & 2.6 & -4.9 & -6.7 & 1.4 & 4.4 & -5.9 & 2.6 & -0.43 & -1.80 & 181 & \begin{tabular}{|l|l|}
0.138 \\
\end{tabular} \\
\hline 45 & -6.1 & -5.2 & 1.7 & 3.2 & -2.7 & -8. & 1.2 & 4.5 & $\begin{array}{l}-7.7 \\
\end{array}$ & -4.8 & 2.1 & 2.7 & -5.0 & -6.7 & 1.4 & 4.4 & \begin{tabular}{|l}
-5.9 \\
\end{tabular} & 2.6 & -0.44 & -1.78 & 181 & \begin{tabular}{|l|l|}
0.144 \\
\end{tabular} \\
\hline 46 & -6.2 & -5.2 & 1.7 & 3.2 & -2.9 & -8.2 & 1.2 & 4.5 & -7.8 & -4.7 & 2.1 & 2.6 & -5.0 & -6.7 & 1.4 & 4.4 & -5.9 & 2.6 & -0.44 & -1.79 & 182 & 0.144 \\
\hline 47 & -6.3 & -5.3 & 1.7 & 3.2 & -2.9 & -8.2 & 1.2 & 4.5 & $\begin{array}{l}-7.7 \\
\end{array}$ & -4.8 & 2.1 & 2.7 & -5.0 & -6.7 & 1.4 & 4.4 & \begin{tabular}{|l}
-6.0 \\
\end{tabular} & 2.6 & -0.43 & \begin{tabular}{|l|l} 
\\
\end{tabular} & 183 & 0.141 \\
\hline 48 & -6.4 & -5.4 & 1.7 & 3.2 & -2.8 & $\begin{array}{l}-7.9 \\
\end{array}$ & 1.2 & 4.6 & \begin{tabular}{|l|}
-7.8 \\
\end{tabular} & $\begin{array}{c}-4.9 \\
\end{array}$ & 2.1 & 2.7 & \begin{tabular}{|l}
-5.0 \\
\end{tabular} & -6.7 & 1.4 & 4.5 & \begin{tabular}{|l}
-6.0 \\
\end{tabular} & 2.6 & -0.43 & -1.80 & 184 & \begin{tabular}{|l}
0.140 \\
\end{tabular} \\
\hline 49 & -6.5 & -5.4 & 1.7 & 3.2 & $\begin{array}{l}-3.0 \\
\end{array}$ & -8.3 & 1.2 & 4.5 & $\begin{array}{l}-7.8 \\
\end{array}$ & -4.9 & 2.1 & 2.7 & \begin{tabular}{|l}
-5.2 \\
\end{tabular} & -6.7 & 1.4 & 4.5 & \begin{tabular}{|l|l}
-6.1 \\
\end{tabular} & 2.6 & -0.43 & -1.81 & 185 & \begin{tabular}{|l}
0.136 \\
\end{tabular} \\
\hline 50 & -6.4 & -5.5 & 1.7 & 3.2 & -3.1 & -8.3 & 1.2 & 4.6 & $\begin{array}{l}-7.8 \\
\end{array}$ & -4.8 & 2.2 & 2.7 & -5.0 & -6.8 & 1.4 & 4.5 & \begin{tabular}{|l|l}
-6.1 \\
\end{tabular} & 2.6 & -0.43 & -1.79 & 185 & \begin{tabular}{|l|}
0.141 \\
\end{tabular} \\
\hline 51 & -6.5 & -5.5 & 1.7 & 3.2 & -3.0 & -8.4 & 1.2 & 4.6 & -8.0 & -4.9 & 2.2 & 2.7 & -5.1 & -6.7 & 1.5 & 4.5 & \begin{tabular}{|l|}
-6.1 \\
\end{tabular} & 2.6 & -0.43 & -1.80 & 187 & \begin{tabular}{|l}
0.138 \\
\end{tabular} \\
\hline 52 & -6.6 & -5.6 & 1.7 & 3.2 & $\begin{array}{l}-3.3 \\
\end{array}$ & -8.8 & 1.2 & 4.5 & \begin{tabular}{|l}
-7.9 \\
\end{tabular} & -5.0 & 2.2 & 2.7 & -5.1 & -6.8 & 1.4 & 4.6 & \begin{tabular}{|l}
-6.2 \\
\end{tabular} & 2.6 & -0.43 & -1.82 & 188 & \begin{tabular}{|l|}
0.134 \\
\end{tabular} \\
\hline 53 & -6.7 & -5.6 & 1.8 & 3.2 & -3.0 & -8.4 & 1.2 & 4.6 & \begin{tabular}{|l|}
-8.0 \\
\end{tabular} & -5.0 & 2.2 & 2.7 & -5.1 & -6.8 & 1.5 & 4.6 & \begin{tabular}{|l|}
-6.2 \\
\end{tabular} & 2.7 & -0.43 & -1.82 & 189 & \begin{tabular}{|l|l|}
0.134 \\
\end{tabular} \\
\hline 54 & -6.7 & -5.7 & 1.8 & 3.3 & -3.3 & -8.8 & 1.2 & 4.6 & \begin{tabular}{|l}
-8.0 \\
\end{tabular} & -5.0 & 2.2 & 2.8 & -5.1 & -6.8 & 1.5 & 4.6 & \begin{tabular}{|l}
-6.2 \\
\end{tabular} & 2.7 & -0.43 & \begin{tabular}{|l|} 
\\
\end{tabular} & 189 & 0.135 \\
\hline 55 & -6.7 & -5.7 & 1.7 & 3.3 & -3.0 & -8.5 & 1.2 & 4.6 & -8.2 & -5.1 & 2.2 & 2.7 & -5.1 & -6.9 & 1.5 & 4.7 & \begin{tabular}{|l|}
-6.3 \\
\end{tabular} & 2.7 & -0.42 & -1.83 & 192 & \begin{tabular}{|l|l|}
0.129 \\
\end{tabular} \\
\hline 56 & -6.7 & -5.7 & 1.8 & 3.3 & -3.3 & -8.6 & 1.2 & 4.6 & -8.1 & -5.1 & 2.2 & 2.8 & -5.2 & -6.9 & 1.5 & 4.6 & \begin{tabular}{|l}
-6.3 \\
\end{tabular} & 2.7 & -0.43 & -1.82 & 191 & 0.133 \\
\hline 57 & -6.9 & -5.8 & 1.8 & 3.3 & -3.0 & -8.5 & 1.2 & 4.7 & -8.1 & -5.2 & 2.2 & 2.8 & \begin{tabular}{|l|}
-5.1 \\
\end{tabular} & -7.0 & 1.5 & 4.7 & \begin{tabular}{|l|}
-6.3 \\
\end{tabular} & 2.7 & -0.42 & -1.83 & 193 & 0.130 \\
\hline 58 & -6.9 & -5.8 & 1.8 & 3.3 & -3.0 & -8.5 & 1. & 4.7 & -8.2 & -5.1 & 2.2 & 2.8 & -5.2 & -6.9 & 1.5 & 4.7 & \begin{tabular}{|l}
-6.3 \\
\end{tabular} & 2.7 & -0.43 & -1.82 & 193 & 0.133 \\
\hline 59 & -6.9 & -5.8 & 1.8 & 3.3 & -3.1 & -8.7 & 1.2 & 4.7 & -8.2 & -5.2 & 2.2 & 2.8 & -5.2 & -6.9 & 1.5 & 4.7 & \begin{tabular}{|l|}
-6.4 \\
\end{tabular} & 2.7 & -0.43 & -1.82 & 194 & \begin{tabular}{|l|l|}
0.132 \\
\end{tabular} \\
\hline 60 & -6.9 & -5.8 & 1.8 & 3.3 & -3.1 & -8.8 & 1.2 & 4.7 & -8.2 & -5.2 & 2.2 & 2.8 & $\begin{array}{ll}-5.2 \\
\end{array}$ & -7.0 & 1.5 & 4.8 & \begin{tabular}{|l|}
-6.4 \\
\end{tabular} & 2.7 & -0.43 & -1.82 & 195 & \begin{tabular}{|l|l}
0.132 \\
\end{tabular} \\
\hline 61 & $\begin{array}{l}-7.0 \\
\end{array}$ & -5.9 & 1.8 & 3.3 & -3.2 & -8. & 1. & 4. & \begin{tabular}{|l}
-8.3 \\
\end{tabular} & -5.3 & 2.2 & 2.8 & \begin{tabular}{|l}
-5.3 \\
\end{tabular} & -6.8 & 1.5 & 4.8 & \begin{tabular}{|l|}
-6.4 \\
\end{tabular} & 2.7 & -0.43 & -1.83 & 195 & \begin{tabular}{|l|}
0.131 \\
\end{tabular} \\
\hline 62 & -7.0 & -5.8 & 1.8 & 3.3 & -3.3 & -8.6 & 1.2 & 4.8 & -8.3 & -5.3 & 2.2 & 2.8 & \begin{tabular}{|l|}
-5.4 \\
\end{tabular} & -7.0 & 1.5 & 4.7 & \begin{tabular}{|l}
-6.5 \\
\end{tabular} & 2.7 & -0.42 & -1.83 & 197 & \begin{tabular}{|l|l}
0.130 \\
\end{tabular} \\
\hline 63 & -7.0 & -5.9 & 1.8 & 3.4 & -3.3 & -8.7 & 1.2 & 4.7 & $\begin{array}{l}-8.3 \\
\end{array}$ & -5.3 & 2.2 & 2.8 & -5.3 & -7.2 & 1.5 & $4 . \varepsilon$ & \begin{tabular}{|l|}
-6.5 \\
\end{tabular} & 2.7 & -0.42 & -1.84 & 198 & \begin{tabular}{|l|}
0.127 \\
\end{tabular} \\
\hline 64 & -7.0 & -5.9 & 1.8 & 3.4 & -3.4 & -8.8 & $\overline{1.2}$ & 4.7 & -8.5 & -5.4 & 2.2 & 2.9 & -5.4 & -7.1 & 1.5 & 4.8 & \begin{tabular}{|l|}
-6.5 \\
\end{tabular} & 2.8 & -0.42 & -1.84 & 199 & 0.126 \\
\hline 65 & -7.0 & -5.9 & 1.8 & 3.4 & -3.3 & -8.8 & 1.2 & 4.8 & $\begin{array}{l}-8.5 \\
\end{array}$ & -5.4 & 2.3 & 2.9 & \begin{tabular}{|l}
-5.3 \\
\end{tabular} & -7.1 & 1.5 & 4.8 & -6.5 & 2.8 & -0.42 & -1.83 & 199 & \begin{tabular}{|l}
0.129 \\
\end{tabular} \\
\hline$\frac{66}{66}$ & -7.0 & -5.9 & 1.8 & 3.4 & -3.4 & -9.0 & 1.3 & 4.8 & \begin{tabular}{|l}
-8.4 \\
\end{tabular} & -5.4 & 2.3 & 2.8 & -5.3 & -7.1 & 1.6 & 4.9 & \begin{tabular}{|l}
-6.5 \\
\end{tabular} & 2.8 & -0.42 & -1.83 & 199 & \begin{tabular}{|l|l|}
0.130 \\
\end{tabular} \\
\hline
\end{tabular}


Table E.12 KL 58-40 Test Data $\left(-20{ }^{\circ} \mathrm{C}\right.$ Tests), continued

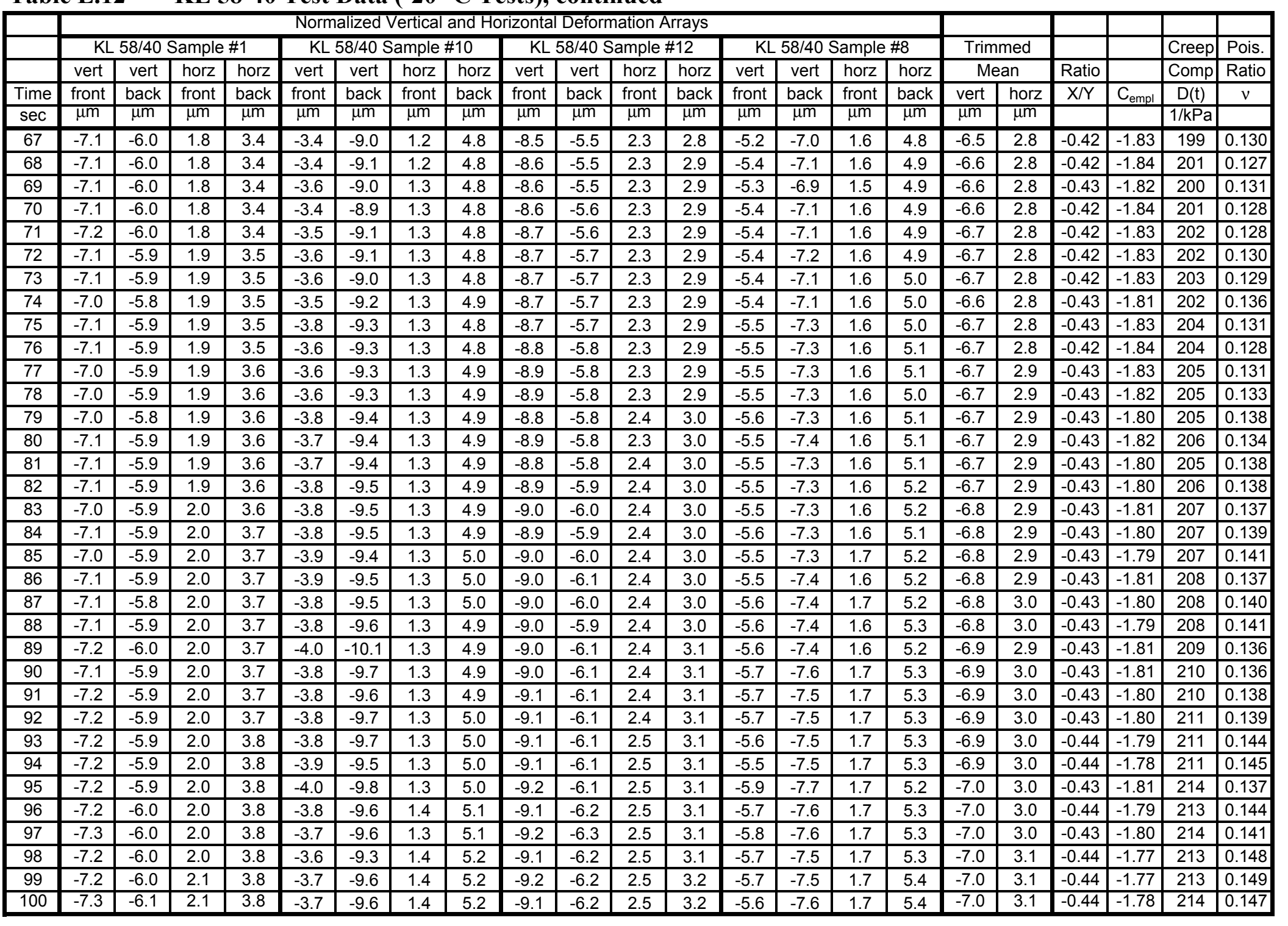


Table E.13 NU 52-34 Sample Data $\left(0^{\circ} \mathrm{C}\right.$ Tests $)$

\begin{tabular}{|c|c|c|c|}
\hline & Thickness & Diameter & Creep Load \\
\hline Sample & $\mathrm{mm}$ & $\mathrm{mm}$ & $\mathrm{kN}$ \\
\hline 6 & 76.85 & 150.2 & -4.27 \\
\hline 8 & 76.71 & 150.3 & -2.33 \\
\hline 9 & 76.72 & 150.1 & -3.44 \\
\hline & & & -3.35 \\
\hline average & 76.76 & 150.2 & \\
\hline
\end{tabular}

\begin{tabular}{|c|c|}
\hline Gage length, $\mathrm{m}$ & 0.038 \\
\hline $\mathrm{C}_{\mathrm{empl}}$ low & 0.595 \\
\hline $\mathrm{C}_{\mathrm{empl}}$ high & 1.466 \\
\hline
\end{tabular}

Table E.14 NU 52-34 Sample Data $\left(-10{ }^{\circ} \mathrm{C}\right.$ Tests $)$

\begin{tabular}{|c|c|c|c|}
\hline & Thickness & Diameter & Creep Load \\
\hline Sample & $\mathrm{mm}$ & $\mathrm{mm}$ & $\mathrm{kN}$ \\
\hline 6 & 76.85 & 150.2 & -5.08 \\
\hline 8 & 76.71 & 150.3 & -4.31 \\
\hline 9 & 76.72 & 150.1 & -6.10 \\
\hline & & & -5.16 \\
\hline average & 76.76 & 150.2 & \\
\hline
\end{tabular}

\begin{tabular}{|c|c|}
\hline Gage length, $\mathrm{m}$ & 0.038 \\
\hline $\mathrm{C}_{\mathrm{empl}}$ low & 0.595 \\
\hline $\mathrm{C}_{\mathrm{empl}}$ high & 1.466 \\
\hline
\end{tabular}

Table E.15 NU 52-34 Sample Data $\left(-20{ }^{\circ} \mathrm{C}\right.$ Tests)

\begin{tabular}{|c|c|c|c|}
\hline & Thickness & Diameter & Creep Load \\
\hline Sample & $\mathrm{mm}$ & $\mathrm{mm}$ & $\mathrm{kN}$ \\
\hline 6 & 76.85 & 150.2 & -8.47 \\
\hline 8 & 76.71 & 150.3 & -7.91 \\
\hline 9 & 76.72 & 150.1 & -10.07 \\
\hline & & & -8.82 \\
\hline average & 76.76 & 150.2 & \\
\hline
\end{tabular}

\begin{tabular}{|c|c|}
\hline Gage length, $\mathrm{m}$ & 0.038 \\
\hline $\mathrm{C}_{\text {empl }}$ low & 0.595 \\
\hline $\mathrm{C}_{\text {empl }}$ high & 1.466 \\
\hline
\end{tabular}


Table E.16 NU 52-34 Test Data $\left(0^{\circ} \mathrm{C}\right.$ Tests)

\begin{tabular}{|c|c|c|c|c|c|c|c|c|c|c|c|c|c|c|c|c|c|c|}
\hline & \multicolumn{12}{|c|}{ Normalized Vertical and Horizontal Deformation Arrays } & \multirow{2}{*}{\multicolumn{2}{|c|}{ Trimmed }} & \multirow[b]{3}{*}{ Ratio } & & \multirow{3}{*}{\begin{tabular}{|l|} 
Creep \\
Comp
\end{tabular}} & \multirow{3}{*}{$\begin{array}{l}\text { Pois } \\
\text { Ratio }\end{array}$} \\
\hline & \multicolumn{4}{|c|}{ NU 52-34 Sample \#6 } & \multicolumn{4}{|c|}{ NU 52-34 Sample \#8 } & \multicolumn{4}{|c|}{ NU 52-34 Sample \#9 } & & & & & & \\
\hline & vert & vert & horz & horz & vert & vert & horz & horz & vert & vert & horz & horz & & & & & & \\
\hline Time & front & back & front & back & front & back & front & back & front & back & front & back & vert & horz & $\bar{X} / \mathrm{Y}$ & $\mathrm{C}_{\mathrm{empl}}$ & $D(t)$ & $\mathrm{v}$ \\
\hline $\mathrm{sec}$ & $\mu \mathrm{m}$ & $\mu \mathrm{m}$ & $\mu \mathrm{m}$ & $\mu \mathrm{m}$ & $\mu \mathrm{m}$ & $\mu \mathrm{m}$ & $\mu \mathrm{m}$ & $\mu \mathrm{m}$ & $\mu \mathrm{m}$ & $\mu \mathrm{m}$ & $\mu \mathrm{m}$ & $\mu \mathrm{m}$ & $\mu \mathrm{m}$ & $\mu \mathrm{m}$ & & & $1 / \mathrm{kPa}$ & \\
\hline 0 & 0.0 & 0.0 & 0.0 & 0.0 & 0.0 & 0.0 & 0.0 & 0.0 & 0.0 & 0.0 & 0.0 & 0.0 & 0.0 & 0.0 & 0.00 & 0.00 & 0 & 0.000 \\
\hline 1 & -2.7 & -2.8 & 0.8 & 1.7 & -1.5 & -5.4 & 0.8 & 2.9 & -2.8 & -3.4 & 0.9 & 1.7 & -2.6 & 1.3 & -0.48 & -1.66 & 190 & 0.191 \\
\hline 2 & -2.9 & -4.0 & 0.9 & 2.3 & -2.1 & $\begin{array}{l}-6.2 \\
\end{array}$ & 1.0 & 3.5 & -3.6 & -4.0 & 1.1 & 2.0 & -3.4 & 1.6 & -0.47 & -1.69 & 245 & 0.181 \\
\hline 3 & -3.2 & -4.9 & 0.9 & 2.7 & -2.6 & -6.9 & 1.1 & 4.0 & -4.0 & -4.3 & 1.3 & 2.3 & -3.9 & 1.8 & -0.47 & -1.69 & 283 & 0.179 \\
\hline 4 & -3.2 & -5.4 & 1.0 & 3.1 & -3.1 & -7.7 & 1.3 & 4.2 & -4.4 & -4.5 & 1.4 & 2.4 & -4.3 & 2.1 & -0.48 & -1.67 & 312 & 0.188 \\
\hline 5 & -3.4 & -5.7 & 1.1 & 3.4 & -3.3 & -7.6 & 1.4 & 4.6 & -5.0 & -5.0 & 1.5 & 2.5 & -4.8 & 2.2 & -0.47 & -1.70 & 340 & 0.176 \\
\hline 6 & $\begin{array}{l}-3.4 \\
\end{array}$ & -6.2 & 1.1 & 3.6 & $\begin{array}{l}-3.3 \\
\end{array}$ & \begin{tabular}{|c|}
-8.1 \\
\end{tabular} & 1.5 & 4.9 & -5.2 & $\begin{array}{c}-5.1 \\
\end{array}$ & 1.6 & 2.7 & -4.9 & 2.4 & -0.48 & -1.66 & 354 & 0.193 \\
\hline 7 & -3.6 & -6.6 & 1.2 & 3.9 & -3.9 & -8.6 & 1.6 & 5.1 & -5.4 & -5.4 & 1.7 & 2.8 & -5.3 & 2.5 & -0.47 & -1.69 & 381 & 0.181 \\
\hline 8 & -3.6 & -6.9 & 1.2 & 4.1 & -4.0 & $\begin{array}{l}-8.8 \\
\end{array}$ & 1.7 & 5.4 & -5.7 & -5.6 & 1.8 & 2.9 & -5.5 & 2.6 & -0.47 & -1.67 & 398 & 0.188 \\
\hline 9 & -3.8 & -7.2 & 1.3 & 4.3 & -4.2 & -9.0 & 1.8 & 5.6 & -6.0 & -5.8 & 1.9 & 3.0 & -5.8 & 2.7 & -0.47 & -1.69 & 417 & 0.180 \\
\hline 10 & -3.9 & $\begin{array}{l}-7.5 \\
\end{array}$ & 1.3 & 4.5 & -4.6 & -9.5 & 1.8 & 5.8 & -6.3 & -6.1 & 1.9 & 3.1 & $\begin{array}{l}-6.1 \\
\end{array}$ & 2.8 & -0.46 & -1.70 & 437 & 0.174 \\
\hline 11 & -4.2 & -7.8 & 1.3 & 4.6 & -4.5 & -9.6 & 1.9 & 6.0 & -6.5 & -6.2 & 2.0 & 3.2 & -6.3 & 2.9 & -0.47 & -1.69 & 450 & 0.179 \\
\hline 12 & -4.2 & -8.1 & 1.4 & 4.8 & -4.7 & -9.7 & 2.0 & 6.1 & -6.7 & -6.4 & 2.1 & 3.2 & -6.5 & 3.0 & -0.47 & -1.69 & 464 & 0.181 \\
\hline 13 & -4.2 & -8.2 & 1.4 & 4.9 & -4.9 & -10.0 & 2.1 & 6.3 & -6.9 & -6.5 & 2.1 & 3.4 & -6.6 & 3.1 & -0.47 & -1.68 & 477 & 0.182 \\
\hline 14 & -4.3 & -8.4 & 1.4 & 5.1 & -5.0 & -10.2 & 2.2 & 6.5 & -7.1 & -6.7 & 2.2 & 3.5 & -6.8 & 3.2 & -0.47 & -1.67 & 489 & 0.188 \\
\hline 15 & -4.5 & -8.7 & 1.5 & 5.2 & -5.3 & -10.4 & 2.2 & 6.6 & -7.4 & -6.9 & 2.2 & 3.5 & -7.1 & 3.3 & -0.47 & -1.69 & 506 & 0.179 \\
\hline 16 & -4.5 & -8.8 & 1.5 & 5.3 & -5.5 & -10.7 & 2.3 & 6.8 & -7.5 & -6.9 & 2.3 & 3.6 & -7.2 & 3.4 & -0.47 & -1.68 & 515 & 0.186 \\
\hline 17 & -4.6 & -9.1 & 1.5 & 5.5 & -5.5 & -10.9 & 2.4 & 6.9 & -7.6 & -7.0 & 2.4 & 3.6 & -7.3 & 3.5 & -0.47 & -1.67 & 524 & 0.188 \\
\hline 18 & -4.7 & -9.2 & 1.6 & 5.6 & -5.7 & -11.0 & 2.4 & 7.1 & -8.0 & -7.2 & 2.4 & 3.7 & -7.5 & 3.6 & -0.47 & -1.68 & 540 & 0.184 \\
\hline 19 & -4.8 & -9.4 & 1.6 & 5.7 & -5.9 & -11.3 & 2.4 & 7.2 & -8.1 & -7.2 & 2.5 & 3.8 & -7.7 & 3.6 & -0.47 & -1.68 & 550 & 0.185 \\
\hline 20 & -5.0 & -9.5 & 1.6 & 5.8 & -5.9 & -11.4 & 2.5 & 7.4 & -8.2 & -7.4 & 2.5 & 3.9 & -7.8 & 3.7 & -0.48 & -1.67 & 558 & 0.189 \\
\hline 21 & -5.2 & -9.7 & 1.7 & 5.9 & -6.1 & -11.5 & 2.6 & 7.5 & -8.4 & -7.6 & 2.6 & 4.0 & -8.0 & 3.8 & -0.47 & -1.67 & 571 & 0.186 \\
\hline 22 & -5.2 & -10.0 & 1.7 & 6.0 & -6.3 & -11.8 & 2.6 & 7.6 & -8.5 & -7.7 & 2.7 & 4.0 & -8.1 & 3.8 & -0.47 & -1.68 & 582 & 0.184 \\
\hline 23 & -5.3 & -10.2 & 1.7 & 6.1 & -6.5 & -11.8 & 2.7 & 7.7 & -8.6 & -7.8 & 2.7 & 4.1 & -8.3 & 3.9 & -0.47 & -1.68 & 592 & 0.184 \\
\hline 24 & -5.3 & -10.3 & 1.8 & 6.2 & -6.5 & -11.8 & 2.7 & 7.8 & -8.7 & -7.9 & 2.7 & 4.1 & -8.3 & 4.0 & -0.47 & -1.67 & 600 & 0.187 \\
\hline 25 & -5.5 & -10.4 & 1.8 & 6.4 & -6.7 & -12.1 & 2.8 & 7.9 & -8.9 & -8.0 & 2.8 & 4.2 & -8.5 & 4.0 & -0.47 & -1.67 & 612 & 0.187 \\
\hline 26 & -5.6 & -10.7 & 1.8 & 6.5 & -6.8 & -12.2 & 2.8 & 8.1 & -9.1 & -8.0 & 2.8 & 4.2 & -8.6 & 4.1 & -0.47 & -1.67 & 620 & 0.186 \\
\hline 27 & -5.8 & -10.7 & 1.8 & 6.6 & -6.9 & -12.4 & 2.9 & 8.2 & -9.2 & -8.1 & 2.9 & 4.3 & -8.7 & 4.2 & -0.48 & -1.66 & 627 & 0.191 \\
\hline 28 & -5.7 & -11.0 & 1.9 & 6.7 & -6.8 & -12.4 & 3.0 & 8.4 & -9.3 & -8.2 & 2.9 & 4.4 & -8.8 & 4.2 & -0.48 & -1.65 & 633 & 0.197 \\
\hline 29 & -5.8 & -11.0 & 1.9 & 6.8 & -7.0 & -12.6 & 3.0 & 8.5 & -9.4 & -8.4 & 3.0 & 4.4 & -8.9 & 4.3 & -0.48 & -1.66 & 644 & 0.193 \\
\hline 30 & -6.0 & -11.3 & 1.9 & 6.8 & -7.2 & -12.7 & 3.0 & 8.6 & -9.5 & -8.4 & 3.0 & 4.5 & -9.1 & 4.3 & -0.48 & -1.67 & 655 & 0.188 \\
\hline 31 & -6.1 & -11.3 & 1.9 & 7.0 & -7.2 & \begin{tabular}{|l|}
-13.1 \\
\end{tabular} & 3.1 & 8.7 & $\begin{array}{l}-9.7 \\
\end{array}$ & -8.5 & 3.1 & 4.5 & -9.2 & 4.4 & -0.48 & -1.66 & 661 & 0.194 \\
\hline 32 & -6.1 & -11.5 & 2.0 & 7.0 & -7.6 & -13.1 & 3.1 & 8.7 & -9.7 & -8.5 & 3.1 & 4.6 & -9.3 & 4.4 & -0.48 & -1.67 & 672 & 0.189 \\
\hline 33 & -6.2 & -11.6 & 2.0 & 7.1 & -7.8 & -13.7 & 3.1 & 8.9 & -9.9 & -8.7 & 3.1 & 4.6 & -9.5 & 4.5 & -0.48 & -1.67 & 683 & 0.189 \\
\hline
\end{tabular}


Table E.16 NU 52-34 Test Data $\left(0{ }^{\circ} \mathrm{C}\right.$ Tests $)$, continued

\begin{tabular}{|c|c|c|c|c|c|c|c|c|c|c|c|c|c|c|c|c|c|c|}
\hline & \multicolumn{12}{|c|}{ Normalized Vertical and Horizontal Deformation Arrays } & \multirow{2}{*}{\multicolumn{2}{|c|}{$\overline{\text { Trimmed }}$}} & \multirow[b]{3}{*}{ Ratio } & & \multirow{3}{*}{\begin{tabular}{|l|} 
Creep \\
Comp \\
\end{tabular}} & \multirow{3}{*}{\begin{tabular}{|l|} 
Pois. \\
Ratio \\
\end{tabular}} \\
\hline & \multicolumn{4}{|c|}{ NU 52-34 Sample \#6 } & \multicolumn{4}{|c|}{ NU 52-34 Sample \#8 } & \multicolumn{4}{|c|}{ NU 52-34 Sample \#9 } & & & & & & \\
\hline & vert & vert & horz & horz & vert & vert & horz & horz & vert & vert & horz & horz & & & & & & \\
\hline Time & front & back & front & back & front & back & front & back & front & back & front & back & vert & horz & $\mathrm{X} / \mathrm{Y}$ & $\mathrm{C}_{\text {empl }}$ & $\mathrm{D}(\mathrm{t})$ & $\mathrm{v}$ \\
\hline sec & $\mu \mathrm{m}$ & $\mu \mathrm{m}$ & $\mu \mathrm{m}$ & $\mu \mathrm{m}$ & $\mu \mathrm{m}$ & $\mu \mathrm{m}$ & $\mu \mathrm{m}$ & $\mu \mathrm{m}$ & $\mu \mathrm{m}$ & $\mu \mathrm{m}$ & $\mu \mathrm{m}$ & 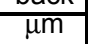 & $\mu \mathrm{m}$ & $\mu \mathrm{m}$ & & & \begin{tabular}{|l|l}
$1 / \mathrm{kPa}$ \\
\end{tabular} & \\
\hline$\overline{34}$ & -6.3 & -11.7 & 2.0 & 7.2 & -7.7 & -13.4 & 3.2 & 8.9 & -10.0 & -8.7 & $\overline{3.2}$ & 4.7 & -9.5 & 4.6 & -0.48 & -1.66 & 687 & 0.193 \\
\hline 35 & -6.3 & -11.9 & 2.0 & 7.3 & -8.1 & \begin{tabular}{|l|}
-13.7 \\
\end{tabular} & 3.2 & 9.0 & -10.1 & -8.7 & 3.2 & 4.7 & -9.7 & 4.6 & \begin{tabular}{|l|}
-0.47 \\
\end{tabular} & -1.67 & 698 & \begin{tabular}{|l|l}
0.188 \\
\end{tabular} \\
\hline 36 & $\begin{array}{l}-6.5 \\
\end{array}$ & -12.0 & 2.1 & 7.4 & \begin{tabular}{|l|}
-7.9 \\
\end{tabular} & \begin{tabular}{|l}
-13.6 \\
\end{tabular} & 3.3 & 9.2 & -10.3 & -8.9 & 3.3 & 4.8 & $\begin{array}{l}-9.8 \\
\end{array}$ & $\begin{array}{l}4.7 \\
\end{array}$ & \begin{tabular}{|l}
-0.48 \\
\end{tabular} & -1.66 & 704 & 0.194 \\
\hline 37 & -6.5 & -12.2 & 2.1 & 7.4 & -8.2 & -14.0 & 3.3 & 9.2 & -10.4 & -9.1 & 3.3 & 4.8 & -10.0 & 4.7 & -0.47 & -1.67 & 716 & 0.186 \\
\hline 38 & -6.6 & -12.3 & 2.1 & 7.6 & -8.1 & -13.9 & 3.4 & 9.4 & -10.4 & -9.1 & 3.4 & 4.9 & -10.0 & 4.8 & -0.48 & -1.66 & 719 & 0.194 \\
\hline 39 & -6.7 & -12.4 & 2.1 & 7.6 & -8.3 & \begin{tabular}{|l|}
-13.9 \\
\end{tabular} & 3.4 & 9.5 & -10.6 & -9.1 & 3.4 & 4.9 & -10.1 & 4.8 & \begin{tabular}{|l|}
-0.48 \\
\end{tabular} & -1.66 & 727 & 0.193 \\
\hline 40 & -6.7 & -12.5 & 2.1 & 7.7 & -8.3 & \begin{tabular}{|l|}
-14.0 \\
\end{tabular} & 3.5 & 9.6 & -10.7 & -9.3 & 3.4 & 5.0 & -10.2 & 4.9 & \begin{tabular}{|l|}
-0.48 \\
\end{tabular} & -1.66 & 735 & \begin{tabular}{|l|}
0.194 \\
\end{tabular} \\
\hline 41 & -6.8 & -12.6 & 2.1 & 7.8 & -8.4 & -14.1 & 3.5 & 9.7 & -10.8 & -9.4 & 3.5 & 5.1 & -10.3 & 5.0 & -0.48 & -1.65 & 743 & 0.196 \\
\hline 42 & -6.9 & -12.7 & 2.2 & 7.9 & -8.5 & -14.3 & 3.5 & 9.7 & -10.9 & -9.5 & 3.5 & 5.1 & -10.4 & 5.0 & -0.48 & -1.65 & 748 & 0.195 \\
\hline 43 & -7.0 & -12.8 & 2.2 & 8.0 & -8.8 & -14.3 & 3.6 & 9.8 & -11.1 & -9.4 & 3.5 & 5.1 & -10.5 & 5.1 & -0.48 & -1.66 & 758 & \begin{tabular}{|l|l|}
0.194 \\
\end{tabular} \\
\hline 44 & -7.1 & -13.1 & 2.2 & 8.0 & -8.9 & \begin{tabular}{|l|}
-14.5 \\
\end{tabular} & 3.6 & 9.9 & -11.0 & -9.5 & 3.6 & 5.2 & -10.6 & 5.1 & \begin{tabular}{|l|}
-0.48 \\
\end{tabular} & -1.65 & 765 & 0.195 \\
\hline 45 & $\begin{array}{l}-7.2 \\
\end{array}$ & -13.0 & 2.2 & 8.1 & -9.0 & \begin{tabular}{|l|l|}
-14.7 \\
\end{tabular} & 3.7 & 10.0 & -11.3 & -9.6 & 3.6 & 5.2 & $\begin{array}{l}-10.7 \\
\end{array}$ & 5.1 & \begin{tabular}{|l}
-0.48 \\
\end{tabular} & -1.66 & 772 & \begin{tabular}{|l}
0.193 \\
\end{tabular} \\
\hline 46 & $\begin{array}{l}-7.2 \\
\end{array}$ & -13.2 & 2.3 & 8.2 & -9.1 & \begin{tabular}{|l}
-14.6 \\
\end{tabular} & 3.7 & 10.1 & -11.4 & -9.7 & 3.6 & 5.2 & -10.8 & 5.2 & \begin{tabular}{|l}
-0.48 \\
\end{tabular} & -1.66 & 780 & 0.193 \\
\hline 47 & $\begin{array}{l}-7.3 \\
\end{array}$ & -13.4 & 2.3 & 8.3 & -9.2 & -14.7 & 3.8 & 10.2 & -11.5 & -9.8 & 3.7 & 5.3 & -11.0 & 5.2 & -0.48 & -1.66 & 788 & \begin{tabular}{|l|l|}
0.192 \\
\end{tabular} \\
\hline 48 & -7.4 & -13.4 & 2.3 & 8.3 & -9.4 & \begin{tabular}{|l|}
-14.9 \\
\end{tabular} & 3.8 & 10.3 & -11.5 & -9.9 & 3.7 & 5.3 & -11.0 & 5.3 & \begin{tabular}{|l|}
-0.48 \\
\end{tabular} & -1.66 & 796 & \begin{tabular}{|l|l|}
0.193 \\
\end{tabular} \\
\hline 49 & \begin{tabular}{|l|}
-7.3 \\
\end{tabular} & -13.5 & 2.3 & 8.4 & \begin{tabular}{|c|}
-9.6 \\
\end{tabular} & -15.1 & 3.8 & 10.4 & -11.6 & -9.9 & 3.8 & 5.4 & -11.1 & 5.3 & \begin{tabular}{|l|l}
-0.48 \\
\end{tabular} & -1.66 & 802 & \begin{tabular}{|l|l|}
0.194 \\
\end{tabular} \\
\hline 50 & \begin{tabular}{|l|l}
-7.4 \\
\end{tabular} & -13.6 & 2.3 & 8.5 & \begin{tabular}{|l|}
-9.5 \\
\end{tabular} & \begin{tabular}{|l}
-15.0 \\
\end{tabular} & 3.9 & 10.4 & -11.7 & -9.9 & 3.8 & 5.4 & -11.2 & 5.4 & \begin{tabular}{|l|l|}
-0.48 \\
\end{tabular} & -1.65 & 806 & 0.196 \\
\hline 51 & $\begin{array}{l}-7.4 \\
\end{array}$ & -13.7 & 2.4 & 8.6 & \begin{tabular}{|c|}
-9.6 \\
\end{tabular} & -15.1 & 3.9 & 10.6 & -11.8 & -10.0 & 3.8 & 5.4 & -11.3 & 5.4 & -0.48 & -1.65 & 813 & 0.196 \\
\hline 52 & -7.5 & -13.9 & 2.4 & 8.6 & -9.9 & -15.5 & 3.9 & 10.6 & -11.9 & -10.2 & 3.8 & 5.5 & -11.5 & 5.5 & -0.48 & -1.67 & 825 & \begin{tabular}{|l}
0.189 \\
\end{tabular} \\
\hline 53 & -7.7 & -14.0 & 2.4 & 8.7 & -9.8 & \begin{tabular}{|l|}
-15.3 \\
\end{tabular} & 4.0 & 10.8 & -11.9 & \begin{tabular}{|l|}
-10.2 \\
\end{tabular} & 3.9 & 5.5 & -11.5 & 5.5 & \begin{tabular}{|l|}
-0.48 \\
\end{tabular} & -1.65 & 827 & \begin{tabular}{|l|l|}
0.195 \\
\end{tabular} \\
\hline 54 & -7.8 & -14.1 & 2.4 & 8.8 & -9.8 & -15.3 & 4.0 & 10.8 & -12.3 & -10.4 & 3.9 & 5.5 & -11.7 & 5.6 & -0.48 & -1.67 & 838 & \begin{tabular}{|l|l|}
0.190 \\
\end{tabular} \\
\hline 55 & -7.8 & -14.2 & 2.4 & 8.8 & -9.9 & -15.3 & 4.1 & 10.9 & -12.3 & -10.3 & $\overline{4.0}$ & 5.6 & -11.7 & 5.6 & -0.48 & -1.65 & 841 & 0.195 \\
\hline 56 & -7.8 & -14.2 & 2.5 & 8.9 & \begin{tabular}{|l}
-10.1 \\
\end{tabular} & -15.6 & 4.1 & 11.0 & -12.4 & -10.4 & 4.0 & 5.6 & -11.8 & 5.7 & \begin{tabular}{|l|l}
-0.48 \\
\end{tabular} & -1.66 & 849 & \begin{tabular}{|l|l|}
0.194 \\
\end{tabular} \\
\hline 57 & -8.0 & -14.3 & 2.5 & 9.0 & -10.0 & -15.5 & 4.1 & 11.1 & -12.4 & \begin{tabular}{|l|}
-10.4 \\
\end{tabular} & 4.0 & 5.7 & -11.8 & 5.7 & -0.48 & -1.64 & 851 & \begin{tabular}{|l}
0.200 \\
\end{tabular} \\
\hline 58 & -8.1 & -14.5 & 2.5 & 9.0 & \begin{tabular}{|l|l}
-10.2 \\
\end{tabular} & \begin{tabular}{|l|}
-15.7 \\
\end{tabular} & 4.2 & 11.2 & -12.5 & \begin{tabular}{|l}
-10.5 \\
\end{tabular} & 4.0 & 5.7 & -11.9 & 5.7 & \begin{tabular}{|l|}
-0.48 \\
\end{tabular} & -1.65 & 859 & \begin{tabular}{|l|l}
0.197 \\
\end{tabular} \\
\hline 59 & -8.1 & -14.5 & 2.5 & 9.1 & -10.3 & -15.8 & 4.2 & 11.3 & -12.5 & -10.6 & 4.1 & 5.7 & -12.0 & 5.8 & -0.48 & -1.65 & 865 & \begin{tabular}{|l|l}
0.197 \\
\end{tabular} \\
\hline 60 & $\begin{array}{l}-8.1 \\
\end{array}$ & -14.5 & 2.5 & 9.2 & \begin{tabular}{|l|l} 
\\
\end{tabular} & -15.8 & 4.2 & 11.3 & -12.9 & -11.0 & 4.1 & 5.7 & -12.2 & 5.8 & \begin{tabular}{|l}
-0.48 \\
\end{tabular} & -1.67 & 877 & \begin{tabular}{|l}
0.190 \\
\end{tabular} \\
\hline 61 & -8.3 & -14.9 & 2.5 & 9.2 & -10.6 & -16.0 & 4.3 & 11.4 & -12.8 & \begin{tabular}{|l|l}
-10.8 \\
\end{tabular} & 4.2 & 5.8 & -12.3 & 5.9 & -0.48 & -1.66 & 883 & \begin{tabular}{|l}
0.192 \\
\end{tabular} \\
\hline$\overline{62}$ & $\begin{array}{l}-8.3 \\
\end{array}$ & -14.8 & 2.6 & 9.3 & \begin{tabular}{|l|}
-10.6 \\
\end{tabular} & \begin{tabular}{|l|l}
-15.9 \\
\end{tabular} & 4.3 & 11.5 & -12.9 & -10.7 & 4.2 & 5.9 & -12.2 & 5.9 & -0.48 & -1.64 & 883 & \begin{tabular}{|l|l|}
0.199 \\
\end{tabular} \\
\hline 63 & -8.3 & -15.0 & 2.6 & 9.3 & -10.6 & -16.1 & 4.3 & 11.6 & -12.9 & -10.9 & 4.2 & 5.9 & -12.3 & 6.0 & -0.48 & -1.65 & 890 & \begin{tabular}{|l}
0.197 \\
\end{tabular} \\
\hline 64 & -8.3 & -15.0 & 2.6 & 9.4 & -10.7 & -16.3 & 4.4 & 11.6 & -13.1 & -10.9 & 4.2 & 5.9 & -12.4 & 6.0 & -0.48 & -1.65 & 895 & 0.199 \\
\hline 65 & -8.4 & -15.2 & 2.6 & 9.5 & \begin{tabular}{|l|}
-10.7 \\
\end{tabular} & -16.3 & 4.4 & 11.7 & -13.2 & -11.0 & 4.3 & 6.0 & -12.5 & 6.0 & -0.48 & -1.65 & 904 & 0.196 \\
\hline 66 & -8.4 & -15.2 & 2.7 & 9.6 & -10.8 & -16.5 & 4.5 & 11.8 & -13.4 & -11.1 & 4.3 & 6.0 & -12.6 & 6.1 & -0.48 & -1.65 & 910 & 0.197 \\
\hline
\end{tabular}


Table E.16 NU 52-34 Test Data $\left({ }^{\circ} \mathrm{C}\right.$ Tests), continued

\begin{tabular}{|c|c|c|c|c|c|c|c|c|c|c|c|c|c|c|c|c|c|c|}
\hline & & & & & & & & & & & & & & & & & & \\
\hline & & $52-34$ & Sample & & & $52-34$ & Sample & & $\mathrm{NU}$ & $52-34$ & Sample & & Trim & ned & & & Creep & Pois \\
\hline & \begin{tabular}{ll|} 
vert \\
\end{tabular} & vert & horz & horz & \begin{tabular}{|l|} 
vert \\
\end{tabular} & vert & horz & horz & \begin{tabular}{|l|} 
vert \\
\end{tabular} & $\begin{array}{l}\text { vert } \\
\end{array}$ & horz & horz & $\mathrm{Me}$ & & Ratio & & Comp & Ratio \\
\hline ime & front & back & front & back & front & back & front & back & & back & front & back & $\overline{\text { vert }}$ & horz & $\bar{X} / \mathrm{Y}$ & $\mathrm{C}_{\mathrm{empl}}$ & & \\
\hline $\mathrm{sec}$ & $\mu \mathrm{m}$ & $\mu \mathrm{m}$ & $\mu \mathrm{m}$ & $\mu \mathrm{m}$ & $\mu \mathrm{m}$ & $\mu \mathrm{m}$ & $\mu \mathrm{m}$ & $\mu \mathrm{m}$ & $\mu \mathrm{m}$ & $\mu \mathrm{m}$ & $\mu \mathrm{m}$ & $\mu \mathrm{m}$ & $\mu \mathrm{m}$ & $\mu \mathrm{m}$ & & & $1 / \mathrm{kPa}$ & \\
\hline 67 & $\begin{array}{l}-8.5 \\
\end{array}$ & -15.2 & 2.7 & 9.6 & -11.0 & -16.5 & 4.5 & 11.9 & -13.4 & -11.1 & 4.3 & 6.1 & -12.7 & 6.1 & 0.48 & -1.65 & $\overline{914}$ & 0.198 \\
\hline 68 & -8.5 & -15.3 & 2.7 & 9.7 & -11.0 & -16.8 & 4.5 & 12.0 & -13.6 & -11.2 & $\sqrt{14}$ & & -12.8 & & 018 & & 21 & \\
\hline 69 & $\begin{array}{l}-8.6 \\
\end{array}$ & -15.4 & 2.7 & 9.8 & \begin{tabular}{|l}
-11.2 \\
\end{tabular} & -16.8 & 4.5 & 12.0 & \begin{tabular}{|l|}
-13.6 \\
\end{tabular} & \begin{tabular}{|l|l|}
-11.2 \\
\end{tabular} & 4.4 & 6.1 & \begin{tabular}{|l|}
-12.9 \\
\end{tabular} & 6.2 & -0.48 & $\begin{array}{l}-1.65 \\
\end{array}$ & 927 & 0.197 \\
\hline 70 & 8.6 & -15.5 & 2.8 & 9.8 & \begin{tabular}{|l|l|} 
\\
\end{tabular} & \begin{tabular}{|l}
-16.8 \\
\end{tabular} & 4.6 & 12.1 & -13.6 & \begin{tabular}{|l|l}
-11.3 \\
\end{tabular} & 4.4 & 6. & -12.9 & 6.3 & -0.49 & -1.64 & 932 & 0.201 \\
\hline 71 & -8.7 & -15.6 & 2.8 & 9.9 & -11.2 & -16.9 & 4. & 12.2 & -13.8 & -11.4 & 4. & 6.2 & -13.0 & 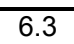 & -0.48 & -1.64 & 937 & \\
\hline 72 & -8.7 & \begin{tabular}{|l}
-15.6 \\
\end{tabular} & 2.8 & 10.0 & \begin{tabular}{|l|l|}
-11.3 \\
\end{tabular} & -16.9 & 4.6 & 12.3 & -13.8 & -11.4 & 4.5 & 6.3 & -13.0 & 6.3 & 0.49 & -1.64 & 941 & 0.202 \\
\hline 73 & -8.7 & -15.7 & 2.8 & 10.0 & -11.4 & -17.1 & 4.7 & 12.4 & -13.9 & -11.5 & 4. & 6.3 & -13.1 & $\overline{6.4}$ & -0.49 & -1.64 & 947 & 0.201 \\
\hline 74 & $\begin{array}{l}-8.8 \\
\end{array}$ & -15.7 & 2.8 & 10.1 & -11.4 & -17.2 & 4.7 & 12.4 & -14.1 & -11.5 & 4.5 & 6.3 & -13.2 & 6.4 & 0.49 & -1.64 & 953 & 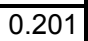 \\
\hline 75 & $\begin{array}{l}-8.9 \\
\end{array}$ & -15.9 & 2.8 & 10.2 & -11.5 & $\begin{array}{l}-17.3 \\
\end{array}$ & 4.8 & 12.5 & -14.0 & -11.6 & $\overline{4.6}$ & 6.3 & -13.3 & 6.5 & -0.49 & -1.64 & 958 & 0.203 \\
\hline 76 & -9.0 & -16.0 & 2.9 & 10.2 & -11.7 & -17.3 & 4. & 12.6 & -14.2 & -11.6 & 4. & & -13.4 & & -0.48 & -1.64 & 966 & \\
\hline 77 & $\begin{array}{l}-8.8 \\
\end{array}$ & -15.9 & 2.9 & 10.3 & \begin{tabular}{|l|l}
-11.7 \\
\end{tabular} & -17.5 & 4.8 & 12.7 & -14.2 & \begin{tabular}{|l|l} 
\\
\end{tabular} & 4.6 & 6. & -13.4 & 6.5 & -0.49 & -1.63 & 966 & 0.20 \\
\hline 78 & -9.1 & -16.2 & 2.9 & 10.3 & -11.8 & -17.6 & 4.8 & 12.7 & -14.4 & -11.9 & 4. & 6.4 & -13.5 & 6.6 & -0.48 & -1.65 & 977 & 0.199 \\
\hline 79 & -9.1 & -16.3 & 2.9 & 10.4 & -11.9 & -17.6 & $\overline{4.8}$ & 12.8 & -14.4 & -11.9 & 4. & & -13.6 & 6. & -0.48 & -1.64 & 983 & 0.19 \\
\hline 80 & -9.1 & \begin{tabular}{|l|}
-16.1 \\
\end{tabular} & 2.9 & 10.5 & -12.0 & -17.7 & 4.9 & 12.9 & -14.5 & -11.9 & 4.7 & 6. & -13.6 & 6. & -0.49 & -1.63 & 984 & 0.205 \\
\hline 81 & $\begin{array}{l}-9.2 \\
\end{array}$ & -16.4 & 2.6 & 10.5 & -12.0 & \begin{tabular}{|l|l}
-17.8 \\
\end{tabular} & 4.9 & 13.0 & -14.5 & -11.9 & 4. & 6. & -13.7 & 6. & -0.49 & -1.64 & 990 & 0.20 \\
\hline 82 & $\begin{array}{l}-9.2 \\
\end{array}$ & \begin{tabular}{|l|}
-16.4 \\
\end{tabular} & 3.0 & 10.6 & \begin{tabular}{|l|}
-12 \\
\end{tabular} & -1 & 4. & 13.0 & -14.7 & \begin{tabular}{|l}
-12.1 \\
\end{tabular} & & & \begin{tabular}{|l|}
-13.8 \\
\end{tabular} & 6. & -0.49 & -1.64 & 991 & 0.20 \\
\hline 83 & -9.3 & -16.5 & 3.0 & 10.6 & -12.0 & -17.8 & 5.0 & 13.1 & -14.6 & -11.9 & 4.8 & 6.6 & \begin{tabular}{|c|}
-13.8 \\
\end{tabular} & 6.7 & -0.49 & -1.63 & 995 & 0.207 \\
\hline 84 & -9.4 & -16.6 & 3.0 & 10.7 & -12.2 & \begin{tabular}{|l|}
-18.1 \\
\end{tabular} & 5.0 & 13.2 & -14.7 & -12.1 & 4. & 6.6 & -13.9 & 6.8 & -0.49 & -1.63 & 1005 & 0.20 \\
\hline 85 & -9.4 & -16.6 & 3.0 & 10.8 & -12 & & 5. & 13.3 & -14.9 & -12.1 & 4. & & -14.0 & 6. & -0.49 & -1.63 & 1011 & 0.20 \\
\hline 86 & -9.5 & \begin{tabular}{|l}
-16.7 \\
\end{tabular} & 3.0 & 10.8 & $\mid-12$ & $\begin{array}{l}-18.3 \\
\end{array}$ & 5.0 & 13.4 & -15.0 & -12.3 & 4.9 & 6.7 & -14.1 & $\overline{6 c}$ & -0.49 & -1.63 & 1017 & 0.20 \\
\hline 87 & $\begin{array}{l}-9.5 \\
\end{array}$ & -16.9 & 3.0 & 10.9 & \begin{tabular}{|l}
-12.4 \\
\end{tabular} & \begin{tabular}{|l|l}
-18.4 \\
\end{tabular} & 5.1 & 13.4 & -15.1 & \begin{tabular}{|l}
-12.3 \\
\end{tabular} & 4.6 & 6. & -14.2 & & 0.49 & -1.63 & 1023 & 0.20 \\
\hline 88 & $\begin{array}{l}-9.6 \\
\end{array}$ & -17.0 & 3.1 & \begin{tabular}{|l|}
10.9 \\
\end{tabular} & \begin{tabular}{|l|l|} 
\\
\end{tabular} & -18.4 & 5.1 & 13.5 & -15.3 & $\begin{array}{l}-12.7 \\
\end{array}$ & 4.9 & 6. & -14.3 & 6.9 & -0.48 & -1.65 & 1034 & 0.197 \\
\hline 89 & -9.6 & $\mid-17.0$ & 3.1 & 11.0 & -12.5 & -18.5 & 5.1 & 13.6 & -15.3 & -12.4 & 5.0 & 6.8 & -14.3 & 7.6 & -0.49 & -1.64 & 1033 & 0.203 \\
\hline 90 & $\begin{array}{l}-9.6 \\
\end{array}$ & $\mid-17.0$ & 3.1 & 11.0 & -12.5 & \begin{tabular}{|l}
-18.6 \\
\end{tabular} & 5. & 13.7 & -15.3 & -12.6 & 5. & 6.8 & -14.4 & 7.0 & -0.49 & -1.63 & 1038 & 0.204 \\
\hline 91 & -9.7 & \begin{tabular}{|l|}
-17.1 \\
\end{tabular} & 3. & 11.1 & $\mid-12$ & \begin{tabular}{|l|}
-18.7 \\
\end{tabular} & 5.2 & 13.7 & -15.3 & -12.6 & & & -14.4 & & -0.49 & -1.63 & 1041 & $8 x$ \\
\hline 92 & -9.7 & \begin{tabular}{|l|l}
-17.2 \\
\end{tabular} & 3.1 & 11.1 & \begin{tabular}{|l}
-12.7 \\
\end{tabular} & -18.7 & 5.2 & 13.8 & -15.5 & \begin{tabular}{|l|}
-12.7 \\
\end{tabular} & 5.0 & 6.9 & -14.5 & 7.1 & -0.49 & -1.64 & 1049 & 0.202 \\
\hline 93 & $\begin{array}{l}-9.8 \\
\end{array}$ & \begin{tabular}{|l|l} 
\\
\end{tabular} & 3.1 & \begin{tabular}{|l|}
11.2 \\
\end{tabular} & \begin{tabular}{|l|l|} 
\\
\end{tabular} & -18.9 & 5.2 & 13.9 & -15.7 & -12.8 & 5.1 & 6.9 & -14.6 & 7.1 & -0.49 & -1.64 & 1056 & 0.201 \\
\hline 94 & -10.0 & -17.5 & 3.2 & 11.2 & 120 & -19.0 & 5.3 & 13.9 & -15.7 & -12.8 & 5.1 & 6. & -14.7 & & -0.48 & -1.64 & 1063 & 0.20 \\
\hline 95 & -9.9 & \begin{tabular}{|l|l}
-17.5 \\
\end{tabular} & 3.2 & 11.3 & \begin{tabular}{|l}
-12.9 \\
\end{tabular} & \begin{tabular}{|l}
-19.0 \\
\end{tabular} & 5.3 & 14.0 & -15.8 & \begin{tabular}{|l}
-12.9 \\
\end{tabular} & 5.1 & 6. & -14.8 & 7.2 & -0.48 & -1.64 & 1068 & $0.19 \mathrm{~s}$ \\
\hline 96 & $\begin{array}{c}-9.9 \\
\end{array}$ & $\mid-17.5$ & 3.2 & 11.4 & \begin{tabular}{|l|}
-13 \\
\end{tabular} & -19.2 & 5. & 14.0 & -16.0 & $\mid-13.1$ & 5. & 6. & -14.9 & 7.2 & -0.48 & -1.65 & 1074 & 0.19 \\
\hline 97 & -10.0 & -17.6 & 3.2 & 11.4 & -13 & -19.4 & 5. & 14.1 & -15.9 & -13.0 & 5 & 7 & -14.9 & & -0.49 & -1.64 & 1076 & 0.2 \\
\hline 98 & -10.0 & \begin{tabular}{|l|}
-17.7 \\
\end{tabular} & 3.3 & 11.5 & -13.2 & -21.9 & 5.4 & 14.1 & -16.0 & -13.0 & 5.2 & 7. & -15.0 & 7.3 & -0.48 & -1.64 & 1081 & 0.20 \\
\hline 99 & \begin{tabular}{l|l}
-10.1 \\
\end{tabular} & $\mid-17.8$ & 3.2 & 11.5 & -13 & \begin{tabular}{|l}
-20.7 \\
\end{tabular} & 5. & 14 & -16.1 & -13.2 & 5.2 & 7. & - & & -0.48 & -1.65 & 1089 & 0.11 \\
\hline 100 & -10.2 & $\mid-17.9$ & 3.3 & 11.6 & \begin{tabular}{|l}
-13.3 \\
\end{tabular} & -20.7 & 5.5 & 14.3 & -16.2 & -13.2 & 5.2 & 7.1 & -15.2 & 7.3 & -0.48 & -1.65 & 1094 & 0.19 \\
\hline
\end{tabular}


Table E.17 NU 52-34 Test Data $\left(-10{ }^{\circ} \mathrm{C}\right.$ Tests)

\begin{tabular}{|c|c|c|c|c|c|c|c|c|c|c|c|c|c|c|c|c|c|c|}
\hline & \multicolumn{12}{|c|}{ Normalized Vertical and Horizontal Deformation Arrays } & \multirow{2}{*}{\multicolumn{2}{|c|}{ Trimmed }} & & & & \\
\hline & \multicolumn{4}{|c|}{ NU 52-34 Sample \#6 } & \multicolumn{4}{|c|}{ NU 52-34 Sample \#8 } & \multicolumn{4}{|c|}{ NU 52-34 Sample \#9 } & & & & & Creep & Pois. \\
\hline & vert & vert & horz & horz & vert & vert & horz & horz & vert & vert & horz & horz & & & Ratio & & Comp & Ratio \\
\hline Time & front & back & front & back & front & back & front & back & front & back & front & back & vert & horz & $\mathrm{X} / \mathrm{Y}$ & $\mathrm{C}_{\mathrm{empl}}$ & $\mathrm{D}(\mathrm{t})$ & $\bar{v}$ \\
\hline $\mathrm{sec}$ & $\mu \mathrm{m}$ & $\mu \mathrm{m}$ & $\mu \mathrm{m}$ & $\mu \mathrm{m}$ & $\mu \mathrm{m}$ & $\mu \mathrm{m}$ & $\mu \mathrm{m}$ & $\mu \mathrm{m}$ & $\mu \mathrm{m}$ & $\mu \mathrm{m}$ & $\mu \mathrm{m}$ & $\mu \mathrm{m}$ & $\mu \mathrm{m}$ & $\mu \mathrm{m}$ & & & $1 / \mathrm{kPa}$ & \\
\hline 0 & 0.0 & 0.0 & 0.0 & 0.0 & 0.0 & 0.0 & 0.0 & 0.0 & 0.0 & 0.0 & 0.0 & 0.0 & 0.0 & 0.0 & 0.00 & 0.00 & 0 & 0.000 \\
\hline 1 & -1.6 & -3.3 & 0.5 & 1.7 & -1.7 & -4.2 & 0.6 & 2.0 & -2.4 & -2.3 & 0.7 & 1.3 & -2.4 & 1.1 & -0.45 & -1.76 & 111 & 0.154 \\
\hline 2 & $\begin{array}{l}-1.8 \\
\end{array}$ & -3.8 & 0.6 & 2.0 & $\begin{array}{c}-1.9 \\
\end{array}$ & -4.8 & 0.8 & 2.4 & -3.0 & -2.8 & 0.8 & 1.5 & -2.9 & 1.3 & -0.44 & -1.78 & 132 & 0.145 \\
\hline 3 & -2.0 & -4.0 & 0.6 & 2.2 & -1.9 & -5.1 & 0.8 & 2.6 & -3.2 & -3.0 & 0.9 & 1.6 & -3.0 & 1.4 & -0.45 & -1.75 & 140 & 0.158 \\
\hline 4 & -2.1 & -4.2 & 0.7 & 2.3 & -2.2 & $\begin{array}{l}-5.3 \\
\end{array}$ & 0.9 & 2.8 & -3.4 & -3.1 & 1.0 & 1.7 & -3.2 & 1.5 & -0.45 & -1.74 & 148 & 0.160 \\
\hline 5 & -2.1 & -4.4 & 0.7 & 2.4 & -2.2 & -5.5 & 0.9 & 3.0 & -3.6 & -3.2 & 1.1 & 1.7 & -3.3 & 1.5 & -0.46 & -1.72 & 155 & 0.169 \\
\hline 6 & -2.3 & -4.7 & 0.7 & 2.4 & -2.2 & -5.5 & 1.0 & 3.1 & -3.7 & -3.3 & 1.1 & 1.8 & -3.5 & 1.6 & -0.45 & -1.74 & 161 & 0.162 \\
\hline 7 & -2.2 & -4.6 & 0.7 & 2.6 & -2.3 & -5.8 & 1.0 & 3.2 & -3.7 & -3.5 & 1.1 & 1.9 & -3.5 & 1.7 & -0.47 & -1.69 & 164 & 0.180 \\
\hline 8 & -2.4 & -4.9 & 0.7 & 2.6 & -2.2 & -5.7 & 1.1 & 3.3 & -3.9 & -3.5 & 1.2 & 1.9 & -3.6 & 1.7 & -0.47 & -1.69 & 169 & 0.181 \\
\hline 9 & -2.4 & -4.9 & 0.8 & 2.7 & -2.3 & -6.0 & 1.1 & 3.4 & -4.0 & -3.6 & 1.2 & 2.0 & -3.7 & 1.7 & -0.47 & -1.68 & 172 & 0.185 \\
\hline 10 & -2.4 & -5.1 & 0.8 & 2.8 & -2.3 & -6.0 & 1.1 & 3.5 & -4.0 & -3.6 & 1.2 & 2.0 & -3.8 & 1.8 & -0.48 & -1.67 & 175 & 0.189 \\
\hline 11 & -2.5 & -5.1 & 0.8 & 2.9 & -2.4 & -6.1 & 1.2 & 3.6 & -4.1 & -3.7 & 1.3 & 2.0 & -3.8 & 1.8 & -0.48 & -1.66 & 179 & 0.193 \\
\hline 12 & -2.4 & -5.2 & 0.8 & 2.9 & -2.5 & -6.1 & 1.2 & 3.7 & -4.1 & -3.7 & 1.3 & 2.1 & -3.9 & 1.9 & -0.48 & -1.64 & 182 & 0.200 \\
\hline 13 & -2.5 & -5.3 & 0.8 & 3.0 & -2.4 & -6.3 & 1.2 & 3.8 & -4.2 & -3.8 & 1.3 & 2.1 & -3.9 & 1.9 & -0.48 & -1.64 & 184 & 0.200 \\
\hline 14 & -2.8 & -5.6 & 0.8 & 3.0 & -2.5 & -6.4 & 1.3 & 3.9 & -4.3 & -3.8 & 1.3 & 2.2 & -4.1 & 2.0 & -0.48 & -1.65 & 189 & 0.196 \\
\hline 15 & -2.7 & -5.5 & 0.9 & 3.1 & -2.6 & -6.4 & 1.3 & 3.9 & -4.4 & -3.9 & 1.3 & 2.2 & -4.1 & 2.0 & -0.48 & -1.64 & 190 & 0.199 \\
\hline 16 & -2.7 & -5.6 & 0.9 & 3.1 & -2.7 & -6.7 & 1.3 & 4.0 & -4.4 & -3.9 & 1.4 & 2.2 & -4.2 & 2.0 & -0.48 & -1.65 & 194 & 0.196 \\
\hline 17 & -2.8 & -5.7 & 0.9 & 3.2 & -2.7 & -6.5 & 1.3 & 4.1 & -4.4 & -4.0 & 1.4 & 2.3 & -4.2 & 2.0 & -0.49 & -1.63 & 196 & 0.206 \\
\hline 18 & -2.8 & -5.7 & 0.9 & 3.2 & -2.7 & -6.8 & 1.3 & 4.1 & -4.6 & -4.0 & 1.4 & 2.3 & -4.3 & 2.1 & -0.48 & -1.64 & 199 & 0.200 \\
\hline 19 & -2.9 & -5.8 & 0.9 & 3.3 & -2.7 & -6.8 & 1.4 & 4.1 & -4.6 & -4.0 & 1.4 & 2.3 & -4.3 & 2.1 & -0.49 & -1.64 & 201 & 0.202 \\
\hline 20 & -2.9 & -5.8 & 0.9 & 3.3 & -2.8 & -7.0 & 1.4 & 4.2 & -4.6 & -4.1 & 1.5 & 2.3 & -4.3 & 2.1 & -0.49 & -1.63 & 203 & 0.206 \\
\hline 21 & -2.9 & -5.9 & 0.9 & 3.4 & -2.9 & -7.1 & 1.4 & 4.2 & -4.6 & -4.0 & 1.5 & 2.4 & -4.4 & 2.2 & -0.49 & -1.62 & 205 & 0.211 \\
\hline 22 & -2.9 & -5.9 & 1.0 & 3.4 & -2.8 & -7.2 & 1.4 & 4.3 & -4.7 & -4.1 & 1.5 & 2.4 & -4.4 & 2.2 & -0.50 & -1.61 & 207 & 0.214 \\
\hline 23 & -3.0 & -6.1 & 1.0 & 3.5 & -3.0 & -7.1 & 1.4 & 4.3 & -4.9 & -4.3 & 1.5 & 2.4 & -4.6 & 2.2 & -0.48 & -1.65 & 213 & 0.196 \\
\hline 24 & -3.0 & -6.1 & 1.0 & 3.5 & -3.0 & -7.2 & 1.4 & 4.4 & -4.9 & -4.3 & 1.5 & 2.4 & -4.6 & 2.2 & -0.49 & -1.64 & 214 & 0.202 \\
\hline 25 & -3.1 & -6.1 & 1.0 & 3.5 & -2.9 & -7.2 & 1.5 & 4.5 & -4.9 & -4.3 & 1.6 & 2.5 & -4.6 & 2.3 & -0.50 & -1.61 & 214 & 0.215 \\
\hline 26 & -3.1 & -6.2 & 1.0 & 3.6 & -3.0 & -7.6 & 1.5 & 4.5 & -4.8 & -4.1 & 1.6 & 2.5 & -4.5 & 2.3 & -0.51 & -1.59 & 214 & 0.228 \\
\hline 27 & -3.1 & -6.2 & 1.0 & 3.6 & -3.0 & -7.5 & 1.5 & 4.5 & -5.1 & -4.3 & 1.6 & 2.5 & -4.6 & 2.3 & -0.50 & -1.61 & 218 & 0.215 \\
\hline 28 & -3.1 & -6.4 & 1.0 & 3.7 & -3.1 & -7.6 & 1.5 & 4.6 & -5.0 & -4.4 & 1.6 & 2.5 & -4.7 & 2.3 & -0.49 & -1.62 & 221 & 0.212 \\
\hline 29 & -3.2 & -6.4 & 1.0 & 3.7 & -3.1 & -7.6 & 1.5 & 4.6 & -5.1 & -4.4 & 1.6 & 2.5 & -4.8 & 2.3 & -0.49 & -1.62 & 223 & 0.209 \\
\hline 30 & -3.3 & -6.6 & 1.0 & 3.7 & -3.1 & -7.6 & 1.6 & 4.7 & -5.1 & -4.4 & 1.6 & 2.6 & -4.8 & 2.4 & -0.49 & -1.62 & 226 & 0.211 \\
\hline 31 & -3.3 & -6.6 & 1.1 & 3.7 & -3.2 & -7.8 & 1.6 & 4.7 & -5.2 & -4.5 & 1.7 & 2.6 & -4.9 & 2.4 & -0.49 & -1.62 & 228 & 0.209 \\
\hline 32 & -3.3 & -6.6 & 1.1 & 3.8 & -3.3 & -7.8 & 1.6 & 4.8 & -5.1 & -4.5 & 1.7 & 2.7 & -4.9 & 2.4 & -0.50 & -1.61 & 229 & 0.215 \\
\hline 33 & -3.3 & -6.8 & 1.1 & 3.8 & -3.3 & -8.0 & 1.6 & 4.8 & -5.2 & -4.5 & 1.7 & 2.7 & -4.9 & 2.4 & -0.49 & -1.62 & 232 & 0.210 \\
\hline
\end{tabular}


Table E.17 NU 52-34 Test Data $\left(-10{ }^{\circ} \mathrm{C}\right.$ Tests $)$, continued

\begin{tabular}{|c|c|c|c|c|c|c|c|c|c|c|c|c|c|c|c|c|c|c|}
\hline & \multicolumn{12}{|c|}{ Normalized Vertical and Horizontal Deformation Arrays } & & & & & & \\
\hline & \multicolumn{4}{|c|}{ NU 52-34 Sample \#6 } & \multicolumn{4}{|c|}{ NU 52-34 Sample \#8 } & \multicolumn{4}{|c|}{ NU 52-34 Sample \#9 } & \multicolumn{2}{|c|}{ Trimmed } & & & Creep & Pois. \\
\hline & vert & vert & horz & horz & vert & vert & horz & horz & vert & vert & horz & horz & & & Ratio & & Comp & Ratio \\
\hline Time & front & back & front & back & front & back & front & back & front & back & front & back & vert & horz & $\mathrm{X} / \mathrm{Y}$ & $\mathrm{C}_{\mathrm{empl}}$ & $D(t)$ & $v$ \\
\hline $\mathrm{sec}$ & $\mu \mathrm{m}$ & $\mu \mathrm{m}$ & $\mu \mathrm{m}$ & $\mu \mathrm{m}$ & $\mu \mathrm{m}$ & $\mu \mathrm{m}$ & $\mu \mathrm{m}$ & $\mu \mathrm{m}$ & $\mu \mathrm{m}$ & $\mu \mathrm{m}$ & $\mu \mathrm{m}$ & $\mu \mathrm{m}$ & $\mu \mathrm{m}$ & $\mu \mathrm{m}$ & & & $1 / \mathrm{kPa}$ & \\
\hline 34 & -3.3 & -6.8 & 1.1 & 3.8 & -3.3 & -8.0 & 1.6 & 4.9 & -5.3 & -4.6 & 1.7 & 2.7 & -5.0 & 2.5 & -0.49 & -1.62 & 234 & 0.210 \\
\hline 35 & -3.4 & -6.8 & 1.1 & 3.9 & -3.4 & -8.0 & 1.6 & 4.9 & -5.3 & -4.6 & 1.7 & 2.7 & -5.0 & 2.5 & -0.49 & -1.63 & 236 & 0.206 \\
\hline 36 & -3.3 & -6.8 & 1.1 & 3.9 & -3.4 & -8.1 & 1.6 & 4.9 & -5.3 & -4.5 & 1.7 & 2.7 & -5.0 & 2.5 & -0.50 & -1.61 & 236 & 0.218 \\
\hline 37 & -3.3 & -6.8 & 1.1 & 3.9 & -3.5 & -8.2 & 1.7 & 5.0 & -5.4 & -4.6 & 1.7 & 2.7 & -5.1 & 2.5 & -0.50 & -1.61 & 238 & 0.215 \\
\hline 38 & -3.4 & -6.9 & 1.1 & 4.0 & -3.5 & -8.2 & 1.6 & 5.0 & -5.3 & -4.6 & 1.8 & 2.8 & -5.1 & 2.5 & -0.50 & -1.60 & 239 & 0.218 \\
\hline 39 & -3.4 & -6.9 & 1.1 & 4.0 & -3.5 & -8.3 & 1.7 & 5.0 & -5.4 & -4.7 & 1.8 & 2.8 & -5.1 & 2.5 & -0.50 & -1.61 & 241 & 0.216 \\
\hline 40 & -3.4 & -6.9 & 1.1 & 4.0 & -3.5 & -8.3 & 1.7 & 5.1 & -5.4 & -4.6 & 1.8 & 2.8 & -5.1 & 2.6 & -0.51 & -1.59 & 240 & 0.226 \\
\hline 41 & -3.4 & $\begin{array}{l}-7.0 \\
\end{array}$ & 1.1 & 4.0 & -3.5 & $\begin{array}{c}-8.4 \\
\end{array}$ & 1.7 & 5.1 & -5.5 & -4.8 & 1.8 & 2.8 & -5.2 & 2.6 & -0.50 & -1.60 & 243 & 0.221 \\
\hline 42 & -3.4 & -7.0 & 1.1 & 4.0 & -3.5 & -8.4 & 1.7 & 5.1 & -5.5 & -4.7 & 1.8 & 2.8 & -5.2 & 2.6 & -0.50 & -1.60 & 244 & 0.223 \\
\hline 43 & -3.6 & -7.0 & 1.1 & 4.1 & -3.7 & -8.5 & 1.7 & 5.2 & -5.5 & -4.7 & 1.8 & 2.9 & -5.2 & 2.6 & -0.50 & -1.60 & 247 & 0.219 \\
\hline 44 & -3.6 & -7.2 & 1.2 & 4.2 & -3.8 & -8.6 & 1.7 & 5.2 & -5.6 & -4.7 & 1.8 & 2.9 & -5.3 & 2.7 & -0.50 & -1.60 & 250 & 0.218 \\
\hline 45 & -3.6 & -7.2 & 1.2 & 4.2 & -3.8 & -8.6 & 1.8 & 5.2 & -5.5 & -4.7 & 1.9 & 2.9 & $\begin{array}{l}-5.3 \\
\end{array}$ & 2.7 & -0.50 & -1.59 & 250 & 0.225 \\
\hline 46 & -3.6 & $\begin{array}{l}-7.2 \\
\end{array}$ & 1.2 & 4.2 & -3.9 & -8.7 & 1.8 & 5.2 & -5.6 & -4.8 & 1.5 & 2.9 & $\begin{array}{l}-5.3 \\
\end{array}$ & 2.7 & -0.50 & -1.60 & 252 & 0.223 \\
\hline 47 & -3.5 & -7.2 & 1.2 & 4.2 & -3.9 & -8.8 & 1.8 & 5.3 & -5.6 & -4.9 & 1.9 & 3.0 & $\begin{array}{l}-5.4 \\
\end{array}$ & 2.7 & -0.50 & -1.60 & 255 & 0.223 \\
\hline 48 & -3.6 & -7.2 & 1.2 & 4.3 & -3.9 & -8.8 & 1.8 & 5.3 & -5.7 & -4.8 & 1.9 & 2.9 & -5.4 & 2.7 & -0.50 & -1.60 & 255 & 0.221 \\
\hline 49 & -3.6 & $\begin{array}{l}-7.3 \\
\end{array}$ & 1.2 & 4.3 & -3.9 & -8.9 & 1.8 & 5.3 & -5.7 & -4.9 & 1.9 & 3.0 & -5.5 & 2.7 & -0.50 & -1.60 & 258 & 0.220 \\
\hline 50 & -3.6 & -7.3 & 1.2 & 4.3 & -4.0 & -8.9 & 1.8 & 5.4 & -5.7 & -4.9 & 1.9 & 3.0 & -5.5 & 2.8 & -0.50 & -1.60 & 258 & 0.222 \\
\hline 51 & -3.7 & -7.4 & 1.2 & 4.3 & -4.0 & -9.0 & 1.8 & 5.4 & -5.8 & -5.1 & 1.9 & 3.0 & -5.6 & 2.8 & -0.49 & -1.62 & 262 & 0.211 \\
\hline 52 & -3.6 & -7.3 & 1.2 & 4.4 & -4.0 & -9.2 & 1.8 & 5.4 & -5.8 & -4.9 & 1.9 & 3.0 & -5.5 & 2.8 & -0.50 & -1.60 & 260 & 0.222 \\
\hline 53 & -3.6 & -7.4 & 1.2 & 4.4 & -4.2 & -9.2 & 1.8 & 5.4 & -5.8 & -4.9 & 1.9 & 3.0 & -5.6 & 2.8 & -0.50 & -1.60 & 263 & 0.221 \\
\hline 54 & -3.6 & -7.4 & 1.2 & 4.4 & -4.3 & -9.4 & 1.8 & 5.5 & -5.7 & -4.9 & 2.0 & 3.0 & -5.6 & 2.8 & -0.50 & -1.60 & 263 & 0.221 \\
\hline 55 & -3.6 & -7.3 & 1.3 & 4.4 & -4.3 & -9.4 & 1.9 & 5.5 & -5.8 & -5.0 & 2.0 & 3.1 & -5.6 & 2.8 & -0.51 & -1.59 & 264 & 0.227 \\
\hline 56 & -3.7 & -7.4 & 1.3 & 4.4 & -4.3 & -9.5 & 1.9 & 5.5 & -6.0 & -5.2 & 2.0 & 3.0 & -5.7 & 2.8 & -0.49 & -1.62 & 269 & 0.210 \\
\hline 57 & -3.6 & -7.5 & 1.2 & 4.5 & -4.5 & -9.6 & 1.9 & 5.5 & -5.8 & -4.9 & 2.0 & 3.1 & -5.7 & 2.9 & -0.50 & -1.60 & 269 & 0.221 \\
\hline 58 & -3.7 & -7.4 & 1.3 & 4.5 & -4.3 & -9.7 & 1.9 & 5.5 & -5.9 & -5.1 & 2.0 & 3.1 & -5.7 & 2.9 & -0.50 & -1.60 & 268 & 0.223 \\
\hline 59 & -3.6 & -7.4 & 1.3 & 4.6 & -4.5 & -9.5 & 1.9 & 5.5 & -5.9 & -5.1 & 2.0 & 3.1 & -5.7 & 2.9 & -0.50 & -1.59 & 271 & 0.225 \\
\hline 60 & -3.7 & -7.6 & 1.3 & 4.6 & -4.5 & -9.7 & 1.9 & 5.6 & -6.0 & -5.1 & 2.0 & 3.1 & -5.8 & 2.9 & -0.50 & -1.60 & 273 & 0.219 \\
\hline 61 & -3.7 & -7.5 & 1.3 & 4.6 & -4.3 & -9.6 & 2.0 & 5.7 & -6.0 & -5.1 & 2.0 & 3.2 & -5.7 & 2.9 & -0.51 & -1.57 & 271 & 0.234 \\
\hline 62 & -3.8 & -7.6 & 1.3 & 4.6 & -4.6 & -9.7 & 2.0 & 5.7 & -6.0 & -5.1 & 2.0 & 3.2 & -5.8 & 2.9 & -0.51 & -1.59 & 275 & 0.227 \\
\hline 63 & -3.7 & -7.7 & 1.3 & 4.6 & -4.5 & -9.8 & 2.0 & 5.8 & -6.0 & -5.1 & 2.1 & 3.2 & -5.8 & 3.0 & -0.51 & -1.58 & 275 & 0.229 \\
\hline 64 & -3.7 & -7.7 & 1.3 & 4.7 & -4.6 & -9.7 & 2.0 & 5.8 & -6.1 & -5.1 & 2.1 & 3.2 & -5.9 & 3.0 & -0.51 & -1.58 & 277 & 0.229 \\
\hline 65 & -3.7 & -7.7 & 1.3 & 4.7 & -4.5 & -9.7 & 2.0 & 5.8 & -6.1 & -5.1 & 2.1 & 3.2 & -5.8 & 3.0 & -0.51 & -1.58 & 276 & 0.233 \\
\hline 66 & -3.7 & -7.7 & 1.3 & 4.7 & -4.7 & -9.9 & 2.0 & 5.9 & -6.0 & -5.2 & 2.1 & 3.2 & -5.9 & 3.0 & -0.51 & -1.58 & 279 & 0.232 \\
\hline
\end{tabular}


Table E.17 NU 52-34 Test Data $\left(-10{ }^{\circ} \mathrm{C}\right.$ Tests $)$, continued

\begin{tabular}{|c|c|c|c|c|c|c|c|c|c|c|c|c|c|c|c|c|c|c|}
\hline & \multicolumn{12}{|c|}{ Normalized Vertical and Horizontal Deformation Arrays } & \multirow{2}{*}{\multicolumn{2}{|c|}{ Trimmed }} & \multirow[b]{3}{*}{ Ratio } & & \multirow{3}{*}{\begin{tabular}{|l|} 
Creep \\
Comp \\
\end{tabular}} & \multirow{3}{*}{\begin{tabular}{|l|} 
Pois. \\
Ratio \\
\end{tabular}} \\
\hline & \multicolumn{4}{|c|}{ NU 52-34 Sample \#6 } & \multicolumn{4}{|c|}{ NU 52-34 Sample \#8 } & \multicolumn{4}{|c|}{ NU 52-34 Sample \#9 } & & & & & & \\
\hline & vert & vert & horz & horz & vert & vert & horz & horz & vert & vert & horz & horz & & & & & & \\
\hline Time & front & back & front & back & front & back & front & back & front & back & front & back & vert & horz & $\mathrm{X} / \mathrm{Y}$ & $\mathrm{C}_{\mathrm{empl}}$ & $D(t)$ & $\bar{v}$ \\
\hline $\mathrm{sec}$ & $\mu \mathrm{m}$ & $\mu \mathrm{m}$ & $\mu \mathrm{m}$ & $\mu \mathrm{m}$ & $\mu \mathrm{m}$ & $\mu \mathrm{m}$ & $\mu \mathrm{m}$ & $\mu \mathrm{m}$ & $\mu \mathrm{m}$ & $\mu \mathrm{m}$ & $\mu \mathrm{m}$ & $\mu \mathrm{m}$ & $\mu \mathrm{m}$ & $\mu \mathrm{m}$ & & & $1 / \mathrm{kPa}$ & \\
\hline 67 & -3.8 & -7.9 & 1.3 & 4.7 & -4.6 & -9.8 & 2.0 & $\overline{5.9}$ & -6.1 & -5.2 & 2.1 & $\overline{3.2}$ & -5.9 & 3.0 & -0.51 & -1.58 & 280 & 0.230 \\
\hline 68 & -3.7 & -7.8 & 1.3 & 4.8 & -4.6 & -9.9 & 2.0 & 5.9 & -6.1 & -5.2 & 2.1 & 3.2 & -5.9 & 3.0 & -0.51 & -1.57 & 281 & 0.235 \\
\hline 69 & $\begin{array}{l}-3.8 \\
\end{array}$ & -7.8 & 1.3 & 4.8 & -4.7 & -10.0 & 2.1 & 6.0 & -6.2 & -5.2 & 2.1 & 3.3 & -6.0 & 3.1 & -0.51 & -1.57 & 282 & 0.234 \\
\hline 70 & -3.8 & -7.9 & 1.3 & 4.8 & -4.6 & -9.9 & 2.1 & 6.0 & -6.1 & -5.3 & 2.1 & 3.3 & -6.0 & 3.1 & -0.51 & -1.57 & 283 & 0.238 \\
\hline 71 & -3.8 & -7.9 & 1.3 & 4.8 & -4.6 & -10.1 & 2.1 & 6.0 & -6.2 & -5.2 & 2.2 & 3.3 & -5.9 & 3.1 & -0.52 & -1.56 & 282 & 0.241 \\
\hline 72 & -3.9 & -8.0 & 1.4 & 4.8 & -4.7 & -10.1 & 2.1 & 6.1 & -6.2 & -5.2 & 2.2 & 3.3 & -6.0 & 3.1 & -0.51 & -1.57 & 285 & 0.236 \\
\hline 73 & $\begin{array}{l}-3.8 \\
\end{array}$ & -7.8 & 1.4 & 4.9 & -4.7 & -10.1 & 2.1 & 6.1 & -6.2 & -5.3 & 2.2 & 3.3 & -6.0 & 3.1 & -0.52 & -1.56 & 285 & 0.241 \\
\hline 74 & -3.9 & -7.9 & 1.4 & 4.9 & -4.7 & -10.1 & 2.1 & 6.1 & -6.2 & -5.3 & 2.2 & 3.3 & -6.0 & 3.1 & -0.52 & -1.56 & 286 & 0.241 \\
\hline 75 & -3.9 & -8.1 & 1.4 & 4.9 & -4.7 & -10.1 & 2.1 & 6.2 & -6.2 & -5.3 & 2.2 & 3.3 & -6.1 & 3.1 & -0.51 & -1.58 & 289 & 0.233 \\
\hline 76 & -3.8 & -8.0 & 1.4 & 4.9 & -4.7 & -10.1 & 2.1 & 6.2 & -6.3 & -5.3 & 2.2 & 3.3 & -6.1 & 3.1 & -0.51 & -1.57 & 289 & 0.239 \\
\hline 77 & -3.9 & -8.0 & 1.4 & 4.9 & -4.7 & -10.2 & 2.1 & 6.2 & -6.3 & -5.4 & 2.2 & 3.4 & -6.1 & 3.2 & -0.52 & -1.56 & 290 & 0.243 \\
\hline 78 & -4.0 & -8.0 & 1.4 & 5.0 & -4.7 & -10.2 & 2.1 & 6.2 & -6.3 & -5.3 & 2.2 & 3.4 & -6.1 & 3.2 & -0.52 & -1.54 & 289 & 0.251 \\
\hline 79 & -3.9 & -8.1 & 1.4 & 4.9 & -4.7 & -10.1 & 2.2 & 6.2 & -6.4 & -5.5 & 2.2 & 3.4 & -6.2 & 3.2 & -0.52 & -1.56 & 292 & 0.240 \\
\hline 80 & -3.8 & -8.1 & 1.4 & 5.0 & -4.7 & -10.3 & 2.2 & 6.2 & -6.4 & -5.4 & 2.2 & 3.4 & -6.1 & 3.2 & -0.52 & -1.54 & 291 & 0.251 \\
\hline 81 & -4.0 & -8.3 & 1.4 & 5.0 & -4.7 & -10.2 & 2.2 & 6.3 & -6.3 & -5.4 & 2.2 & 3.4 & -6.2 & 3.2 & -0.52 & -1.56 & 294 & 0.243 \\
\hline 82 & -4.0 & -8.1 & 1.4 & 5.0 & -4.8 & -10.3 & 2.2 & 6.3 & -6.4 & -5.5 & 2.3 & 3.5 & -6.2 & 3.2 & -0.52 & -1.55 & 295 & 0.247 \\
\hline 83 & -3.9 & -8.1 & 1.4 & 5.1 & -4.7 & -10.3 & 2.2 & 6.3 & -6.4 & -5.5 & 2.3 & 3.5 & -6.2 & 3.3 & -0.53 & -1.53 & 293 & 0.257 \\
\hline 84 & -3.9 & -8.1 & 1.4 & 5.1 & -4.7 & -10.3 & 2.2 & 6.4 & -6.5 & -5.6 & 2.3 & 3.5 & -6.2 & 3.3 & -0.52 & -1.55 & 297 & 0.247 \\
\hline 85 & -3.9 & -8.1 & 1.4 & 5.1 & -4.7 & -10.3 & 2.2 & 6.4 & -6.5 & -5.5 & 2.3 & 3.5 & -6.2 & 3.3 & -0.53 & -1.53 & 295 & 0.258 \\
\hline 86 & -3.8 & -8.2 & 1.5 & 5.1 & -4.7 & -10.4 & 2.2 & 6.4 & -6.5 & -5.5 & 2.3 & 3.5 & -6.2 & 3.3 & -0.53 & -1.53 & 296 & 0.260 \\
\hline 87 & -4.0 & -8.2 & 1.5 & 5.2 & -4.8 & -10.3 & 2.3 & 6.5 & -6.5 & -5.5 & 2.3 & 3.5 & -6.3 & 3.3 & -0.53 & -1.53 & 298 & 0.261 \\
\hline 88 & -3.9 & -8.1 & 1.5 & 5.2 & -4.7 & -10.2 & 2.3 & 6.5 & -6.5 & -5.6 & 2.3 & 3.5 & -6.2 & 3.3 & -0.53 & -1.52 & 298 & 0.264 \\
\hline 89 & -3.9 & -8.2 & 1.5 & 5.2 & -4.8 & -10.3 & 2.3 & 6.5 & -6.6 & -5.6 & 2.3 & 3.6 & -6.3 & 3.3 & -0.53 & -1.53 & 301 & 0.257 \\
\hline 90 & -3.9 & -8.2 & 1.5 & 5.2 & -4.7 & -10.5 & 2.3 & 6.5 & -6.6 & -5.6 & 2.3 & 3.6 & -6.3 & 3.4 & -0.53 & -1.52 & 300 & 0.265 \\
\hline 91 & -3.9 & -8.3 & 1.5 & 5.2 & -4.8 & -10.4 & 2.3 & 6.6 & -6.5 & -5.6 & 2.3 & 3.6 & -6.3 & 3.4 & -0.53 & -1.53 & 301 & 0.262 \\
\hline 92 & -3.9 & -8.3 & 1.5 & 5.2 & -4.7 & -10.3 & 2.3 & 6.6 & -6.6 & -5.6 & 2.3 & 3.6 & -6.3 & 3.4 & -0.54 & -1.52 & 302 & 0.266 \\
\hline 93 & -4.0 & -8.3 & 1.5 & 5.3 & -4.7 & -10.5 & 2.3 & 6.6 & -6.7 & -5.6 & 2.3 & 3.6 & -6.3 & 3.4 & -0.53 & -1.52 & 302 & 0.265 \\
\hline 94 & -3.9 & -8.3 & 1.5 & 5.3 & -4.7 & -10.4 & 2.3 & 6.6 & -6.6 & -5.7 & 2.4 & 3.6 & -6.3 & 3.4 & -0.54 & -1.51 & 303 & 0.269 \\
\hline 95 & -4.0 & -8.4 & 1.5 & 5.3 & -4.7 & -10.4 & 2.3 & 6.7 & -6.6 & -5.6 & 2.4 & 3.6 & -6.4 & 3.4 & -0.54 & -1.52 & 304 & 0.266 \\
\hline 96 & -3.9 & -8.3 & 1.5 & 5.3 & -4.7 & -10.5 & 2.3 & 6.7 & -6.7 & -5.7 & 2.4 & 3.6 & -6.3 & 3.4 & -0.54 & -1.51 & 304 & 0.272 \\
\hline 97 & -4.0 & -8.4 & 1.5 & 5.3 & -4.6 & -10.5 & 2.4 & 6.8 & -6.7 & -5.7 & 2.4 & 3.6 & -6.4 & 3.4 & -0.54 & -1.51 & 304 & 0.273 \\
\hline 98 & -3.9 & -8.4 & 1.5 & 5.4 & -4.8 & -10.6 & 2.3 & 6.7 & -6.7 & -5.7 & 2.4 & 3.6 & -6.4 & 3.4 & -0.54 & -1.52 & 307 & 0.266 \\
\hline 99 & -4.0 & -8.5 & 1.5 & 5.4 & -4.7 & -10.5 & 2.4 & 6.8 & -6.8 & -5.8 & 2.4 & 3.7 & -6.4 & 3.5 & -0.54 & -1.51 & 307 & 0.270 \\
\hline 100 & -4.1 & -8.5 & 1.5 & 5.4 & -4.7 & -10.5 & 2.4 & 6.8 & -6.8 & -5.7 & 2.4 & 3.7 & -6.4 & 3.5 & -0.54 & -1.51 & 307 & 0.271 \\
\hline
\end{tabular}


Table E.18 NU 52-34 Test Data $\left(-20{ }^{\circ} \mathrm{C}\right.$ Tests)

\begin{tabular}{|c|c|c|c|c|c|c|c|c|c|c|c|c|c|c|c|c|c|c|}
\hline & \multicolumn{12}{|c|}{ Normalized Vertical and Horizontal Deformation Arrays } & \multirow{2}{*}{\multicolumn{2}{|c|}{ Trimmed }} & & & & \\
\hline & \multicolumn{4}{|c|}{ NU 52-34 Sample \#6 } & \multicolumn{4}{|c|}{ NU 52-34 Sample \#8 } & \multicolumn{4}{|c|}{ NU 52-34 Sample \#9 } & & & & & Creep & Pois. \\
\hline & vert & vert & horz & horz & vert & vert & horz & horz & vert & vert & horz & horz & & & Ratio & & Comp & Ratio \\
\hline Time & front & back & front & back & front & back & front & back & front & back & front & back & vert & horz & $\mathrm{X} / \mathrm{Y}$ & $\mathrm{C}_{\mathrm{empl}}$ & $\mathrm{D}(\mathrm{t})$ & $v$ \\
\hline $\sec$ & $\mu \mathrm{m}$ & $\mu \mathrm{m}$ & $\mu \mathrm{m}$ & $\mu \mathrm{m}$ & $\mu \mathrm{m}$ & $\mu \mathrm{m}$ & $\mu \mathrm{m}$ & $\mu \mathrm{m}$ & $\mu \mathrm{m}$ & $\mu \mathrm{m}$ & $\mu \mathrm{m}$ & $\mu \mathrm{m}$ & $\mu \mathrm{m}$ & $\mu \mathrm{m}$ & & & $1 / \mathrm{kPa}$ & \\
\hline 0 & 0.0 & 0.0 & 0.0 & 0.0 & 0.0 & 0.0 & 0.0 & 0.0 & 0.0 & 0.0 & 0.0 & 0.0 & 0.0 & 0.0 & 0.00 & 0.00 & 0 & 0.000 \\
\hline 1 & -1.6 & -3.4 & 0.5 & 1.6 & -1.4 & -4.6 & 0.6 & 1.8 & -2.1 & -2.3 & 0.6 & 1.2 & -1.8 & 1.0 & -0.54 & -1.51 & 51 & 0.270 \\
\hline 2 & -2.1 & -4.1 & 0.6 & 2.0 & -1.6 & -5.1 & 0.6 & 2.1 & -2.8 & -3.2 & 0.8 & 1.6 & -2.4 & 1.3 & -0.53 & -1.53 & 67 & 0.256 \\
\hline 3 & -2.2 & -4.3 & 0.6 & 2.1 & -1.8 & -5.5 & 0.7 & 2.2 & -3.1 & -3.5 & 0.9 & 1.7 & -2.6 & 1.3 & -0.52 & -1.55 & 71 & 0.247 \\
\hline 4 & -2.2 & -4.4 & 0.7 & 2.2 & -1.8 & -5.6 & 0.7 & 2.3 & -3.2 & -3.6 & 0.9 & 1.8 & -2.6 & 1.4 & -0.53 & -1.53 & 73 & 0.260 \\
\hline 5 & -2.3 & -4.5 & 0.7 & 2.2 & -1.9 & -5.8 & 0.7 & 2.4 & -3.3 & -3.8 & 1.0 & 1.8 & -2.7 & 1.4 & -0.52 & -1.54 & 76 & 0.252 \\
\hline 6 & -2.3 & -4.6 & 0.7 & 2.3 & -1.9 & -5.8 & 0.7 & 2.5 & -3.3 & -3.8 & 1.0 & 1.8 & -2.7 & 1.5 & -0.54 & -1.51 & 77 & 0.269 \\
\hline 7 & -2.4 & -4.8 & 0.7 & 2.4 & -1.9 & -6.0 & 0.8 & 2.6 & -3.4 & -3.9 & 1.0 & 1.9 & -2.8 & 1.5 & -0.53 & -1.52 & 79 & 0.264 \\
\hline 8 & -2.5 & -4.8 & 0.7 & 2.4 & -2.2 & -6.1 & 0.8 & 2.6 & -3.5 & -3.9 & 1.0 & 1.9 & -2.9 & 1.5 & -0.53 & -1.54 & 81 & 0.253 \\
\hline 9 & -2.5 & -4.8 & 0.7 & 2.4 & -2.0 & -6.1 & 0.8 & 2.7 & -3.6 & -4.0 & 1.0 & 1.9 & -2.9 & 1.5 & -0.53 & -1.53 & 82 & 0.257 \\
\hline 10 & -2.5 & -4.9 & 0.7 & 2.4 & -2.2 & -6.2 & 0.8 & 2.7 & -3.6 & -4.0 & 1.0 & 2.0 & -3.0 & 1.6 & -0.53 & -1.53 & 83 & 0.260 \\
\hline 11 & -2.5 & -5.0 & 0.8 & 2.5 & -2.0 & -6.3 & 0.8 & 2.8 & -3.8 & -4.0 & 1.1 & 2.0 & -3.0 & 1.6 & -0.53 & -1.53 & 84 & 0.262 \\
\hline 12 & -2.6 & -5.0 & 0.7 & 2.5 & -2.2 & -6.3 & 0.8 & 2.8 & -3.8 & -4.1 & 1.1 & 2.0 & -3.0 & 1.6 & -0.53 & -1.53 & 85 & 0.258 \\
\hline 13 & -2.6 & -5.1 & 0.8 & 2.5 & -2.2 & -6.4 & 0.8 & 2.8 & -3.8 & -4.2 & 1.1 & 2.0 & -3.1 & 1.6 & -0.53 & -1.53 & 86 & 0.257 \\
\hline 14 & -2.7 & -5.2 & 0.7 & 2.5 & -2.2 & -6.4 & 0.9 & 2.9 & -3.9 & -4.2 & 1.1 & 2.0 & -3.1 & 1.6 & -0.52 & -1.55 & 87 & 0.250 \\
\hline 15 & -2.6 & -5.2 & 0.8 & 2.6 & -2.2 & -6.5 & 0.9 & 3.0 & -3.9 & -4.2 & 1.1 & 2.1 & -3.1 & 1.7 & -0.53 & -1.53 & 88 & 0.259 \\
\hline 16 & -2.6 & -5.2 & 0.8 & 2.6 & -2.3 & -6.5 & 0.9 & 3.0 & -4.0 & -4.3 & 1.1 & 2.1 & -3.2 & 1.7 & -0.53 & -1.54 & 89 & 0.254 \\
\hline 17 & -2.7 & -5.3 & 0.8 & 2.7 & -2.2 & -6.4 & 0.9 & 3.0 & -4.0 & -4.3 & 1.2 & 2.1 & -3.2 & 1.7 & -0.53 & -1.52 & 89 & 0.263 \\
\hline 18 & -2.7 & -5.3 & 0.8 & 2.7 & -2.2 & -6.6 & 0.9 & 3.0 & -4.0 & -4.3 & 1.2 & 2.1 & -3.2 & 1.7 & -0.53 & -1.52 & 90 & 0.264 \\
\hline 19 & -2.7 & -5.3 & 0.8 & 2.7 & -2.2 & -6.6 & 0.9 & 3.0 & -4.0 & -4.3 & 1.2 & 2.1 & -3.2 & 1.7 & -0.54 & -1.50 & 90 & 0.275 \\
\hline 20 & -2.6 & -5.4 & 0.8 & 2.8 & -2.4 & -6.8 & 0.9 & 3.1 & -4.2 & -4.4 & 1.2 & 2.2 & -3.3 & 1.8 & -0.54 & -1.52 & 92 & 0.267 \\
\hline 21 & -2.7 & -5.3 & 0.8 & 2.8 & -2.3 & -6.7 & 0.9 & 3.1 & -4.3 & -4.6 & 1.2 & 2.1 & -3.3 & 1.8 & -0.53 & -1.54 & 93 & 0.255 \\
\hline 22 & -2.6 & -5.3 & 0.9 & 2.8 & -2.4 & -6.8 & 0.9 & 3.1 & -4.2 & -4.4 & 1.2 & 2.2 & -3.2 & 1.8 & -0.55 & -1.49 & 91 & 0.287 \\
\hline 23 & -2.6 & -5.3 & 0.8 & 2.9 & -2.3 & -6.9 & 0.9 & 3.1 & -4.2 & -4.6 & 1.2 & 2.2 & -3.3 & 1.8 & -0.54 & -1.51 & 93 & 0.274 \\
\hline 24 & -2.6 & -5.3 & 0.9 & 2.9 & -2.3 & -6.9 & 0.9 & 3.2 & -4.3 & -4.5 & 1.2 & 2.2 & -3.3 & 1.8 & -0.55 & -1.48 & 93 & 0.289 \\
\hline 25 & -2.6 & -5.3 & 0.9 & 2.9 & -2.4 & -7.0 & 0.9 & 3.2 & -4.3 & -4.5 & 1.2 & 2.2 & -3.3 & 1.8 & -0.56 & -1.47 & 93 & 0.298 \\
\hline 26 & -2.8 & -5.5 & 0.9 & 2.9 & -2.5 & -7.1 & 0.9 & 3.3 & -4.2 & -4.6 & 1.2 & 2.2 & -3.4 & 1.8 & -0.54 & -1.50 & 95 & 0.276 \\
\hline 27 & -2.6 & -5.3 & 0.9 & 2.9 & -2.5 & -7.0 & 1.0 & 3.3 & -4.3 & -4.5 & 1.3 & 2.2 & -3.3 & 1.8 & -0.56 & -1.46 & 93 & 0.303 \\
\hline 28 & -2.6 & -5.3 & 0.9 & 3.0 & -2.5 & -7.0 & 1.0 & 3.3 & -4.3 & -4.5 & 1.3 & 2.2 & -3.3 & 1.9 & -0.57 & -1.45 & 93 & 0.311 \\
\hline 29 & -2.5 & -5.3 & 0.9 & 3.0 & -2.6 & -7.2 & 1.0 & 3.3 & -4.3 & -4.6 & 1.3 & 2.2 & -3.3 & 1.9 & -0.56 & -1.46 & 94 & 0.304 \\
\hline 30 & -2.5 & -5.3 & 0.9 & 3.0 & -2.7 & -7.2 & 1.0 & 3.3 & -4.4 & -4.7 & 1.3 & 2.3 & -3.4 & 1.9 & -0.56 & -1.48 & 95 & 0.294 \\
\hline 31 & -2.5 & -5.3 & 0.9 & 3.1 & -2.8 & -7.2 & 1.0 & 3.3 & -4.3 & -4.7 & 1.3 & 2.3 & -3.4 & 1.9 & -0.56 & -1.46 & 95 & 0.307 \\
\hline 32 & -2.5 & -5.5 & 0.9 & 3.0 & -2.8 & -7.3 & 1.0 & 3.3 & -4.4 & -4.6 & 1.3 & 2.3 & -3.4 & 1.9 & -0.56 & -1.47 & 96 & 0.297 \\
\hline 33 & -2.5 & -5.3 & 0.9 & 3.1 & -2.8 & -7.3 & 1.0 & 3.3 & -4.4 & -4.6 & 1.3 & 2.3 & -3.3 & 1.9 & -0.57 & -1.45 & 95 & 0.316 \\
\hline
\end{tabular}


Table E.18 NU 52-34 Test Data $\left(-20{ }^{\circ} \mathrm{C}\right.$ Tests $)$, continued

\begin{tabular}{|c|c|c|c|c|c|c|c|c|c|c|c|c|c|c|c|c|c|c|}
\hline & \multicolumn{12}{|c|}{ Normalized Vertical and Horizontal Deformation Arrays } & & & & & & \\
\hline & \multicolumn{4}{|c|}{ NU 52-34 Sample \#6 } & \multicolumn{4}{|c|}{ NU 52-34 Sample \#8 } & \multicolumn{4}{|c|}{ NU 52-34 Sample \#9 } & \multicolumn{2}{|c|}{ Trimmed } & & & Creep & Pois. \\
\hline & vert & vert & horz & horz & vert & vert & horz & horz & vert & vert & horz & horz & & & Ratio & & Comp & Ratio \\
\hline Time & front & back & front & back & front & back & front & back & front & back & front & back & vert & horz & $\mathrm{X} / \mathrm{Y}$ & $\mathrm{C}_{\mathrm{empl}}$ & $\mathrm{D}(\mathrm{t})$ & $v$ \\
\hline $\mathrm{sec}$ & $\mu \mathrm{m}$ & $\mu \mathrm{m}$ & $\mu \mathrm{m}$ & $\mu \mathrm{m}$ & $\mu \mathrm{m}$ & $\mu \mathrm{m}$ & $\mu \mathrm{m}$ & $\mu \mathrm{m}$ & $\mu \mathrm{m}$ & $\mu \mathrm{m}$ & $\mu \mathrm{m}$ & $\mu \mathrm{m}$ & $\mu \mathrm{m}$ & $\mu \mathrm{m}$ & & & $1 / \mathrm{kPa}$ & \\
\hline 34 & -2.7 & -5.7 & 0.9 & 3.0 & -2.8 & -7.4 & 1.0 & 3.3 & -4.4 & -4.7 & 1.3 & 2.3 & -3.5 & 1.9 & -0.55 & -1.49 & 98 & 0.285 \\
\hline 35 & -2.5 & -5.4 & 0.9 & 3.1 & -2.8 & -7.5 & 1.0 & 3.4 & -4.5 & -4.7 & 1.3 & 2.3 & -3.4 & 1.9 & -0.56 & -1.46 & 96 & 0.305 \\
\hline 36 & -2.5 & -5.3 & 1.0 & 3.1 & -2.8 & -7.4 & 1.0 & 3.4 & -4.4 & -4.6 & 1.3 & 2.3 & -3.4 & 1.9 & -0.58 & -1.43 & 95 & 0.326 \\
\hline 37 & -2.5 & -5.3 & 1.0 & 3.1 & -3.0 & -7.6 & 1.0 & 3.4 & -4.5 & -4.6 & 1.3 & 2.3 & -3.4 & 1.9 & -0.58 & -1.43 & 96 & 0.325 \\
\hline 38 & -2.5 & -5.4 & 1.0 & 3.1 & -2.9 & -7.6 & 1.0 & 3.4 & -4.4 & -4.6 & 1.3 & 2.4 & -3.4 & 2.0 & -0.58 & -1.42 & 96 & 0.332 \\
\hline 39 & -2.6 & -5.4 & 1.0 & 3.1 & -3.0 & -7.7 & 1.0 & 3.4 & -4.5 & -4.7 & 1.3 & 2.4 & -3.4 & 2.0 & -0.58 & -1.43 & 97 & 0.329 \\
\hline 40 & -2.5 & -5.5 & 1.0 & 3.2 & -3.0 & -7.7 & 1.0 & 3.4 & -4.5 & -4.7 & 1.4 & 2.4 & -3.4 & 2.0 & -0.58 & -1.42 & 97 & 0.334 \\
\hline 41 & -2.5 & -5.5 & 1.0 & 3.2 & -3.0 & -7.7 & 1.0 & 3.4 & -4.5 & -4.6 & 1.4 & 2.4 & $\begin{array}{l}-3.4 \\
\end{array}$ & 2.0 & -0.59 & -1.41 & 97 & 0.341 \\
\hline 42 & -2.4 & -5.5 & 1.0 & 3.2 & -3.1 & -7.8 & 1.0 & 3.4 & -4.4 & -4.7 & 1.4 & 2.4 & -3.4 & 2.0 & -0.59 & -1.40 & 97 & 0.349 \\
\hline 43 & -2.5 & -5.5 & 1.0 & 3.2 & -3.1 & -7.8 & 1.0 & 3.4 & -4.5 & -4.6 & 1.4 & 2.5 & $\begin{array}{l}-3.4 \\
\end{array}$ & 2.0 & -0.59 & -1.41 & 98 & 0.344 \\
\hline 44 & -2.5 & -5.5 & 1.0 & 3.2 & -3.1 & -7.9 & 1.0 & 3.4 & -4.5 & -4.6 & 1.4 & 2.5 & -3.4 & 2.0 & -0.59 & -1.40 & 98 & 0.349 \\
\hline 45 & -2.5 & -5.6 & 1.0 & 3.3 & -3.0 & -7.8 & 1.0 & 3.5 & -4.5 & -4.6 & 1.4 & 2.5 & $\begin{array}{l}-3.4 \\
\end{array}$ & 2.0 & -0.60 & -1.40 & 98 & 0.354 \\
\hline 46 & -2.5 & -5.6 & 1.0 & 3.3 & -3.1 & \begin{tabular}{|c|}
-7.9 \\
\end{tabular} & 1.0 & 3.5 & -4.4 & -4.6 & 1.4 & 2.5 & \begin{tabular}{|l|}
-3.4 \\
\end{tabular} & 2.0 & -0.60 & -1.39 & 98 & 0.360 \\
\hline 47 & -2.5 & -5.6 & 1.0 & 3.3 & -3.1 & -7.9 & 1.0 & 3.5 & -4.4 & -4.6 & 1.4 & 2.5 & $\begin{array}{l}-3.4 \\
\end{array}$ & 2.1 & -0.60 & -1.39 & 98 & 0.365 \\
\hline 48 & -2.5 & -5.5 & 1.0 & 3.3 & -3.1 & -7.8 & 1.0 & 3.5 & -4.4 & -4.6 & 1.4 & 2.5 & -3.4 & 2.1 & -0.62 & -1.36 & 97 & 0.386 \\
\hline 49 & -2.5 & -5.7 & 1.0 & 3.3 & -3.1 & -7.9 & 1.0 & 3.6 & -4.4 & -4.6 & 1.4 & 2.5 & $\begin{array}{l}-3.4 \\
\end{array}$ & 2.1 & -0.61 & -1.37 & 99 & 0.376 \\
\hline 50 & -2.6 & -5.7 & 1.0 & 3.4 & -3.1 & -7.9 & 1.1 & 3.6 & -4.3 & -4.6 & 1.4 & 2.5 & $\begin{array}{l}-3.4 \\
\end{array}$ & 2.1 & -0.62 & -1.35 & 98 & 0.397 \\
\hline 51 & -2.6 & -5.6 & 1.0 & 3.3 & -3.1 & -7.9 & 1.1 & 3.6 & -4.4 & -4.7 & 1.4 & 2.6 & $\begin{array}{l}-3.4 \\
\end{array}$ & 2.1 & -0.61 & -1.37 & 99 & 0.382 \\
\hline 52 & -2.6 & -5.7 & 1.0 & 3.4 & -3.1 & -7.9 & 1.1 & 3.6 & -4.4 & -4.6 & 1.5 & 2.6 & -3.4 & 2.1 & -0.62 & -1.35 & 99 & 0.397 \\
\hline 53 & -2.5 & -5.7 & 1.0 & 3.4 & -3.2 & -8.0 & 1.1 & 3.6 & -4.4 & -4.5 & 1.5 & 2.6 & -3.4 & 2.1 & -0.63 & -1.35 & 98 & 0.399 \\
\hline 54 & -2.6 & -5.7 & 1.0 & 3.3 & -3.1 & -8.0 & 1.1 & 3.6 & -4.3 & -4.6 & 1.5 & 2.6 & -3.4 & 2.1 & -0.62 & -1.36 & 99 & 0.390 \\
\hline 55 & -2.7 & -5.7 & 1.0 & 3.3 & -3.1 & -8.0 & 1.1 & 3.7 & -4.4 & -4.6 & 1.5 & 2.6 & -3.4 & 2.1 & -0.62 & -1.35 & 99 & 0.393 \\
\hline 56 & -2.7 & -6.0 & 1.0 & 3.3 & -3.1 & -8.1 & 1.1 & 3.7 & -4.4 & -4.6 & 1.5 & 2.6 & -3.5 & 2.1 & -0.61 & -1.37 & 100 & 0.381 \\
\hline 57 & -2.8 & -6.0 & 1.0 & 3.3 & -3.2 & -8.1 & 1.1 & 3.7 & -4.3 & -4.6 & 1.5 & 2.6 & -3.5 & 2.1 & -0.62 & -1.36 & 100 & 0.384 \\
\hline 58 & -2.9 & -6.0 & 1.0 & 3.3 & -3.1 & -8.0 & 1.1 & 3.7 & -4.4 & -4.5 & 1.5 & 2.6 & -3.5 & 2.1 & -0.61 & -1.37 & 101 & 0.382 \\
\hline 59 & -2.9 & -6.0 & 1.0 & 3.3 & -3.1 & -8.0 & 1.1 & 3.7 & -4.4 & -4.6 & 1.5 & 2.6 & -3.5 & 2.1 & -0.62 & -1.36 & 101 & 0.386 \\
\hline 60 & -2.9 & -6.1 & 1.0 & 3.4 & -3.1 & -8.0 & 1.1 & 3.7 & -4.4 & -4.5 & 1.5 & 2.6 & -3.5 & 2.2 & -0.62 & -1.36 & 101 & 0.391 \\
\hline 61 & -2.9 & -6.0 & 1.0 & 3.3 & -3.1 & -8.1 & 1.1 & 3.7 & -4.4 & -4.6 & 1.5 & 2.7 & -3.5 & 2.2 & -0.62 & -1.36 & 101 & 0.388 \\
\hline 62 & -3.1 & -6.3 & 1.0 & 3.3 & -3.2 & -8.1 & 1.1 & 3.8 & -4.3 & -4.5 & 1.5 & 2.7 & -3.5 & 2.2 & -0.61 & -1.37 & 102 & 0.375 \\
\hline 63 & -3.0 & -6.2 & 1.0 & 3.4 & -3.2 & -8.1 & 1.1 & 3.8 & -4.3 & -4.6 & 1.5 & 2.7 & -3.5 & 2.2 & -0.61 & -1.37 & 102 & 0.382 \\
\hline 64 & -3.0 & -6.2 & 1.0 & 3.4 & -3.1 & -8.2 & 1.1 & 3.8 & -4.3 & -4.6 & 1.5 & 2.7 & -3.5 & 2.2 & -0.62 & -1.35 & 102 & 0.394 \\
\hline 65 & -3.2 & -6.4 & 1.0 & 3.3 & -3.2 & -8.1 & 1.1 & 3.8 & -4.4 & -4.6 & 1.5 & 2.7 & -3.6 & 2.2 & -0.61 & -1.38 & 103 & 0.372 \\
\hline 66 & -2.9 & -6.4 & 1.0 & 3.4 & -3.1 & -8.1 & 1.1 & 3.8 & -4.4 & -4.6 & 1.5 & 2.7 & -3.6 & 2.2 & -0.61 & -1.37 & 103 & 0.381 \\
\hline
\end{tabular}


Table E.18 NU 52-34 Test Data $\left(-20^{\circ} \mathrm{C}\right.$ Tests $)$, continued

\begin{tabular}{|c|c|c|c|c|c|c|c|c|c|c|c|c|c|c|c|c|c|c|}
\hline & \multicolumn{12}{|c|}{ Normalized Vertical and Horizontal Deformation Arrays } & & & & & & \\
\hline & \multicolumn{4}{|c|}{ NU 52-34 Sample \#6 } & \multicolumn{4}{|c|}{ NU 52-34 Sample \#8 } & \multicolumn{4}{|c|}{ NU 52-34 Sample \#9 } & \multicolumn{2}{|c|}{ Trimmed } & & & Creep & Pois. \\
\hline & vert & vert & horz & horz & vert & vert & horz & horz & vert & vert & horz & horz & & & Ratio & & Comp & Ratio \\
\hline Time & front & back & front & back & front & back & front & back & front & back & front & back & vert & horz & $\mathrm{X} / \mathrm{Y}$ & $\mathrm{C}_{\mathrm{empl}}$ & $\mathrm{D}(\mathrm{t})$ & $\bar{v}$ \\
\hline $\mathrm{sec}$ & $\mu \mathrm{m}$ & $\mu \mathrm{m}$ & $\mu \mathrm{m}$ & $\overline{\mu m}$ & $\mu \mathrm{m}$ & $\mu \mathrm{m}$ & $\mu \mathrm{m}$ & $\overline{\mu m}$ & $\mu \mathrm{m}$ & $\mu \mathrm{m}$ & $\mu \mathrm{m}$ & $\mu \mathrm{m}$ & $\mu \mathrm{m}$ & $\mu \mathrm{m}$ & & & $1 / \mathrm{kPa}$ & \\
\hline 67 & -3.0 & -6.3 & 1.0 & 3.4 & -3.1 & -8.1 & 1.1 & 3.8 & -4.4 & -4.6 & 1.5 & 2.7 & -3.6 & 2.2 & -0.62 & -1.36 & 103 & 0.386 \\
\hline 68 & -3.0 & -6.4 & 1.1 & 3.4 & -3.1 & -8.0 & 1.1 & 3.9 & -4.5 & -4.7 & 1.5 & 2.8 & -3.6 & 2.2 & -0.61 & -1.37 & 104 & 0.377 \\
\hline 69 & -3.2 & -6.4 & 1.1 & 3.4 & -3.2 & -8.2 & 1.1 & 3.8 & -4.5 & -4.6 & 1.6 & 2.8 & -3.6 & 2.2 & -0.62 & -1.36 & 104 & 0.390 \\
\hline 70 & -3.0 & -6.4 & 1.1 & 3.4 & -3.1 & -8.2 & 1.1 & 3.9 & -4.5 & -4.7 & 1.6 & 2.7 & -3.6 & 2.2 & -0.61 & -1.38 & 105 & 0.369 \\
\hline 71 & -3.2 & -6.5 & 1.1 & 3.4 & -3.4 & -8.5 & 1.1 & 3.8 & -4.6 & -4.7 & 1.5 & 2.7 & -3.7 & 2.2 & -0.60 & -1.39 & 106 & 0.361 \\
\hline 72 & -3.1 & -6.4 & 1.1 & 3.5 & -3.2 & -8.3 & 1.1 & 3.9 & -4.5 & -4.7 & 1.6 & 2.7 & -3.6 & 2.2 & -0.61 & -1.37 & 105 & 0.378 \\
\hline 73 & -3.2 & -6.4 & 1.1 & 3.5 & $\begin{array}{l}-3.1 \\
\end{array}$ & -8.1 & 1.2 & 3.9 & -4.6 & -4.8 & 1.6 & 2.8 & -3.7 & 2.2 & -0.61 & -1.38 & 106 & 0.373 \\
\hline 74 & -3.2 & -6.5 & 1.1 & 3.4 & -3.1 & -8.1 & 1.2 & 3.9 & -4.6 & -4.7 & 1.6 & 2.7 & -3.7 & 2.2 & -0.60 & -1.38 & 106 & 0.365 \\
\hline 75 & -3.2 & -6.5 & 1.1 & 3.4 & -3.2 & -8.2 & 1.2 & 4.0 & -4.6 & -4.8 & 1.6 & 2.7 & -3.7 & 2.2 & -0.60 & -1.39 & 107 & 0.363 \\
\hline 76 & -3.2 & -6.6 & 1.1 & 3.5 & -3.1 & -8.2 & 1.2 & 4.0 & -4.7 & -4.8 & 1.6 & 2.7 & -3.7 & 2.2 & -0.60 & -1.39 & 107 & 0.360 \\
\hline 77 & -3.2 & -6.7 & 1.1 & 3.4 & -3.1 & -8.2 & 1.2 & 4.0 & -4.6 & -4.9 & 1.6 & 2.7 & -3.8 & 2.2 & -0.59 & -1.41 & 108 & 0.345 \\
\hline 78 & -3.2 & -6.5 & 1.1 & 3.4 & -3.1 & -8.3 & 1.2 & 4.0 & -4.7 & -4.9 & 1.6 & 2.8 & -3.8 & 2.2 & -0.60 & -1.40 & 108 & 0.354 \\
\hline 79 & -3.2 & -6.5 & 1.1 & 3.4 & -3.1 & -8.2 & 1.2 & 4.0 & -4.6 & -4.8 & 1.6 & 2.8 & -3.7 & 2.2 & -0.60 & -1.39 & 107 & 0.365 \\
\hline 80 & -3.1 & -6.6 & 1.1 & 3.4 & -3.2 & -8.2 & 1.2 & 4.0 & -4.7 & -4.8 & 1.6 & 2.8 & -3.8 & 2.3 & -0.60 & -1.39 & 108 & 0.362 \\
\hline 81 & -3.2 & -6.5 & 1.1 & 3.5 & -3.2 & -8.2 & 1.2 & 4.0 & -4.8 & -4.9 & 1.6 & 2.8 & -3.8 & 2.3 & -0.60 & -1.39 & 109 & 0.358 \\
\hline 82 & -3.2 & -6.6 & 1.1 & 3.4 & -3.1 & -8.1 & 1.2 & 4.1 & -4.8 & -5.0 & 1.6 & 2.8 & -3.8 & 2.2 & -0.59 & -1.41 & 109 & 0.341 \\
\hline 83 & -3.2 & -6.6 & 1.1 & 3.5 & -3.1 & -8.1 & 1.2 & 4.1 & -4.9 & -5.0 & 1.6 & 2.8 & -3.9 & 2.3 & -0.59 & -1.42 & 110 & 0.339 \\
\hline 84 & -3.2 & -6.6 & 1.1 & 3.5 & -3.1 & -8.1 & 1.2 & 4.1 & -4.9 & -5.1 & 1.6 & 2.8 & -3.9 & 2.3 & -0.58 & -1.42 & 111 & 0.336 \\
\hline 85 & -3.2 & -6.7 & 1.1 & 3.5 & -3.1 & -8.2 & 1.2 & 4.1 & -4.9 & -5.1 & 1.6 & 2.8 & -3.9 & 2.3 & -0.58 & -1.42 & 111 & 0.333 \\
\hline 86 & -3.2 & -6.7 & 1.1 & 3.5 & -3.0 & -8.2 & 1.2 & 4.1 & -4.9 & -5.0 & 1.6 & 2.8 & -3.9 & 2.3 & -0.59 & -1.41 & 111 & 0.342 \\
\hline 87 & -3.1 & -6.6 & 1.1 & 3.6 & -2.9 & -8.1 & 1.2 & 4.2 & -5.0 & -5.0 & 1.6 & 2.8 & -3.9 & 2.3 & -0.59 & -1.41 & 111 & 0.343 \\
\hline 88 & -3.2 & -6.7 & 1.1 & 3.5 & -2.8 & -8.1 & 1.2 & 4.2 & -4.9 & -5.2 & 1.6 & 2.8 & -3.9 & 2.3 & -0.58 & -1.42 & 112 & 0.334 \\
\hline 89 & -3.2 & -6.7 & 1.1 & 3.6 & -2.9 & -8.1 & 1.2 & 4.2 & -5.0 & -5.0 & 1.6 & 2.8 & -3.9 & 2.3 & -0.59 & -1.41 & 112 & 0.342 \\
\hline 90 & -3.2 & -6.7 & 1.1 & 3.6 & -2.8 & -8.1 & 1.2 & 4.2 & -5.0 & -5.2 & 1.6 & 2.8 & -3.9 & 2.3 & -0.58 & -1.42 & 113 & 0.334 \\
\hline 91 & -3.1 & -6.7 & 1.1 & 3.6 & -2.9 & -8.2 & 1.2 & 4.2 & -5.0 & -5.2 & 1.6 & 2.8 & -3.9 & 2.3 & -0.59 & -1.41 & 113 & 0.342 \\
\hline 92 & -3.1 & -6.7 & 1.1 & 3.6 & -2.8 & -8.1 & 1.3 & 4.3 & -5.0 & -5.1 & 1.7 & 2.8 & -3.9 & 2.3 & -0.59 & -1.40 & 112 & 0.348 \\
\hline 93 & -3.2 & -6.6 & 1.1 & 3.6 & -2.7 & -7.9 & 1.3 & 4.3 & -5.0 & -5.2 & 1.6 & 2.8 & -3.9 & 2.3 & -0.59 & -1.40 & 113 & 0.351 \\
\hline 94 & -3.1 & -6.7 & 1.1 & 3.6 & -2.8 & -7.9 & 1.3 & 4.3 & -5.1 & -5.2 & 1.6 & 2.8 & -4.0 & 2.3 & -0.59 & -1.41 & 113 & 0.348 \\
\hline 95 & -3.0 & -6.7 & 1.1 & 3.7 & -2.8 & -7.9 & 1.3 & 4.3 & -5.0 & -5.2 & 1.7 & 2.8 & -3.9 & 2.4 & -0.60 & -1.39 & 113 & 0.360 \\
\hline 96 & -3.2 & -6.5 & 1.1 & 3.7 & -2.7 & -7.9 & 1.3 & 4.3 & -5.1 & -5.2 & 1.7 & 2.8 & -3.9 & 2.4 & -0.60 & -1.39 & 113 & 0.360 \\
\hline 97 & -3.0 & -6.5 & 1.1 & 3.7 & -2.9 & -8.2 & 1.3 & 4.3 & -5.1 & -5.3 & 1.7 & 2.8 & -3.9 & 2.4 & -0.60 & -1.40 & 113 & 0.356 \\
\hline 98 & -3.0 & -6.5 & 1.1 & 3.7 & -2.7 & -7.9 & 1.3 & 4.3 & -5.0 & -5.1 & 1.7 & 2.8 & -3.9 & 2.4 & -0.61 & -1.37 & 112 & 0.377 \\
\hline 99 & -3.1 & -6.6 & 1.1 & 3.7 & -2.7 & -7.9 & 1.3 & 4.4 & -5.2 & -5.4 & 1.7 & 2.8 & -4.0 & 2.4 & -0.59 & -1.40 & 115 & 0.348 \\
\hline 100 & -3.0 & -6.5 & 1.1 & 3.7 & $\begin{array}{l}-2.8 \\
\end{array}$ & \begin{tabular}{|c|}
-7.9 \\
\end{tabular} & 1.3 & 4.4 & -5.2 & -5.3 & 1.7 & 2.8 & -4.0 & 2.4 & -0.60 & -1.39 & 114 & 0.359 \\
\hline
\end{tabular}


Table E.19 NU 58-40 Sample Data $\left(0^{\circ} \mathrm{C}\right.$ Tests $)$

\begin{tabular}{|c|c|c|c|}
\hline & Thickness & Diameter & Creep Load \\
\hline Sample & $\mathrm{mm}$ & $\mathrm{mm}$ & $\mathrm{kN}$ \\
\hline 11 & 76.94 & 150.1 & -2.93 \\
\hline 12 & 76.81 & 150.0 & -2.60 \\
\hline 9 & 76.79 & 150.1 & -2.39 \\
\hline & & & -2.64 \\
\hline average & 76.85 & 150.1 & \\
\hline
\end{tabular}

\begin{tabular}{|c|c|}
\hline Gage length, $\mathrm{m}$ & 0.038 \\
\hline $\mathrm{C}_{\mathrm{empl}}$ low & 0.595 \\
\hline $\mathrm{C}_{\mathrm{empl}}$ high & 1.466 \\
\hline
\end{tabular}

Table E.20 NU 58-40 Sample Data $\left(-10{ }^{\circ} \mathrm{C}\right.$ Tests)

\begin{tabular}{|c|c|c|c|}
\hline & Thickness & Diameter & Creep Load \\
\hline Sample & $\mathrm{mm}$ & $\mathrm{mm}$ & $\mathrm{kN}$ \\
\hline 11 & 76.94 & 150.1 & -4.70 \\
\hline 12 & 76.81 & 150.0 & -3.65 \\
\hline 9 & 76.79 & 150.1 & -3.80 \\
\hline & & & \\
\hline average & 76.85 & 150.1 & -4.05 \\
\hline
\end{tabular}

\begin{tabular}{|c|c|}
\hline Gage length, $\mathrm{m}$ & 0.038 \\
\hline $\mathrm{C}_{\text {empl }}$ low & 0.595 \\
\hline $\mathrm{C}_{\text {empl }}$ high & 1.466 \\
\hline
\end{tabular}

Table E.21 NU 58-40 Sample Data $\left(-20{ }^{\circ} \mathrm{C}\right.$ Tests)

\begin{tabular}{|c|c|c|c|}
\hline & Thickness & Diameter & Creep Load \\
\hline Sample & $\mathrm{mm}$ & $\mathrm{mm}$ & $\mathrm{kN}$ \\
\hline 11 & 76.94 & 150.1 & -10.21 \\
\hline 12 & 76.81 & 150.0 & -6.53 \\
\hline 9 & 76.79 & 150.1 & -7.71 \\
\hline & & & -8.15 \\
\hline average & 76.85 & 150.1 & \\
\hline
\end{tabular}

\begin{tabular}{|c|c|}
\hline Gage length, $\mathrm{m}$ & 0.038 \\
\hline $\mathrm{C}_{\text {empl }}$ low & 0.595 \\
\hline $\mathrm{C}_{\text {empl }}$ high & 1.466 \\
\hline
\end{tabular}


Table E.22 NU 58-40 Test Data $\left(0^{\circ} \mathrm{C}\right.$ Tests)

\begin{tabular}{|c|c|c|c|c|c|c|c|c|c|c|c|c|c|c|c|c|c|c|}
\hline & \multicolumn{12}{|c|}{ Normalized Vertical and Horizontal Deformation Arrays } & \multirow{2}{*}{\multicolumn{2}{|c|}{ Trimmed }} & \multirow[b]{3}{*}{ Ratio } & & \multirow{3}{*}{\begin{tabular}{|l|} 
Creep \\
Comp \\
\end{tabular}} & \multirow{3}{*}{$\begin{array}{l}\text { Pois. } \\
\text { Ratio }\end{array}$} \\
\hline & \multicolumn{4}{|c|}{ NU 58-40 Sample \#11 } & \multicolumn{4}{|c|}{ NU 58-40 Sample \#12 } & \multicolumn{4}{|c|}{ NU 58-40 Sample \#9 } & & & & & & \\
\hline & vert & vert & horz & horz & vert & vert & horz & horz & vert & vert & horz & horz & & & & & & \\
\hline Time & front & back & front & back & front & back & front & back & front & back & front & back & vert & horz & $\mathrm{X} / \mathrm{Y}$ & $\mathrm{C}_{\mathrm{empl}}$ & $\mathrm{D}(\mathrm{t})$ & \\
\hline sec & $\mu \mathrm{m}$ & $\mu \mathrm{m}$ & & $\mu \mathrm{m}$ & $\mu \mathrm{m}$ & $\mu \mathrm{m}$ & $\mu \mathrm{m}$ & $\mu \mathrm{m}$ & $\mu \mathrm{m}$ & $\mu \mathrm{m}$ & $\mu \mathrm{m}$ & $\mu \mathrm{m}$ & $\mu \mathrm{m}$ & $\mu \mathrm{m}$ & & & $1 / \mathrm{kPa}$ & \\
\hline$\overline{0}$ & $\overline{0.0}$ & 0.0 & 0.0 & 0.0 & 0.0 & 0.0 & 0.0 & 0.0 & 0.0 & 0.0 & 0.0 & 0.0 & 0.0 & 0.0 & 0.00 & 0.00 & $\overline{0}$ & 0.000 \\
\hline 1 & -4.5 & -2.5 & 1.2 & 1.5 & \begin{tabular}{|l|}
-2.8 \\
\end{tabular} & -4.8 & 0.9 & 2.6 & -4.6 & \begin{tabular}{l|l}
-6.4 \\
\end{tabular} & 1.7 & 3.7 & -4.2 & 1.7 & -0.42 & $\begin{array}{l}-1.86 \\
\end{array}$ & 372 & 0.122 \\
\hline 2 & -5.8 & -3.3 & 1.5 & 1.9 & -3.5 & -6.0 & 1.1 & 3.4 & -5.8 & -8.4 & 2.3 & 4.9 & -5.3 & 2.2 & -0.42 & -1.83 & 473 & 0.129 \\
\hline 3 & -6.8 & $\begin{array}{l}-3.8 \\
\end{array}$ & 1.7 & 2.2 & $\begin{array}{l}-4.0 \\
\end{array}$ & $\begin{array}{l}7.0 \\
\end{array}$ & 1.3 & 3.9 & -6.8 & -9.9 & 2.7 & 5.8 & -6.2 & 2.6 & -0.42 & -1.84 & 550 & \\
\hline 4 & \begin{tabular}{|c|}
-7.4 \\
\end{tabular} & -4.1 & 1.9 & 2.4 & -4.6 & $\begin{array}{l}-7.7 \\
\end{array}$ & 1.4 & 4.3 & \begin{tabular}{|c|}
-7.6 \\
\end{tabular} & -11.1 & 3.0 & 6.5 & -6.8 & 2.9 & -0.42 & -1.83 & 609 & 0.130 \\
\hline 5 & -8.0 & $\begin{array}{l}-4.4 \\
\end{array}$ & 2.1 & 2.7 & \begin{tabular}{|c|}
-4.9 \\
\end{tabular} & -8.4 & 1.6 & 4.7 & -8.1 & \begin{tabular}{|c|}
-12.1 \\
\end{tabular} & 3.3 & 7.1 & -7.4 & 3.2 & -0.43 & -1.81 & 659 & \begin{tabular}{|l|}
0.137 \\
\end{tabular} \\
\hline 6 & -8.5 & -4.7 & 2.3 & 2.8 & -5.2 & -8.9 & 1.7 & 5.0 & -8.7 & -13.0 & 3.5 & 7.7 & -7.8 & 3.4 & -0.43 & -1.80 & 703 & 0.138 \\
\hline 7 & -9.0 & -5.0 & 2.4 & 3.0 & 5.5 & -9.5 & 1.8 & 5.3 & -9.2 & -13.7 & 3.7 & 8.1 & -8.3 & $\begin{array}{l}3.6 \\
\end{array}$ & -0.43 & $\begin{array}{l}1.79 \\
\end{array}$ & 743 & 0.141 \\
\hline$=$ & -9.5 & -5.2 & 2.5 & 3.2 & 5.9 & $\begin{array}{l}9.9 \\
\end{array}$ & 1.9 & 5.6 & -9.6 & \begin{tabular}{|l|l}
-14.4 \\
\end{tabular} & 3.9 & 8.5 & -8.7 & $\begin{array}{l}3.8 \\
\end{array}$ & -0.43 & $\begin{array}{l}-1.79 \\
\end{array}$ & 782 & \begin{tabular}{|l|l|}
0.141 \\
\end{tabular} \\
\hline 9 & -9.8 & -5.6 & 2.6 & 3.3 & -6.1 & -10.3 & 2.0 & 5.8 & -10.1 & -15.2 & 4.1 & 8.9 & -9.1 & 3.9 & -0.44 & -1.79 & 815 & 0.141 \\
\hline 10 & -10.2 & $\begin{array}{l}-5.7 \\
\end{array}$ & 2.8 & 3.5 & \begin{tabular}{|l|}
-6.2 \\
\end{tabular} & -10.4 & 2.1 & 6.0 & -10.5 & -15.8 & 4.2 & 9.3 & -9.3 & 4.1 & -0.44 & -1.77 & 841 & \begin{tabular}{|l|}
0.149 \\
\end{tabular} \\
\hline 11 & \begin{tabular}{|c|}
-10.6 \\
\end{tabular} & -5.8 & 2.9 & 3.6 & -6.7 & -11.0 & 2.2 & 6.2 & -10.9 & \begin{tabular}{|c|}
-16.3 \\
\end{tabular} & 4.4 & 9.6 & $\begin{array}{l}-9.8 \\
\end{array}$ & 4.3 & -0.44 & $\begin{array}{l}-1.79 \\
\end{array}$ & 880 & 0.142 \\
\hline$\overline{12}$ & \begin{tabular}{|c|}
-10.9 \\
\end{tabular} & $\begin{array}{l}-6.1 \\
\end{array}$ & 3.0 & 3.8 & \begin{tabular}{|l|}
-6.8 \\
\end{tabular} & -11.3 & 2.2 & 6.5 & -11.2 & \begin{tabular}{|c|}
-16.9 \\
\end{tabular} & 4.5 & 9.9 & -10.1 & 4.4 & -0.44 & -1.77 & 905 & 0.149 \\
\hline 13 & -11.3 & $\begin{array}{l}-6.3 \\
\end{array}$ & 3.1 & 3.9 & \begin{tabular}{|l|}
-7.0 \\
\end{tabular} & -11.7 & 2.3 & 6.7 & -11.5 & $\begin{array}{l}-17.3 \\
\end{array}$ & 4.7 & $\begin{array}{ll}10.3 \\
\end{array}$ & -10.4 & 4.6 & -0.44 & $\begin{array}{l}-1.77 \\
\end{array}$ & 935 & \begin{tabular}{|l|l|}
0.149 \\
\end{tabular} \\
\hline 14 & -11.7 & $\begin{array}{l}-6.5 \\
\end{array}$ & 3.1 & 4.0 & \begin{tabular}{|l|}
-7.2 \\
\end{tabular} & -12.0 & 2.4 & 6.8 & -11.8 & -17.8 & 4.8 & $\begin{array}{l}10.6 \\
\end{array}$ & -10.7 & 4.7 & -0.44 & $\begin{array}{l}-1.78 \\
\end{array}$ & 960 & \begin{tabular}{|l|l|}
0.147 \\
\end{tabular} \\
\hline 15 & -12.0 & -6.7 & 3.2 & 4.1 & $\begin{array}{l}-7.5 \\
\end{array}$ & -12.3 & 2.5 & 7.0 & -12.1 & \begin{tabular}{|c|}
-18.4 \\
\end{tabular} & 4.9 & $\begin{array}{l}10.8 \\
\end{array}$ & -11.0 & $\begin{array}{l}4.8 \\
\end{array}$ & -0.44 & $\begin{array}{l}-1.77 \\
\end{array}$ & 987 & \begin{tabular}{|l|l|}
0.148 \\
\end{tabular} \\
\hline 16 & -12.2 & $\begin{array}{l}-6.8 \\
\end{array}$ & 3.3 & 4.2 & $\begin{array}{l}-7.6 \\
\end{array}$ & -12.7 & 2.5 & 7.2 & -12.4 & \begin{tabular}{|c|}
-18.7 \\
\end{tabular} & 5.1 & 11.1 & -11.2 & 5.0 & -0.44 & $\begin{array}{l}-1.77 \\
\end{array}$ & 1010 & \begin{tabular}{|l|l|}
0.148 \\
\end{tabular} \\
\hline 17 & -12.5 & $\begin{array}{l}-7.2 \\
\end{array}$ & 3.4 & 4.3 & \begin{tabular}{l|l|}
-7.8 \\
\end{tabular} & -12.9 & 2.6 & 7.4 & \begin{tabular}{|c|}
-12.6 \\
\end{tabular} & \begin{tabular}{|c|}
-19.1 \\
\end{tabular} & 5.2 & \begin{tabular}{|l|l}
11.4 \\
\end{tabular} & -11.5 & \begin{tabular}{|l}
5.1 \\
\end{tabular} & -0.44 & $\begin{array}{l}-1.77 \\
\end{array}$ & 1032 & \begin{tabular}{|l|l|}
0.150 \\
\end{tabular} \\
\hline 18 & \begin{tabular}{|c|}
-12.9 \\
\end{tabular} & $\begin{array}{l}-7.3 \\
\end{array}$ & 3.5 & 4.5 & \begin{tabular}{|c|}
-8.0 \\
\end{tabular} & -13.2 & 2.7 & 7.5 & -12.8 & \begin{tabular}{|c|}
-19.5 \\
\end{tabular} & 5.3 & $\begin{array}{ll}11.6 \\
\end{array}$ & -11.7 & 5.2 & -0.44 & -1.76 & 1053 & \begin{tabular}{|l|}
0.151 \\
\end{tabular} \\
\hline 19 & -13.0 & $\begin{array}{l}-7.4 \\
\end{array}$ & 3.6 & 4.6 & \begin{tabular}{|l|}
-8.2 \\
\end{tabular} & -13.5 & 2.7 & 7.7 & -13.1 & -19.9 & 5.4 & 11.9 & -12.0 & 5.3 & -0.45 & -1.76 & 1077 & \begin{tabular}{|l|}
0.154 \\
\end{tabular} \\
\hline 20 & -13.4 & -7.5 & 3.6 & 4.7 & $\begin{array}{l}-8.3 \\
\end{array}$ & -13.8 & 2.8 & 7.9 & -13.3 & -20.2 & 5.5 & \begin{tabular}{|l|}
12.1 \\
\end{tabular} & -12.2 & 5.4 & -0.44 & $\begin{array}{l}-1.76 \\
\end{array}$ & 1097 & \begin{tabular}{|l|l|}
0.152 \\
\end{tabular} \\
\hline 21 & $\begin{array}{l}-13.6 \\
\end{array}$ & $\begin{array}{l}-7.8 \\
\end{array}$ & 3.7 & 4.8 & $\begin{array}{l}-8.5 \\
\end{array}$ & -14.0 & 2.9 & 80 & \begin{tabular}{|l|}
-13.5 \\
\end{tabular} & $\begin{array}{l}-20.6 \\
\end{array}$ & 5.6 & $\begin{array}{l}12.3 \\
\end{array}$ & -12.4 & 5.5 & -0.45 & -1.76 & 1118 & 0.154 \\
\hline 22 & \begin{tabular}{|c|}
-14.0 \\
\end{tabular} & $\begin{array}{l}-7.9 \\
\end{array}$ & 3.8 & 4.8 & -8.7 & -14.3 & 2.9 & 8.2 & -13.8 & \begin{tabular}{|c|}
-21.0 \\
\end{tabular} & 5.7 & 12.5 & -12.7 & 5.6 & -0.44 & $\begin{array}{l}-1.77 \\
\end{array}$ & 1145 & 0.150 \\
\hline 23 & -14.2 & $\begin{array}{l}-8.1 \\
\end{array}$ & 3.9 & 5.0 & \begin{tabular}{|l|}
-8.9 \\
\end{tabular} & -14.5 & 3.0 & 8.3 & \begin{tabular}{|c|}
-14.0 \\
\end{tabular} & -21.2 & 5.8 & 12.7 & -12.9 & $\begin{array}{l}5.7 \\
\end{array}$ & -0.44 & -1.76 & 1161 & \begin{tabular}{|l|}
0.152 \\
\end{tabular} \\
\hline 24 & \begin{tabular}{|l|}
-14.3 \\
\end{tabular} & $\begin{array}{l}-8.1 \\
\end{array}$ & 3.9 & 5.1 & $\begin{array}{l}-9.1 \\
\end{array}$ & $\begin{array}{l}-14.7 \\
\end{array}$ & 3.0 & 8.4 & -14.2 & -21.5 & 5.9 & \begin{tabular}{|l|l}
12.9 \\
\end{tabular} & -13.1 & 5.8 & -0.45 & -1.76 & 1179 & \begin{tabular}{|l|}
0.154 \\
\end{tabular} \\
\hline 25 & \begin{tabular}{|c|}
-14.6 \\
\end{tabular} & $\begin{array}{l}-8.3 \\
\end{array}$ & 4.0 & 5.2 & \begin{tabular}{|l|}
-9.1 \\
\end{tabular} & -14.9 & 3.1 & 8.6 & \begin{tabular}{|c|}
-14.4 \\
\end{tabular} & \begin{tabular}{|c|}
-22.0 \\
\end{tabular} & 6.0 & 13.1 & -13.3 & 5.9 & -0.45 & -1.75 & 1197 & 0.155 \\
\hline 26 & $\begin{array}{l}-14.8 \\
\end{array}$ & $\begin{array}{l}-8.4 \\
\end{array}$ & 4.1 & 5.3 & -9.3 & -15.2 & 3.1 & 8.7 & -14.5 & \begin{tabular}{l|l}
-22.1 \\
\end{tabular} & 6.1 & 13.3 & -13.5 & 6.0 & -0.45 & -1.75 & 1214 & \begin{tabular}{|l|l|}
0.157 \\
\end{tabular} \\
\hline 27 & -15.0 & $\begin{array}{l}-8.5 \\
\end{array}$ & 4.1 & 5.4 & \begin{tabular}{|l|}
-9.5 \\
\end{tabular} & -15.5 & 3.2 & 8.9 & -14.7 & -22.4 & 6.2 & 13.5 & -13.7 & 6.1 & -0.45 & -1.75 & 1235 & \begin{tabular}{|l|}
0.156 \\
\end{tabular} \\
\hline 28 & \begin{tabular}{|c|}
-15.3 \\
\end{tabular} & $\begin{array}{l}-8.7 \\
\end{array}$ & 4.2 & 5.4 & \begin{tabular}{|l|}
-9.7 \\
\end{tabular} & -15.7 & 3.2 & 8.9 & \begin{tabular}{|c|}
-14.8 \\
\end{tabular} & -22.7 & 6.2 & \begin{tabular}{|l|l}
13.7 \\
\end{tabular} & -13.9 & 6.2 & -0.45 & -1.75 & 1250 & \begin{tabular}{|l|}
0.156 \\
\end{tabular} \\
\hline 29 & -15.5 & $\begin{array}{l}-8.8 \\
\end{array}$ & 4.3 & 5.5 & -9.9 & -15.9 & 3.3 & 9.1 & -15.0 & \begin{tabular}{|c|}
-22.9 \\
\end{tabular} & 6.3 & 13.9 & -14.1 & 6.3 & -0.45 & -1.75 & 1269 & 0.156 \\
\hline 30 & -15.7 & $\begin{array}{l}-9.0 \\
\end{array}$ & 4.3 & 5.6 & $\begin{array}{l}-9.9 \\
\end{array}$ & -16.2 & 3.3 & 9.2 & -15.3 & -23.4 & 6.4 & 14.0 & -14.3 & $\begin{array}{ll}6.4 \\
\end{array}$ & -0.45 & -1.75 & 1288 & 0.156 \\
\hline 31 & \begin{tabular}{|c|}
-15.9 \\
\end{tabular} & \begin{tabular}{|l|}
-9.1 \\
\end{tabular} & 4.4 & 5.7 & -10.2 & -16.4 & 3.4 & 9.3 & -15.2 & -23.5 & 6.5 & 14.2 & -14.4 & 6.5 & -0.45 & -1.75 & 1302 & \begin{tabular}{|l|l|}
0.157 \\
\end{tabular} \\
\hline 32 & -16.0 & $\begin{array}{l}-9.2 \\
\end{array}$ & 4.4 & 5.8 & \begin{tabular}{|c|}
-10.3 \\
\end{tabular} & -16.7 & 3.4 & 9.5 & -15.5 & -23.8 & 0.6 & 14.4 & -14.6 & 0.0 & -0.45 & -1.75 & 1320 & \begin{tabular}{|l|}
0.157 \\
\end{tabular} \\
\hline 33 & -16.2 & -9.3 & 4.5 & 5.9 & -10.4 & -16.8 & 3.5 & 9.6 & -15.6 & -23.9 & 6.7 & \begin{tabular}{|l|l}
14.6 \\
\end{tabular} & -14.8 & 6.6 & -0.45 & -1.74 & 1333 & \begin{tabular}{|l|}
0.159 \\
\end{tabular} \\
\hline
\end{tabular}


Table E.22 NU 58-40 Test Data $\left(0{ }^{\circ} \mathrm{C}\right.$ Tests $)$, continued

\begin{tabular}{|c|c|c|c|c|c|c|c|c|c|c|c|c|c|c|c|c|c|c|}
\hline & & & & & & & & 111 & ravic & trrays & & & & & & & & \\
\hline & $\mathrm{NU}$ & $58-40$ & sample & & $\mathrm{NU}$ & $58-40 \mathrm{~s}$ & ample & $\# 12$ & & $58-40$ & Sample & & Trim & ned & & & Creep & Pois. \\
\hline & \begin{tabular}{l|l} 
vert \\
\end{tabular} & vert & horz & horz & \begin{tabular}{l|l} 
vert \\
\end{tabular} & \begin{tabular}{|l|} 
vert \\
\end{tabular} & horz & horz & vert & \begin{tabular}{|l|} 
vert \\
\end{tabular} & horz & horz & $\overline{\mathrm{Me}}$ & & Ratio & & Comp & \begin{tabular}{|l|} 
Ratio \\
\end{tabular} \\
\hline Time & front & back & front & back & front & back & front & back & front & back & front & back & vert & horz & $\mathrm{X} / \mathrm{Y}$ & $\mathrm{C}_{\text {empl }}$ & $\mathrm{D}(\mathrm{t})$ & \\
\hline $\mathrm{sec}$ & $\mu \mathrm{m}$ & $\mu \mathrm{m}$ & $\mu \mathrm{m}$ & $\mu \mathrm{m}$ & $\mu \mathrm{m}$ & $\mu \mathrm{m}$ & $\mu \mathrm{m}$ & $\mu \mathrm{m}$ & $\mu \mathrm{m}$ & $\mu \mathrm{m}$ & $\mu \mathrm{m}$ & $\mu \mathrm{m}$ & $\mu \mathrm{m}$ & $\mu \mathrm{m}$ & & & $1 / \mathrm{kPa}$ & \\
\hline 34 & -16.4 & -9.4 & 4.6 & 6.0 & -10.6 & -17.0 & 3.5 & 9.7 & -15.9 & -24.3 & 6.7 & 14.7 & -15.0 & 6.7 & -0.45 & -1.74 & 1350 & 0.159 \\
\hline 35 & -16.7 & -9.6 & 4.6 & 6.0 & \begin{tabular}{|c|}
-10.6 \\
\end{tabular} & $\begin{array}{l}-17.2 \\
\end{array}$ & 3.6 & 9.8 & -16.0 & -24.4 & 6.8 & $\begin{array}{l}14.9 \\
\end{array}$ & $\mid-15.1$ & 6.8 & -0.45 & -1.75 & 1367 & \\
\hline 36 & -16.7 & -9.5 & 4.7 & 6.1 & -11.0 & -17.4 & 3.6 & 9.9 & \begin{tabular}{|l|}
-16.1 \\
\end{tabular} & $\mid-24.7$ & 6.9 & 15.0 & -15.3 & 6.9 & -0.45 & -1.75 & 1383 & 0.158 \\
\hline 37 & \begin{tabular}{|c|}
-17.0 \\
\end{tabular} & -9.6 & 4.7 & 6.2 & -11.0 & \begin{tabular}{|l|l}
-17.6 \\
\end{tabular} & 3.7 & 10.0 & -16.3 & -24.9 & 6.9 & 15.2 & $\begin{array}{l}-15.5 \\
\end{array}$ & 7.0 & \begin{tabular}{|l|}
-0.45 \\
\end{tabular} & -1.74 & 1397 & \begin{tabular}{|l|}
0.158 \\
\end{tabular} \\
\hline 38 & -17.3 & -9.8 & 4.8 & 6.3 & -11.1 & -17.8 & 3.7 & $\begin{array}{l}10.1 \\
\end{array}$ & \begin{tabular}{|l|l} 
\\
\end{tabular} & -25.4 & 7.0 & \begin{tabular}{|l|}
15.3 \\
\end{tabular} & \begin{tabular}{|l|l|}
-15.7 \\
\end{tabular} & 7.0 & \begin{tabular}{|l}
-0.45 \\
\end{tabular} & -1.75 & 1416 & \begin{tabular}{|l|}
0.155 \\
\end{tabular} \\
\hline 39 & \begin{tabular}{|l|}
-17.4 \\
\end{tabular} & -9.9 & 4.8 & 6.3 & -11.2 & \begin{tabular}{|l|}
-18.1 \\
\end{tabular} & 3.7 & 10.2 & -16.6 & -25.3 & 7.1 & $\begin{array}{ll}15.5 \\
\end{array}$ & $\begin{array}{l}-15.8 \\
\end{array}$ & 7.1 & -0.45 & -1.75 & 1428 & 0.158 \\
\hline 40 & -17.5 & -10.1 & 4.9 & 6.5 & -11.3 & -18.1 & 3.8 & 10.3 & -16.7 & -25.5 & 7.1 & 15.6 & \begin{tabular}{|l|}
-15.9 \\
\end{tabular} & 7.2 & \begin{tabular}{|l|}
-0.45 \\
\end{tabular} & -1.74 & 1439 & 0.161 \\
\hline 41 & \begin{tabular}{|c|}
-17.7 \\
\end{tabular} & -10.2 & 4.9 & 6.5 & -11.4 & -18.4 & 3.8 & 10.4 & -16.8 & \begin{tabular}{|l|}
-25.8 \\
\end{tabular} & 7.2 & $\begin{array}{ll}15.8 \\
\end{array}$ & \begin{tabular}{|l}
-16.1 \\
\end{tabular} & 7.3 & -0.45 & -1.74 & 1453 & \begin{tabular}{|l|}
0.160 \\
\end{tabular} \\
\hline 42 & -17.8 & -10.2 & 5.0 & 6.6 & -11.6 & -18.5 & 3.9 & $\begin{array}{l}10.5 \\
\end{array}$ & -17.0 & \begin{tabular}{|l|}
-25.9 \\
\end{tabular} & 7.3 & 15.9 & -16.2 & 7.3 & \begin{tabular}{|l|}
-0.45 \\
\end{tabular} & -1.74 & 1466 & \begin{tabular}{|l|}
0.161 \\
\end{tabular} \\
\hline 43 & -18.0 & -10.2 & 5.1 & 6.7 & -11.7 & -18.7 & 3.9 & \begin{tabular}{|l|}
10.6 \\
\end{tabular} & -17.1 & -26.2 & 7.3 & \begin{tabular}{|l|}
16.1 \\
\end{tabular} & \begin{tabular}{|l|l|} 
\\
\end{tabular} & 7.4 & -0.45 & -1.74 & 1480 & \begin{tabular}{|l|l}
0.162 \\
\end{tabular} \\
\hline 44 & \begin{tabular}{|l|}
-18.1 \\
\end{tabular} & -10.4 & 5.1 & 6.7 & -11.8 & -18.8 & 4.0 & 10.6 & -17.2 & -26.4 & 7.4 & 16.2 & $\begin{array}{l}-16.5 \\
\end{array}$ & 7.5 & \begin{tabular}{|l|}
-0.45 \\
\end{tabular} & -1.73 & 1489 & \begin{tabular}{|l|l|}
0.162 \\
\end{tabular} \\
\hline 45 & \begin{tabular}{|c|}
-18.3 \\
\end{tabular} & -10.5 & 5.2 & 6.8 & -11.9 & -19.0 & 4.0 & \begin{tabular}{|l|}
10.8 \\
\end{tabular} & -17.3 & -26.6 & 7.5 & \begin{tabular}{|l|}
16.4 \\
\end{tabular} & \begin{tabular}{|l}
-16.6 \\
\end{tabular} & 7.5 & -0.45 & -1.73 & 1505 & 0.163 \\
\hline 46 & -18.4 & -10.6 & 5.2 & 6.9 & -12.0 & -19.2 & 4.0 & 10.9 & -17.5 & \begin{tabular}{|l}
-26.7 \\
\end{tabular} & 7.5 & 16.5 & \begin{tabular}{|l}
-16.8 \\
\end{tabular} & 7.6 & \begin{tabular}{|l|}
-0.45 \\
\end{tabular} & -1.73 & 1516 & \begin{tabular}{|l|}
0.164 \\
\end{tabular} \\
\hline 47 & -18.6 & -10.7 & 5.3 & 7.0 & -12.1 & -19.4 & 4.1 & $\begin{array}{ll}10.9 \\
\end{array}$ & -17.6 & -27.0 & 7.6 & $\begin{array}{ll}16.6 \\
\end{array}$ & \begin{tabular}{|l|l|}
-16.9 \\
\end{tabular} & 7.7 & \begin{tabular}{|l}
-0.45 \\
\end{tabular} & -1.73 & 1531 & \begin{tabular}{|l|}
0.163 \\
\end{tabular} \\
\hline 48 & -18.7 & -10.8 & 5.3 & 7.1 & -12.2 & -19.5 & 4.1 & 11.0 & -17.8 & \begin{tabular}{|l|}
-27.2 \\
\end{tabular} & 7.6 & \begin{tabular}{|l|}
16.7 \\
\end{tabular} & -17.0 & 7.8 & -0.46 & -1.73 & 1542 & \begin{tabular}{|l|l}
0.165 \\
\end{tabular} \\
\hline 49 & \begin{tabular}{|c|}
-18.9 \\
\end{tabular} & -10.9 & 5.3 & $\begin{array}{l}7.1 \\
\end{array}$ & \begin{tabular}{|c|}
-12.3 \\
\end{tabular} & $\begin{array}{l}-19.7 \\
\end{array}$ & 4.1 & $\begin{array}{ll}11.1 \\
\end{array}$ & \begin{tabular}{|l|l|}
-17.8 \\
\end{tabular} & \begin{tabular}{|l|l|}
-27.2 \\
\end{tabular} & 7.7 & $\begin{array}{ll}16.9 \\
\end{array}$ & \begin{tabular}{|l|l|l} 
\\
\end{tabular} & 7.8 & \begin{tabular}{|l|}
-0.46 \\
\end{tabular} & $\begin{array}{l}-1.73 \\
\end{array}$ & 1553 & \begin{tabular}{|l|}
0.165 \\
\end{tabular} \\
\hline 50 & \begin{tabular}{|c|}
-19.0 \\
\end{tabular} & -11.0 & 5.4 & 7.2 & -12.5 & -19.9 & 4.2 & 11.2 & \begin{tabular}{|l}
-17.9 \\
\end{tabular} & \begin{tabular}{|l}
-27.4 \\
\end{tabular} & 7.8 & 17.0 & \begin{tabular}{|l}
-17.3 \\
\end{tabular} & 7.9 & \begin{tabular}{|l|}
-0.46 \\
\end{tabular} & \begin{tabular}{|c|}
-1.73 \\
\end{tabular} & 1567 & \begin{tabular}{|l|}
0.165 \\
\end{tabular} \\
\hline 51 & -19.2 & -11.0 & 5.5 & 7.3 & -12.5 & -20.1 & 4.2 & \begin{tabular}{|l|l}
11.3 \\
\end{tabular} & \begin{tabular}{|l}
-18.1 \\
\end{tabular} & \begin{tabular}{|l|}
-27.7 \\
\end{tabular} & 7.8 & \begin{tabular}{|l|l|}
17.1 \\
\end{tabular} & \begin{tabular}{|l}
-17.5 \\
\end{tabular} & 8.0 & \begin{tabular}{|l|}
-0.46 \\
\end{tabular} & -1.73 & \begin{tabular}{|l|}
1582 \\
\end{tabular} & \begin{tabular}{|l|}
0.165 \\
\end{tabular} \\
\hline 52 & \begin{tabular}{|c|}
-19.3 \\
\end{tabular} & -11.1 & 5.5 & 7.3 & -12.7 & -20.2 & 4.3 & \begin{tabular}{|l|l}
11.4 \\
\end{tabular} & \begin{tabular}{|l|l|}
-18.1 \\
\end{tabular} & \begin{tabular}{|l|}
-27.9 \\
\end{tabular} & 7.9 & \begin{tabular}{|l|l}
17.3 \\
\end{tabular} & \begin{tabular}{|l|l|}
-17.6 \\
\end{tabular} & 8.0 & \begin{tabular}{|c|}
-0.46 \\
\end{tabular} & -1.72 & \begin{tabular}{|l|}
1592 \\
\end{tabular} & \begin{tabular}{|l|}
0.166 \\
\end{tabular} \\
\hline 53 & -19.5 & -11.2 & 5.5 & 7.4 & -12.8 & -20.3 & 4.3 & 11.5 & -18.3 & \begin{tabular}{|c|}
-28.1 \\
\end{tabular} & 7.9 & 17.4 & -17.7 & 8.1 & -0.46 & -1.72 & 1604 & \begin{tabular}{|l|}
0.166 \\
\end{tabular} \\
\hline 54 & $\begin{array}{l}-19.6 \\
\end{array}$ & -11.3 & 5.6 & $\begin{array}{l}7.5 \\
\end{array}$ & \begin{tabular}{|c|}
-12.9 \\
\end{tabular} & -20.5 & 4.3 & $\begin{array}{l}11.6 \\
\end{array}$ & \begin{tabular}{|l|l|} 
\\
\end{tabular} & \begin{tabular}{|l|}
-28.2 \\
\end{tabular} & 8.0 & $\begin{array}{ll}17.5 \\
\end{array}$ & \begin{tabular}{|l|l|}
-17.9 \\
\end{tabular} & 8.2 & \begin{tabular}{|c|}
-0.46 \\
\end{tabular} & -1.72 & 1619 & \begin{tabular}{|l|}
0.166 \\
\end{tabular} \\
\hline 55 & \begin{tabular}{|c|}
-19.8 \\
\end{tabular} & -11.3 & 5.6 & 7.5 & -13.1 & -20.6 & 4.4 & $\begin{array}{l}11.6 \\
\end{array}$ & -18.4 & \begin{tabular}{|l}
-28.4 \\
\end{tabular} & 8.1 & \begin{tabular}{|l|l}
17.6 \\
\end{tabular} & -18.0 & 8.2 & \begin{tabular}{|l|}
-0.46 \\
\end{tabular} & -1.72 & \begin{tabular}{|l|}
1627 \\
\end{tabular} & \begin{tabular}{|l|}
0.166 \\
\end{tabular} \\
\hline 56 & -19.9 & -11.5 & 5.7 & 7.6 & -13.2 & -20.7 & 4.4 & $\begin{array}{ll}11.7 \\
\end{array}$ & -18.6 & -28.4 & 8.1 & \begin{tabular}{|l|}
17.7 \\
\end{tabular} & \begin{tabular}{|l|l|}
-18.1 \\
\end{tabular} & 8.3 & \begin{tabular}{|l}
-0.46 \\
\end{tabular} & -1.72 & \begin{tabular}{|l|}
1639 \\
\end{tabular} & \begin{tabular}{|l|}
0.166 \\
\end{tabular} \\
\hline 57 & \begin{tabular}{|c|}
-19.9 \\
\end{tabular} & -11.5 & 5.7 & $\begin{array}{l}7.7 \\
\end{array}$ & \begin{tabular}{|c|}
-13.4 \\
\end{tabular} & -21.0 & 4.4 & $\begin{array}{ll}11.8 \\
\end{array}$ & -18.7 & \begin{tabular}{|c|}
-28.6 \\
\end{tabular} & 8.1 & \begin{tabular}{|l|l}
17.9 \\
\end{tabular} & $\mid-18.2$ & 8.3 & -0.46 & -1.72 & 1652 & \begin{tabular}{|l|l|}
0.167 \\
\end{tabular} \\
\hline 58 & -20.2 & -11.6 & 5.8 & 7.7 & -13.4 & -21.1 & 4.5 & $\begin{array}{ll}11.9 \\
\end{array}$ & -18.7 & -28.8 & 8.2 & 18.0 & -18.3 & 8.4 & -0.46 & -1.72 & 1662 & \begin{tabular}{|l|l}
0.167 \\
\end{tabular} \\
\hline 59 & -20.4 & -11.6 & 5.8 & 7.8 & -13.5 & -21.3 & 4.5 & 11.9 & -18.8 & \begin{tabular}{|l|}
-29.0 \\
\end{tabular} & 8.3 & $\begin{array}{ll}18.1 \\
\end{array}$ & \begin{tabular}{|l}
-18.5 \\
\end{tabular} & 8.4 & \begin{tabular}{|c|}
-0.46 \\
\end{tabular} & -1.72 & \begin{tabular}{|l|}
1675 \\
\end{tabular} & \begin{tabular}{|l|}
0.166 \\
\end{tabular} \\
\hline 60 & -20.4 & -11.8 & 5.9 & 7.9 & -13.6 & -21.4 & 4.5 & 12.0 & -19.0 & \begin{tabular}{|l}
-29.1 \\
\end{tabular} & 8.3 & $\begin{array}{l}18.2 \\
\end{array}$ & \begin{tabular}{|l}
-18.6 \\
\end{tabular} & 8.5 & \begin{tabular}{|l|}
-0.46 \\
\end{tabular} & -1.72 & \begin{tabular}{|l|}
1685 \\
\end{tabular} & \begin{tabular}{|l|}
0.167 \\
\end{tabular} \\
\hline 61 & -20.6 & -11.8 & 5.9 & 7.9 & -13.7 & -21.5 & 4.6 & \begin{tabular}{|l|}
12.1 \\
\end{tabular} & -19.1 & \begin{tabular}{|l|} 
\\
\end{tabular} & 8.3 & \begin{tabular}{|l|}
18.3 \\
\end{tabular} & -18.7 & 8.6 & \begin{tabular}{|l|}
-0.46 \\
\end{tabular} & -1.72 & \begin{tabular}{|l|}
1697 \\
\end{tabular} & 0.168 \\
\hline 62 & -20.7 & -11.9 & 5.9 & 8.0 & -13.8 & -21.7 & 4.6 & 12.2 & \begin{tabular}{|l}
-19.1 \\
\end{tabular} & -29.4 & 8.4 & \begin{tabular}{|l|l}
18.4 \\
\end{tabular} & \begin{tabular}{|l}
-18.8 \\
\end{tabular} & 8.6 & \begin{tabular}{|c|}
-0.46 \\
\end{tabular} & -1.72 & \begin{tabular}{|l|}
1705 \\
\end{tabular} & \begin{tabular}{|l}
0.168 \\
\end{tabular} \\
\hline 63 & -20.7 & -11.8 & 6.0 & 8.1 & -14.0 & -21.9 & 4.6 & 12.2 & $\mid-19.2$ & \begin{tabular}{|l|l|} 
\\
\end{tabular} & 8.4 & 18.5 & -18.9 & 8.7 & -0.46 & -1.72 & 1717 & 0.169 \\
\hline 64 & -21.0 & -12.1 & 6.0 & 8.1 & -14.0 & -22.0 & 4.7 & 12.3 & -19.3 & \begin{tabular}{|l|}
-29.8 \\
\end{tabular} & 8.5 & $\begin{array}{l}18.6 \\
\end{array}$ & \begin{tabular}{|l}
-19.1 \\
\end{tabular} & 8.7 & \begin{tabular}{|c|c|}
-0.46 \\
\end{tabular} & -1.72 & \begin{tabular}{|l|}
1728 \\
\end{tabular} & \begin{tabular}{|l|}
0.168 \\
\end{tabular} \\
\hline 65 & -21.1 & -12.1 & 6.1 & 8.2 & -14.0 & -22.1 & 4.7 & 12.4 & -19.4 & -29.8 & 8.5 & 18.8 & -19.2 & 8.8 & -0.46 & -1.72 & 1736 & 0.169 \\
\hline 66 & -21.2 & -12.2 & 6.1 & $\overline{8.2}$ & -14.2 & -22.2 & 4.7 & 12.5 & -19.5 & -29.9 & 8.6 & 18.9 & 9.3 & 8.9 & -0.46 & -1.7 & 1747 & 0.17 \\
\hline
\end{tabular}


Table E.22 NU 58-40 Test Data $\left(0^{\circ} \mathrm{C}\right.$ Tests), continued

\begin{tabular}{|c|c|c|c|c|c|c|c|c|c|c|c|c|c|c|c|c|c|c|}
\hline & & & & & & & & Deforn & ne & Arrays & & & & & & & & \\
\hline & $\mathrm{NU}$ & $58-40$ & sample & $\# 11$ & $\mathrm{NU}$ & $58-40$ & ample & $\# 12$ & & $158-40$ & Sample & & Trim & $\mathrm{med}$ & & & Creep & Pois. \\
\hline & \begin{tabular}{l|l} 
vert \\
\end{tabular} & \begin{tabular}{|l|} 
vert \\
\end{tabular} & horz & horz & vert & vert & horz & horz & vert & vert & horz & horz & $\overline{\mathrm{Me}}$ & & Ratio & & Comp & \begin{tabular}{|l|l|} 
Ratio \\
\end{tabular} \\
\hline Time & front & back & front & back & \begin{tabular}{|l|} 
front \\
\end{tabular} & back & front & back & \begin{tabular}{|l|} 
front \\
\end{tabular} & back & front & back & \begin{tabular}{|l|} 
vert \\
\end{tabular} & horz & $\mathrm{X} / \mathrm{Y}$ & $\mathrm{C}_{\mathrm{emp}}$ & & \\
\hline $\mathrm{sec}$ & $\mu \mathrm{m}$ & $\mu \mathrm{m}$ & $\mu \mathrm{m}$ & $\mu \mathrm{m}$ & $\mu \mathrm{m}$ & $\mu \mathrm{m}$ & $\mu \mathrm{m}$ & $\mu \mathrm{m}$ & $\mu \mathrm{m}$ & $\mu \mathrm{m}$ & $\mu \mathrm{m}$ & $\mu \mathrm{m}$ & $\mu \mathrm{m}$ & $\mu \mathrm{m}$ & & & $1 / \mathrm{kPa}$ & \\
\hline 67 & -21.3 & -12.2 & 6.2 & 8.3 & -14.3 & -22.4 & 4.8 & 12.6 & -19.8 & -30.3 & 8.6 & 18.9 & -19.4 & 8.9 & -0.46 & -1.72 & 1761 & 0.167 \\
\hline 68 & -21.5 & -12.4 & 6.2 & 8.4 & -14.4 & -22.6 & 4.8 & 12.6 & \begin{tabular}{|l|}
-19.8 \\
\end{tabular} & -30.3 & 8.7 & 19.0 & \begin{tabular}{|l}
-19.5 \\
\end{tabular} & 8.9 & -0.46 & -1.72 & 1770 & 0.168 \\
\hline 69 & -21.6 & -12.4 & 6.2 & 8.4 & -14.6 & -22.8 & 4.8 & 12.7 & -19.7 & -30.4 & 8.7 & 19.2 & \begin{tabular}{|l|l|}
-19.7 \\
\end{tabular} & 9.0 & -0.46 & -1.72 & 1781 & \begin{tabular}{|l|l|}
0.169 \\
\end{tabular} \\
\hline 70 & -21.6 & -12.5 & 6.3 & 8.5 & \begin{tabular}{|l}
-14.6 \\
\end{tabular} & -22.8 & 4.9 & 12.7 & \begin{tabular}{|l|}
-19.8 \\
\end{tabular} & -30.4 & 8.8 & 19.2 & \begin{tabular}{|l|}
-19.7 \\
\end{tabular} & 9.1 & -0.46 & \begin{tabular}{|l}
-1.71 \\
\end{tabular} & 1786 & \begin{tabular}{|l|}
0.172 \\
\end{tabular} \\
\hline 71 & -21.8 & -12.6 & 6.3 & 8.5 & -14.5 & -22.7 & 4.9 & 12.9 & -19.8 & -30.6 & 8.8 & 19.3 & \begin{tabular}{|l|l} 
\\
\end{tabular} & 9.1 & -0.46 & \begin{tabular}{|l}
-1.70 \\
\end{tabular} & 1790 & \begin{tabular}{|l|l}
0.174 \\
\end{tabular} \\
\hline 72 & -21.9 & -12.6 & 6.4 & 8.6 & -14.9 & -23.1 & 4.9 & 12.9 & $\begin{array}{l}-20.0 \\
\end{array}$ & -30.7 & 8.9 & 19.4 & $\begin{array}{l}-20.0 \\
\end{array}$ & 9.2 & -0.46 & \begin{tabular}{|l}
-1.71 \\
\end{tabular} & 1811 & \begin{tabular}{|l|l}
0.170 \\
\end{tabular} \\
\hline 73 & -22.2 & $\begin{array}{l}-12.9 \\
\end{array}$ & 6.4 & 8.6 & \begin{tabular}{|l}
-14.9 \\
\end{tabular} & -23.3 & 4.9 & 13.0 & \begin{tabular}{|l}
-20.0 \\
\end{tabular} & \begin{tabular}{|l|}
-31.1 \\
\end{tabular} & 8.9 & 19.6 & \begin{tabular}{|l}
-20.1 \\
\end{tabular} & 9.2 & -0.46 & \begin{tabular}{|l|l|}
-1.72 \\
\end{tabular} & 1823 & 0.168 \\
\hline 74 & -22.1 & -12.8 & 6.4 & 8.7 & -14.9 & -23.3 & 5.0 & 13.1 & \begin{tabular}{|l}
-20.1 \\
\end{tabular} & $\mid-31.0$ & 9.0 & 19.7 & \begin{tabular}{|l}
-20.1 \\
\end{tabular} & 9.3 & -0.46 & \begin{tabular}{|l}
-1.70 \\
\end{tabular} & 1823 & \begin{tabular}{|l|l|}
0.173 \\
\end{tabular} \\
\hline 75 & -22.3 & -12.9 & 6.5 & 8.8 & -15.1 & -23.5 & 5.0 & \begin{tabular}{|l|}
13.1 \\
\end{tabular} & \begin{tabular}{|l}
-20.2 \\
\end{tabular} & -31.2 & 9.0 & 19.8 & \begin{tabular}{|l|}
-20.3 \\
\end{tabular} & 9.3 & -0.46 & $\begin{array}{l}-1.71 \\
\end{array}$ & 1838 & \begin{tabular}{|l|l}
0.170 \\
\end{tabular} \\
\hline 76 & -22.4 & -13.0 & 6.5 & 8.9 & -15.1 & -23.7 & 5.0 & 13.2 & -20.1 & -31.2 & 9.0 & 19.9 & -20.4 & 9.4 & -0.46 & -1.71 & 1847 & \begin{tabular}{|l|l}
0.172 \\
\end{tabular} \\
\hline 77 & -22.5 & -13.0 & 6.6 & 8.9 & -15.1 & -23.8 & 5.1 & 13.2 & \begin{tabular}{|l}
-20.3 \\
\end{tabular} & -31.5 & 9.1 & 20.0 & \begin{tabular}{|l}
-20.4 \\
\end{tabular} & 9.4 & -0.46 & $\begin{array}{l}-1.71 \\
\end{array}$ & 1853 & \begin{tabular}{|l|}
0.173 \\
\end{tabular} \\
\hline 78 & -22.7 & -13.1 & 6.6 & 8.9 & -15.3 & -23.9 & 5.1 & 13.3 & -20.5 & -31.5 & 9.1 & 20.0 & -20.6 & 9.5 & -0.46 & -1.71 & 1866 & \begin{tabular}{|l|l}
0.172 \\
\end{tabular} \\
\hline 79 & -22.7 & -13.2 & 6.6 & 9.0 & -15.4 & -23.9 & 5.1 & \begin{tabular}{|l|l}
13.4 \\
\end{tabular} & $\begin{array}{l}-20.5 \\
\end{array}$ & -31.8 & 9.2 & 20.1 & \begin{tabular}{|l}
-20.6 \\
\end{tabular} & 9.5 & -0.46 & \begin{tabular}{|l}
-1.71 \\
\end{tabular} & 1873 & \begin{tabular}{|l|l|}
0.173 \\
\end{tabular} \\
\hline 80 & -22.9 & -13.3 & 6.7 & 9.0 & -15.5 & -24.1 & 5.2 & 13.4 & -20.6 & -31.8 & 9.2 & 20.2 & -20.8 & 9.6 & -0.46 & \begin{tabular}{|l}
-1.71 \\
\end{tabular} & 1883 & \begin{tabular}{|l|l|}
0.172 \\
\end{tabular} \\
\hline 81 & -23.0 & -13.3 & 6.7 & 9.1 & -15.5 & -24.2 & 5.2 & 13.5 & -20.6 & \begin{tabular}{|l|}
-31.9 \\
\end{tabular} & 9.3 & 20.3 & -20.8 & 9.6 & -0.46 & -1.70 & 1892 & \begin{tabular}{|l|l|}
0.173 \\
\end{tabular} \\
\hline 82 & -23.0 & $\begin{array}{l}-13.2 \\
\end{array}$ & 6.8 & 9.2 & -15.6 & -24.3 & 5.2 & $\begin{array}{ll}13.6 \\
\end{array}$ & \begin{tabular}{|l}
-20.7 \\
\end{tabular} & \begin{tabular}{|l|}
-32.1 \\
\end{tabular} & 9.3 & 20.4 & \begin{tabular}{|l}
-20.9 \\
\end{tabular} & 9.7 & -0.46 & \begin{tabular}{|l|l|}
-1.70 \\
\end{tabular} & 1900 & \begin{tabular}{|l|l|}
0.174 \\
\end{tabular} \\
\hline 83 & -23.3 & -13.5 & 6.8 & 9.2 & -15.7 & -24.5 & 5.3 & 13.6 & -20.8 & $\mid-32.1$ & 9.3 & 20.5 & \begin{tabular}{|l}
-21.1 \\
\end{tabular} & 9.7 & -0.46 & \begin{tabular}{|l}
-1.71 \\
\end{tabular} & 1914 & \begin{tabular}{|l|l|}
0.173 \\
\end{tabular} \\
\hline 84 & -23.5 & -13.6 & 6.8 & 9.3 & -15.8 & -24.5 & 5.3 & 13.7 & -20.8 & -32.3 & 9.4 & 20.6 & \begin{tabular}{|l|l}
-21.2 \\
\end{tabular} & 9.8 & -0.46 & -1.70 & 1920 & \begin{tabular}{|l|l}
0.174 \\
\end{tabular} \\
\hline 85 & -23.5 & -13.6 & 6.9 & 9.3 & -15.8 & -24.6 & 5.3 & \begin{tabular}{|l|l}
13.8 \\
\end{tabular} & -20.9 & \begin{tabular}{|l|}
-32.3 \\
\end{tabular} & 9.4 & 20.7 & \begin{tabular}{|l}
-21.2 \\
\end{tabular} & 9.9 & -0.46 & $\begin{array}{l}-1.70 \\
\end{array}$ & 1928 & 0.175 \\
\hline 86 & -23.6 & -13.8 & 6.9 & 9.4 & -15.9 & -24.7 & 5.3 & \begin{tabular}{|l|}
13.9 \\
\end{tabular} & -21.0 & -32.6 & 9.5 & 20.8 & \begin{tabular}{|l|l}
-21.3 \\
\end{tabular} & 9.9 & -0.46 & -1.70 & 1938 & \begin{tabular}{|l|l|}
0.175 \\
\end{tabular} \\
\hline 87 & -23.7 & -13.9 & 6.9 & 9.4 & -16.0 & -24.9 & 5.4 & 13.9 & \begin{tabular}{|l}
-21.1 \\
\end{tabular} & -32.6 & 9.5 & 20.9 & -21.4 & 9.9 & -0.46 & $\begin{array}{l}-1.70 \\
\end{array}$ & 1947 & 0.175 \\
\hline 88 & -23.9 & -13.9 & 7.0 & 9.5 & \begin{tabular}{|l|}
-16.1 \\
\end{tabular} & -25.0 & 5.4 & $\begin{array}{l}14.0 \\
\end{array}$ & -21.2 & -32.8 & 9.5 & 20.9 & \begin{tabular}{|l}
-21.6 \\
\end{tabular} & 10.0 & -0.46 & -1.70 & 1958 & \begin{tabular}{|l|l|}
0.175 \\
\end{tabular} \\
\hline 89 & -23.9 & -14.0 & 7.0 & 9.5 & -16.2 & -25.1 & 5.5 & 14.0 & -21.2 & -32.8 & 9.6 & 21.1 & -21.6 & 10.0 & -0.46 & -1.70 & 1964 & \begin{tabular}{|l|l}
0.175 \\
\end{tabular} \\
\hline 90 & -24.1 & -14.1 & 7.0 & 9.6 & -16.4 & -25.2 & 5.5 & 14.1 & \begin{tabular}{|l}
-21.3 \\
\end{tabular} & -33.0 & 9.6 & 21.1 & \begin{tabular}{|l}
-21.8 \\
\end{tabular} & 10.1 & -0.46 & \begin{tabular}{|l}
-1.70 \\
\end{tabular} & 1975 & \begin{tabular}{|l|l|}
0.173 \\
\end{tabular} \\
\hline 91 & -24.2 & -14.1 & 7.1 & 9.7 & -16.4 & -25.4 & 5.5 & 14.2 & -21.4 & -33.1 & 9.7 & 21.2 & \begin{tabular}{|l|l}
-21.8 \\
\end{tabular} & 10.1 & -0.46 & \begin{tabular}{|l}
-1.70 \\
\end{tabular} & 1984 & \begin{tabular}{|l|l}
0.175 \\
\end{tabular} \\
\hline 92 & -24.2 & -14.1 & 7.1 & 9.7 & -16.5 & -25.4 & 5.5 & 14.2 & \begin{tabular}{|l|l|} 
\\
\end{tabular} & -33.2 & 9.7 & 21.3 & \begin{tabular}{|l}
-21.9 \\
\end{tabular} & 10.2 & -0.47 & \begin{tabular}{|l}
-1.70 \\
\end{tabular} & 1988 & \begin{tabular}{|l|l|}
0.177 \\
\end{tabular} \\
\hline 93 & -24.4 & -14.2 & 7.1 & 9.7 & -16.5 & -25.6 & 5.5 & 14.3 & -21.4 & -33.2 & 9.7 & 21.4 & -22.0 & 10.2 & -0.47 & -1.70 & 1995 & \begin{tabular}{|l|l}
0.177 \\
\end{tabular} \\
\hline 94 & -24.4 & -14.4 & 7.2 & 9.8 & -16.6 & -25.7 & 5.6 & 14.3 & -21.6 & -33.4 & 9.8 & 21.5 & \begin{tabular}{|l|}
-22.1 \\
\end{tabular} & 10.3 & -0.46 & -1.70 & 2007 & \begin{tabular}{|l}
0.176 \\
\end{tabular} \\
\hline 95 & -24.6 & -14.4 & 7.2 & 9.9 & -16.7 & -25.7 & 5.6 & \begin{tabular}{|c|}
14.4 \\
\end{tabular} & -21.6 & -33.5 & 9.8 & 21.5 & -22.1 & 10.3 & -0.47 & -1.69 & 2013 & \begin{tabular}{|l|l}
0.178 \\
\end{tabular} \\
\hline 96 & -24.6 & -14.4 & 7.3 & 9.9 & \begin{tabular}{|l}
-16.8 \\
\end{tabular} & -25.8 & $\overline{5.6}$ & 14.4 & \begin{tabular}{|l|}
-21.8 \\
\end{tabular} & -33.6 & 9.8 & 21.6 & $\begin{array}{l}-22.2 \\
\end{array}$ & 10.4 & -0.47 & $\begin{array}{l}-1.70 \\
\end{array}$ & 2022 & \begin{tabular}{|l|l|}
0.177 \\
\end{tabular} \\
\hline 97 & -24.7 & -14.5 & 7.3 & 10.0 & -16.8 & -26.0 & 5.6 & 14.5 & \begin{tabular}{|l}
-21.8 \\
\end{tabular} & -33.6 & 9.9 & 21.7 & \begin{tabular}{|l}
-22.3 \\
\end{tabular} & 10.4 & -0.47 & \begin{tabular}{|l}
-1.69 \\
\end{tabular} & 2030 & \begin{tabular}{|l|l}
0.178 \\
\end{tabular} \\
\hline 98 & -24.8 & -14.5 & 7.3 & 10.0 & -16.8 & -26.0 & 5.7 & 14.6 & -21.8 & $\mid-33.8$ & 9.9 & 21.8 & -22.4 & 10.5 & -0.47 & -1.69 & 2034 & \begin{tabular}{|l|l}
0.180 \\
\end{tabular} \\
\hline 99 & -24.9 & -14.8 & 7.3 & 10.0 & -17.1 & -26.3 & 5.7 & 14.6 & -21.9 & \begin{tabular}{|l|} 
\\
\end{tabular} & 9.9 & 21.9 & -22.6 & 10.5 & -0.46 & -1.70 & 2050 & \begin{tabular}{|l|}
0.176 \\
\end{tabular} \\
\hline 100 & -24.9 & -14.7 & 7.4 & \begin{tabular}{|l|}
10.1 \\
\end{tabular} & -17.0 & -26.3 & 5.7 & \begin{tabular}{|l|}
14.7 \\
\end{tabular} & -21.9 & -33.8 & 10.0 & 21.9 & -22.5 & 10.5 & -0.47 & -1.69 & 2051 & \begin{tabular}{|l|l|}
0.179 \\
\end{tabular} \\
\hline
\end{tabular}


Table E.23 NU 58-40 Test Data $\left(-10{ }^{\circ} \mathrm{C}\right.$ Tests)

\begin{tabular}{|c|c|c|c|c|c|c|c|c|c|c|c|c|c|c|c|c|c|c|}
\hline & \multicolumn{12}{|c|}{ Normalized Vertical and Horizontal Deformation Arrays } & & & & & & \\
\hline & \multicolumn{4}{|c|}{ NU 58-40 Sample \#11 } & \multicolumn{4}{|c|}{ NU 58-40 Sample \#12 } & \multicolumn{4}{|c|}{ NU 58-40 Sample \#9 } & \multicolumn{2}{|c|}{ Trimmed } & & & Creep & Pois. \\
\hline & vert & vert & horz & horz & vert & vert & horz & horz & vert & vert & horz & horz & & & Ratio & & Comp & Ratio \\
\hline Time & front & back & front & back & front & back & front & back & front & back & front & back & vert & horz & $\mathrm{X} / \mathrm{Y}$ & $\mathrm{C}_{\mathrm{empl}}$ & $D(t)$ & $v$ \\
\hline $\sec$ & $\mu \mathrm{m}$ & $\mu \mathrm{m}$ & $\mu \mathrm{m}$ & $\mu \mathrm{m}$ & $\mu \mathrm{m}$ & $\mu \mathrm{m}$ & $\mu \mathrm{m}$ & $\mu \mathrm{m}$ & $\mu \mathrm{m}$ & $\mu \mathrm{m}$ & $\mu \mathrm{m}$ & $\mu \mathrm{m}$ & $\mu \mathrm{m}$ & $\mu \mathrm{m}$ & & & $1 / \mathrm{kPa}$ & \\
\hline 0 & 0.0 & 0.0 & 0.0 & 0.0 & 0.0 & 0.0 & 0.0 & 0.0 & 0.0 & 0.0 & 0.0 & 0.0 & 0.0 & 0.0 & 0.00 & 0.00 & 0 & 0.000 \\
\hline 1 & -3.5 & -1.7 & 0.9 & 1.1 & -2.1 & -3.5 & 0.7 & 1.9 & -2.9 & -3.1 & 1.0 & 1.9 & -3.0 & 1.2 & -0.40 & -1.91 & 173 & 0.108 \\
\hline 2 & -4.3 & -2.1 & 1.2 & 1.3 & -2.5 & -4.1 & 0.8 & 2.2 & -3.6 & -3.9 & 1.2 & 2.3 & -3.6 & 1.5 & -0.40 & -1.91 & 210 & 0.107 \\
\hline 3 & -4.8 & -2.3 & 1.3 & 1.4 & -2.8 & -4.5 & 0.9 & 2.5 & -3.9 & -4.3 & 1.3 & 2.6 & -4.0 & 1.6 & -0.41 & -1.90 & 233 & 0.111 \\
\hline 4 & -5.2 & -2.5 & 1.4 & 1.6 & -3.0 & -4.8 & 1.0 & 2.7 & -4.1 & -4.7 & 1.5 & 2.8 & -4.3 & 1.8 & -0.42 & -1.85 & 247 & 0.123 \\
\hline 5 & -5.3 & -2.7 & 1.5 & 1.7 & -3.2 & -4.9 & 1.1 & 2.8 & -4.4 & -4.9 & 1.6 & 3.0 & -4.5 & 1.9 & -0.43 & -1.83 & 259 & 0.130 \\
\hline 6 & -5.7 & -3.0 & 1.6 & 1.8 & -3.2 & -4.8 & 1.1 & 3.1 & -4.6 & -5.3 & 1.6 & 3.1 & -4.6 & 2.0 & -0.44 & -1.78 & 269 & 0.146 \\
\hline 7 & -5.9 & -3.0 & 1.6 & 1.9 & -3.4 & -5.4 & 1.2 & 3.1 & -4.7 & -5.4 & 1.7 & 3.3 & -4.9 & 2.1 & -0.43 & -1.81 & 283 & 0.136 \\
\hline 8 & -6.1 & -3.1 & 1.7 & 2.0 & -3.5 & -5.5 & 1.2 & 3.2 & -5.0 & -5.8 & 1.8 & 3.5 & -5.0 & 2.2 & -0.43 & -1.80 & 293 & 0.138 \\
\hline 9 & -6.4 & -3.3 & 1.7 & 2.0 & -3.7 & -5.7 & 1.3 & 3.4 & -5.1 & -6.1 & 1.9 & 3.6 & -5.2 & 2.2 & -0.43 & -1.81 & 305 & 0.135 \\
\hline 10 & -6.5 & -3.4 & 1.8 & 2.1 & -3.8 & -5.8 & 1.3 & 3.5 & -5.2 & -6.2 & 1.9 & 3.7 & -5.3 & 2.3 & -0.44 & -1.79 & 311 & 0.142 \\
\hline 11 & -6.6 & -3.4 & 1.9 & 2.1 & -3.9 & -5.8 & 1.3 & 3.6 & -5.4 & -6.4 & 2.0 & 3.8 & -5.4 & 2.4 & -0.44 & -1.77 & 317 & 0.149 \\
\hline 12 & -6.8 & -3.6 & 1.9 & 2.2 & -3.9 & -6.0 & 1.4 & 3.7 & -5.4 & -6.6 & 2.1 & 3.9 & -5.6 & 2.5 & -0.44 & -1.77 & 326 & 0.149 \\
\hline 13 & -6.9 & -3.7 & 1.9 & 2.2 & -4.2 & -6.4 & 1.4 & 3.7 & -5.6 & -6.8 & 2.1 & 4.0 & -5.8 & 2.5 & -0.43 & -1.80 & 337 & 0.138 \\
\hline 14 & -7.1 & -3.7 & 2.0 & 2.3 & -4.1 & -6.4 & 1.4 & 3.8 & -5.7 & -6.9 & 2.2 & 4.2 & -5.8 & 2.6 & -0.44 & -1.77 & 341 & 0.149 \\
\hline 15 & -7.3 & -3.8 & 2.0 & 2.4 & -4.2 & -6.5 & 1.5 & 3.9 & -5.9 & -7.1 & 2.2 & 4.3 & -6.0 & 2.6 & -0.44 & -1.77 & 349 & 0.147 \\
\hline 16 & -7.4 & -3.8 & 2.1 & 2.4 & -4.3 & -6.8 & 1.5 & 4.0 & -5.9 & -7.0 & 2.3 & 4.4 & -6.1 & 2.7 & -0.44 & -1.77 & 357 & 0.148 \\
\hline 17 & -7.5 & -3.9 & 2.1 & 2.4 & -4.3 & -6.7 & 1.5 & 4.0 & -6.1 & -7.3 & 2.3 & 4.4 & -6.2 & 2.7 & -0.44 & -1.77 & 361 & 0.148 \\
\hline 18 & -7.7 & -4.1 & 2.1 & 2.5 & -4.5 & -6.8 & 1.6 & 4.1 & -6.1 & -7.6 & 2.3 & 4.5 & -6.3 & 2.8 & -0.44 & -1.77 & 369 & 0.149 \\
\hline 19 & -7.9 & -4.2 & 2.2 & 2.5 & -4.7 & -6.9 & 1.6 & 4.2 & -6.4 & -7.9 & 2.4 & 4.6 & -6.5 & 2.8 & -0.43 & -1.80 & 378 & 0.140 \\
\hline 20 & -7.9 & -4.1 & 2.2 & 2.6 & -4.8 & -7.1 & 1.6 & 4.2 & -6.4 & -7.9 & 2.4 & 4.7 & -6.5 & 2.9 & -0.44 & -1.78 & 382 & 0.147 \\
\hline 21 & -8.1 & -4.5 & 2.2 & 2.7 & -4.8 & -7.1 & 1.6 & 4.3 & -6.4 & -7.9 & 2.5 & 4.8 & -6.6 & 2.9 & -0.44 & -1.78 & 387 & 0.146 \\
\hline 22 & -8.1 & -4.3 & 2.3 & 2.7 & -4.9 & -7.2 & 1.7 & 4.4 & -6.6 & -8.1 & 2.5 & 4.9 & -6.7 & 3.0 & -0.44 & -1.77 & 393 & 0.149 \\
\hline 23 & -8.2 & -4.3 & 2.3 & 2.8 & -4.8 & -7.3 & 1.7 & 4.4 & -6.6 & -8.2 & 2.5 & 4.9 & -6.7 & 3.0 & -0.45 & -1.76 & 396 & 0.154 \\
\hline 24 & -8.3 & -4.3 & 2.3 & 2.8 & -5.1 & -7.5 & 1.7 & 4.5 & -6.6 & -8.1 & 2.6 & 5.0 & -6.9 & 3.0 & -0.44 & -1.77 & 404 & 0.150 \\
\hline 25 & -8.3 & -4.5 & 2.4 & 2.8 & -5.2 & -7.9 & 1.7 & 4.4 & -6.7 & -8.4 & 2.6 & 5.1 & -7.0 & 3.1 & -0.44 & -1.79 & 412 & 0.142 \\
\hline 26 & -8.5 & -4.6 & 2.4 & 2.9 & -5.3 & -7.7 & 1.7 & 4.5 & -6.9 & -8.5 & 2.6 & 5.2 & -7.1 & 3.1 & -0.44 & -1.78 & 416 & 0.144 \\
\hline 27 & -8.6 & -4.6 & 2.4 & 2.9 & -5.2 & -7.9 & 1.8 & 4.6 & -6.8 & -8.6 & 2.7 & 5.2 & -7.1 & 3.1 & -0.44 & -1.77 & 417 & 0.149 \\
\hline 28 & -8.6 & -4.6 & 2.5 & 2.9 & -5.4 & -7.8 & 1.8 & 4.7 & -6.9 & -8.7 & 2.7 & 5.3 & -7.2 & 3.2 & -0.45 & -1.76 & 421 & 0.154 \\
\hline 29 & -8.8 & -4.6 & 2.5 & 3.0 & -5.4 & -8.0 & 1.8 & 4.7 & -7.0 & -8.8 & 2.8 & 5.4 & -7.3 & 3.2 & -0.45 & -1.76 & 427 & 0.153 \\
\hline 30 & -8.8 & -4.6 & 2.5 & 3.0 & -5.5 & -8.0 & 1.8 & 4.8 & -7.1 & -8.9 & 2.8 & 5.4 & $\begin{array}{l}-7.4 \\
\end{array}$ & 3.3 & -0.45 & -1.76 & 432 & 0.153 \\
\hline 31 & -8.9 & -4.8 & 2.6 & 3.1 & -5.6 & -8.1 & 1.9 & 4.8 & -7.1 & -8.9 & 2.8 & 5.5 & -7.4 & 3.3 & -0.45 & -1.76 & 436 & 0.154 \\
\hline 32 & -9.0 & -4.6 & 2.6 & 3.1 & -5.6 & -8.2 & 1.9 & 4.9 & -7.2 & -9.1 & 2.8 & 5.6 & -7.5 & 3.4 & -0.45 & -1.75 & 440 & 0.155 \\
\hline 33 & -9.0 & -4.8 & 2.6 & 3.2 & -5.7 & -8.3 & 1.9 & 4.9 & -7.5 & -9.4 & 2.9 & 5.6 & -7.6 & 3.4 & -0.44 & -1.76 & 447 & 0.151 \\
\hline
\end{tabular}


Table E.23 NU 58-40 Test Data $\left(-10{ }^{\circ} \mathrm{C}\right.$ Tests $)$, continued

\begin{tabular}{|c|c|c|c|c|c|c|c|c|c|c|c|c|c|c|c|c|c|c|}
\hline & \multicolumn{12}{|c|}{ Normalized Vertical and Horizontal Deformation Arrays } & & & & & & \\
\hline & \multicolumn{4}{|c|}{ NU 58-40 Sample \#11 } & \multicolumn{4}{|c|}{ NU 58-40 Sample \#12 } & \multicolumn{4}{|c|}{ NU 58-40 Sample \#9 } & \multicolumn{2}{|c|}{ Trimmed } & & & Creep & Pois. \\
\hline & vert & vert & horz & horz & vert & vert & horz & horz & vert & vert & horz & horz & & & Ratio & & Comp & Ratio \\
\hline Time & front & back & front & back & front & back & front & back & front & back & front & back & vert & horz & $\mathrm{X} / \mathrm{Y}$ & $\mathrm{C}_{\mathrm{empl}}$ & $\mathrm{D}(\mathrm{t})$ & $\bar{v}$ \\
\hline $\mathrm{sec}$ & $\mu \mathrm{m}$ & $\mu \mathrm{m}$ & $\mu \mathrm{m}$ & $\mu \mathrm{m}$ & $\mu \mathrm{m}$ & $\mu \mathrm{m}$ & $\mu \mathrm{m}$ & $\mu \mathrm{m}$ & $\mu \mathrm{m}$ & $\mu \mathrm{m}$ & $\mu \mathrm{m}$ & $\mu \mathrm{m}$ & $\mu \mathrm{m}$ & $\mu \mathrm{m}$ & & & $1 / \mathrm{kPa}$ & \\
\hline 34 & -9.1 & -4.8 & 2.6 & 3.2 & -5.9 & -8.9 & 1.9 & 4.9 & -7.4 & -9.4 & 2.9 & 5.7 & -7.8 & 3.4 & -0.44 & -1.78 & 456 & 0.144 \\
\hline 35 & -9.2 & -5.0 & 2.7 & 3.2 & -5.8 & -8.6 & 1.9 & 5.0 & -7.3 & -9.4 & 2.9 & 5.8 & -7.7 & 3.5 & -0.45 & -1.76 & 454 & 0.154 \\
\hline 36 & -9.2 & -4.8 & 2.7 & 3.3 & -5.8 & -8.7 & 1.9 & 5.0 & -7.6 & -9.5 & 3.0 & 5.8 & $\begin{array}{l}-7.8 \\
\end{array}$ & 3.5 & -0.44 & -1.76 & 459 & 0.152 \\
\hline 37 & -9.3 & -4.9 & 2.7 & 3.3 & -6.1 & -8.9 & 2.0 & 5.0 & -7.6 & -9.8 & 3.0 & 5.8 & -8.0 & 3.5 & -0.44 & -1.77 & 467 & 0.148 \\
\hline 38 & -9.3 & -4.9 & 2.7 & 3.4 & -6.0 & -8.8 & 2.0 & 5.1 & -7.6 & $\begin{array}{l}-9.7 \\
\end{array}$ & 3.0 & 5.9 & $\begin{array}{l}-7.9 \\
\end{array}$ & 3.6 & -0.45 & -1.74 & 467 & 0.158 \\
\hline 39 & -9.4 & -4.9 & 2.8 & 3.4 & -6.4 & -9.1 & 2.0 & 5.1 & -7.6 & -9.8 & 3.0 & 6.0 & -8.1 & 3.6 & -0.44 & -1.78 & 476 & 0.147 \\
\hline 40 & -9.3 & -4.9 & 2.8 & 3.4 & -6.3 & -8.9 & 2.0 & 5.2 & -7.8 & -9.9 & 3.1 & 6.1 & -8.1 & 3.6 & -0.45 & -1.75 & 475 & 0.157 \\
\hline 41 & -9.5 & -4.9 & 2.8 & 3.5 & -6.3 & -9.0 & 2.0 & 5.2 & -7.8 & -10.0 & 3.1 & 6.1 & -8.1 & 3.7 & -0.45 & -1.75 & 479 & 0.157 \\
\hline 42 & -9.6 & -5.1 & 2.8 & 3.5 & -6.4 & -9.2 & 2.0 & 5.2 & -7.9 & -10.1 & 3.1 & 6.1 & -8.2 & 3.7 & -0.45 & -1.76 & 484 & 0.154 \\
\hline 43 & -9.7 & -5.1 & 2.9 & 3.5 & -6.4 & -9.2 & 2.1 & 5.3 & -7.9 & -10.3 & 3.1 & 6.2 & -8.3 & 3.7 & -0.45 & -1.76 & 487 & 0.154 \\
\hline 44 & -9.7 & -5.2 & 2.9 & 3.5 & -6.4 & -9.4 & 2.1 & 5.3 & -8.0 & -10.3 & 3.2 & 6.2 & -8.4 & 3.7 & -0.45 & -1.76 & 492 & 0.153 \\
\hline 45 & -9.7 & -5.1 & 2.9 & 3.6 & -6.6 & -9.3 & 2.1 & 5.3 & -8.1 & -10.3 & 3.2 & 6.3 & -8.4 & 3.8 & -0.45 & -1.76 & 494 & 0.154 \\
\hline 46 & -9.8 & -5.2 & 2.9 & 3.6 & -6.6 & -9.5 & 2.1 & 5.4 & -8.1 & -10.4 & 3.2 & 6.3 & -8.5 & 3.8 & -0.45 & -1.75 & 499 & 0.155 \\
\hline 47 & -9.9 & -5.2 & 3.0 & 3.6 & -6.6 & -9.6 & 2.1 & 5.4 & -8.3 & -10.7 & 3.2 & 6.3 & -8.6 & 3.8 & -0.44 & -1.77 & 504 & 0.150 \\
\hline 48 & -9.9 & -5.3 & 3.0 & 3.7 & -6.8 & -9.8 & 2.1 & 5.4 & -8.1 & -10.5 & 3.3 & 6.4 & -8.6 & 3.8 & -0.44 & -1.76 & 506 & 0.151 \\
\hline 49 & -10.0 & -5.3 & 3.0 & 3.7 & -6.7 & -9.8 & 2.2 & 5.5 & -8.2 & -10.7 & 3.3 & 6.5 & -8.7 & 3.9 & -0.45 & -1.76 & 509 & 0.154 \\
\hline 50 & \begin{tabular}{|l|}
-10.1 \\
\end{tabular} & -5.4 & 3.0 & 3.7 & -6.8 & -9.8 & 2.2 & 5.5 & -8.3 & -10.8 & 3.3 & 6.5 & -8.7 & 3.9 & -0.44 & -1.76 & 513 & 0.152 \\
\hline 51 & \begin{tabular}{|l|}
-10.1 \\
\end{tabular} & -5.4 & 3.0 & 3.7 & -6.8 & -9.9 & 2.2 & 5.5 & -8.3 & -10.7 & 3.4 & 6.6 & -8.8 & 3.9 & -0.44 & -1.76 & 516 & 0.153 \\
\hline 52 & -10.1 & -5.4 & 3.0 & 3.8 & -6.9 & -9.9 & 2.2 & 5.6 & -8.4 & -10.9 & 3.4 & 6.6 & -8.8 & 3.9 & -0.45 & -1.75 & 519 & 0.154 \\
\hline 53 & -10.4 & -5.6 & 3.0 & 3.8 & -6.9 & -10.1 & 2.2 & 5.6 & -8.4 & -10.9 & 3.4 & 6.7 & -9.0 & 3.9 & -0.44 & -1.77 & 525 & 0.148 \\
\hline 54 & -10.3 & -5.5 & 3.1 & 3.8 & -7.0 & -10.1 & 2.2 & 5.7 & -8.4 & -10.9 & 3.4 & 6.8 & -9.0 & 4.0 & -0.45 & -1.76 & 527 & 0.154 \\
\hline 55 & -10.5 & -5.6 & 3.1 & 3.8 & -7.1 & -10.2 & 2.2 & 5.7 & -8.6 & -11.1 & 3.4 & 6.8 & -9.1 & 4.0 & -0.44 & -1.78 & 534 & 0.145 \\
\hline 56 & -10.4 & -5.6 & 3.1 & 3.9 & -7.1 & -10.2 & 2.3 & 5.7 & -8.6 & -11.2 & 3.4 & 6.8 & -9.1 & 4.0 & -0.44 & -1.76 & 534 & 0.151 \\
\hline 57 & -10.5 & -5.7 & 3.2 & 3.9 & $\begin{array}{l}-7.1 \\
\end{array}$ & -10.2 & 2.3 & 5.8 & $\begin{array}{l}-8.7 \\
\end{array}$ & -11.2 & 3.5 & 6.8 & -9.1 & 4.1 & -0.45 & -1.76 & 536 & 0.153 \\
\hline 58 & -10.5 & -5.7 & 3.2 & 3.9 & -7.1 & -10.3 & 2.3 & 5.8 & -8.6 & -11.2 & 3.5 & 6.9 & -9.1 & 4.1 & -0.45 & -1.75 & 537 & 0.158 \\
\hline 59 & -10.7 & -5.7 & 3.2 & 3.9 & -7.1 & -10.1 & 2.3 & 5.9 & -8.7 & -11.3 & 3.5 & 7.0 & -9.2 & 4.1 & -0.45 & -1.74 & 539 & 0.158 \\
\hline 60 & -10.7 & -5.7 & 3.2 & 4.0 & -7.1 & -10.3 & 2.3 & 5.9 & -8.7 & -11.4 & 3.5 & 7.0 & -9.2 & 4.1 & -0.45 & -1.74 & 542 & 0.158 \\
\hline 61 & \begin{tabular}{|l|l}
-10.8 \\
\end{tabular} & -5.9 & 3.2 & 4.0 & $\begin{array}{l}-7.1 \\
\end{array}$ & -10.2 & 2.4 & 6.0 & -8.6 & -11.4 & 3.6 & $\begin{array}{l}7.1 \\
\end{array}$ & -9.2 & 4.2 & -0.46 & -1.73 & 541 & 0.165 \\
\hline 62 & -10.8 & -5.9 & 3.2 & 4.0 & -7.1 & -10.5 & 2.4 & 6.0 & -8.8 & -11.5 & 3.6 & 7.1 & -9.3 & 4.2 & -0.45 & -1.74 & 548 & 0.160 \\
\hline 63 & -11.0 & -6.0 & 3.2 & 4.0 & -7.2 & -10.5 & 2.4 & 6.0 & -8.8 & -11.5 & 3.6 & 7.2 & -9.4 & 4.2 & -0.45 & -1.75 & 551 & 0.157 \\
\hline 64 & -11.0 & -6.0 & 3.3 & 4.1 & -7.3 & -10.5 & 2.4 & 6.0 & -8.9 & -11.6 & 3.6 & 7.2 & -9.4 & 4.2 & -0.45 & -1.74 & 554 & 0.160 \\
\hline 65 & -11.0 & -6.1 & 3.3 & 4.1 & -7.3 & -10.6 & 2.4 & 6.0 & -8.9 & -11.7 & 3.7 & 7.2 & -9.5 & 4.3 & -0.45 & -1.74 & 556 & 0.160 \\
\hline 66 & -11.1 & -6.1 & 3.3 & 4.1 & -7.4 & -10.7 & 2.4 & 6.1 & -9.0 & -11.7 & 3.7 & 7.2 & -9.6 & 4.3 & -0.45 & -1.75 & 562 & 0.156 \\
\hline
\end{tabular}


Table E.23 NU 58-40 Test Data $\left(-10{ }^{\circ} \mathrm{C}\right.$ Tests $)$, continued

\begin{tabular}{|c|c|c|c|c|c|c|c|c|c|c|c|c|c|c|c|c|c|c|}
\hline & \multicolumn{12}{|c|}{ Normalized Vertical and Horizontal Deformation Arrays } & & & & & & \\
\hline & \multicolumn{4}{|c|}{ NU 58-40 Sample \#11 } & \multicolumn{4}{|c|}{ NU 58-40 Sample \#12 } & \multicolumn{4}{|c|}{ NU 58-40 Sample \#9 } & \multicolumn{2}{|c|}{ Trimmed } & & & Creep & Pois. \\
\hline & vert & vert & horz & horz & vert & vert & horz & horz & vert & vert & horz & horz & & & Ratio & & Comp & Ratio \\
\hline Time & front & back & front & back & front & back & front & back & front & back & front & back & vert & horz & $\mathrm{X} / \mathrm{Y}$ & $\mathrm{C}_{\mathrm{empl}}$ & $\mathrm{D}(\mathrm{t})$ & $\bar{v}$ \\
\hline $\mathrm{sec}$ & $\mu \mathrm{m}$ & $\mu \mathrm{m}$ & $\mu \mathrm{m}$ & $\mu \mathrm{m}$ & $\mu \mathrm{m}$ & $\mu \mathrm{m}$ & $\mu \mathrm{m}$ & $\mu \mathrm{m}$ & $\mu \mathrm{m}$ & $\mu \mathrm{m}$ & $\mu \mathrm{m}$ & $\mu \mathrm{m}$ & $\mu \mathrm{m}$ & $\mu \mathrm{m}$ & & & $1 / \mathrm{kPa}$ & \\
\hline 67 & -11.2 & -6.2 & 3.3 & 4.1 & -7.4 & -10.7 & 2.4 & 6.1 & -9.0 & -11.8 & 3.7 & 7.3 & -9.6 & 4.3 & -0.45 & -1.75 & 564 & 0.158 \\
\hline 68 & -11.1 & -6.2 & 3.3 & 4.2 & -7.7 & -11.0 & 2.4 & 6.1 & -8.9 & -11.8 & 3.7 & 7.3 & -9.7 & 4.3 & -0.45 & -1.76 & 569 & 0.154 \\
\hline 69 & -11.3 & -6.2 & 3.3 & 4.2 & $\begin{array}{l}-7.4 \\
\end{array}$ & -10.8 & 2.5 & 6.2 & -9.1 & -11.9 & 3.7 & 7.4 & -9.7 & 4.4 & -0.45 & $\begin{array}{l}-1.74 \\
\end{array}$ & 568 & 0.159 \\
\hline 70 & -11.4 & -6.3 & 3.3 & 4.2 & -7.5 & -10.9 & 2.5 & 6.2 & -9.1 & -12.0 & 3.7 & 7.4 & -9.7 & 4.4 & -0.45 & -1.74 & 572 & 0.159 \\
\hline 71 & -11.5 & -6.3 & 3.4 & 4.2 & -7.4 & -10.9 & 2.5 & 6.3 & -9.1 & -11.9 & 3.8 & 7.5 & -9.7 & 4.4 & -0.45 & -1.73 & 573 & 0.162 \\
\hline 72 & -11.6 & -6.4 & 3.4 & 4.2 & -7.5 & -10.9 & 2.5 & 6.3 & -9.1 & -12.1 & 3.8 & 7.5 & -9.8 & 4.4 & -0.45 & -1.73 & 575 & 0.162 \\
\hline 73 & -11.6 & -6.5 & 3.4 & 4.3 & -7.6 & -10.9 & 2.5 & 6.3 & -9.1 & -12.1 & 3.8 & 7.5 & -9.8 & 4.5 & -0.45 & -1.73 & 578 & 0.163 \\
\hline 74 & -11.6 & -6.5 & 3.4 & 4.2 & -7.6 & -11.0 & 2.5 & 6.3 & -9.2 & -12.2 & 3.8 & 7.6 & -9.9 & 4.5 & -0.45 & -1.74 & 581 & 0.161 \\
\hline 75 & -11.8 & -6.6 & 3.4 & 4.3 & -7.6 & -11.0 & 2.6 & 6.4 & -9.1 & -12.2 & 3.8 & 7.6 & -9.9 & 4.5 & -0.45 & -1.73 & 583 & 0.162 \\
\hline 76 & -11.8 & -6.5 & 3.4 & 4.3 & -7.6 & -10.9 & 2.6 & 6.4 & -9.2 & -12.2 & 3.9 & 7.6 & -9.9 & 4.5 & -0.46 & -1.73 & 583 & 0.164 \\
\hline 77 & -11.9 & -6.7 & 3.5 & 4.3 & -7.8 & -11.3 & 2.6 & 6.4 & -9.2 & -12.4 & 3.9 & 7.7 & -10.1 & 4.5 & -0.45 & -1.75 & 592 & 0.157 \\
\hline 78 & -11.9 & -6.7 & 3.5 & 4.3 & -7.7 & -11.1 & 2.6 & 6.5 & -9.4 & -12.5 & 3.9 & 7.7 & -10.1 & 4.5 & -0.45 & -1.74 & 592 & 0.161 \\
\hline 79 & -12.1 & -6.8 & 3.5 & 4.3 & -7.6 & -11.1 & 2.6 & 6.6 & -9.4 & -12.4 & 3.9 & 7.8 & -10.0 & 4.6 & -0.46 & -1.73 & 592 & 0.165 \\
\hline 80 & -12.1 & -6.8 & 3.5 & 4.3 & -7.5 & -11.2 & 2.6 & 6.6 & -9.4 & -12.4 & 3.9 & 7.8 & -10.0 & 4.6 & -0.46 & -1.72 & 593 & 0.167 \\
\hline 81 & -12.2 & -6.8 & 3.5 & 4.4 & -7.5 & -11.1 & 2.7 & 6.6 & -9.4 & -12.5 & 4.0 & 7.8 & -10.1 & 4.6 & -0.46 & -1.71 & 594 & 0.170 \\
\hline 82 & -12.2 & -7.0 & 3.5 & 4.4 & -7.6 & -11.1 & 2.7 & 6.7 & -9.5 & -12.6 & 4.0 & 7.9 & -10.1 & 4.6 & -0.46 & -1.71 & 596 & 0.170 \\
\hline 83 & -12.3 & -7.0 & 3.5 & 4.4 & -7.6 & -11.2 & 2.7 & 6.7 & -9.5 & -12.6 & 4.0 & 7.9 & -10.1 & 4.6 & -0.46 & -1.72 & 598 & 0.168 \\
\hline 84 & -12.3 & -7.0 & 3.5 & 4.4 & -7.6 & -11.2 & 2.7 & 6.7 & -9.5 & -12.7 & 4.0 & 8.0 & -10.2 & 4.7 & -0.46 & -1.71 & 600 & 0.170 \\
\hline 85 & -12.4 & -7.1 & 3.6 & 4.4 & -7.6 & -11.2 & 2.7 & 6.8 & -9.6 & -12.7 & 4.0 & 8.0 & -10.2 & 4.7 & -0.46 & -1.71 & 602 & 0.171 \\
\hline 86 & -12.4 & -7.0 & 3.6 & 4.5 & -7.6 & -11.1 & 2.7 & 6.8 & -9.5 & -12.8 & 4.0 & 8.0 & -10.1 & 4.7 & -0.47 & -1.69 & 601 & 0.178 \\
\hline 87 & -12.4 & -7.1 & 3.6 & 4.5 & -7.6 & -11.2 & 2.8 & 6.8 & -9.7 & -12.7 & 4.1 & 8.1 & -10.2 & 4.8 & -0.47 & -1.69 & 604 & 0.177 \\
\hline 88 & -12.5 & -7.1 & 3.6 & 4.5 & -7.7 & -11.2 & 2.8 & 6.9 & -9.7 & -12.9 & 4.1 & 8.1 & -10.3 & 4.8 & -0.46 & -1.70 & 608 & 0.175 \\
\hline 89 & -12.6 & -7.1 & 3.6 & 4.5 & -7.7 & -11.3 & 2.8 & 6.9 & -9.7 & -12.9 & 4.1 & 8.1 & -10.3 & 4.8 & -0.46 & -1.71 & 611 & 0.172 \\
\hline 90 & -12.6 & -7.2 & 3.7 & 4.6 & -7.7 & -11.2 & 2.8 & 6.9 & -9.7 & -13.2 & 4.1 & 8.1 & -10.3 & 4.8 & -0.47 & -1.69 & 611 & 0.179 \\
\hline 91 & -12.7 & -7.2 & 3.7 & 4.6 & -7.7 & -11.3 & 2.8 & 7.0 & -9.8 & -13.2 & 4.1 & 8.2 & -10.4 & 4.8 & -0.47 & -1.70 & 614 & 0.177 \\
\hline 92 & -12.7 & -7.2 & 3.7 & 4.6 & -7.7 & -11.2 & 2.8 & 7.0 & -9.8 & -13.2 & 4.1 & 8.2 & -10.3 & 4.9 & -0.47 & -1.68 & 613 & 0.183 \\
\hline 93 & -12.7 & -7.3 & 3.7 & 4.6 & -7.7 & -11.3 & 2.9 & 7.0 & -9.8 & -13.2 & 4.2 & 8.3 & -10.4 & 4.9 & -0.47 & -1.69 & 616 & 0.181 \\
\hline 94 & -12.7 & -7.3 & 3.7 & 4.7 & -7.7 & -11.4 & 2.8 & 7.1 & -9.9 & -13.3 & 4.2 & 8.3 & -10.5 & 4.9 & -0.47 & -1.69 & 620 & 0.181 \\
\hline 95 & -12.8 & -7.3 & 3.7 & 4.7 & -7.7 & -11.5 & 2.9 & 7.1 & -9.9 & -13.3 & 4.2 & 8.3 & -10.5 & 4.9 & -0.47 & -1.68 & 622 & 0.182 \\
\hline 96 & -12.7 & -7.2 & 3.7 & 4.7 & -7.7 & -11.5 & 2.9 & 7.1 & -9.9 & -13.4 & 4.2 & 8.4 & -10.5 & 4.9 & -0.47 & -1.68 & 622 & 0.184 \\
\hline 97 & -12.9 & -7.2 & 3.8 & 4.7 & -7.9 & -11.4 & 2.9 & 7.1 & -9.9 & -13.4 & 4.2 & 8.4 & -10.5 & 5.0 & -0.47 & -1.68 & 624 & 0.184 \\
\hline 98 & -12.9 & -7.3 & 3.8 & 4.8 & -7.8 & -11.5 & 2.9 & 7.1 & -9.9 & -13.4 & 4.2 & 8.4 & -10.5 & 5.0 & -0.47 & -1.68 & 625 & 0.185 \\
\hline 99 & -12.9 & -7.4 & 3.8 & 4.8 & -7.7 & -11.5 & 2.9 & 7.2 & -9.9 & -13.5 & 4.3 & 8.5 & -10.5 & 5.0 & -0.47 & -1.67 & 626 & 0.187 \\
\hline 100 & -12.9 & -7.4 & 3.8 & 4.8 & -7.9 & -11.5 & 3.0 & 7.2 & -10.0 & -13.5 & 4.3 & 8.5 & -10.6 & 5.0 & -0.47 & -1.67 & 628 & 0.186 \\
\hline
\end{tabular}


Table E.24 NU 58-40 Test Data $\left(-20{ }^{\circ} \mathrm{C}\right.$ Tests)

\begin{tabular}{|c|c|c|c|c|c|c|c|c|c|c|c|c|c|c|c|c|c|c|}
\hline & \multicolumn{12}{|c|}{ Normalized Vertical and Horizontal Deformation Arrays } & \multirow{2}{*}{\multicolumn{2}{|c|}{ Trimmed }} & & & & \\
\hline & $\mathrm{NU}$ & $58-40$ & Sample & \#11 & $\mathrm{NU}$ & $58-40$ & ample & $\# 12$ & & $58-40$ & Sampl & & & & & & Creep & Pois. \\
\hline & vert & vert & horz & horz & vert & vert & horz & horz & vert & vert & horz & horz & & & Ratio & & Comp & Ratio \\
\hline Time & front & back & front & back & front & back & front & back & front & back & front & back & vert & horz & $\mathrm{X} / \mathrm{Y}$ & $\mathrm{C}_{\mathrm{empl}}$ & $\mathrm{D}(\mathrm{t})$ & $\bar{v}$ \\
\hline $\mathrm{sec}$ & $\mu \mathrm{m}$ & $\mu \mathrm{m}$ & $\mu \mathrm{m}$ & $\mu \mathrm{m}$ & $\mu \mathrm{m}$ & $\mu \mathrm{m}$ & $\mu \mathrm{m}$ & $\mu \mathrm{m}$ & $\mu \mathrm{m}$ & $\mu \mathrm{m}$ & $\mu \mathrm{m}$ & $\mu \mathrm{m}$ & $\mu \mathrm{m}$ & $\mu \mathrm{m}$ & & & $1 / \mathrm{kPa}$ & \\
\hline 0 & 0.0 & 0.0 & 0.0 & 0.0 & 0.0 & 0.0 & 0.0 & 0.0 & 0.0 & 0.0 & 0.0 & 0.0 & 0.0 & 0.0 & 0.00 & 0.00 & 0 & 0.000 \\
\hline 1 & -3.2 & -1.6 & 0.7 & 0.9 & -2.2 & -4.5 & 0.6 & 2.0 & -2.7 & -3.9 & 0.7 & 1.8 & -3.3 & 1.0 & -0.31 & -2.39 & 91 & 0.022 \\
\hline 2 & -4.6 & -2.5 & 1.1 & 1.4 & -2.4 & -5.0 & 0.8 & 2.3 & -2.9 & -4.3 & 0.9 & 2.1 & -3.7 & 1.4 & -0.38 & -2.01 & 104 & 0.082 \\
\hline 3 & -4.9 & -2.7 & 1.2 & 1.5 & -2.7 & -5.2 & 0.8 & 2.5 & -3.0 & -4.5 & 1.0 & 2.3 & -3.9 & 1.5 & -0.39 & -1.97 & 110 & 0.093 \\
\hline 4 & -5.2 & -2.8 & 1.3 & 1.6 & -2.7 & -5.4 & 0.9 & 2.7 & -3.3 & -4.8 & 1.0 & 2.4 & -4.0 & 1.6 & \begin{tabular}{|l|}
-0.39 \\
\end{tabular} & -1.94 & 115 & 0.099 \\
\hline 5 & -5.2 & -2.8 & 1.4 & 1.7 & -2.8 & -5.5 & 0.9 & 2.7 & -3.3 & -4.9 & 1.1 & 2.6 & -4.2 & 1.7 & -0.41 & -1.90 & 119 & 0.110 \\
\hline 6 & -5.5 & -3.1 & 1.4 & 1.7 & -3.0 & -5.8 & 0.9 & 2.8 & -3.4 & -5.1 & 1.1 & 2.7 & -4.3 & 1.7 & -0.40 & -1.91 & 124 & 0.106 \\
\hline 7 & -5.6 & -3.1 & 1.4 & 1.8 & -3.0 & -5.8 & 1.0 & 2.9 & -3.5 & -5.2 & 1.2 & 2.7 & -4.4 & 1.8 & -0.41 & -1.89 & 125 & 0.113 \\
\hline 8 & -6.0 & -3.2 & 1.5 & 1.8 & -3.1 & -5.9 & 1.0 & 2.9 & -3.5 & -5.4 & 1.2 & 2.8 & -4.5 & 1.8 & -0.41 & -1.89 & 129 & 0.111 \\
\hline 9 & -6.0 & -3.2 & 1.5 & 1.9 & -3.2 & -6.0 & 1.0 & 3.0 & -3.5 & -5.4 & 1.2 & 2.9 & -4.5 & 1.9 & -0.41 & -1.87 & 131 & 0.119 \\
\hline 10 & -6.1 & -3.3 & 1.6 & 1.9 & -3.3 & -6.2 & 1.0 & 3.0 & -3.5 & -5.5 & 1.3 & 2.9 & -4.6 & 1.9 & -0.41 & -1.87 & 133 & 0.118 \\
\hline 11 & -6.2 & -3.3 & 1.6 & 2.0 & -3.4 & -6.3 & 1.0 & 3.1 & -3.6 & -5.5 & 1.3 & 3.0 & -4.7 & 2.0 & -0.42 & -1.86 & 136 & 0.120 \\
\hline 12 & -6.2 & -3.4 & 1.6 & 2.0 & -3.4 & -6.2 & 1.1 & 3.2 & -3.8 & -5.5 & 1.3 & 3.0 & -4.7 & 2.0 & -0.42 & -1.84 & 137 & 0.128 \\
\hline 13 & -6.4 & -3.4 & 1.6 & 2.0 & -3.5 & -6.4 & 1.1 & 3.2 & -3.8 & -5.5 & 1.3 & 3.1 & -4.8 & 2.0 & -0.42 & -1.83 & 139 & 0.128 \\
\hline 14 & -6.5 & -3.5 & 1.7 & 2.1 & -3.5 & -6.4 & 1.1 & 3.3 & -3.8 & -5.7 & 1.3 & 3.2 & -4.8 & 2.1 & -0.43 & -1.82 & 140 & 0.131 \\
\hline 15 & -6.6 & -3.5 & 1.7 & 2.1 & -3.7 & -6.4 & 1.1 & 3.3 & -3.8 & -5.7 & 1.4 & 3.2 & -4.9 & 2.1 & -0.43 & -1.81 & 142 & 0.136 \\
\hline 16 & -6.6 & -3.6 & 1.7 & 2.1 & -3.6 & -6.5 & 1.2 & 3.4 & -3.7 & -5.7 & 1.4 & 3.3 & -4.9 & 2.1 & -0.44 & -1.79 & 142 & 0.142 \\
\hline 17 & -6.7 & -3.5 & 1.8 & 2.2 & -3.6 & -6.6 & 1.1 & 3.4 & -3.8 & -5.7 & 1.4 & 3.3 & -4.9 & 2.2 & -0.44 & -1.77 & 143 & 0.148 \\
\hline 18 & -6.9 & -3.7 & 1.8 & 2.2 & -3.7 & -6.6 & 1.2 & 3.4 & -3.8 & -5.9 & 1.4 & 3.4 & -5.0 & 2.2 & -0.44 & -1.78 & 145 & 0.145 \\
\hline 19 & -6.9 & -3.6 & 1.8 & 2.2 & -3.7 & -6.7 & 1.2 & 3.5 & -3.8 & -5.9 & 1.4 & 3.4 & -5.0 & 2.2 & -0.44 & -1.77 & 146 & 0.150 \\
\hline 20 & -6.9 & -3.6 & 1.8 & 2.3 & -3.9 & -6.8 & 1.2 & 3.5 & -3.8 & -5.9 & 1.5 & 3.4 & -5.1 & 2.2 & -0.44 & -1.77 & 148 & 0.148 \\
\hline 21 & -7.0 & -3.8 & 1.8 & 2.3 & -3.9 & -6.8 & 1.2 & 3.6 & -3.8 & -6.0 & 1.5 & 3.5 & -5.1 & 2.3 & -0.45 & -1.75 & 148 & 0.155 \\
\hline 22 & -7.0 & -3.8 & 1.8 & 2.3 & -3.9 & -6.6 & 1.2 & 3.6 & -3.8 & -6.0 & 1.5 & 3.5 & -5.1 & 2.3 & -0.45 & -1.74 & 149 & 0.159 \\
\hline 23 & -7.0 & -3.8 & 1.9 & 2.3 & -3.9 & -6.9 & 1.2 & 3.7 & -3.9 & -6.2 & 1.5 & 3.5 & -5.2 & 2.3 & -0.45 & -1.76 & 152 & 0.153 \\
\hline 24 & -7.1 & -3.8 & 1.9 & 2.4 & -4.1 & -7.2 & 1.2 & 3.6 & -3.9 & -6.1 & 1.6 & 3.6 & -5.3 & 2.4 & -0.44 & -1.76 & 155 & 0.151 \\
\hline 25 & -7.2 & -4.0 & 1.9 & 2.4 & -4.0 & -7.1 & 1.2 & 3.7 & -3.9 & -6.2 & 1.6 & 3.7 & -5.3 & 2.4 & -0.45 & -1.75 & 155 & 0.157 \\
\hline 26 & -7.3 & -3.9 & 1.9 & 2.4 & -4.0 & -7.1 & 1.3 & 3.7 & -3.9 & -6.2 & 1.6 & 3.7 & -5.3 & 2.4 & -0.45 & -1.74 & 156 & 0.160 \\
\hline 27 & -7.3 & -4.0 & 1.9 & 2.4 & -4.0 & -7.3 & 1.3 & 3.7 & -3.8 & -6.2 & 1.6 & 3.8 & -5.3 & 2.4 & -0.45 & -1.73 & 156 & 0.162 \\
\hline 28 & -7.3 & -4.0 & 2.0 & 2.5 & -4.0 & -7.3 & 1.3 & 3.8 & -3.9 & -6.2 & 1.6 & 3.8 & -5.4 & 2.4 & -0.46 & -1.72 & 157 & 0.166 \\
\hline 29 & -7.5 & -4.0 & 2.0 & 2.4 & -4.0 & -7.3 & 1.3 & 3.8 & -4.0 & -6.4 & 1.6 & 3.8 & -5.4 & 2.4 & -0.45 & -1.74 & 159 & 0.159 \\
\hline 30 & -7.5 & -4.1 & 2.0 & 2.5 & -4.2 & -7.4 & 1.3 & 3.8 & -4.0 & -6.3 & 1.6 & 3.8 & -5.5 & 2.5 & -0.46 & -1.73 & 160 & 0.165 \\
\hline 31 & -7.5 & -4.0 & 2.0 & 2.5 & -4.2 & -7.4 & 1.3 & 3.9 & -4.0 & -6.4 & 1.6 & 3.8 & -5.5 & 2.5 & -0.45 & -1.73 & 161 & 0.164 \\
\hline 32 & -7.6 & -4.2 & 2.0 & 2.5 & -4.1 & -7.3 & 1.3 & 3.9 & -4.0 & -6.5 & 1.6 & 3.9 & -5.5 & 2.5 & -0.46 & -1.72 & 160 & 0.167 \\
\hline 33 & -7.6 & -4.1 & 2.0 & 2.5 & -4.2 & -7.5 & 1.3 & 3.9 & -4.0 & -6.5 & 1.7 & 3.9 & -5.5 & 2.5 & -0.46 & -1.72 & 162 & 0.166 \\
\hline
\end{tabular}


Table E.24 NU 58-40 Test Data $\left(-20{ }^{\circ} \mathrm{C}\right.$ Tests), continued

\begin{tabular}{|c|c|c|c|c|c|c|c|c|c|c|c|c|c|c|c|c|c|c|}
\hline & \multicolumn{12}{|c|}{ Normalized Vertical and Horizontal Deformation Arrays } & & & & & & \\
\hline & \multicolumn{4}{|c|}{ NU 58-40 Sample \#11 } & \multicolumn{4}{|c|}{ NU 58-40 Sample \#12 } & \multicolumn{4}{|c|}{ NU 58-40 Sample \#9 } & \multicolumn{2}{|c|}{ Trimmed } & & & Creep & Pois. \\
\hline & vert & vert & horz & horz & vert & vert & horz & horz & vert & vert & horz & horz & & & Ratio & & Comp & Ratio \\
\hline Time & front & back & front & back & front & back & front & back & front & back & front & back & vert & horz & $\mathrm{X} / \mathrm{Y}$ & $\mathrm{C}_{\mathrm{empl}}$ & $\mathrm{D}(\mathrm{t})$ & $v$ \\
\hline $\mathrm{sec}$ & $\mu \mathrm{m}$ & $\mu \mathrm{m}$ & $\mu \mathrm{m}$ & $\mu \mathrm{m}$ & $\mu \mathrm{m}$ & $\mu \mathrm{m}$ & $\mu \mathrm{m}$ & $\mu \mathrm{m}$ & $\mu \mathrm{m}$ & $\mu \mathrm{m}$ & $\mu \mathrm{m}$ & $\mu \mathrm{m}$ & $\mu \mathrm{m}$ & $\mu \mathrm{m}$ & & & $1 / \mathrm{kPa}$ & \\
\hline 34 & -7.6 & -4.1 & 2.0 & 2.6 & -4.3 & -7.6 & 1.3 & 3.9 & -4.0 & -6.6 & 1.7 & 3.9 & -5.6 & 2.6 & -0.45 & -1.73 & 165 & 0.163 \\
\hline 35 & -7.6 & -4.1 & 2.1 & 2.6 & -4.2 & -7.5 & 1.4 & 4.0 & -4.1 & -6.6 & 1.7 & 3.9 & -5.6 & 2.6 & -0.46 & -1.73 & 164 & 0.165 \\
\hline 36 & -7.7 & -4.2 & 2.1 & 2.6 & -4.1 & -7.6 & 1.4 & 4.0 & -4.1 & -6.7 & 1.7 & 4.0 & -5.6 & 2.6 & -0.46 & -1.72 & 165 & 0.168 \\
\hline 37 & -7.7 & -4.2 & 2.1 & 2.6 & -4.4 & -7.6 & 1.4 & 4.0 & -4.0 & -6.6 & 1.7 & 4.0 & -5.7 & 2.6 & -0.46 & -1.72 & 166 & 0.169 \\
\hline 38 & -7.7 & -4.2 & 2.1 & 2.7 & -4.3 & -7.7 & 1.4 & 4.0 & -4.3 & -6.7 & 1.7 & 4.0 & -5.7 & 2.6 & -0.46 & -1.72 & 168 & 0.166 \\
\hline 39 & -7.8 & -4.2 & 2.1 & 2.7 & -4.4 & -7.6 & 1.4 & 4.0 & -4.3 & -6.7 & 1.7 & 4.0 & -5.8 & 2.6 & -0.46 & -1.72 & 169 & 0.166 \\
\hline 40 & -7.9 & -4.2 & 2.1 & 2.7 & -4.4 & -7.6 & 1.4 & 4.0 & -4.4 & -7.0 & 1.7 & 4.0 & -5.8 & 2.6 & -0.45 & -1.74 & 170 & 0.160 \\
\hline 41 & $\begin{array}{l}-7.8 \\
\end{array}$ & -4.3 & 2.1 & 2.7 & -4.4 & -7.7 & 1.4 & 4.1 & -4.5 & -7.0 & 1.7 & 4.0 & -5.9 & 2.6 & -0.45 & -1.75 & 171 & 0.158 \\
\hline 42 & -7.9 & -4.2 & 2.2 & 2.7 & -4.4 & -7.7 & 1.4 & 4.0 & -4.3 & -6.8 & 1.7 & 4.1 & -5.8 & 2.7 & -0.46 & -1.71 & 170 & 0.170 \\
\hline 43 & $\begin{array}{l}-7.9 \\
\end{array}$ & -4.3 & 2.2 & 2.7 & -4.5 & -7.8 & 1.4 & 4.1 & -4.3 & -7.0 & 1.8 & 4.1 & $\begin{array}{l}-5.9 \\
\end{array}$ & 2.7 & -0.46 & -1.73 & 173 & 0.164 \\
\hline 44 & -7.9 & -4.3 & 2.2 & 2.8 & -4.4 & -7.8 & 1.4 & 4.1 & -4.4 & -7.0 & 1.8 & 4.1 & -5.9 & 2.7 & -0.46 & -1.71 & 172 & 0.172 \\
\hline 45 & -8.0 & -4.3 & 2.2 & 2.8 & -4.4 & -7.8 & 1.4 & 4.1 & -4.3 & -7.0 & 1.8 & 4.1 & $\begin{array}{l}-5.9 \\
\end{array}$ & 2.7 & -0.46 & -1.70 & 173 & 0.173 \\
\hline 46 & -8.0 & -4.3 & 2.2 & 2.8 & -4.4 & -7.9 & 1.5 & 4.1 & -4.4 & -7.0 & 1.8 & 4.1 & -5.9 & 2.7 & -0.46 & -1.72 & 174 & 0.168 \\
\hline 47 & -8.0 & -4.4 & 2.2 & 2.8 & -4.5 & -8.0 & 1.5 & 4.2 & -4.4 & $\begin{array}{l}-7.1 \\
\end{array}$ & 1.8 & 4.2 & $\begin{array}{l}-6.0 \\
\end{array}$ & 2.7 & -0.46 & -1.73 & 176 & 0.165 \\
\hline 48 & -8.2 & -4.4 & 2.2 & 2.8 & -4.5 & -7.9 & 1.5 & 4.2 & -4.4 & -7.0 & 1.8 & 4.2 & -6.0 & 2.8 & -0.46 & -1.70 & 175 & 0.175 \\
\hline 49 & -8.2 & -4.4 & 2.2 & 2.8 & -4.5 & -7.9 & 1.5 & 4.2 & -4.6 & -7.2 & $\overline{1.8}$ & 4.2 & $\begin{array}{l}-6.0 \\
\end{array}$ & 2.8 & -0.46 & -1.71 & 177 & 0.170 \\
\hline 50 & -8.3 & -4.4 & 2.2 & 2.8 & -4.5 & -8.0 & 1.5 & 4.2 & -4.6 & -7.2 & 1.8 & 4.3 & -6.1 & 2.8 & -0.46 & -1.71 & 178 & 0.169 \\
\hline 51 & -8.2 & -4.5 & 2.3 & 2.8 & -4.7 & -8.1 & 1.5 & 4.2 & -4.5 & -7.2 & 1.8 & 4.3 & $\begin{array}{l}-6.1 \\
\end{array}$ & 2.8 & -0.46 & -1.73 & 180 & 0.164 \\
\hline 52 & -8.3 & -4.5 & 2.3 & 2.9 & -4.7 & -8.0 & 1.5 & 4.3 & -4.5 & -7.3 & 1.8 & 4.3 & -6.1 & 2.8 & -0.46 & -1.72 & 180 & 0.167 \\
\hline 53 & -8.3 & -4.4 & 2.3 & 2.9 & -4.6 & -8.0 & 1.5 & 4.3 & -4.7 & -7.3 & $\overline{1.9}$ & 4.3 & -6.2 & 2.8 & -0.46 & -1.72 & 180 & 0.166 \\
\hline 54 & -8.3 & -4.5 & 2.3 & 2.9 & -4.6 & -8.0 & 1.5 & 4.3 & -4.7 & -7.4 & 1.9 & 4.3 & -6.2 & 2.8 & -0.46 & -1.72 & 182 & 0.167 \\
\hline 55 & -8.4 & -4.5 & 2.3 & 2.9 & -4.5 & -8.0 & 1.5 & 4.4 & -4.7 & -7.4 & 1.9 & 4.3 & -6.2 & 2.8 & -0.46 & -1.71 & 181 & 0.171 \\
\hline 56 & -8.4 & -4.5 & 2.3 & 2.9 & -4.5 & -8.2 & 1.5 & 4.3 & -4.6 & -7.4 & 1.9 & 4.4 & -6.2 & 2.9 & -0.46 & -1.70 & 182 & 0.174 \\
\hline 57 & -8.4 & -4.5 & 2.3 & 2.9 & -4.7 & -8.1 & 1.5 & 4.4 & -4.7 & -7.5 & 1.9 & 4.4 & -6.3 & 2.9 & -0.46 & -1.71 & 183 & 0.170 \\
\hline 58 & -8.5 & -4.5 & 2.3 & 3.0 & -4.6 & -8.2 & 1.5 & 4.4 & -4.8 & -7.5 & 1.9 & 4.4 & -6.3 & 2.9 & -0.46 & -1.71 & 185 & 0.171 \\
\hline 59 & -8.5 & -4.6 & 2.3 & 3.0 & -4.7 & -8.2 & 1.5 & 4.4 & -4.7 & -7.5 & 1.9 & 4.4 & -6.3 & 2.9 & -0.46 & -1.70 & 184 & 0.174 \\
\hline 60 & -8.5 & -4.5 & 2.3 & 3.0 & -4.7 & -8.3 & 1.5 & 4.4 & -4.8 & -7.7 & 1.9 & 4.4 & -6.3 & 2.9 & -0.46 & -1.72 & 186 & 0.169 \\
\hline 61 & -8.7 & -4.6 & 2.4 & 3.0 & -4.7 & -8.3 & 1.5 & 4.4 & -4.7 & -7.6 & 1.9 & 4.4 & -6.3 & 2.9 & -0.46 & -1.70 & 186 & 0.175 \\
\hline 62 & -8.6 & -4.7 & 2.4 & 3.0 & -4.7 & -8.2 & 1.5 & 4.5 & -4.7 & -7.6 & 1.9 & 4.5 & -6.3 & 2.9 & -0.47 & $\begin{array}{l}-1.69 \\
\end{array}$ & 185 & 0.179 \\
\hline 63 & -8.7 & -4.6 & 2.4 & 3.0 & -4.7 & -8.3 & 1.6 & 4.5 & -4.9 & -7.9 & 1.9 & 4.4 & -6.5 & 2.9 & -0.46 & -1.73 & 189 & 0.164 \\
\hline 64 & -8.7 & -4.7 & 2.4 & 3.0 & -4.7 & -8.3 & 1.6 & 4.5 & -4.8 & -7.7 & 2.0 & 4.5 & -6.4 & 3.0 & -0.46 & -1.70 & 188 & 0.174 \\
\hline 65 & -8.8 & -4.7 & 2.4 & 3.1 & -4.7 & -8.3 & 1.6 & 4.5 & -5.0 & -7.8 & 2.0 & 4.5 & -6.4 & 3.0 & -0.46 & -1.71 & 189 & 0.173 \\
\hline 66 & -8.8 & -4.7 & 2.4 & 3.1 & -4.8 & -8.2 & 1.6 & 4.5 & -4.9 & -7.8 & 1.9 & 4.5 & -6.4 & 3.0 & -0.46 & -1.70 & 189 & 0.174 \\
\hline
\end{tabular}


Table E.24 NU 58-40 Test Data $\left(-20^{\circ} \mathrm{C}\right.$ Tests $)$, continued

\begin{tabular}{|c|c|c|c|c|c|c|c|c|c|c|c|c|c|c|c|c|c|c|}
\hline & \multicolumn{12}{|c|}{ Normalized Vertical and Horizontal Deformation Arrays } & & & & & & \\
\hline & \multicolumn{4}{|c|}{ NU 58-40 Sample \#11 } & \multicolumn{4}{|c|}{ NU 58-40 Sample \#12 } & \multicolumn{4}{|c|}{ NU 58-40 Sample \#9 } & \multicolumn{2}{|c|}{ Trimmed } & & & Creep & Pois. \\
\hline & vert & vert & horz & horz & vert & vert & horz & horz & vert & vert & horz & horz & & & Ratio & & Comp & Ratio \\
\hline Time & front & back & front & back & front & back & front & back & front & back & front & back & vert & horz & $\mathrm{X} / \mathrm{Y}$ & $\mathrm{C}_{\mathrm{empl}}$ & $D(t)$ & $v$ \\
\hline $\mathrm{sec}$ & $\mu \mathrm{m}$ & $\mu \mathrm{m}$ & $\mu \mathrm{m}$ & $\mu \mathrm{m}$ & $\mu \mathrm{m}$ & $\mu \mathrm{m}$ & $\mu \mathrm{m}$ & $\mu \mathrm{m}$ & $\mu \mathrm{m}$ & $\mu \mathrm{m}$ & $\mu \mathrm{m}$ & $\mu \mathrm{m}$ & $\mu \mathrm{m}$ & $\mu \mathrm{m}$ & & & $1 / \mathrm{kPa}$ & \\
\hline 67 & -8.8 & -4.7 & 2.4 & 3.1 & -4.8 & -8.3 & 1.6 & 4.6 & -5.0 & -8.0 & 1.9 & 4.5 & -6.5 & 3.0 & -0.46 & -1.72 & 191 & 0.169 \\
\hline 68 & -8.9 & -4.7 & 2.4 & 3.1 & -4.8 & -8.5 & 1.6 & 4.6 & -5.1 & -7.9 & 2.0 & 4.6 & -6.6 & 3.0 & -0.46 & -1.73 & 193 & 0.165 \\
\hline 69 & -9.0 & -4.9 & 2.4 & 3.1 & -4.8 & -8.5 & 1.6 & 4.5 & -5.0 & -8.0 & 2.0 & 4.6 & -6.6 & 3.0 & -0.46 & -1.71 & 192 & 0.170 \\
\hline 70 & -9.0 & -4.8 & 2.4 & 3.1 & -4.9 & -8.5 & 1.6 & 4.6 & -5.0 & -8.2 & 2.0 & 4.6 & -6.6 & 3.0 & -0.45 & -1.73 & 194 & 0.164 \\
\hline 71 & -9.0 & -4.7 & 2.4 & 3.1 & -5.0 & -8.5 & 1.6 & 4.6 & -5.2 & -8.2 & 2.0 & 4.6 & -6.7 & 3.0 & -0.45 & -1.74 & 196 & 0.160 \\
\hline 72 & -9.0 & -4.8 & 2.5 & 3.1 & -4.9 & -8.5 & 1.6 & 4.6 & -5.1 & -8.3 & 2.0 & 4.6 & -6.7 & 3.0 & -0.45 & -1.74 & 197 & 0.161 \\
\hline 73 & -9.0 & -4.8 & 2.5 & 3.2 & -4.8 & -8.5 & 1.6 & 4.7 & -5.1 & -8.3 & 2.0 & 4.6 & -6.7 & 3.1 & -0.46 & -1.72 & 195 & 0.169 \\
\hline 74 & -9.1 & -4.8 & 2.5 & 3.2 & -4.9 & -8.5 & 1.6 & 4.7 & -5.1 & -8.4 & 2.0 & 4.6 & -6.7 & 3.1 & -0.46 & -1.72 & 196 & 0.166 \\
\hline 75 & -9.1 & -4.8 & 2.5 & 3.2 & -4.9 & -8.6 & 1.6 & 4.6 & -5.1 & -8.3 & 2.0 & 4.6 & -6.7 & 3.1 & -0.46 & -1.72 & 197 & 0.166 \\
\hline 76 & -9.1 & -4.8 & 2.5 & 3.2 & -4.9 & -8.6 & 1.6 & 4.7 & -5.2 & -8.4 & 2.0 & 4.6 & -6.8 & 3.1 & -0.45 & -1.73 & 198 & 0.164 \\
\hline 77 & -9.1 & -4.8 & 2.5 & 3.2 & -4.9 & -8.5 & 1.7 & 4.7 & -5.3 & -8.4 & 2.0 & 4.6 & -6.8 & 3.1 & -0.45 & -1.73 & 199 & 0.163 \\
\hline 78 & -9.2 & -4.9 & 2.5 & 3.2 & -4.9 & -8.6 & 1.7 & 4.7 & -5.2 & -8.4 & 2.0 & 4.7 & -6.8 & 3.1 & -0.46 & -1.73 & 199 & 0.165 \\
\hline 79 & -9.2 & -4.9 & 2.5 & 3.2 & -4.9 & -8.7 & 1.7 & 4.7 & -5.2 & -8.5 & 2.0 & 4.7 & -6.9 & 3.1 & -0.45 & -1.73 & 201 & 0.164 \\
\hline 80 & -9.2 & -4.8 & 2.5 & 3.2 & -4.9 & -8.7 & 1.7 & 4.7 & -5.2 & -8.4 & 2.0 & 4.7 & -6.8 & 3.1 & -0.46 & -1.73 & 200 & 0.165 \\
\hline 81 & -9.2 & -4.9 & 2.5 & 3.2 & -5.0 & -8.7 & 1.7 & 4.8 & -5.3 & -8.6 & 2.0 & 4.7 & -6.9 & 3.1 & -0.45 & -1.73 & 202 & 0.162 \\
\hline 82 & -9.2 & -4.9 & 2.6 & 3.2 & -5.0 & -8.8 & 1.7 & 4.8 & -5.3 & -8.6 & 2.0 & 4.7 & -6.9 & 3.1 & -0.45 & -1.74 & 203 & 0.161 \\
\hline 83 & -9.2 & -4.9 & 2.6 & 3.3 & -5.1 & -9.0 & 1.7 & 4.8 & -5.3 & -8.6 & 2.0 & 4.7 & -7.0 & 3.1 & -0.45 & -1.75 & 204 & 0.156 \\
\hline 84 & -9.3 & -4.9 & 2.6 & 3.3 & -5.0 & -8.8 & 1.7 & 4.8 & -5.3 & -8.6 & 2.0 & 4.7 & -6.9 & 3.1 & -0.45 & -1.73 & 203 & 0.164 \\
\hline 85 & -9.3 & -5.0 & 2.6 & 3.3 & -5.0 & -8.8 & 1.7 & 4.8 & -5.4 & -8.7 & 2.1 & 4.8 & -7.0 & 3.2 & -0.45 & -1.73 & 204 & 0.163 \\
\hline 86 & -9.3 & -5.0 & 2.6 & 3.3 & -5.0 & -8.8 & 1.7 & 4.8 & -5.5 & -8.9 & 2.1 & 4.7 & -7.0 & 3.2 & -0.45 & -1.75 & 205 & 0.157 \\
\hline 87 & -9.3 & -5.0 & 2.6 & 3.3 & -5.1 & -8.9 & 1.7 & 4.9 & -5.4 & -8.7 & 2.1 & 4.8 & -7.0 & 3.2 & -0.45 & -1.73 & 206 & 0.162 \\
\hline 88 & -9.4 & -5.0 & 2.6 & 3.3 & -5.0 & -8.8 & 1.7 & 4.9 & -5.4 & -8.8 & 2.1 & 4.8 & -7.0 & 3.2 & -0.45 & -1.73 & 205 & 0.164 \\
\hline 89 & -9.3 & -5.0 & 2.6 & 3.3 & -5.1 & -9.0 & 1.7 & 4.9 & -5.4 & -8.8 & 2.1 & 4.8 & -7.1 & 3.2 & -0.45 & -1.73 & 207 & 0.164 \\
\hline 90 & -9.4 & -5.0 & 2.6 & 3.3 & -5.1 & -8.9 & 1.7 & 4.9 & -5.5 & -8.8 & 2.1 & 4.9 & -7.1 & 3.2 & -0.46 & -1.73 & 207 & 0.165 \\
\hline 91 & -9.5 & -5.0 & 2.6 & 3.3 & -5.1 & -9.0 & 1.8 & 4.9 & -5.5 & -8.9 & 2.1 & 4.9 & -7.1 & 3.2 & -0.46 & -1.73 & 208 & 0.165 \\
\hline 92 & -9.6 & -5.0 & 2.6 & 3.4 & -5.2 & -9.0 & 1.8 & 4.9 & -5.5 & $\begin{array}{l}-8.9 \\
\end{array}$ & 2.1 & 4.9 & -7.1 & 3.2 & -0.45 & -1.73 & 209 & 0.162 \\
\hline 93 & -9.5 & -5.0 & 2.6 & 3.4 & -5.0 & -8.7 & 1.8 & 5.0 & -5.4 & -8.9 & 2.1 & 4.9 & -7.0 & 3.3 & -0.46 & -1.70 & 206 & 0.176 \\
\hline 94 & -9.5 & -5.0 & 2.6 & 3.4 & -5.1 & -9.0 & 1.8 & 5.0 & -5.5 & -8.9 & 2.1 & 4.9 & -7.1 & 3.3 & -0.46 & -1.72 & 209 & 0.168 \\
\hline 95 & -9.4 & -5.1 & 2.6 & 3.4 & -5.2 & -9.0 & 1.8 & 5.0 & -5.6 & -8.9 & 2.1 & 4.9 & -7.2 & 3.3 & -0.46 & -1.73 & 210 & 0.165 \\
\hline 96 & -9.6 & -5.1 & 2.7 & 3.4 & -5.3 & -9.1 & 1.8 & 5.0 & -5.5 & $\begin{array}{l}-8.9 \\
\end{array}$ & 2.1 & 4.9 & -7.2 & 3.3 & -0.45 & -1.73 & 211 & 0.163 \\
\hline 97 & -9.6 & -5.1 & 2.7 & 3.4 & -5.3 & -9.1 & 1.8 & 5.0 & -5.4 & -8.9 & 2.2 & 4.9 & -7.2 & 3.3 & -0.46 & -1.72 & 211 & 0.167 \\
\hline 98 & -9.6 & -5.2 & 2.7 & 3.4 & -5.1 & -9.0 & 1.8 & 5.0 & -5.5 & -8.9 & 2.2 & 5.0 & -7.1 & 3.3 & -0.46 & -1.70 & 209 & 0.175 \\
\hline 99 & -9.7 & -5.3 & 2.7 & 3.4 & -5.1 & -9.0 & 1.8 & 5.0 & -5.4 & -8.9 & 2.2 & 4.9 & -7.1 & 3.3 & -0.46 & -1.70 & 209 & 0.174 \\
\hline 100 & -9.6 & -5.2 & 2.7 & 3.4 & -5.2 & -9.0 & 1.8 & 5.1 & -5.5 & -8.8 & 2.2 & 5.0 & -7.1 & 3.3 & -0.47 & -1.69 & 209 & 0.180 \\
\hline
\end{tabular}




\section{APPENDIX F IDT STRENGTH DATA}


Table F.1 IDT Specimen Geometry

\begin{tabular}{|c|c|c|c|c|}
\hline & & Thickness & Diameter & Failure Load \\
\hline Mix & Sample \# & $\mathbf{m m}$ & $\mathbf{~ m m}$ & $\mathbf{k N}$ \\
\hline KL 52-34 & 2 & 76.88 & 150.38 & -57.9 \\
\hline KL 52-34 & 5 & 76.91 & 150.33 & -67.7 \\
\hline KL 58-40 & 1 & 76.82 & 150.10 & -70.3 \\
\hline KL 58-40 & 10 & 76.74 & 150.23 & -70.3 \\
\hline KL 58-40 & 12 & 76.85 & 150.00 & -75.3 \\
\hline KL 58-40 & 8 & 76.80 & 150.03 & -61.5 \\
\hline NU 52-34 & 6 & 76.85 & 150.24 & -72.6 \\
\hline NU 52-34 & 8 & 76.71 & 150.29 & -71.4 \\
\hline NU 52-34 & 9 & 76.72 & 150.13 & -71.7 \\
\hline NU 58-40 & 11 & 76.94 & 150.11 & -68.4 \\
\hline NU 58-40 & 12 & 76.81 & 150.02 & -70.4 \\
\hline NU 58-40 & 9 & 76.79 & 150.10 & -66.8 \\
\hline
\end{tabular}

Gage Length $=38 \mathrm{~mm}$

Test Temperature $=-10^{\circ} \mathrm{C}$

Displacement Rate $=12.5 \mathrm{~mm} / \mathrm{min}$. 
Table F.2 IDT Strength Data (KL 52-34 \#2)

\begin{tabular}{|c|c|c|c|c|c|c|}
\hline Time & Axial Force & Axial Displ. & Vert front & Vert back & Horz front & Horz back \\
\hline $\mathrm{Sec}$ & $\mathrm{kN}$ & $\mathrm{mm}$ & $\mu \mathrm{m}$ & $\mu \mathrm{m}$ & $\mu \mathrm{m}$ & $\mu \mathrm{m}$ \\
\hline 0.00 & -0.2 & -0.93 & 0.34 & 0.67 & 0.20 & -0.08 \\
\hline 0.05 & -0.3 & -0.94 & 0.34 & 0.34 & 0.18 & 0.03 \\
\hline 0.10 & -0.5 & -0.95 & 0.47 & 0.13 & 0.18 & 0.15 \\
\hline 0.15 & -0.8 & -0.96 & 0.54 & -0.27 & 0.18 & 0.37 \\
\hline 0.20 & -1.0 & -0.97 & 0.74 & -0.74 & 0.18 & 0.52 \\
\hline 0.25 & -1.2 & -0.98 & 0.87 & -1.07 & 0.19 & 0.72 \\
\hline 0.30 & -1.5 & $\begin{array}{l}-0.99 \\
\end{array}$ & 0.87 & -1.61 & 0.17 & 0.92 \\
\hline 0.35 & -1.7 & -1.00 & 0.87 & -2.08 & 0.19 & 1.16 \\
\hline 0.40 & -2.1 & -1.01 & 1.01 & -2.55 & 0.20 & 1.43 \\
\hline 0.45 & -2.7 & -1.02 & 0.81 & -3.36 & 0.22 & 1.75 \\
\hline 0.50 & -3.0 & -1.03 & 0.94 & -3.83 & 0.27 & 2.00 \\
\hline 0.55 & -3.4 & -1.04 & 1.01 & -4.57 & 0.30 & 2.38 \\
\hline 0.60 & -3.8 & -1.05 & 0.87 & -5.30 & 0.32 & 2.65 \\
\hline 0.65 & -4.1 & -1.06 & 0.94 & -5.84 & 0.34 & 2.92 \\
\hline 0.70 & -4.7 & -1.08 & 0.94 & -6.51 & 0.40 & 3.37 \\
\hline 0.75 & -5.1 & -1.09 & 0.87 & -7.52 & 0.44 & 3.69 \\
\hline 0.80 & -5.5 & -1.09 & 0.87 & -8.06 & 0.50 & 4.06 \\
\hline 0.85 & -5.9 & -1.10 & 0.67 & -8.86 & 0.51 & 4.38 \\
\hline 0.90 & -6.4 & -1.11 & 0.60 & -9.80 & 0.54 & 4.82 \\
\hline 0.95 & -6.7 & -1.13 & 0.81 & -10.07 & 0.61 & 5.20 \\
\hline 1.00 & -7.1 & -1.14 & 0.60 & -11.08 & 0.65 & 5.61 \\
\hline 1.05 & -7.5 & -1.15 & 0.74 & -11.62 & 0.71 & 5.93 \\
\hline 1.10 & -7.7 & -1.16 & 0.54 & -12.42 & 0.75 & 6.28 \\
\hline 1.15 & -8.3 & -1.17 & 0.34 & -13.23 & 0.81 & 6.66 \\
\hline 1.20 & -8.8 & -1.18 & 0.40 & -13.97 & 0.90 & 7.13 \\
\hline 1.25 & -9.1 & -1.19 & 0.20 & -14.70 & 0.97 & 7.49 \\
\hline 1.30 & -9.7 & -1.20 & 0.00 & -15.51 & 1.04 & 7.97 \\
\hline 1.35 & -10.1 & -1.21 & -0.13 & -16.32 & 1.12 & 8.38 \\
\hline 1.40 & -10.5 & -1.22 & -0.27 & -17.12 & 1.19 & 8.81 \\
\hline 1.45 & -10.9 & -1.23 & -0.54 & -17.73 & 1.28 & 9.18 \\
\hline 1.50 & -11.2 & -1.24 & -0.67 & -18.53 & 1.35 & 9.58 \\
\hline 1.55 & -11.6 & -1.25 & -0.81 & -19.14 & 1.43 & 10.00 \\
\hline 1.60 & -12.1 & -1.26 & -1.07 & -19.94 & 1.53 & 10.41 \\
\hline 1.65 & -12.5 & -1.27 & -1.28 & -20.75 & 1.61 & 10.84 \\
\hline 1.70 & -13.0 & -1.28 & -1.61 & -21.49 & 1.74 & 11.28 \\
\hline 1.75 & -13.5 & -1.29 & -1.88 & -22.36 & 1.85 & 11.75 \\
\hline 1.80 & -13.8 & -1.31 & -2.22 & -22.96 & 1.95 & 12.15 \\
\hline 1.85 & -14.2 & -1.31 & -2.55 & -23.70 & 2.06 & 12.59 \\
\hline 1.90 & -14.6 & -1.32 & -2.69 & -24.37 & 2.17 & 13.03 \\
\hline 1.95 & -15.1 & -1.34 & -3.16 & -25.18 & 2.32 & 13.51 \\
\hline 2.00 & -15.7 & -1.35 & -3.56 & -26.05 & 2.44 & 14.00 \\
\hline 2.05 & -16.1 & -1.36 & -3.96 & -26.79 & 2.60 & 14.47 \\
\hline 2.10 & -16.6 & -1.37 & -4.30 & -27.59 & 2.74 & 14.92 \\
\hline 2.15 & -17.1 & -1.38 & -4.70 & -28.40 & 2.89 & 15.41 \\
\hline 2.20 & -17.4 & -1.38 & -5.04 & -29.00 & 3.04 & 15.86 \\
\hline 2.25 & -18.2 & -1.40 & -5.64 & -30.01 & 3.23 & 16.45 \\
\hline
\end{tabular}


Table F.2 IDT Strength Data (KL 52-34 \#2), continued

\begin{tabular}{|c|c|c|c|c|c|c|}
\hline Time & Axial Force & Axial Displ. & Vert front & Vert back & Horz front & Horz back \\
\hline $\mathrm{Sec}$ & $\mathrm{kN}$ & $\mathrm{mm}$ & $\mu \mathrm{m}$ & $\mu \mathrm{m}$ & $\mu \mathrm{m}$ & $\mu \mathrm{m}$ \\
\hline 2.30 & -18.7 & -1.41 & -6.11 & -30.82 & 3.39 & 16.97 \\
\hline 2.35 & -19.0 & -1.42 & -6.65 & -31.62 & 3.55 & 17.46 \\
\hline 2.40 & -19.4 & -1.43 & -6.92 & -32.36 & 3.71 & 17.93 \\
\hline 2.45 & -19.8 & -1.44 & -7.52 & -33.10 & 3.86 & 18.41 \\
\hline 2.50 & -20.2 & -1.45 & -7.99 & -33.84 & 4.05 & 18.93 \\
\hline 2.55 & -20.8 & -1.46 & -8.59 & -34.78 & 4.25 & 19.47 \\
\hline 2.60 & -21.3 & -1.47 & -9.06 & -35.58 & 4.45 & 20.01 \\
\hline 2.65 & -21.8 & -1.48 & -9.60 & -36.39 & 4.62 & 20.53 \\
\hline 2.70 & -22.1 & -1.49 & -10.14 & -37.20 & 4.83 & 21.05 \\
\hline 2.75 & -22.5 & -1.50 & -10.74 & -37.93 & 5.01 & 21.59 \\
\hline 2.80 & -23.0 & -1.51 & -11.35 & -38.87 & 5.21 & 22.12 \\
\hline 2.85 & -23.3 & -1.52 & -11.75 & -39.55 & 5.40 & 22.61 \\
\hline 2.90 & -24.0 & -1.53 & -12.49 & -40.49 & 5.62 & 23.23 \\
\hline 2.95 & -24.2 & -1.55 & -13.03 & -41.22 & 5.81 & 23.73 \\
\hline 3.00 & -24.7 & -1.55 & -13.63 & -42.16 & 6.03 & 24.32 \\
\hline 3.05 & -25.4 & -1.56 & -14.23 & -43.10 & 6.28 & 24.93 \\
\hline 3.10 & -25.9 & -1.58 & -15.11 & -44.04 & 6.52 & 25.56 \\
\hline 3.15 & -26.5 & -1.59 & -15.85 & -45.12 & 6.77 & 26.22 \\
\hline 3.20 & -26.7 & -1.59 & -16.45 & -45.92 & 6.98 & 26.76 \\
\hline 3.25 & -27.0 & -1.61 & -17.05 & -46.60 & 7.22 & 27.34 \\
\hline 3.30 & -27.4 & -1.62 & -17.73 & -47.60 & 7.44 & 27.93 \\
\hline 3.35 & -28.0 & -1.63 & -18.46 & -48.54 & 7.70 & 28.57 \\
\hline 3.40 & -28.3 & -1.64 & -19.20 & -49.55 & 7.93 & 29.17 \\
\hline 3.45 & -28.8 & -1.65 & -19.81 & -50.29 & 8.20 & 29.81 \\
\hline 3.50 & -29.2 & -1.66 & -20.61 & -51.23 & 8.46 & 30.48 \\
\hline 3.55 & -29.8 & -1.67 & -21.49 & -52.24 & 8.75 & 31.12 \\
\hline 3.60 & -30.3 & -1.68 & -22.29 & -53.38 & 9.01 & 31.82 \\
\hline 3.65 & -30.5 & -1.69 & -22.96 & -54.32 & 9.27 & 32.43 \\
\hline 3.70 & -30.8 & -1.70 & -23.70 & -55.12 & 9.53 & 33.05 \\
\hline 3.75 & -31.3 & -1.71 & -24.51 & -56.20 & 9.79 & 33.74 \\
\hline 3.80 & -31.9 & -1.72 & -25.45 & -57.14 & 10.10 & 34.46 \\
\hline 3.85 & -32.2 & -1.73 & -26.25 & -58.21 & 10.38 & 35.11 \\
\hline 3.90 & -32.8 & -1.74 & -27.06 & -59.22 & 10.69 & 35.85 \\
\hline 3.95 & -33.4 & -1.75 & -28.00 & -60.49 & 11.04 & 36.66 \\
\hline 4.00 & -33.9 & -1.76 & -29.00 & -61.57 & 11.36 & 37.43 \\
\hline 4.05 & -34.2 & -1.77 & -29.88 & -62.51 & 11.64 & 38.10 \\
\hline 4.10 & -34.5 & -1.78 & -30.68 & -63.52 & 11.95 & 38.82 \\
\hline 4.15 & -34.8 & -1.79 & -31.42 & -64.46 & 12.26 & 39.53 \\
\hline 4.20 & -35.2 & -1.80 & -32.43 & -65.53 & 12.57 & 40.30 \\
\hline 4.25 & -35.8 & -1.82 & -33.50 & -66.81 & 12.93 & 41.11 \\
\hline 4.30 & -36.0 & -1.82 & -34.31 & -67.74 & 13.24 & 41.80 \\
\hline 4.35 & -36.3 & -1.83 & -35.32 & -68.68 & 13.56 & 42.53 \\
\hline 4.40 & -36.8 & -1.85 & -36.19 & -69.89 & 13.91 & 43.34 \\
\hline 4.45 & -37.5 & -1.86 & -37.40 & -71.17 & 14.29 & 44.20 \\
\hline 4.50 & -37.9 & -1.86 & -38.40 & -72.31 & 14.66 & 45.02 \\
\hline
\end{tabular}


Table F.2 IDT Strength Data (KL 52-34 \#2), continued

\begin{tabular}{|c|c|c|c|c|c|c|}
\hline Time & Axial Force & Axial Displ. & Vert front & Vert back & Horz front & Horz back \\
\hline $\mathrm{Sec}$ & $\mathrm{kN}$ & $\mathrm{mm}$ & $\mu \mathrm{m}$ & $\mu \mathrm{m}$ & $\mu \mathrm{m}$ & $\mu \mathrm{m}$ \\
\hline 4.55 & -38.2 & -1.88 & -39.41 & -73.52 & 15.01 & 45.84 \\
\hline 4.60 & -38.3 & -1.89 & -40.35 & -74.53 & 15.35 & 46.58 \\
\hline 4.65 & -38.6 & -1.90 & -41.29 & -75.53 & 15.71 & 47.37 \\
\hline 4.70 & -39.1 & -1.91 & -42.50 & -76.81 & 16.11 & 48.22 \\
\hline 4.75 & -39.8 & -1.92 & -43.51 & -78.08 & 16.53 & 49.18 \\
\hline 4.80 & -40.0 & -1.93 & -44.58 & -79.23 & 16.90 & 49.95 \\
\hline 4.85 & -40.2 & -1.94 & -45.59 & -80.23 & 17.26 & 50.81 \\
\hline 4.90 & -40.5 & -1.95 & -46.66 & -81.37 & 17.66 & 51.63 \\
\hline 4.95 & -40.9 & -1.96 & -47.74 & -82.58 & 18.05 & 52.52 \\
\hline 5.00 & -41.2 & -1.97 & -48.74 & -83.86 & 18.46 & 53.36 \\
\hline 5.05 & -41.4 & -1.98 & -49.95 & -84.93 & 18.86 & 54.22 \\
\hline 5.10 & -41.8 & -1.99 & -51.03 & -86.07 & 19.23 & 55.07 \\
\hline 5.16 & -42.3 & -2.00 & -52.17 & -87.55 & 19.70 & 56.03 \\
\hline 5.21 & -42.6 & -2.01 & -53.38 & -88.69 & 20.12 & 56.94 \\
\hline 5.26 & -43.0 & -2.02 & -54.52 & -89.97 & 20.55 & 57.86 \\
\hline 5.31 & -43.1 & -2.03 & -55.66 & -91.18 & 20.97 & 58.76 \\
\hline 5.36 & -43.6 & -2.04 & -57.07 & -92.59 & 21.41 & 59.65 \\
\hline 5.41 & -43.8 & -2.05 & -58.08 & -93.66 & 21.87 & 60.63 \\
\hline 5.46 & -44.4 & -2.06 & -59.69 & -95.34 & 22.32 & 61.65 \\
\hline 5.51 & -44.6 & -2.08 & -60.63 & -96.35 & 22.79 & 62.64 \\
\hline 5.56 & -44.6 & -2.09 & -61.84 & -97.69 & 23.22 & 63.48 \\
\hline 5.61 & -44.8 & -2.09 & -63.05 & -98.83 & 23.68 & 64.42 \\
\hline 5.66 & -45.2 & -2.10 & -64.32 & -100.17 & 24.15 & 65.48 \\
\hline 5.71 & -45.7 & -2.12 & -65.60 & -101.65 & 24.65 & 66.50 \\
\hline 5.76 & -46.1 & -2.13 & -67.07 & -103.13 & 25.18 & 67.59 \\
\hline 5.81 & -46.0 & -2.14 & -68.15 & -104.20 & 25.64 & 68.48 \\
\hline 5.86 & -46.3 & -2.15 & -69.36 & -105.55 & 26.13 & 69.49 \\
\hline 5.91 & -46.5 & -2.16 & -70.83 & -107.02 & 26.60 & 70.46 \\
\hline 5.96 & -47.1 & -2.17 & -72.04 & -108.50 & 27.17 & 71.66 \\
\hline 6.01 & -47.0 & -2.18 & -73.45 & -109.78 & 27.65 & 72.60 \\
\hline 6.06 & -47.7 & -2.19 & -74.86 & -111.25 & 28.22 & 73.77 \\
\hline 6.11 & -48.0 & -2.20 & -76.34 & -112.86 & 28.77 & 74.90 \\
\hline 6.16 & -48.1 & -2.21 & -77.75 & -114.14 & 29.28 & 75.95 \\
\hline 6.21 & -48.6 & -2.22 & -79.23 & -115.75 & 29.88 & 77.16 \\
\hline 6.26 & -48.7 & -2.23 & -80.70 & -117.16 & 30.39 & 78.24 \\
\hline 6.31 & -48.8 & -2.24 & -81.98 & -118.50 & 30.96 & 79.34 \\
\hline 6.36 & -49.1 & -2.25 & -83.52 & -120.05 & 31.52 & 80.48 \\
\hline 6.41 & -49.4 & -2.26 & -85.00 & -121.52 & 32.12 & 81.69 \\
\hline 6.46 & -49.8 & -2.27 & -86.61 & -123.20 & 32.72 & 82.90 \\
\hline 6.51 & -49.9 & -2.28 & -88.09 & -124.61 & 33.29 & 84.06 \\
\hline 6.56 & -50.1 & -2.30 & -89.57 & -126.22 & 33.89 & 85.25 \\
\hline 6.61 & -50.1 & -2.30 & -91.04 & -127.63 & 34.45 & 86.39 \\
\hline 6.66 & -50.3 & -2.31 & -92.65 & -129.18 & 35.06 & 87.59 \\
\hline 6.71 & -50.7 & -2.32 & -94.20 & -130.86 & 35.67 & 88.86 \\
\hline 6.76 & -50.7 & -2.34 & -95.61 & -132.27 & 36.26 & 90.02 \\
\hline
\end{tabular}


Table F.2 IDT Strength Data (KL 52-34 \#2), continued

\begin{tabular}{|c|c|c|c|c|c|c|}
\hline Time & Axial Force & Axial Displ. & Vert front $\mathrm{A}$ & Vert back B & Horz front $\mathrm{C}$ & Horz back D \\
\hline $\mathrm{Sec}$ & $\mathrm{kN}$ & $\mathrm{mm}$ & $\mu \mathrm{m}$ & $\mu \mathrm{m}$ & $\mu \mathrm{m}$ & $\mu \mathrm{m}$ \\
\hline 6.81 & -50.8 & -2.34 & -97.15 & -133.81 & 36.85 & 91.23 \\
\hline 6.86 & -51.1 & -2.36 & -98.83 & -135.42 & 37.50 & 92.54 \\
\hline 6.91 & -51.1 & -2.37 & -100.17 & -136.90 & 38.10 & 93.73 \\
\hline 6.96 & -51.3 & -2.37 & -101.72 & -138.38 & 38.75 & 95.04 \\
\hline 7.01 & -51.8 & -2.38 & -103.53 & -140.39 & 39.41 & 96.38 \\
\hline 7.06 & -52.2 & -2.40 & -105.28 & -142.00 & 40.12 & 97.79 \\
\hline 7.11 & -52.4 & -2.41 & -106.89 & -143.82 & 40.81 & 99.20 \\
\hline 7.16 & -52.6 & -2.42 & -108.63 & -145.43 & 41.50 & 100.58 \\
\hline 7.21 & -52.6 & -2.43 & -110.25 & -147.04 & 42.18 & 101.94 \\
\hline 7.26 & -52.7 & -2.44 & -111.92 & -148.72 & 42.86 & 103.23 \\
\hline 7.31 & -52.7 & -2.45 & -113.47 & -150.33 & 43.51 & 104.55 \\
\hline 7.36 & -52.9 & -2.46 & -115.15 & -151.94 & 44.23 & 105.91 \\
\hline 7.41 & -53.3 & -2.47 & -116.96 & -153.89 & 44.97 & 107.41 \\
\hline 7.46 & -53.4 & -2.48 & -118.70 & -155.50 & 45.67 & 108.84 \\
\hline 7.51 & -53.5 & -2.50 & -120.38 & -157.31 & 46.39 & 110.21 \\
\hline 7.56 & -53.4 & -2.50 & -121.99 & -158.79 & 47.10 & 111.59 \\
\hline 7.61 & -53.7 & -2.51 & -123.81 & -160.73 & 47.86 & 113.08 \\
\hline 7.66 & -53.8 & -2.52 & -125.75 & -162.75 & 48.62 & 114.54 \\
\hline 7.71 & -54.0 & -2.53 & -127.50 & -164.56 & 49.39 & 116.02 \\
\hline 7.76 & -54.0 & -2.55 & -129.18 & -165.90 & 50.15 & 117.58 \\
\hline 7.81 & -54.3 & -2.55 & -130.72 & -167.72 & 50.95 & 119.07 \\
\hline 7.86 & -54.5 & -2.57 & -132.80 & -169.73 & 51.73 & 120.64 \\
\hline 7.91 & -54.7 & -2.58 & -134.62 & -171.68 & 52.58 & 122.26 \\
\hline 7.96 & -54.8 & -2.58 & -136.50 & -173.42 & 53.39 & 123.84 \\
\hline 8.01 & -55.0 & -2.60 & -138.31 & -175.37 & 54.23 & 125.47 \\
\hline 8.06 & -55.3 & -2.60 & -140.32 & -177.52 & 55.13 & 127.20 \\
\hline 8.11 & -55.4 & -2.62 & -142.14 & -179.40 & 55.98 & 128.84 \\
\hline 8.16 & -55.5 & -2.63 & -144.02 & -181.28 & 56.85 & 130.56 \\
\hline 8.21 & -55.6 & -2.64 & -145.90 & -183.16 & 57.72 & 132.18 \\
\hline 8.26 & -55.7 & -2.65 & -147.91 & -185.17 & 58.62 & 133.91 \\
\hline 8.31 & -55.8 & -2.66 & -149.86 & -187.19 & 59.52 & 135.66 \\
\hline 8.36 & -56.0 & -2.67 & -151.87 & -189.27 & 60.46 & 137.44 \\
\hline 8.41 & -56.0 & -2.68 & -153.89 & -191.22 & 61.39 & 139.17 \\
\hline 8.46 & -56.3 & -2.69 & -155.90 & -193.30 & 62.37 & 141.08 \\
\hline 8.51 & -56.4 & -2.70 & -157.98 & -195.45 & 63.32 & 142.93 \\
\hline 8.56 & -56.3 & -2.71 & -159.79 & -197.26 & 64.28 & 144.72 \\
\hline 8.61 & -56.4 & -2.72 & -161.81 & -199.41 & 65.24 & 146.53 \\
\hline 8.66 & -56.5 & -2.73 & -163.89 & -201.42 & 66.25 & 148.46 \\
\hline 8.71 & -56.6 & -2.74 & -165.77 & -203.57 & 67.24 & 150.34 \\
\hline 8.76 & -56.7 & -2.76 & -167.85 & -205.65 & 68.27 & 152.36 \\
\hline 8.81 & -56.5 & -2.76 & -169.80 & -207.46 & 69.23 & 154.19 \\
\hline 8.86 & -56.5 & -2.77 & -171.75 & -209.48 & 70.24 & 156.07 \\
\hline 8.91 & -56.7 & -2.78 & -173.76 & -211.76 & 71.29 & 158.05 \\
\hline 8.96 & -56.8 & -2.80 & -175.91 & -213.84 & 72.34 & 160.10 \\
\hline 9.01 & -56.9 & -2.80 & -177.99 & -215.99 & 73.40 & 162.14 \\
\hline
\end{tabular}


Table F.2 IDT Strength Data (KL 52-34 \#2), continued

\begin{tabular}{|c|c|c|c|c|c|c|}
\hline Time & Axial Force & Axial Displ. & Vert front $\mathrm{A}$ & Vert back B & Horz front $\mathrm{C}$ & Horz back D \\
\hline $\mathrm{Sec}$ & $\mathrm{kN}$ & $\mathrm{mm}$ & $\mu \mathrm{m}$ & $\mu \mathrm{m}$ & $\mu \mathrm{m}$ & $\mu \mathrm{m}$ \\
\hline 9.06 & -57.1 & -2.82 & -180.21 & -218.27 & 74.51 & 164.28 \\
\hline 9.11 & -57.3 & -2.82 & -182.42 & -220.56 & 75.63 & 166.48 \\
\hline 9.16 & -57.7 & -2.84 & -184.64 & -222.91 & 76.76 & 168.77 \\
\hline 9.21 & -57.6 & -2.85 & -186.85 & -225.26 & 77.91 & 170.97 \\
\hline 9.26 & -57.4 & -2.85 & -188.93 & -227.41 & 79.02 & 173.12 \\
\hline 9.31 & -57.8 & -2.87 & -191.28 & -229.82 & 80.22 & 175.49 \\
\hline 9.36 & -57.7 & -2.88 & -193.30 & -231.97 & 81.42 & 177.81 \\
\hline 9.41 & -57.6 & -2.89 & -195.51 & -234.19 & 82.61 & 180.09 \\
\hline 9.46 & -57.6 & -2.90 & -197.80 & -236.60 & 83.79 & 182.45 \\
\hline 9.51 & -57.5 & -2.91 & -199.95 & -238.75 & 84.95 & 184.69 \\
\hline 9.56 & -57.5 & -2.92 & -202.16 & -241.10 & 86.20 & 187.07 \\
\hline 9.61 & -57.6 & -2.93 & -204.44 & -243.45 & 87.43 & 189.49 \\
\hline 9.66 & -57.5 & -2.94 & -206.59 & -245.67 & 88.68 & 191.96 \\
\hline 9.71 & -57.5 & -2.95 & -208.67 & -247.95 & 89.96 & 194.41 \\
\hline 9.76 & -57.8 & -2.96 & -211.09 & -250.50 & 91.29 & 197.07 \\
\hline 9.81 & -57.8 & -2.97 & -213.51 & -253.05 & 92.66 & 199.69 \\
\hline 9.86 & -57.9 & -2.99 & -215.86 & -255.47 & 94.05 & 202.40 \\
\hline 9.91 & -57.6 & -3.00 & -217.94 & -257.82 & 95.34 & 204.91 \\
\hline 9.96 & -57.7 & -3.01 & -220.29 & -260.24 & 96.72 & 207.63 \\
\hline 10.01 & -57.6 & -3.02 & -222.71 & -262.79 & 98.12 & 210.35 \\
\hline 10.06 & -57.6 & -3.03 & -224.99 & -265.34 & 99.52 & 213.10 \\
\hline 10.11 & -57.5 & -3.03 & -227.41 & -267.89 & 100.94 & 215.87 \\
\hline 10.16 & -57.6 & -3.05 & -229.49 & -270.11 & 102.47 & 218.88 \\
\hline 10.21 & -57.7 & -3.06 & -231.97 & -272.59 & 103.97 & 221.85 \\
\hline 10.26 & -57.5 & -3.07 & -234.59 & -275.75 & 105.43 & 224.69 \\
\hline 10.31 & -57.6 & -3.08 & -236.87 & -277.96 & 106.99 & 227.76 \\
\hline 10.36 & -57.5 & -3.09 & -239.29 & -280.38 & 108.56 & 230.86 \\
\hline 10.41 & -57.8 & -3.10 & -241.84 & -283.27 & 110.20 & 234.14 \\
\hline 10.46 & -57.8 & -3.11 & -244.26 & -286.02 & 111.86 & 237.44 \\
\hline 10.51 & -57.7 & -3.12 & -246.54 & -288.71 & 113.48 & 240.72 \\
\hline 10.56 & -57.3 & -3.13 & -248.89 & -291.19 & 115.11 & 244.01 \\
\hline 10.61 & -57.4 & -3.14 & -251.37 & -293.94 & 116.82 & 247.45 \\
\hline 10.66 & -57.5 & -3.15 & -253.79 & -296.76 & 118.59 & 250.99 \\
\hline 10.71 & -57.4 & -3.16 & -256.21 & -301.06 & 120.32 & 254.50 \\
\hline 10.76 & -57.7 & -3.17 & -258.83 & -303.81 & 122.25 & 258.44 \\
\hline 10.81 & -57.7 & -3.18 & -261.31 & -306.90 & 124.11 & 262.23 \\
\hline 10.86 & -57.6 & -3.19 & -263.86 & -310.26 & 126.03 & 266.21 \\
\hline 10.91 & -57.5 & -3.20 & -266.28 & -313.35 & 127.94 & 270.16 \\
\hline 10.96 & -57.5 & -3.22 & -268.83 & -316.84 & 129.94 & 274.34 \\
\hline 11.01 & -57.4 & -3.22 & -271.45 & -320.13 & 131.96 & 278.60 \\
\hline 11.06 & -57.5 & -3.23 & -274.07 & -323.75 & 134.04 & 283.12 \\
\hline 11.11 & -57.3 & -3.25 & -276.89 & -326.17 & 135.99 & 288.77 \\
\hline 11.16 & -57.3 & -3.26 & -279.64 & -327.92 & 137.75 & 298.76 \\
\hline 11.21 & -53.7 & -3.27 & -284.74 & -332.75 & 159.74 & 418.92 \\
\hline
\end{tabular}


Table F.3 IDT Strength Data (KL 52-34 \#5)

\begin{tabular}{|c|c|c|c|c|c|c|}
\hline Time & Axial Force & Axial Displ. & Vert front & Vert back & Horz front & Horz back \\
\hline $\mathrm{Sec}$ & $\mathrm{N}$ & $\mathrm{mm}$ & $\mu \mathrm{m}$ & $\mu \mathrm{m}$ & $\mu \mathrm{m}$ & $\mu \mathrm{m}$ \\
\hline 0.00 & -0.1 & -0.92 & 0.27 & 0.13 & 0.25 & 0.08 \\
\hline 0.05 & -0.4 & -0.93 & 0.00 & -0.13 & 0.27 & 0.08 \\
\hline 0.10 & -0.7 & -0.94 & -0.07 & 0.00 & 0.31 & 0.15 \\
\hline 0.15 & -1.0 & -0.95 & -0.27 & -0.20 & 0.37 & 0.18 \\
\hline 0.20 & -1.3 & -0.96 & -0.47 & -0.40 & 0.41 & 0.27 \\
\hline 0.25 & -2.0 & -0.97 & -0.81 & -0.60 & 0.50 & 0.40 \\
\hline 0.30 & -2.5 & -0.98 & -1.14 & -0.81 & 0.56 & 0.55 \\
\hline 0.35 & -3.2 & -0.99 & -1.41 & -1.14 & 0.62 & 0.72 \\
\hline 0.40 & -3.9 & -1.00 & -1.68 & -1.48 & 0.71 & 0.94 \\
\hline 0.45 & -4.5 & -1.02 & -2.01 & -1.81 & 0.77 & 1.09 \\
\hline 0.50 & -5.3 & -1.02 & -2.15 & -2.15 & 0.88 & 1.34 \\
\hline 0.55 & -5.9 & -1.04 & -2.55 & -2.55 & 0.97 & 1.54 \\
\hline 0.60 & -6.7 & -1.05 & -2.75 & -2.95 & 1.07 & 1.83 \\
\hline 0.65 & -7.6 & -1.06 & -3.16 & -3.42 & 1.16 & 2.06 \\
\hline 0.70 & -8.4 & -1.07 & -3.49 & -3.96 & 1.27 & 2.37 \\
\hline 0.75 & -9.2 & -1.08 & -3.76 & -4.50 & 1.36 & 2.64 \\
\hline 0.80 & -9.9 & -1.09 & -4.03 & -4.90 & 1.44 & 2.89 \\
\hline 0.85 & -11.1 & -1.09 & -4.63 & -5.57 & 1.58 & 3.26 \\
\hline 0.90 & -11.8 & -1.11 & $\begin{array}{l}-5.04 \\
\end{array}$ & -6.11 & 1.69 & 3.59 \\
\hline 0.95 & -12.7 & -1.12 & -5.30 & -6.58 & 1.79 & 3.88 \\
\hline 1.00 & -13.5 & -1.13 & -5.71 & -7.25 & 1.91 & 4.23 \\
\hline 1.05 & -14.0 & -1.15 & -5.98 & -7.59 & 1.98 & 4.45 \\
\hline 1.10 & -14.7 & -1.15 & -6.31 & -7.99 & 2.10 & 4.77 \\
\hline 1.15 & -15.7 & -1.16 & -6.71 & -8.80 & 2.22 & 5.10 \\
\hline 1.20 & -16.4 & -1.17 & -7.05 & -9.27 & 2.33 & 5.44 \\
\hline 1.25 & -17.1 & -1.18 & -7.59 & -9.94 & 2.42 & 5.71 \\
\hline 1.30 & -17.9 & -1.19 & -7.92 & -10.27 & 2.53 & 6.08 \\
\hline 1.35 & -18.6 & -1.20 & -8.33 & -11.15 & 2.62 & 6.38 \\
\hline 1.40 & -19.2 & -1.21 & -8.46 & -11.48 & 2.75 & 6.70 \\
\hline 1.45 & -20.3 & -1.23 & -8.93 & -12.09 & 2.90 & 7.17 \\
\hline 1.50 & -21.0 & -1.24 & $\begin{array}{l}-9.33 \\
\end{array}$ & -12.62 & 3.00 & 7.47 \\
\hline 1.55 & -21.7 & -1.24 & -9.74 & -13.23 & 3.11 & 7.84 \\
\hline 1.60 & -22.6 & -1.25 & -9.94 & -13.97 & 3.25 & 8.19 \\
\hline 1.65 & -23.3 & -1.27 & -10.47 & -14.50 & 3.35 & 8.54 \\
\hline 1.70 & -24.1 & -1.28 & -10.74 & -15.11 & 3.50 & 8.96 \\
\hline 1.75 & -24.9 & -1.29 & -11.28 & -15.78 & 3.59 & 9.33 \\
\hline 1.80 & -25.9 & -1.30 & -11.75 & -16.45 & 3.76 & 9.80 \\
\hline 1.85 & -26.8 & -1.31 & -12.09 & -17.12 & 3.89 & 10.24 \\
\hline 1.90 & -27.3 & -1.32 & -12.49 & -17.86 & 4.01 & 10.59 \\
\hline 1.95 & -28.1 & -1.33 & -12.89 & -18.53 & 4.14 & 11.03 \\
\hline 2.00 & -28.7 & -1.34 & -13.29 & -19.07 & 4.26 & 11.41 \\
\hline 2.05 & -29.4 & -1.35 & -13.70 & -19.74 & 4.36 & 11.80 \\
\hline 2.10 & -30.3 & -1.36 & -14.03 & -20.48 & 4.50 & 12.25 \\
\hline 2.15 & -30.9 & -1.37 & -14.37 & -21.02 & 4.62 & 12.66 \\
\hline 2.20 & -32.0 & -1.38 & -14.97 & -21.82 & 4.77 & 13.16 \\
\hline 2.25 & -32.5 & -1.39 & -15.31 & -22.49 & 4.88 & 13.56 \\
\hline
\end{tabular}


Table F.3 IDT Strength Data (KL 52-34 \#5), continued

\begin{tabular}{|c|c|c|c|c|c|c|}
\hline Time & Axial Force & Axial Displ. & Vert front & Vert back & Horz front & Horz back \\
\hline $\mathrm{Sec}$ & $\mathrm{N}$ & $\mathrm{mm}$ & $\mu \mathrm{m}$ & $\mu \mathrm{m}$ & $\mu \mathrm{m}$ & $\mu \mathrm{m}$ \\
\hline 2.30 & -33.2 & -1.40 & -15.71 & -23.16 & 5.01 & 14.03 \\
\hline 2.35 & -34.0 & -1.41 & -16.25 & -23.83 & 5.15 & 14.47 \\
\hline 2.40 & -34.8 & -1.42 & -16.65 & -24.71 & 5.28 & 14.96 \\
\hline 2.45 & -35.5 & -1.43 & -17.05 & -25.38 & 5.42 & 15.43 \\
\hline 2.50 & -36.3 & -1.44 & -17.59 & -26.18 & 5.56 & 15.93 \\
\hline 2.55 & -37.1 & -1.45 & -17.93 & -26.86 & 5.69 & 16.42 \\
\hline 2.60 & -37.7 & -1.46 & -18.40 & -27.53 & 5.84 & 16.92 \\
\hline 2.65 & -38.5 & -1.47 & -18.93 & -28.53 & 5.98 & 17.42 \\
\hline 2.70 & -39.2 & -1.49 & -19.40 & -29.14 & 6.13 & 17.89 \\
\hline 2.75 & -40.0 & -1.49 & -19.94 & -29.94 & 6.29 & 18.46 \\
\hline 2.80 & -40.9 & -1.50 & -20.34 & -30.75 & 6.45 & 19.07 \\
\hline 2.85 & -41.6 & -1.51 & -20.88 & -31.56 & 6.61 & 19.59 \\
\hline 2.90 & -42.4 & -1.53 & -21.42 & -32.50 & 6.75 & 20.14 \\
\hline 2.95 & -43.0 & -1.54 & -21.96 & -33.17 & 6.91 & 20.70 \\
\hline 3.00 & -43.7 & -1.55 & -22.43 & -33.97 & 7.07 & 21.23 \\
\hline 3.05 & -44.2 & -1.56 & -22.89 & -34.85 & 7.23 & 21.75 \\
\hline 3.10 & -45.0 & -1.57 & -23.50 & -35.65 & 7.49 & 22.32 \\
\hline 3.15 & -45.6 & -1.58 & -23.70 & -36.19 & 7.70 & 22.93 \\
\hline 3.20 & -46.3 & -1.59 & -24.44 & -37.20 & 7.87 & 23.50 \\
\hline 3.25 & -47.0 & -1.59 & -25.11 & -38.07 & 8.04 & 24.05 \\
\hline 3.30 & -47.9 & -1.61 & -25.65 & -39.01 & 8.26 & 24.77 \\
\hline 3.35 & -48.6 & -1.62 & -26.25 & -39.88 & 8.43 & 25.41 \\
\hline 3.40 & -49.2 & -1.63 & -26.65 & -40.75 & 8.62 & 26.03 \\
\hline 3.45 & -49.8 & -1.64 & -27.33 & -41.69 & 8.80 & 26.67 \\
\hline 3.50 & -50.4 & -1.65 & -27.80 & -42.50 & 8.98 & 27.31 \\
\hline 3.55 & -50.6 & -1.66 & -28.27 & -43.17 & 9.15 & 27.86 \\
\hline 3.60 & $\begin{array}{l}-51.3 \\
\end{array}$ & -1.67 & -28.87 & -44.11 & 9.32 & 28.55 \\
\hline 3.65 & -52.1 & -1.68 & -29.47 & -44.98 & 9.53 & 29.24 \\
\hline 3.70 & -52.7 & -1.69 & -30.08 & -45.92 & 9.71 & 29.93 \\
\hline 3.75 & -53.1 & -1.70 & -30.55 & -46.80 & 9.89 & 30.57 \\
\hline 3.80 & $\begin{array}{l}-53.8 \\
\end{array}$ & -1.71 & -31.15 & -47.74 & 10.08 & 31.29 \\
\hline 3.85 & -54.4 & -1.72 & -31.76 & -48.68 & 10.28 & 32.04 \\
\hline 3.90 & -55.1 & -1.73 & -32.43 & -49.68 & 10.50 & 32.80 \\
\hline 3.95 & -55.7 & -1.74 & -32.97 & -50.56 & 10.70 & 33.60 \\
\hline 4.00 & -56.2 & -1.76 & -33.64 & -51.56 & 10.90 & 34.36 \\
\hline 4.05 & -56.6 & -1.76 & -34.17 & -52.50 & 11.08 & 35.11 \\
\hline 4.10 & $\begin{array}{l}-57.3 \\
\end{array}$ & -1.77 & -34.85 & -53.44 & 11.30 & 35.92 \\
\hline 4.15 & -58.0 & -1.79 & -35.58 & -54.45 & 11.52 & 36.81 \\
\hline 4.20 & -58.4 & -1.80 & -36.32 & -55.53 & 11.73 & 37.63 \\
\hline 4.25 & $\begin{array}{l}-58.8 \\
\end{array}$ & -1.81 & -36.86 & -56.40 & 11.98 & 38.44 \\
\hline 4.30 & -59.4 & -1.81 & -37.60 & -57.47 & 12.23 & 39.31 \\
\hline 4.35 & -60.1 & -1.82 & -38.34 & -58.68 & 12.45 & 40.27 \\
\hline 4.40 & -60.6 & -1.84 & -39.21 & -59.62 & 12.70 & 41.22 \\
\hline 4.45 & -61.0 & -1.84 & -39.68 & -60.70 & 12.90 & 42.11 \\
\hline 4.50 & $\begin{array}{l}-61.4 \\
\end{array}$ & -1.86 & -40.35 & -61.70 & 13.17 & 43.12 \\
\hline
\end{tabular}


Table F.3 IDT Strength Data (KL 52-34 \#5), continued

\begin{tabular}{|c|c|c|c|c|c|c|}
\hline Time & Axial Force & Axial Displ. & Vert front & Vert back & Horz front & Horz back \\
\hline Sec & $\mathrm{N}$ & $\mathrm{mm}$ & $\mu \mathrm{m}$ & $\mu \mathrm{m}$ & $\mu \mathrm{m}$ & $\mu \mathrm{m}$ \\
\hline 4.55 & -61.9 & -1.86 & -41.22 & -62.91 & 13.44 & 44.13 \\
\hline 4.60 & -62.3 & -1.88 & -41.83 & -63.99 & 13.65 & 45.14 \\
\hline 4.65 & -63.0 & -1.89 & -42.70 & -65.13 & 13.91 & 46.29 \\
\hline 4.70 & -63.6 & -1.90 & -43.51 & -66.34 & 14.19 & 47.42 \\
\hline 4.75 & -64.0 & -1.91 & -44.25 & -67.48 & 14.46 & 48.56 \\
\hline 4.80 & -64.2 & -1.92 & -44.98 & -68.35 & 14.72 & 49.87 \\
\hline 4.85 & -64.7 & -1.93 & -45.72 & -69.69 & 14.98 & 51.31 \\
\hline 4.90 & -65.0 & -1.94 & -46.60 & -70.83 & 15.24 & 52.64 \\
\hline 4.95 & -65.6 & -1.95 & -47.40 & -72.11 & 15.53 & 54.10 \\
\hline 5.00 & -65.9 & -1.96 & -48.27 & -73.18 & 15.79 & 55.44 \\
\hline 5.05 & -66.3 & -1.97 & -49.15 & -74.46 & 16.13 & 56.87 \\
\hline 5.10 & -66.8 & -1.98 & -50.22 & -75.60 & 16.60 & 58.38 \\
\hline 5.16 & -66.8 & -1.99 & -51.63 & -76.81 & 17.37 & 59.79 \\
\hline 5.21 & -67.2 & -2.00 & -52.77 & -79.02 & 18.15 & 61.55 \\
\hline 5.26 & -67.6 & -2.02 & -54.12 & -80.97 & 18.85 & 63.92 \\
\hline 5.31 & -67.4 & -2.03 & -56.94 & -83.32 & 19.06 & 92.99 \\
\hline 5.36 & -66.8 & -2.04 & -61.30 & -89.36 & 22.18 & 129.58 \\
\hline 5.41 & -65.2 & -2.05 & -70.90 & -106.75 & 36.79 & 190.19 \\
\hline 5.46 & -61.4 & -2.06 & -81.58 & -132.40 & 70.88 & 268.29 \\
\hline
\end{tabular}


Table F.4 IDT Strength Data (KL 58-40 \#1)

\begin{tabular}{|c|c|c|c|c|c|c|}
\hline Time & Axial Force & Axial Displ. & Vert front & Vert back & Horz front & Horz back \\
\hline $\mathrm{Sec}$ & $\mathrm{kN}$ & $\mathrm{mm}$ & $\mu \mathrm{m}$ & $\mu \mathrm{m}$ & $\mu \mathrm{m}$ & $\mu \mathrm{m}$ \\
\hline 0.00 & -0.2 & -0.81 & 0.81 & 0.47 & 0.16 & 0.03 \\
\hline 0.05 & -0.4 & -0.82 & 0.67 & 0.27 & 0.19 & 0.12 \\
\hline 0.10 & -0.7 & -0.83 & 0.54 & 0.20 & 0.22 & 0.17 \\
\hline 0.15 & -1.1 & -0.84 & 0.34 & 0.00 & 0.28 & 0.27 \\
\hline 0.20 & -1.8 & -0.85 & 0.00 & -0.27 & 0.36 & 0.45 \\
\hline 0.25 & -2.4 & -0.86 & -0.34 & -0.54 & 0.44 & 0.60 \\
\hline 0.30 & -3.0 & -0.87 & -0.67 & -0.81 & 0.52 & 0.79 \\
\hline 0.35 & -3.5 & -0.88 & -1.01 & -1.07 & 0.63 & 0.96 \\
\hline 0.40 & -4.2 & -0.89 & -1.48 & -1.41 & 0.76 & 1.21 \\
\hline 0.45 & -4.9 & -0.90 & -1.88 & -1.75 & 0.86 & 1.36 \\
\hline 0.50 & -5.6 & -0.91 & -2.35 & -2.15 & 0.97 & 1.61 \\
\hline 0.55 & -6.4 & -0.92 & -2.69 & -2.28 & 1.11 & 1.90 \\
\hline 0.60 & $\begin{array}{l}-7.1 \\
\end{array}$ & -0.93 & -3.36 & -3.02 & 1.23 & 2.11 \\
\hline 0.65 & -7.9 & -0.94 & -3.83 & -3.49 & 1.36 & 2.37 \\
\hline 0.70 & -8.7 & -0.95 & -4.30 & -3.89 & 1.50 & 2.69 \\
\hline 0.75 & -9.5 & -0.97 & -4.83 & -4.43 & 1.65 & 3.00 \\
\hline 0.80 & -10.1 & -0.97 & -5.24 & -4.83 & 1.77 & 3.24 \\
\hline 0.85 & -11.3 & -0.99 & -5.98 & -5.51 & 1.96 & 3.66 \\
\hline 0.90 & -12.0 & -1.00 & -6.58 & -6.11 & 2.11 & 3.98 \\
\hline 0.95 & -12.9 & -1.01 & -7.18 & -6.65 & 2.28 & 4.36 \\
\hline 1.00 & -13.4 & -1.02 & -7.72 & -7.12 & 2.42 & 4.63 \\
\hline 1.05 & -14.1 & -1.02 & -8.26 & -7.65 & 2.56 & 4.93 \\
\hline 1.10 & -14.6 & -1.04 & -8.73 & -8.06 & 2.69 & 5.25 \\
\hline 1.15 & -15.5 & -1.04 & -9.27 & -8.66 & 2.89 & 5.64 \\
\hline 1.20 & -16.2 & -1.06 & -9.80 & -9.27 & 3.02 & 5.94 \\
\hline 1.25 & -17.1 & -1.07 & -10.54 & -9.94 & 3.23 & 6.36 \\
\hline 1.30 & -17.8 & -1.08 & -11.21 & -10.47 & 3.40 & 6.75 \\
\hline 1.35 & -18.6 & -1.09 & -11.82 & -11.01 & 3.60 & 7.12 \\
\hline 1.40 & -19.1 & -1.10 & -12.42 & -11.62 & 3.76 & 7.44 \\
\hline 1.45 & -19.8 & -1.11 & -12.89 & -12.29 & 3.93 & 7.87 \\
\hline 1.50 & -20.6 & -1.12 & -13.70 & -12.69 & 4.13 & 8.24 \\
\hline 1.55 & -21.1 & -1.12 & -14.37 & -13.36 & 4.30 & 8.59 \\
\hline 1.60 & -21.7 & -1.14 & -14.97 & -13.90 & 4.46 & 8.96 \\
\hline 1.65 & -22.4 & -1.15 & -15.58 & -14.57 & 4.65 & 9.33 \\
\hline 1.70 & -23.2 & -1.16 & -16.32 & -15.17 & 4.86 & 9.75 \\
\hline 1.75 & -23.9 & -1.17 & -16.85 & -15.85 & 5.06 & 10.19 \\
\hline 1.80 & -24.4 & -1.18 & -17.59 & -16.45 & 5.25 & 10.59 \\
\hline 1.85 & -25.1 & -1.19 & -18.33 & -17.12 & 5.46 & 11.01 \\
\hline 1.90 & -25.8 & -1.20 & -19.00 & -17.73 & 5.66 & 11.45 \\
\hline 1.95 & -26.5 & -1.21 & -19.74 & -18.40 & 5.88 & 11.88 \\
\hline 2.00 & -27.5 & -1.22 & -20.55 & -19.07 & 6.11 & 12.42 \\
\hline 2.05 & -28.0 & -1.24 & -21.22 & -19.87 & 6.34 & 12.89 \\
\hline 2.10 & -28.8 & -1.25 & -22.22 & -20.68 & 6.56 & 13.34 \\
\hline 2.15 & -29.2 & -1.26 & -22.76 & -21.22 & 6.78 & 13.78 \\
\hline 2.20 & -29.9 & -1.27 & -23.50 & -21.82 & 7.01 & 14.23 \\
\hline 2.25 & -30.4 & -1.28 & -24.30 & -22.63 & 7.21 & 14.70 \\
\hline
\end{tabular}


Table F.4 IDT Strength Data (KL 58-40 \#1), continued

\begin{tabular}{|c|c|c|c|c|c|c|}
\hline Time & Axial Force & Axial Displ. & Vert front & Vert back & Horz front & Horz back \\
\hline $\mathrm{Sec}$ & $\mathrm{kN}$ & $\mathrm{mm}$ & $\mu \mathrm{m}$ & $\mu \mathrm{m}$ & $\mu \mathrm{m}$ & $\mu \mathrm{m}$ \\
\hline 2.30 & -31.2 & -1.29 & -25.04 & -23.36 & 7.48 & 15.22 \\
\hline 2.35 & -31.9 & -1.30 & -25.98 & -24.10 & 7.71 & 15.71 \\
\hline 2.40 & -32.7 & -1.31 & -26.92 & -24.91 & 7.99 & 16.26 \\
\hline 2.45 & -33.3 & -1.32 & -27.73 & -25.65 & 8.25 & 16.80 \\
\hline 2.50 & -33.9 & -1.33 & -28.47 & -26.12 & 8.51 & 17.37 \\
\hline 2.55 & -34.6 & -1.34 & -29.47 & -27.39 & 8.76 & 17.84 \\
\hline 2.60 & -35.3 & -1.35 & -30.28 & -28.00 & 9.03 & 18.43 \\
\hline 2.65 & -36.0 & -1.36 & -31.35 & -28.80 & 9.32 & 19.03 \\
\hline 2.70 & -36.2 & -1.37 & -32.16 & -29.47 & 9.53 & 19.45 \\
\hline 2.75 & -36.9 & -1.38 & -32.97 & -30.35 & 9.81 & 20.06 \\
\hline 2.80 & -37.6 & -1.39 & -33.77 & -31.09 & 10.10 & 20.63 \\
\hline 2.85 & -38.1 & -1.40 & -34.78 & -31.96 & 10.38 & 21.18 \\
\hline 2.90 & -38.7 & -1.41 & -35.72 & -32.90 & 10.68 & 21.77 \\
\hline 2.95 & -39.2 & -1.43 & -36.59 & -33.64 & 10.94 & 22.36 \\
\hline 3.00 & -39.8 & -1.43 & -37.60 & -34.44 & 11.25 & 22.93 \\
\hline 3.05 & -40.0 & -1.44 & -38.34 & -35.18 & 11.50 & 23.47 \\
\hline 3.10 & -40.8 & -1.45 & -39.34 & -36.19 & 11.82 & 24.10 \\
\hline 3.15 & -41.5 & -1.46 & -40.35 & -36.93 & 12.14 & 24.76 \\
\hline 3.20 & -42.3 & -1.47 & -41.63 & -38.07 & 12.50 & 25.50 \\
\hline 3.25 & -43.1 & -1.48 & -42.70 & -39.01 & 12.85 & 26.22 \\
\hline 3.30 & -43.3 & -1.49 & -43.57 & -39.88 & 13.13 & 26.81 \\
\hline 3.35 & -43.6 & -1.51 & -44.51 & -40.62 & 13.44 & 27.41 \\
\hline 3.40 & -44.2 & -1.52 & -45.59 & -41.49 & 13.77 & 28.08 \\
\hline 3.45 & -44.6 & -1.52 & -46.73 & -42.63 & 14.07 & 28.69 \\
\hline 3.50 & -45.1 & -1.54 & -47.60 & -43.24 & 14.43 & 29.39 \\
\hline 3.55 & -45.9 & -1.54 & -48.74 & -44.31 & 14.80 & 30.13 \\
\hline 3.60 & -46.4 & -1.56 & -49.95 & -45.32 & 15.17 & 30.85 \\
\hline 3.65 & -47.1 & -1.57 & -51.09 & -46.33 & 15.56 & 31.62 \\
\hline 3.70 & -47.4 & -1.58 & -52.24 & -47.33 & 15.91 & 32.33 \\
\hline 3.75 & -47.8 & -1.59 & -53.31 & -48.27 & 16.27 & 33.05 \\
\hline 3.80 & -48.3 & -1.60 & -54.45 & -49.15 & 16.63 & 33.79 \\
\hline 3.85 & -48.7 & -1.61 & -55.59 & -50.09 & 17.04 & 34.53 \\
\hline 3.90 & -49.4 & -1.62 & -56.80 & -51.30 & 17.43 & 35.33 \\
\hline 3.95 & -49.8 & -1.63 & -58.01 & -52.24 & 17.83 & 36.14 \\
\hline 4.00 & -50.3 & -1.64 & -59.22 & -53.24 & 18.24 & 36.93 \\
\hline 4.05 & -50.8 & -1.65 & -60.43 & -54.32 & 18.67 & 37.75 \\
\hline 4.10 & -51.4 & -1.66 & -61.77 & -55.46 & 19.08 & 38.59 \\
\hline 4.15 & -51.8 & -1.67 & -62.98 & -56.47 & 19.50 & 39.41 \\
\hline 4.20 & -52.3 & -1.69 & -64.32 & -57.67 & 19.97 & 40.28 \\
\hline 4.25 & -52.6 & -1.69 & -65.60 & -58.68 & 20.39 & 41.09 \\
\hline 4.30 & -53.1 & -1.70 & -66.94 & -59.76 & 20.83 & 41.96 \\
\hline 4.35 & -53.5 & -1.71 & -68.28 & -60.90 & 21.29 & 42.84 \\
\hline 4.40 & -54.0 & -1.72 & -69.83 & -62.24 & 21.75 & 43.69 \\
\hline 4.45 & -54.1 & -1.73 & -70.90 & -63.11 & 22.20 & 44.55 \\
\hline 4.50 & -54.8 & -1.74 & -72.31 & -64.25 & 22.72 & 45.50 \\
\hline
\end{tabular}


Table F.4 IDT Strength Data (KL 58-40 \#1), continued

\begin{tabular}{|c|c|c|c|c|c|c|}
\hline Time & Axial Force & Axial Displ. & Vert front & Vert back & Horz front & Horz back \\
\hline $\mathrm{Sec}$ & $\mathrm{kN}$ & $\mathrm{mm}$ & $\mu \mathrm{m}$ & $\mu \mathrm{m}$ & $\mu \mathrm{m}$ & $\mu \mathrm{m}$ \\
\hline 4.55 & -55.1 & -1.76 & -73.65 & -65.46 & 23.20 & 46.39 \\
\hline 4.60 & -55.5 & -1.77 & -75.00 & -66.60 & 23.70 & 47.35 \\
\hline 4.65 & -55.9 & -1.78 & -76.41 & -67.68 & 24.20 & 48.27 \\
\hline 4.70 & -56.0 & -1.78 & -77.82 & -68.75 & 24.67 & 49.15 \\
\hline 4.75 & -56.6 & -1.80 & -79.23 & -70.03 & 25.20 & 50.14 \\
\hline 4.80 & -57.0 & -1.81 & -80.77 & -71.10 & 25.71 & 51.09 \\
\hline 4.85 & -57.3 & -1.82 & -82.25 & -72.51 & 26.23 & 52.05 \\
\hline 4.90 & -57.6 & -1.83 & -83.66 & -73.52 & 26.76 & 53.04 \\
\hline 4.95 & -58.0 & -1.84 & -85.07 & -74.53 & 27.33 & 54.07 \\
\hline 5.00 & -58.5 & -1.85 & -86.81 & -76.00 & 27.89 & 55.07 \\
\hline 5.05 & -59.0 & -1.86 & -88.29 & -77.28 & 28.49 & 56.20 \\
\hline 5.10 & -59.0 & -1.87 & -89.77 & -78.49 & 29.03 & 57.19 \\
\hline 5.16 & -59.5 & -1.88 & -91.38 & -79.76 & 29.62 & 58.24 \\
\hline 5.21 & -60.0 & -1.89 & -93.06 & -81.11 & 30.26 & 59.40 \\
\hline 5.26 & -60.1 & -1.90 & -94.60 & -82.52 & 30.85 & 60.48 \\
\hline 5.31 & -60.7 & -1.91 & -96.28 & -83.72 & 31.54 & 61.64 \\
\hline 5.36 & -60.9 & -1.93 & -97.96 & -85.07 & 32.17 & 62.78 \\
\hline 5.41 & -61.2 & -1.93 & -99.57 & -86.48 & 32.82 & 63.93 \\
\hline 5.46 & -61.5 & -1.94 & -101.32 & -87.75 & 33.47 & 65.13 \\
\hline 5.51 & -61.7 & -1.95 & -102.99 & -89.23 & 34.17 & 66.28 \\
\hline 5.56 & -62.2 & -1.96 & -104.81 & -90.64 & 34.88 & 67.59 \\
\hline 5.61 & -62.4 & -1.97 & -106.55 & -91.92 & 35.60 & 68.82 \\
\hline 5.66 & -62.9 & -1.98 & -108.50 & -93.53 & 36.35 & 70.13 \\
\hline 5.71 & -63.2 & -1.99 & -110.18 & -94.94 & 37.10 & 71.49 \\
\hline 5.76 & -63.7 & -2.01 & -112.19 & -96.48 & 37.90 & 72.90 \\
\hline 5.81 & -63.8 & -2.02 & -113.87 & -97.96 & 38.69 & 74.27 \\
\hline 5.86 & -63.8 & -2.03 & -115.75 & -99.23 & 39.45 & 75.62 \\
\hline 5.91 & -64.2 & -2.04 & -117.83 & -101.05 & 40.24 & 76.99 \\
\hline 5.96 & -64.6 & -2.05 & -119.64 & -102.46 & 41.12 & 78.54 \\
\hline 6.01 & -64.8 & -2.06 & -121.52 & -104.00 & 41.98 & 80.10 \\
\hline 6.06 & -64.8 & -2.07 & -123.34 & -105.41 & 42.78 & 81.54 \\
\hline 6.11 & -65.2 & -2.08 & -125.28 & -107.09 & 43.67 & 83.07 \\
\hline 6.16 & -65.3 & -2.09 & -127.16 & -108.70 & 44.56 & 84.65 \\
\hline 6.21 & -65.6 & -2.10 & -129.18 & -110.51 & 45.48 & 86.28 \\
\hline 6.26 & -66.0 & -2.11 & -131.13 & -112.13 & 46.45 & 87.95 \\
\hline 6.31 & -66.2 & -2.12 & -133.27 & -113.94 & 47.40 & 89.68 \\
\hline 6.36 & -66.3 & -2.13 & -135.15 & -115.62 & 48.40 & 91.41 \\
\hline 6.41 & -66.3 & -2.14 & -137.03 & -117.23 & 49.39 & 93.09 \\
\hline 6.46 & -66.4 & -2.15 & -139.12 & -119.17 & 50.38 & 94.75 \\
\hline 6.51 & -66.7 & -2.16 & -141.13 & -120.85 & 51.45 & 96.65 \\
\hline 6.56 & -66.9 & -2.17 & -143.14 & -122.53 & 52.54 & 98.50 \\
\hline 6.61 & -67.1 & -2.18 & -145.16 & -124.34 & 53.64 & 100.41 \\
\hline 6.66 & -67.4 & -2.19 & -147.44 & -126.29 & 54.83 & 102.47 \\
\hline 6.71 & -67.6 & -2.20 & -149.46 & -128.37 & 56.00 & 104.49 \\
\hline 6.76 & -67.8 & -2.21 & -151.60 & -130.32 & 57.25 & 106.59 \\
\hline
\end{tabular}


Table F.4 IDT Strength Data (KL 58-40 \#1), continued

\begin{tabular}{|c|c|c|c|c|c|c|}
\hline Time & Axial Force & Axial Displ. & Vert front & Vert back & Horz front & Horz back \\
\hline $\mathrm{Sec}$ & $\mathrm{kN}$ & $\mathrm{mm}$ & $\mu \mathrm{m}$ & $\mu \mathrm{m}$ & $\mu \mathrm{m}$ & $\mu \mathrm{m}$ \\
\hline 6.81 & -68.1 & -2.23 & -153.89 & -132.47 & 58.52 & 108.78 \\
\hline 6.86 & -68.2 & -2.24 & -156.17 & -134.55 & 59.81 & 111.00 \\
\hline 6.91 & -68.3 & -2.25 & -158.25 & -136.56 & 61.15 & 113.23 \\
\hline 6.96 & -68.5 & -2.25 & -160.47 & -138.58 & 62.51 & 115.53 \\
\hline 7.01 & -68.6 & -2.26 & -162.82 & -140.59 & 63.92 & 117.95 \\
\hline 7.06 & -68.8 & -2.28 & -164.96 & -142.67 & 65.35 & 120.37 \\
\hline 7.11 & -69.0 & -2.29 & -167.31 & -145.09 & 66.86 & 122.88 \\
\hline 7.16 & -69.2 & -2.30 & -169.66 & -147.17 & 68.42 & 125.55 \\
\hline 7.21 & -69.4 & -2.31 & -172.15 & -149.52 & 70.04 & 128.36 \\
\hline 7.26 & -69.6 & -2.32 & -174.63 & -151.87 & 71.71 & 131.18 \\
\hline 7.31 & -69.5 & -2.33 & -176.92 & -154.09 & 73.42 & 134.03 \\
\hline 7.36 & -69.8 & -2.34 & -179.47 & -156.37 & 75.21 & 137.12 \\
\hline 7.41 & -69.7 & -2.35 & -181.88 & -158.72 & 77.05 & 140.16 \\
\hline 7.46 & -69.8 & -2.36 & -184.37 & -161.07 & 78.97 & 143.33 \\
\hline 7.51 & -70.0 & -2.37 & -186.92 & -163.62 & 81.01 & 146.75 \\
\hline 7.56 & -69.8 & -2.38 & -189.54 & -166.04 & 83.09 & 150.16 \\
\hline 7.61 & -70.0 & -2.39 & -192.22 & -168.46 & 85.31 & 153.80 \\
\hline 7.66 & -70.2 & -2.41 & -195.11 & -171.34 & 87.68 & 157.58 \\
\hline 7.71 & -70.1 & -2.42 & -197.86 & -173.96 & 90.15 & 161.52 \\
\hline 7.76 & -70.0 & -2.42 & -200.82 & -176.71 & 92.73 & 165.55 \\
\hline 7.81 & -70.2 & -2.43 & -203.84 & -179.53 & 95.53 & 169.83 \\
\hline 7.86 & -70.2 & -2.44 & -206.73 & -182.62 & 98.49 & 174.26 \\
\hline 7.91 & -70.3 & -2.45 & -209.75 & -185.58 & 101.67 & 178.95 \\
\hline 7.96 & -70.0 & -2.47 & -212.63 & -188.73 & 105.00 & 183.75 \\
\hline 8.01 & -70.1 & -2.48 & -215.86 & -192.09 & 108.62 & 188.83 \\
\hline 8.06 & -69.8 & -2.49 & -218.81 & -195.38 & 112.44 & 194.07 \\
\hline 8.11 & -70.0 & -2.50 & -221.97 & -198.94 & 116.72 & 199.81 \\
\hline 8.16 & -69.9 & -2.51 & -224.99 & -202.56 & 121.35 & 205.90 \\
\hline 8.21 & -69.8 & -2.52 & -228.08 & -206.39 & 126.38 & 212.40 \\
\hline 8.26 & -69.5 & -2.53 & -230.70 & -210.35 & 131.94 & 219.58 \\
\hline 8.31 & -69.1 & -2.54 & -233.31 & -214.51 & 138.01 & 227.10 \\
\hline 8.36 & -68.7 & -2.55 & -235.80 & -219.68 & 144.84 & 237.66 \\
\hline 8.41 & -68.3 & -2.56 & -238.08 & -225.26 & 152.81 & 248.64 \\
\hline 8.46 & -67.5 & -2.57 & -240.30 & -232.84 & 161.83 & 271.95 \\
\hline 8.51 & -64.4 & -2.59 & -244.86 & -253.12 & 179.95 & 373.30 \\
\hline
\end{tabular}


Table F.5 IDT Strength Data (KL 58-40 \#10)

\begin{tabular}{|c|c|c|c|c|c|c|}
\hline Time & Axial Force & Axial Displ. & Vert front & Vert back & Horz front & Horz back \\
\hline $\mathrm{Sec}$ & $\mathrm{kN}$ & $\mathrm{mm}$ & $\mu \mathrm{m}$ & $\mu \mathrm{m}$ & $\mu \mathrm{m}$ & $\mu \mathrm{m}$ \\
\hline 0.00 & -0.4 & -0.36 & 1.07 & 0.60 & 0.18 & 0.15 \\
\hline 0.05 & -0.7 & -0.37 & 0.94 & 0.20 & 0.20 & 0.30 \\
\hline 0.10 & -1.1 & -0.38 & 1.07 & -0.13 & 0.24 & 0.50 \\
\hline 0.15 & -1.7 & -0.39 & 0.94 & -0.74 & 0.27 & 0.79 \\
\hline 0.20 & -2.2 & -0.40 & 0.81 & -1.14 & 0.33 & 1.04 \\
\hline 0.25 & -2.6 & -0.41 & 0.74 & -1.61 & 0.38 & 1.24 \\
\hline 0.30 & -3.3 & -0.42 & 0.47 & -2.22 & 0.45 & 1.54 \\
\hline 0.35 & -4.0 & -0.44 & 0.13 & -2.69 & 0.54 & 1.86 \\
\hline 0.40 & -4.7 & -0.45 & -0.07 & -3.36 & 0.63 & 2.18 \\
\hline 0.45 & -5.4 & -0.46 & -0.40 & -3.83 & 0.74 & 2.53 \\
\hline 0.50 & -6.2 & -0.47 & -0.67 & -4.77 & 0.84 & 2.95 \\
\hline 0.55 & -7.0 & -0.48 & -1.21 & -5.44 & 0.96 & 3.31 \\
\hline 0.60 & -7.8 & -0.49 & -1.41 & -6.04 & 1.10 & 3.68 \\
\hline 0.65 & -8.5 & -0.50 & -1.68 & -6.65 & 1.23 & 4.10 \\
\hline 0.70 & -9.0 & -0.51 & -2.15 & -7.25 & 1.34 & 4.46 \\
\hline 0.75 & -9.7 & -0.51 & -2.62 & -8.06 & 1.50 & 4.87 \\
\hline 0.80 & -10.5 & -0.53 & -3.09 & -8.73 & 1.61 & 5.25 \\
\hline 0.85 & -11.2 & -0.53 & -3.36 & -9.40 & 1.76 & 5.64 \\
\hline 0.90 & -11.8 & -0.55 & -3.76 & -10.07 & 1.91 & 6.04 \\
\hline 0.95 & -12.7 & -0.56 & -4.23 & -10.88 & 2.08 & 6.55 \\
\hline 1.00 & -13.4 & -0.57 & -4.90 & -11.75 & 2.22 & 6.93 \\
\hline 1.05 & -14.1 & -0.58 & -5.24 & -12.35 & 2.39 & 7.39 \\
\hline 1.10 & -14.8 & -0.59 & -5.77 & -13.09 & 2.58 & 7.89 \\
\hline 1.15 & -15.5 & -0.60 & -6.24 & -13.90 & 2.75 & 8.38 \\
\hline 1.20 & -16.3 & -0.61 & -6.78 & -14.70 & 2.93 & 8.83 \\
\hline 1.25 & -16.8 & -0.62 & -7.25 & -15.51 & 3.09 & 9.30 \\
\hline 1.30 & -17.5 & -0.63 & -7.79 & -16.32 & 3.28 & 9.77 \\
\hline 1.35 & -18.2 & -0.64 & -8.39 & -17.05 & 3.47 & 10.27 \\
\hline 1.40 & -19.2 & -0.65 & -9.00 & -17.93 & 3.68 & 10.83 \\
\hline 1.45 & -20.0 & -0.66 & -9.60 & -18.67 & 3.94 & 11.45 \\
\hline 1.50 & -20.8 & -0.67 & -10.27 & -19.81 & 4.15 & 11.97 \\
\hline 1.55 & -21.4 & -0.69 & -11.01 & -20.61 & 4.34 & 12.49 \\
\hline 1.60 & -21.9 & -0.70 & -11.55 & -21.49 & 4.56 & 12.99 \\
\hline 1.65 & -22.7 & -0.70 & -12.22 & -22.43 & 4.78 & 13.56 \\
\hline 1.70 & -23.3 & -0.71 & -12.76 & -23.16 & 5.01 & 14.08 \\
\hline 1.75 & -24.3 & -0.72 & -13.70 & -24.37 & 5.25 & 14.79 \\
\hline 1.80 & -24.8 & -0.74 & -14.30 & -25.24 & 5.49 & 15.27 \\
\hline 1.85 & -25.2 & -0.75 & -14.84 & -25.92 & 5.69 & 15.79 \\
\hline 1.90 & -26.1 & -0.76 & -15.64 & -26.99 & 5.97 & 16.50 \\
\hline 1.95 & -26.8 & -0.76 & -16.38 & -27.86 & 6.22 & 17.07 \\
\hline 2.00 & -27.6 & -0.78 & -17.19 & -29.00 & 6.50 & 17.74 \\
\hline 2.05 & -28.0 & -0.79 & -17.86 & -29.61 & 6.74 & 18.31 \\
\hline 2.10 & -28.6 & -0.80 & -18.53 & -30.75 & 6.98 & 18.93 \\
\hline 2.15 & -29.4 & -0.81 & -19.34 & -31.69 & 7.28 & 19.62 \\
\hline 2.20 & -30.1 & -0.82 & -20.28 & -32.76 & 7.54 & 20.29 \\
\hline 2.25 & -31.1 & -0.82 & -21.02 & -33.91 & 7.86 & 21.05 \\
\hline
\end{tabular}


Table F.5 IDT Strength Data (KL 58-40 \#10), continued

\begin{tabular}{|c|c|c|c|c|c|c|}
\hline Time & Axial Force & Axial Displ. & Vert front & Vert back & Horz front & Horz back \\
\hline $\mathrm{Sec}$ & $\mathrm{kN}$ & $\mathrm{mm}$ & $\mu \mathrm{m}$ & $\mu \mathrm{m}$ & $\mu \mathrm{m}$ & $\mu \mathrm{m}$ \\
\hline 2.30 & -31.6 & -0.84 & -21.89 & -34.98 & 8.16 & 21.70 \\
\hline 2.35 & -32.2 & -0.85 & -22.69 & -35.99 & 8.44 & 22.41 \\
\hline 2.40 & -32.5 & -0.86 & -23.70 & -37.26 & 8.67 & 22.95 \\
\hline 2.45 & -33.3 & -0.87 & -24.44 & -38.00 & 8.99 & 23.70 \\
\hline 2.50 & -33.9 & -0.88 & -25.04 & -38.94 & 9.29 & 24.44 \\
\hline 2.55 & -34.7 & -0.89 & -26.05 & -40.15 & 9.63 & 25.23 \\
\hline 2.60 & -35.4 & -0.90 & -27.06 & -41.29 & 9.94 & 26.00 \\
\hline 2.65 & -35.9 & -0.92 & -27.80 & -42.37 & 10.26 & 26.72 \\
\hline 2.70 & -36.4 & -0.92 & -28.74 & -43.37 & 10.57 & 27.43 \\
\hline 2.75 & -36.9 & -0.94 & -29.61 & -44.58 & 10.87 & 28.15 \\
\hline 2.80 & -37.7 & -0.94 & -30.62 & -45.79 & 11.23 & 28.99 \\
\hline 2.85 & -38.3 & -0.95 & -31.56 & -46.86 & 11.57 & 29.81 \\
\hline 2.90 & -38.8 & -0.97 & -32.50 & -48.14 & 11.88 & 30.60 \\
\hline 2.95 & -39.2 & -0.97 & -33.23 & -49.01 & 12.22 & 31.34 \\
\hline 3.00 & -39.7 & -0.99 & -34.17 & -50.15 & 12.54 & 32.11 \\
\hline 3.05 & -40.4 & -1.00 & -35.18 & -51.43 & 12.92 & 32.95 \\
\hline 3.10 & -41.0 & -1.01 & -36.19 & -52.77 & 13.29 & 33.82 \\
\hline 3.15 & -41.5 & -1.02 & -37.20 & -53.85 & 13.62 & 34.68 \\
\hline 3.20 & -42.1 & -1.03 & -38.27 & -55.19 & 14.01 & 35.53 \\
\hline 3.25 & -42.6 & -1.04 & -39.28 & -56.60 & 14.38 & 36.42 \\
\hline 3.30 & -43.2 & -1.05 & -40.28 & -58.28 & 14.75 & 37.31 \\
\hline 3.35 & -43.7 & -1.06 & -41.49 & -59.55 & 15.12 & 38.14 \\
\hline 3.40 & -44.3 & -1.07 & -42.43 & -60.83 & 15.53 & 39.08 \\
\hline 3.45 & -44.8 & -1.08 & -43.44 & -62.11 & 15.92 & 40.03 \\
\hline 3.50 & -45.5 & -1.09 & -44.58 & -63.45 & 16.34 & 41.02 \\
\hline 3.55 & -45.9 & -1.10 & -45.59 & -64.72 & 16.74 & 41.95 \\
\hline 3.60 & -46.3 & -1.11 & -46.80 & -66.07 & 17.13 & 42.87 \\
\hline 3.65 & -46.7 & -1.12 & -47.80 & -67.34 & 17.52 & 43.79 \\
\hline 3.70 & -47.2 & -1.13 & -49.01 & -68.68 & 17.94 & 44.78 \\
\hline 3.75 & -47.4 & -1.14 & -50.02 & -69.89 & 18.32 & 45.64 \\
\hline 3.80 & -48.1 & -1.15 & -51.30 & -71.57 & 18.74 & 46.65 \\
\hline 3.85 & -48.5 & -1.16 & -52.37 & -72.78 & 19.18 & 47.65 \\
\hline 3.90 & -49.0 & -1.17 & -53.44 & -74.19 & 19.60 & 48.66 \\
\hline 3.95 & -49.6 & -1.19 & -54.72 & -75.60 & 20.05 & 49.70 \\
\hline 4.00 & -50.0 & -1.20 & -55.79 & -76.94 & 20.50 & 50.76 \\
\hline 4.05 & -50.5 & -1.21 & -57.00 & -78.35 & 20.95 & 51.80 \\
\hline 4.10 & -51.0 & -1.22 & -58.28 & -79.90 & 21.39 & 52.92 \\
\hline 4.15 & -51.4 & -1.23 & -59.49 & -81.37 & 21.86 & 54.01 \\
\hline 4.20 & -51.8 & -1.24 & -60.70 & -82.85 & 22.31 & 55.07 \\
\hline 4.25 & -52.0 & -1.24 & -61.90 & -84.26 & 22.74 & 56.16 \\
\hline 4.30 & -52.7 & -1.26 & -63.11 & -85.60 & 23.25 & 57.37 \\
\hline 4.35 & -53.0 & -1.27 & -64.46 & -87.28 & 23.70 & 58.50 \\
\hline 4.40 & -53.4 & -1.27 & -65.73 & -88.83 & 24.16 & 59.62 \\
\hline 4.45 & -54.1 & -1.29 & -67.07 & -90.44 & 24.67 & 60.93 \\
\hline 4.50 & -54.2 & -1.30 & -68.35 & -91.85 & 25.14 & 62.11 \\
\hline
\end{tabular}


Table F.5 IDT Strength Data (KL 58-40 \#10), continued

\begin{tabular}{|c|c|c|c|c|c|c|}
\hline Time & Axial Force & Axial Displ. & Vert front & Vert back & Horz front & Horz back \\
\hline $\mathrm{Sec}$ & $\mathrm{kN}$ & $\mathrm{mm}$ & $\mu \mathrm{m}$ & $\mu \mathrm{m}$ & $\mu \mathrm{m}$ & $\mu \mathrm{m}$ \\
\hline 4.55 & -54.4 & -1.31 & -69.49 & -93.26 & 25.60 & 63.25 \\
\hline 4.60 & -55.0 & -1.32 & -70.90 & -94.87 & 26.11 & 64.52 \\
\hline 4.65 & -55.4 & -1.33 & -72.38 & -96.48 & 26.60 & 65.83 \\
\hline 4.70 & -55.7 & -1.34 & -73.59 & -98.16 & 27.10 & 67.06 \\
\hline 4.75 & -56.1 & -1.35 & -74.93 & -99.64 & 27.63 & 68.40 \\
\hline 4.80 & -56.6 & -1.36 & -76.47 & -101.52 & 28.16 & 69.73 \\
\hline 4.85 & -56.7 & -1.37 & -77.75 & -102.99 & 28.65 & 71.00 \\
\hline 4.90 & -56.9 & -1.38 & -79.09 & -104.47 & 29.16 & 72.31 \\
\hline 4.95 & -57.3 & -1.39 & -80.30 & -106.02 & 29.68 & 73.67 \\
\hline 5.00 & -57.7 & -1.40 & -81.78 & -107.76 & 30.23 & 75.05 \\
\hline 5.05 & -58.3 & -1.41 & -83.32 & -109.64 & 30.81 & 76.54 \\
\hline 5.10 & -58.7 & -1.42 & -84.80 & -111.39 & 31.39 & 78.08 \\
\hline 5.16 & -59.0 & -1.43 & -86.28 & -113.20 & 31.96 & 79.54 \\
\hline 5.21 & -59.5 & -1.44 & -87.75 & -115.01 & 32.55 & 81.14 \\
\hline 5.26 & -59.8 & -1.45 & -89.30 & -116.82 & 33.14 & 82.70 \\
\hline 5.31 & -60.2 & -1.46 & -90.91 & -118.84 & 33.74 & 84.28 \\
\hline 5.36 & -60.4 & -1.47 & -92.39 & -120.58 & 34.33 & 85.81 \\
\hline 5.41 & -60.5 & -1.48 & -93.80 & -122.20 & 34.91 & 87.33 \\
\hline 5.46 & -60.9 & -1.50 & -95.34 & -124.01 & 35.53 & 88.96 \\
\hline 5.51 & -61.2 & -1.51 & -96.95 & -125.89 & 36.15 & 90.62 \\
\hline 5.56 & -61.5 & -1.52 & -98.50 & -127.84 & 36.78 & 92.27 \\
\hline 5.61 & -61.8 & -1.52 & -100.04 & -129.72 & 37.41 & 93.96 \\
\hline 5.66 & -62.1 & -1.54 & -101.65 & -131.60 & 38.08 & 95.74 \\
\hline 5.71 & -62.5 & -1.55 & -103.40 & -133.61 & 38.75 & 97.51 \\
\hline 5.76 & -62.8 & -1.56 & -105.08 & -135.56 & 39.41 & 99.32 \\
\hline 5.81 & -62.9 & -1.57 & -106.69 & -137.44 & 40.07 & 101.13 \\
\hline 5.86 & -63.2 & -1.58 & -108.37 & -139.52 & 40.76 & 102.98 \\
\hline 5.91 & -63.4 & -1.59 & -109.91 & -141.26 & 41.42 & 104.79 \\
\hline 5.96 & -63.7 & -1.60 & -111.52 & -143.35 & 42.15 & 106.72 \\
\hline 6.01 & -64.1 & -1.61 & -113.27 & -145.43 & 42.87 & 108.70 \\
\hline 6.06 & -64.2 & -1.62 & -115.08 & -147.44 & 43.57 & 110.68 \\
\hline 6.11 & -64.5 & -1.63 & -116.69 & -149.46 & 44.32 & 112.71 \\
\hline 6.16 & -64.7 & -1.64 & -118.57 & -151.60 & 45.05 & 114.74 \\
\hline 6.21 & -65.1 & -1.65 & -120.45 & -153.95 & 45.82 & 116.89 \\
\hline 6.26 & -65.3 & -1.66 & -122.13 & -156.04 & 46.60 & 119.09 \\
\hline 6.31 & -65.7 & -1.67 & -124.21 & -158.38 & 47.38 & 121.29 \\
\hline 6.36 & -66.0 & -1.69 & -126.02 & -160.73 & 48.21 & 123.66 \\
\hline 6.41 & -66.1 & -1.70 & -127.77 & -162.75 & 49.01 & 125.92 \\
\hline 6.46 & -66.0 & -1.71 & -129.45 & -164.76 & 49.76 & 128.09 \\
\hline 6.51 & -66.3 & -1.71 & -131.39 & -166.91 & 50.61 & 130.49 \\
\hline 6.56 & -66.5 & -1.73 & -133.27 & -169.26 & 51.41 & 132.89 \\
\hline 6.61 & -66.3 & -1.73 & -135.09 & -171.28 & 52.20 & 135.12 \\
\hline 6.66 & -66.6 & -1.75 & -136.83 & -173.49 & 53.05 & 137.59 \\
\hline 6.71 & -66.8 & -1.75 & -138.78 & -175.71 & 53.89 & 140.06 \\
\hline 6.76 & -67.0 & -1.77 & -140.79 & -178.06 & 54.76 & 142.66 \\
\hline
\end{tabular}


Table F.5 IDT Strength Data (KL 58-40 \#10), continued

\begin{tabular}{|c|c|c|c|c|c|c|}
\hline Time & Axial Force & Axial Displ. & Vert front & Vert back & Horz front & Horz back \\
\hline $\mathrm{Sec}$ & $\overline{\mathrm{kN}}$ & $\mathrm{mm}$ & $\mu \mathrm{m}$ & $\mu \mathrm{m}$ & $\mu \mathrm{m}$ & $\mu \mathrm{m}$ \\
\hline 6.81 & -67.1 & -1.78 & -142.61 & -180.27 & 55.64 & 145.24 \\
\hline 6.86 & -67.5 & -1.79 & -144.76 & -182.62 & 56.56 & 147.94 \\
\hline 6.91 & -67.4 & -1.80 & -146.64 & -184.91 & 57.45 & 150.61 \\
\hline 6.96 & -67.6 & -1.81 & -148.58 & -187.26 & 58.38 & 153.40 \\
\hline 7.01 & -67.8 & -1.82 & -150.53 & -189.61 & 59.32 & 156.24 \\
\hline 7.06 & -68.1 & -1.83 & -152.68 & $\begin{array}{l}-192.09 \\
\end{array}$ & 60.31 & 159.22 \\
\hline 7.11 & -68.1 & -1.84 & -154.76 & -194.51 & 61.28 & 162.23 \\
\hline 7.16 & -68.3 & -1.85 & -156.91 & -196.99 & 62.26 & 165.28 \\
\hline 7.21 & -68.5 & -1.86 & -158.99 & -199.48 & 63.27 & 168.47 \\
\hline 7.26 & -68.6 & -1.87 & -161.07 & -201.96 & 64.30 & 171.66 \\
\hline 7.31 & -68.8 & -1.88 & -163.35 & -204.51 & 65.34 & 175.00 \\
\hline 7.36 & -68.9 & -1.89 & -165.64 & -207.13 & 66.40 & 178.41 \\
\hline 7.41 & $\begin{array}{l}-68.9 \\
\end{array}$ & -1.91 & -167.58 & -209.48 & 67.47 & 181.88 \\
\hline 7.46 & -69.1 & -1.91 & -169.73 & -212.03 & 68.60 & 185.53 \\
\hline 7.51 & -69.2 & -1.92 & -172.01 & -214.65 & 69.70 & 189.24 \\
\hline 7.56 & -69.3 & -1.93 & -174.23 & -217.33 & 70.84 & 193.05 \\
\hline 7.61 & -69.2 & -1.95 & -176.45 & -219.82 & 71.94 & 196.87 \\
\hline 7.66 & -69.4 & -1.95 & -178.73 & -222.44 & 73.13 & 200.85 \\
\hline 7.71 & -69.6 & -1.96 & -181.08 & -225.19 & 74.36 & 205.05 \\
\hline 7.76 & -69.7 & -1.98 & -183.43 & -227.88 & 75.59 & 209.38 \\
\hline 7.81 & -69.7 & -1.98 & -185.85 & -230.63 & 76.84 & 213.79 \\
\hline 7.86 & -69.9 & -2.00 & -188.46 & -233.45 & 78.10 & 218.43 \\
\hline 7.91 & -69.7 & -2.01 & -190.81 & -236.00 & 79.39 & 223.09 \\
\hline 7.96 & -69.8 & -2.01 & -193.10 & -238.75 & 80.69 & 227.96 \\
\hline 8.01 & -69.7 & -2.03 & -195.58 & -241.24 & 82.01 & 232.94 \\
\hline 8.06 & -70.0 & -2.04 & -198.13 & -244.19 & 83.41 & 238.25 \\
\hline 8.11 & -70.0 & -2.05 & -200.68 & -247.01 & 84.81 & 243.77 \\
\hline 8.16 & -70.0 & -2.06 & -203.23 & -249.63 & 86.23 & 249.39 \\
\hline 8.21 & -70.1 & -2.07 & -205.92 & -252.52 & 87.68 & 255.39 \\
\hline 8.26 & -70.2 & -2.08 & -208.61 & -255.34 & 89.18 & 261.75 \\
\hline 8.31 & -70.1 & -2.09 & -211.36 & -258.09 & 90.65 & 268.18 \\
\hline 8.36 & -70.0 & -2.10 & -214.11 & -260.71 & 92.14 & 275.06 \\
\hline 8.41 & -70.0 & -2.11 & -216.80 & -263.33 & 93.69 & 282.41 \\
\hline 8.46 & -69.9 & -2.13 & -219.55 & -265.88 & 95.24 & 290.30 \\
\hline 8.51 & -69.7 & -2.14 & -222.44 & -268.50 & 96.78 & 298.69 \\
\hline 8.56 & -69.7 & -2.14 & -225.32 & -271.11 & 98.37 & 308.01 \\
\hline 8.61 & -69.5 & -2.15 & -228.41 & -273.73 & 99.92 & 318.21 \\
\hline 8.66 & -69.5 & -2.16 & -231.50 & -276.62 & 101.47 & 329.90 \\
\hline 8.71 & -69.3 & -2.18 & -234.66 & -279.44 & 102.98 & 343.31 \\
\hline 8.76 & -69.3 & -2.19 & -238.15 & -282.80 & 104.47 & 358.90 \\
\hline 8.81 & -69.2 & -2.20 & -241.71 & -285.95 & 105.95 & 376.64 \\
\hline 8.86 & -68.7 & -2.21 & -245.60 & -288.44 & 107.32 & 396.10 \\
\hline 8.91 & -68.4 & -2.22 & -249.63 & -287.43 & 108.63 & 417.93 \\
\hline 8.96 & -67.6 & -2.23 & -253.46 & -285.75 & 109.85 & 441.77 \\
\hline 9.01 & -67.1 & -2.24 & -257.62 & -286.36 & 111.08 & 467.64 \\
\hline
\end{tabular}


Table F.5 IDT Strength Data (KL 58-40 \#10), continued

\begin{tabular}{|c|c|c|c|c|c|c|}
\hline Time & Axial Force & Axial Displ. & Vert front & Vert back & Horz front & Horz back \\
\hline Sec & $\mathrm{kN}$ & $\mathrm{mm}$ & $\mu \mathrm{m}$ & $\mu \mathrm{m}$ & $\mu \mathrm{m}$ & $\mu \mathrm{m}$ \\
\hline 9.06 & -66.5 & -2.26 & -262.05 & -287.63 & 112.46 & 495.73 \\
\hline 9.11 & -65.7 & -2.27 & -266.68 & -289.65 & 114.00 & 525.09 \\
\hline 9.16 & -64.9 & -2.27 & -271.38 & -291.46 & 115.71 & 556.55 \\
\hline 9.21 & -64.2 & -2.28 & -276.35 & -295.89 & 117.62 & 590.39 \\
\hline 9.26 & -63.2 & -2.30 & -281.45 & -302.00 & 119.65 & 626.20 \\
\hline
\end{tabular}


Table F.6 IDT Strength Data (KL 58-40 \#12)

\begin{tabular}{|c|c|c|c|c|c|c|}
\hline Time & Axial Force & Axial Displ. & Vert front & Vert back & Horz front & Horz back \\
\hline $\mathrm{Sec}$ & $\mathrm{kN}$ & $\mathrm{mm}$ & $\mu \mathrm{m}$ & $\mu \mathrm{m}$ & $\mu \mathrm{m}$ & $\mu \mathrm{m}$ \\
\hline 0.00 & -0.5 & -0.92 & 0.54 & 0.54 & 0.24 & 0.20 \\
\hline 0.05 & -0.8 & -0.93 & 0.27 & 0.60 & 0.30 & 0.23 \\
\hline 0.10 & -1.2 & -0.94 & -0.07 & 0.47 & 0.39 & 0.32 \\
\hline 0.15 & -1.7 & -0.95 & -0.40 & 0.34 & 0.48 & 0.39 \\
\hline 0.20 & -2.2 & -0.96 & -0.81 & 0.20 & 0.58 & 0.50 \\
\hline 0.25 & -2.8 & -0.97 & -1.28 & -0.07 & 0.68 & 0.65 \\
\hline 0.30 & -3.4 & -0.98 & -1.68 & -0.40 & 0.79 & 0.79 \\
\hline 0.35 & -4.2 & -0.99 & -2.28 & -0.94 & 0.93 & 1.02 \\
\hline 0.40 & -5.0 & -1.00 & -2.75 & -1.28 & 1.06 & 1.24 \\
\hline 0.45 & -5.5 & -1.01 & -3.22 & -1.68 & 1.17 & 1.44 \\
\hline 0.50 & -6.4 & -1.02 & -3.83 & -2.35 & 1.33 & 1.73 \\
\hline 0.55 & -7.1 & -1.03 & -4.36 & -2.82 & 1.47 & 1.96 \\
\hline 0.60 & -8.0 & -1.04 & -4.90 & -3.36 & 1.64 & 2.28 \\
\hline 0.65 & -8.8 & -1.05 & -5.51 & -4.03 & 1.79 & 2.62 \\
\hline 0.70 & -9.7 & -1.06 & -6.11 & -4.70 & 1.96 & 2.89 \\
\hline 0.75 & -10.4 & -1.07 & -6.71 & -5.30 & 2.11 & 3.22 \\
\hline 0.80 & -11.3 & -1.08 & -7.25 & -6.04 & 2.30 & 3.56 \\
\hline 0.85 & -12.1 & -1.09 & -7.86 & -6.71 & 2.46 & 3.89 \\
\hline 0.90 & -13.1 & -1.10 & -8.59 & -7.45 & 2.67 & 4.28 \\
\hline 0.95 & -13.6 & -1.11 & -9.20 & -8.06 & 2.83 & 4.60 \\
\hline 1.00 & -14.5 & -1.12 & -9.80 & -8.86 & 2.99 & 4.97 \\
\hline 1.05 & -15.4 & -1.13 & -10.54 & -9.74 & 3.21 & 5.42 \\
\hline 1.10 & -16.1 & -1.15 & -11.21 & -10.34 & 3.38 & 5.72 \\
\hline 1.15 & -16.8 & -1.16 & -11.88 & -11.15 & 3.56 & 6.09 \\
\hline 1.20 & -17.5 & -1.16 & -12.42 & -11.75 & 3.77 & 6.48 \\
\hline 1.25 & -18.3 & -1.18 & -13.16 & -12.62 & 3.96 & 6.85 \\
\hline 1.30 & -19.1 & -1.19 & -13.90 & -13.43 & 4.15 & 7.30 \\
\hline 1.35 & -19.9 & -1.20 & -14.64 & -14.23 & 4.36 & 7.70 \\
\hline 1.40 & -20.5 & -1.21 & -15.31 & -15.04 & 4.57 & 8.11 \\
\hline 1.45 & -21.2 & -1.22 & -16.11 & -15.71 & 4.77 & 8.49 \\
\hline 1.50 & -21.9 & -1.23 & -16.85 & -16.65 & 4.97 & 8.91 \\
\hline 1.55 & -22.6 & -1.24 & -17.52 & -17.46 & 5.19 & 9.37 \\
\hline 1.60 & -23.6 & -1.25 & -18.33 & -18.53 & 5.41 & 9.87 \\
\hline 1.65 & -24.1 & -1.26 & -19.00 & -19.20 & 5.61 & 10.29 \\
\hline 1.70 & -24.9 & -1.27 & -19.81 & -20.21 & 5.86 & 10.78 \\
\hline 1.75 & -25.4 & -1.28 & -20.61 & -21.15 & 6.03 & 11.16 \\
\hline 1.80 & -26.2 & -1.29 & -21.28 & -22.02 & 6.29 & 11.72 \\
\hline 1.85 & -26.8 & -1.30 & -22.02 & -22.83 & 6.50 & 12.19 \\
\hline 1.90 & -27.8 & -1.31 & -22.89 & -23.97 & 6.76 & 12.72 \\
\hline 1.95 & -28.6 & -1.32 & -23.70 & -24.98 & 7.03 & 13.29 \\
\hline 2.00 & -29.3 & -1.33 & -24.57 & -25.98 & 7.26 & 13.83 \\
\hline 2.05 & -29.9 & -1.34 & -25.31 & -26.99 & 7.51 & 14.32 \\
\hline 2.10 & -30.7 & -1.35 & -26.12 & -28.06 & 7.76 & 14.87 \\
\hline 2.15 & -31.3 & -1.36 & -26.92 & -29.07 & 8.01 & 15.43 \\
\hline 2.20 & -32.0 & -1.37 & -27.93 & -30.35 & 8.22 & 15.91 \\
\hline 2.25 & -32.5 & -1.39 & -28.47 & -31.09 & 8.50 & 16.47 \\
\hline
\end{tabular}


Table F.6 IDT Strength Data (KL 58-40 \#12), continued

\begin{tabular}{|c|c|c|c|c|c|c|}
\hline Time & Axial Force & Axial Displ. & Vert front & Vert back & Horz front & Horz back \\
\hline $\mathrm{Sec}$ & $\mathrm{kN}$ & $\mathrm{mm}$ & $\mu \mathrm{m}$ & $\mu \mathrm{m}$ & $\mu \mathrm{m}$ & $\mu \mathrm{m}$ \\
\hline 2.30 & -33.1 & -1.40 & -29.27 & -32.09 & 8.73 & 16.99 \\
\hline 2.35 & -34.0 & -1.40 & -30.15 & -33.10 & 9.04 & 17.71 \\
\hline 2.40 & -34.7 & -1.42 & -31.22 & -34.58 & 9.30 & 18.26 \\
\hline 2.45 & -35.2 & -1.43 & -31.96 & -35.58 & 9.55 & 18.85 \\
\hline 2.50 & -36.0 & -1.44 & -32.90 & -36.66 & 9.84 & 19.49 \\
\hline 2.55 & -36.7 & -1.45 & -33.91 & -37.87 & 10.13 & 20.09 \\
\hline 2.60 & -37.2 & -1.46 & -34.78 & -39.01 & 10.38 & 20.71 \\
\hline 2.65 & -37.9 & -1.47 & -35.72 & -40.15 & 10.68 & 21.37 \\
\hline 2.70 & -38.4 & -1.48 & -36.46 & -41.36 & 10.96 & 21.97 \\
\hline 2.75 & -39.0 & -1.49 & -37.40 & -42.50 & 11.23 & 22.61 \\
\hline 2.80 & -39.7 & -1.50 & -38.34 & -43.71 & 11.53 & 23.28 \\
\hline 2.85 & -40.4 & -1.51 & -39.21 & -44.98 & 11.85 & 23.99 \\
\hline 2.90 & -40.7 & -1.52 & -40.08 & -46.13 & 12.11 & 24.57 \\
\hline 2.95 & -41.5 & -1.53 & -41.22 & -47.54 & 12.42 & 25.26 \\
\hline 3.00 & -42.2 & -1.54 & -42.16 & -48.74 & 12.73 & 26.02 \\
\hline 3.05 & -42.8 & -1.55 & -43.17 & -50.02 & 13.04 & 26.72 \\
\hline 3.10 & -43.4 & -1.56 & -44.25 & -51.43 & 13.36 & 27.44 \\
\hline 3.15 & -43.8 & -1.57 & -45.05 & -52.57 & 13.64 & 28.15 \\
\hline 3.20 & -44.5 & -1.58 & -46.19 & -53.91 & 13.97 & 29.24 \\
\hline 3.25 & -44.9 & -1.59 & -47.13 & -55.19 & 14.28 & 29.94 \\
\hline 3.30 & -45.6 & -1.60 & -48.14 & -56.40 & 14.61 & 30.75 \\
\hline 3.35 & -46.5 & -1.61 & -49.42 & -58.14 & 14.96 & 31.59 \\
\hline 3.40 & -46.9 & -1.62 & -50.49 & -59.35 & 15.29 & 32.36 \\
\hline 3.45 & -47.4 & -1.63 & -51.50 & -60.70 & 15.63 & 33.62 \\
\hline 3.50 & -48.1 & -1.64 & -52.64 & -62.24 & 15.98 & 34.86 \\
\hline 3.55 & -48.7 & -1.65 & -53.78 & -63.78 & 16.33 & 35.80 \\
\hline 3.60 & -49.1 & -1.66 & -54.92 & -65.19 & 16.68 & 36.63 \\
\hline 3.65 & -49.7 & -1.67 & -56.00 & -66.54 & 17.03 & 37.48 \\
\hline 3.70 & -50.0 & -1.69 & -57.20 & -68.08 & 17.36 & 38.27 \\
\hline 3.75 & -50.4 & -1.70 & -58.21 & -69.42 & 17.68 & 39.08 \\
\hline 3.80 & -51.0 & -1.71 & -59.42 & -70.90 & 18.04 & 39.93 \\
\hline 3.85 & -51.5 & -1.72 & -60.49 & -72.44 & 18.40 & 40.77 \\
\hline 3.90 & -52.2 & -1.72 & -61.77 & -73.99 & 18.80 & 41.73 \\
\hline 3.95 & -52.7 & -1.74 & -62.91 & -75.60 & 19.18 & 42.62 \\
\hline 4.00 & -53.2 & -1.75 & -64.19 & -77.14 & 19.55 & 43.47 \\
\hline 4.05 & -53.6 & -1.76 & -65.46 & -78.55 & 19.92 & 44.31 \\
\hline 4.10 & -54.0 & -1.76 & -66.67 & -80.17 & 20.29 & 45.15 \\
\hline 4.15 & -54.5 & -1.78 & -67.81 & -81.58 & 20.67 & 46.02 \\
\hline 4.20 & -55.1 & -1.79 & -69.15 & -83.32 & 21.11 & 47.00 \\
\hline 4.25 & -55.7 & -1.80 & -70.50 & -85.00 & 21.51 & 47.90 \\
\hline 4.30 & -56.2 & -1.81 & -71.77 & -86.95 & 21.92 & 48.90 \\
\hline 4.35 & -56.6 & -1.82 & -72.98 & -88.36 & 22.35 & 49.89 \\
\hline 4.40 & -56.9 & -1.83 & -74.26 & -89.90 & 22.75 & 50.76 \\
\hline 4.45 & -57.5 & -1.84 & -75.67 & -91.71 & 23.18 & 51.80 \\
\hline 4.50 & -57.8 & -1.85 & -76.94 & -93.39 & 23.62 & 52.76 \\
\hline
\end{tabular}


Table F.6 IDT Strength Data (KL 58-40 \#12), continued

\begin{tabular}{|c|c|c|c|c|c|c|}
\hline Time & Axial Force & Axial Displ. & Vert front & Vert back & Horz front & Horz back \\
\hline $\mathrm{Sec}$ & $\mathrm{kN}$ & $\mathrm{mm}$ & $\mu \mathrm{m}$ & $\mu \mathrm{m}$ & $\mu \mathrm{m}$ & $\mu \mathrm{m}$ \\
\hline 4.55 & -58.4 & -1.86 & -78.29 & -95.14 & 24.07 & 53.80 \\
\hline 4.60 & -58.9 & -1.87 & -79.70 & -96.88 & 24.51 & 54.87 \\
\hline 4.65 & -59.2 & -1.89 & -80.97 & -98.56 & 24.94 & 55.88 \\
\hline 4.70 & -59.4 & -1.90 & -82.25 & -100.24 & 25.38 & 56.90 \\
\hline 4.75 & -59.7 & -1.91 & -83.46 & -101.85 & 25.81 & 57.89 \\
\hline 4.80 & -60.2 & -1.91 & -84.66 & -103.73 & 26.29 & 58.98 \\
\hline 4.85 & -60.7 & -1.92 & -85.94 & -105.41 & 26.76 & 60.09 \\
\hline 4.90 & -61.0 & -1.94 & -87.22 & -107.22 & 27.23 & 61.18 \\
\hline 4.95 & -61.4 & -1.95 & -88.42 & -108.97 & 27.70 & 62.29 \\
\hline 5.00 & -61.6 & -1.96 & -89.97 & -110.92 & 28.13 & 63.28 \\
\hline 5.05 & -62.1 & -1.96 & -91.04 & -112.59 & 28.68 & 64.56 \\
\hline 5.10 & -62.5 & -1.98 & -92.52 & -114.47 & 29.17 & 65.68 \\
\hline 5.16 & -62.9 & -1.99 & -93.86 & -116.42 & 29.68 & 66.87 \\
\hline 5.21 & -63.4 & -2.00 & -95.34 & -118.30 & 30.22 & 68.08 \\
\hline 5.26 & -63.7 & -2.01 & -96.68 & -120.18 & 30.75 & 69.27 \\
\hline 5.31 & -64.0 & -2.02 & -98.09 & -121.93 & 31.28 & 70.48 \\
\hline 5.36 & -64.4 & -2.03 & -99.70 & -123.94 & 31.82 & 71.66 \\
\hline 5.41 & -64.8 & -2.04 & -101.32 & -126.02 & 32.38 & 72.93 \\
\hline 5.46 & -65.0 & -2.05 & -102.79 & -127.77 & 32.94 & 74.17 \\
\hline 5.51 & -65.4 & -2.06 & -104.34 & -129.78 & 33.51 & 75.43 \\
\hline 5.56 & -65.8 & -2.07 & -106.15 & -132.07 & 34.10 & 76.71 \\
\hline 5.61 & -65.8 & -2.08 & -107.36 & -133.61 & 34.67 & 77.97 \\
\hline 5.66 & -66.1 & -2.10 & -108.97 & -135.62 & 35.24 & 79.24 \\
\hline 5.71 & -66.5 & -2.10 & -110.65 & -137.77 & 35.84 & 80.55 \\
\hline 5.76 & -67.0 & -2.11 & -112.33 & -139.85 & 36.50 & 81.98 \\
\hline 5.81 & -67.3 & -2.12 & -114.00 & -142.00 & 37.12 & 83.39 \\
\hline 5.86 & -67.5 & -2.14 & -115.55 & -143.95 & 37.78 & 84.83 \\
\hline 5.91 & -67.7 & -2.14 & -117.23 & -146.03 & 38.42 & 86.18 \\
\hline 5.96 & -67.9 & -2.16 & -118.70 & -147.98 & 39.09 & 87.62 \\
\hline 6.01 & -68.1 & -2.17 & -120.38 & -150.06 & 39.72 & 88.98 \\
\hline 6.06 & -68.2 & -2.17 & -121.99 & -152.21 & 40.39 & 90.39 \\
\hline 6.11 & -68.5 & -2.19 & -123.67 & -154.22 & 41.06 & 91.85 \\
\hline 6.16 & -68.8 & -2.19 & -125.35 & -156.37 & 41.78 & 93.39 \\
\hline 6.21 & -69.2 & -2.21 & -127.30 & -158.65 & 42.52 & 94.97 \\
\hline 6.26 & -69.4 & -2.22 & -128.98 & -160.87 & 43.23 & 96.51 \\
\hline 6.31 & -69.7 & -2.23 & -130.72 & -163.02 & 43.97 & 98.08 \\
\hline 6.36 & -69.9 & -2.24 & -132.54 & -165.17 & 44.73 & 99.69 \\
\hline 6.41 & -70.1 & -2.25 & -134.28 & -167.31 & 45.52 & 101.35 \\
\hline 6.46 & -70.4 & -2.26 & -135.69 & -169.66 & 46.29 & 103.01 \\
\hline 6.51 & -70.7 & -2.27 & -135.49 & -172.15 & 47.12 & 104.72 \\
\hline 6.56 & -70.9 & -2.28 & -130.12 & -174.63 & 47.94 & 106.50 \\
\hline 6.61 & -71.3 & -2.28 & -132.27 & -177.12 & 48.78 & 108.26 \\
\hline 6.66 & -71.4 & -2.30 & -134.42 & -179.74 & 49.63 & 110.09 \\
\hline 6.71 & -71.7 & -2.31 & -136.50 & -182.35 & 50.55 & 111.96 \\
\hline 6.76 & -71.8 & -2.32 & -138.65 & -184.91 & 51.41 & 113.80 \\
\hline
\end{tabular}


Table F.6 IDT Strength Data (KL 58-40 \#12), continued

\begin{tabular}{|c|c|c|c|c|c|c|}
\hline Time & Axial Force & Axial Displ. & Vert front & Vert back & Horz front & Horz back \\
\hline $\mathrm{Sec}$ & $\mathrm{kN}$ & $\mathrm{mm}$ & $\mu \mathrm{m}$ & $\mu \mathrm{m}$ & $\mu \mathrm{m}$ & $\mu \mathrm{m}$ \\
\hline 6.81 & -71.8 & -2.33 & -140.73 & -187.39 & 52.26 & 115.63 \\
\hline 6.86 & -72.2 & -2.35 & -142.94 & -190.14 & 53.24 & 117.66 \\
\hline 6.91 & -72.4 & -2.35 & -145.16 & -192.76 & 54.19 & 119.64 \\
\hline 6.96 & -72.5 & -2.36 & -147.37 & -195.45 & 55.14 & 121.63 \\
\hline 7.01 & -72.7 & -2.37 & -149.79 & -198.20 & 56.11 & 123.72 \\
\hline 7.06 & -73.1 & -2.38 & -152.14 & -201.15 & 57.18 & 125.92 \\
\hline 7.11 & -73.2 & -2.39 & -154.49 & -203.97 & $\begin{array}{l}58.20 \\
\end{array}$ & 128.10 \\
\hline 7.16 & $\begin{array}{l}-73.3 \\
\end{array}$ & -2.40 & -156.77 & -206.66 & \begin{tabular}{|l|l}
59.28 \\
\end{tabular} & 130.32 \\
\hline 7.21 & -73.5 & -2.42 & -159.32 & -209.61 & 60.37 & 132.57 \\
\hline 7.26 & -73.4 & -2.42 & -161.81 & -212.50 & 61.43 & 134.75 \\
\hline 7.31 & -73.7 & -2.44 & -164.16 & -215.39 & 62.60 & 137.20 \\
\hline 7.36 & -73.8 & -2.45 & -166.71 & -218.27 & 63.77 & 139.55 \\
\hline 7.41 & $\begin{array}{l}-73.8 \\
\end{array}$ & -2.45 & -169.19 & -221.23 & 64.95 & 141.97 \\
\hline 7.46 & -74.1 & -2.47 & -171.68 & -224.32 & 66.18 & 144.52 \\
\hline 7.51 & -74.5 & -2.48 & -174.57 & -227.67 & 67.53 & 147.26 \\
\hline 7.56 & -74.6 & -2.49 & -177.18 & -230.70 & 68.86 & 150.01 \\
\hline 7.61 & -74.6 & -2.50 & -179.94 & -233.85 & 70.20 & 152.73 \\
\hline 7.66 & -74.6 & -2.51 & -182.42 & -237.01 & 71.61 & 155.53 \\
\hline 7.71 & -74.7 & -2.52 & -184.97 & -240.23 & 73.04 & 158.42 \\
\hline 7.76 & -74.7 & -2.53 & -186.38 & -243.45 & 74.51 & 161.42 \\
\hline 7.81 & -74.8 & -2.54 & -182.09 & -246.74 & 76.05 & 164.53 \\
\hline 7.86 & -74.8 & -2.55 & -185.04 & -250.17 & 77.63 & 167.75 \\
\hline 7.91 & -74.9 & -2.56 & -187.99 & -253.66 & 79.28 & 171.07 \\
\hline 7.96 & -74.9 & -2.57 & -190.81 & -257.15 & 80.96 & 174.52 \\
\hline 8.01 & -75.1 & -2.58 & -193.10 & -260.71 & 82.74 & 178.06 \\
\hline 8.06 & -75.2 & -2.59 & -195.78 & -264.33 & 84.63 & 181.82 \\
\hline 8.11 & -75.0 & -2.60 & -198.80 & -267.96 & 86.53 & 185.53 \\
\hline 8.16 & -75.0 & -2.62 & -201.69 & -271.45 & 88.55 & 189.42 \\
\hline 8.21 & -75.0 & -2.63 & -204.64 & -275.28 & 90.66 & 193.43 \\
\hline 8.26 & -75.1 & -2.63 & -207.87 & -279.24 & 92.89 & 197.81 \\
\hline 8.31 & -75.1 & -2.65 & -210.89 & -283.27 & 95.25 & 202.36 \\
\hline 8.36 & -75.1 & -2.65 & -213.91 & -287.30 & 97.77 & 207.13 \\
\hline 8.41 & -74.9 & -2.67 & -217.00 & -291.32 & 100.39 & 212.10 \\
\hline 8.46 & -75.1 & -2.68 & -220.15 & -295.62 & 103.27 & 217.55 \\
\hline 8.51 & -75.1 & -2.69 & -223.44 & -300.19 & 106.35 & 223.46 \\
\hline 8.56 & $\begin{array}{l}-74.9 \\
\end{array}$ & -2.70 & -226.67 & -304.55 & 109.62 & 229.72 \\
\hline 8.61 & -74.7 & -2.71 & -230.16 & -309.18 & 113.21 & 236.55 \\
\hline 8.66 & -74.6 & -2.72 & -233.38 & -313.88 & 117.32 & 244.14 \\
\hline 8.71 & -74.2 & -2.74 & -236.67 & -318.65 & 121.84 & 252.58 \\
\hline 8.76 & -73.9 & -2.74 & -239.96 & -323.42 & 126.92 & 262.13 \\
\hline 8.81 & -73.7 & -2.75 & -243.86 & -328.65 & 132.79 & 273.26 \\
\hline 8.86 & $\begin{array}{l}-73.2 \\
\end{array}$ & -2.77 & -248.62 & -333.62 & 139.35 & 285.97 \\
\hline 8.91 & -72.8 & -2.77 & -254.13 & -338.86 & 146.64 & 301.16 \\
\hline 8.96 & -72.4 & -2.79 & -260.04 & -344.16 & 154.69 & 320.60 \\
\hline 9.01 & -71.6 & -2.79 & -266.62 & -349.67 & 163.74 & 344.10 \\
\hline
\end{tabular}


Table F.6 IDT Strength Data (KL 58-40 \#12), continued

\begin{tabular}{|c|c|c|c|c|c|c|}
\hline Time & Axial Force & Axial Displ. & Vert front & Vert back & Horz front & Horz back \\
\hline Sec & $\mathrm{kN}$ & $\mathrm{mm}$ & $\mu \mathrm{m}$ & $\mu \mathrm{m}$ & $\mu \mathrm{m}$ & $\mu \mathrm{m}$ \\
\hline 9.06 & -70.7 & -2.81 & -274.14 & -355.38 & 174.41 & 369.81 \\
\hline 9.11 & -69.3 & -2.82 & -281.92 & -360.68 & 185.56 & 397.10 \\
\hline 9.16 & -68.3 & -2.83 & -290.18 & -366.12 & 197.57 & 427.01 \\
\hline
\end{tabular}


Table F.7 IDT Strength Data (KL 58-40 \#8)

\begin{tabular}{|c|c|c|c|c|c|c|}
\hline Time & Axial Force & Axial Displ. & Vert front & Vert back & Horz front & Horz back \\
\hline $\mathrm{Sec}$ & $\mathrm{kN}$ & $\mathrm{mm}$ & $\mu \mathrm{m}$ & $\mu \mathrm{m}$ & $\mu \mathrm{m}$ & $\mu \mathrm{m}$ \\
\hline 0.00 & -0.1 & -1.57 & 1.41 & 0.81 & 0.53 & 0.25 \\
\hline 0.05 & -0.1 & -1.58 & 1.48 & 0.94 & 0.54 & 0.25 \\
\hline 0.10 & -0.1 & -1.59 & 1.34 & 0.81 & 0.55 & 0.27 \\
\hline 0.15 & -0.2 & -1.61 & 1.34 & 0.67 & 0.56 & 0.29 \\
\hline 0.20 & -0.4 & -1.61 & 1.28 & 0.81 & 0.56 & 0.34 \\
\hline 0.25 & -0.6 & -1.62 & 1.21 & 0.47 & 0.61 & 0.44 \\
\hline 0.30 & -1.0 & -1.63 & 0.87 & 0.27 & 0.67 & 0.54 \\
\hline 0.35 & -1.3 & -1.64 & 0.60 & 0.07 & 0.72 & 0.70 \\
\hline 0.40 & -1.8 & -1.65 & 0.34 & -0.20 & 0.79 & 0.91 \\
\hline 0.45 & -2.4 & -1.67 & -0.07 & -0.60 & 0.87 & 1.19 \\
\hline 0.50 & -3.0 & -1.67 & -0.40 & -1.01 & 0.98 & 1.43 \\
\hline 0.55 & -3.5 & -1.69 & -0.74 & -1.48 & 1.05 & 1.75 \\
\hline 0.60 & -4.0 & $\begin{array}{l}-1.69 \\
\end{array}$ & -1.07 & -1.95 & 1.12 & 2.00 \\
\hline 0.65 & -4.7 & -1.70 & -1.48 & -2.55 & 1.28 & 2.40 \\
\hline 0.70 & -5.6 & -1.72 & -2.08 & -3.29 & 1.38 & 2.82 \\
\hline 0.75 & -6.2 & -1.73 & -2.62 & -3.96 & 1.52 & 3.21 \\
\hline 0.80 & -6.8 & -1.74 & -3.22 & -4.63 & 1.64 & 3.58 \\
\hline 0.85 & -7.5 & -1.75 & -3.56 & -5.17 & 1.79 & 4.08 \\
\hline 0.90 & -8.0 & -1.76 & -4.03 & $\begin{array}{l}-5.71 \\
\end{array}$ & 1.88 & 4.48 \\
\hline 0.95 & -8.9 & -1.77 & -4.70 & -6.65 & 2.06 & 4.95 \\
\hline 1.00 & -9.6 & -1.78 & -5.24 & -7.39 & 2.24 & 5.46 \\
\hline 1.05 & -10.1 & -1.79 & -5.71 & -7.86 & 2.38 & 5.94 \\
\hline 1.10 & -10.9 & -1.80 & -6.45 & -8.66 & 2.57 & 6.46 \\
\hline 1.15 & -11.5 & -1.81 & -6.98 & -9.40 & 2.75 & 6.92 \\
\hline 1.20 & -12.1 & -1.82 & -7.52 & -10.14 & 2.93 & 7.44 \\
\hline 1.25 & -12.7 & -1.83 & -8.19 & -10.94 & 3.08 & 7.99 \\
\hline 1.30 & -13.3 & -1.84 & -8.86 & -11.68 & 3.26 & 8.48 \\
\hline 1.35 & -14.1 & -1.85 & -9.67 & -12.56 & 3.50 & 9.10 \\
\hline 1.40 & -14.6 & -1.86 & -10.14 & -13.29 & 3.68 & 9.60 \\
\hline 1.45 & -15.1 & -1.87 & -10.81 & -14.17 & 3.85 & 10.17 \\
\hline 1.50 & -15.8 & -1.88 & -11.55 & -14.97 & 4.07 & 10.76 \\
\hline 1.55 & -16.6 & -1.90 & -12.29 & -16.05 & 4.30 & 11.43 \\
\hline 1.60 & -17.2 & -1.91 & -13.09 & -16.92 & 4.50 & 12.03 \\
\hline 1.65 & -17.4 & -1.91 & -13.50 & -17.52 & 4.66 & 12.54 \\
\hline 1.70 & -18.0 & -1.92 & -14.17 & -18.40 & 4.86 & 13.13 \\
\hline 1.75 & -18.8 & -1.94 & -14.91 & -19.47 & 5.10 & 13.88 \\
\hline 1.80 & -19.4 & -1.95 & -15.71 & -20.48 & 5.31 & 14.54 \\
\hline 1.85 & -20.0 & -1.96 & -16.38 & -21.49 & 5.53 & 15.19 \\
\hline 1.90 & -20.6 & -1.97 & -17.12 & -22.43 & 5.77 & 15.91 \\
\hline 1.95 & -21.1 & -1.97 & -17.93 & -23.30 & 5.98 & 16.58 \\
\hline 2.00 & -21.9 & -1.99 & -18.73 & -24.57 & 6.25 & 17.41 \\
\hline 2.05 & -22.3 & -2.00 & -19.47 & -25.45 & 6.45 & 18.06 \\
\hline 2.10 & -22.9 & -2.01 & -20.28 & -26.45 & 6.69 & 18.78 \\
\hline 2.15 & -23.6 & -2.02 & -21.02 & -27.66 & 6.95 & 19.62 \\
\hline 2.20 & -24.2 & -2.03 & -21.89 & -28.74 & 7.18 & 20.44 \\
\hline 2.25 & -24.5 & -2.04 & -22.49 & -29.68 & 7.41 & 21.15 \\
\hline
\end{tabular}


Table F.7 IDT Strength Data (KL 58-40 \#8), continued

\begin{tabular}{|c|c|c|c|c|c|c|}
\hline Time & Axial Force & Axial Displ. & Vert front & Vert back & Horz front & Horz back \\
\hline $\mathrm{Sec}$ & $\mathrm{kN}$ & $\mathrm{mm}$ & $\mu \mathrm{m}$ & $\mu \mathrm{m}$ & $\mu \mathrm{m}$ & $\mu \mathrm{m}$ \\
\hline 2.30 & -24.8 & -2.05 & -23.16 & -30.75 & 7.62 & 21.80 \\
\hline 2.35 & -25.7 & -2.06 & -24.24 & -31.89 & 7.90 & 22.64 \\
\hline 2.40 & -26.5 & -2.07 & -25.18 & -33.30 & 8.21 & 23.65 \\
\hline 2.45 & -26.8 & -2.08 & -25.98 & -34.44 & 8.44 & 24.41 \\
\hline 2.50 & -27.6 & -2.09 & -26.99 & -35.72 & 8.73 & 25.38 \\
\hline 2.55 & -27.9 & -2.10 & -27.66 & -36.73 & 8.98 & 26.18 \\
\hline 2.60 & -28.7 & -2.11 & -28.60 & -38.14 & 9.27 & 27.18 \\
\hline 2.65 & -29.2 & -2.12 & -29.54 & -39.14 & 9.57 & 28.06 \\
\hline 2.70 & -29.5 & -2.13 & -30.55 & -40.49 & 9.82 & 28.94 \\
\hline 2.75 & -30.2 & -2.14 & -31.35 & -41.83 & 10.13 & 29.89 \\
\hline 2.80 & -30.8 & -2.15 & -32.36 & -43.10 & 10.40 & 30.88 \\
\hline 2.85 & -31.4 & -2.17 & -33.30 & -44.65 & 10.73 & 31.91 \\
\hline 2.90 & -31.9 & -2.17 & -34.31 & -45.92 & 11.04 & 32.90 \\
\hline 2.95 & -32.2 & -2.18 & -35.25 & -47.13 & 11.33 & 33.82 \\
\hline 3.00 & -33.0 & -2.20 & -36.32 & -48.74 & 11.67 & 34.96 \\
\hline 3.05 & -33.4 & -2.21 & -37.40 & -49.95 & 11.96 & 35.97 \\
\hline 3.10 & -33.8 & -2.22 & -38.27 & -51.43 & 12.26 & 36.91 \\
\hline 3.15 & -34.3 & -2.23 & -39.34 & -52.71 & 12.59 & 37.97 \\
\hline 3.20 & -34.7 & -2.24 & -40.28 & -54.18 & 12.91 & 39.03 \\
\hline 3.25 & -35.2 & -2.25 & -41.56 & -55.73 & 13.23 & 40.05 \\
\hline 3.30 & -35.5 & -2.26 & -42.30 & -56.80 & 13.55 & 41.11 \\
\hline 3.35 & -36.3 & -2.27 & -43.44 & -58.55 & 13.95 & 42.35 \\
\hline 3.40 & -36.8 & -2.28 & -44.58 & -60.02 & 14.28 & 43.49 \\
\hline 3.45 & -37.0 & -2.29 & -45.45 & -61.30 & 14.63 & 44.55 \\
\hline 3.50 & -37.5 & -2.30 & -46.73 & -62.84 & 14.97 & 45.69 \\
\hline 3.55 & -37.9 & -2.31 & -47.80 & -64.32 & 15.32 & 46.85 \\
\hline 3.60 & -38.3 & -2.32 & -48.95 & -65.87 & 15.68 & 47.99 \\
\hline 3.65 & -38.8 & -2.33 & -50.09 & -67.34 & 16.05 & 49.20 \\
\hline 3.70 & -39.0 & -2.34 & -51.23 & -69.02 & 16.38 & 50.27 \\
\hline 3.75 & -39.7 & -2.35 & -52.37 & -70.50 & 16.78 & 51.55 \\
\hline 3.80 & -40.0 & -2.36 & -53.58 & -72.18 & 17.15 & 52.71 \\
\hline 3.85 & -40.6 & -2.37 & -54.85 & -73.72 & 17.53 & 54.01 \\
\hline 3.90 & -40.9 & -2.38 & -55.86 & -75.26 & 17.93 & 55.24 \\
\hline 3.95 & -41.2 & -2.39 & -57.00 & -76.74 & 18.31 & 56.47 \\
\hline 4.00 & -41.8 & -2.40 & -58.35 & -78.49 & 18.73 & 57.82 \\
\hline 4.05 & -42.1 & -2.41 & -59.49 & -80.10 & 19.14 & 59.10 \\
\hline 4.10 & -42.7 & -2.42 & -60.83 & -81.98 & 19.57 & 60.49 \\
\hline 4.15 & -43.1 & -2.44 & -62.17 & -83.66 & 20.01 & 61.85 \\
\hline 4.20 & -43.5 & -2.45 & -63.45 & -85.40 & 20.44 & 63.18 \\
\hline 4.25 & -43.8 & -2.45 & -64.72 & -87.01 & 20.84 & 64.54 \\
\hline 4.30 & -44.1 & -2.47 & -65.93 & -88.56 & 21.30 & 65.88 \\
\hline 4.35 & -44.4 & -2.48 & -67.41 & -90.37 & 21.70 & 67.17 \\
\hline 4.40 & -44.9 & -2.49 & -68.82 & -92.18 & 22.16 & 68.62 \\
\hline 4.45 & -45.3 & -2.50 & -70.09 & -94.00 & 22.62 & 70.09 \\
\hline 4.50 & -45.5 & -2.51 & -71.44 & -95.68 & 23.06 & 71.47 \\
\hline
\end{tabular}


Table F.7 IDT Strength Data (KL 58-40 \#8), continued

\begin{tabular}{|c|c|c|c|c|c|c|}
\hline Time & Axial Force & Axial Displ. & Vert front & Vert back & Horz front & Horz back \\
\hline $\mathrm{Sec}$ & $\mathrm{kN}$ & $\mathrm{mm}$ & $\mu \mathrm{m}$ & $\mu \mathrm{m}$ & $\mu \mathrm{m}$ & $\mu \mathrm{m}$ \\
\hline 4.55 & -45.9 & -2.52 & -72.91 & -97.42 & 23.53 & 72.85 \\
\hline 4.60 & -46.1 & -2.53 & -74.12 & -99.17 & 23.96 & 74.26 \\
\hline 4.65 & -46.5 & -2.54 & -75.60 & -100.98 & 24.44 & 75.73 \\
\hline 4.70 & -46.8 & -2.55 & -76.94 & -102.66 & 24.93 & 77.18 \\
\hline 4.75 & -47.0 & -2.56 & -78.22 & -104.47 & 25.39 & 78.62 \\
\hline 4.80 & -47.6 & -2.57 & -79.76 & -106.15 & 25.89 & 80.25 \\
\hline 4.85 & -47.9 & -2.58 & -81.37 & -108.37 & 26.38 & 81.79 \\
\hline 4.90 & -48.3 & -2.59 & -82.92 & -110.38 & 26.87 & 83.37 \\
\hline 4.95 & -48.8 & -2.60 & -84.53 & -112.33 & 27.41 & 85.03 \\
\hline 5.00 & -49.0 & -2.61 & -86.14 & -114.21 & 27.92 & 86.58 \\
\hline 5.05 & -49.3 & -2.62 & -87.69 & -116.09 & 28.45 & 88.24 \\
\hline 5.10 & -49.3 & -2.63 & -89.03 & -117.83 & 28.95 & 89.75 \\
\hline 5.16 & -49.8 & -2.64 & -90.64 & -119.85 & 29.51 & 91.43 \\
\hline 5.21 & -50.1 & -2.65 & -92.18 & -121.86 & 30.04 & 93.16 \\
\hline 5.26 & -50.2 & -2.67 & -93.73 & -123.74 & 30.57 & 94.72 \\
\hline 5.31 & -50.5 & -2.67 & -95.27 & -125.69 & 31.10 & 96.38 \\
\hline 5.36 & -51.0 & -2.68 & -97.09 & -127.90 & 31.67 & 98.16 \\
\hline 5.41 & -51.1 & -2.70 & -98.63 & -129.72 & 32.22 & 99.87 \\
\hline 5.46 & -51.7 & -2.71 & -100.38 & -131.93 & 32.84 & 101.79 \\
\hline 5.51 & -51.9 & -2.72 & -102.05 & -133.88 & 33.40 & 103.51 \\
\hline 5.56 & -52.2 & -2.73 & -103.87 & -136.03 & 34.02 & 105.34 \\
\hline 5.61 & -52.5 & -2.74 & -105.61 & -138.24 & 34.62 & 107.21 \\
\hline 5.66 & -53.0 & -2.75 & -107.43 & -140.46 & 35.27 & 109.19 \\
\hline 5.71 & -53.1 & -2.76 & -109.10 & -142.27 & 35.88 & 111.07 \\
\hline 5.76 & -53.1 & -2.77 & -110.98 & -144.49 & 36.47 & 112.85 \\
\hline 5.81 & -53.5 & -2.78 & -112.80 & -146.77 & 37.11 & 114.76 \\
\hline 5.86 & -53.5 & -2.79 & -114.54 & -148.72 & 37.72 & 116.64 \\
\hline 5.91 & -53.6 & -2.80 & -116.29 & -150.73 & 38.35 & 118.47 \\
\hline 5.96 & -53.8 & -2.81 & -118.17 & -152.81 & 38.96 & 120.30 \\
\hline 6.01 & -54.1 & -2.82 & -119.78 & -154.96 & 39.62 & 122.30 \\
\hline 6.06 & -54.6 & -2.83 & -121.79 & -157.31 & 40.31 & 124.36 \\
\hline 6.11 & -54.8 & -2.84 & -123.67 & -159.53 & 41.00 & 126.43 \\
\hline 6.16 & -55.0 & -2.85 & -125.69 & -161.81 & 41.67 & 128.42 \\
\hline 6.21 & -55.1 & -2.86 & -127.50 & -163.82 & 42.36 & 130.47 \\
\hline 6.26 & -55.4 & -2.87 & -129.45 & -166.24 & 43.05 & 132.54 \\
\hline 6.31 & -55.6 & -2.88 & -131.46 & -168.52 & 43.78 & 134.65 \\
\hline 6.36 & -55.9 & -2.90 & -133.41 & -170.87 & 44.51 & 136.83 \\
\hline 6.41 & -56.0 & -2.90 & -135.42 & -173.22 & 45.22 & 138.93 \\
\hline 6.46 & -56.3 & -2.92 & -137.37 & -175.57 & 45.97 & 140.88 \\
\hline 6.51 & -56.5 & -2.93 & -139.38 & -177.86 & 46.75 & 142.67 \\
\hline 6.56 & -56.7 & -2.93 & -141.53 & -180.34 & 47.47 & 144.82 \\
\hline 6.61 & -56.9 & -2.95 & -143.41 & -182.62 & 48.27 & 147.11 \\
\hline 6.66 & -56.9 & -2.96 & -145.36 & -184.91 & 49.02 & 149.22 \\
\hline 6.71 & -57.2 & -2.97 & -147.51 & -187.39 & 49.84 & 151.47 \\
\hline 6.76 & -57.2 & -2.98 & -149.59 & -189.61 & 50.64 & 153.74 \\
\hline
\end{tabular}


Table F.7 IDT Strength Data (KL 58-40 \#8), continued

\begin{tabular}{|c|c|c|c|c|c|c|}
\hline Time & Axial Force & Axial Displ. & Vert front & Vert back & Horz front & Horz back \\
\hline $\mathrm{Sec}$ & $\mathrm{kN}$ & $\mathrm{mm}$ & $\mu \mathrm{m}$ & $\mu \mathrm{m}$ & $\mu \mathrm{m}$ & $\mu \mathrm{m}$ \\
\hline 6.81 & -57.4 & -2.99 & -151.60 & -191.96 & 51.43 & 156.05 \\
\hline 6.86 & -57.1 & -3.00 & -153.55 & -194.17 & 52.18 & 158.18 \\
\hline 6.91 & -57.3 & -3.01 & -155.57 & -196.45 & 52.97 & 160.40 \\
\hline 6.96 & -57.6 & -3.02 & -157.71 & -198.94 & 53.80 & 162.72 \\
\hline 7.01 & -57.9 & -3.03 & -160.06 & -201.56 & 54.67 & 165.12 \\
\hline 7.06 & -58.1 & -3.04 & -162.14 & -203.97 & 55.55 & 167.53 \\
\hline 7.11 & -58.3 & -3.05 & -164.49 & -206.52 & 56.46 & 169.93 \\
\hline 7.16 & -58.5 & -3.06 & -166.71 & -209.08 & 57.36 & 172.38 \\
\hline 7.21 & -58.7 & -3.07 & -168.99 & -211.90 & 58.28 & 174.92 \\
\hline 7.26 & -58.7 & -3.08 & -171.75 & -214.38 & 59.18 & 177.34 \\
\hline 7.31 & -58.8 & -3.09 & -174.03 & -217.07 & 60.16 & 179.85 \\
\hline 7.36 & -59.0 & -3.10 & -176.38 & -219.75 & 61.11 & 182.44 \\
\hline 7.41 & -58.9 & -3.11 & -178.59 & -222.10 & 62.05 & 184.86 \\
\hline 7.46 & -59.3 & -3.12 & -181.15 & -225.06 & 63.05 & 187.51 \\
\hline 7.51 & -59.6 & -3.14 & -183.70 & -227.88 & 64.12 & 190.38 \\
\hline 7.56 & -59.5 & -3.15 & -186.11 & -230.56 & 65.11 & 192.93 \\
\hline 7.61 & -59.8 & -3.16 & -188.67 & -233.45 & 66.16 & 195.70 \\
\hline 7.66 & -60.0 & -3.16 & -191.28 & -236.34 & 67.28 & 198.57 \\
\hline 7.71 & -60.4 & -3.17 & -193.90 & -239.36 & 68.42 & 201.47 \\
\hline 7.76 & -60.4 & -3.19 & -196.52 & -242.24 & 69.53 & 204.43 \\
\hline 7.81 & -60.2 & -3.19 & -199.01 & -245.13 & 70.65 & 207.23 \\
\hline 7.86 & -60.4 & -3.21 & -201.62 & -247.95 & 71.79 & 210.18 \\
\hline 7.91 & -60.2 & -3.22 & -204.11 & -250.70 & 72.91 & 212.99 \\
\hline 7.96 & -60.5 & -3.23 & -206.79 & -253.72 & 74.11 & 216.13 \\
\hline 8.01 & -60.5 & -3.24 & -209.61 & -257.01 & 75.26 & 219.15 \\
\hline 8.06 & -60.7 & -3.25 & -212.23 & -259.90 & 76.52 & 222.42 \\
\hline 8.11 & -60.8 & -3.26 & -214.92 & -262.86 & 77.77 & 225.61 \\
\hline 8.16 & -60.7 & -3.27 & -217.47 & -265.74 & 79.02 & 228.83 \\
\hline 8.21 & -60.9 & -3.28 & -220.22 & -268.83 & 80.34 & 232.19 \\
\hline 8.26 & -60.7 & -3.29 & -222.97 & -271.72 & 81.62 & 235.45 \\
\hline 8.31 & -60.9 & -3.30 & -225.79 & -274.94 & 82.94 & 238.95 \\
\hline 8.36 & -61.0 & -3.32 & -228.55 & -278.03 & 84.31 & 242.41 \\
\hline 8.41 & -60.9 & -3.32 & -231.23 & -281.12 & 85.69 & 245.92 \\
\hline 8.46 & -61.0 & -3.34 & -234.05 & -284.21 & 87.10 & 249.56 \\
\hline 8.51 & -61.0 & -3.34 & -236.94 & -287.43 & 88.53 & 253.17 \\
\hline 8.56 & -61.1 & -3.36 & -239.76 & -290.59 & 90.01 & 257.01 \\
\hline 8.61 & -61.1 & -3.36 & -242.85 & -293.94 & 91.53 & 260.99 \\
\hline 8.66 & -61.0 & -3.38 & -245.80 & -296.96 & 93.00 & 264.87 \\
\hline 8.71 & -61.2 & -3.38 & -248.69 & -300.32 & 94.60 & 268.98 \\
\hline 8.76 & -61.5 & -3.40 & -251.84 & -303.81 & 96.25 & 273.40 \\
\hline 8.81 & -61.4 & -3.41 & -255.00 & -307.24 & 97.92 & 277.81 \\
\hline 8.86 & -61.3 & -3.42 & -258.09 & -310.53 & 99.59 & 282.26 \\
\hline 8.91 & -61.3 & -3.43 & -261.24 & -313.95 & 101.32 & 286.91 \\
\hline 8.96 & -61.4 & -3.44 & -264.60 & -317.44 & 103.09 & 291.78 \\
\hline 9.01 & -61.2 & -3.45 & -267.82 & -320.73 & 104.82 & 296.49 \\
\hline
\end{tabular}


Table F.7 IDT Strength Data (KL 58-40 \#8), continued

\begin{tabular}{|c|c|c|c|c|c|c|}
\hline Time & Axial Force & Axial Displ. & Vert front & Vert back & Horz front & Horz back \\
\hline $\mathrm{Sec}$ & $\mathrm{kN}$ & $\mathrm{mm}$ & $\mu \mathrm{m}$ & $\mu \mathrm{m}$ & $\mu \mathrm{m}$ & $\mu \mathrm{m}$ \\
\hline 9.06 & -61.1 & -3.46 & -271.05 & -324.16 & 106.66 & 301.50 \\
\hline 9.11 & -61.1 & -3.47 & -274.27 & -327.65 & 108.54 & 306.65 \\
\hline 9.16 & -60.9 & -3.48 & -277.69 & -331.14 & 110.44 & 311.95 \\
\hline 9.21 & -60.9 & -3.49 & -281.12 & -334.63 & 112.39 & 317.44 \\
\hline 9.26 & -60.8 & -3.50 & -284.61 & -338.25 & 114.37 & 323.15 \\
\hline 9.31 & -61.1 & -3.51 & -288.17 & -342.08 & 116.55 & 329.51 \\
\hline 9.36 & -60.7 & -3.52 & -291.86 & -345.91 & 118.65 & 335.74 \\
\hline 9.41 & -60.7 & -3.53 & -295.42 & -349.67 & 120.87 & 342.42 \\
\hline 9.46 & -60.6 & -3.54 & -299.25 & -353.63 & 123.18 & 349.45 \\
\hline 9.51 & -60.5 & -3.55 & -303.01 & -357.46 & 125.54 & 356.74 \\
\hline 9.56 & -60.4 & -3.57 & -306.83 & -361.42 & 128.00 & 364.52 \\
\hline 9.61 & -60.3 & -3.57 & -310.73 & -365.45 & 130.50 & 372.68 \\
\hline 9.66 & -60.2 & -3.59 & -314.76 & -369.74 & 133.15 & 381.41 \\
\hline 9.71 & -60.1 & -3.60 & -318.85 & -374.11 & 135.84 & 390.71 \\
\hline 9.76 & -60.0 & -3.61 & -323.22 & -378.54 & 138.65 & 400.63 \\
\hline 9.81 & -59.6 & -3.62 & -327.31 & -382.90 & 141.50 & 410.99 \\
\hline 9.86 & -59.3 & -3.63 & -331.74 & -387.33 & 144.40 & 422.03 \\
\hline 9.91 & -59.0 & -3.64 & -336.31 & -392.10 & 147.41 & 433.88 \\
\hline 9.96 & -59.0 & -3.65 & -340.94 & -397.07 & 150.59 & 446.80 \\
\hline 10.01 & -58.8 & -3.66 & -345.77 & -402.71 & 153.87 & 460.69 \\
\hline 10.06 & -58.4 & -3.67 & -350.74 & -408.15 & 157.26 & 475.41 \\
\hline 10.11 & -57.8 & -3.68 & -355.44 & -413.12 & 160.68 & 490.38 \\
\hline 10.16 & -57.5 & -3.69 & -360.61 & -417.95 & 164.28 & 506.21 \\
\hline 10.21 & -57.1 & -3.70 & -365.85 & -422.58 & 168.02 & 522.74 \\
\hline 10.26 & -56.8 & -3.71 & -371.42 & -426.88 & 171.96 & 540.10 \\
\hline 10.31 & -56.3 & -3.72 & -376.93 & -430.77 & 176.08 & 545.37 \\
\hline 10.36 & -55.9 & -3.73 & -382.50 & -433.66 & 180.42 & 545.37 \\
\hline 10.41 & -55.4 & -3.74 & -388.07 & -435.81 & 184.98 & 545.37 \\
\hline
\end{tabular}


Table F.8 IDT Strength Data (NU 52-34 \#6)

\begin{tabular}{|c|c|c|c|c|c|c|}
\hline Time & Axial Force & Axial Displ. & Vert front & Vert back & Horz front & Horz back \\
\hline $\mathrm{Sec}$ & $\mathrm{kN}$ & $\mathrm{mm}$ & $\mu \mathrm{m}$ & $\mu \mathrm{m}$ & $\mu \mathrm{m}$ & $\mu \mathrm{m}$ \\
\hline 0.00 & 0.0 & -0.73 & 0.47 & 0.81 & 0.18 & 0.00 \\
\hline 0.05 & -0.1 & -0.74 & 0.54 & 0.87 & 0.19 & 0.00 \\
\hline 0.10 & -0.1 & -0.75 & 0.47 & 0.81 & 0.19 & 0.02 \\
\hline 0.15 & -0.3 & -0.76 & 0.47 & 0.81 & 0.22 & 0.03 \\
\hline 0.20 & -0.6 & -0.77 & 0.34 & 0.67 & 0.23 & 0.12 \\
\hline 0.25 & -1.1 & -0.78 & 0.27 & 0.47 & 0.28 & 0.25 \\
\hline 0.30 & -1.6 & -0.79 & 0.27 & 0.13 & 0.30 & 0.40 \\
\hline 0.35 & -2.0 & -0.80 & -0.07 & -0.13 & 0.34 & 0.50 \\
\hline 0.40 & -2.6 & -0.81 & -0.13 & -0.40 & 0.39 & 0.69 \\
\hline 0.45 & -3.3 & -0.82 & -0.34 & -1.01 & 0.44 & 0.84 \\
\hline 0.50 & -4.1 & -0.83 & -0.54 & -1.41 & 0.52 & 1.11 \\
\hline 0.55 & -4.9 & -0.84 & -0.81 & -1.88 & 0.58 & 1.34 \\
\hline 0.60 & $\begin{array}{l}-5.7 \\
\end{array}$ & -0.85 & -1.07 & -2.35 & 0.65 & 1.58 \\
\hline 0.65 & -6.5 & -0.86 & -1.21 & -2.89 & 0.74 & 1.83 \\
\hline 0.70 & -7.4 & -0.88 & -1.61 & -3.42 & 0.83 & 2.11 \\
\hline 0.75 & -8.1 & -0.89 & -1.95 & -3.83 & 0.89 & 2.35 \\
\hline 0.80 & -8.9 & -0.89 & -2.22 & -4.23 & 0.97 & 2.62 \\
\hline 0.85 & -9.7 & -0.90 & -2.48 & -4.83 & 1.07 & 2.89 \\
\hline 0.90 & -10.9 & -0.91 & -2.82 & $\begin{array}{l}-5.51 \\
\end{array}$ & 1.17 & 3.26 \\
\hline 0.95 & -11.8 & -0.93 & -3.22 & -6.11 & 1.29 & 3.51 \\
\hline 1.00 & -12.9 & -0.94 & -3.42 & -6.71 & 1.41 & 3.89 \\
\hline 1.05 & -13.6 & -0.95 & -3.76 & -7.18 & 1.49 & 4.16 \\
\hline 1.10 & -14.6 & -0.96 & -4.23 & -7.99 & 1.60 & 4.55 \\
\hline 1.15 & -15.3 & -0.97 & -4.57 & -8.39 & 1.70 & 4.83 \\
\hline 1.20 & -16.2 & -0.98 & -4.90 & -9.06 & 1.80 & 5.14 \\
\hline 1.25 & -17.0 & -0.99 & -5.37 & -9.53 & 1.91 & 5.47 \\
\hline 1.30 & -17.6 & -1.00 & -5.57 & -10.14 & 1.99 & 5.74 \\
\hline 1.35 & -18.7 & -1.01 & -6.04 & -10.81 & 2.11 & 6.11 \\
\hline 1.40 & -19.4 & -1.02 & -6.38 & -11.28 & 2.22 & 6.43 \\
\hline 1.45 & -20.4 & -1.03 & -6.78 & -12.09 & 2.32 & 6.78 \\
\hline 1.50 & -21.0 & -1.04 & -6.85 & -12.42 & 2.45 & 7.17 \\
\hline 1.55 & -21.9 & -1.05 & -7.45 & -13.36 & 2.54 & 7.47 \\
\hline 1.60 & -22.8 & -1.06 & -7.86 & -13.83 & 2.67 & 7.84 \\
\hline 1.65 & -23.5 & -1.08 & -8.19 & -14.44 & 2.76 & 8.19 \\
\hline 1.70 & -24.4 & -1.08 & -8.53 & -15.17 & 2.89 & 8.56 \\
\hline 1.75 & -25.3 & -1.09 & -9.00 & -15.78 & 3.03 & 9.00 \\
\hline 1.80 & -26.2 & -1.10 & -9.40 & -16.52 & 3.14 & 9.38 \\
\hline 1.85 & -27.2 & -1.11 & -9.87 & -17.26 & 3.27 & 9.82 \\
\hline 1.90 & -28.1 & -1.12 & -10.21 & -17.99 & 3.40 & 10.26 \\
\hline 1.95 & -29.0 & -1.14 & -10.61 & -18.73 & 3.53 & 10.69 \\
\hline 2.00 & -29.8 & -1.14 & -11.15 & -19.54 & 3.65 & 11.08 \\
\hline 2.05 & -30.6 & -1.15 & -11.48 & -20.14 & 3.79 & 11.51 \\
\hline 2.10 & -31.3 & -1.16 & -11.95 & -20.95 & 3.91 & 11.92 \\
\hline 2.15 & -32.3 & -1.17 & -12.35 & -21.62 & 4.06 & 12.39 \\
\hline 2.20 & -33.0 & -1.19 & -12.76 & -22.36 & 4.15 & 12.82 \\
\hline 2.25 & -33.6 & -1.20 & -13.29 & -22.96 & 4.29 & 13.21 \\
\hline
\end{tabular}


Table F.8 IDT Strength Data (NU 52-34 \#6), continued

\begin{tabular}{|c|c|c|c|c|c|c|}
\hline Time & Axial Force & Axial Displ. & Vert front & Vert back & Horz front & Horz back \\
\hline $\mathrm{Sec}$ & $\mathrm{kN}$ & $\mathrm{mm}$ & $\mu \mathrm{m}$ & $\mu \mathrm{m}$ & $\mu \mathrm{m}$ & $\mu \mathrm{m}$ \\
\hline 2.30 & -34.5 & -1.21 & -13.70 & -23.77 & 4.41 & 13.66 \\
\hline 2.35 & -35.6 & -1.21 & -14.10 & -24.57 & 4.57 & 14.22 \\
\hline 2.40 & -36.6 & -1.23 & -14.57 & -25.45 & 4.72 & 14.74 \\
\hline 2.45 & -37.3 & -1.24 & -15.04 & -26.25 & 4.86 & 15.22 \\
\hline 2.50 & -38.0 & -1.25 & -15.44 & -26.92 & 4.98 & 15.71 \\
\hline 2.55 & -38.6 & -1.26 & -15.91 & -27.73 & 5.09 & 16.13 \\
\hline 2.60 & -39.4 & -1.27 & -16.38 & -28.40 & 5.25 & 16.63 \\
\hline 2.65 & -40.5 & -1.28 & -16.92 & -29.47 & 5.40 & 17.22 \\
\hline 2.70 & -41.2 & -1.29 & -17.39 & -30.21 & 5.54 & 17.74 \\
\hline 2.75 & -41.9 & -1.30 & -17.79 & -30.95 & 5.68 & 18.21 \\
\hline 2.80 & -42.6 & -1.31 & -18.26 & -31.76 & 5.82 & 18.78 \\
\hline 2.85 & -43.3 & -1.32 & -18.73 & -32.63 & 5.94 & 19.27 \\
\hline 2.90 & -44.1 & -1.33 & -19.27 & -33.44 & 6.10 & 19.86 \\
\hline 2.95 & -44.8 & -1.34 & -19.74 & -34.38 & 6.24 & 20.43 \\
\hline 3.00 & -45.9 & -1.35 & -20.41 & -35.38 & 6.40 & 21.08 \\
\hline 3.05 & -46.4 & -1.36 & -20.81 & -36.12 & 6.55 & 21.64 \\
\hline 3.10 & -47.1 & -1.37 & -21.28 & -36.99 & 6.68 & 22.22 \\
\hline 3.15 & -47.8 & -1.38 & -21.82 & -37.73 & 6.83 & 22.81 \\
\hline 3.20 & -48.6 & -1.39 & -22.22 & -38.67 & 6.99 & 23.42 \\
\hline 3.25 & -49.1 & -1.40 & -22.89 & -39.61 & 7.13 & 24.04 \\
\hline 3.30 & -49.8 & -1.41 & -23.30 & -40.49 & 7.26 & 24.64 \\
\hline 3.35 & -50.5 & -1.42 & -23.57 & -41.09 & 7.45 & 25.35 \\
\hline 3.40 & -51.1 & -1.43 & -24.51 & -42.43 & 7.57 & 25.93 \\
\hline 3.45 & -51.6 & -1.45 & -24.77 & -43.04 & 7.74 & 26.57 \\
\hline 3.50 & $\begin{array}{l}-53.0 \\
\end{array}$ & -1.46 & -25.38 & -44.25 & 7.93 & 27.38 \\
\hline 3.55 & $\begin{array}{l}-53.5 \\
\end{array}$ & -1.46 & -26.05 & -45.05 & 8.07 & 28.12 \\
\hline 3.60 & -54.0 & -1.48 & -26.59 & -46.13 & 8.23 & 28.77 \\
\hline 3.65 & -54.7 & -1.48 & -27.12 & -47.00 & 8.41 & 29.49 \\
\hline 3.70 & -55.5 & -1.50 & -27.80 & -48.01 & 8.56 & 30.30 \\
\hline 3.75 & -56.1 & -1.51 & -28.33 & -48.95 & 8.75 & 31.07 \\
\hline 3.80 & -56.9 & -1.52 & -29.00 & -50.09 & 8.90 & 31.94 \\
\hline 3.85 & $\begin{array}{l}-57.4 \\
\end{array}$ & -1.53 & -29.61 & -51.09 & 9.08 & 32.80 \\
\hline 3.90 & $\begin{array}{l}-57.8 \\
\end{array}$ & -1.54 & -30.01 & -51.90 & 9.22 & 33.54 \\
\hline 3.95 & -58.6 & -1.55 & -30.68 & -53.04 & 9.40 & 34.43 \\
\hline 4.00 & -59.1 & -1.56 & -31.22 & -54.05 & 9.57 & 35.32 \\
\hline 4.05 & -59.9 & -1.57 & -31.89 & -55.12 & 9.75 & 36.19 \\
\hline 4.10 & -60.5 & -1.58 & -32.50 & -56.26 & 9.93 & 37.10 \\
\hline 4.15 & -61.1 & -1.59 & -33.17 & -57.41 & 10.12 & 38.04 \\
\hline 4.20 & -61.5 & -1.60 & -33.77 & -58.28 & 10.27 & 38.91 \\
\hline 4.25 & -61.8 & -1.61 & -34.38 & -59.35 & 10.45 & 39.80 \\
\hline 4.30 & -62.4 & -1.62 & -35.05 & -60.36 & 10.62 & 40.70 \\
\hline 4.35 & -62.9 & -1.63 & -35.58 & -61.50 & 10.80 & 41.66 \\
\hline 4.40 & -63.7 & -1.64 & -36.32 & -62.64 & 11.01 & 42.70 \\
\hline 4.45 & -64.2 & -1.65 & -37.06 & -63.92 & 11.19 & 43.74 \\
\hline 4.50 & -64.7 & -1.66 & -37.73 & -64.99 & 11.38 & 44.78 \\
\hline
\end{tabular}


Table F.8 IDT Strength Data (NU 52-34 \#6), continued

\begin{tabular}{|c|c|c|c|c|c|c|}
\hline Time & Axial Force & Axial Displ. & Vert front & Vert back & Horz front & Horz back \\
\hline $\mathrm{Sec}$ & $\mathrm{kN}$ & $\mathrm{mm}$ & $\mu \mathrm{m}$ & $\mu \mathrm{m}$ & $\mu \mathrm{m}$ & $\mu \mathrm{m}$ \\
\hline 4.55 & -65.3 & -1.67 & -38.47 & -66.20 & 11.57 & 45.87 \\
\hline 4.60 & -65.8 & -1.68 & -39.14 & -67.41 & 11.77 & 46.93 \\
\hline 4.65 & -66.0 & -1.70 & -39.81 & -68.55 & 11.96 & 48.02 \\
\hline 4.70 & -66.6 & -1.70 & -40.55 & -69.62 & 12.17 & 49.16 \\
\hline 4.75 & -67.2 & -1.71 & -41.36 & -71.03 & 12.35 & 50.36 \\
\hline 4.80 & -67.6 & -1.73 & -42.16 & -72.18 & 12.56 & 51.60 \\
\hline 4.85 & -68.0 & -1.74 & -42.77 & -73.32 & 12.76 & 52.81 \\
\hline 4.90 & -68.3 & -1.75 & -43.51 & -74.66 & 12.94 & 54.08 \\
\hline 4.95 & -68.6 & -1.75 & -44.25 & -75.67 & 13.14 & 55.34 \\
\hline 5.00 & -69.1 & -1.76 & -45.12 & -77.21 & 13.34 & 56.72 \\
\hline 5.05 & -69.3 & -1.77 & -45.86 & -78.29 & 13.55 & 58.06 \\
\hline 5.10 & -69.8 & -1.78 & -46.53 & -79.63 & 13.76 & 59.52 \\
\hline 5.16 & -70.3 & -1.80 & -47.47 & -81.11 & 13.97 & 61.06 \\
\hline 5.21 & -70.9 & -1.81 & -48.34 & -82.65 & 14.22 & 62.73 \\
\hline 5.26 & -71.1 & -1.82 & -49.15 & -83.99 & 14.43 & 64.37 \\
\hline 5.31 & -71.5 & -1.83 & -50.09 & -85.54 & 14.63 & 66.03 \\
\hline 5.36 & -71.8 & -1.84 & -50.96 & -86.95 & 14.84 & 67.90 \\
\hline 5.41 & -72.0 & -1.85 & -51.97 & -88.36 & 15.27 & 69.79 \\
\hline 5.46 & -72.5 & -1.86 & -53.11 & -89.77 & 15.45 & 74.26 \\
\hline 5.51 & -72.5 & -1.87 & -55.32 & -91.78 & 15.31 & 99.84 \\
\hline 5.56 & -72.2 & -1.88 & -59.82 & -93.73 & 17.67 & 118.57 \\
\hline 5.61 & -71.8 & -1.89 & -64.72 & -95.74 & 22.86 & 138.34 \\
\hline 5.66 & -71.0 & -1.91 & -69.15 & -98.50 & 30.73 & 164.73 \\
\hline 5.71 & -69.5 & -1.92 & -76.61 & -101.65 & 50.37 & 196.39 \\
\hline 5.76 & -67.6 & -1.93 & -84.46 & -103.46 & 69.14 & 234.69 \\
\hline 5.81 & -65.7 & -1.94 & -93.26 & -103.80 & 84.87 & 278.20 \\
\hline
\end{tabular}


Table F.9 IDT Strength Data (NU 52-34 \#8)

\begin{tabular}{|c|c|c|c|c|c|c|}
\hline Time & Axial Force & Axial Displ. & Vert front & Vert back & Horz front & Horz back \\
\hline $\mathrm{Sec}$ & $\overline{\mathrm{kN}}$ & $\mathrm{mm}$ & $\mu \mathrm{m}$ & $\mu \mathrm{m}$ & $\mu \mathrm{m}$ & $\mu \mathrm{m}$ \\
\hline 0.00 & -0.4 & -0.97 & 1.48 & 1.41 & 0.26 & 0.35 \\
\hline 0.05 & -0.9 & -0.98 & 1.41 & 1.14 & 0.30 & 0.47 \\
\hline 0.10 & -1.4 & -0.99 & 1.21 & 0.67 & 0.34 & 0.64 \\
\hline 0.15 & -2.2 & -1.00 & 1.07 & 0.13 & 0.41 & 0.86 \\
\hline 0.20 & -2.9 & -1.01 & 0.94 & -0.20 & 0.50 & 1.09 \\
\hline 0.25 & -3.6 & -1.02 & 0.60 & -0.81 & 0.58 & 1.34 \\
\hline 0.30 & -4.5 & -1.04 & 0.34 & -1.34 & 0.68 & 1.58 \\
\hline 0.35 & -5.0 & -1.05 & 0.07 & -1.75 & 0.74 & 1.78 \\
\hline 0.40 & -6.0 & -1.05 & -0.13 & -2.42 & 0.86 & 2.08 \\
\hline 0.45 & -6.7 & -1.06 & -0.34 & -2.95 & 0.95 & 2.33 \\
\hline 0.50 & -7.6 & -1.07 & -0.81 & -3.56 & 1.06 & 2.64 \\
\hline 0.55 & -8.5 & -1.09 & -1.01 & -4.16 & 1.19 & 2.95 \\
\hline 0.60 & -9.4 & -1.09 & -1.54 & -4.77 & 1.29 & 3.27 \\
\hline 0.65 & -10.2 & -1.10 & -1.75 & -5.44 & 1.39 & 3.58 \\
\hline 0.70 & -11.1 & -1.11 & -2.15 & -6.18 & 1.53 & 3.93 \\
\hline 0.75 & -12.1 & -1.13 & -2.48 & -6.85 & 1.65 & 4.31 \\
\hline 0.80 & -13.1 & -1.14 & -2.89 & -7.79 & 1.80 & 4.73 \\
\hline 0.85 & -13.8 & -1.15 & -3.22 & -8.33 & 1.92 & 5.02 \\
\hline 0.90 & -14.7 & -1.16 & -3.63 & -9.06 & 2.05 & 5.39 \\
\hline 0.95 & -15.4 & -1.17 & -3.96 & -9.67 & 2.17 & 5.69 \\
\hline 1.00 & -16.1 & -1.18 & -4.30 & -10.34 & 2.27 & 6.03 \\
\hline 1.05 & -16.7 & -1.19 & -4.57 & -10.81 & 2.39 & 6.34 \\
\hline 1.10 & -17.8 & -1.20 & -5.04 & -11.75 & 2.55 & 6.73 \\
\hline 1.15 & -18.7 & -1.21 & -5.44 & -12.56 & 2.69 & 7.17 \\
\hline 1.20 & -19.7 & -1.22 & -5.91 & -13.29 & 2.85 & 7.57 \\
\hline 1.25 & -20.3 & -1.23 & -6.24 & -14.03 & 2.98 & 7.92 \\
\hline 1.30 & -21.0 & -1.24 & -6.58 & -14.64 & 3.09 & 8.29 \\
\hline 1.35 & -21.7 & -1.25 & -6.85 & -15.31 & 3.24 & 8.64 \\
\hline 1.40 & -22.6 & -1.26 & -7.32 & -16.11 & 3.38 & 9.06 \\
\hline 1.45 & -23.3 & -1.27 & $\begin{array}{l}-7.79 \\
\end{array}$ & -16.79 & 3.52 & 9.48 \\
\hline 1.50 & -24.1 & $\begin{array}{l}-1.29 \\
\end{array}$ & -8.26 & -17.59 & 3.68 & 9.89 \\
\hline 1.55 & -24.9 & $\begin{array}{l}-1.29 \\
\end{array}$ & -8.66 & -18.06 & 3.84 & 10.31 \\
\hline 1.60 & -25.5 & -1.30 & -9.20 & -19.00 & 3.95 & 10.66 \\
\hline 1.65 & -26.3 & -1.31 & -9.53 & -19.67 & 4.10 & 11.06 \\
\hline 1.70 & -27.1 & -1.32 & -10.07 & -20.55 & 4.28 & 11.55 \\
\hline 1.75 & -28.2 & -1.34 & -10.54 & -21.35 & 4.47 & 12.03 \\
\hline 1.80 & -28.7 & -1.35 & -11.01 & -22.02 & 4.63 & 12.44 \\
\hline 1.85 & -29.7 & -1.36 & -11.48 & -23.03 & 4.80 & 12.96 \\
\hline 1.90 & -30.4 & -1.37 & -12.09 & -23.70 & 4.98 & 13.38 \\
\hline 1.95 & -31.4 & -1.38 & -12.62 & -24.64 & 5.16 & 13.91 \\
\hline 2.00 & -32.0 & -1.39 & -13.16 & -25.31 & 5.33 & 14.35 \\
\hline 2.05 & -32.6 & -1.40 & -13.56 & -25.78 & 5.52 & 14.85 \\
\hline 2.10 & -33.4 & -1.41 & -14.23 & -26.86 & 5.67 & 15.31 \\
\hline 2.15 & -34.1 & -1.42 & -14.70 & -27.66 & 5.86 & 15.79 \\
\hline 2.20 & \begin{tabular}{|l|}
-34.9 \\
\end{tabular} & $\begin{array}{l}-1.43 \\
\end{array}$ & -15.31 & -28.47 & 6.05 & 16.33 \\
\hline 2.25 & -35.7 & -1.44 & -15.85 & -29.34 & 6.24 & 16.84 \\
\hline
\end{tabular}


Table F.9 IDT Strength Data (NU 52-34 \#8), continued

\begin{tabular}{|c|c|c|c|c|c|c|}
\hline Time & Axial Force & Axial Displ. & Vert front & Vert back & Horz front & Horz back \\
\hline $\mathrm{Sec}$ & $\mathrm{kN}$ & $\mathrm{mm}$ & $\mu \mathrm{m}$ & $\mu \mathrm{m}$ & $\mu \mathrm{m}$ & $\mu \mathrm{m}$ \\
\hline 2.30 & -36.4 & -1.45 & -16.52 & -30.08 & 6.43 & 17.34 \\
\hline 2.35 & -37.1 & -1.46 & -16.99 & -30.95 & 6.61 & 17.86 \\
\hline 2.40 & -38.0 & -1.47 & -17.66 & -31.82 & 6.84 & 18.48 \\
\hline 2.45 & -38.7 & -1.48 & -18.20 & -32.63 & 7.02 & 19.02 \\
\hline 2.50 & -39.9 & -1.49 & -19.14 & -33.84 & 7.27 & 19.66 \\
\hline 2.55 & -40.6 & -1.50 & -19.47 & -34.38 & 7.49 & 20.28 \\
\hline 2.60 & -41.3 & -1.51 & -20.28 & -35.45 & 7.70 & 20.83 \\
\hline 2.65 & -41.9 & -1.52 & -20.81 & -36.39 & 7.91 & 21.42 \\
\hline 2.70 & -42.4 & -1.53 & -21.49 & -36.99 & 8.09 & 21.94 \\
\hline 2.75 & -43.0 & -1.54 & -22.02 & -38.00 & 8.29 & 22.54 \\
\hline 2.80 & -43.9 & -1.55 & -22.69 & -38.87 & 8.53 & 23.15 \\
\hline 2.85 & -44.6 & -1.56 & -23.43 & -39.75 & 8.75 & 23.80 \\
\hline 2.90 & -45.3 & -1.58 & -24.04 & -40.55 & 8.97 & 24.46 \\
\hline 2.95 & -46.1 & -1.58 & -24.71 & -41.56 & 9.20 & 25.14 \\
\hline 3.00 & -46.7 & -1.59 & -25.51 & -42.57 & 9.42 & 25.73 \\
\hline 3.05 & -47.6 & -1.61 & -26.12 & -43.24 & 9.70 & 26.50 \\
\hline 3.10 & -47.9 & -1.61 & -26.86 & -44.38 & 9.91 & 27.09 \\
\hline 3.15 & -48.5 & -1.63 & -27.46 & -45.32 & 10.10 & 27.70 \\
\hline 3.20 & -49.3 & -1.64 & -28.33 & -46.19 & 10.37 & 28.38 \\
\hline 3.25 & -49.7 & -1.65 & -28.87 & -47.13 & 10.58 & 29.06 \\
\hline 3.30 & -50.8 & -1.66 & -29.88 & -48.27 & 10.88 & 29.89 \\
\hline 3.35 & -51.5 & -1.67 & -30.68 & -49.28 & 11.12 & 30.72 \\
\hline 3.40 & -51.9 & -1.68 & -31.29 & -50.15 & 11.36 & 31.42 \\
\hline 3.45 & -52.4 & -1.69 & -32.09 & -51.09 & 11.60 & 32.18 \\
\hline 3.50 & $\begin{array}{l}-53.1 \\
\end{array}$ & -1.70 & -32.97 & -52.24 & 11.85 & 32.98 \\
\hline 3.55 & $\begin{array}{l}-53.7 \\
\end{array}$ & -1.71 & -33.84 & -53.51 & 12.09 & 33.72 \\
\hline 3.60 & -54.3 & -1.72 & -34.51 & -54.32 & 12.37 & 34.59 \\
\hline 3.65 & -55.0 & -1.73 & -35.25 & -55.46 & 12.64 & 35.47 \\
\hline 3.70 & -55.6 & -1.74 & -36.05 & -56.47 & 12.92 & 36.29 \\
\hline 3.75 & -56.2 & -1.75 & -36.93 & -57.61 & 13.18 & 37.16 \\
\hline 3.80 & -56.8 & -1.77 & -37.73 & -58.68 & 13.46 & 38.07 \\
\hline 3.85 & $\begin{array}{l}-57.0 \\
\end{array}$ & -1.77 & -38.61 & -59.76 & 13.71 & 38.89 \\
\hline 3.90 & -57.7 & -1.78 & -39.34 & -61.03 & 13.99 & 39.76 \\
\hline 3.95 & -58.1 & -1.79 & -40.22 & -62.24 & 14.26 & 40.69 \\
\hline 4.00 & -58.7 & -1.80 & -41.22 & -63.72 & 14.53 & 41.59 \\
\hline 4.05 & -59.3 & -1.81 & -41.96 & -64.79 & 14.85 & 42.58 \\
\hline 4.10 & $\begin{array}{l}-59.8 \\
\end{array}$ & -1.83 & -42.84 & -66.20 & 15.13 & 43.62 \\
\hline 4.15 & -60.4 & -1.83 & -43.78 & -67.48 & 15.45 & 44.67 \\
\hline 4.20 & -60.7 & -1.85 & -44.65 & -68.82 & 15.72 & 45.64 \\
\hline 4.25 & $\begin{array}{l}-61.3 \\
\end{array}$ & -1.86 & -45.52 & -70.23 & 16.02 & 46.71 \\
\hline 4.30 & -61.9 & -1.86 & -46.46 & -71.57 & 16.33 & 47.82 \\
\hline 4.35 & -62.2 & -1.88 & -47.33 & -73.05 & 16.63 & 48.95 \\
\hline 4.40 & -62.4 & -1.89 & -48.27 & -74.26 & 16.92 & 49.95 \\
\hline 4.45 & -62.7 & -1.90 & -49.21 & -76.00 & 17.18 & $\begin{array}{l}51.01 \\
\end{array}$ \\
\hline 4.50 & $\begin{array}{l}-63.4 \\
\end{array}$ & -1.91 & -50.15 & -77.21 & 17.56 & 52.27 \\
\hline
\end{tabular}


Table F.9 IDT Strength Data (NU 52-34 \#8), continued

\begin{tabular}{|c|c|c|c|c|c|c|}
\hline Time & Axial Force & Axial Displ. & Vert front & Vert back & Horz front & Horz back \\
\hline $\mathrm{Sec}$ & $\mathrm{kN}$ & $\mathrm{mm}$ & $\mu \mathrm{m}$ & $\mu \mathrm{m}$ & $\mu \mathrm{m}$ & $\mu \mathrm{m}$ \\
\hline 4.55 & -63.9 & -1.92 & -51.16 & -78.69 & 17.91 & 53.46 \\
\hline 4.60 & -64.4 & -1.93 & -52.17 & -80.30 & 18.25 & 54.72 \\
\hline 4.65 & -64.7 & -1.94 & -53.18 & -81.84 & 18.59 & 55.93 \\
\hline 4.70 & -65.0 & -1.95 & -54.18 & -83.46 & 18.93 & 57.22 \\
\hline 4.75 & -65.4 & -1.96 & -55.19 & -84.87 & 19.27 & 58.55 \\
\hline 4.80 & -65.7 & -1.97 & -56.06 & -86.54 & 19.61 & 59.89 \\
\hline 4.85 & -66.2 & -1.97 & -57.14 & -88.22 & 19.93 & 61.30 \\
\hline 4.90 & -66.7 & -1.99 & -58.21 & -89.97 & 20.31 & 62.76 \\
\hline 4.95 & -67.0 & -2.00 & -59.29 & -91.78 & 20.66 & 64.27 \\
\hline 5.00 & -67.4 & -2.01 & -60.36 & -93.66 & 21.02 & 65.75 \\
\hline 5.05 & -67.8 & -2.02 & -61.57 & -95.47 & 21.42 & 67.33 \\
\hline 5.10 & -68.1 & -2.03 & -62.64 & -97.29 & 21.78 & 68.90 \\
\hline 5.16 & -68.4 & -2.04 & -63.78 & -98.97 & 22.18 & 70.60 \\
\hline 5.21 & -68.6 & -2.05 & -64.86 & -100.85 & 22.56 & 72.33 \\
\hline 5.26 & -69.0 & -2.06 & -66.13 & -102.79 & 22.95 & 74.11 \\
\hline 5.31 & -69.1 & -2.07 & -67.21 & -104.67 & 23.34 & 75.90 \\
\hline 5.36 & -69.6 & -2.08 & -68.42 & -106.69 & 23.78 & 77.85 \\
\hline 5.41 & -69.9 & -2.09 & -69.69 & -108.77 & 24.21 & 79.90 \\
\hline 5.46 & -70.2 & -2.11 & -71.03 & -110.92 & 24.64 & 81.96 \\
\hline 5.51 & -70.2 & -2.12 & -72.24 & -112.93 & 25.07 & 84.09 \\
\hline 5.56 & -70.5 & -2.12 & -73.38 & -115.15 & 25.51 & 86.33 \\
\hline 5.61 & -70.8 & -2.14 & -74.79 & -117.43 & 26.01 & 88.64 \\
\hline 5.66 & -71.2 & -2.15 & -76.34 & -119.85 & 26.54 & 91.40 \\
\hline 5.71 & -71.4 & -2.16 & -77.61 & -122.60 & 27.05 & 94.50 \\
\hline 5.76 & -71.4 & -2.17 & -80.50 & -122.93 & 27.54 & 117.95 \\
\hline 5.81 & -70.2 & -2.18 & -84.40 & -127.57 & 39.77 & 160.67 \\
\hline 5.86 & -68.1 & -2.19 & -96.15 & -123.74 & 60.97 & 220.61 \\
\hline 5.91 & -65.2 & -2.20 & -110.04 & -119.85 & 86.35 & 281.60 \\
\hline
\end{tabular}


Table F.10 IDT Strength Data (NU 52-34 \#9)

\begin{tabular}{|c|c|c|c|c|c|c|}
\hline Time & Axial Force & Axial Displ. & Vert front & Vert back & Horz front & Horz back \\
\hline $\mathrm{Sec}$ & $\mathrm{kN}$ & $\mathrm{mm}$ & $\mu \mathrm{m}$ & $\mu \mathrm{m}$ & $\mu \mathrm{m}$ & $\mu \mathrm{m}$ \\
\hline 0.00 & -0.4 & -1.01 & 1.14 & 0.67 & 0.19 & 0.13 \\
\hline 0.05 & -0.7 & -1.02 & 0.94 & 0.47 & 0.23 & 0.22 \\
\hline 0.10 & -1.3 & -1.03 & 0.74 & 0.27 & 0.29 & 0.29 \\
\hline 0.15 & -1.8 & -1.04 & 0.54 & 0.07 & 0.35 & 0.42 \\
\hline 0.20 & -2.5 & -1.05 & 0.20 & -0.20 & 0.44 & 0.54 \\
\hline 0.25 & -3.0 & -1.06 & -0.07 & -0.34 & 0.52 & 0.67 \\
\hline 0.30 & -3.6 & -1.06 & -0.40 & -0.60 & 0.59 & 0.79 \\
\hline 0.35 & -4.7 & -1.08 & -0.94 & -1.14 & 0.70 & 0.97 \\
\hline 0.40 & -5.4 & -1.09 & -1.21 & -1.34 & 0.81 & 1.16 \\
\hline 0.45 & -6.1 & -1.10 & -1.61 & -1.68 & 0.90 & 1.33 \\
\hline 0.50 & -6.9 & -1.11 & -1.95 & -2.01 & 1.02 & 1.53 \\
\hline 0.55 & $\begin{array}{l}-7.9 \\
\end{array}$ & -1.12 & -2.42 & -2.55 & 1.16 & 1.78 \\
\hline 0.60 & -8.6 & -1.13 & -2.69 & -2.95 & 1.26 & 2.00 \\
\hline 0.65 & -9.6 & -1.14 & -3.22 & -3.56 & 1.39 & 2.20 \\
\hline 0.70 & -10.4 & -1.15 & -3.56 & -3.96 & 1.53 & 2.42 \\
\hline 0.75 & -11.8 & -1.16 & -4.30 & -4.50 & 1.69 & 2.74 \\
\hline 0.80 & -12.4 & -1.17 & -4.70 & -4.90 & 1.80 & 2.97 \\
\hline 0.85 & -13.4 & -1.18 & -5.17 & -5.37 & 1.95 & 3.24 \\
\hline 0.90 & -14.5 & -1.19 & $\begin{array}{l}-5.64 \\
\end{array}$ & -6.04 & 2.11 & 3.52 \\
\hline 0.95 & -15.4 & -1.20 & -6.11 & -6.51 & 2.24 & 3.78 \\
\hline 1.00 & -16.5 & -1.21 & -6.71 & -6.98 & 2.40 & 4.10 \\
\hline 1.05 & -17.1 & -1.22 & -7.05 & -7.59 & 2.54 & 4.33 \\
\hline 1.10 & -17.9 & -1.23 & -7.59 & -7.99 & 2.69 & 4.58 \\
\hline 1.15 & -18.8 & -1.25 & -8.12 & -8.53 & 2.83 & 4.87 \\
\hline 1.20 & -19.6 & -1.26 & -8.66 & -9.00 & 2.99 & 5.14 \\
\hline 1.25 & -20.4 & -1.26 & -9.13 & -9.60 & 3.14 & 5.40 \\
\hline 1.30 & -21.4 & -1.27 & -9.87 & -10.34 & 3.26 & 5.66 \\
\hline 1.35 & -22.2 & -1.29 & -10.21 & -10.68 & 3.45 & 5.99 \\
\hline 1.40 & -23.0 & -1.30 & -10.74 & -11.21 & 3.58 & 6.26 \\
\hline 1.45 & -23.8 & -1.31 & -11.21 & -11.75 & 3.76 & 6.55 \\
\hline 1.50 & -24.9 & -1.32 & -11.82 & -12.29 & 3.94 & 6.92 \\
\hline 1.55 & -25.9 & $\begin{array}{l}-1.33 \\
\end{array}$ & -12.49 & -12.96 & 4.11 & 7.25 \\
\hline 1.60 & -26.4 & -1.34 & -12.82 & -13.29 & 4.28 & 7.52 \\
\hline 1.65 & -27.5 & -1.35 & -13.50 & -14.10 & 4.44 & 7.82 \\
\hline 1.70 & -28.3 & -1.36 & -14.03 & -14.70 & 4.62 & 8.14 \\
\hline 1.75 & -29.3 & -1.37 & -14.70 & -15.38 & 4.80 & 8.51 \\
\hline 1.80 & -29.8 & -1.38 & -15.31 & -15.85 & 4.95 & 8.80 \\
\hline 1.85 & -31.0 & -1.39 & -15.98 & -16.58 & 5.16 & 9.15 \\
\hline 1.90 & -31.6 & -1.40 & -16.38 & -16.99 & 5.35 & 9.52 \\
\hline 1.95 & -32.8 & -1.41 & -17.12 & -17.93 & 5.55 & 9.85 \\
\hline 2.00 & -33.2 & -1.42 & -17.66 & -18.26 & 5.69 & 10.12 \\
\hline 2.05 & -34.1 & -1.43 & -18.33 & -18.93 & 5.88 & 10.47 \\
\hline 2.10 & -35.2 & -1.44 & -19.00 & -19.67 & 6.12 & 10.91 \\
\hline 2.15 & -35.9 & -1.45 & -19.67 & -20.21 & 6.28 & 11.26 \\
\hline 2.20 & -37.0 & -1.46 & -20.48 & -21.15 & 6.49 & 11.62 \\
\hline 2.25 & -37.9 & -1.47 & -21.08 & -21.75 & 6.71 & 12.07 \\
\hline
\end{tabular}


Table F.10 IDT Strength Data (NU 52-34 \#9), continued

\begin{tabular}{|c|c|c|c|c|c|c|}
\hline Time & Axial Force & Axial Displ. & Vert front & Vert back & Horz front & Horz back \\
\hline $\mathrm{Sec}$ & $\mathrm{kN}$ & $\mathrm{mm}$ & $\mu \mathrm{m}$ & $\mu \mathrm{m}$ & $\mu \mathrm{m}$ & $\mu \mathrm{m}$ \\
\hline 2.30 & -38.7 & -1.48 & -21.75 & -22.36 & 6.92 & 12.44 \\
\hline 2.35 & -39.6 & -1.50 & -22.49 & -23.16 & 7.14 & 12.84 \\
\hline 2.40 & -40.3 & -1.50 & -23.10 & -23.70 & 7.35 & 13.21 \\
\hline 2.45 & -41.2 & -1.51 & -23.83 & -24.51 & 7.58 & 13.65 \\
\hline 2.50 & -42.0 & -1.52 & -24.64 & -25.11 & 7.77 & 14.02 \\
\hline 2.55 & -42.7 & -1.54 & -25.31 & -25.78 & 7.99 & 14.45 \\
\hline 2.60 & -43.5 & -1.55 & -25.85 & -26.45 & 8.22 & 14.84 \\
\hline 2.65 & -44.1 & -1.55 & -26.65 & -27.12 & 8.43 & 15.22 \\
\hline 2.70 & -45.0 & -1.56 & -27.33 & -27.86 & 8.66 & 15.63 \\
\hline 2.75 & -45.9 & -1.58 & -28.06 & -28.53 & 8.91 & 16.06 \\
\hline 2.80 & -46.4 & -1.58 & -28.80 & -29.27 & 9.11 & 16.47 \\
\hline 2.85 & -47.4 & -1.59 & -29.68 & -30.01 & 9.34 & 16.80 \\
\hline 2.90 & -48.2 & -1.61 & -30.41 & -30.82 & 9.60 & 17.26 \\
\hline 2.95 & -49.1 & -1.62 & -31.22 & -31.56 & 9.87 & 17.73 \\
\hline 3.00 & -49.7 & -1.63 & -31.96 & -32.36 & 10.10 & 18.14 \\
\hline 3.05 & -50.4 & -1.64 & -32.70 & -32.97 & 10.35 & 18.56 \\
\hline 3.10 & -51.2 & -1.65 & -33.50 & -33.91 & 10.59 & 19.08 \\
\hline 3.15 & -51.6 & -1.65 & -34.11 & -34.44 & 10.83 & 19.47 \\
\hline 3.20 & -52.4 & -1.67 & -35.05 & -35.25 & 11.09 & 19.99 \\
\hline 3.25 & -53.2 & -1.68 & -35.92 & -36.05 & 11.36 & 20.46 \\
\hline 3.30 & -54.1 & -1.69 & -36.79 & -36.93 & 11.64 & 21.03 \\
\hline 3.35 & -54.5 & -1.70 & -37.53 & -37.60 & 11.91 & 21.50 \\
\hline 3.40 & -55.1 & -1.71 & -38.34 & -38.27 & 12.18 & 21.99 \\
\hline 3.45 & -55.9 & -1.72 & -39.28 & -39.21 & 12.43 & 22.49 \\
\hline 3.50 & -56.7 & -1.73 & -40.15 & -39.88 & 12.74 & 23.05 \\
\hline 3.55 & -57.6 & -1.74 & -41.02 & -40.96 & 13.05 & 23.68 \\
\hline 3.60 & -58.0 & -1.75 & -41.90 & -41.76 & 13.34 & 24.22 \\
\hline 3.65 & -58.6 & -1.76 & -42.84 & -42.57 & 13.60 & 24.77 \\
\hline 3.70 & -59.3 & -1.77 & -43.57 & -43.24 & 13.90 & 25.38 \\
\hline 3.75 & -59.9 & -1.79 & -44.51 & -44.04 & 14.20 & 26.00 \\
\hline 3.80 & -60.3 & -1.80 & -45.52 & -44.85 & 14.47 & 26.57 \\
\hline 3.85 & -60.9 & -1.81 & -46.39 & -45.66 & 14.76 & 27.16 \\
\hline 3.90 & -61.3 & -1.82 & -47.20 & -46.39 & 15.04 & 27.76 \\
\hline 3.95 & -62.2 & -1.82 & -48.14 & -47.47 & 15.37 & 28.59 \\
\hline 4.00 & -62.7 & -1.83 & -49.21 & -48.34 & 15.65 & 29.36 \\
\hline 4.05 & -63.2 & -1.84 & -50.15 & -49.21 & 15.98 & 30.15 \\
\hline 4.10 & -63.8 & -1.86 & -51.16 & -50.15 & 16.27 & 30.94 \\
\hline 4.15 & -64.1 & -1.87 & -52.03 & -50.83 & 16.58 & 31.67 \\
\hline 4.20 & -64.6 & -1.88 & -53.04 & -51.83 & 16.90 & 32.48 \\
\hline 4.25 & -65.2 & -1.89 & -54.05 & -52.71 & 17.25 & 33.37 \\
\hline 4.30 & -65.6 & -1.90 & -55.06 & -53.58 & 17.57 & 34.14 \\
\hline 4.35 & -66.3 & -1.91 & -56.06 & -54.52 & 17.93 & 35.01 \\
\hline 4.40 & -66.9 & -1.92 & -57.14 & -55.53 & 18.28 & 35.92 \\
\hline 4.45 & -67.5 & -1.93 & -58.21 & -56.47 & 18.66 & 36.93 \\
\hline 4.50 & -68.0 & -1.94 & -59.49 & -57.41 & 19.00 & 37.87 \\
\hline
\end{tabular}


Table F.10 IDT Strength Data (NU 52-34 \#9), continued

\begin{tabular}{|c|c|c|c|c|c|c|}
\hline Time & Axial Force & Axial Displ. & Vert front & Vert back & Horz front & Horz back \\
\hline Sec & $\mathrm{kN}$ & $\mathrm{mm}$ & $\mu \mathrm{m}$ & $\mu \mathrm{m}$ & $\mu \mathrm{m}$ & $\mu \mathrm{m}$ \\
\hline 4.55 & -68.2 & -1.95 & -60.36 & -58.35 & 19.35 & 38.81 \\
\hline 4.60 & -68.5 & -1.97 & -61.43 & -59.22 & 19.70 & 39.81 \\
\hline 4.65 & -69.0 & -1.97 & -62.51 & -60.23 & 20.08 & 40.89 \\
\hline 4.70 & -69.5 & -1.98 & -63.65 & -61.17 & 20.49 & 42.03 \\
\hline 4.75 & -69.8 & -1.99 & -64.86 & -62.24 & 20.86 & 43.21 \\
\hline 4.80 & -70.2 & -2.00 & -66.07 & -63.31 & 21.23 & 44.41 \\
\hline 4.85 & -70.7 & -2.01 & -67.21 & -64.25 & 21.65 & 45.77 \\
\hline 4.90 & -71.1 & -2.02 & -68.42 & -65.40 & 22.06 & 47.13 \\
\hline 4.95 & -71.6 & -2.03 & -69.76 & -66.47 & 22.48 & 48.58 \\
\hline 5.00 & -71.7 & -2.04 & -70.90 & -67.41 & 22.87 & 50.34 \\
\hline 5.05 & -70.4 & -2.06 & -78.08 & -74.39 & 21.91 & 136.01 \\
\hline 5.10 & -67.8 & -2.07 & -85.27 & -93.06 & 32.11 & 220.83 \\
\hline 5.16 & -65.3 & -2.09 & -90.84 & -108.37 & 48.50 & 278.77 \\
\hline
\end{tabular}


Table F.11 IDT Strength Data (NU 58-40 \#11)

\begin{tabular}{|c|c|c|c|c|c|c|}
\hline Time & Axial Force & Axial Displ. & Vert front & Vert back & Horz front & Horz back \\
\hline $\mathrm{Sec}$ & $\mathrm{kN}$ & $\mathrm{mm}$ & $\mu \mathrm{m}$ & $\mu \mathrm{m}$ & $\mu \mathrm{m}$ & $\mu \mathrm{m}$ \\
\hline 0.00 & -0.1 & -1.00 & 3.02 & 1.61 & 0.13 & 0.34 \\
\hline 0.05 & -0.3 & -1.02 & 2.95 & 1.68 & 0.18 & 0.32 \\
\hline 0.10 & -0.5 & -1.03 & 2.69 & 1.61 & 0.22 & 0.34 \\
\hline 0.15 & -0.8 & -1.03 & 2.55 & 1.61 & 0.29 & 0.39 \\
\hline 0.20 & -1.4 & -1.05 & 2.22 & 1.41 & 0.39 & 0.50 \\
\hline 0.25 & -1.8 & -1.06 & 1.68 & 1.07 & 0.44 & 0.55 \\
\hline 0.30 & -2.4 & -1.07 & 1.41 & 1.14 & 0.55 & 0.70 \\
\hline 0.35 & -3.0 & -1.08 & 1.07 & 0.87 & 0.68 & 0.86 \\
\hline 0.40 & -3.6 & -1.09 & 0.40 & 0.60 & 0.80 & 0.97 \\
\hline 0.45 & -4.4 & -1.10 & -0.07 & 0.27 & 0.93 & 1.14 \\
\hline 0.50 & -4.9 & -1.11 & -0.47 & -0.13 & 1.06 & 1.34 \\
\hline 0.55 & -5.7 & -1.12 & -1.01 & -0.54 & 1.19 & 1.58 \\
\hline 0.60 & -6.5 & -1.13 & -1.54 & -0.94 & 1.34 & 1.80 \\
\hline 0.65 & -7.1 & -1.14 & -2.01 & -1.48 & 1.50 & 2.00 \\
\hline 0.70 & -7.9 & -1.15 & -2.69 & -1.88 & 1.66 & 2.28 \\
\hline 0.75 & -8.9 & -1.16 & -3.36 & -2.55 & 1.83 & 2.57 \\
\hline 0.80 & -9.8 & -1.17 & -4.03 & -3.16 & 2.02 & 2.87 \\
\hline 0.85 & -10.5 & -1.18 & -4.63 & -3.63 & 2.18 & 3.17 \\
\hline 0.90 & -11.3 & -1.19 & -5.17 & -4.03 & 2.38 & 3.49 \\
\hline 0.95 & -12.1 & -1.20 & -5.84 & $\begin{array}{l}-4.83 \\
\end{array}$ & 2.55 & 3.81 \\
\hline 1.00 & -12.7 & -1.21 & -6.51 & -5.37 & 2.72 & 4.08 \\
\hline 1.05 & -13.6 & -1.22 & -7.18 & -6.04 & 2.94 & 4.46 \\
\hline 1.10 & -14.2 & -1.23 & -7.92 & -6.51 & 3.11 & 4.73 \\
\hline 1.15 & -15.0 & -1.25 & -8.53 & -7.18 & 3.32 & 5.07 \\
\hline 1.20 & -15.6 & -1.26 & -9.27 & -7.79 & 3.51 & 5.39 \\
\hline 1.25 & -16.5 & -1.27 & -10.00 & -8.46 & 3.73 & 5.76 \\
\hline 1.30 & -17.0 & -1.28 & -10.68 & -9.06 & 3.91 & 6.09 \\
\hline 1.35 & -17.9 & -1.29 & -11.35 & -9.60 & 4.14 & 6.48 \\
\hline 1.40 & -18.6 & -1.30 & -12.09 & -10.27 & 4.36 & 6.85 \\
\hline 1.45 & -19.5 & -1.31 & -12.96 & -11.08 & 4.59 & 7.27 \\
\hline 1.50 & -20.1 & -1.32 & -13.70 & -11.82 & 4.81 & 7.64 \\
\hline 1.55 & -20.6 & -1.33 & -14.50 & -12.49 & 4.99 & 7.96 \\
\hline 1.60 & -21.3 & -1.33 & -15.17 & -13.23 & 5.21 & 8.36 \\
\hline 1.65 & -22.2 & -1.35 & -15.98 & -13.90 & 5.48 & 8.85 \\
\hline 1.70 & -22.6 & -1.36 & -16.85 & -14.50 & 5.68 & 9.20 \\
\hline 1.75 & -23.4 & -1.37 & -17.66 & -15.38 & 5.93 & 9.63 \\
\hline 1.80 & -24.0 & -1.38 & -18.40 & -16.05 & 6.15 & 10.05 \\
\hline 1.85 & -24.6 & -1.39 & -19.14 & -16.72 & 6.38 & 10.52 \\
\hline 1.90 & -25.1 & -1.40 & -19.87 & -17.39 & 6.60 & 10.88 \\
\hline 1.95 & -25.6 & -1.41 & -20.68 & -17.99 & 6.83 & 11.36 \\
\hline 2.00 & -26.3 & -1.42 & -21.49 & -18.93 & 7.07 & 11.78 \\
\hline 2.05 & -26.9 & -1.43 & -22.22 & -19.74 & 7.32 & 12.25 \\
\hline 2.10 & -27.5 & -1.44 & -23.10 & -20.55 & 7.54 & 12.71 \\
\hline 2.15 & -28.2 & -1.45 & -24.10 & -21.28 & 7.81 & 13.21 \\
\hline 2.20 & -28.6 & -1.46 & -24.71 & -22.09 & 8.05 & 13.63 \\
\hline 2.25 & -29.5 & -1.47 & -25.71 & -22.96 & 8.33 & 14.20 \\
\hline
\end{tabular}


Table F.11 IDT Strength Data (NU 58-40 \#11), continued

\begin{tabular}{|c|c|c|c|c|c|c|}
\hline Time & Axial Force & Axial Displ. & Vert front & Vert back & Horz front & Horz back \\
\hline $\mathrm{Sec}$ & $\mathrm{kN}$ & $\mathrm{mm}$ & $\mu \mathrm{m}$ & $\mu \mathrm{m}$ & $\mu \mathrm{m}$ & $\mu \mathrm{m}$ \\
\hline 2.30 & -30.2 & -1.48 & -26.59 & -23.90 & 8.60 & 14.74 \\
\hline 2.35 & -30.9 & -1.50 & -27.53 & -24.77 & 8.88 & 15.26 \\
\hline 2.40 & -31.3 & -1.50 & -28.47 & -25.71 & 9.15 & 15.79 \\
\hline 2.45 & -32.0 & -1.52 & -29.41 & -26.65 & 9.42 & 16.38 \\
\hline 2.50 & -32.3 & -1.52 & -30.21 & -27.33 & 9.67 & 16.85 \\
\hline 2.55 & -32.9 & -1.53 & -31.22 & -28.20 & 9.95 & 17.37 \\
\hline 2.60 & -33.7 & -1.54 & -32.23 & -29.34 & 10.24 & 17.98 \\
\hline 2.65 & -34.1 & -1.56 & -33.10 & -30.08 & 10.51 & 18.56 \\
\hline 2.70 & -34.9 & -1.57 & -34.11 & -31.15 & 10.83 & 19.17 \\
\hline 2.75 & -35.3 & -1.58 & -35.18 & -32.03 & 11.12 & 19.84 \\
\hline 2.80 & -36.0 & -1.59 & -36.12 & -33.03 & 11.41 & 20.46 \\
\hline 2.85 & -36.5 & -1.60 & -37.13 & -33.97 & 11.71 & 21.07 \\
\hline 2.90 & -36.6 & -1.61 & -38.07 & -34.85 & 11.97 & 21.67 \\
\hline 2.95 & -37.3 & -1.62 & -38.94 & -35.92 & 12.25 & 22.34 \\
\hline 3.00 & -38.0 & -1.63 & -40.02 & -36.86 & 12.60 & 23.01 \\
\hline 3.05 & -38.5 & -1.64 & -41.22 & -37.93 & 12.89 & 23.63 \\
\hline 3.10 & -38.9 & -1.65 & -42.10 & -38.74 & 13.20 & 24.32 \\
\hline 3.15 & -39.3 & -1.66 & -43.17 & -39.88 & 13.49 & 24.96 \\
\hline 3.20 & -39.9 & -1.67 & -44.18 & -40.89 & 13.81 & 25.66 \\
\hline 3.25 & -40.5 & $\begin{array}{l}-1.69 \\
\end{array}$ & -45.45 & -42.03 & 14.15 & 26.39 \\
\hline 3.30 & -41.0 & -1.69 & -46.39 & -42.97 & 14.48 & 27.12 \\
\hline 3.35 & -41.4 & -1.70 & -47.47 & -43.98 & 14.79 & 27.83 \\
\hline 3.40 & -41.9 & -1.71 & -48.68 & -45.05 & 15.12 & 28.64 \\
\hline 3.45 & -42.3 & -1.72 & -49.75 & -46.06 & 15.46 & 29.34 \\
\hline 3.50 & -42.7 & $\begin{array}{l}-1.73 \\
\end{array}$ & -50.89 & -47.13 & 15.79 & 30.05 \\
\hline 3.55 & -43.1 & -1.74 & -51.97 & -48.14 & 16.10 & 30.77 \\
\hline 3.60 & -43.6 & -1.75 & -53.11 & -49.28 & 16.46 & 31.54 \\
\hline 3.65 & -43.9 & -1.77 & -54.12 & -50.29 & 16.78 & 32.29 \\
\hline 3.70 & -44.4 & -1.78 & -55.32 & -51.36 & 17.15 & 33.08 \\
\hline 3.75 & -45.0 & -1.79 & -56.60 & -52.50 & 17.49 & 33.91 \\
\hline 3.80 & -45.2 & -1.79 & -57.54 & -53.58 & 17.83 & 34.66 \\
\hline 3.85 & -45.9 & -1.81 & -58.95 & -54.72 & 18.20 & 35.53 \\
\hline 3.90 & -46.4 & -1.81 & -60.36 & -56.13 & 18.56 & 36.36 \\
\hline 3.95 & -46.6 & -1.83 & -61.43 & -57.00 & 18.97 & 37.23 \\
\hline 4.00 & -46.8 & -1.84 & -62.44 & -57.94 & 19.29 & 38.00 \\
\hline 4.05 & -47.4 & -1.85 & -63.72 & -59.22 & 19.69 & 38.91 \\
\hline 4.10 & -47.7 & -1.86 & -64.99 & -60.43 & 20.05 & 39.75 \\
\hline 4.15 & -48.2 & -1.87 & -66.27 & -61.57 & 20.44 & 40.65 \\
\hline 4.20 & -48.5 & -1.88 & -67.41 & -62.58 & 20.83 & 41.54 \\
\hline 4.25 & -49.1 & -1.89 & -68.75 & -63.85 & 21.24 & 42.45 \\
\hline 4.30 & -49.4 & -1.90 & -70.09 & -65.06 & 21.64 & 43.39 \\
\hline 4.35 & -49.9 & -1.91 & -71.44 & -66.34 & 22.05 & 44.36 \\
\hline 4.40 & -50.2 & -1.92 & -72.85 & -67.54 & 22.44 & 45.29 \\
\hline 4.45 & -50.5 & -1.93 & -73.99 & -68.68 & 22.84 & 46.19 \\
\hline 4.50 & $\begin{array}{l}-51.2 \\
\end{array}$ & -1.94 & -75.40 & -69.96 & 23.29 & 47.25 \\
\hline
\end{tabular}


Table F.11 IDT Strength Data (NU 58-40 \#11), continued

\begin{tabular}{|c|c|c|c|c|c|c|}
\hline Time & Axial Force & Axial Displ. & Vert front & Vert back & Horz front & Horz back \\
\hline $\mathrm{Sec}$ & $\mathrm{kN}$ & $\mathrm{mm}$ & $\mu \mathrm{m}$ & $\mu \mathrm{m}$ & $\mu \mathrm{m}$ & $\mu \mathrm{m}$ \\
\hline 4.55 & -51.6 & -1.95 & -76.94 & -71.30 & 23.73 & 48.27 \\
\hline 4.60 & -51.6 & -1.96 & -78.15 & -72.44 & 24.13 & 49.26 \\
\hline 4.65 & -51.9 & -1.97 & -79.56 & -73.65 & 24.52 & 50.22 \\
\hline 4.70 & -52.4 & -1.98 & -80.90 & -74.86 & 24.98 & 51.28 \\
\hline 4.75 & -52.8 & -2.00 & -82.25 & -76.14 & 25.42 & 52.34 \\
\hline 4.80 & -53.3 & -2.01 & -83.86 & -77.61 & 25.88 & 53.46 \\
\hline 4.85 & -53.6 & -2.02 & -85.27 & -78.82 & 26.34 & 54.52 \\
\hline 4.90 & \begin{tabular}{|l|}
-53.9 \\
\end{tabular} & -2.02 & -86.75 & -80.03 & 26.79 & 55.64 \\
\hline 4.95 & $\begin{array}{l}-54.3 \\
\end{array}$ & -2.04 & -88.29 & -81.31 & 27.27 & 56.77 \\
\hline 5.00 & -54.3 & -2.05 & -89.57 & -82.45 & 27.70 & 57.81 \\
\hline 5.05 & -54.8 & -2.06 & -91.11 & -83.72 & 28.17 & 59.00 \\
\hline 5.10 & -55.4 & -2.07 & -92.72 & -85.40 & 28.69 & 60.21 \\
\hline 5.16 & -55.7 & -2.08 & -94.33 & -86.68 & 29.18 & 61.40 \\
\hline 5.21 & -55.8 & -2.09 & -95.88 & -87.95 & 29.65 & 62.51 \\
\hline 5.26 & -56.2 & -2.10 & -97.35 & -89.30 & 30.15 & 63.75 \\
\hline 5.31 & -56.3 & -2.11 & -98.90 & -90.57 & 30.62 & 64.93 \\
\hline 5.36 & -56.2 & -2.12 & -100.24 & -91.65 & 31.09 & 66.03 \\
\hline 5.41 & -57.1 & -2.13 & -101.99 & -93.26 & 31.64 & 67.34 \\
\hline 5.46 & -57.4 & -2.14 & -103.73 & -94.67 & 32.17 & 68.65 \\
\hline 5.51 & $\begin{array}{l}-57.6 \\
\end{array}$ & -2.15 & -105.28 & -96.08 & 32.70 & $\begin{array}{l}69.98 \\
\end{array}$ \\
\hline 5.56 & $\begin{array}{l}-57.9 \\
\end{array}$ & -2.16 & -106.96 & -97.42 & 33.23 & 71.29 \\
\hline 5.61 & -58.1 & -2.18 & -108.57 & -98.83 & 33.77 & 72.60 \\
\hline 5.66 & -58.6 & -2.18 & -110.25 & -100.38 & 34.33 & 74.02 \\
\hline 5.71 & -58.7 & -2.19 & -111.72 & -101.45 & 34.88 & 75.37 \\
\hline 5.76 & $\begin{array}{l}-59.1 \\
\end{array}$ & -2.20 & -113.67 & -103.20 & 35.43 & 76.73 \\
\hline 5.81 & -59.2 & -2.21 & -115.28 & -104.40 & 35.98 & 78.14 \\
\hline 5.86 & -59.5 & -2.23 & -117.03 & -105.95 & 36.54 & 79.53 \\
\hline 5.91 & -59.6 & -2.23 & -118.70 & -107.29 & 37.09 & 80.95 \\
\hline 5.96 & -59.9 & -2.24 & -120.45 & -108.84 & 37.66 & 82.38 \\
\hline 6.01 & -60.4 & -2.25 & -122.26 & -110.31 & 38.30 & 83.99 \\
\hline 6.06 & -60.5 & -2.27 & -124.01 & -111.72 & 38.88 & 85.50 \\
\hline 6.11 & -61.0 & -2.28 & -125.89 & -113.40 & 39.50 & 87.06 \\
\hline 6.16 & -61.0 & -2.29 & -127.57 & -114.61 & 40.10 & 88.64 \\
\hline 6.21 & -61.3 & -2.30 & -129.38 & -116.22 & 40.72 & 90.24 \\
\hline 6.26 & -61.6 & -2.31 & -131.33 & -117.76 & 41.35 & 91.88 \\
\hline 6.31 & -62.0 & -2.32 & -133.14 & -119.38 & 42.02 & 93.63 \\
\hline 6.36 & -62.2 & -2.33 & -135.29 & -121.05 & 42.65 & 95.27 \\
\hline 6.41 & $\begin{array}{l}-62.4 \\
\end{array}$ & -2.34 & -136.97 & -122.46 & 43.30 & 97.07 \\
\hline 6.46 & -62.7 & -2.35 & -138.98 & -124.21 & 43.97 & 98.95 \\
\hline 6.51 & -62.9 & -2.36 & -140.93 & -125.82 & 44.66 & 100.85 \\
\hline 6.56 & -63.0 & -2.37 & -142.81 & -127.30 & 45.30 & 102.69 \\
\hline 6.61 & -63.1 & -2.38 & -144.76 & -128.84 & 45.97 & 104.55 \\
\hline 6.66 & -63.4 & -2.39 & -146.77 & -130.59 & 46.69 & 106.49 \\
\hline 6.71 & -63.5 & -2.41 & -148.72 & -132.20 & 47.38 & 108.48 \\
\hline 6.76 & $\begin{array}{l}-63.6 \\
\end{array}$ & -2.41 & -150.73 & -133.81 & 48.06 & 110.40 \\
\hline
\end{tabular}


Table F.11 IDT Strength Data (NU 58-40 \#11), continued

\begin{tabular}{|c|c|c|c|c|c|c|}
\hline Time & Axial Force & Axial Displ. & Vert front & Vert back & Horz front & Horz back \\
\hline $\mathrm{Sec}$ & $\mathrm{kN}$ & $\mathrm{mm}$ & $\mu \mathrm{m}$ & $\mu \mathrm{m}$ & $\mu \mathrm{m}$ & $\mu \mathrm{m}$ \\
\hline 6.81 & -63.9 & -2.42 & -152.75 & -135.56 & 48.77 & 112.46 \\
\hline 6.86 & -63.8 & -2.43 & -154.63 & -137.17 & 49.46 & 114.49 \\
\hline 6.91 & $\begin{array}{l}-63.9 \\
\end{array}$ & -2.44 & -156.57 & -138.85 & 50.15 & 116.52 \\
\hline 6.96 & -64.2 & -2.45 & -158.65 & -140.53 & 50.88 & 118.69 \\
\hline 7.01 & -64.4 & -2.46 & -160.80 & -142.34 & 51.63 & 120.84 \\
\hline 7.06 & -64.4 & -2.48 & -162.82 & -144.02 & 52.34 & 123.04 \\
\hline 7.11 & -64.6 & -2.49 & -164.76 & -145.49 & 53.10 & 125.23 \\
\hline 7.16 & -65.0 & -2.49 & -166.91 & -147.51 & 53.89 & 127.58 \\
\hline 7.21 & -65.1 & -2.50 & -169.06 & -149.25 & 54.68 & 129.92 \\
\hline 7.26 & -65.4 & -2.51 & -171.34 & -151.13 & 55.49 & 132.42 \\
\hline 7.31 & -65.7 & -2.53 & -173.63 & -153.01 & 56.30 & 134.90 \\
\hline 7.36 & -65.6 & -2.54 & -175.71 & -154.83 & 57.10 & 137.39 \\
\hline 7.41 & $\begin{array}{l}-65.9 \\
\end{array}$ & -2.55 & -177.99 & -156.64 & 57.96 & 140.02 \\
\hline 7.46 & $\begin{array}{l}-66.1 \\
\end{array}$ & -2.56 & -180.27 & -158.59 & 58.80 & 142.74 \\
\hline 7.51 & -66.3 & -2.57 & -182.56 & -160.53 & 59.66 & 145.46 \\
\hline 7.56 & -66.3 & -2.58 & -184.84 & -162.48 & 60.51 & 148.16 \\
\hline 7.61 & -66.5 & -2.59 & -187.12 & -164.43 & 61.41 & 151.05 \\
\hline 7.66 & -66.4 & -2.60 & -189.27 & -166.17 & 62.26 & 153.84 \\
\hline 7.71 & -66.4 & -2.61 & -191.69 & -168.32 & 63.13 & 156.67 \\
\hline 7.76 & -66.7 & -2.62 & -194.04 & -170.20 & 64.05 & 159.79 \\
\hline 7.81 & -66.8 & -2.63 & -196.32 & -172.28 & 65.01 & 162.87 \\
\hline 7.86 & -66.9 & -2.64 & -198.74 & -174.43 & 65.92 & 166.02 \\
\hline 7.91 & -66.9 & -2.65 & -201.15 & -176.38 & 66.85 & 169.19 \\
\hline 7.96 & -67.2 & -2.66 & -203.64 & -178.66 & 67.82 & 172.53 \\
\hline 8.01 & \begin{tabular}{|l|}
-67.3 \\
\end{tabular} & -2.67 & -206.05 & -180.74 & 68.82 & 176.03 \\
\hline 8.06 & \begin{tabular}{|l|}
-67.3 \\
\end{tabular} & -2.68 & -208.54 & -182.96 & 69.79 & 179.48 \\
\hline 8.11 & -67.4 & -2.69 & -210.89 & -185.11 & 70.80 & 183.08 \\
\hline 8.16 & -67.5 & -2.70 & -213.51 & -187.32 & 71.81 & 186.75 \\
\hline 8.21 & -67.5 & -2.71 & -216.06 & -189.67 & 72.83 & 190.53 \\
\hline 8.26 & -67.6 & -2.72 & -218.68 & -192.09 & 73.86 & 194.41 \\
\hline 8.31 & -67.8 & -2.74 & -221.23 & -194.37 & 74.95 & 198.45 \\
\hline 8.36 & $\begin{array}{l}-67.8 \\
\end{array}$ & -2.74 & -223.78 & -196.79 & 76.00 & 202.51 \\
\hline 8.41 & -68.0 & -2.76 & -226.60 & -199.81 & 77.11 & 206.86 \\
\hline 8.46 & -68.0 & -2.77 & -229.29 & -202.76 & 78.23 & 211.28 \\
\hline 8.51 & -68.0 & -2.78 & -231.90 & -205.58 & 79.34 & 215.76 \\
\hline 8.56 & -68.1 & -2.79 & -234.66 & -208.67 & 80.47 & 220.41 \\
\hline 8.61 & -67.8 & -2.80 & -237.34 & -211.29 & 81.58 & 225.07 \\
\hline 8.66 & $\begin{array}{l}-68.0 \\
\end{array}$ & -2.81 & -240.30 & -214.51 & 82.74 & 230.01 \\
\hline 8.71 & -68.2 & -2.82 & -242.98 & -217.13 & 83.96 & 235.23 \\
\hline 8.76 & -68.1 & -2.83 & -245.80 & -219.95 & 85.16 & 240.46 \\
\hline 8.81 & -68.1 & -2.84 & -248.62 & -222.97 & 86.34 & 245.89 \\
\hline 8.86 & -68.0 & -2.85 & -251.58 & -225.66 & 87.57 & 251.49 \\
\hline 8.91 & -68.1 & -2.86 & -254.60 & -228.61 & 88.81 & 257.38 \\
\hline 8.96 & \begin{tabular}{|l|}
-68.3 \\
\end{tabular} & -2.87 & -257.62 & -231.50 & 90.13 & 263.68 \\
\hline 9.01 & $\begin{array}{l}-68.2 \\
\end{array}$ & -2.88 & -260.64 & -234.25 & 91.41 & 270.11 \\
\hline
\end{tabular}


Table F.11 IDT Strength Data (NU 58-40 \#11), continued

\begin{tabular}{|c|c|c|c|c|c|c|}
\hline Time & Axial Force & Axial Displ. & Vert front & Vert back & Horz front & Horz back \\
\hline Sec & $\mathrm{kN}$ & $\mathrm{mm}$ & $\mu \mathrm{m}$ & $\mu \mathrm{m}$ & $\mu \mathrm{m}$ & $\mu \mathrm{m}$ \\
\hline 9.06 & -67.9 & -2.89 & -263.66 & -237.21 & 92.65 & 276.59 \\
\hline 9.11 & -68.1 & -2.90 & -266.68 & -239.69 & 94.01 & 283.59 \\
\hline 9.16 & -67.9 & -2.91 & -269.70 & -242.24 & 95.31 & 290.77 \\
\hline 9.21 & -68.0 & -2.93 & -272.99 & -245.20 & 96.67 & 298.37 \\
\hline 9.26 & -67.9 & -2.94 & -276.15 & -247.88 & 98.04 & 306.36 \\
\hline 9.31 & -67.9 & -2.94 & -279.51 & -250.70 & 99.42 & 314.79 \\
\hline 9.36 & -67.7 & -2.96 & -282.86 & -253.39 & 100.84 & 323.74 \\
\hline 9.41 & -67.8 & -2.97 & -286.36 & -256.07 & 102.26 & 333.39 \\
\hline 9.46 & -67.5 & -2.98 & -289.85 & -258.69 & 103.65 & 343.73 \\
\hline 9.51 & -67.4 & -2.99 & -293.34 & -261.04 & 105.02 & 355.02 \\
\hline 9.56 & -67.2 & -3.00 & -296.96 & -262.92 & 106.36 & 367.53 \\
\hline 9.61 & -66.9 & -3.01 & -300.59 & -264.53 & 107.64 & 381.17 \\
\hline 9.66 & -66.6 & -3.02 & -304.42 & -266.15 & 108.89 & 396.21 \\
\hline 9.71 & -66.2 & -3.03 & -308.24 & -267.56 & 110.08 & 412.60 \\
\hline 9.76 & -66.0 & -3.05 & -312.81 & -269.64 & 111.28 & 430.29 \\
\hline 9.81 & -65.4 & -3.06 & -316.77 & -271.58 & 112.40 & 448.82 \\
\hline 9.86 & -65.0 & -3.06 & -320.73 & -273.80 & 113.52 & 468.51 \\
\hline 9.91 & -64.6 & -3.07 & -325.03 & -276.35 & 114.66 & 489.59 \\
\hline 9.96 & -64.2 & -3.09 & -329.46 & -278.97 & 115.80 & 511.85 \\
\hline 10.01 & -63.8 & -3.09 & -333.89 & -282.06 & 116.99 & 535.33 \\
\hline 10.06 & -63.1 & -3.11 & -338.39 & -285.42 & 118.18 & 559.69 \\
\hline 10.11 & -62.6 & -3.11 & -342.95 & -288.71 & 119.44 & 584.83 \\
\hline 10.16 & -62.0 & -3.12 & -347.79 & -291.53 & 120.79 & 611.13 \\
\hline
\end{tabular}


Table F.12 IDT Strength Data (NU 58-40 \#12)

\begin{tabular}{|c|c|c|c|c|c|c|}
\hline Time & Axial Force & Axial Displ. & Vert front & Vert back & Horz front & Horz back \\
\hline $\mathrm{Sec}$ & $\mathrm{kN}$ & $\mathrm{mm}$ & $\mu \mathrm{m}$ & $\mu \mathrm{m}$ & $\mu \mathrm{m}$ & $\mu \mathrm{m}$ \\
\hline 0.00 & -0.1 & -0.92 & 0.94 & 0.34 & 0.09 & 0.03 \\
\hline 0.05 & -0.1 & -0.93 & 0.94 & 0.34 & 0.09 & 0.07 \\
\hline 0.10 & -0.2 & -0.94 & 0.94 & 0.54 & 0.12 & 0.12 \\
\hline 0.15 & -0.4 & -0.95 & 0.81 & 0.07 & 0.13 & 0.15 \\
\hline 0.20 & -0.7 & -0.96 & 0.67 & -0.13 & 0.16 & 0.25 \\
\hline 0.25 & -1.1 & -0.98 & 0.60 & -0.34 & 0.18 & 0.35 \\
\hline 0.30 & -1.7 & -0.98 & 0.34 & -0.60 & 0.27 & 0.57 \\
\hline 0.35 & -2.2 & -0.99 & 0.07 & -1.01 & 0.34 & 0.77 \\
\hline 0.40 & -2.5 & -1.00 & -0.27 & -1.34 & 0.39 & 0.89 \\
\hline 0.45 & -3.2 & -1.02 & -0.40 & -1.75 & 0.48 & 1.12 \\
\hline 0.50 & -3.7 & -1.03 & -0.67 & -2.08 & 0.55 & 1.38 \\
\hline 0.55 & -4.3 & -1.04 & -1.07 & -2.69 & 0.67 & 1.59 \\
\hline 0.60 & -4.9 & -1.05 & -1.34 & -2.95 & 0.75 & 1.85 \\
\hline 0.65 & -5.8 & -1.06 & -1.68 & -3.76 & 0.88 & 2.15 \\
\hline 0.70 & -6.5 & -1.07 & -2.22 & -4.23 & 0.98 & 2.47 \\
\hline 0.75 & -7.2 & -1.08 & -2.55 & -4.83 & 1.12 & 2.80 \\
\hline 0.80 & -7.9 & -1.09 & -3.09 & -5.37 & 1.23 & 3.11 \\
\hline 0.85 & -8.4 & -1.10 & -3.42 & -5.98 & 1.33 & 3.36 \\
\hline 0.90 & -9.1 & -1.11 & -3.83 & -6.58 & 1.48 & 3.71 \\
\hline 0.95 & -9.9 & -1.12 & -4.36 & -7.32 & 1.61 & 4.06 \\
\hline 1.00 & -10.7 & -1.13 & -4.83 & -7.99 & 1.76 & 4.46 \\
\hline 1.05 & -11.4 & -1.14 & -5.44 & -8.73 & 1.89 & 4.85 \\
\hline 1.10 & -12.0 & -1.15 & -5.91 & -9.20 & 2.04 & 5.19 \\
\hline 1.15 & -12.7 & -1.16 & -6.38 & -10.07 & 2.18 & 5.59 \\
\hline 1.20 & -13.3 & -1.17 & -6.85 & -10.74 & 2.31 & 5.94 \\
\hline 1.25 & -14.0 & -1.18 & -7.39 & -11.41 & 2.48 & 6.33 \\
\hline 1.30 & -14.6 & -1.19 & -7.86 & -12.09 & 2.62 & 6.71 \\
\hline 1.35 & -15.1 & -1.20 & -8.33 & -12.62 & 2.76 & 7.07 \\
\hline 1.40 & -15.9 & -1.22 & -9.06 & -13.43 & 2.94 & 7.50 \\
\hline 1.45 & -16.5 & -1.22 & -9.47 & -14.23 & 3.09 & 7.91 \\
\hline 1.50 & -17.1 & -1.23 & -10.00 & -14.97 & 3.24 & 8.33 \\
\hline 1.55 & -17.8 & -1.24 & -10.54 & -15.85 & 3.42 & 8.73 \\
\hline 1.60 & -18.6 & -1.26 & -11.35 & -16.58 & 3.59 & 9.23 \\
\hline 1.65 & -19.3 & -1.26 & -11.75 & -17.39 & 3.78 & 9.70 \\
\hline 1.70 & -19.7 & -1.28 & -12.42 & -18.13 & 3.94 & 10.10 \\
\hline 1.75 & -20.4 & -1.29 & -13.03 & -19.07 & 4.10 & 10.54 \\
\hline 1.80 & -21.1 & -1.29 & -13.70 & -19.74 & 4.27 & 10.99 \\
\hline 1.85 & -21.7 & -1.31 & -14.23 & -20.75 & 4.47 & 11.51 \\
\hline 1.90 & -22.3 & -1.32 & -14.84 & -21.49 & 4.66 & 11.98 \\
\hline 1.95 & -22.9 & -1.33 & -15.44 & -22.36 & 4.84 & 12.47 \\
\hline 2.00 & -23.4 & -1.34 & -16.05 & -23.10 & 5.03 & 12.94 \\
\hline 2.05 & -24.1 & -1.35 & -16.72 & -23.97 & 5.22 & 13.46 \\
\hline 2.10 & -24.5 & -1.36 & -17.32 & -24.84 & 5.38 & 13.88 \\
\hline 2.15 & -25.1 & -1.37 & -18.13 & -25.92 & 5.57 & 14.38 \\
\hline 2.20 & -25.9 & -1.38 & -18.73 & -26.59 & 5.80 & 14.96 \\
\hline 2.25 & -26.4 & -1.39 & -19.54 & -27.59 & 5.99 & 15.49 \\
\hline
\end{tabular}


Table F.12 IDT Strength Data (NU 58-40 \#12), continued

\begin{tabular}{|c|c|c|c|c|c|c|}
\hline Time & Axial Force & Axial Displ. & Vert front & Vert back & Horz front & Horz back \\
\hline $\mathrm{Sec}$ & $\mathrm{kN}$ & $\mathrm{mm}$ & $\mu \mathrm{m}$ & $\mu \mathrm{m}$ & $\mu \mathrm{m}$ & $\mu \mathrm{m}$ \\
\hline 2.30 & -27.1 & -1.40 & -20.01 & -28.53 & 6.21 & 16.03 \\
\hline 2.35 & -27.6 & -1.41 & -20.88 & -29.41 & 6.42 & 16.53 \\
\hline 2.40 & -28.2 & -1.42 & -21.62 & -30.41 & 6.61 & 17.07 \\
\hline 2.45 & -28.8 & -1.43 & -22.29 & -31.35 & 6.84 & 17.62 \\
\hline 2.50 & -29.4 & -1.44 & -23.16 & -32.43 & 7.06 & 18.20 \\
\hline 2.55 & -29.8 & -1.45 & -23.83 & -33.17 & 7.25 & 18.68 \\
\hline 2.60 & -30.6 & -1.46 & -24.64 & -34.44 & 7.50 & 19.35 \\
\hline 2.65 & -31.2 & -1.48 & -25.45 & -35.38 & 7.73 & 19.94 \\
\hline 2.70 & -31.8 & -1.49 & -26.25 & -36.39 & 7.96 & 20.56 \\
\hline 2.75 & -32.5 & -1.50 & -27.06 & -37.46 & 8.20 & 21.18 \\
\hline 2.80 & -32.8 & -1.51 & -27.93 & -38.54 & 8.42 & 21.75 \\
\hline 2.85 & -33.4 & -1.51 & -28.74 & -39.48 & 8.66 & 22.39 \\
\hline 2.90 & -34.1 & -1.53 & -29.47 & -40.49 & 8.90 & 23.05 \\
\hline 2.95 & -34.5 & -1.54 & -30.35 & -41.69 & 9.15 & 23.63 \\
\hline 3.00 & -35.0 & -1.55 & -31.15 & -42.70 & 9.39 & 24.27 \\
\hline 3.05 & -35.4 & -1.56 & -31.89 & -43.71 & 9.63 & 24.86 \\
\hline 3.10 & -36.2 & -1.57 & -32.76 & -44.98 & 9.91 & 25.56 \\
\hline 3.15 & -36.7 & -1.58 & -33.77 & -46.06 & 10.15 & 26.22 \\
\hline 3.20 & -37.0 & -1.59 & -34.51 & -47.00 & 10.38 & 26.79 \\
\hline 3.25 & -37.5 & -1.60 & -35.45 & -48.27 & 10.63 & 27.46 \\
\hline 3.30 & -38.2 & -1.61 & -36.32 & -49.42 & 10.90 & 28.15 \\
\hline 3.35 & -38.6 & -1.62 & -37.26 & -50.49 & 11.17 & 28.85 \\
\hline 3.40 & -39.1 & -1.63 & -38.14 & -51.63 & 11.46 & 29.51 \\
\hline 3.45 & -39.2 & -1.64 & -38.94 & -52.64 & 11.68 & 30.10 \\
\hline 3.50 & -40.2 & -1.65 & -40.15 & -53.98 & 11.98 & 30.90 \\
\hline 3.55 & -40.4 & -1.66 & -40.89 & -55.06 & 12.24 & 31.57 \\
\hline 3.60 & -40.9 & -1.67 & -41.90 & -56.20 & 12.53 & 32.28 \\
\hline 3.65 & -41.6 & -1.68 & -42.77 & -57.54 & 12.81 & 33.03 \\
\hline 3.70 & -41.9 & -1.69 & -43.78 & -58.68 & 13.07 & 33.70 \\
\hline 3.75 & -42.3 & -1.70 & -44.78 & -59.89 & 13.37 & 34.48 \\
\hline 3.80 & -42.9 & -1.71 & -45.72 & -61.10 & 13.65 & 35.22 \\
\hline 3.85 & -43.4 & -1.72 & -46.73 & -62.44 & 13.95 & 35.95 \\
\hline 3.90 & -43.6 & -1.73 & -47.67 & -63.52 & 14.23 & 36.64 \\
\hline 3.95 & -44.0 & -1.75 & -48.68 & -64.66 & 14.52 & 37.40 \\
\hline 4.00 & -44.5 & -1.76 & -49.82 & -66.07 & 14.82 & 38.19 \\
\hline 4.05 & -45.1 & -1.77 & -51.03 & -67.48 & 15.12 & 38.96 \\
\hline 4.10 & -45.0 & -1.78 & -51.77 & -68.35 & 15.38 & 39.60 \\
\hline 4.15 & -45.5 & -1.79 & -52.77 & -69.49 & 15.69 & 40.33 \\
\hline 4.20 & -46.0 & -1.80 & -53.65 & -70.83 & 16.00 & 41.16 \\
\hline 4.25 & -46.4 & -1.81 & -54.92 & -72.11 & 16.31 & 41.96 \\
\hline 4.30 & -46.8 & -1.81 & -55.86 & -73.38 & 16.62 & 42.75 \\
\hline 4.35 & -47.3 & -1.83 & -56.94 & -74.73 & 16.95 & 43.57 \\
\hline 4.40 & -47.8 & -1.84 & -58.21 & -76.34 & 17.25 & 44.40 \\
\hline 4.45 & -48.5 & -1.85 & -59.22 & -77.55 & 17.62 & 45.35 \\
\hline 4.50 & -48.6 & -1.85 & -60.29 & -78.82 & 17.93 & 46.14 \\
\hline
\end{tabular}


Table F.12 IDT Strength Data (NU 58-40 \#12), continued

\begin{tabular}{|c|c|c|c|c|c|c|}
\hline Time & Axial Force & Axial Displ. & Vert front & Vert back & Horz front & Horz back \\
\hline $\mathrm{Sec}$ & $\mathrm{kN}$ & $\mathrm{mm}$ & $\mu \mathrm{m}$ & $\mu \mathrm{m}$ & $\mu \mathrm{m}$ & $\mu \mathrm{m}$ \\
\hline 4.55 & -49.3 & -1.87 & -61.57 & -80.30 & 18.29 & 47.07 \\
\hline 4.60 & -49.5 & -1.88 & -62.58 & -81.64 & 18.63 & 47.92 \\
\hline 4.65 & -50.0 & -1.89 & -63.78 & -83.19 & 18.98 & 48.81 \\
\hline 4.70 & -50.5 & -1.90 & -65.06 & -84.66 & 19.34 & 49.77 \\
\hline 4.75 & -50.7 & -1.91 & -66.20 & -85.81 & 19.68 & 50.59 \\
\hline 4.80 & -51.0 & -1.92 & -67.28 & -87.15 & 20.03 & 51.48 \\
\hline 4.85 & -51.1 & -1.93 & -68.35 & -88.36 & 20.36 & 52.27 \\
\hline 4.90 & -51.8 & -1.94 & -69.56 & -90.10 & 20.74 & 53.23 \\
\hline 4.95 & -52.2 & -1.95 & -70.90 & -91.51 & 21.11 & 54.20 \\
\hline 5.00 & -52.5 & -1.96 & -71.97 & -92.79 & 21.47 & 55.07 \\
\hline 5.05 & -52.9 & -1.97 & -73.18 & -94.33 & 21.85 & 56.10 \\
\hline 5.10 & -53.4 & -1.98 & -74.39 & -95.81 & 22.23 & 57.02 \\
\hline 5.16 & -53.5 & -1.99 & -75.73 & -97.22 & 22.58 & 57.93 \\
\hline 5.21 & -53.9 & -2.00 & -76.88 & -98.70 & 22.98 & 58.88 \\
\hline 5.26 & -54.2 & -2.01 & -78.08 & -100.11 & 23.36 & 59.86 \\
\hline 5.31 & -54.7 & -2.03 & -79.29 & -101.65 & 23.77 & 60.90 \\
\hline 5.36 & -54.9 & -2.03 & -80.50 & -102.99 & 24.15 & 61.82 \\
\hline 5.41 & -55.0 & -2.04 & -81.71 & -104.40 & 24.51 & 62.74 \\
\hline 5.46 & -55.3 & -2.06 & -82.85 & -105.81 & 24.92 & 63.70 \\
\hline 5.51 & -55.8 & -2.07 & -84.33 & -107.56 & 25.33 & 64.76 \\
\hline 5.56 & -56.0 & -2.08 & -85.47 & -108.90 & 25.74 & 65.76 \\
\hline 5.61 & -56.3 & -2.09 & -86.68 & -110.31 & 26.14 & 66.75 \\
\hline 5.66 & -56.7 & -2.09 & -87.95 & -111.92 & 26.57 & 67.80 \\
\hline 5.71 & -57.1 & -2.11 & -89.36 & -113.53 & 27.00 & 68.87 \\
\hline 5.76 & -57.6 & -2.12 & -90.71 & -115.28 & 27.45 & 70.01 \\
\hline 5.81 & -57.7 & -2.13 & -91.98 & -116.69 & 27.86 & 71.02 \\
\hline 5.86 & -58.1 & -2.14 & -93.39 & -118.44 & 28.30 & 72.09 \\
\hline 5.91 & -58.1 & -2.15 & -94.53 & -119.78 & 28.73 & 73.15 \\
\hline 5.96 & -58.4 & -2.16 & -95.81 & -121.39 & 29.18 & 74.22 \\
\hline 6.01 & -58.9 & -2.17 & -97.15 & -123.00 & 29.63 & 75.35 \\
\hline 6.06 & -59.0 & -2.18 & -98.43 & -124.55 & 30.08 & 76.46 \\
\hline 6.11 & -59.2 & -2.19 & -99.84 & -126.16 & 30.52 & 77.56 \\
\hline 6.16 & -59.2 & -2.20 & -101.18 & -127.50 & 30.97 & 78.55 \\
\hline 6.21 & -59.4 & -2.21 & -102.52 & -129.04 & 31.42 & 79.61 \\
\hline 6.26 & -60.0 & -2.22 & -104.07 & -130.79 & 31.90 & 80.79 \\
\hline 6.31 & -60.1 & -2.23 & -105.28 & -132.33 & 32.37 & 82.00 \\
\hline 6.36 & -60.4 & -2.24 & -106.82 & -133.95 & 32.84 & 83.10 \\
\hline 6.41 & -60.7 & -2.26 & -108.16 & -135.62 & 33.34 & 84.31 \\
\hline 6.46 & -61.1 & -2.27 & -109.84 & -137.30 & 33.85 & 85.55 \\
\hline 6.51 & -61.3 & -2.27 & -111.25 & -138.98 & 34.35 & 86.76 \\
\hline 6.56 & -61.8 & -2.28 & -112.73 & -140.79 & 34.85 & 88.02 \\
\hline 6.61 & -62.0 & -2.30 & -114.27 & -142.47 & 35.39 & 89.31 \\
\hline 6.66 & -61.9 & -2.31 & -115.75 & -144.08 & 35.87 & 90.46 \\
\hline 6.71 & -62.1 & -2.32 & -117.23 & -145.76 & 36.39 & 91.65 \\
\hline 6.76 & -62.5 & -2.33 & -118.77 & -147.37 & 36.92 & 92.94 \\
\hline
\end{tabular}


Table F.12 IDT Strength Data (NU 58-40 \#12), continued

\begin{tabular}{|c|c|c|c|c|c|c|}
\hline Time & Axial Force & Axial Displ. & Vert front & Vert back & Horz front & Horz back \\
\hline $\mathrm{Sec}$ & $\overline{\mathrm{kN}}$ & $\mathrm{mm}$ & $\mu \mathrm{m}$ & $\mu \mathrm{m}$ & $\mu \mathrm{m}$ & $\mu \mathrm{m}$ \\
\hline 6.81 & -63.0 & -2.34 & -120.52 & -149.25 & 37.47 & 94.37 \\
\hline 6.86 & -63.2 & -2.35 & -121.99 & -150.93 & 38.03 & 95.68 \\
\hline 6.91 & -63.4 & -2.36 & -123.67 & -152.81 & 38.58 & 97.00 \\
\hline 6.96 & -63.6 & -2.37 & -125.28 & -154.56 & 39.13 & 98.33 \\
\hline 7.01 & -63.9 & -2.38 & -126.96 & -156.30 & 39.71 & 99.74 \\
\hline 7.06 & -63.9 & -2.39 & -128.44 & -157.98 & 40.26 & 101.03 \\
\hline 7.11 & -64.1 & -2.40 & -129.98 & -159.79 & 40.85 & 102.42 \\
\hline 7.16 & -64.0 & -2.41 & -131.60 & -161.54 & 41.39 & 103.68 \\
\hline 7.21 & -64.3 & -2.42 & -133.21 & -163.35 & 41.99 & 105.09 \\
\hline 7.26 & -64.7 & -2.43 & -134.95 & -165.17 & 42.59 & 106.57 \\
\hline 7.31 & -65.0 & -2.44 & -136.63 & -166.98 & 43.22 & 108.06 \\
\hline 7.36 & -65.2 & -2.45 & -138.44 & -168.93 & 43.83 & 109.49 \\
\hline 7.41 & \begin{tabular}{|c|}
-65.3 \\
\end{tabular} & -2.46 & -140.19 & -170.87 & 44.45 & 110.98 \\
\hline 7.46 & -65.3 & -2.47 & -141.80 & -172.55 & 45.06 & 112.44 \\
\hline 7.51 & -65.5 & -2.48 & -143.48 & -174.36 & 45.69 & 113.95 \\
\hline 7.56 & -65.8 & -2.50 & -145.29 & -176.24 & 46.34 & 115.50 \\
\hline 7.61 & -65.9 & -2.50 & -147.04 & -178.19 & 47.01 & 117.04 \\
\hline 7.66 & -66.0 & -2.51 & -148.85 & -180.47 & 47.67 & 118.62 \\
\hline 7.71 & -66.3 & -2.53 & -150.60 & -182.22 & 48.36 & 120.22 \\
\hline 7.76 & -66.4 & -2.54 & -152.41 & -184.23 & 49.02 & 121.83 \\
\hline 7.81 & -66.5 & -2.55 & -154.16 & -186.11 & 49.70 & 123.46 \\
\hline 7.86 & -66.7 & -2.56 & -155.90 & -188.13 & 50.41 & 125.10 \\
\hline 7.91 & -67.0 & -2.57 & -157.71 & -190.28 & 51.13 & 126.83 \\
\hline 7.96 & -67.2 & -2.58 & -159.66 & -192.49 & 51.87 & 128.54 \\
\hline 8.01 & \begin{tabular}{|c|}
-67.3 \\
\end{tabular} & -2.59 & -161.54 & -194.57 & 52.64 & 130.30 \\
\hline 8.06 & -67.4 & -2.60 & -163.35 & -196.79 & 53.39 & 132.07 \\
\hline 8.11 & -67.3 & -2.61 & -165.23 & -198.80 & 54.14 & 133.78 \\
\hline 8.16 & -67.4 & -2.62 & -167.05 & -200.89 & 54.91 & 135.46 \\
\hline 8.21 & -67.5 & -2.63 & -168.99 & -202.97 & 55.70 & 137.27 \\
\hline 8.26 & -67.9 & -2.64 & -170.94 & -205.32 & 56.53 & 139.15 \\
\hline 8.31 & $\begin{array}{l}-68.0 \\
\end{array}$ & -2.65 & -172.95 & -207.80 & $\begin{array}{l}57.38 \\
\end{array}$ & 141.05 \\
\hline 8.36 & -68.2 & -2.66 & -175.10 & -210.15 & 58.19 & 142.93 \\
\hline 8.41 & $\begin{array}{l}-68.4 \\
\end{array}$ & -2.67 & -177.12 & -212.30 & 59.09 & 144.96 \\
\hline 8.46 & -68.6 & -2.68 & -179.20 & -214.65 & 60.00 & 146.95 \\
\hline 8.51 & -68.8 & -2.69 & -181.35 & -217.07 & 60.92 & 149.05 \\
\hline 8.56 & -68.8 & -2.70 & -183.36 & -219.35 & 61.85 & 151.05 \\
\hline 8.61 & -69.0 & -2.71 & -185.44 & -221.63 & 62.78 & 153.13 \\
\hline 8.66 & -69.0 & -2.73 & -187.66 & -223.98 & 63.76 & 155.28 \\
\hline 8.71 & -69.0 & -2.74 & -189.74 & -226.33 & 64.74 & 157.33 \\
\hline 8.76 & -69.0 & -2.75 & -191.89 & -228.75 & 65.75 & 159.49 \\
\hline 8.81 & -69.1 & -2.76 & -194.04 & -231.03 & 66.77 & 161.66 \\
\hline 8.86 & -69.3 & -2.77 & -196.39 & -233.65 & 67.84 & 163.92 \\
\hline 8.91 & -69.3 & -2.77 & -198.54 & -236.13 & 68.89 & 166.11 \\
\hline 8.96 & -69.4 & -2.79 & -200.55 & -238.55 & 70.04 & 168.46 \\
\hline 9.01 & -69.5 & -2.80 & -202.70 & -240.90 & 71.16 & 170.79 \\
\hline
\end{tabular}


Table F.12 IDT Strength Data (NU 58-40 \#12), continued

\begin{tabular}{|c|c|c|c|c|c|c|}
\hline Time & Axial Force & Axial Displ. & Vert front & Vert back & Horz front & Horz back \\
\hline $\mathrm{Sec}$ & $\mathrm{kN}$ & $\mathrm{mm}$ & $\mu \mathrm{m}$ & $\mu \mathrm{m}$ & $\mu \mathrm{m}$ & $\mu \mathrm{m}$ \\
\hline 9.06 & -69.6 & -2.81 & -204.91 & -243.52 & 72.34 & 173.27 \\
\hline 9.11 & -69.6 & -2.82 & -207.06 & -245.87 & 73.53 & 175.64 \\
\hline 9.16 & -69.6 & -2.83 & -209.21 & -248.42 & 74.74 & 178.07 \\
\hline 9.21 & -69.8 & -2.84 & -211.29 & -251.04 & 75.99 & 180.59 \\
\hline 9.26 & -69.8 & -2.85 & -213.37 & -253.59 & 77.28 & 183.16 \\
\hline 9.31 & -70.0 & -2.86 & -215.32 & -256.41 & 78.63 & 185.86 \\
\hline 9.36 & $\begin{array}{l}-69.9 \\
\end{array}$ & -2.87 & -217.54 & -258.83 & 79.96 & 188.46 \\
\hline 9.41 & -70.1 & -2.88 & -219.82 & -261.85 & 81.36 & 191.28 \\
\hline 9.46 & -70.1 & -2.89 & -222.37 & -264.74 & 82.80 & 194.10 \\
\hline 9.51 & -70.2 & -2.90 & -224.85 & -267.29 & 84.30 & 197.04 \\
\hline 9.56 & -70.1 & -2.91 & -227.20 & -269.91 & 85.83 & 199.93 \\
\hline 9.61 & -70.1 & -2.92 & -229.89 & -272.73 & 87.40 & 202.90 \\
\hline 9.66 & -70.0 & -2.93 & -232.44 & -275.41 & 89.03 & 205.92 \\
\hline 9.71 & -70.1 & -2.94 & -235.13 & -278.37 & 90.70 & 209.03 \\
\hline 9.76 & -70.3 & -2.96 & -237.68 & -281.12 & 92.47 & 212.23 \\
\hline 9.81 & -70.2 & -2.96 & -240.43 & -284.01 & 94.32 & 215.56 \\
\hline 9.86 & -70.3 & -2.97 & -243.12 & -287.03 & 96.20 & 218.85 \\
\hline 9.91 & -70.2 & -2.99 & -245.87 & -289.78 & 98.16 & 222.27 \\
\hline 9.96 & $\begin{array}{l}-70.2 \\
\end{array}$ & -3.00 & -248.62 & $\begin{array}{l}-292.40 \\
\end{array}$ & 100.22 & 225.79 \\
\hline 10.01 & -70.0 & -3.01 & -251.51 & -295.28 & 102.31 & 229.25 \\
\hline 10.06 & -70.0 & -3.02 & -254.13 & -298.24 & 104.53 & 232.84 \\
\hline 10.11 & -70.0 & -3.02 & -257.08 & -301.33 & 106.86 & 236.54 \\
\hline 10.16 & -70.2 & -3.04 & -259.90 & -304.68 & 109.37 & 240.50 \\
\hline 10.21 & -69.9 & -3.05 & -262.79 & -307.71 & 111.93 & 244.31 \\
\hline 10.26 & -70.0 & -3.07 & -265.61 & -310.93 & 114.71 & 248.42 \\
\hline 10.31 & $\begin{array}{l}-69.9 \\
\end{array}$ & -3.07 & -268.50 & -314.15 & 117.62 & 252.52 \\
\hline 10.36 & $\begin{array}{l}-69.9 \\
\end{array}$ & -3.08 & -271.45 & -317.51 & 120.74 & 256.75 \\
\hline 10.41 & -69.7 & -3.09 & -274.14 & -320.60 & 124.04 & 260.99 \\
\hline 10.46 & -69.7 & -3.10 & -276.89 & -323.89 & 127.68 & 265.49 \\
\hline 10.51 & -69.6 & -3.11 & -279.64 & -327.38 & 131.64 & 270.04 \\
\hline 10.56 & -69.3 & -3.12 & -282.13 & -330.74 & 135.87 & 274.49 \\
\hline 10.61 & -69.3 & -3.13 & -284.54 & -334.36 & 140.61 & 279.34 \\
\hline 10.66 & -69.1 & -3.14 & -286.09 & -337.92 & 145.75 & 284.11 \\
\hline 10.71 & -68.7 & -3.16 & -287.83 & -341.95 & 151.28 & 288.92 \\
\hline 10.76 & -68.7 & -3.17 & -288.91 & -345.51 & 157.28 & 293.96 \\
\hline 10.81 & -68.4 & -3.18 & -280.04 & -349.47 & 163.70 & 299.06 \\
\hline 10.86 & -68.1 & -3.19 & -281.66 & -353.63 & 170.52 & 304.25 \\
\hline 10.91 & $\begin{array}{l}-67.9 \\
\end{array}$ & -3.20 & -283.54 & -357.66 & 177.76 & 309.60 \\
\hline 10.96 & -67.4 & -3.21 & -285.28 & -361.82 & 185.36 & 314.92 \\
\hline 11.01 & -67.0 & -3.22 & -287.43 & -366.05 & 193.43 & 320.43 \\
\hline 11.06 & -66.8 & -3.23 & -289.51 & -370.55 & 202.04 & 326.25 \\
\hline 11.11 & -66.4 & -3.24 & -291.93 & -375.12 & 211.12 & 332.13 \\
\hline 11.16 & -66.1 & -3.25 & -294.75 & -379.82 & 219.64 & 338.47 \\
\hline 11.21 & -65.6 & -3.26 & -297.97 & -384.92 & 219.64 & 344.82 \\
\hline 11.26 & $\begin{array}{l}-64.9 \\
\end{array}$ & -3.27 & -301.86 & -389.89 & 219.64 & 351.30 \\
\hline 11.31 & -64.6 & -3.28 & -305.29 & -395.26 & 219.64 & 358.28 \\
\hline 11.36 & -63.9 & -3.29 & -310.12 & -400.43 & 219.64 & 365.36 \\
\hline 11.41 & -63.5 & -3.30 & -319.46 & -405.93 & 219.64 & 373.03 \\
\hline
\end{tabular}


Table F.13 IDT Strength Data (NU 58-40 \#9)

\begin{tabular}{|c|c|c|c|c|c|c|}
\hline Time & Axial Force & Axial Displ. & Vert front & Vert back & Horz front & Horz back \\
\hline $\mathrm{Sec}$ & $\mathrm{kN}$ & $\mathrm{mm}$ & $\mu \mathrm{m}$ & $\mu \mathrm{m}$ & $\mu \mathrm{m}$ & $\mu \mathrm{m}$ \\
\hline 0.00 & -0.1 & -0.56 & 1.07 & 0.81 & 0.08 & -0.02 \\
\hline 0.05 & -0.3 & -0.58 & 0.94 & 0.60 & 0.13 & 0.02 \\
\hline 0.10 & -0.7 & -0.59 & 0.74 & 0.60 & 0.19 & 0.10 \\
\hline 0.15 & -1.1 & -0.60 & 0.47 & 0.40 & 0.25 & 0.22 \\
\hline 0.20 & -1.6 & -0.61 & 0.20 & 0.13 & 0.34 & 0.34 \\
\hline 0.25 & -2.0 & -0.62 & -0.07 & -0.20 & 0.43 & 0.49 \\
\hline 0.30 & -2.6 & -0.63 & -0.34 & -0.47 & 0.51 & 0.74 \\
\hline 0.35 & -3.2 & -0.64 & -0.81 & -1.01 & 0.62 & 0.97 \\
\hline 0.40 & -4.0 & -0.65 & -1.14 & -1.48 & 0.77 & 1.28 \\
\hline 0.45 & -4.8 & -0.66 & -1.61 & -2.15 & 0.91 & 1.61 \\
\hline 0.50 & -5.5 & -0.67 & -1.88 & -2.69 & 1.05 & 1.95 \\
\hline 0.55 & -6.2 & -0.68 & -2.42 & -3.29 & 1.20 & 2.25 \\
\hline 0.60 & -6.8 & -0.69 & -2.95 & -4.23 & 1.32 & 2.55 \\
\hline 0.65 & -7.7 & -0.70 & -3.36 & -4.70 & 1.53 & 3.00 \\
\hline 0.70 & -8.5 & -0.71 & -3.63 & -5.24 & 1.72 & 3.44 \\
\hline 0.75 & -9.0 & -0.72 & -4.30 & -6.31 & 1.82 & 3.71 \\
\hline 0.80 & -9.9 & -0.73 & -4.83 & -7.05 & 2.03 & 4.25 \\
\hline 0.85 & -10.6 & -0.74 & -5.17 & -7.59 & 2.24 & 4.70 \\
\hline 0.90 & -11.2 & -0.75 & -5.71 & -8.39 & 2.39 & 5.04 \\
\hline 0.95 & -12.1 & -0.76 & -6.38 & -9.33 & 2.61 & 5.51 \\
\hline 1.00 & -12.7 & -0.77 & -6.78 & -10.07 & 2.80 & 5.98 \\
\hline 1.05 & -13.4 & -0.78 & -7.25 & -10.81 & 3.00 & 6.46 \\
\hline 1.10 & -14.0 & -0.79 & -7.92 & -11.68 & 3.20 & 6.93 \\
\hline 1.15 & -14.8 & -0.80 & -8.39 & -12.56 & 3.42 & 7.39 \\
\hline 1.20 & -15.4 & -0.82 & -8.93 & -13.43 & 3.62 & 7.89 \\
\hline 1.25 & -16.0 & -0.83 & -9.47 & -14.23 & 3.83 & 8.31 \\
\hline 1.30 & -16.7 & -0.83 & -10.07 & -15.11 & 4.06 & 8.85 \\
\hline 1.35 & -17.3 & -0.85 & -10.47 & -15.98 & 4.28 & 9.40 \\
\hline 1.40 & -18.0 & -0.86 & -11.28 & -16.92 & 4.51 & 9.87 \\
\hline 1.45 & -18.8 & -0.87 & -11.82 & -17.86 & 4.74 & 10.46 \\
\hline 1.50 & -19.4 & -0.88 & -12.56 & -18.73 & 4.97 & 11.01 \\
\hline 1.55 & -20.3 & -0.89 & -13.03 & -19.81 & 5.26 & 11.63 \\
\hline 1.60 & -20.8 & -0.90 & -13.76 & -20.75 & 5.51 & 12.22 \\
\hline 1.65 & -21.5 & -0.91 & -14.23 & -21.69 & 5.77 & 12.79 \\
\hline 1.70 & -22.2 & -0.92 & -15.04 & -22.69 & 6.03 & 13.38 \\
\hline 1.75 & -22.7 & -0.93 & -15.64 & -23.83 & 6.28 & 14.00 \\
\hline 1.80 & -23.3 & -0.94 & -16.32 & -24.71 & 6.54 & 14.55 \\
\hline 1.85 & -24.0 & -0.95 & -16.99 & -25.78 & 6.81 & 15.22 \\
\hline 1.90 & -24.7 & -0.96 & -17.59 & -26.86 & 7.10 & 15.88 \\
\hline 1.95 & -25.1 & -0.97 & -18.26 & -27.93 & 7.36 & 16.45 \\
\hline 2.00 & -25.9 & -0.98 & -19.00 & -29.14 & 7.65 & 17.14 \\
\hline 2.05 & -26.9 & -0.99 & -19.87 & -30.41 & 7.98 & 17.93 \\
\hline 2.10 & -27.2 & -1.00 & -20.55 & -31.49 & 8.28 & 18.58 \\
\hline 2.15 & -27.5 & -1.01 & -21.02 & -32.36 & 8.52 & 19.12 \\
\hline 2.20 & -28.4 & -1.02 & -21.89 & -33.64 & 8.85 & 19.94 \\
\hline 2.25 & -28.8 & -1.04 & -22.49 & -34.71 & 9.16 & 20.60 \\
\hline
\end{tabular}


Table F.13 IDT Strength Data (NU 58-40 \#9), continued

\begin{tabular}{|c|c|c|c|c|c|c|}
\hline Time & Axial Force & Axial Displ. & Vert front & Vert back & Horz front & Horz back \\
\hline $\mathrm{Sec}$ & $\mathrm{kN}$ & $\mathrm{mm}$ & $\mu \mathrm{m}$ & $\mu \mathrm{m}$ & $\mu \mathrm{m}$ & $\mu \mathrm{m}$ \\
\hline 2.30 & -29.5 & -1.04 & -23.36 & -35.85 & 9.48 & 21.33 \\
\hline 2.35 & -30.0 & -1.05 & -24.04 & -37.06 & 9.77 & 22.07 \\
\hline 2.40 & -30.8 & -1.06 & -24.71 & -38.34 & 10.12 & 22.84 \\
\hline 2.45 & -31.4 & -1.08 & -25.65 & -39.61 & 10.47 & 23.63 \\
\hline 2.50 & -31.8 & -1.09 & -26.25 & -40.82 & 10.78 & 24.41 \\
\hline 2.55 & -32.5 & -1.10 & -27.06 & -42.03 & 11.12 & 25.18 \\
\hline 2.60 & -33.0 & -1.11 & -27.80 & -43.17 & 11.46 & 25.93 \\
\hline 2.65 & -33.6 & -1.12 & -28.74 & -44.58 & 11.82 & 26.74 \\
\hline 2.70 & -34.4 & -1.12 & -29.61 & -45.86 & 12.21 & 27.66 \\
\hline 2.75 & -34.9 & -1.14 & -30.41 & -47.27 & 12.57 & 28.43 \\
\hline 2.80 & -35.4 & -1.15 & -31.22 & -48.54 & 12.96 & 29.29 \\
\hline 2.85 & -36.0 & -1.16 & -32.03 & -49.89 & 13.33 & 30.20 \\
\hline 2.90 & -36.6 & -1.17 & -32.97 & -51.30 & 13.72 & 31.10 \\
\hline 2.95 & -37.1 & -1.18 & -33.70 & -52.50 & 14.12 & 31.99 \\
\hline 3.00 & -37.6 & -1.19 & -34.58 & -54.05 & 14.51 & 32.87 \\
\hline 3.05 & -38.1 & -1.20 & -35.45 & -55.32 & 14.92 & 33.81 \\
\hline 3.10 & -38.8 & -1.21 & -36.39 & -56.73 & 15.33 & 34.76 \\
\hline 3.15 & -39.5 & -1.22 & -37.33 & -58.35 & 15.79 & 35.80 \\
\hline 3.20 & -39.7 & -1.23 & -38.14 & -59.55 & 16.18 & 36.68 \\
\hline 3.25 & -40.3 & -1.24 & -39.08 & -61.10 & 16.61 & 37.63 \\
\hline 3.30 & -40.8 & -1.25 & -39.95 & -62.51 & 17.05 & 38.66 \\
\hline 3.35 & -41.3 & -1.26 & -40.82 & -63.92 & 17.50 & 39.65 \\
\hline 3.40 & -41.9 & -1.27 & -41.69 & -65.46 & 17.94 & 40.72 \\
\hline 3.45 & -42.6 & -1.28 & -42.77 & -66.94 & 18.45 & 41.85 \\
\hline 3.50 & -42.8 & -1.29 & -43.64 & -68.42 & 18.90 & 42.84 \\
\hline 3.55 & -43.5 & -1.30 & -44.65 & -69.96 & 19.38 & 43.93 \\
\hline 3.60 & -43.8 & -1.31 & -45.52 & -71.44 & 19.83 & 44.97 \\
\hline 3.65 & -44.4 & -1.32 & -46.66 & -72.98 & 20.33 & 46.11 \\
\hline 3.70 & -45.0 & -1.34 & -47.67 & -74.59 & 20.86 & 47.28 \\
\hline 3.75 & -45.2 & -1.35 & -48.54 & -76.07 & 21.33 & 48.36 \\
\hline 3.80 & -45.6 & -1.35 & -49.55 & -77.55 & 21.81 & 49.45 \\
\hline 3.85 & -46.1 & -1.36 & -50.49 & -79.09 & 22.32 & 50.64 \\
\hline 3.90 & -46.5 & -1.38 & -51.50 & -80.77 & 22.85 & 51.78 \\
\hline 3.95 & -46.9 & -1.38 & -52.57 & -82.31 & 23.36 & 52.96 \\
\hline 4.00 & -47.5 & -1.40 & -53.58 & -83.86 & 23.89 & 54.18 \\
\hline 4.05 & -48.0 & -1.41 & -54.59 & -85.47 & 24.45 & 55.42 \\
\hline 4.10 & -48.2 & -1.42 & -55.53 & -86.88 & 24.96 & 56.57 \\
\hline 4.15 & -48.7 & -1.43 & -56.67 & -88.42 & 25.51 & 57.84 \\
\hline 4.20 & -49.1 & -1.44 & -57.67 & -90.17 & 26.09 & 59.10 \\
\hline 4.25 & -49.2 & -1.45 & -58.61 & -91.58 & 26.61 & 60.29 \\
\hline 4.30 & -49.7 & -1.46 & -59.69 & -93.19 & 27.18 & 61.57 \\
\hline 4.35 & -50.2 & -1.47 & -60.76 & -94.80 & 27.76 & 62.93 \\
\hline 4.40 & -50.6 & -1.48 & -61.84 & -96.55 & 28.35 & 64.22 \\
\hline 4.45 & -51.2 & -1.49 & -63.05 & -98.23 & 28.99 & 65.66 \\
\hline 4.50 & -51.5 & -1.50 & -64.12 & -99.91 & 29.58 & 67.02 \\
\hline
\end{tabular}


Table F.13 IDT Strength Data (NU 58-40 \#9), continued

\begin{tabular}{|c|c|c|c|c|c|c|}
\hline Time & Axial Force & Axial Displ. & Vert front & Vert back & Horz front & Horz back \\
\hline $\mathrm{Sec}$ & $\overline{\mathrm{kN}}$ & $\mathrm{mm}$ & $\mu \mathrm{m}$ & $\mu \mathrm{m}$ & $\mu \mathrm{m}$ & $\mu \mathrm{m}$ \\
\hline 4.55 & -51.8 & -1.52 & -65.26 & -101.45 & 30.17 & 68.37 \\
\hline 4.60 & -52.2 & -1.53 & -66.34 & -103.20 & 30.81 & 69.78 \\
\hline 4.65 & -52.4 & -1.53 & -67.41 & -104.74 & 31.41 & 71.15 \\
\hline 4.70 & -52.7 & -1.55 & -68.55 & -106.28 & 32.02 & 72.53 \\
\hline 4.75 & -53.2 & -1.55 & -69.49 & -107.90 & 32.65 & 74.01 \\
\hline 4.80 & -53.7 & -1.57 & -70.90 & -109.78 & 33.33 & 75.57 \\
\hline 4.85 & $\begin{array}{l}-54.1 \\
\end{array}$ & -1.58 & -72.11 & -111.52 & 34.02 & 77.09 \\
\hline 4.90 & -54.6 & -1.58 & -73.25 & -113.33 & 34.68 & 78.66 \\
\hline 4.95 & -54.9 & -1.60 & -74.46 & -115.01 & 35.36 & 80.20 \\
\hline 5.00 & -55.1 & -1.61 & -75.67 & -116.76 & 36.06 & 81.79 \\
\hline 5.05 & -55.5 & -1.62 & -76.88 & -118.57 & 36.76 & 83.42 \\
\hline 5.10 & -55.8 & -1.62 & -78.02 & -120.25 & 37.46 & 85.05 \\
\hline 5.16 & -56.0 & -1.64 & -79.23 & -121.99 & 38.17 & 86.65 \\
\hline 5.21 & -56.1 & -1.65 & -80.37 & -123.61 & 38.86 & 88.21 \\
\hline 5.26 & -56.4 & -1.66 & -81.58 & -125.28 & 39.54 & 89.80 \\
\hline 5.31 & -56.8 & -1.67 & -82.78 & -127.10 & 40.29 & 91.53 \\
\hline 5.36 & -57.2 & -1.68 & -84.13 & -128.84 & 41.06 & 93.29 \\
\hline 5.41 & -57.5 & -1.69 & -85.27 & -130.72 & 41.80 & 95.02 \\
\hline 5.46 & $\begin{array}{l}-57.7 \\
\end{array}$ & -1.70 & -86.48 & -132.40 & 42.57 & 96.78 \\
\hline 5.51 & -58.3 & -1.71 & -87.89 & -134.42 & 43.36 & 98.70 \\
\hline 5.56 & -58.4 & -1.72 & -89.10 & -136.23 & 44.13 & 100.48 \\
\hline 5.61 & -58.6 & -1.73 & -90.37 & -138.04 & 44.93 & 102.32 \\
\hline 5.66 & -58.7 & -1.74 & -91.58 & -139.65 & 45.71 & 104.12 \\
\hline 5.71 & -58.8 & -1.75 & -92.86 & -141.40 & 46.49 & 105.91 \\
\hline 5.76 & -59.2 & -1.76 & -94.13 & -143.35 & 47.31 & 107.83 \\
\hline 5.81 & -59.7 & -1.77 & -95.54 & -145.36 & 48.16 & 109.83 \\
\hline 5.86 & -60.0 & -1.79 & -96.88 & -147.17 & 49.02 & 111.82 \\
\hline 5.91 & -60.1 & -1.79 & -98.16 & -148.92 & 49.85 & 113.80 \\
\hline 5.96 & -60.0 & -1.80 & -99.30 & -150.53 & 50.66 & 115.65 \\
\hline 6.01 & -60.2 & -1.82 & -100.58 & -152.28 & 51.50 & 117.65 \\
\hline 6.06 & -60.5 & -1.82 & -101.99 & -154.29 & 52.39 & 119.71 \\
\hline 6.11 & -60.9 & -1.83 & -103.13 & -155.97 & 53.30 & 121.88 \\
\hline 6.16 & -61.3 & -1.85 & -104.81 & -158.38 & 54.22 & 124.06 \\
\hline 6.21 & -61.4 & -1.86 & -106.22 & -160.13 & 55.10 & 126.16 \\
\hline 6.26 & -61.5 & -1.87 & -107.43 & -161.94 & 56.05 & 128.37 \\
\hline 6.31 & -61.6 & -1.88 & -108.70 & -163.62 & 57.00 & 130.54 \\
\hline 6.36 & -61.8 & -1.89 & -110.11 & -165.70 & 57.93 & 132.72 \\
\hline 6.41 & -61.9 & -1.90 & -111.52 & -167.52 & 58.86 & 134.90 \\
\hline 6.46 & -62.5 & -1.91 & -113.00 & -169.73 & 59.90 & 137.40 \\
\hline 6.51 & -62.5 & -1.92 & -114.21 & -171.41 & 60.89 & 139.69 \\
\hline 6.56 & -62.9 & -1.93 & -115.68 & -173.49 & 61.92 & 142.15 \\
\hline 6.61 & -63.1 & -1.94 & -117.29 & -175.77 & 62.97 & 144.67 \\
\hline 6.66 & $\begin{array}{l}-63.3 \\
\end{array}$ & -1.95 & -118.64 & -177.52 & $\begin{array}{l}64.01 \\
\end{array}$ & 147.17 \\
\hline 6.71 & -63.4 & -1.96 & -120.05 & -179.53 & 65.08 & 149.72 \\
\hline 6.76 & -63.4 & -1.97 & -121.26 & -181.21 & 66.14 & 152.29 \\
\hline
\end{tabular}


Table F.13 IDT Strength Data (NU 58-40 \#9), continued

\begin{tabular}{|c|c|c|c|c|c|c|}
\hline Time & Axial Force & Axial Displ. & Vert front & Vert back & Horz front & Horz back \\
\hline $\mathrm{Sec}$ & $\overline{\mathrm{kN}}$ & $\mathrm{mm}$ & $\mu \mathrm{m}$ & $\mu \mathrm{m}$ & $\mu \mathrm{m}$ & $\mu \mathrm{m}$ \\
\hline 6.81 & -63.6 & -1.98 & -122.80 & -183.29 & 67.19 & 154.84 \\
\hline 6.86 & -63.7 & -2.00 & -124.21 & -185.24 & 68.27 & 157.41 \\
\hline 6.91 & -64.1 & -2.00 & -125.75 & -187.32 & 69.42 & 160.21 \\
\hline 6.96 & -64.4 & -2.02 & -127.30 & -189.47 & 70.58 & 163.07 \\
\hline 7.01 & -64.4 & -2.03 & -128.78 & -191.49 & 71.76 & 165.90 \\
\hline 7.06 & -64.4 & -2.04 & -130.12 & -193.43 & 72.89 & 168.67 \\
\hline 7.11 & -64.7 & -2.04 & -131.73 & -195.58 & 74.09 & 171.66 \\
\hline 7.16 & -64.8 & -2.06 & -133.34 & -197.66 & 75.31 & 174.65 \\
\hline 7.21 & -64.9 & -2.06 & -134.68 & -199.54 & 76.51 & 177.62 \\
\hline 7.26 & -65.2 & -2.08 & -136.36 & -201.69 & 77.77 & 180.74 \\
\hline 7.31 & -65.1 & -2.09 & -137.77 & -203.77 & 79.00 & 183.80 \\
\hline 7.36 & -65.1 & -2.10 & -139.25 & -205.72 & 80.27 & 186.97 \\
\hline 7.41 & $\begin{array}{l}-65.4 \\
\end{array}$ & -2.10 & -140.86 & -207.80 & 81.57 & 190.24 \\
\hline 7.46 & -65.4 & -2.12 & -142.34 & -209.88 & 82.87 & 193.55 \\
\hline 7.51 & -65.6 & -2.13 & -143.95 & -212.10 & 84.23 & 196.99 \\
\hline 7.56 & -65.6 & -2.14 & -145.49 & -214.18 & 85.57 & 200.38 \\
\hline 7.61 & -66.0 & -2.15 & -147.11 & -216.33 & 87.01 & 204.07 \\
\hline 7.66 & -65.9 & -2.16 & -148.65 & -218.41 & 88.39 & 207.65 \\
\hline 7.71 & -66.2 & -2.17 & -150.33 & -220.76 & 89.89 & 211.53 \\
\hline 7.76 & -66.1 & -2.18 & -151.87 & -222.77 & 91.36 & 215.29 \\
\hline 7.81 & -66.2 & -2.19 & -153.48 & -224.99 & 92.86 & 219.18 \\
\hline 7.86 & -66.2 & -2.20 & -155.16 & -227.20 & 94.38 & 223.14 \\
\hline 7.91 & -66.2 & -2.21 & -156.64 & -229.15 & 95.89 & 227.12 \\
\hline 7.96 & -66.4 & -2.22 & -158.32 & -231.37 & 97.48 & 231.32 \\
\hline 8.01 & -66.4 & -2.23 & -159.86 & -233.52 & 99.07 & 235.58 \\
\hline 8.06 & -66.4 & -2.24 & -161.47 & -235.80 & 100.73 & 239.98 \\
\hline 8.11 & -66.4 & -2.25 & -163.15 & -238.15 & 102.38 & 244.43 \\
\hline 8.16 & -66.4 & -2.26 & -164.83 & -240.23 & 104.08 & 249.04 \\
\hline 8.21 & -66.6 & -2.28 & -166.58 & -242.51 & 105.84 & 253.84 \\
\hline 8.26 & -66.7 & -2.29 & -168.25 & -244.80 & 107.63 & 258.81 \\
\hline 8.31 & -66.8 & -2.30 & -170.00 & -247.08 & 109.48 & 263.95 \\
\hline 8.36 & -66.7 & -2.31 & -171.75 & -249.29 & 111.32 & 269.08 \\
\hline 8.41 & -66.6 & -2.32 & -173.36 & -251.51 & 113.20 & 274.34 \\
\hline 8.46 & -66.8 & -2.33 & -175.17 & -253.79 & 115.17 & 279.93 \\
\hline 8.51 & -66.6 & -2.34 & -176.92 & -256.01 & 117.14 & 285.55 \\
\hline 8.56 & -66.6 & -2.35 & -178.66 & -258.36 & 119.19 & 291.36 \\
\hline 8.61 & -66.6 & -2.36 & -180.41 & -260.57 & 121.29 & 297.42 \\
\hline 8.66 & -66.6 & -2.37 & -182.29 & -263.06 & 123.45 & 303.64 \\
\hline 8.71 & -66.3 & -2.38 & -183.90 & -264.74 & 125.62 & 309.97 \\
\hline 8.76 & -66.2 & -2.39 & -185.71 & -267.22 & 127.84 & 316.42 \\
\hline 8.81 & -66.4 & -2.40 & -187.59 & -269.64 & 130.22 & 323.35 \\
\hline 8.86 & -66.4 & -2.41 & -189.40 & -271.85 & 132.71 & 330.60 \\
\hline 8.91 & -66.2 & -2.42 & -191.49 & -274.14 & 135.19 & 337.97 \\
\hline 8.96 & -66.1 & -2.43 & -193.16 & -276.35 & 137.77 & 345.66 \\
\hline 9.01 & $\begin{array}{l}-66.1 \\
\end{array}$ & -2.44 & -195.18 & -278.50 & 140.48 & 353.73 \\
\hline
\end{tabular}


Table F.13 IDT Strength Data (NU 58-40 \#9), continued

\begin{tabular}{|c|c|c|c|c|c|c|}
\hline Time & Axial Force & Axial Displ. & Vert front & Vert back & Horz front & Horz back \\
\hline Sec & $\mathrm{kN}$ & $\mathrm{mm}$ & $\mu \mathrm{m}$ & $\mu \mathrm{m}$ & $\mu \mathrm{m}$ & $\mu \mathrm{m}$ \\
\hline 9.06 & -66.0 & -2.46 & -196.92 & -280.78 & 143.30 & 362.12 \\
\hline 9.11 & -65.7 & -2.47 & -198.80 & -282.86 & 146.17 & 370.80 \\
\hline 9.16 & -65.6 & -2.47 & -200.82 & -285.01 & 149.17 & 379.78 \\
\hline 9.21 & -65.7 & -2.49 & -202.90 & -287.16 & 152.43 & 389.68 \\
\hline 9.26 & -65.5 & -2.49 & -204.85 & -289.18 & 155.75 & 399.87 \\
\hline 9.31 & -65.3 & -2.51 & -206.93 & -291.32 & 159.20 & 410.62 \\
\hline 9.36 & -65.0 & -2.52 & -208.87 & -293.14 & 162.82 & 421.90 \\
\hline 9.41 & -64.8 & -2.53 & -210.96 & -294.88 & 166.61 & 433.83 \\
\hline 9.46 & -64.5 & -2.54 & -213.17 & -296.49 & 170.59 & 446.49 \\
\hline 9.51 & -64.2 & -2.55 & -215.25 & -297.90 & 174.79 & 459.90 \\
\hline 9.56 & -63.9 & -2.56 & -217.47 & -299.25 & 179.20 & 474.20 \\
\hline 9.61 & -63.5 & -2.57 & -219.68 & -300.39 & 183.86 & 489.49 \\
\hline 9.66 & -63.0 & -2.58 & -221.77 & -301.39 & 188.75 & 505.84 \\
\hline 9.71 & -62.7 & -2.59 & -224.18 & -302.47 & 194.03 & 523.71 \\
\hline 9.76 & -62.1 & -2.60 & -226.40 & -303.21 & 199.62 & 542.90 \\
\hline 9.81 & -61.5 & -2.61 & -228.61 & -304.21 & 205.52 & 563.08 \\
\hline 9.86 & -61.0 & -2.62 & -230.96 & -305.02 & 211.99 & 585.01 \\
\hline 9.91 & -60.3 & -2.64 & -233.18 & -306.16 & 218.75 & 608.43 \\
\hline 9.96 & -59.6 & -2.64 & -235.26 & -306.97 & 226.04 & 632.90 \\
\hline
\end{tabular}

